\title{
SYNTHESIS AND CHARACTERISATION OF COPPER (I) OXIDE AND ZINC OXIDE QUANTUM DOTS \\ AND \\ DEVELOPMENT OF NEW FLUORESCENT FIBRE COMPOSITES
}

By

Andreas Josef Zeller

A thesis

submitted to the Victoria University of Wellington in fulfilment of the requirements for the degree of Doctor of Philosophy in Chemistry 



\section{ABSTRACT}

The use of nanotechnology in the fabrication of everyday products has increased significantly in the last years. Materials prepared in the form of very small particles, at nano-scale, change their physical and chemical properties and differ significantly from their bulk form. These new, size-dependent properties can improve and enhance the properties and functionality of different materials. Nanotechnology, however, is a relatively new science and many of its potential applications are just being explored.

This thesis deals with the development and characterisation of new fluorescent hybrid materials made of quantum dots and NZ wool and paper fibres for potential use in fabrics, and packaging and labelling papers, respectively. To develop these hybrid materials, the metal oxide semiconductors $\mathrm{Cu}_{2} \mathrm{O}$ and $\mathrm{ZnO}$ were chosen because they can easily be synthesised using aqueous solution and sol-gel methods, and they are likely to be compatible with human skin avoiding negative reactions such as skin irritation. Fluorescence of these quantum dots was tuned by varying the particle size of the quantum dot (controlled by changes in process parameters such as reaction concentrations, temperature, time, $\mathrm{pH}$ and use of stabilizer agent) or by introducing small quantities of dopants into the crystal lattice of the quantum dot material.

$\mathrm{Cu}_{2} \mathrm{O}$ quantum dots were synthesised after developing a wet chemical reduction method. The method allows the controlled formation of cubic shaped $\mathrm{Cu}_{2} \mathrm{O}$ nanocrystals in aqueous solution. TEM images revealed that these nanocubes were formed by an assemblage of small spherical nanocrystals $(5 \mathrm{~nm})$ with a super lattice structure. This arrangement has a strong effect on the light absorption and light scattering properties of the $\mathrm{Cu}_{2} \mathrm{O}$ suspension. Suspensions containing mainly large polycrystalline nanocubes showed a dichroic effect; the suspension was yellow-green in reflected light and redpurple and transparent in transmitted light. This phenomenon is caused by Surface Plasmon Resonance (SPR). Thereby, the size of the nanocubes and the intermolecular interaction between the nanocubes determined the wavelength (range) of the adsorbed and scattered light and the ratio of light absorption and light scattering. Fluorescence of these quantum dots was of very low intensity and therefore not suitable for the development of hybrids materials. 
A sol-gel method was developed to prepare $\mathrm{ZnO}$ quantum dots. This method produced polycrystalline particles in the shape of round rice grains of $100 \mathrm{~nm}$ and $1 \mu \mathrm{m}$ in the size. TEM images revealed that these polycrystalline particles consist of small spherical and ellipsoidal nanocrystals 10 - $12 \mathrm{~nm}$. The ZnO suspension was white under ambient light and yellow-orange under UV light. When excited with UV-light $\left(\lambda_{\text {exc }}=260 \mathrm{~nm}\right)$, the $\mathrm{ZnO}$ suspension showed two distinct emission peaks in the UV ( $380 \mathrm{~nm})$ and visible range $(\sim 560 \mathrm{~nm})$.

To develop quantum dots -fibre composites, the properties of the $\mathrm{ZnO}$ quantum dots were combined with the bulk properties of NZ merino wool and paper fibre substrates. This was achieved by using the in-situ and building block approaches. In the in-situ approach, quantum dots were formed in the chemical surrounding of the substrate material. This method relied on cross-link reactions between the quantum dots and wool fibre during nucleation and particle growth. On the other hand, in the building block approach, quantum dots were directly attached to the substrate material or with the use of linker molecules. To ensure long-life stability of the composite material this approach relies on the creation of strong chemical bonding between both components. Different processing strategies were conducted to improve the chemical bonding between the inorganic and organic phase. This was achieved by modifying the interface between both components either by functionalising the surface area of the substrate material or exchanging functional groups at the quantum dot surface. The building block and in-situ approach produced $\mathrm{ZnO}$ quantum dot - wool composites with a sharp emission peak in the UV range. This emission peak (c. $380 \mathrm{~nm}$ ) referred to the band-edge emission of the $\mathrm{ZnO}$ quantum dots. The attachment of ZnO quantum dots to bleached Kraft paper did not result in the functionalisation of the paper fibre with fluorescence; however, the fibres were covered with a surface layer that exhibit UV filtering properties.

The use of 8-Hydroxyquinoline (8-HQ) to obtain fluorescence in the visible range of the spectrum was also explored here. 8-HQ was used as a fluorescent chelate agent for zinc cations at the $\mathrm{ZnO}$ quantum dot surface. $\mathrm{ZnO}$ quantum dots functionalised with 8-HQ conferred the wool and paper fibre with strong fluorescence in the visible range. Depending on the concentration of 8-HQ and other parameters (e.g. choice of solvent, soaking temperature and time) the fluorescence of $\mathrm{ZnO}$ quantum dot - wool and paper composites were tuned from white-light green to yellow-green. 
An alternative procedure for the development of fluorescent wool fibre composites was also developed. Wool was functionalised by the uptake of zinc and 8-HQ in two separate batch processes. 8-HQ is bi-dentate chelating agent and forms with zinc cations a planar coordination complex, chelate. This complex formation creates fluorescence in the blue and green region of the visible spectrum. Two methods, which differed in the order of uptake of the precursor material onto the wool, were used to dye the wool. The optical fluorescence of the wool fibre was tuned between white-bluish, white, whitegreenish and green-yellowish by modifying the dyeing bath conditions used (e.g. concentration of 8-HQ and choice of solvent).

Fluorescent composite materials have numerous potential applications and further research is necessary to optimise and develop preparation procedures. Composite materials comprising quantum dots and wool and paper were developed as part of this thesis. However, it is evident from this study that the synthesis of metal oxide quantum dots with intense, stable, spectroscopically pure fluorescent colours is difficult to achieve. This is because the synthesis of size and shaped controlled metal oxides at nanoscale is challenging and can be affected by agglomeration reactions in aqueous solution. Furthermore, it should be kept in mind that chemical interaction between quantum dots and organic substrate material can create different non-radiative energy transfer processes which will quench the fluorescence of the quantum dots. Finally, future studies should focus on developing methods to successfully incorporate dopants inside the lattice structure of metal oxide nanoparticles to obtain and tune the fluorescence at different wavelengths and hence colours in the visible range. 


\section{ACKNOWLEDGEMENTS}

I would like to thank my supervisors, Professor James H. Johnston and Dr. Thomas Borrmann for all their support over the course of my Ph.D.

My sincere thanks also go to all members of LB 107 and the numerous people I met in New Zealand. I am extremely grateful to all of them for their friendship and support. This made my time in New Zealand feel like a great adventure.

My thanks are also due to all the staff of the School of Chemical and Physical Sciences, especially David Flynn for his help with the electron microscope. Thanks to everybody who has made my time at university an enjoyable experience.

I would like to thank my family for their love and help during my time overseas working on this thesis. I also would like to thank Carlos Lehnebach for his support and advice and just for being there for me through the good and bad times of writing my thesis.

Finally I would like to acknowledge the financial support for this work provided to Prof. J. H. Johnston, New Zealand FRST NERF Quantum Dot - Fibre Composites Grant. The Victoria University Submission Scholarship is also gratefully acknowledged. 


\section{TABLE OF CONTENTS}

\section{CHAPTER 1 - INTRODUCTION}

$\begin{array}{lll}1.1 & \text { Hybrid materials; general background } & 1\end{array}$

1.2 Fluorophores 4

1.1.1 Organic fluorophores 4

$\begin{array}{ll}\text { 1.1.2 Coordination complexes } & 9\end{array}$

$\begin{array}{ll}\text { 1.1.3 Quantum dots } & 11\end{array}$

1.3 Quantum dot - fibre composites $\quad 24$

$\begin{array}{ll}\text { 1.3.1 Choice of the quantum dot material } & 24\end{array}$

$\begin{array}{ll}\text { 1.3.2 Synthesis of quantum dots } & 25\end{array}$

1.3.3 Choice of fibrous substrate material 33

$\begin{array}{lll}\text { 1.3.3.1 } & \text { Merino wool } & 34\end{array}$

1.3.3.2 Paper fibre 38

1.3.4 Theoretical background for the development of quantum dots-fibre composites 41

1.3.4.1 Building block approach 41

$\begin{array}{lll}\text { 1.3.4.2 In-situ approach } & 43\end{array}$

$\begin{array}{lll}1.4 & \text { Research goals } & 45\end{array}$

CHAPTER 2 - EXPERIMENTAL PROCEDURE $\quad 50$

2.1 Synthesis of quantum dots

$\begin{array}{lll}2.1 .1 & \text { Experimental setup } & 51\end{array}$

2.1.2 Materials used for the synthesis of quantum dots 53

$\begin{array}{lll}\text { 2.1.2.1 Capping agents } & 53\end{array}$

2.1.2.2 Copper (I) oxide $\quad 54$

2.1.2.3 Zinc oxide $\quad 55$

2.1.2.4 Zinc sulphide 55

2.1.2.5 Dopants used for doping of quantum dots 56

2.1.3 Methods used to produce quantum dots 56

2.1.3.1 Chemical reduction method - Copper (I) oxide nanocrystals 56

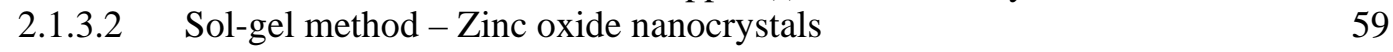

$\begin{array}{lll}\text { 2.1.3.3 Precipitation method - Zinc sulphide nanocrystals } & 61\end{array}$

2.2 Methods used to prepare quantum dot - fibre composites 63

2.2.1 In-situ binding of $\mathrm{ZnO}$ quantum dots to wool 63

2.2.2 Building block approach for $\mathrm{ZnO}$ quantum dot - fibre composites 64

2.2.2.1 Merino wool and surface treatment methods 64

2.2.2.1.1 Alcoholic alkali treatment $\quad 65$

2.2.2.1.2 Acid treatment $\quad 65$

2.2.2.1.3 Mercaptosuccinic acid treatment 65

2.2.2.1.4 8-Hydroxyquinoline treatment 65

2.2.2.2 Kraft paper fibres and pre-treatment methods 66

2.2.2.2.1 Mercaptosuccinic acid treatment 66

2.2.2.2.2 Washing procedure of paper composite materials 67

2.2.2.3 Attachment of $\mathrm{ZnO}$ quantum dots onto wool 68

2.2.2.4 Attachment of $\mathrm{ZnO}$ quantum dots to paper fibre 68 
$2.3 \quad$ In-situ dyeing of wool using the fluorescent chelate $\mathrm{Zn}(8-\mathrm{Q})_{2} \quad 69$

2.3.1 Materials used for the complex formation of $\mathrm{Zn}(8-\mathrm{Q})_{2}$ chelate $\quad 70$

$\begin{array}{lll}\text { 2.3.2 Preparation route } & 70\end{array}$

$\begin{array}{lll}\text { 2.3.3 } \mathrm{Zn}^{2+} \text { uptake on wool } & 71\end{array}$

$\begin{array}{lll}\text { 2.3.4 } & \text { 8-HQ uptake on wool } & 72\end{array}$

2.3.5 8-HQ uptake on zinc functionalised wool $\quad 74$

2.3.6 Zinc uptake on 8-HQ functionalised wool 75

$2.4 \quad$ Characterisation methods $\quad \mathbf{7 6}$

$\begin{array}{lll}\text { 2.4.1 Particle size and morphology analysis } & 76\end{array}$

2.4.1.1 Zetasizer $\quad 76$

2.4.1.2 Scanning Electron Microscope (SEM) 76

2.4.1.3 Transmission Electron Microscope (TEM) 77

$\begin{array}{lll}\text { 2.4.2 Chemical composition } & 77\end{array}$

$\begin{array}{lll}\text { 2.4.2.1 Energy dispersive X-ray spectroscopy (EDS) } & 77\end{array}$

$\begin{array}{lll}\text { 2.4.2.2 X-ray photoelectron spectroscopy (XPS) } & 78\end{array}$

$\begin{array}{lll}\text { 2.4.2.3 X-ray diffraction (XRD) } & 78\end{array}$

$\begin{array}{lll}2.4 .3 & \text { Spectroscopy } & 79\end{array}$

$\begin{array}{lll}\text { 2.4.3.1 Fluorescence spectroscopy } & 79\end{array}$

$\begin{array}{lll}\text { 2.4.3.2 UV-Visible spectroscopy } & 79\end{array}$

2.4.4 Anti-microbial testing $\quad 80$

CHAPTER 3 - SYNTHESIS AND CHARACTERISATION OF COPPER (I) OXIDE QUANTUM DOTS 81

3.1 Introduction; $\mathrm{Cu}_{2} \mathrm{O}$ quantum dots $\quad 82$

$\begin{array}{lll}3.1 .1 & \text { Chemical reduction method } & 83\end{array}$

3.1.2 Effect of capping agents on particle growth 84

$\begin{array}{llr}3.2 & \text { Preparation of } \mathrm{Cu}_{2} \mathrm{O} & \mathbf{8 8}\end{array}$

$\begin{array}{llr}3.3 & \text { Results } & \mathbf{8 9}\end{array}$

$\begin{array}{lll}\text { 3.3.1 Chemical composition } & 92\end{array}$

$\begin{array}{lll}\text { 3.3.1.1 } & \text { EDS mapping } & 92\end{array}$

3.3.1.2 X-ray diffraction spectroscopy (XRD) 93

3.3.1.3 X-ray photoelectron spectroscopy 93

3.3.2 Morphology and size 95

3.3.2.1 Transmission Electron Microscope (TEM) 95

$\begin{array}{llr}\text { 3.3.2.2 Scanning Electron Microscope (SEM) } & 97\end{array}$

$\begin{array}{lll}\text { 3.3.2.3 Zetasizer } & 101\end{array}$

$\begin{array}{lll}\text { 3.3.3 Spectroscopy } & 105\end{array}$

$\begin{array}{lll}\text { 3.3.3.1 UV-vis spectroscopy of } \mathrm{Cu}_{2} \mathrm{O} & 105\end{array}$

$\begin{array}{lll}\text { 3.3.3.2 UV-vis spectroscopy of } \mathrm{Cu}_{2} \mathrm{O} / \mathrm{Zn} & 111\end{array}$

$\begin{array}{lll}\text { 3.3.3.3 Photoluminescence spectroscopy } & 116\end{array}$

$\begin{array}{lll}3.4 & \text { Discussion } & 117\end{array}$

3.4.1 Mechanism of particle growth - Wet chemical reduction method 118

$\begin{array}{ll}\text { 3.4.1.1 Nucleation and Condensation } & 118\end{array}$

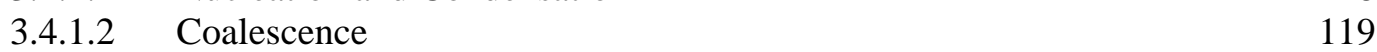

$\begin{array}{ll}\text { 3.4.1.3 Self-assembled agglomeration } & 121\end{array}$

$\begin{array}{ll}\text { 3.4.2 Effect of process parameters on particle growth } & 124\end{array}$

$\begin{array}{lll}\text { 3.4.2.1 } & \mathrm{NaBH}_{4} \text { concentration } & 125\end{array}$

$\begin{array}{ll}\text { 3.4.2.2 Trisodium citrate concentration } & 126\end{array}$ 
$\begin{array}{lll}\text { 3.4.2.3 } & \text { Reaction time } & 128\end{array}$

$\begin{array}{lll}3.4 .2 .4 & \text { Reaction temperature } & 129\end{array}$

$\begin{array}{lll}3.4 .2 .5 & \text { Effect of } \mathrm{pH} & 129\end{array}$

3.4.2.6 The choice of the Cu precursor material 130

3.4.2.7 The choice of the polymeric dispersant 131

$\begin{array}{lll}\text { 3.4.3 Optical properties } & 133\end{array}$

$\begin{array}{lll}\text { 3.4.3.1 Surface Plasmon Resoance } & 135\end{array}$

$\begin{array}{ll}\text { 3.4.4 Doping procedure } & 139\end{array}$

$\begin{array}{lll}3.5 & \text { Conclusions } & 142\end{array}$

\section{CHAPTER 4 - SYNTHESIS AND CHARACTERISATION OF ZINC OXIDE QUANTUM DOTS}

4.1 Introduction; ZnO quantum dots 145

$\begin{array}{lll}\text { 4.1.1 Sol-gel method } & 146\end{array}$

$\begin{array}{lll}\text { 4.1.2 } & \text { Forced alkaline hydrolysis } & 147\end{array}$

$\begin{array}{lll}4.2 & \text { Preparation of } \mathrm{ZnO} & 151\end{array}$

$\begin{array}{lll}4.3 & \text { Results } & 152\end{array}$

4.3.1 Size and morphology 153

4.3.1.1 Electron microscopy - TEM 153

4.3.1.2 Electron microscopy - SEM 154

$\begin{array}{lll}\text { 4.3.1.3 Zetasizer } & 157\end{array}$

$\begin{array}{lll}\text { 4.3.3 Optical properties } & 158\end{array}$

4.3.3.1 UV-Visible spectroscopy 158

$\begin{array}{lll}\text { 4.3.3.2 } & \text { Fluorescence spectroscopy } & 159\end{array}$

$\begin{array}{lll}\text { 4.4 Discussion } & \mathbf{1 6 2}\end{array}$

$\begin{array}{lll}\text { 4.4.1 Different steps of the particle growth } & 162\end{array}$

4.4.1.1 Forced alkaline hydrolysis 163

4.4.1.2 Confinement of particle growth 166

4.4.1.3 Formation of self-assembled structures 167

$\begin{array}{ll}\text { 4.4.2 Optical properties } & 171\end{array}$

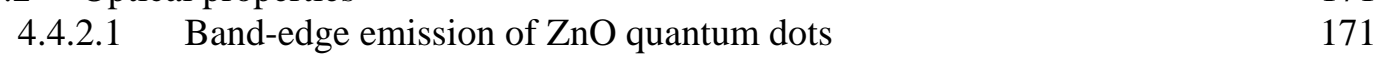

4.4.2.2 Defect emission of $\mathrm{ZnO}$ quantum dots 172

$\begin{array}{ll}\text { 4.4.3 Effect of process parameters on optical properties } & 175\end{array}$

$\begin{array}{lll}\text { 4.4.3.1 PVP and PMAA-Na } & 176\end{array}$

4.4.3.2 Uptake of polymeric surfactant 176

4.4.3.3 Effect of $\mathrm{NaBH}_{4}$ concentration on the defect emission 178

4.4.3.4 Effect of temperature on the defect emission 180

4.4.3.5 Doping of $\mathrm{ZnO}$ quantum dots 182

$\begin{array}{lll}4.5 & \text { Conclusions } & 183\end{array}$ 
CHAPTER 5 - CO-PRECIPITATION OF METALLIC NANOCLUSTERS WITH ZINC SULPHIDE

$\begin{array}{lll}5.1 & \text { Introduction; Co-precipitation method } & 186\end{array}$

$\begin{array}{llr}5.2 & \text { Methods } & 187\end{array}$

$\begin{array}{lll}5.3 & \text { Results } & 187\end{array}$

$\begin{array}{lll}5.4 & \text { Discussion } & 189\end{array}$

$\begin{array}{lll}5.5 & \text { Conclusions } & 190\end{array}$

\section{CHAPTER 6 - DEVELOPMENT OF ZINC OXIDE QUANTUM DOT - FIBRE COMPOSITES}

6.1 Introduction; Quantum dot - fibre composites 192

$\begin{array}{ll}\text { 6.1.1 Choice of quantum dot material } & 195\end{array}$

6.2 Methods 196

6.2.1 In-situ binding of $\mathrm{ZnO}$ quantum dots to wool 196

6.2.2 Building block approach 198

6.2.2.1 Surface modification of $\mathrm{ZnO}$ quantum dots 200

6.2.2.2 Merino wool and pre-treatment methods 201

6.2.2.3 Kraft paper fibres and pre-treatment methods 204

$\begin{array}{lll}6.3 & \text { Results } & 204\end{array}$

6.3.1 In-situ binding of $\mathrm{ZnO}$ quantum dot onto wool 204

$\begin{array}{ll}\text { 6.3.2 Building block approach } & 205\end{array}$

6.3.2.1 Attachment of ZnO quantum dot onto wool and pre-treated with

$\mathrm{NaOH}$ and $\mathrm{HCl}$

6.3.2.2 Attachment of $\mathrm{ZnO}$ quantum dot onto wool functionalised with
linker molecules

6.3.2.3 Surface modification of $\mathrm{ZnO}$ quantum dots and attachment on wool 208

6.3.2.4 ZnO quantum dot - paper fibre composites 211

$\begin{array}{lll}\text { 6.4 Discussion } & \mathbf{2 1 4}\end{array}$

$\begin{array}{lll}\text { 6.4.1 ZnO quantum dot - wool fibre composites } & 215\end{array}$

6.4.1.1 In-situ binding of $\mathrm{ZnO}$ quantum dots to wool 215

$\begin{array}{ll}\text { 6.4.1.2 Building block approach } & 217\end{array}$

6.4.1.2.1 Attachment of $\mathrm{ZnO}$ quantum dot onto wool 217

6.4.1.2.2 Attachment of $\mathrm{ZnO}$ quantum dot onto wool using linker molecules $\quad 218$

6.4.1.2.3 Attachment of $\mathrm{ZnO}$ quantum dot onto wool using 8-HQ 220

6.4.2 ZnO quantum dot - paper fibre composites 223

6.4.3 Different factors affecting the fluorescence efficiency of fibre composites
functionalised with ZnO quantum dots and 8-HQ

6.4.4 Anti-microbial coating of wool with $\mathrm{ZnO} 226$

$\begin{array}{lll}6.5 & \text { Conclusions } & 226\end{array}$ 


\section{CHAPTER 7 - DYEING WOOL WITH ZINC AND 8-HQ USING IN-SITU APPROACH}

\subsection{Introduction; Fluorescent chelates}

7.1.1 Use of fluorescent chelates for dyeing fibrous substrate materials 234

7.1.1.1 Soaking fibrous substrate in chelate solution 235

7.1.1.2 In-situ dyeing approach 236

$\begin{array}{lll}\text { 7.1.2 Choice of fluorescent chelate - Zn(8-Q) } 236 & 236\end{array}$

$\begin{array}{lll}7.2 & \text { Methods } & 238\end{array}$

$\begin{array}{lll}\text { 7.2.1 Preparation of } \mathrm{Zn}(8-\mathrm{Q})_{2} \text { chelate solution } & 238\end{array}$

7.2.2 Dyeing of wool fibre with $\mathrm{Zn}(8-\mathrm{Q})_{2}$ using in-situ approach 238

7.2.2.1 In-situ method 1- Zinc uptake onto 8-HQ-wool 239

7.2.2.2 In-situ method 2-8-HQ uptake onto zinc-wool 240

$\begin{array}{lll}\text { 7.2.2.3 Process parameters } & 242\end{array}$

7.2.2.3.1 Uptake of 8-HQ onto wool 243

7.2.2.3.2 Uptake of $\mathrm{Zn}^{2+}$ onto 8-HQ-wool 243

7.2.2.3.3 Uptake of $\mathrm{Zn}^{2+}$ onto wool 244

7.2.2.3.4 Uptake of 8-HQ onto Zn-wool in EtOH solution 245

7.2.2.3.5 Uptake of 8-HQ onto Zn-wool in gas atmosphere 245

$\begin{array}{lll}7.3 & \text { Results } & \mathbf{2 4 6}\end{array}$

$\begin{array}{lll}\text { 7.3.1 Preparation of Zn(8-Q) })_{2} \text { chelate solution } & 246\end{array}$

7.3.1.1 $\mathrm{Zn}(8-\mathrm{Q})_{2}$ in EtOH solution $\quad 246$

7.3.1.2 $\mathrm{Zn}(8-\mathrm{Q})_{2}$ in $\mathrm{H}_{2} \mathrm{O}$ solution $\quad 250$

$\begin{array}{ll}\text { 7.3.2 Dyeing of wool fibre using in-situ method } 1 & 251\end{array}$

7.3.2.1 Uptake of 8-HQ (EtOH) onto wool 252

7.3.2.2 Uptake of 8-HQ $\left(\mathrm{H}_{2} \mathrm{O}\right)$ onto wool 255

7.3.2.3 Uptake of $\mathrm{ZnCl}_{2}(\mathrm{EtOH})$ onto 8-HQ-wool 258

$\begin{array}{lll}\text { 7.3.3 Dyeing of wool fibre using in-situ method } 2 & 261\end{array}$

$\begin{array}{lll}\text { 7.3.3.1 Uptake of zinc onto wool } & 261\end{array}$

7.3.3.2 Uptake of 8-HQ (EtOH) onto $\mathrm{ZnCl}_{2}(\mathrm{EtOH})$ doped wool 263

7.3.3.3 Uptake of 8-HQ (EtOH) onto $\mathrm{ZnCl}_{2}(\mathrm{H} 2 \mathrm{O})$ doped wool 266

7.3.3.4 Uptake of 8-HQ (EtOH) onto $\mathrm{Zn}(\mathrm{Ac})_{2}$ (H2O) doped wool 268

7.3.3.5 Uptake of 8-HQ onto zinc doped wool in a gas atmosphere 272

$\begin{array}{ll}\text { 7.3.4 Process parameters of in-situ dyeing method } 2 & 274\end{array}$

$\begin{array}{lll}\text { 7.3.4.1 Choice of zinc precursor material; } \mathrm{ZnCl}_{2} \text { and } \mathrm{Zn}(\mathrm{Ac})_{2} & 274\end{array}$

7.3.4.2 Effect of $\mathrm{H}_{2} \mathrm{O}$ added to 8-HQ (EtOH) soaking solution 278

7.3.4.3 Uptake of 8-HQ onto zinc doped wool in water solution 280

\subsection{Discussion}

$\begin{array}{lll}\text { 7.4.1 Preparation of } \mathrm{Zn}(8-\mathrm{Q})_{2} \text { chelate solution } & 283\end{array}$

7.4.1.1 Preparation of $\mathrm{Zn}(8-\mathrm{Q})_{2}$ chelate EtOH solution 283

7.4.1.2 Preparation of $\mathrm{Zn}(8-\mathrm{Q})_{2}$ chelate aqueous solution 286

7.4.2 Dyeing wool with Zn(8-Q $)_{2} \cdot 2 \mathrm{H}_{2} \mathrm{O}$ using in-situ approach 289

$\begin{array}{lll}\text { 7.4.2.1 In-situ method } 1 & 292\end{array}$

7.4.2.2 In-situ method $2 \quad 293$

$\begin{array}{lll}\text { 7.4.2.3 In-situ mehtod } 3 & 294\end{array}$

7.4.3 Process parameters of in-situ dyeing method $1 \quad 296$

7.4.3.1 Effect of 8-HQ concentration onto fluorescence properties 296

7.4.3.2 Effect of water during the uptake of 8-HQ onto wool 298

7.4.4 Process parameters of in-situ dyeing method $2 \quad 299$

7.4.4.1 Effect of 8-HQ concentration onto fluorescence properties 299

7.4.4.2 Effect of water during the uptake of 8-HQ onto zinc-wool 302

7.4.4.3 Effect of drying after dyeing process 
7.4.4.4 Effect of the zinc precursor material onto 8-HQ uptake 303

7.4.4.5 Pre-treatment of wool fibre 307

7.4.5 Ageing processes and sun light bleaching 309

7.4.6 Anti-microbial activity of wool dyed with Zn(8-Q) 2

$\begin{array}{llr}7.5 & \text { Conclusions } & 311\end{array}$

CHAPTER 8 - CONCLUDING REMARKS $\quad 315$

$\begin{array}{ll}\text { REFERENCES } & 321\end{array}$

$\begin{array}{ll}\text { APPENDIX - LIST OF SAMPLES } & 328\end{array}$ 


\section{LIST OF FIGURES}

Figure 1.1: Church window glass where the red colour originates from the incorporation of gold nano-particles.

Figure 1.2: Colour variation of nanogold wool fibre composites sorted by the uptake of gold nanoparticles [4].

Figure 1.3: $\quad$ Resonance structure of cyanine.

Figure 1.4: Molecular orbital diagram for the $\pi$ - $M O$ of a cyanine molecule and graphical illustration of the electronic transition of a $\pi$ - electron from HOMO to LUMO ( $\psi_{n}=$ molecular orbital wave function).

Figure 1.5: $\quad$ MO-diagrams of the five d- orbitals splitting of a $\mathrm{d}^{6}$ metal in an octahedral field.

Figure 1.6: $\quad$ Electronic band structure of a macroscopic semiconductor crystal showing continuous bands of energy formed by the overlap of orbital's from adjacent atoms.

Figure 1.7: Electronic band structure of bulk semiconductor and nanometric semiconductor crystals [30].

Figure 1.8: Illustration of the relationship between light emission and band gap energy. Arrow 1: jump of the electron over the band gap by the adsorption of energy through UV irradiation or thermal excitation; Arrow 2: fall of the excited electron to the LUMO level through different heat-emitting steps; Arrow 3: radiative recombination of the electron-hole pair between the $L U M O$ and HOMO level.

Figure 1.9: Quantum dots of CdSe arranged by size emitting light in different colours. The quantum dot suspensions have been excited simultaneously by a single excitation wavelength [copyright NASA, USA].

Figure 1.10: Energy-momentum dispersion of a direct band gap (right) and indirect band gap (left). $E(k)_{\text {Номо }}$ represents the energy maximum of the HOMO energy state; $E(k)_{L U M O}$ represents the energy minimum of the LUMO energy state.

Figure 1.11: Band structures of a n-type (A) and p-type (B) semiconductor. Semiconductor A: donor impurities create energy states within the band gap near the conduction band. Semiconductor B: acceptor impurities create energy states within the band gap near the valence band.

Figure 1.12: Graphic illustration of the solvation effect (solvatochromic effect) at the energy states $E_{L U M O}$ and $E_{\text {Номо }}$, depending on the polarity of the solvent used (polar and non-polar, respectively).

Figure 1.13: Different bonding characteristics between particle surfaces and capping agents [52].

Figure 1.14: Diversity of chemical bonds found in wool fibre [53].

Figure 1.15: $\quad$ Physical structure of fine wool [53].

Figure 1.16: Schematic model of wool fibre composition. 
Figure 1.17: Illustration of wool fibre surface showing overlapping scales allowing intercellular diffusion for dyes [66].

Figure 1.18: Chemical composition of wood [55].

Figure 1.19: Chemical structure of cellulose $\left(\mathrm{C}_{6} \mathrm{H}_{10} \mathrm{O}_{5}\right)_{\mathrm{n}}$.

Figure 1.20: Average composition of softwoods and hardwoods [55].

Figure 1.21: SEM image of paper fibres with fines (A, white arrow) produced by the RMP method and SEM image of “clean” fibres (B) produced by the Kraft method [55].

Figure 1.22: Schematic illustration of the development of new hybrid materials using the building block approach.

Figure 1.23: Schematic illustration of the development of new hybrid materials using the in-situ approach.

Figure 2.1: $\quad$ Flow chart for the synthesis of quantum dots and development of fluorescent composite materials.

Figure 2.2: Experimental setup for the synthesis of quantum dots carried out in a fume hood.

Figure 3.1: Formula of trisodium citrate - ionic capping agent (A) and PMAA-Na polymeric dispersant (B).

Figure 3.2: Schematic representation of particle growth by coalescence of small nanoclusters (A) and by surface reduction after nucleation and condensation (B).

Figure 3.3: $\quad$ Final colour of the $\mathrm{Cu}_{2} \mathrm{O}$ nanocrystal suspension.

Figure 3.4: Dichroic effect of $\mathrm{Cu}_{2} \mathrm{O}$ nanocrystals in transmitted light (right) and reflected light (left).

Figure 3.5: $\quad$ Lycurgus cup from $4^{\text {th }}$ century AD in reflected light (left) and illuminated with light from inside the cup (right) [Copy rights of British Museum].

Figure 3.6: Aqueous suspension of $\mathrm{Cu}_{2} \mathrm{O}$ nanocrystals doped with $\mathrm{Zn}^{2+}$ ions in reflected (left) and transmitted light (right).

Figure 3.7: $\quad \mathrm{Cu}_{2} \mathrm{O}$ nanocrystals doped with $\mathrm{Zn}^{2+}$ ions incorporated in hydroxyl-ethyl cellulose sheets. The sheets show an orange colour in reflected (left) and purple colour in transmitted light (right).

Figure 3.8: $\quad \mathrm{Cu}_{2} \mathrm{O}$ nanocubes of $70-250 \mathrm{~nm}$. TEM image shows these are regular and well-dispersed.

Figure 3.9: $\quad$ Energy dispersive X-ray spectroscopy (EDS) mapping of copper (left) and oxygen (right) of the nanocubes.

Figure 3.10: X-ray powder diffraction of $\mathrm{Cu}_{2} \mathrm{O}$ nanocrystals synthesised following the wet chemical reduction method. 
Figure 3.11: Binding energy of copper atoms in the cuprite crystal structure of $\mathrm{Cu}_{2} \mathrm{O}$ nanocrystals (A) formed with $0.3 \cdot 10^{-3} \mathrm{M}$ TSC and binding energy of copper atoms in a cupric oxide crystal structure of $\mathrm{CuO}$ nanocrystals (B) formed with $0.48 \cdot 10^{-3} \mathrm{M}$ TSC.

Figure 3.12: TEM image of regular shaped nanocubes obtained by the reduction of $\mathrm{Cu}\left(\mathrm{NO}_{3}\right)_{2}$ with $\mathrm{NaBH}_{4}$ in the presence of PMAA-Na.

Figure 3.13: TEM images of small colloidal $\mathrm{Cu}_{2} \mathrm{O}$ nanocrystals forming regular shaped nanocubes.

Figure 3.14: TEM images of colloidal $\mathrm{Cu}_{2} \mathrm{O}$ nano-crystals of $5-10 \mathrm{~nm}$ in diameter. Note superlattice structure encircled.

Figure 3.15: $\quad$ SEM and backs-scatter images of the preparation of $\mathrm{Cu}_{2} \mathrm{O}$ nanocrystals at 96 ${ }^{\circ} \mathrm{C}(\mathrm{A})$, with $\mathrm{CuCl}_{2}$ as copper precursor (B) and PVP as polymeric surfactant (C). Details of sample preparation are listed in the appendix in table A.2.

Figure 3.16: SEM and back-scatter image of $\mathrm{Cu}_{2} \mathrm{O}$ nanocrystals doped with zinc and nickel ions. Note that the doped $\mathrm{Cu}_{2} \mathrm{O}$ nanocrystals form larger pseudododecahedron shapes.

Figure 3.17: SEM images of $\mathrm{Cu}_{2} \mathrm{O}$ nanocrystals prepared according to the doping procedure without zinc (A) and with the addition of $\mathrm{Zn}^{2+}$ and $\mathrm{Ni}^{2+}$ and overheated in a silicone oil bath at $\sim 140{ }^{\circ} \mathrm{C}$ (B). Details of sample preparation are listed in the appendix in table A.3.

Figure 3.18: Size distribution of the $\mathrm{Cu}_{2} \mathrm{O}$ suspension (given in volume \%) prepared according to the standard procedure (green line) and diluted with water in a ratio of 1:5 (red line).

Figure 3.19: Size distribution of $\mathrm{Cu}_{2} \mathrm{O}$ nanocrystals doped with zinc and nickel (given in number \%) after several steps of dilution with water and centrifugations.

Figure 3.20: Size distribution of $\mathrm{Cu}_{2} \mathrm{O}$ suspension by number (A), volume (B) and intensity (C) given in \% prepared following the standard procedure with different concentration of $\mathrm{NaBH}_{4}$ (see table 3.3).

Figure 3.21: Absorbance scan of $\mathrm{Cu}_{2} \mathrm{O}$ samples with different concentration of $\mathrm{NaBH}_{4}$ listed in table 3.3. ${ }^{*} \mathrm{Cu}_{2} \mathrm{O}$ suspension prepared with $\mathrm{CuCl}_{2}$ as copper precursor material.

Figure 3.22: $\quad \mathrm{Cu}_{2} \mathrm{O}$ samples $\mathrm{C}, \mathrm{D}$ and $\mathrm{E}$ (listed in table 3.4) observed in transmitted light. Pictures were taken in a different angle to the transmitted light. Note reflection of the light is higher at the top photographs.

Figure 3.23: UV-vis scans of $\mathrm{Cu}_{2} \mathrm{O}$ prepared following the standard procedure without and with the addition of $0.25 \mathrm{~mL} 0.01 \mathrm{M} \mathrm{NaOH}$ after $\mathrm{NaBH}_{4}$ (blue line and red line, respectively).

Figure 3.24 Correlation between nanocubes size and their corresponding absorbance peak.

Figure 3.25: Absorbance scan of $\mathrm{Cu}_{2} \mathrm{O}$ samples doped with $\mathrm{Zn}^{2+}$ and $\mathrm{Ni}^{2+}$ carried out at 92, 94, 96, 98 and $99^{\circ} \mathrm{C}$. 
Figure 3.26: $\quad$ Reflectance scan of $\mathrm{Cu}_{2} \mathrm{O}$ samples doped with $\mathrm{Zn}^{2+}$ and $\mathrm{Ni}^{2+}$ carried out at 92, 94, 96, 98 and $99^{\circ} \mathrm{C}$.

Figure 3.27: Absorbance scan of $\mathrm{Cu}_{2} \mathrm{O}$ samples doped with $\mathrm{Zn}^{2+}, \mathrm{Zn}^{2+}$ and $\mathrm{Mn}^{2+}, \mathrm{Zn}^{2+}$ and $\mathrm{Co}^{2+}$ and using different stirring times. Details of sample preparation are listed in the appendix in table A.5.

Figure 3.28: Absorbance scan of $\mathrm{Cu}_{2} \mathrm{O}$ samples doped with $\mathrm{Zn}^{2+}$, and $\mathrm{Zn}^{2+} /$ Citrate, stirred for $30 \mathrm{~min}$. Details of sample preparation are listed in the appendix in table A.5.

Figure 3.29: Change of the optical properties of $\mathrm{Cu}_{2} \mathrm{O}$ nanocrystal suspension doped with zinc before and after the addition of $2 \mathrm{~mL} 0.001 \mathrm{M} \mathrm{Na}_{2} \mathrm{SO}_{3}$.

Figure 3.30: $\quad$ PL scans of $\mathrm{Cu}_{2} \mathrm{O}$ aqueous suspensions undoped (A) and doped with $\mathrm{Zn}^{2+}$ and $\mathrm{Ni}^{2+}$ (B and C; B shows the dichroic effect) $\left(\lambda_{\text {exc }}=300 \mathrm{~nm}\right.$; exc. / em. slit $=10 \mathrm{~nm})$.

Figure 3.31: Illustration of particle growth of spherical $\mathrm{Cu}_{2} \mathrm{O}$ nanocrystals with a superlattice structure.

Figure 3.32: Illustration of the $\mathrm{Cu}_{2} \mathrm{O}$ particle growth starting from mono-dispersed nanocrystals with a superlattice structure followed by an agglomeration processes to the transformation in regular cube-shaped particles.

Figure 3.33 Absorption and scattering of incident light at the surface of cube-shaped $\mathrm{Cu}_{2} \mathrm{O}$ nanocrystals.

Figure 3.34: Graphic illustration of the periodical displacement of the surface conduction band's electrons in spherical metal nanoparticles induced by a resonant incident electric field of incoming light [98].

Figure 4.1: $\quad$ Schematic illustration of the oxalation (A) and olation (B) condensation reactions for hydrolysed zinc ions.

Figure 4.2: $\quad$ Schematic illustration of the addition of a droplet of alkali to a metal salt solution causing a concentration gradient.

Figure 4.3: Optical fluorescence of $\mathrm{ZnO}$ nanocrystals suspended in water (left) and attached to the base of the plastic receptacle (right) $\left(\lambda_{\text {exc }}=254 \mathrm{~nm}\right)$.

Figure 4.4: $\quad$ TEM image of ZnO self-assembled structures (A) and detailed view of the small colloidal nanoclusters $(\mathrm{B})$ that form them.

Figure 4.5: $\quad$ TEM images of ellipsoidal $\mathrm{ZnO}$ nanoclusters adhering loosely together.

Figure 4.6: $\quad$ SEM images of $\mathrm{ZnO}$ nanoparticles formed with $\mathrm{NaBH}_{4}$ (A) and $\mathrm{LiBH}_{4}$ (B) as reducing and alkali agent, respectively. Details of sample preparation are listed in the appendix in table A.6.

Figure 4.7: High magnification SEM images of ZnO nanoparticles prepared with PVP (A), PMAA-Na (B) and 1:1 PVP / PMAA-NA (C). Details of sample preparation are listed in the appendix in table A.7.

Figure 4.8: $\quad$ Size distribution of $\mathrm{ZnO}$ nanocrystals (given in intensity / \%) after several steps of dilution with water and centrifugation; green curve corresponds to a dilution ratio of 1:5 and red curve to 1:10. 
Figure 4.9: $\quad$ Absorbance scan of $\mathrm{ZnO}$ nanocrystals undoped (blue line) and doped with $\mathrm{Ce}^{3+}$ (red line) in aqueous solution. Details of sample preparation are listed in the appendix in table A.8.

Figure 4.10: $\mathrm{PL}$ scan of $\mathrm{ZnO}$ suspension doped with $\mathrm{CeCl}_{3}$ (blue line) and undoped, measured right after preparation, 1 and 10 days of ageing $\left(\lambda_{\mathrm{exc}}=260 \mathrm{~nm}\right.$, exc. $/$ em. slit $=10 \mathrm{~nm}$ ).

Figure 4.11: Emission spectra of rice grain shaped $\mathrm{ZnO}$ nanocrystals in aqueous solution at different ageing stages and $\mathrm{ZnO}$ nanocrystals doped with $\mathrm{Ce}^{3+}$ (blue line) $\left(\lambda_{\text {exc }}=350 \mathrm{~nm}(\right.$ exc. $/$ em. slit $\left.=10 \mathrm{~nm})\right)$.

Figure 4.12: Effect of PVP concentration on the PL intensity of the maxima emission wavelength in the visible range $\left(\mathrm{T}=70^{\circ} \mathrm{C}\right)\left(\lambda_{\text {exc }}=350 \mathrm{~nm}\right.$; exc. $/ \mathrm{em}$. slit $=$ $10 \mathrm{~nm})$. Details of sample preparation are listed in the appendix in table A.9.

Figure 4.13: Effect of $\mathrm{NaBH}_{4}$ concentration on the PL intensity of the maxima emission wavelength in the visible range $\left(\mathrm{T}=70^{\circ} \mathrm{C}\right)\left(\lambda_{\text {exc }}=350 \mathrm{~nm}\right.$; exc. $/ \mathrm{em}$. slit $=$ $10 \mathrm{~nm})$. Details of sample preparation are listed in the appendix in table A.10.

Figure 4.14: Effect of temperature on the PL intensity of the maxima emission wavelength in the visible range $\left(\lambda_{\text {exc }}=350 \mathrm{~nm}\right.$; exc. $/$ em. slit $\left.=10 \mathrm{~nm}\right)$.

Figure 5.1: Nucleation centres (seeds) formed instantaneously after the addition of $\mathrm{NaBH}_{4}$. These can form nanoclusters which is promoted by stirring time or arrested into the chemical composition of $\mathrm{ZnS}$ quantum dots.

Figure 5.2: $\quad$ Fluorescence colours of $\mathrm{ZnS}$ quantum dot suspension obtained by the coprecipitation of $\mathrm{ZnO}(\mathrm{A}), \mathrm{Cu}(\mathrm{B}), \mathrm{Mn}(\mathrm{C})$ and $\mathrm{Mn} / \mathrm{Ni}(\mathrm{D})$ nanoclusters ( $\lambda_{\text {exc }}$ $=256 \mathrm{~nm})$. Details of sample preparation are listed in the appendix in table A.11.

Figure 5.3: $\quad$ PL scans of ZnS quantum dot suspensions precipitated from solution in the presence of $\mathrm{ZnO}(\mathrm{A}), \mathrm{Cu}(\mathrm{B}), \mathrm{Mn}(\mathrm{C})$ and $\mathrm{Mn} / \mathrm{Ni}(\mathrm{D})$ nanoclusters ( $\lambda_{\text {exc }}=$ $260 \mathrm{~nm}$, slit $=5 \mathrm{~nm}$ ).

Figure 5.4: Electron-hole recombination in $\mathrm{ZnS}: \mathrm{Cu}^{2+}$ quantum dots with intermediate green fluorescence.

Figure 5.5: Electron-hole recombination in $\mathrm{ZnS}: \mathrm{Mn}^{2+}$ quantum dots with intermediate orange fluorescence.

Figure 6.1: Schematic illustration of the development of new hybrid materials comprising quantum dots and wool and paper fibres.

Figure 6.2: $\quad$ Chemical structure of mercaptosuccinic acid.

Figure 6.3: $\quad$ Chemical structure of L-cysteine.

Figure 6.4: $\quad$ Tautomerization of 8-Hydroxyquinoline.

Figure 6.5: $\quad$ Fluorescence scan of $\mathrm{ZnO}$ quantum dot - wool composite prepared using the in-situ approach and different capping agents $\left(\lambda_{\text {exc }}=260 \mathrm{~nm}\right.$, exc. $/$ em. slit $=$ $5 \mathrm{~nm})$. Details of sample preparation are listed in the appendix in table A.12. 
Figure 6.6: Fluorescence scans of $\mathrm{ZnO}$ quantum dot - wool composited prepared using the building block approach and the effect of 3, 5, 7 and 9 days ageing on the fluorescence scans $\left(\lambda_{\text {exc }}=260 \mathrm{~nm}\right.$, exc. $/$ em. slit $\left.=5 \mathrm{~nm}\right)$. Details of the sample preparation are listed in the appendix in table A.13.

Figure 6.7: Attachment of $\mathrm{ZnO}$ quantum dots to wool using 8-HQ as linker molecule and fluorescent coordination ligand $(\lambda$ exc $=260 \mathrm{~nm}$; exc. $/$ em. slit $=5 \mathrm{~nm})$. Details of sample preparation are listed in the appendix in table A.14.

Figure 6.8: Different fluorescence colours of $\mathrm{ZnO}$ quantum dots under UV light $\left(\lambda_{\text {exc }}=\right.$ $366 \mathrm{~nm}$ ). A: ZnO quantum dots functionalised with 18-MEA (fatty acid monolayer of the wool fibre); $\mathrm{B}$ : ZnO quantum dots functionalised with 8HQ and C: ZnO quantum dots coated with PVP.

Figure 6.9: Fluorescence scan of $\mathrm{ZnO}$ quantum dot suspensions functionalised with MEA (A), 8-HQ (B) and PVP (C). A: 0.5 g merino soaked into $75 \mathrm{~mL}$ ZnO aqueous suspension + $0.5 \mathrm{~mL} 0.5 \mathrm{M}$ MSA. B: $75 \mathrm{~mL} \mathrm{ZnO}+0.5 \mathrm{~mL} 0.025$ M 8-HQ (EtOH). C: ZnO quantum dot suspension. $\left(\lambda_{\text {exc }}=260 \mathrm{~nm}\right.$, exc $/ \mathrm{em}$. slit $=10 \mathrm{~nm}$ ). Details of sample preparation are listed in the appendix in table A.15.

Figure 6.10: Fluorescence scan of $0.1 \mathrm{~g} \mathrm{ZnO}$ quantum dot wool fibre soaked in $20 \mathrm{~mL}$ $1.25 \cdot 10^{-3} \mathrm{M} 8-\mathrm{HQ}(\mathrm{EtOH})$ at $50^{\circ} \mathrm{C}$ for 3 days (red) and aged under ambient atmosphere at room temperature for 15 days (green) and untreated wool (blue) $\left(\lambda_{\text {exc }}=260 \mathrm{~nm}\right.$, exc /em. slit $=5 \mathrm{~nm}$.) Details of sample preparation are listed in the appendix in table A.16.

Figure 6.11: ZnO-paper fibre aqueous suspension functionalised with 8 -HQ $(+1 \mathrm{~mL}$ $0.025 \mathrm{M} 8$ 8-HQ at $70{ }^{\circ} \mathrm{C}$ ) in aqueous suspension (right) and dried (left) observed under UV-light $\left(\lambda_{\text {exc }}=254 \mathrm{~nm}\right)$.

Figure 6.12: $\quad$ Paper fibre functionalised with $\mathrm{ZnO}$ quantum dots (A) and $\mathrm{ZnO}$-paper fibre composites functionalised with different concentration of 8-HQ (B, C and D) under UV light (top, $\lambda_{\text {exc }}=254 \mathrm{~nm}$ ) and ambient light (bottom). Details of sample preparation are listed in the appendix in table A.17.

Figure 6.13: PL scan of bleached Kraft paper (blue line), ZnO quantum dot - paper composite (A, red line) and $\mathrm{ZnO}$ quantum dot - paper composites functionalised with different concentration of 8-HQ (B, black line: V(0.025 M 8-HQ $(\mathrm{EtOH}))=0.1 \mathrm{~mL}, 70{ }^{\circ} \mathrm{C}$; C, green line: $\mathrm{V}(0.025 \mathrm{M}$ 8-HQ $(\mathrm{EtOH}))$ $=1 \mathrm{~mL}, 70{ }^{\circ} \mathrm{C} ; \mathrm{D}$, brown line: $\mathrm{V}(0.025 \mathrm{M}$ 8-HQ $\left.(\mathrm{EtOH}))=1 \mathrm{~mL}, 100{ }^{\circ} \mathrm{C}\right)$ $\left(\lambda_{\text {exc }}=260 \mathrm{~nm}\right.$; exc /em. slit $\left.=5 \mathrm{~nm}\right)$.

Figure 6.14: Upper and lower side of $\mathrm{ZnO}$ quantum dot - paper fibre obtained after ageing ( $~ 3$ months). Note the $\mathrm{ZnO}$ quantum dots leaching out at the fringes of the paper sheets and the fluorescence of the bleached Kraft paper ( $\lambda_{\text {exc }}=$ $366 \mathrm{~nm})$.

Figure 7.1: Graphic illustration of the uptake of 8-HQ onto wool through transcellular and intercellular diffusion.

Figure 7.2: $\quad$ PL scan of homogeneously distributed crystals of the $\mathrm{Zn}(8-\mathrm{Q})_{2}$ chelate $(0.001 \mathrm{M})$ in EtOH solution excited with wavelengths in the range of $\lambda_{\text {exc }}=$ $315-370 \mathrm{~nm}$; (exciting / emission slit width = $5 \mathrm{~nm}$ ). Details of sample preparation are listed in the appendix in table A.18; A. 
Figure 7.3: $\quad$ PL scan of $\mathrm{Zn}(8-\mathrm{Q})_{2}$ EtOH suspension (0.001 M) before (solid square) and after (solid triangle) precipitation of the crystals in solution. Exciting wavelength: 330 and $370 \mathrm{~nm}$; (exciting / emission slit width = $5 \mathrm{~nm}$ ).

Figure 7.4: $\quad$ PL scans of diluted $\mathrm{Zn}(8-\mathrm{Q})_{2}$ EtOH solution without crystals at different exciting wavelengths $\lambda_{\text {exc }}$; (exciting / emission slit width $=5 \mathrm{~nm}$ ).

Figure 7.5: PL scans of 8-HQ EtOH solution ( $0.25 \mathrm{M})$ excited with wavelengths in the range of $240-350 \mathrm{~nm}$ (exciting / emission slit width $=5 \mathrm{~nm}$ ).

Figure 7.6: $\quad$ Stoke shift of 8-HQ (EtOH) (red curve - absorbance curve; black curve PL scan). The PL scan of 8-HQ was recorded with an excitation wavelength of $315 \mathrm{~nm}$ and the excitation / emission slit was set up with $5 \mathrm{~nm}$.

Figure 7.7: $\quad$ PL scans of $\mathrm{Zn}(8-\mathrm{Q})_{2} \mathrm{H}_{2} \mathrm{O}$ suspension in different zinc to 8-HQ ratio; 1:2 (black line); 1:4 (red line) and 1:2 diluted in EtOH solution (blue line) (exciting / emission slit width $=5 \mathrm{~nm}$ ). Details of sample preparation are shown in the appendix in table A.18; B.

Figure 7.8: $\quad$ PL scans of 8-HQ (EtOH) uptake onto $0.1 \mathrm{~g}$ merino wool with different mass fraction. A and B excited with $\lambda_{\text {exc }}=260 \mathrm{~nm}$ (exciting/emission slit $=5$ $\mathrm{nm}$ ); C and D excited with $\lambda_{\text {exc }}=350 \mathrm{~nm}$ (exciting / emission slit width $=3$ $\mathrm{nm}$ ). Details of sample preparation are listed in the appendix in table A.19.

Figure 7.9: $\quad$ PL scans of 8- $\mathrm{HQ}\left(\mathrm{H}_{2} \mathrm{O}\right)$ uptake onto $0.1 \mathrm{~g}$ merino wool with different mass fraction. $A$ and $B$ excited with $\lambda_{\text {exc }}=260 \mathrm{~nm}$ (exciting/emission slit $=5 \mathrm{~nm}$ ); $\mathrm{C}$ and D excited with $\lambda_{\text {exc }}=350 \mathrm{~nm}$ (exciting $/$ emission slit width $=3 \mathrm{~nm}$ ). Details of sample preparation are listed in the appendix in table A.20.

Figure 7.10: $\quad$ PL scans of wool fibre composites produced by the uptake of $\mathrm{ZnCl}_{2}(\mathrm{EtOH})$ onto 8-HQ treated wool $(\mathrm{wt}(8-\mathrm{HQ})=0.1-8 \%)\left(\lambda_{\mathrm{exc}}=260 \mathrm{~nm}\right.$; exciting $/$ emission slit width $=5 \mathrm{~nm})$. Details of sample preparation are listed in appendix in table A.21.

Figure 7.11: $\quad$ PL scans of wool fibre composites produced by the uptake of $\mathrm{ZnCl}_{2}(\mathrm{EtOH})$ onto 8-HQ treated wool $(\mathrm{wt}(8-\mathrm{HQ})=0.1-8 \%)\left(\lambda_{\text {exc }}=350 \mathrm{~nm}\right.$; exciting / emission slit width $=3 \mathrm{~nm}$ ). Details of sample preparation are liseted in the appendix in table A.21.

Figure 7.12: $\quad$ Zinc - (8-HQ wool) fibres prepared according dyeing method 1, crushed with liquid nitrogen and observed under UV light $\left(\lambda_{\text {exc }}=366 \mathrm{~nm}\right)$. Maximum emission wavelength and 8-HQ content indicated.

Figure 7.13: $\quad$ EDS elemental analysis of $\mathrm{ZnCl}_{2}(\mathrm{EtOH})$ doped wool pre-treated with MSA.

Figure 7.14: $\quad$ PL scan of wool fibre composites produced by soaking $\mathrm{ZnCl}_{2}(\mathrm{EtOH})$ doped wool (zinc content is constant) in $10 \mathrm{~mL} \mathrm{8-HQ} \mathrm{(EtOH)} \mathrm{solution} \mathrm{([8-HQ]} \mathrm{=}$ $\left.6.25 \cdot 10^{-5}-2.5 \cdot 10^{-3} \mathrm{M}\right)$ at $50{ }^{\circ} \mathrm{C}$ for 3 days $\left(\lambda_{\text {exc }}=350 \mathrm{~nm}\right.$; exciting / emission slit width $=3 \mathrm{~nm}$ ). Details of sample preparation are listed in the appendix in table A.23.

Figure .715: PL scan of wool fibre composites with a high uptake of 8-HQ and the effect of ageing on the fluorescence intensity and emission wavelength. The composites were prepared by soaking $0.1 \mathrm{~g} \mathrm{Zn}$ doped wool into $10 \mathrm{~mL}$ 8$\mathrm{HQ}(\mathrm{EtOH})$ solution $(0.0025 \mathrm{M})$ at $50^{\circ} \mathrm{C}$ for 3 days $\left(\lambda_{\text {exc }}=350 \mathrm{~nm}\right.$; exciting / emission slit width $=3 \mathrm{~nm})$. 
Figure 7.16: 8-HQ - (Zn wool) fibres prepared according dyeing method 2, crushed with liquid nitrogen and observed under UV light $\left(\lambda_{\text {exc }}=366 \mathrm{~nm}\right)$. Maximum emission wavelength and 8-HQ content indicated.

Figure 7.17: $\quad$ PL scans of wool fibre composites produced by soaking $\mathrm{ZnCl}_{2}\left(\mathrm{H}_{2} \mathrm{O}\right)$ doped wool (zinc content is constant) in $10 \mathrm{~mL}$ 8-HQ (EtOH) solution ([8-HQ] = $\left.6.25 \cdot 10^{-5}-1.875 \cdot 10^{-3} \mathrm{M}\right)$ at $50^{\circ} \mathrm{C}$ for 3 days, The PL scans were obtained after 6 days of ageing $\left(\lambda_{\text {exc }}=350 \mathrm{~nm}\right.$; exciting / emission slit width $\left.=3 \mathrm{~nm}\right)$. Details of sample preparation are listed in the appendix in table A.24.

Figure 7.18: Wool doped with $\mathrm{ZnCl}_{2}\left(\mathrm{H}_{2} \mathrm{O}\right)$ and soaked in $10 \mathrm{~mL} 6.25 \cdot 10^{-5} \mathrm{M}$ 8-HQ (EtOH) (A), $1.25 \cdot 10^{-4} \mathrm{M} 8-\mathrm{HQ}(\mathrm{EtOH})(\mathrm{B})$ and $1.25 \cdot 10^{-3} \mathrm{M} 8-\mathrm{HQ}$ (EtOH) (C) for 3 days at $50{ }^{\circ} \mathrm{C}$ observed under UV-light (above, $\lambda_{\text {exc }}=256 \mathrm{~nm}$ ) and ambient light (below). Pictures were taken after 2 months of ageing.

Figure 7.19: Wool doped with $\mathrm{Zn}(\mathrm{Ac})_{2}\left(\mathrm{H}_{2} \mathrm{O}\right)$ and soaked in $10 \mathrm{~mL} 2.5 \cdot 10^{-3} \mathrm{M}$ 8-HQ EtOH solution for 3 days at $50{ }^{\circ} \mathrm{C}$. Photographs taken under ambient light (A) and UV-light $\left(B ; \lambda_{\text {exc }}=366 \mathrm{~nm}\right)$.

Figure 7.20: PL scan of wool fibre, untreated (solid square, triangle and rhomboid) and pre-treated with MSA (open square, triangle and rhomboid), doped with $\mathrm{Zn}(\mathrm{Ac})_{2}\left(\mathrm{H}_{2} \mathrm{O}\right)$ and soaked in 8-HQ EtOH solution $\left([8-\mathrm{HQ}]=1.25 \cdot 10^{-3}-\right.$ $\left.2.5 \cdot 10^{-3} \mathrm{M}\right)$ and wool fibre doped with $\mathrm{Zn}(\mathrm{Cl})_{2}(\mathrm{EtOH})$ (plus sign) soaked in 8-HQ EtOH solution $\left([8-\mathrm{HQ}]=1.875 \cdot 10^{-3} \mathrm{M}\right)\left(\lambda_{\text {exc }}=350 \mathrm{~nm}\right.$; exciting / emission slit width $=3 \mathrm{~nm}$ ). Details of sample preparation are listed in the appendix in table A.25.

Figure 7.21: $\quad$ PL scan of wool fibre doped with $\mathrm{Zn}(\mathrm{Ac})_{2}\left(\mathrm{H}_{2} \mathrm{O}\right)$ and soaked in $10 \mathrm{~mL} 1.875$ $\cdot 10^{-3} \mathrm{M} 8-\mathrm{HQ}$ EtOH solution (wt(8-HQ) $=2.7 \%$ on $0.1 \mathrm{~g}$ wool) at $50{ }^{\circ} \mathrm{C}$ for 3 days. The PL scans were obtained daily after preparation for 6 days $\left(\lambda_{\text {exc }}=\right.$ $350 \mathrm{~nm}$; exciting / emission slit width $=3 \mathrm{~nm}$ ). The wool fibre was treated with MSA before $\mathrm{Zn}(\mathrm{Ac})_{2}\left(\mathrm{H}_{2} \mathrm{O}\right)$ was absorbed. Details of the sample are listed in the appendix in table A.25; D.

Figure 7.22: Effect of ageing (6 days sun light) on wool pre-treated with MSA, doped with $\mathrm{Zn}(\mathrm{Ac})_{2}\left(\mathrm{H}_{2} \mathrm{O}\right)$ and soaked in $10 \mathrm{~mL} 1.875 \cdot 10^{-3} \mathrm{M}$ 8-HQ EtOH solution for 3 days at $50{ }^{\circ} \mathrm{C}$. Photographs taken under ambient light (A) and UV-light (B; $\left.\lambda_{\mathrm{exc}}=366 \mathrm{~nm}\right)$.

Figure 7.23: PL scan of wool doped with $\mathrm{ZnCl}_{2}(\mathrm{EtOH})(\mathrm{A}), \mathrm{ZnCl}_{2}\left(\mathrm{H}_{2} \mathrm{O}\right)$ (B) and $\mathrm{Zn}(\mathrm{Ac})_{2}\left(\mathrm{H}_{2} \mathrm{O}\right)(\mathrm{C})$ and exposed to 8-HQ (EtOH) gas for 3 days at $50{ }^{\circ} \mathrm{C}$ $\left(\lambda_{\text {exc }}=260 \mathrm{~nm}\right.$; exciting / emission slit width $\left.=5 \mathrm{~nm}\right)$. Details of the Zndoped wool fibres are listed in the appendix in table A.22.

Figure 7.24 PL scans of wool fibre composites dyed using three methods. Method 1: 0.1 g wool treated with 8-HQ $(\mathrm{EtOH})\left([8-\mathrm{HQ}]=2.5 \cdot 10^{-4} \mathrm{M}\right)$ in a total volume of $10 \mathrm{~mL}$ EtOH and then soaked in $10 \mathrm{~mL} 0.01 \mathrm{M} \mathrm{ZnCl}_{2}$ (EtOH) for 3 days at $50{ }^{\circ} \mathrm{C}$ (sample details are listed in the appendix in table A.21; wt(8-HQ) = $0.36 \%)$. Method 2: $0.1 \mathrm{~g}$ wool doped with $\mathrm{ZnCl}_{2}(\mathrm{EtOH})$ and then soaked in $10 \mathrm{~mL} 8-\mathrm{HQ}(\mathrm{EtOH})$ solution $\left([8-\mathrm{HQ}]=1.25 \cdot 10^{-4} \mathrm{M}\right)$ (sample details are listed in the appendix in table A.23; wt(8-HQ) $=0.18 \%)$. Method 3: $0.1 \mathrm{~g}$ wool doped with $\mathrm{ZnCl}_{2}(\mathrm{EtOH})$ and exposed in 8-HQ gas for 1 day at 50 (blue line) and $80{ }^{\circ} \mathrm{C}$ (green line) (procedure for 8-HQ gas uptake is described in chapter 7; 7.2.2.3.5); $\left(\lambda_{\text {exc }}=260 \mathrm{~nm}\right.$; exciting / emission slit width $=5 \mathrm{~nm}$ ). 
Figure 7.25: Fluorescence of merino wool (A) and wool doped with $\mathrm{ZnCl}_{2}(\mathrm{EtOH})$ and exposed to $8-\mathrm{HQ}$ gas at $80^{\circ} \mathrm{C}$ for 1 day (B), 2 days (C) and 3 days (D) $\left(\lambda_{\text {exc }}\right.$ $=366 \mathrm{~nm})$.

Figure 7.26: Wool pre-treated with MSA and doped with $\mathrm{Zn}(\mathrm{Ac})_{2}\left(\mathrm{H}_{2} \mathrm{O}\right)(\mathrm{A}, \mathrm{B})$ and $\mathrm{ZnCl}_{2}(\mathrm{EtOH})$ doped wool $(\mathrm{C}, \mathrm{D})$ soaked in $1.875 \cdot 10^{-3} \mathrm{M} 8-\mathrm{HQ} \mathrm{EtOH}$ solution at $50{ }^{\circ} \mathrm{C}$ for 3 days. Left: ambient light. Right: UV-light $\left(\lambda_{\mathrm{exc}}=256\right.$ $\mathrm{nm})$. Samples shown in A and B are listed in the appendix in table A.25; D and $\mathrm{G}$, respectively.

Figure 7.27: Absorbance scan of wool fibre composites functionalised with $\mathrm{ZnCl}_{2}\left(\mathrm{H}_{2} \mathrm{O}\right)$ and $\mathrm{Zn}(\mathrm{Ac})_{2}\left(\mathrm{H}_{2} \mathrm{O}\right)$ and soaked in 8-HQ EtOH solution $\left(\left([8-\mathrm{HQ}]=1.25 \cdot 10^{-4}\right.\right.$ $\mathrm{M}$ and $\left.1.875 \cdot 10^{-4} \mathrm{M}\right)$. Sample details of $\mathrm{wt}(8-\mathrm{HQ})=1.8 \%, \mathrm{ZnCl}_{2}-\operatorname{wt}(8-$ $\mathrm{HQ})=1.8$ and $2.7 \%$ are listed in appendix, table A.19 and A.24, respectively. Sample details of $\mathrm{Zn}(\mathrm{Ac})_{2}-\mathrm{wt}(8-\mathrm{HQ})=1.8$ and $2.7 \%$ are listed in table A.25; A and C, respectively.

Figure 7.28: Stoke shift of $\mathrm{ZnCl}_{2}(\mathrm{EtOH})$ doped wool soaked in $1.25 \cdot 10^{-3} \mathrm{M}$ 8-HQ $(\mathrm{EtOH})(\mathrm{wt}(8-\mathrm{HQ})=1.8 \%)$ at $50{ }^{\circ} \mathrm{C}$ for 3 days. Red: Absorbances scan. Black: PL scan. Details of sample preparation are listed in the appendix in table A.23; wt $(8-\mathrm{HQ})=1.8 \%$.

Figure 7.29: Blue shift of the emission wavelength after adding water $\left(2 \times 2.5 \mathrm{~mL} \mathrm{H}_{2} \mathrm{O}\right.$ in a $2 \mathrm{hrs}$ time difference) to $10 \mathrm{~mL} 0.0025 \mathrm{M}$ 8-HQ EtOH solution containing wool doped with $\mathrm{ZnCl}_{2}\left(\mathrm{H}_{2} \mathrm{O}\right)$ (solid red square before, open red square after) and $\mathrm{Zn}(\mathrm{Ac})_{2} \quad\left(\mathrm{H}_{2} \mathrm{O}\right)$ ((solid green rhomboid before, open green rhomboid after $)\left(\lambda_{\text {exc }}=350 \mathrm{~nm}\right.$; exciting $/$ emission slit width $\left.=3 \mathrm{~nm}\right)$. Sample details of $\mathrm{ZnCl}_{2}\left(\mathrm{H}_{2} \mathrm{O}\right)$ - wool are listed in the appendix in table A.24; wt $(8-\mathrm{HQ})=3.5 \%$. Sample details of $\mathrm{Zn}(\mathrm{Ac})_{2}\left(\mathrm{H}_{2} \mathrm{O}\right)-$ wool are listed in table A.25; E.

Figure 7.30: Aqueous suspension of $\mathrm{Zn}(8-\mathrm{Q})_{2}$ particles (A) and hydrolysed $\mathrm{Zn}(8-\mathrm{Q})_{2}$ particles around wool (B) under UV-light $\left(\lambda_{\text {exc }}=366 \mathrm{~nm}\right)$. Bright fluorescent particles were formed around the wool fibre, when $2 \times 2.5 \mathrm{~mL}$ water were added to $10 \mathrm{~mL} 2.5 \cdot 10^{-3} \mathrm{M} 8-\mathrm{HQ}(\mathrm{EtOH})$ soaking solution containing $\mathrm{ZnCl}_{2}(\mathrm{EtOH})$ wool fibre at $50{ }^{\circ} \mathrm{C}$. The aqueous suspension of $\mathrm{Zn}(8-\mathrm{Q})_{2}$ was obtained by removing the particles around the wool using ultrasound.

Figure 7.31: Absorbance scans of wool fibre composites dyed with zinc and 8-HQ in the presence of water. A: Wool soaked in aqueous suspension of $\mathrm{Zn}(8-\mathrm{Q})_{2}$ $\left(\mathrm{H}_{2} \mathrm{O}\right)$ ( see table A.18 in appendix). B: Wool soaked in $\mathrm{Zn}^{2+}$ aqueous suspension and followed by the addition of 8-HQ (EtOH). C: Wool doped with zinc and soaked in 8-HQ EtOH solution (see sample $\mathrm{E}$ in the appendix in table A.25) followed by the addition of $\mathrm{H}_{2} \mathrm{O}$.

Figure 7.32: Emission shift between 460 and $505 \mathrm{~nm}$ of the maximum emission wavelength of wool fibre composites produced following in-situ approach 1 $\left(\mathrm{Zn}^{2+}\right.$ uptake onto 8-HQ treated wool) and 2 (8-HQ uptake onto zinc doped wool) using non-aqueous dyeing bath condition and different 8-HQ concentration ( $\lambda_{\text {exc }}=350 \mathrm{~nm}$; exciting / emission slit width $\left.=3 \mathrm{~nm}\right)$. Sample details are listed in the appendix in table A.21 (dyeing method 1) and table A.23 (dyeing method 2), respectively.

Figure 7.33: Fluorescent colours observed in wool fibre composites functionalised with different concentration of $\mathrm{Zn}(8-\mathrm{Q})_{2}$ using the in-situ dyeing approach 1 and 2 . The concentration varies from small (right) to large (left) $\left(\lambda_{\text {exc }}=366 \mathrm{~nm}\right)$. 


\section{LIST OF TABLES}

Table 1.1: List of non-polar aprotic, polar aprotic and polar protic solvents with corresponding dielectric constants $\left(20^{\circ} \mathrm{C}\right)$.

Table 1.2: $\quad$ Physical and chemical techniques commonly used to prepare quantum dots [46-51].

Table 1.3: Selection of ionic and polymeric stabiliser used for the synthesis of nanocrystals [46-51].

Table 2.1: $\quad$ Separation and washing procedure for synthesised quantum dot materials.

Table 2.2: $\quad$ Instructions for the synthesis of copper (I) oxide nanocrystals.

Table 2.3: Instructions for the synthesis of $\mathrm{Zn}^{2+}$ and $\mathrm{Ni}^{2+}$ doped copper (I) oxide nanocrystals.

Table 2.4: $\quad$ Instructions for the synthesis of zinc oxide nanocrystals.

Table 2.5: Instruction for the synthesis of zinc sulfide nanocrystals containing Mnnanocluster

Table 2.6: Instruction for the synthesis of zinc sulfide nanocrystals containing $\mathrm{Mn} / \mathrm{Ni}$ nanoclusters

Table 2.7: $\quad$ List of capping agents used to modify the surface area of wool fibres.

Table 2.8: Functionalisation of wool fibre using 8-HQ as linker molecule in EtOH solution.

Table 2.9: $\quad$ Washing and drying procedure for paper fibre.

Table 2.10: List of precursor materials and reaction conditions for the development of $\mathrm{ZnO}$ quantum dot - wool composite.

Table 2.11: List of precursor materials and reaction conditions for the development of $\mathrm{ZnO}$ quantum dot - paper composite materials.

Table 2.12: $\quad$ List of precursor materials and solutions used for the in-situ dyeing of wool with $\mathrm{Zn}(8-\mathrm{Q})_{2}$.

Table 2.13: Instructions for the uptake of $\mathrm{Zn}^{2+}$ ions onto wool fibre.

Table 2.14: $\quad$ Process parameters used for the uptake of $\mathrm{Zn}^{2+}$ ions onto wool fibre.

Table 2.15: Instructions for the uptake of 8-HQ onto wool fibre.

Table 2.16: $\quad$ Process parameters investigated for the uptake of 8-HQ onto wool fibre.

Table 2.17: Instructions for the uptake of 8-HQ onto wool functionalised with $\mathrm{Zn}^{2+}$.

Table 2.18: $\quad$ Process parameters used for the uptake of 8-HQ onto wool functionalised with $\mathrm{Zn}^{2+}$. 
Table 2.19: Instructions for the uptake of $\mathrm{Zn}^{2+}$ ions onto wool treated with 8-HQ.

Table 2.20: $\quad$ Process parameters used for the uptake of $\mathrm{Zn}^{2+}$ ions onto wool treated with 8-HQ.

Table 3.1: $\quad$ List and details of process parameters investigated for the synthesis of doped and undoped $\mathrm{Cu}_{2} \mathrm{O}$ nanoparticles.

Table 3.2: Binding energy of copper atoms obtained after adding different concentrations of TSC to the reaction medium. Details of sample preparation are listed in the appendix in A.1; appendix.

Table 3.3: $\quad$ Size of the particles (d.nm) in figure 3.19 obtained by adding $\mathrm{NaBH}_{4}$ in different concentration of to the standard reaction. ${ }^{*} \mathrm{Cu}_{2} \mathrm{O}$ suspension prepared with $\mathrm{CuCl}_{2}$ as copper precursor material. Details of sample preparation are listed in the appendix in table A.4.

Table 3.4: $\quad$ List of $\mathrm{Cu}_{2} \mathrm{O}$ samples showing particle size, absorbance wavelengths and their colour in reflected and transmitted light. ${ }^{*} \mathrm{Cu}_{2} \mathrm{O}$ suspension prepared with $\mathrm{CuCl}_{2}$ as copper precursor material.

Table 3.5: Absorbance range of visible light with the corresponding complementary colour.

Table 3.6: Correlation between particle sizes and absorbance peaks.

Table 4.1: $\quad$ List and details of process parameters investigated for the synthesis of $\mathrm{ZnO}$ nanoparticles.

Table 5.1: List of ZnS quantum dot suspension precipitated from solution in the presence of $\mathrm{ZnO}, \mathrm{Cu}, \mathrm{Mn}$ and $\mathrm{Mn} / \mathrm{Ni}$ nanoclusters with corresponding maximum emission wavelength obtained with exciting wavelengths $\lambda_{\mathrm{exc}}=$ 260 and $350 \mathrm{~nm}$.

Table 6.1: $\quad$ Reaction parameters used for the in-situ formation of $\mathrm{ZnO}$ quantum dot wool fibre composites.

Table 6.2: List of ionic surfactants and linker molecules used to modify the surface area of quantum dots.

Table 7.1: $\quad$ Process parameters used for the uptake of 8-HQ molecules onto wool.

Table 7.2: $\quad$ Process parameters used for the uptake of $\mathrm{Zn}^{2+}$ ions onto 8-HQ treated wool.

Table 7.3: $\quad$ Process parameters used for the uptake of $\mathrm{Zn}^{2+}$ ions onto wool.

Table 7.4: $\quad$ Process parameters used for the uptake of 8-HQ onto zinc doped wool.

Table 7.5: $\quad$ Volume and concentration of 8-HQ (EtOH) used for the uptake of 8-HQ onto wool and 8-HQ mass percentage on weight of wool (wt \%), assuming 8-HQ was completely absorbed by the wool.

Table 7.6: $\quad$ Volume and concentration of 8-HQ $\left(\mathrm{H}_{2} \mathrm{O}\right)$ used for the uptake of 8-HQ onto wool and 8-HQ mass percentage on weight of wool (wt \%), assuming 8-HQ was completely absorbed by the wool. 
Table 7.7: $\quad$ Volume and concentration of 8-HQ (EtOH) used for the in-situ dyeing method, and 8-HQ mass percentage on weight of wool (wt \%), assuming 8HQ was completely absorbed by the wool.

Table A.1: $\quad \mathrm{Cu}_{2} \mathrm{O}$ quantum dots samples prepared according to a wet chemical reduction method (see chapter 2; 2.1.3.1). Variable process parameter: trisodium citrate concentrations. Constant process parameters: $\mathrm{T}=99^{\circ} \mathrm{C}$ and stirring time $=30 \mathrm{~min}$.

Table A.2: $\quad \mathrm{Cu}_{2} \mathrm{O}$ quantum dots samples prepared according to a wet chemical reduction method (see chapter 2; 2.1.3.1). Variable process parameters: copper salt precursor, polymeric dispersant and reaction temperature. Constant process parameter: stirring time $=30 \mathrm{~min}$.

Table A.3: $\quad \mathrm{Cu}_{2} \mathrm{O}$ quantum dots samples prepared according to a wet chemical reduction method (see chapter 2; 2.1.3.1). Variable process parameters: copper salt precursor, polymeric dispersant and reaction temperature. Constant process parameter: stirring time $=30 \mathrm{~min}$.

Table A.4: $\quad \mathrm{Cu}_{2} \mathrm{O}$ quantum dots samples prepared according to a wet chemical reduction method (see chapter 2; 2.1.3.1). Variable process parameter: sodium borohydride. Constant process parameters: $\mathrm{T}=99^{\circ} \mathrm{C}$ and stirring time $=30$ min.

Table A.5: $\quad \mathrm{Cu}_{2} \mathrm{O}$ quantum dots samples doped with impurities (see chapter 2; 2.1.3.1). Variable process parameters: dopants, stirring time and addition of trisodium citrate. Constant process parameter: $\mathrm{T}=99^{\circ} \mathrm{C}$.

Table A.6: $\quad \mathrm{ZnO}$ quantum dots samples prepared according to a sol-gel method (see chapter 2; 2.1.3.2). Variable process parameter: borohydride precursor. Constant process parameters: $\mathrm{T}=70^{\circ} \mathrm{C}$ and stirring time $=30 \mathrm{~min}$.

Table A.7: $\quad$ ZnO quantum dots samples prepared according to a sol-gel method (see chapter 2; 2.1.3.2). Variable process parameter: polymeric dispersant. Constant process parameters: $\mathrm{T}=70^{\circ} \mathrm{C}$ and stirring time $=30 \mathrm{~min}$.

Table A.8: $\quad$ ZnO quantum dots samples prepared according to a sol-gel method (see chapter 2; 2.1.3.2). Variable process parameter: dopants. Constant process parameters: $\mathrm{T}=70^{\circ} \mathrm{C}$ and stirring time $=30 \mathrm{~min}$.

Table A.9: $\quad$ ZnO quantum dots samples prepared according to a sol-gel method (see chapter 2; 2.1.3.2). Variable process parameter: PVP concentration. Constant process parameters: $\mathrm{T}=70^{\circ} \mathrm{C}$ and stirring time $=30 \mathrm{~min}$.

Table A.10: ZnO quantum dots samples prepared according to a sol-gel method (see chapter 2; 2.1.3.2). Variable process parameter: $\mathrm{NaBH}_{4}$ concentration. Constant process parameters: $\mathrm{T}=70^{\circ} \mathrm{C}$ and stirring time $=30 \mathrm{~min}$.

Table A.11: ZnS quantum dots samples prepared according to a wet chemical precipitation method (see chapter 2; 2.1.3.3). Variable process parameter: dopants.

Table A.12: ZnO quantum dot - wool fibre composites developed according to the insitu dyeing method (see chapter 2; 2.2.1). Variable process parameter: capping agents. Constant process parameters: $\mathrm{m}($ wool $)=0.2 \mathrm{~g}, \mathrm{~V}_{\text {tot }}=50 \mathrm{~mL}$, soaking temperature $=70{ }^{\circ} \mathrm{C}$ for $24 \mathrm{hrs}$ and then room temperature for 7 days. 
Table A.13: ZnO quantum dot - wool fibre composites developed according to the building block dyeing method (chapter 2; 2.2.2.3). Variable process parameter: merino wool (untreated and pre-treated with $\mathrm{NaOH}, \mathrm{HCl}, \mathrm{MSA}$ and 8-HQ). Constant process parameters: $\mathrm{m}($ wool $)=0.1 \mathrm{~g}, \mathrm{~V}_{\text {tot }}=20 \mathrm{~mL}$ and soaking temperature $=70^{\circ} \mathrm{C}$ for 3 days.

Table A.14: $\quad$ 8-HQ - wool fibre composites (see preparation in chapter 2; 2.2.2.1.4). Variable process parameter: 8-HQ uptake. Constant process parameters: $\mathrm{m}($ wool $)=0.1 \mathrm{~g}, \mathrm{~V}_{\text {tot }}=10 \mathrm{~mL}$ and soaking temperature $=50^{\circ} \mathrm{C}$ for 1 day.

Table A.15: $\quad$ Surface modification of $\mathrm{ZnO}$ quantum dots with MSA-wool and 8-HQ.

Table A.16: ZnO quantum dot - wool fibre composites functionalised with 8-HQ (procedure described in chapter 2; 2.2.2.3). Variable process parameter: 8HQ concentration. Constant process parameters: $\mathrm{m}($ wool $)=0.1 \mathrm{~g}, \mathrm{~V}_{\text {tot }}=20$ $\mathrm{mL}$ and soaking temperature $=50^{\circ} \mathrm{C}$ for 3 days.

Table A.17: ZnO quantum dot - paper fibre composites developed according to the building block dyeing methods (see chapter 2; 2.2.2.4). Variable process parameter: 8-HQ concentration and soaking temperature. Constant process parameters: $\mathrm{m}$ (paper) $=0.1 \mathrm{~g}$ and $\mathrm{V}_{\text {tot }}=80 \mathrm{~mL}$.

Table A.18: $\quad$ Preparation of $0.001 \mathrm{M} \mathrm{Zn(8-Q)})_{2} \cdot 2 \mathrm{H}_{2} \mathrm{O}$ in EtOH (A) and $\mathrm{H}_{2} \mathrm{O}$ (B) solution. Constant process parameter: $\mathrm{V}_{\text {tot }}=10 \mathrm{~mL}$.

Table A.19: List of 8-HQ - wool fibre composites with different uptake of 8-HQ (EtOH) onto wool (see procedure in chapter 2; 2.3.4). Variable process parameter: 8HQ uptake. Constant process parameters: $\mathrm{m}($ wool $)=0.1 \mathrm{~g}, \mathrm{~V}_{\text {tot }}=10 \mathrm{~mL}$, solvent $=\mathrm{EtOH}$ and soaking temperature $=50{ }^{\circ} \mathrm{C}$ for 3 days.

Table A.20: List of 8-HQ - wool fibre composites with different uptake of 8-HQ $\left(\mathrm{H}_{2} \mathrm{O}\right)$ onto wool (see procedure in chapter 2 ; 2.3.4). Variable process parameter: 8HQ uptake. Constant process parameters: $\mathrm{m}($ wool $)=0.1 \mathrm{~g}, \mathrm{~V}_{\text {tot }}=10 \mathrm{~mL}$, solvent $=\mathrm{H}_{2} \mathrm{O}$ and soaking temperature $=50^{\circ} \mathrm{C}$ for 7 days.

Table A.21: List of 8-HQ - wool fibre composites soaked in $10 \mathrm{~mL} 0.01 \mathrm{M} \mathrm{ZnCl}_{2}$ $(\mathrm{EtOH})$ solutions at $50{ }^{\circ} \mathrm{C}$ for 3 days (see procedure in chapter 2; 2.3.6). Variable process parameter: 8-HQ uptake onto wool. Constant process parameters: $\mathrm{m}($ wool $)=0.1 \mathrm{~g}, \mathrm{~V}_{\text {tot }}=10 \mathrm{~mL}$ and soaking temperature $=50{ }^{\circ} \mathrm{C}$ for 3 days.

Table A.22: $\quad$ List of $\mathrm{Zn}$ - wool fibre composites with different zinc precursor materials (see procedure in chapter 2; 2.3.3). Variable process parameters: wool (untreated, $\mathrm{NaOH}-, \mathrm{HCl}-, \mathrm{MSA}-$ and L-cysteine pre-treated) and zinc precursor $\left(\mathrm{ZnCl}_{2}(\mathrm{EtOH}), \mathrm{ZnCl}_{2}\left(\mathrm{H}_{2} \mathrm{O}\right)\right.$ and $\left.\mathrm{ZnAc}_{2}\left(\mathrm{H}_{2} \mathrm{O}\right)\right)$. Constant process parameters: $\mathrm{m}($ wool $)=0.1 \mathrm{~g}, \mathrm{~V}_{\text {tot }}=10 \mathrm{~mL}, \mathrm{c}\left(\mathrm{Zn}^{2+}\right)=0.01 \mathrm{M}$ and soaking temperature $=50^{\circ} \mathrm{C}$ for 2 days.

Table A.23: List of $\mathrm{ZnCl}_{2}(\mathrm{EtOH})$ - wool fibre composites soaked in $10 \mathrm{~mL}$ 8-HQ $(\mathrm{EtOH})$ solutions at $50{ }^{\circ} \mathrm{C}$ for 3 days (see procedure in chapter 2 ; 2.3.5). Variable process parameter: c(8-HQ (EtOH). Constant process parameters: $\mathrm{m}($ wool $)=0.1 \mathrm{~g}, \mathrm{~V}_{\text {tot }}=10 \mathrm{~mL}$ and soaking temperature $=50^{\circ} \mathrm{C}$ for 3 days.

Table A.24: $\quad$ List of $\mathrm{ZnCl}_{2}\left(\mathrm{H}_{2} \mathrm{O}\right)$ - wool fibre composites soaked in $10 \mathrm{~mL}$ 8-HQ (EtOH) solutions at $50{ }^{\circ} \mathrm{C}$ for 3 days (see procedure in chapter 2; 2.3.5). Variable process parameter: $\mathrm{c}(8-\mathrm{HQ}(\mathrm{EtOH})$. Constant process parameters: $\mathrm{m}($ wool $)=$ $0.1 \mathrm{~g}, \mathrm{~V}_{\text {tot }}=10 \mathrm{~mL}$ and soaking temperature $=50^{\circ} \mathrm{C}$ for 3 days. 
Table A.25: $\quad$ List of $\mathrm{Zn}(\mathrm{Ac})_{2}\left(\mathrm{H}_{2} \mathrm{O}\right)$ - wool fibre composites soaked in $10 \mathrm{~mL}$ 8-HQ $(\mathrm{EtOH})$ solutions at $50{ }^{\circ} \mathrm{C}$ for 3 days (see procedure in chapter 2; 2.3.5). Variable process parameter: c(8-HQ (EtOH). Constant process parameters: $\mathrm{m}($ wool $)=0.1 \mathrm{~g}, \mathrm{~V}_{\text {tot }}=10 \mathrm{~mL}$ and soaking temperature $=50^{\circ} \mathrm{C}$ for 3 days. 


\section{LIST OF ABBREVIATIONS}

\section{Chemical abbreviations}

$\begin{array}{ll}\text { 8-HQ } & \text { 8-Hydroxyquinoline } \\ \text { 18-MEA } & \text { 18-methyleicosanoic acid } \\ \mathrm{Ac} & \text { Acetate } \\ \mathrm{Alq}_{3} & \text { Tris-(8-hydroxyquinolinato) aluminium } \\ \mathrm{CdSe} & \text { Cadmium selenide } \\ \mathrm{Cu}_{2} \mathrm{O} & \text { Copper (I) oxide } \\ \mathrm{EtOH} & \text { Ethanol } \\ \mathrm{H}_{2} \mathrm{O} & \text { Water } \\ \mathrm{HEC} & \text { Hydroxyethyl cellulose } \\ \mathrm{MgO} & \text { Magnesium oxide } \\ \mathrm{MSA} & \text { Mercaptosuccinic acid } \\ \mathrm{PbS} & \text { Lead sulfide } \\ \mathrm{PMAA}-\mathrm{Na}- & \text { Poly(methacrylic acid, sodium salt) } \\ \mathrm{PVP} & \text { Poly(vinyl-pyrrolidone) } \\ \mathrm{TiO} & \text { Titanium oxide } \\ \mathrm{TSC} & \text { Sodium citrate tribasic dihydrate }\left(\mathrm{Na}_{3} \mathrm{C}_{6} \mathrm{H}_{5} \mathrm{O}_{7} \cdot 2 \mathrm{H}_{2} \mathrm{O} \text { ) }\right. \\ \mathrm{Zn}(8-\mathrm{Q})_{2} \cdot 2 \mathrm{H}{ }_{2} \mathrm{O} & \text { Zinc(II)-Bis(8-hydroxyquinoline) } \\ \mathrm{ZnO} & \text { Zinc oxide } \\ \mathrm{ZnS} & \text { Zinc sulfide } \\ \mathrm{ZnS} / \mathrm{Cu} & \text { Zinc sulfide doped with copper } \\ \mathrm{ZnS} / \mathrm{Mn} & \text { Zinc sulfide doped with manganese } \\ & \end{array}$

\section{General abbreviations}

$A_{\mathrm{N}}$

Nucleophilic addition mechanism

CT

Charge transfer

DLS

Dynamic light scattering

C

Speed of light in a vacuum

EDS

Energy dispersive spectroscopy

e.g.

exempli gratia

$E_{\text {em }}$

Energy of the emitted light

$E_{g}$

Band gap energy 


$\begin{array}{ll}E_{\text {HOMO }} & \text { Energy level of the highest occupied molecular orbital } \\ E_{\text {kin }} & \text { Kinetic energy the electron } \\ E_{L U M O} & \text { Energy level of the lowest occupied molecular orbital } \\ E_{\text {pot }} & \text { Potential energy } \\ \text { etc. } & \text { et cetera } \\ \text { FC } & \text { Fluorescent chelate } \\ \text { H } & \text { Hydrogen } \\ \text { HOMO } & \text { Highest occupied molecular orbital } \\ \text { hrs } & \text { Hours } \\ \text { IPC } & \text { Industrial Process Control } \\ \text { LMCT } & \text { Ligand-to-metal charge-transfer } \\ L U M O & \text { Lowest unoccupied molecular orbital } \\ \text { M } & \text { Metal / metal oxide } \\ \text { min } & \text { Minutes } \\ \text { MLCT } & \text { Metal-to-ligand charge-transfer } \\ M O & \text { Molecular orbital } \\ \text { OLED } & \text { Organic light emitting diodes } \\ \text { PL } & \text { Photoluminescence } \\ \text { R } & \text { Organic ligand } \\ \text { RT } & \text { Room temperature } \\ \text { SEM } & \text { Scanning electron microscopy } \\ S_{\mathrm{N}} & \text { Eucleophilic substitution mechanism } \\ \text { SPR } & \text { Surface Plasmon Resonance } \\ \text { TEM } & \text { Transmission electron microscopy } \\ \text { UV } & \text { Ultraviolet } \\ \text { UV-vis } & \text { Ultraviolet-visible } \\ \text { wt \% } & \text { Weight percent } \\ \text { XPS } & \text { X-ray photoelectron spectroscopy } \\ \lambda_{\text {exc }} & \text { X-ray diffraction } \\ \lambda_{\text {ebs }} & \text { Absorbance wavelength } \\ \text { ERD } & \end{array}$






\section{Chapter 1}

\section{Introduction}

\subsection{Hybrid materials; general background}

Nanoscience has developed various methods to produce nanosized particles with interesting chemical or physical properties, such properties can be conductivity, magnetic behaviour, thermal, switching, fluorescence and optical [1]. The unique size and shaped dependent properties of nanoparticles can be used to functionalise a range of bulk materials. By doing so, new hybrid materials with multifunctional properties can be created. In fact, the process of combining nanosized materials together with bulk materials present a nearly unlimited opportunity for the development of new materials that can be used in ordinary products such as textiles, tools, cleaning products and so on. For this reason, research involving nanotechnology to create new hybrid materials with multifunctional properties is of great industrial interest.

A hybrid material is described as a material that includes at least two components combined together. One component is inorganic and the other one organic in nature. The new material is expected to possess the properties of both components or even enhanced properties. For example, the attachment of nanomaterials with excellent catalytic properties to a sponge - like network structure, can improve catalytic processes of a chemical reaction by increasing the surface area between the catalyst and the reaction medium. For the development of hybrid materials, the organic and inorganic units have to be compatible with each other. The compatibility is determined by the active forces prevailing at the interface between both units. When these forces are attractive to each other, then the organic and inorganic components can be combined. These attractive forces also determine the stability of the hybrid material. For example, when the chemical interaction between the adsorbed nanomaterial and the network structure of the substrate material is weak, diffusion effects (leaching) can occur over time resulting in the damage of the hybrid material. For this reason, strong chemical interactions such as covalent bonds are always favoured. 
Nevertheless, stable hybrid materials can still be created if the chemical interaction is weak. To do this, nanomaterials can be enclosed in a cross-linked polymer matrix, where physical interactions such as weak cross-links can occur between trapped nanoparticles and the network structure. The final product is called "blend". Nowadays, inorganic nanomaterials are already embedded in a variety of polymers, whereby the final blend exhibit higher mechanical strength compared to the pure polymer. Such polymer fibres, reinforced with nanoparticles, for instance found application in lightweight materials used in the fabrication of vehicles and sport gear.

Nanoparticles can also be used to give colours to materials. For instance gold nanoparticles have been unknowingly incorporated as colourants (pigments) in the production of stained glass and ceramic from centuries ago. Small colloidal gold nanoparticles, formed by the reduction of gold salt during the glass processing, by combining aqua regia gold solution with tin, give the typical red colour to window glasses as shown in figure 1.1.

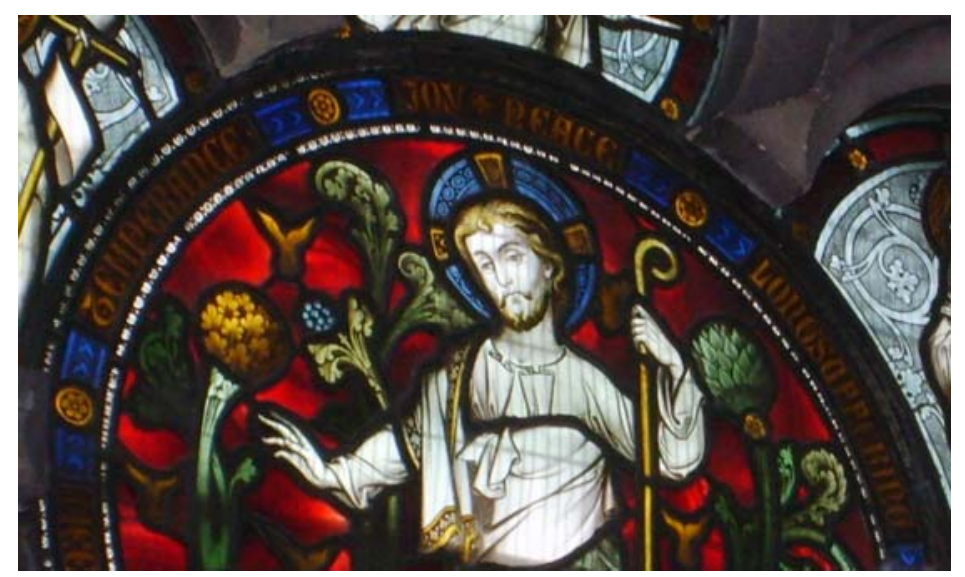

Figure 1.1: Church window glass where the red colour originates from the incorporation of gold nano-particles.

This red colour is caused by a Surface Plasmon Resonance (SPR) effect. This effect is strongly dependent on the size and shape of the colloid gold nanoparticles. By varying the size and shape of the gold colloids, it is possible to change the colour in the visible ramge. Similarly, the SPR effect of nanogold and nanosilver has been used to dye wool fibre. Studies by previous researchers [2-4] have shown that the uptake of gold nanoparticles onto merino wool in different concentration $(\mu \mathrm{g}(\mathrm{Au}) / \mathrm{g}(\mathrm{wool})$ can produce colours varying from shades of purple to grey (figure 1.2). 


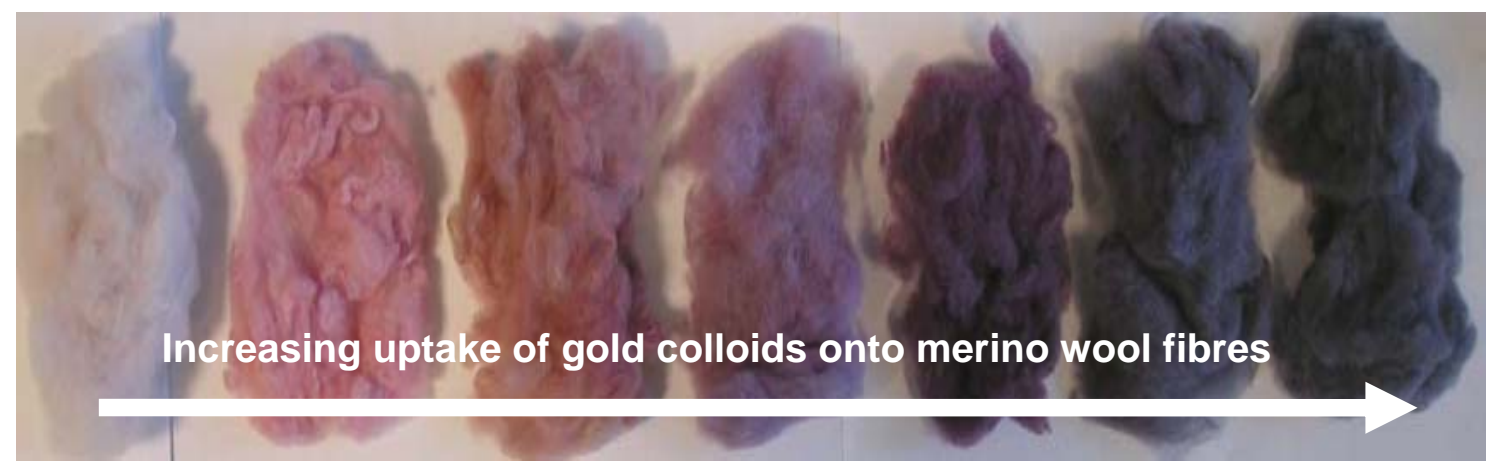

Figure 1.2: Colour variation of nanogold wool fibre composites sorted by the uptake of gold nanoparticles [4].

The chemical bonding between wool fibre and nanogold and nanosilver colloids has been proven to be covalent, providing long term stability for the final composite material. Furthermore, nanogold- and nanosilver wool fibres composites exhibit antimicrobial activities and show a higher photostability compared to wool fabrics dyed with organic dyes.

Nowadays, nanotechnology has made enormous progress in the development of new nano-sized semiconductor materials (quantum dots) showing bright stable fluorescence colours. Fluorescence is the emission of light or the radiative release of energy (excess energy) in the form of electromagnetic waves after electronic, thermal or photoelectrical excitation. Becaue of the fluorescence, quantum dots belong to the chemical group of fluorophores. There are different types of fluorophores such as organic fluorophores (e.g. small molecules and proteins), coordination complexes and quantum dots. Flurophores have a wide field of applications ranging from scientific research projects to industrial usages. For example, fluorophores find application in biotechnology, biochemistry and protein studies, were they are used as fluorescent dyes for biomedical imaging of non-fluorescent molecules or proteins [5-7]. Fluorophores, however, also find application in everyday objects. They are often used as as additive for the production of paper and textiles or dyeing of clothing [8-10].

Organic fluorophores are commonly used to functionalise materials with optical fluorescence. However, quantum dots and fluorescent coordination complexes also have the potential to be used as entities to functionalise a wide range of bulk materials with fluorescence. Furthermore, these fluorophores combined with different substrate 
material could create hybrid materials which have properties of interest in science and industrial areas. For example, quantum dots show higher stability towards external environmental influences such as moisture and sun light exposure. Therefore, the fluorescence life time of quantum dot can easily exceed those of organic fluorophores. Furthermore, quantum dots showing bright stable fluorescence colours may also have other size-related properties which can add extra functionality to materials. Besides quantum dots, fluorescent coordination complexes such as chelates are known for the bright fluorescence colours. Hence, chelates have also the potential to replace a wide range of commercially available organic fluorophores.

Quantum dots and fluorescent chelates, however, are widely unstudied for the creation of new hybrid materials. For this reason, this project explores quantum dots and fluorescent chelates as alternative to organic fluorophores to dye wool and paper fibre with optical properties. To obtain a better understanding of the different groups of fluorophores and their fluorescence activity, each group is briefly discussed in the following sections.

\subsection{Flurophores}

As mentioned before, there are different types of fluorophores. These fluorophores can be organic fluorophores, coordination complexes or quantum dots. Each of these fluorophores has its unique absorption and emission spectrum.

\subsubsection{Organic fluorophores}

Organic fluorophores are grouped according to their molecular structure in linear or aromatic organic fluorophores. Linear organic fluorophores such cyanine and its derivates are commonly used as fluorescent dyes for biomedical imaging due to their optical properties [11]. Aromatic fluorophores such as derivatives of fluoresceine and rhodamine are also used for the labelling of non-fluorescent molecules in biotechnology [12]. By attaching them to non-fluorescent molecules, new functionalised molecules with optical fluorescence are created. Organic fluorophores are also commercially used as optical brighteners. Optical brighteners such as derivatives of coumarin, imidazolines, diazoles, triazine-stilbenes are commonly used as additives in the 
production for paper, textiles and detergents $[8,9,13,14]$. They enhance the appearance of colour of fabrics and papers, causing a "whitening" effect, making material look less yellow by increasing the overall amount of light reflected. Optical brighteners have also found application in cosmetic products (shampoos, conditioners, eye make-up), and safety equipment and clothing. In general, optical brighteners or fluorescent whiting agents are dyes which absorb light in the UV-light region $(340-370 \mathrm{~nm})$ and emit light in the blue region $(420-470 \mathrm{~nm})$. The added blue tone in the reflected light gives a feeling of increased whiteness.

The electronic and optical properties of organic fluorophores are based on their molecular electronic structure. Organic fluorophores exhibit an extended conjugated or an aromatic electronic structure with delocalized $\pi$ - electrons. Here, the $\pi$ - electrons are not located to a single bond or atom, but distributed over a shared $\pi$ - electronic system which is formed by the overlap of adjacent aligned p- orbitals. Due to the uniform distribution of the $\pi$ - electrons in a conjugated electronic structure, the covalent C-C bondings obtain an equal length and the molecule have an overall lower potential energy, which makes it more stable. These molecules can be represented by several contributing structures; also termed resonance structures. However, the real electronic structure in organic fluorophores lies between the resonance structures. Figure 1.3 shows two possible resonance structure of cyanine as an example of a linear conjugated $\pi$ - system.

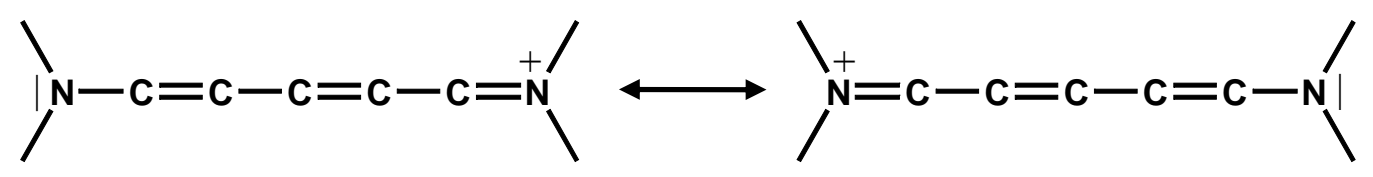

Figure 1.3: Resonance structure of cyanine.

Compounds having several conjugated double bonds show colour. This is based on the molecular orbital configuration of such compounds which allows the absorption of light in the visible range. Figure 1.4 illustrates the molecular orbital digram for the $\pi$-MOs of cyanine and the electronic transition between the highest occupied molecular orbital $(H O M O)$ and the lowest unoccupied molecular orbital (LUMO). 


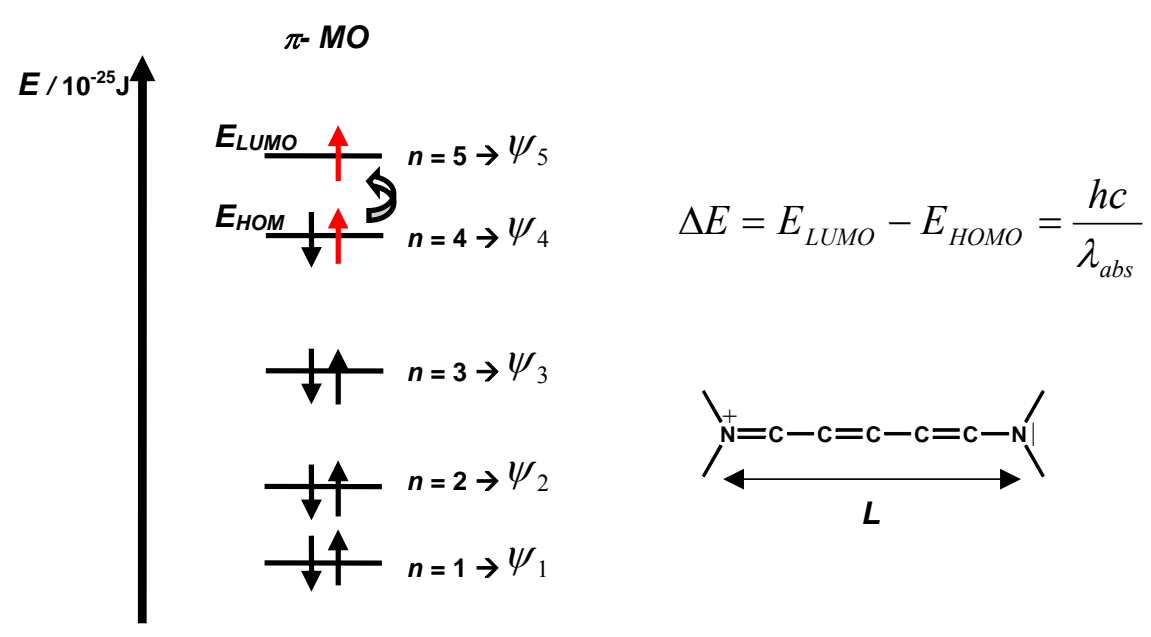

Figure 1.4: Molecular orbital diagram for the $\pi$ - $M O$ of a cyanine molecule and graphical illustration of the electronic transition of a $\pi$ - electron from HOMO to LUMO ( $\psi_{n}=$ molecular orbital wave function).

The difference in energy between HOMO and $L U M O$ determines the wavelength of the absorbed light and the colour they produce in solution. In this cyanine example, around $4.3 \cdot 10^{-25} \mathrm{~J}$ is necessary to excite an electron from HOMO to LUMO. This energy corresponds to light in the visble spectrum with a wavelength of around $461 \mathrm{~nm}$. Due to the absorption of light with $\lambda_{\mathrm{abs}}=461 \mathrm{~nm}$, the cyanine solution shows a yellow colour, which is the complementary colour of the absorbed light. The wavelength of the absorbed light can be easily determined experimentally by UV-vis spectroscopy.

Cyanine, however, can also show fluorescence. Fluorescence is only observed when the excess energy of the excited electron is not exchanged with surrounding molecules or converted into vibration, rotation and/or translation energies inside the molecule. Under this condition, the relaxation of the $\pi$ - electron from the higher energy state $\left(E_{L U M O}\right)$ to the energetic stable energy state $\left(E_{\text {НОмо }}\right)$ will result in emission of light. The energy content of the emitted light corresponds to the energy gap between the LUMO and HOMO energy level. Hence, the energy gap can be calculated with the wavelength of the emitted light using equation (1). 
Energy gap between $E_{L U M O}$ and $E_{\text {Hомо }}$

$$
\Delta E=E_{L U M O}-E_{\text {HOMO }}=\frac{h c}{\lambda_{e m}}
$$

Where

$$
\begin{aligned}
& \Delta E \text { is the energy gap between } E_{L U M O} \text { and } E_{\text {HOMO }}(\mathrm{eV}) \\
& h \text { is the Planck constant }\left(h=6.626 \cdot 10^{-34} \mathrm{Js}\right), \\
& c \text { is the speed of light in a vacuum }\left(c=299,792,458 \mathrm{~ms}^{-1}\right) \\
& \lambda_{e m} \text { is the wavelength of the emitted light }(\mathrm{m}) \\
& E_{L U M O} \text { is the energy level of the lowest occupied molecular orbital }(\mathrm{eV}) \\
& E_{H O M O} \text { is the energy level of the highest occupied molecular orbital }(\mathrm{eV})
\end{aligned}
$$

The wavelength of the emitted light can be dertermined experimentally by flurorescence spectroscopy. It is important to note that the absorption wavelength is always smaller than the emission wavelength.

The wavelength of the absorbed or emitted light of organic flurophores with a linear conjugated $\pi$ - electron system can also be estimated mathematically by the "particle in a one-dimensional box" model [15]. The "particle in a one-dimensional box" is a simplified model of quantum mechanics used to describe the properties of very small particles and molecules. The basis of this model is that the energy of any type of motion such as electron, vibration, rotation and translation is quantised to discrete energy levels given by the quantum number $n(n=1,2,3 \ldots)$. This model provides approximations of the energy levels in molecules with a conjugated $\pi$ - system.

\section{Quantised energy levels for an electron described in a one-dimensional box [15]}

$$
E=\frac{h^{2}}{8 m_{e} L^{2}} \cdot n^{2}
$$

Where

$$
\begin{aligned}
& E \text { is the energy level of a } \pi \text { - electron in a linear conjugated system }(\mathrm{eV}) \\
& h \text { is the Planck constant }\left(h=4.135667516 \cdot 10^{-15} \mathrm{eV} \cdot \mathrm{s}\right), \\
& L \text { is the length of a one-dimensional box }(\mathrm{m}), \\
& m_{e} \text { is the mass of the electron }(\mathrm{kg}), \\
& n \text { is the quantum number }(n=1,2,3 \ldots)
\end{aligned}
$$

Here, the length $L$ is defined by the length of the conjugated electronic structure of the molecule and each quantum number $n(n=1,2,3 \ldots)$ corresponds to a wave function 
$\left(\psi_{n}\right)$. Each wave function can be referred to as a molecular orbital $(M O)$ and its respective energy is the orbital energy. As shown in figure 1.4, each $\pi$ - $M O$ is filled up by a maximum of two $\pi$ - electrons with opposite spin starting from the lowest to the highest molecular orbital following the Pauli Exclusion Principle. After filling the $\pi$ $M O$ with $\pi$ - electrons, the energy gap between the quantised molecular orbital of $L U M O$ and $H O M O$ can be expressed according to the following equation (3).

\section{Energy gap between the energy levels LUMO and HOMO [16]}

$$
\Delta E=E_{L U M O}-E_{\text {HOMO }}=\frac{h^{2}}{8 m_{e} L^{2}}\left(n_{L U M O}^{2}-n_{\text {HOMO }}^{2}\right)
$$

Where

$$
\begin{aligned}
& n_{L U M O} \text { is the quantum number of the wave function presenting } \operatorname{LUMO}(n=1,2,3 \ldots) \\
& n_{\text {HOMO }} \text { is the quantum number of the wave function presenting } \operatorname{HOMO}(n=1,2,3 \ldots)
\end{aligned}
$$

This mathematical model is only valid by assuming that there are no intramolecular interactions between the $\pi$ - electrons such as electron repulsion and they move through a $\pi$ - electron cloud above and below the covalent $\sigma$ - bondings of the molecular structure. According to this model, the energy levels of LUMO $\left(E_{L U M O}\right)$ and HOMO $\left(E_{\text {НОмO }}\right)$ in a linear conjugated system is dependent on the length $L$ of the delocalised $\pi$ electron system and the number of delocalised $\pi$ - electrons. The attachment of additional double or triple bonds or aromatic rings at the molecule will increase the conjugated electron structure and hence the length $L$ of the one-dimensional box in equation (2 and 3). Such extensions will result in the lowering of the energy levels of the $\pi$ - $M O$ and the molecule obtains a lower potential energy which in turn increases the stability of the molecule. However, the lowering of the energy levels also affects the energy gap between $L U M O$ and HOMO. For example, by adding additional double and triple bonds to the cyanine molecule, the energy gap between LUMO and HOMO decreases allowing the absorption of light with lower energy content. Hence, cyanine molecules of different length show different optical properties. In summary, it can be stated that the wavelength of the absorbed and/or emitted light is strongly dependent on the number of double and triple bonds in a delocalised $\pi$-electron system of an organic fluorophore. 


\subsubsection{Coordination complexes}

Another group of fluorophores are certain types of coordination complexes. In a coordination complex, a ligand is attached to a central metal cation by a "coordinate covalent bond". This bonding between the ligand and central atom is usually formed by a Lewis acid / base reaction. The ligand has a lone pair of electrons (= Lewis base electron pair donor) which is able to donate into an empty metal orbital (= Lewis acid electron pair acceptor) and form thereby a coordination complex (=Lewis adduct). The donation of an electron pair from a donor to an acceptor is usually indicated by an arrow, for example $D \rightarrow A(D=$ Donor; $A=$ Acceptor $)$.

A typical feature of coordination complexes is their intense bright colour. The colour is based on the absorption of light with a certain wavelength in the visible spectrum and the transmission of others. The absorption of light in a coordination complex is strongly dependent on the energy splitting of the atomic orbitals of the metal ion and their occupation with electrons. The energy splitting or arrangement of the atomic orbitals in a coordination complex is usually described with Crystal Field Theory [17]. This theory implies that ligands, characterised as point charges, have a strong influence on the electron configuration of metal atoms, since they induce a static electric field. Depending on the type of ligand surrounding the metal ion, the energy levels of the degenerate d- orbitals can split differently producing two d orbital states. The loss of degeneracy of those orbitals is also the reason for the formation of different geometrical structures of metal complexes [18].

The difference in energy between the two d orbital states is referred to as crystal field splitting energy $(\Delta)$. Depending on the crystal field splitting energy induced by the ligand, the two d orbital states in a transition metal complex can be filled by electrons in two different ways. In the presence of a strong ligand field $\left(\Delta_{\text {large }}\right)$, electrons will first fill up orbitals with a lower energy (see figure 1.5; right). Here, it is important to note that pairing energy is needed in order to force an electron to fill an orbital that is already occupied with an electron. In the presence of a weak ligand field $\left(\Delta_{\text {small }}\right)$, the electron can also fill higher energy orbitals to avoid the pairing energy (see figure 1.5; center). 


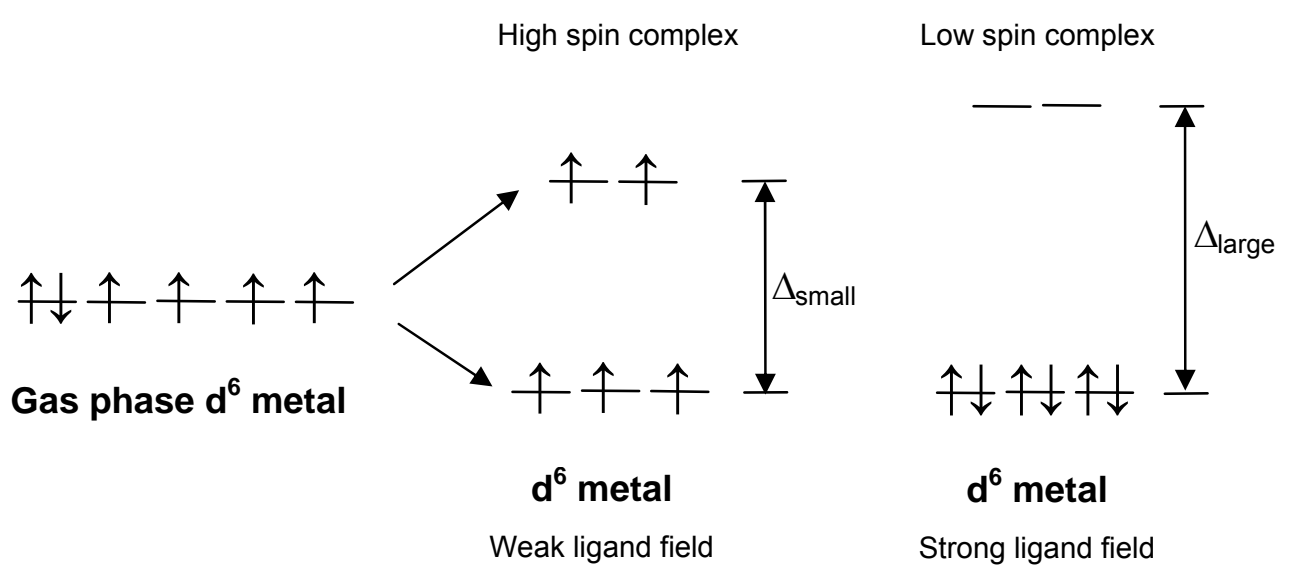

Figure 1.5: MO-diagrams of the five $d$ - orbitals splitting of a $\mathrm{d}^{6}$ metal in an octahedral field.

The loss of degeneracy of the d- orbitals in transition metal complex allows electronic transitions between the d orbital states. For example, electrons occupying d- orbitals of a lower energy can be transferred to an empty d- orbital with a higher content of energy under the absorbtion of light. This electronic transition is shown by a colour of the complex due to the absorption of light in the visible spectrum. The wavelength of the absorbed light is determined by the energy difference between the $\mathrm{d}$ orbital states. Hence, equation (1) can be used to relate the wavelength of the absorbed light to the energy difference between d-orbitals of the metal which have been split due to the ligand field.

The loss of degeneracy of the d- orbitals in transition metal complex allows charge transfer (CT) processes shown by a bright intense colour. A charge transfer process is a phenomenon that can occur in organic and inorganic compounds. In a coordination complex, the charge transfer occurs due to the absorption of light and involves electron transfer between molecular orbitals of the metal atom and its ligands. The electronic transition can occur from the metal atom to the ligand (electron transfer is referred to as a metal-to-ligand charge-transfer process; MLCT) or from ligand to metal atom (electron transfer is referred to as a ligand-to-metal charge-transfer process; LMCT). Independently from the electron transfer process, the wavelength of the absorbed light determines the energy difference between the $M O$ s. The electronic transition transfers the coordination complex into an excited state. The excited state, however, is not stable and the charge-transfer-complex will return into its ground state. 
A charge transfer process can also occur between an organic compound acting as a bior multidentate ligand and a transition metal ion (such as $\mathrm{Mn}^{2+}, \mathrm{Fe}^{2+}, \mathrm{Ni}^{2+}, \mathrm{Cu}^{2+}$ and $\mathrm{Zn}^{2+}$ ). Coordination complexes consisting of a metal centre and organic ligands, which are bound to the metal centre by two or more coordinate covalent bonds, are known as chelate complexes. Some chelates show optical fluorescence, especially when the organic ligand has a conjugated $\pi$ - electron structure. A typical example is tris-(8hydroxyquinolinato) aluminium $\left(\mathrm{Alq}_{3}\right) . \mathrm{Alq}_{3}$ has excellent electroluminescence properties and therefore it is used in organic light-emitting diodes (OELD) [19].

In chelates, the fluorescence is caused by electron transfer between $\sigma$ - or $\pi$-MOs of the organic ligand and d- orbital of a transition metal ion (LMCT). Depending on the conjugated $\pi$ - electron structure of the ligand, different contents of energies can be absorbed through photo-excitation. As mentioned before, organic molecules with a greater extended conjugated and / or an aromatic electronic structure can absorb light with a lower content of energy (see cyanine example). Under the absorption of light, a $\pi$ - electron leaves its molecular orbital (HOMO) and occupy a d-orbital of the metal atom with a higher energy content $(L U M O)$. The electron transfer converts the chelate in an excited state. In order to return to the ground state, the excited electron releases its excess energy by emitting light. By doing so the electron returns to its initial molecular orbital $(H O M O)$.

The fluorescence colour in chelates is usually determined by several factors such as the crystal field splitting energy $(\Delta)$, chemical interaction with the surrounding medium (solvent molecules) and intermolecular interaction between adjacent chelates. These factors, however, will be discussed more in detail in the introduction part of chapter 7.

\subsubsection{Quantum dots}

Quantum dots are nano-scale inorganic semiconductor materials typically 1-10 nm in size, which exhibit interesting optical, electronic, magnetic and catalytic properties. Fluorescence, in particular, is a prominent feature of quantum dots. The fluorescence activity of quantum dots is due to the diverse size- and shape-dependent characteristics of inorganic semiconductor materials, which are a completely different in their physical and chemical properties from their bulk form [20-23]. In the recent decades, great effort 
has been put into the production of quantum dot materials with intense, stable, spectroscopically pure fluorescent colours, when excited electronically or with UV light. For this reason, quantum dots are now widely used and have applications ranging from optoelectronic devices such as light-emitting diodes [24], laser diodes [25] and solar cells [26], fluorescent labelling in biotechnology (e.g. biochips) and medical imaging (optical detection of certain cancerous tumours) [27].

The fluorescence activity of quantum dots is caused by the quantum confinement effect of an inorganic semiconductor material [28]. To understand the quantum confinement effect, a basic description of the electronic band structure of a macroscopic semiconductor material is required. The electronic band structure (or simply band structure) of an inorganic semiconductor is typically characterised by a band gap between its valence and its conduction band as shown in figure 1.6. This gap between both bands corresponds to a forbidden zone, which can not be occupied by electrons.

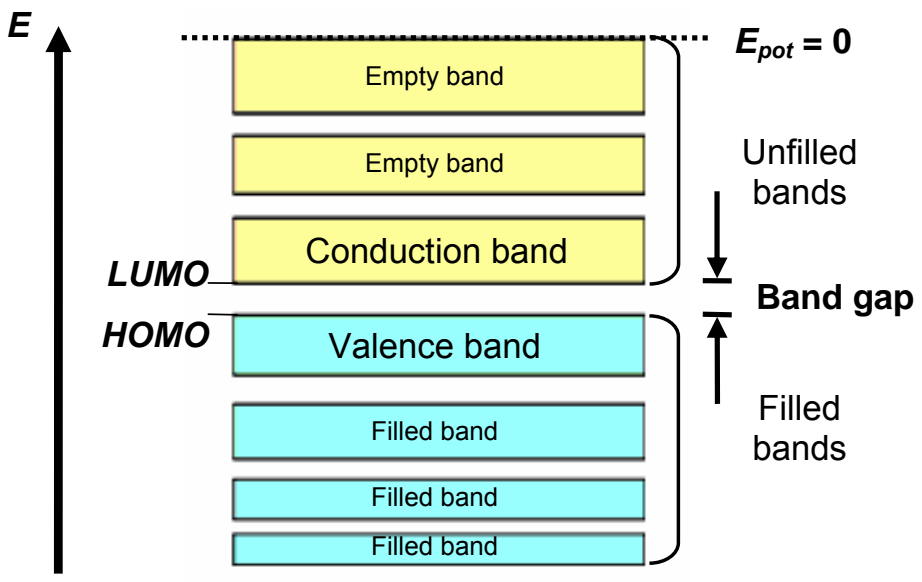

Figure 1.6: Electronic band structure of a macroscopic semiconductor crystal showing continuous bands of energy formed by the overlap of orbital's from adjacent atoms.

The valence band corresponds to the highest energy band that is fully occupied with electrons in the ground state, while the conduction band is the lowest energetic band with unoccupied energy states. The band gap in a solid can also be expressed by the molecular orbital $(M O)$ theory.In this theory, the band gap is defined by the zone between the lowest unoccupied molecular orbital (LUMO) from the conduction band and the highest occupied molecular orbital from the valence band (HOMO). The energy difference between the $L U M O$ and HOMO level represents the band gap energy which is calculated by the formula in equation (4). 


\section{Band gap energy}

$$
E_{g}=E_{\text {LUMO }}-E_{\text {НОМО }}
$$

Where

$E_{g}$ is the band gap energy $(\mathrm{eV})$

$E_{L U M O}$ is the energy level of the lowest occupied molecular orbital (eV)

$E_{\text {НОмо }}$ is the energy level of the highest occupied molecular orbital (eV)

The band gap of semiconductor materials is considerably smaller than that of insulating materials. Even at room temperature, a small number of electrons are able to gain enough thermal energy to leap the band gap from the valence band to the conduction band. By crossing over the band gap, an electron will leave a hole behind in the valence band. Due to the high affinity of electrons to occupy a low energy state by being closer to the nucleus, the hole is refilled by surrounding electrons with a higher content of energy inside the valence band. As a result, the hole is displaced to the top of the valence band $(H O M O)$ and the excess energy of the electrons filling the intermediates holes is exchanged in the form of lattice vibrations [28].

In solid-state physics, the electronic band structure describes those ranges of energy that an electron can attain in a solid. From quantum mechanics view, the energy bands correspond to a large number of discrete quantum states. The bands are formed by the overlap of atomic orbitals much as molecular orbitals are formed by the overlap of atomic orbitals in small molecules. The overlap of the atomic orbitals depends on the distances between the atoms. When the distance between two atoms is small enough to interact with each other, the discrete energy levels of each atom $(1 \mathrm{~s}, 2 \mathrm{~s}, 2 \mathrm{p}, 3 \mathrm{~s} . .$.$) split$ and form two MOs with high and low energy, repectively. As more atoms interact with each other, more orbitals combine to form MOs. The energy differences among the $M O \mathrm{~s}$ decreases as the number of atoms increases. In general, the interaction of $N$ atoms will split an energy level into $N$ levels. In a macroscopic semiconductor crystal, the energy level splitting of the discrete energy levels of $N$ atoms causes a continuous band of energy. By decreasing the size of the semiconductor crystal, the number of atoms declines in the crystal as well as the number of MOs. This will increase the energy differences between individual orbital energy levels, and also the gap between energy bands as shown in figure 1.7. 


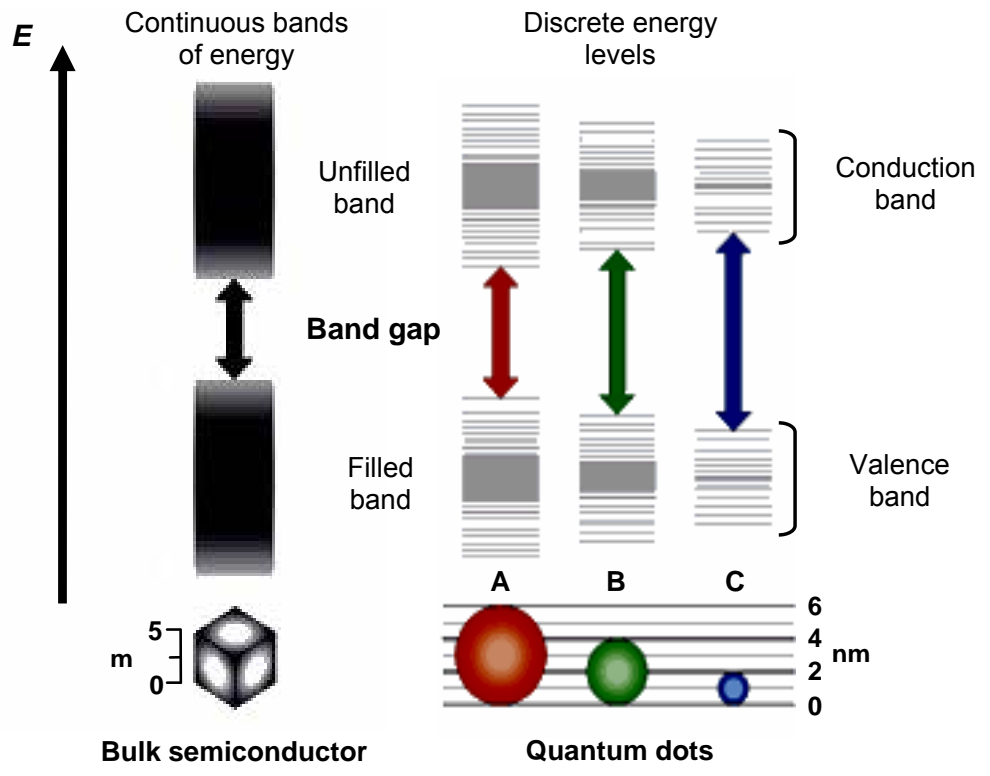

Figure 1.7: Electronic band structure of bulk semiconductor and nanometric semiconductor crystals [30].

With the reduction in size of the semiconductor crystal, electrons are restricted to certain energy levels. This restriction is also known as the "quantum confinement effect", and it can be determined experimentally by spectroscopy (UV-vis and Fluorescecne spectroscopy). Due to the quantum confinement effect, quantum dots show electroluminescent and photoluminescent features, when excited thermally, electrically or photo electrically [30]. Figure 1.8 illustrates the different steps for the fluorescence occurrence in quantum dots.

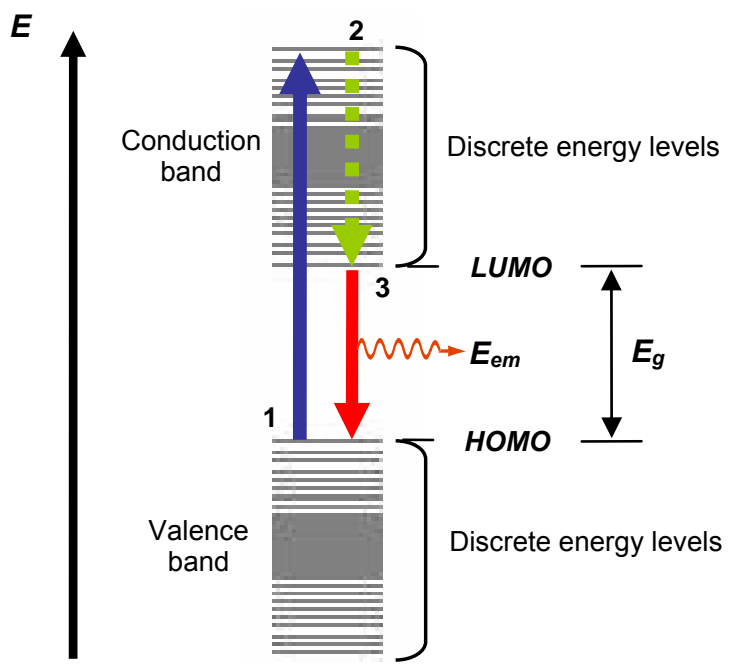

Figure 1.8: Illustration of the relationship between light emission and band gap energy. Arrow 1: jump of the electron over the band gap by the adsorption of energy through UV irradiation or thermal excitation; Arrow 2: fall of the excited electron to the LUMO level through different heatemitting steps; Arrow 3: radiative recombination of the electron-hole pair between the LUMO and HOMO level. 
When irradiated with ultraviolet light, an electron gains sufficient energy to leap the band gap from the valence band to the conduction band (figure 1.8; Arrow 1). Due to quantum confinement effect, however, the energy states are quantised which means that the electron can only absorb certain amount of energy to reach superior orbitals of the conduction band. By doing so, a hole remain in the valence band which is relocated at the top of the valence band (HOMO). After different heat-emitting steps the excited electron in the conduction band falls back to the lower edge of the conduction band (LUMO) (figure 1.8; Arrow 2). In this state, an electron-hole pair (exciton) is formed and the band gap is represented by the energy difference of the electron at the lower edge of the conduction band (LUMO) and the hole at the top of the valence band $(H O M O)$. Finally, optical fluorescence appears due to the radiative recombination of the electron-hole pair (figure 1.8; Arrow 3). The excess energy of the electron is converted into light and it is directly related to the band gap energy $E_{g}$ of the inorganic semiconductor crystal. Hence, the band gap energy $E_{g}$ can also be associated with the wavelength of the emitted light according to equation (1). This formula correlates the energy of the emitted light with the energy difference between LUMO and HOMO, which defines the band gap energy $E_{g}$ of the quantum dot.

The band gap energy in a quantum dot can be calculated using equation (5), which considers the different forces acting on an electron in semiconductor material. It is important to note that the movement of an electron through a crystal is not only affected by the potential of the atomic nuclei, but also by internal forces such as electronelectron interactions and nucleus-nucleus interaction. These forces also determine the energy states of quantum dots.

Band gap energy of spherical quantum dots [23]

$$
E_{g}=E_{g}(\infty)+\underbrace{\frac{h^{2}}{8 L^{2}}\left(\frac{1}{m_{e}^{*} m_{e}}+\frac{1}{m_{h}^{*} m_{e}}\right)}-\underbrace{\frac{1.8 e^{2}}{\varepsilon} k_{c} \frac{1}{L}}
$$

(A)

(B)

Where $\quad E_{g}(\infty)$ is the band gap of the macroscopic semiconductor crystal $(\mathrm{eV})$

$m_{e}{ }^{*}$ is the effective mass of the electron (dimensionless variable)

$m_{e}$ is the mass of the electron $(\mathrm{kg})$

$m_{h}{ }^{*}$ is the effective mass of the hole (dimensionless variable)

$h$ is the Planck constant $\left(h=4.135667516 \cdot 10^{-15} \mathrm{eV} \cdot \mathrm{s}\right)$ 


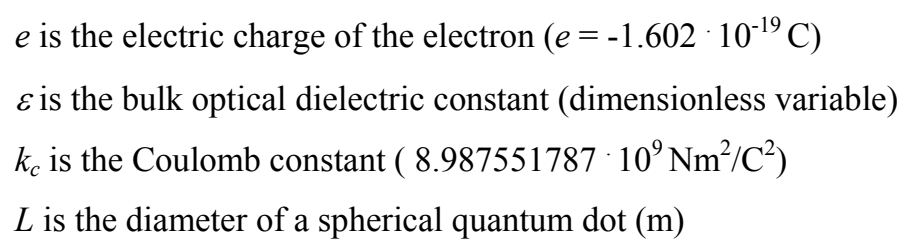

The term (A) in equation (5) corresponds to the kinetic energy of the electron and hole, described as particles in a spherical box with diameter $L$ (see equation (2)). The term (B) corresponds to the Coulomb attraction between electron and hole, idealised as two point charges, presented in the exciton state. By introducing the dimensionless variables $m_{e}{ }^{*}$ and $m_{h}{ }^{*}$, the mass of the electron and the hole in the exciton state is expressed by an effective mass, which considers the effects of internal forces acting on the electron moving through a solid. The electron and hole effective masses are different for each semiconductor material. The term $E_{g}(\infty)$ correspond to the band gap of the macroscopic semiconductor crystal.

Equation (5) indicates that the band gap energy is directly related to the size of the quantum dot $(L)$. By decreasing the size of the nanocrystals, the term $\left(1 / L^{2}\right)$ becomes more significant. This is more evident when the particle size is smaller than the Bohr exciton radius $\left(\alpha_{B}\right)$ shown in equation (6).

\section{Bohr exciton radius [31]}

$$
\alpha_{B}=\frac{h^{2} \varepsilon}{e^{2}}\left(\frac{1}{m_{e}^{*} \cdot m_{e}}+\frac{1}{m_{h}^{*} \cdot m_{e}}\right)
$$

By approaching a size similar to the Bohr exciton radius, the motion of an electron will be confined in all three spatial directions. Once the particle size becomes smaller than the Bohr exciton radius, the band gap energy of the semiconductor material will progressively increase according to equation (5). The increment of the band gap energy is referred to the quantum confinement effect. The quantum dot size effect is also illustrated in figure 1.7. The reduction in the size of the quantum dot results in the increment of the band gap energy, which in turn change the light absorption and emission properties of the quantum dots. Hence, quantum dots of the same semiconductor material, but of different sizes, can emit light of different colours. 
Semiconductors of the group II-VI and III-V are typical quantum dot materials which can be used to examine the quantum dot size effects. For example, semiconductor material formed from elements II-VI such as chalcogenides of lead and cadmium (PbE and $\mathrm{CdE}$ with $\mathrm{E}=\mathrm{Se}, \mathrm{S}$ and $\mathrm{Te}$ ) exhibit bright fluorescent colours which depends strongly on the particle size. Despite their excellent luminescence quantum yield and high photostability, a great drawback of these materials is their high toxicity. Fluorescence colours of cadmium selenide (CdSe) quantum dots sorted by size $(\sim 2-8$ $\mathrm{nm})$ are shown in figure 1.9. The emission wavelength shifts with the increment of the particle size from the blue to the red end of the visible spectrum.

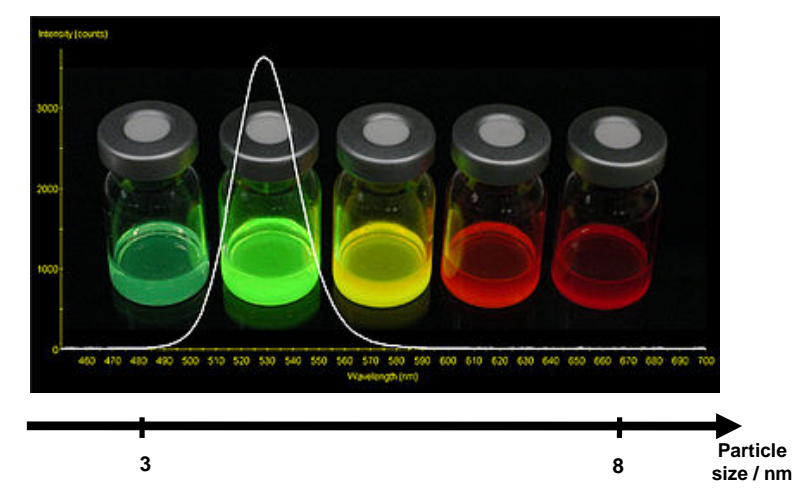

Figure 1.9: Quantum dots of CdSe arranged by size emitting light in different colours. The quantum dot suspensions have been excited simultaneously by a single excitation wavelength [copyright NASA, USA].

Light emission and absorption are not only dependent on the physical dimension of the nanostructure, other factors such as shape, chemical composition, type of semiconductor material and nature of the surrounding medium also affect the energy value of the band gap and hence the colour of the light emitted by a semiconductor material.

Inorganic semiconductors are classified into two groups - direct and indirect semiconductors [32]. The difference of the materials is shown in their dispersion relation $E(k)$ or "energy-momentum dispersion", which illustrates the energy states of the valence and conduction band as a function of $k$. In quantum mechanics, the pseudomomentum of an electron is usually represented by a wave number $k$. The wave number $k$ is commonly used in quantum mechanics in preference to the momentum.

The movement of an electron in a macroscopic crystal is very complex and effects such as nucleus vibration, nucleus-nucleus interaction, electron-nucleus attraction, electron- 
electron interactions and electron spin have to be considered. This complexity is reflected in the energy-momentum diagram $E(k)$ showing the energy states as curves with a non-parabolic character (see figure 1.10). The non-parabolic characters of the edges of the valence and conduction band can be explained by assuming that electrons and holes behave like particles showing a mass-dependency on their direction of movement within a crystal. Because of this reason, dimensionless variables $m_{e}{ }^{*}$ and $m_{h}{ }^{*}$ are introduced for each semiconductor material. These variables act as corrective factors in equation (5). The difference of the dispersion relation $E(k)$ in a direct and indirect semiconductor is illustrated in figure 1.10.
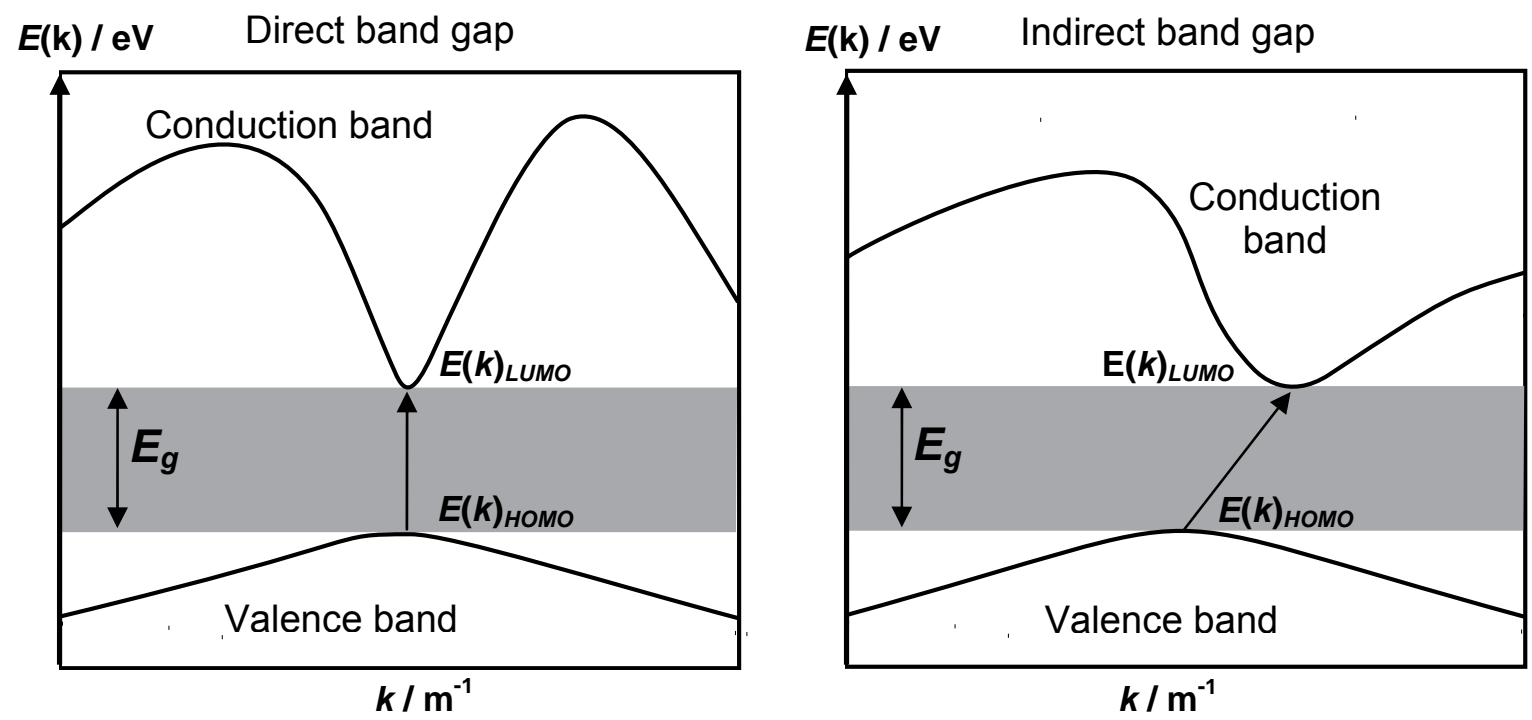

Figure 1.10: Energy-momentum dispersion of a direct band gap (right) and indirect band gap (left).

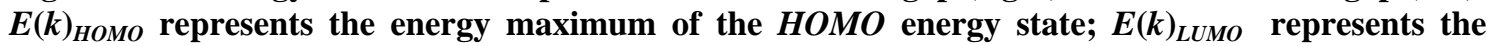
energy minimum of the LUMO energy state.

The energy diagrams $E(k)$ of a direct and indirect semiconductor show that the top of the valence band has at least one energy maximum and the bottom of the conduction band shows respectively one energy minimum. The energy maximum at the top of the valence band is referred to the highest occupied molecular orbital $E(k)_{\text {Номо }}$ and the energy minimum at the bottom of the conduction band is referred to the lowest unoccupied molecular orbital $E(k)_{L U M O}$ (see figure 1.10). Although the energy states changes with the $k$-value, the energy-momentum diagram $E(k)$ provides enegy difference between the energy states $E(k)_{L U M O}$ and $E(k)_{\text {HOMO }}$, which defines the band gap energy of a semiconductor material. 
In order to excite an electron from the valence band to the conduction band, the energy required has to be at least the difference between $E(k)_{L U M O}$ and $E(k)_{\text {HOMO. If these }}$ energies have the same $k$-value, which occurs in a direct band gap semiconductor, it is easy to excite an electron with a photon. The electron has to undergo a change in its energy level but not in its momentum. However, if $E(k)_{\text {НОMO }}$ and $E(k)_{L U M O}$ appear at different $k$-values, which exist in an indirect band gap semiconductor, then the electron has to undergo a change in its momentum before it can reach the conduction band. Thereby, an electron has to interact with a phonon. Phonons are connected to the vibration energy of the crystal lattice for which the vibration motion is also quantised [33]. By interacting with a phonon, the electrons either gain or lose discrete values of momentum. Basically the same effects occur for the recombination of the electron-hole pair. Before the excited electron can return from the lower edge of the conduction band to the hole on the top of the valence band, it has to interact first with a phonon to change its $k$-value and then can release the excess energy by emitting a photon.

It is important to note that a photon has a very low momentum compared to the lattice vibration. By interacting with phonons, an excited electron is able to release the excess energy completely to the lattice vibration which causes heat. By converting the excess energy in heat, however, the electron-hole recombination will take place without light emission. For this reason, direct semiconductors are preferred in optoelectronics because they are more efficient as light emitters than indirect gap semiconductors materials. All these processes of gaining and releasing of energy are determined by the physical law of Conservation of Energy and the Conservation of Momentum respectively [33].

Defects in the crystal structure, created by impurities for example, also influence the radiative recombination of the electron-hole pair. The incorporation of impurity ions into the crystal lattice of quantum dots leads to additional energy levels within the band gap where an electron or hole can be trapped. The process of adding impurities in a controlled manner to a semiconductor material is known as "doping" [34]. Depending on the type of the dopants (donor or acceptor), the introduced energy states are either very close to the conduction band (donor) or near the valence band (acceptor). Semiconductor material doped with electron donor impurities are called "n-type", while those doped with electron acceptor impurities are known as "p-type". The band gap 
between the introduced energy states and the nearest energy band is referred to as dopant-site binding energy $E_{B}$.

Figure 1.11 shows the electronic band structure of an n-type and p-type semiconductor material and the corresponding dopant-site binding energy $E_{B}$.

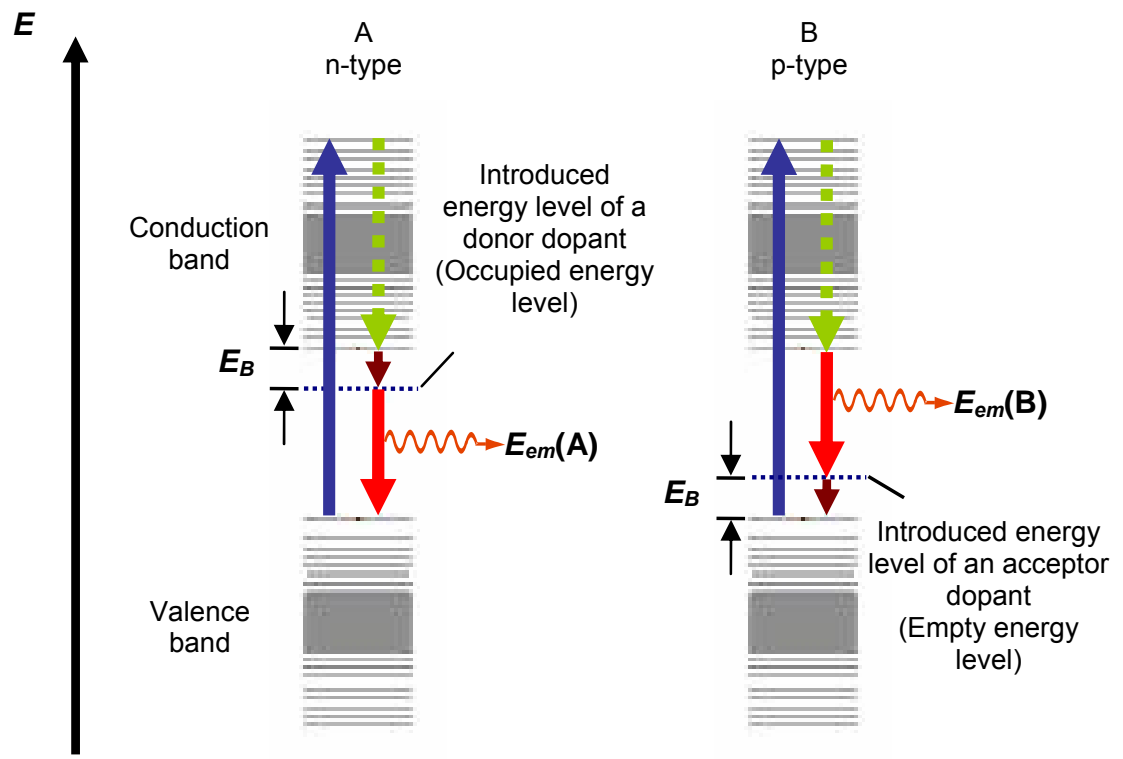

Figure 1.11: Band structures of a n-type (A) and p-type (B) semiconductor. Semiconductor A: donor impurities create energy states within the band gap near the conduction band. Semiconductor B: acceptor impurities create energy states within the band gap near the valence band.

It is important to note that the incorporation of additional doping levels in the crystal structure can eventually transform a direct semiconductor into an indirect semiconductor by changing their energy-momentum dispersion relation [32]. Nevertheless, the controlled addition of small quantities of dopants into the crystal lattice can be used to shift the emission wavelength within the visible range. To dope semiconductor materials, small quantities of impurities such as transition metal ions $\left(\mathrm{Co}^{2+}, \mathrm{Ni}^{2+}, \mathrm{Mn}^{2+}, \mathrm{Cu}^{2+}\right)$ and rare earth metal ions $\left(\mathrm{Ce}^{3+}, \mathrm{Eu}^{3+}, \mathrm{Er}^{2+/ 3+}\right)$ are used to obtain additional energy level within the band gap. Transition metal ions are characterised for partially filled d-orbitals with a few exception (i.e. $\mathrm{Zn}^{2+}, \mathrm{Cd}^{2+}$ and $\mathrm{Hg}^{2+}$ ). Rare earth metal ions are the lanthanide series. These elements have incomplete 4f- orbitals, which provide unoccupied orbitals for electron transitions. 
A change of the medium surrounding an inorganic semiconductor can also cause a shift of its emission wavelength. Nanocrystals dispersed in a solvent can undergo solvation processes which can result in the stabilization or destabilization of the particles in the dispersion medium. The solvation usually results in the stabilization of the energy states and an increment of the band gap energy. The enlargement of the band gap, however, will lead to the emission of light with a shorter wavelength such as seen in figure 1.12.

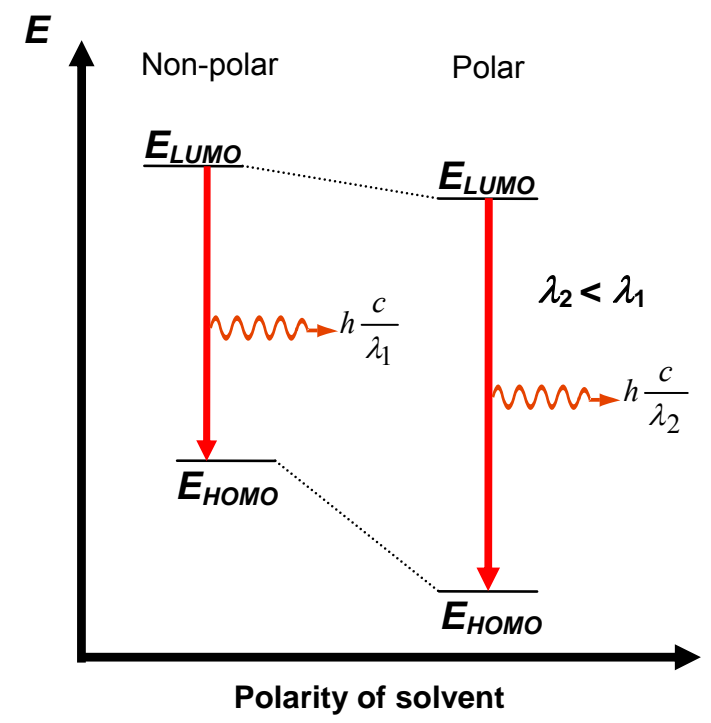

Figure 1.12: Graphic illustration of the solvation effect (solvatochromic effect) at the energy states $E_{L U M O}$ and $E_{\text {Hомо }}$, depending on the polarity of the solvent used (polar and non-polar, respectively).

Hence, nanocrystals of the same semiconductor material may show different fluorescence colours depending on the solvent they are dispersed in. This effect is known as solvatochromism. The extent of the stabilization of the energy states is usually determined by the polarity of the solvent given by the dielectric constant and the polarity of the ground and excited state of the nanocrystals. A polar solvent will usually stabilize the energy levels in a nanometric crystal to a greater extent than non-polar solvents.

However, solvents with a high dielectric constant can destabilize the particles in the suspension causing agglomeration and precipitation of the nanocrystals in solution. In general, inorganic nanocrystals contain a surface charge due to the presence of metal ions at the surface. When immersed in a liquid medium, they will attract other ionic species with opposite charge present in the solution. These ionic species, which can be ions or ionic molecules, will be tightly attached by electrostatic forces to the surface of the particles and form an electrically charged layer at the interface of the particles. This 
electrically charged layer prevents the particles from coming into close contact with each other through Coulomb repulsion. The repulsive force is dependent on the thickness and charge of the electrostatic layer around the particles. The thicker the electrolyte layers with similar charge, the stronger are the electrostatic repulsion between the particles.

The thickness of the electrostatic repulsion layer, however, is dependent on the ionic strength of the dispersion medium. In general, the higher the ionic strength of the suspension, the lower is the uptake of ionic species at particle interface and the thinner the electrostatic layer around the particles is. This, however, will destabilize the particles in the dispersion medium due to the reduction of repulsive forces between the particles. As a consequence, solvated particles tend to agglomerate in solution and form polycrystalline particles. By sticking together, larger particles / aggregates can be formed, which can eventually precipitate out from the suspension under the influence of gravity (phase separation). To avoid aggregation / agglomeration processes, the choice of the solvent is crucial. In general, the ionic strength of the dispersion medium is determined by the polarity of the solvent and the presence of ions and / or ionic molecules. Solvents with a high dielectric constant (polar solvent) will reduce the thickness of the charged repelling layer at the interface of particle surface. A list of solvents, grouped into non-polar aprotic, polar aprotic and polar protic solvents, is presented by increasing polarity in the table 1.1 .

Table 1.1: List of non-polar aprotic, polar aprotic and polar protic solvents with corresponding dielectric constants $\left(20^{\circ} \mathrm{C}\right)$.

\begin{tabular}{lclclc}
\hline $\begin{array}{c}\text { Non-polar } \\
\text { aprotic solvent }\end{array}$ & $\begin{array}{c}\text { Dielectric } \\
\text { constant }\end{array}$ & Polar aprotic solvent & $\begin{array}{c}\text { Dielectric } \\
\text { constant }\end{array}$ & $\begin{array}{c}\text { Polar protic } \\
\text { solvent }\end{array}$ & $\begin{array}{c}\text { Dielectric } \\
\text { constant }\end{array}$ \\
\hline Hexane & 2 & Ethyl acetate & 6 & Acetic acid & 6.2 \\
$\begin{array}{l}\text { Tetrachloro- } \\
\text { methane }\end{array}$ & 2.2 & Tetrahydrofuran & 7.5 & Ammonia & 16.5 \\
1,4 Dioxane & 2.3 & Dichloromethane & 9.1 & n-Butanol & 18 \\
Benzene & 2.3 & Acetone & 21 & Isopropanol & 18 \\
Toluene & 2.4 & $\begin{array}{l}\text { Hexamethyl- } \\
\text { phosphorotriamide }\end{array}$ & 30 & n-Propanol & 20 \\
Diethyl ether & 4.3 & Acetronitrile & 37 & Ethanol & 30 \\
Chloroform & 4.8 & Dimethylformamide & 38 & Methanol & 33 \\
& & Dimethylsulfoxide & 47 & Formic acid & 58 \\
& & & & Water & 80 \\
\hline
\end{tabular}


By adding additional ionic species to the dispersion medium (concentration of ions increases in the dispersion medium), the ionic strength of the dispersion medium will also increase, which in turn will reduce the thickness of the electrostatic layer around the particles. As a consequence, particle show a higher chemical reactivity to adhere together by van der Waals forces. Particle growth via aggregation or agglomeration, however, has negative effects on the fluorescent properties of quantum dots. By sticking together, the energy of excited quantum dots can be released by non-radiative transition processes. These processes occur at the interface of the particles via an energy transfer mechanism that involves vibration and electronic transitions. The energy transfer results in several heat-emitting steps, whereby an excited quantum dot returns to the ground state without emitting light. Processes that decrease the fluorescence intensity of optical active substances are generally termed quenching.

There are several processes that lead to quenching such as charge transfer reactions, excited state reactions or complex formations in the ground state [35]. Quenching also occurs in the presence of chemical reactive molecules such as molecular oxygen, chloride, iodine and acrylamide. These quencher molecules exchange energy by collision according the donor-acceptor principle. Thus, energy will be transferred from the excited particle (donor) to the quencher molecule (acceptor). The extent of quenching depends on the donor-acceptor distance and the type of chemical interaction. Quenching processes are also highly dependent on ambient conditions such as pressure and temperature.

In summary, the fluorescence colour of quantum dots is directly related to the band gap energy which is defined by the the energy difference between the energy states of $L U M O$ and HOMO. These discrete energy states are determined by the particle dimension due to the quantum confinement effect. As a consequence, by changing the size of the quantum dots, the colour of the fluorescence can be tuned. Furthermore, the the wavelength of the emitted light relased by the radiative recombination of the electron-hole pair can be influenced by the incorporation of additional energy levels of dopant ions within the band gap of the quantum dots. Neverthless, the fluorescence mechanism of quantum dots is quite complex and different processes can happen simultaneously, which often leads to quenching of the fluorescence. 


\subsection{Quantum dot - fibre composites}

Quantum dots showing bright fluorescence colours under UV-light can be embedded in a fibrous substrate material such as wool and paper. When the chemical interaction between the quantum dots and the fibrous material is attractive, the fibre will be covered by a surface layer of quantum dots which will functionalise it with optical fluorescence under UV-light. Because of the additional chemical and physical properties that the quantum dots give to the substrate material, the fabrication of such materials can be of great interest for the fashion and paper industry.

For example, quantum dot - composites materials with tuneable optical fluorescence and electronic properties may have multiple applications ranging from security packaging and labels, in the paper industry, to new photoluminescent textiles and product authentication, in the textile and fashion industry [36]. They can also be incorporated into plastic products and used to create discrete barcodes to mark valuables or commercial goods.

New Zealand is known for its export of wood pulp, paper and wool products [37]. However, competition in international market is strong. To stand out, New Zealand has to add significant value to their primary resources. New Zealand paper and wool industry can greatly benefit from the use of nanotechnology in the fabrication of their products. In general, wool and paper fibres make excellent substrate materials to combine with the properties of nanosized materials. In fact, the adsorption of quantum dots onto wool and paper could be facilitated by the presence of functional groups and the large surface area of these fibrous substrate materials. Exploring the possibility to produce new composite materials comprising quantum dots and NZ wool and paper will be one of the main objectives of this thesis.

\subsubsection{Choice of the quantum dot material}

To develop new quantum dot - natural fibre composites, however, choosing the correct quantum dot material is essential for meeting the final criteria of the hybrid material. A great concern with many quantum dot materials is their toxicity. The composition of some well-known quantum dots (such as $\mathrm{CdSe}$ and $\mathrm{PbS}$ ) is often highly toxic or they have been synthesised with high toxic materials. The release of toxic quantum dots into 
the environment by leaching, over longer time, also presents a high health risk to the end user and the water systems. From a commercial and health point of view, the production of quantum dots should be economically and cost-effective. Synthesis methods should make use of simple and cheap equipment and avoid expensive apparatus in order to minimize incidental expenses. Furthermore, the synthesis method selected should have the potential to be used in a scale-up production in view of industrial processing. The preparation of the quantum dot material should also be in line with health and safety regulation and minimise environmental impacts. For the further processing, the quantum dots should be preferably homogeneously dispersed in a solution. The quantum dots should be able to react with the substrate material and form chemical bonds between the surface area of the quantum dots and network structure of the substrate material. To fulfil all these requirements, the choice of quantum dot materials which can be used is greatly limited. There is a demand for quantum dot materials made of non-toxic, or at least less toxic, materials showing bright and intense fluorescence colours after electrochemical stimulation or irradiation with UV light.

For the development of quantum dot - fibre composites, metal oxide semiconductors present appropriate additives for textiles and surfaces that come into contact with human skin. They are also known for their simple production using sol-gel methods [38-44]. Actually, the textile industry already uses metal oxide nanoparticles such as $\mathrm{TiO}_{2}$ and $\mathrm{MgO}$ for their textile finishing [45]. These metal oxides possess photo-catalytic activity to break down harmful chemical and biological agents. Therefore, fabrics previously treated with active carbon can be replaced by fabrics with $\mathrm{TiO}_{2}$ and $\mathrm{MgO}$.

\subsubsection{Synthesis of quantum dots}

The synthesis of inorganic semiconductor materials at nano-scale is difficult and often results in a mixture of crystals of different sizes and shapes. If materials are prepared at nanometer level, they will change their physical and chemical properties significantly in contrast to their bulk form. In particular, the chemical reactivity increases by the reduction in the size of the particles. The chemical activity and physical properties of solid materials are determined by their surface area, which in turn is strongly dependent on the size and structure of the particle. For example, by decreasing the size of an inorganic semiconductor material, the surface area increases considerably with the 
surface-to-volume ratio. This, however, exposes a greater number of atoms at the interfacial surface area, which can strongly interact with the surrounding medium. Furthermore, attractive correlation forces, also known as cohesive forces, among the atoms in a bulk material will subsequently decrease with the reduction in size. These intermolecular forces held the atoms together and prevent them from breaking apart from the solid, especially at the surface. They can also initiate the reorganisation of the atoms at the interfacial surface area. Crystals of very small size can adopt a modified morphology and/or crystal structure so that atoms, especially at the surface, can receive the most stable configuration of minimal energy at nanoscale.

Due to these size-related effects, materials prepared at nanolevel become instable. This is also reflected in the increment of the surface energy by the transition from macroscopic to nanoscopic objects. The driving force of the surface energy is to stabilise the solid phase by minimising the surface area. Therefore, nano-objects with a high surface energy show a higher tendency to undergo chemical reactions such as agglomeration and oxidation reactions. Agglomeration reactions are principally induced by attractive forces between the particles in a dispersion medium. Small particles in a colloidal system are permanently under the influence of Brownian motion, which result in the frequent collision of the particle with each other. Thereby, the stability of the colloidal system is determined by the interaction between the particles during the collision. These interactions can be either attractive or repulsive. Repulsive interactions between the particles will stabilise the colloidal system and the particles remain in a dispersed state. Attractive interaction, however, will result in formation of agglomerates. Here, the particles adhere with each other and this can lead to the coalescence of the entire dispersion. The attraction between the particles originates from van der Waals forces, which are always present between particles of similar compositions. These forces are created either by permanent dipole-permanent dipole interaction (Keesom force), permanent dipole and the corresponding induced dipole interaction (Debye force) or by interaction between two instantaneously induced dipoles (London dispersion force). By sticking together through van der Waals forces, the particles decrease their exposed surface area and obtain a lower level of energy at the surface. Thus, to stabilise colloidal particles in a dispersion medium, van der Waals forces between the particles have to be neutralised. 
Small nanometric crystals are also more likely affected by oxidation processes. Oxidation reactions at the surface usually reduce the surface energy by forming a stable oxide layer around the crystals. Due to the passivation of the surface area with a hard and non-reactive surface film, further particle growth via coalescence is inhibited and the nanocrystals obtain a higher stability at nano-scale. Furthermore, the size and shape of nanoparticles in suspension is also affected by ageing processes. Over time smaller particles may dissolve back into solution and dissolved atoms aggregate onto larger crystals through an "Ostwald ripening" process [46]. This process result in the growth of the particles in the dispersion medium and the overall energy of the colloidal system is reduced due to the decline of the surface-to-volume ratio of the particles. All these processes complicate the production of shape- and size-controlled nanocrystals and make it challenging to maintain stability of the particle size for long-term storage. However, in the recent years many studies have focused in the development of synthetic methods to control size, shape, purity, composition and homogeneity of nanocrystalline semiconductor materials. Nowadays, a variety of nanocrystals with tuneable electronic and optical properties have been described in the literature [47]. Some of these materials are also commercially available and can be handled like bulk chemical substances.

There are basically two synthetic routes available for the production of quantum dots. Synthetic routes are distinguished by the techniques used, which may be either chemical or physical. A tabulation of these techniques is given below.

Table 1.2: Physical and chemical techniques commonly used to prepare quantum dots [46-51].

\begin{tabular}{ll}
\hline Chemical Methods & Physical Methods \\
\hline Heat evaporation & PVD - physical vapour deposition \\
Decomposition of organometallic compounds & CVD - chemical vapour deposition \\
Solvothermal chemical method & ALD - atomic layer deposition \\
Polymerisation method & ALE - atomic layer epitaxy \\
Micro emulsion & MBE - molecular beam epitaxy \\
Chemical reduction & LLIRT - liq.-liq. interface reaction tech. \\
Sol-gel method & ECD - electrochemical deposition \\
Wet chemical precipitation method: & LBL - layer by layer assembly \\
$-\quad$ Arrested precipitation method & CBD - chemical bath deposition \\
$-\quad$ Co-precipitation & Electrochemical reduction \\
& Spray (sputter) deposition \\
\hline
\end{tabular}


In the physical route, small crystals are grown for example by lithographic or molecular beam techniques on a suitable substrate material or host matrix. Generally, the quantum dots, once grown on a substrate, become fixed and can not be removed. The physical route requires the use of special and expensive apparatus and the synthesis is often carried out at high temperature over a long period of time. This can be a potential risk when toxic reagents are involved or created during the procedure. The physical route is also unsuitable for the production of quantum dots on a large scale, because of the costly and complicated apparatus needed for the crystal growth on host matrices.

In the chemical route, on the contrary, nanostructure crystals are dispersed in a liquid phase formed either by particle growth inside restricted matrices or by the methods of colloidal chemistry such as aqueous based precipitation method or chemical reduction methods in solution [47]. Such quantum dot suspensions can be easily purified by centrifugation, filtration or decanting from all unwanted by-products and unreacted chemicals. By doing so, quantum dots can be re-dispersed in solution or transformed in powder by different drying process such as freeze-drying techniques. The chemical route and the wet chemistry methods in particular, have significant advantages because of its easy procedure and the requirement of simple equipment. Furthermore, many chemical methods are carried out under mild reaction conditions with stable agents, which can be non-toxic or have a low toxicity. Chemical methods are basically cheap, efficient and environmentally friendly compared to physical techniques. There is also the possibility to change the procedure from bench top condition to a large scale up production. Consequently, the wet chemical methods for the synthesis of quantum dots at bench top conditions using simple glassware appear the most appealing.

There are many different wet chemical techniques available to prepare colloidal quantum dots (see table 1.2). All these methods are either based on the kinetic control of nucleation and growth of the quantum dots, or stabilization of the quantum dots in a liquid phase. The final product is a dispersion of colloidal particles in a solution in which the particles are homogenously arranged with a preferable narrow size distribution.

A typical chemical colloidal method to produce quantum dots is the reduction of a metal salt in solution with a reducing agent. Here, the nucleation and growth of the quantum 
dots are controlled by the adsorption of a particle stabilizer on the surface. Particle stabilizers, also called capping agents, play an important role in the wet chemistry method. As indicated before, very small particles show higher chemical reactivity and strong affinity to undergo agglomeration and oxidation reactions due to the high surface energy. This, however, can be inhibited by shielding the semiconductor surface with suitable capping agents. If the interaction of the capping agent with the particle surface is attractive, then it will lead to a reduction of the surface energy and hence reduce the chemical reactivity of the particles in the dispersion medium. The passivation of the particle surface can be achieved by any type of attractive correlation forces, such as Hbonding, coordinative interaction, van der Waals interaction, between the capping agent and the crystal. The different binding modes between capping agents and particle surface are illustrated in figure 1.12 .

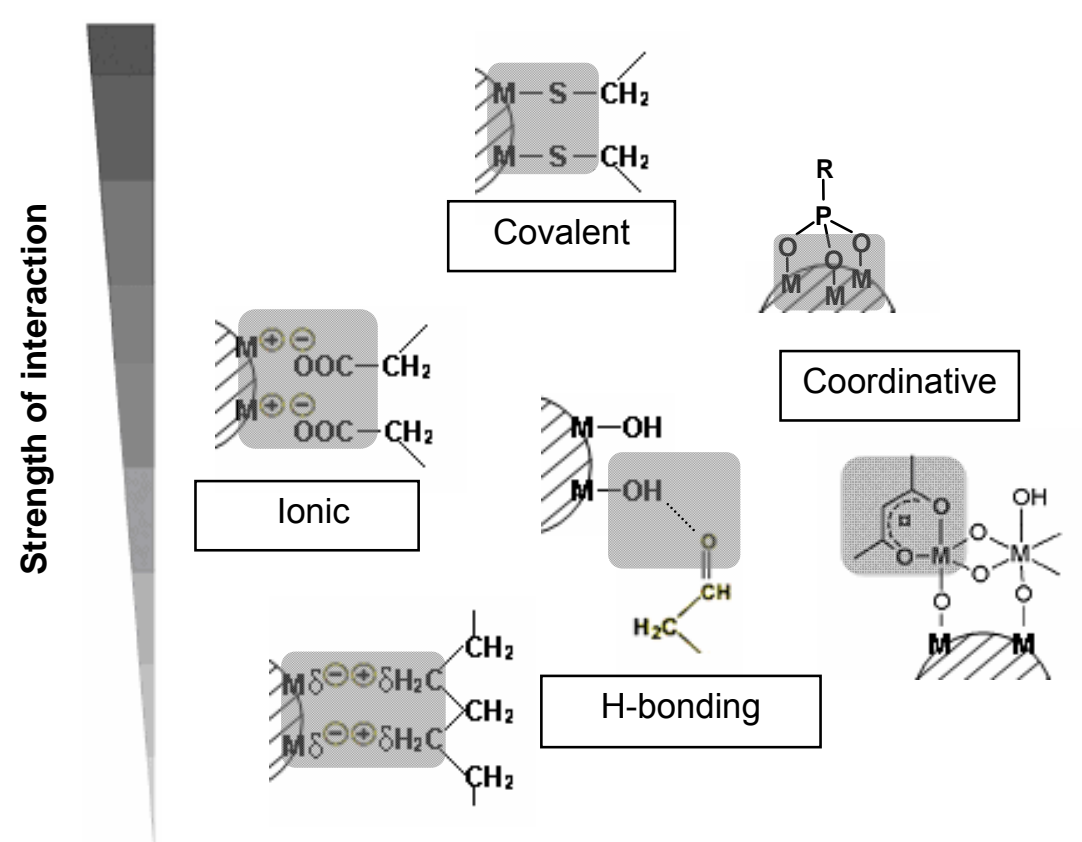

Figure 1.13: Different bonding characteristics between particle surfaces and capping agents [52].

Based on different chemical binding modes as shown in figure 1.13, the surface area of nanocrystals can be coated / functionalised using different capping agents. The capping agents are usually grouped into ionic ligands or spatial constraints. A selection of ionic and spatial (polymeric) stabilisers commonly used for the synthesis of quantum dots is listed in table 1.3. 
Table 1.3: Selection of ionic and polymeric stabiliser used for the synthesis of nanocrystals [46-51].

\begin{tabular}{ll}
\hline lonic stabilizer & Polymeric dispersants \\
\hline Citrate & PEG (polyethylene glycol) \\
Thioglycerol & PC (pyrocatechol) \\
MAA (mercaptoacetic acid) & PVA (poly(vinylalcohol)) \\
Mercaptoethanol & PVB (poly(vinylbutryal)) \\
Pentaflourothiophenol & PAA (poly(acylicacid)) \\
TOP (trioctylphosphine) & PVP (poly(vinyl-pyrrolidone) \\
TOPO (trioctylphosphine oxide) & PMMA (poly(methyl methacrylate)) \\
TDPA (n-tetradecylphosphonic acid) & AOT (dioctysulfosuccinate sodium salt) \\
HPA (hexyl-phosphonic acid) & Poly(oxyethylene)5nonylphenol \\
CTAB (cetyltrimethylammonium bromide) & Poly(oxyethylene)9nonylphenol \\
Oleic acid & \\
Lauric acid & \\
Tris(2-ethylhexyl) phosphate & \\
4-(trifluoromethyl) thiophenol & \\
\hline
\end{tabular}

As illustrated in figure 1.13, the adsorption of capping agents on the surface of the nanocrystals results in the formation of a surface layer around the particles. The properties of these nanocrystallites are determined not only by the composition of the inorganic core but also by the properties of the organic surface layer surrounding the particle. Because of this, crystalline nanoparticles functionalised with organic ligands are also known as nanocomposites [34, 46-52]. The organic surface layer stabilizes the particles in the dispersion medium through electrostatic or steric forces, depending on the capping agents used. For example, ionic capping agents with a charge opposite to that of the particle surface's are reversibly adsorbed onto the surface of the nanocrystals and form a shell which is electrostatically charged. This results in a Coulomb repulsion between approaching nanoparticles. By tailoring the particle surface area with spatial constraints (polymeric dispersant), attraction between the particles is inhibited by steric hindrance. Consequently, the formation of an ionic or steric repelling layer around the particles prevents them from agglomerating and forming larger structures. The stabilization effect of the repelling layer is strongly dependent on the adsorption / uptake of the capping agent on the particle surface area. When the repelling layer is not properly built up, the particles will interact with each other due to van der Waals forces. Because of these forces, particles tend to agglomerate in solution and form polycrystalline particles which are hold together by van der Waals forces too. 
In the wet chemistry method, the stability of the particle dispersion is also dependent on the temperature and solvent used [26]. If the interaction between the capping agent and the solvent is favourable, the nanocrystals will remain isolated and will not agglomerate. However, the introduction of an additional solvent, which is miscible with the first solvent, but has an unfavourable interaction with the capping group, can destabilize the particle dispersion and lead to further particle growth via agglomeration [26].

To maintain the stability of the nanocomposites over time in the dispersion medium, the chemical bonding between the capping agent and particle surface has to be strong. The strength of the chemical bondings decreases from covalent, coordinative, ionic to hydrogen bonds as shown in figure 1.13. Nevertheless, the strength of bonding can also vary between the different bonding characteristics. For example, there are hydrogen bonds which are certainly stronger than weak coordinative bonds. Furthermore, the chemical interaction between the capping agent and particle surface can vary in strength, depending on the composition of the inorganic semiconductor material.

Depending on the composition of the inorganic semiconductor, the surface area can be heterogeneous or homogeneous. Attaching capping agents to a heterogeneous surface is often more complicated than to a homogeneous surface. Metal oxides are typical examples of particles with heterogeneous surface. They are usually characterized by a lack of reactivity because the oxygen atoms on the surface are less reactive [52]. The charge of metal oxides is also known to be frequently delocalized over the whole particle surface and can change over a wide area depending on the metal and $\mathrm{pH}$ value. For this reason, usually a weak interaction is formed between a capping agent and metal oxide nanoparticles. The formation of weak interactions such as H-bonding or weak electrostatic interaction can lead to ligand exchange reactions with solvent molecules over time. This reduces the surface coverage around the nanocrystals and without a strong repelling layer, the nanocrystals tend to agglomerate and form larger polycrystalline particles. This can be avoided by using a capping agent that undergoes a stronger chemical interaction with the particle surface. Positive charged metal oxide particles will undergo a stronger chemical interaction with capping agents that contain functional groups such as carboxylate, sulfonates and phosphonates. The bonding 
between the particle surface and these functional groups are based on ionic and coordinative interaction.

Another approach to increase particle stability in solution, over longer time, is the modification of the particle surface with specific surface ligands after its particle preparation. For example, the surface area of a metal oxide particle can be functionalised with organic molecules / ligands that are able to undergo the following $S_{n}$ reaction:

\section{$S_{n}$ reaction between a metal oxide surface and surfactant containing hydroxyl groups}

$$
\mathrm{M}-\mathrm{OH}+\mathrm{HO}-\mathrm{R} \stackrel{\mathrm{H}_{2} \mathrm{O}}{\longrightarrow} \quad \mathrm{M}-\mathrm{O}-\mathrm{R}+\mathrm{H}_{2} \mathrm{O}
$$

The presence of hydroxyl groups on the surface of metal oxide particles (M) allows the attachment of different organic ligands $(\mathrm{R})$ through covalent bonds. Covalent bondings are preferred due to higher chemical stability. Furthermore, the particle surface can be further functionalised by ligand exchange reactions. Here, capping agents used during the synthesis can be replaced by a capping agent that shows a better bonding capability with the particle surface. In that way, the stability and dispersion properties of the nanocomposites can be enhanced significantly and a homogeneous suspension is guaranteed over a longer time.

The properties of inorganic semiconductor materials can be easily changed by the incorporation of surface ligands or capping agents. Based on the different techniques, inorganic semiconductor materials can be functionalized by a variety of additional functional groups, which are not reacting with the particle's surface, but interacting with the surrounding medium. Thereby different nanocomposites can be created with hydrophobic or lipophobic properties or nanocomposites which exhibit higher resistance to acid media than the coatless inorganic semiconductor material itself. For example, nanoparticles can be stabilized in an organic solvent (with hydrophobic character) by shielding the surface area with a capping agent that contain two segments, one of which interact with the surface of the inorganic particle, for example by electrostatic or hydrogen bonding, and the other one, commonly a non-polar block consisting of long alkyl chains, interacts with the surrounding organic phase. 
However, it is important to note that the bonding character of the capping agent to the particle's surface can also have an influence on the optical properties of the quantum dot. For example, if a coordinated complex is formed or an electron transfer process is enabled by the interaction between the capping agent and the particle surface, the fluorescence intensity of a quantum dot can be enhanced or quenched.

In summary, the choice of capping agents in the synthesis of quantum dots should be based on their strength of adsorption at the surface of the particles. Capping agents which stabilize and control the size of the particle during the preparation have to withstand different reaction conditions such as high temperature, high or low $\mathrm{pH}$ or strong oxidizing conditions. Furthermore, the chemical interaction between the capping agent and surface area should not result in quenching of the fluorescence intensity.

\subsubsection{Choice of fibrous substrate material}

Merino wool and Kraft paper fibres make excellent fibrous substrate materials to combine with the properties of quantum dots. Merino wool is a common woven textile and appreciated due to its well known properties such as high elasticity, thermal insulation, softness, low flammability and many more. It consists of over $90 \%$ of protein, whereby the main component is keratin - a structured protein made up of 22 naturally occurring amino acids $[53,54]$. It is likely that the quantum dots are easily absorbed onto the framework structure of wool fibres, because of the electrostatic interaction between the positively charged quantum dots (positively charged metal ions at the surface) and functional groups of wool's keratin located on the surface of the fibres. Thereby, the nitrogen, oxygen and sulphur atoms of the amino acids are the sites targeted for the attachment of the quantum dots to produce quantum dot - fibre composites.

Paper is a widely used material with many applications, e.g. printing, writing and packaging. Paper fibres consist mainly of cellulose, which is also the target component for the attachment of quantum dots. Cellulose has surface hydroxyl groups that allow the binding of various materials through hydrogen bonding [55]. Other properties of cellulose, which make it as an excellent substrate material for the development of quantum dot - fibre composites are high tensile strength, resistance to plastic 
deformation, hydrophilic, water dispersible, inherent bonding ability (H-bonding), ability to absorb modifying additives, chemical stability, relatively colourless, etc. [55].

For the development of quantum dot - wool and paper fibre composites, it is desired that the new fluorescent composite material exhibit the inherent properties of both precursor materials. For this reason, a brief introduction of the chemical and physical composition of wool and paper fibres as well as processing methods of wool and paper are included below. This will also provide an understanding of possible bonding modes between quantum dots and the organic substrate materials.

\subsubsection{Merino wool}

Merino wool consists of over $90 \%$ protein, with the remainder consisting of lipids, waxes, inorganic salts and polysaccharides. Like other proteins, merino wool proteins are made up of long chains of various amino acids, which are joined together via peptide bonds. These polypeptide chains in turn are linked together by a variety of bonds such as hydrogen bonds, disulphide and isopeptide cross-links or by hydrophobic and ionic interaction (see figure 1.14). These bonds and intermolecular interactions construct the final protein structure.

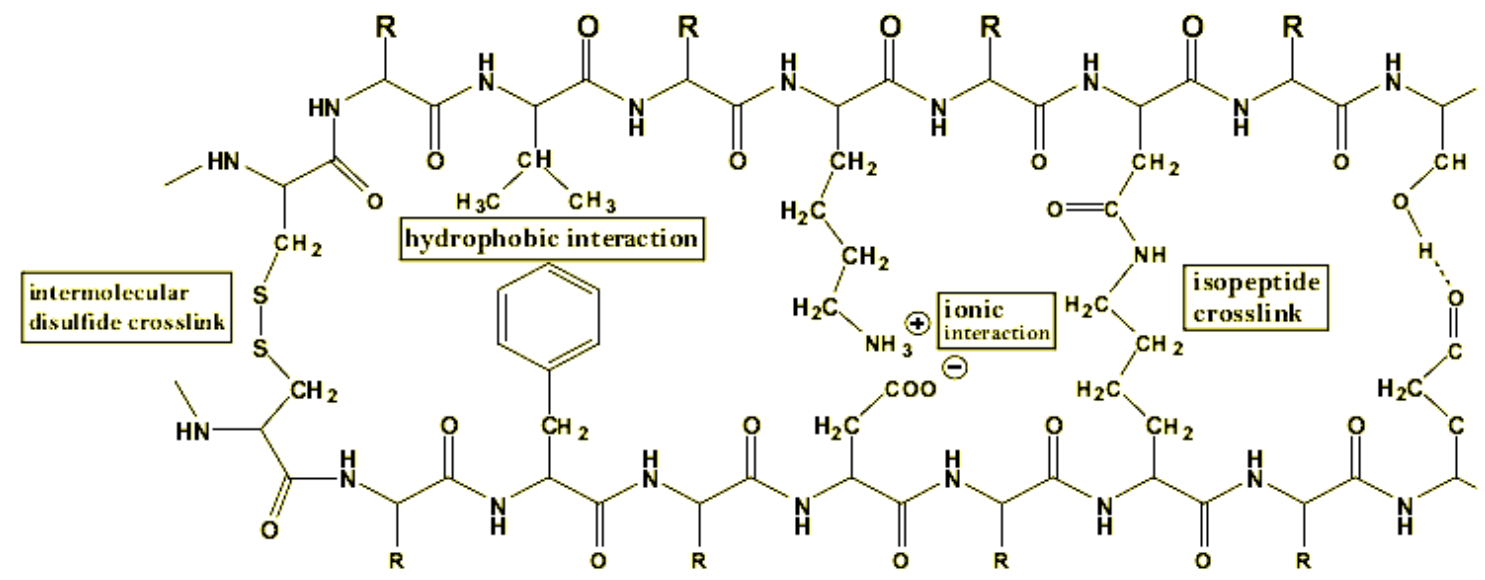

Figure 1.14: Diversity of chemical bonds found in wool fibre [53].

The main component of merino wool is keratin, a structural protein. Here, long polypeptide chains are folded into a final 3-dimensional form, the alpha helix. The alpha helix structure is held in place by hydrogen bonds between N-H groups and oxygen of $\mathrm{C}=\mathrm{O}$ groups. Furthermore, the alpha helix of keratin is reinforced by intermolecular 
cross-link reaction between two cysteine amino acids forming a disulphide bond. Other proteins in wool have a more amorphous structure and are responsible for absorbing a relatively large amount of water without feeling wet; up to around $30 \%$ of the mass of the dry fibre. The physical structure of wool fibres is very complex as seen in figure 1.15 .

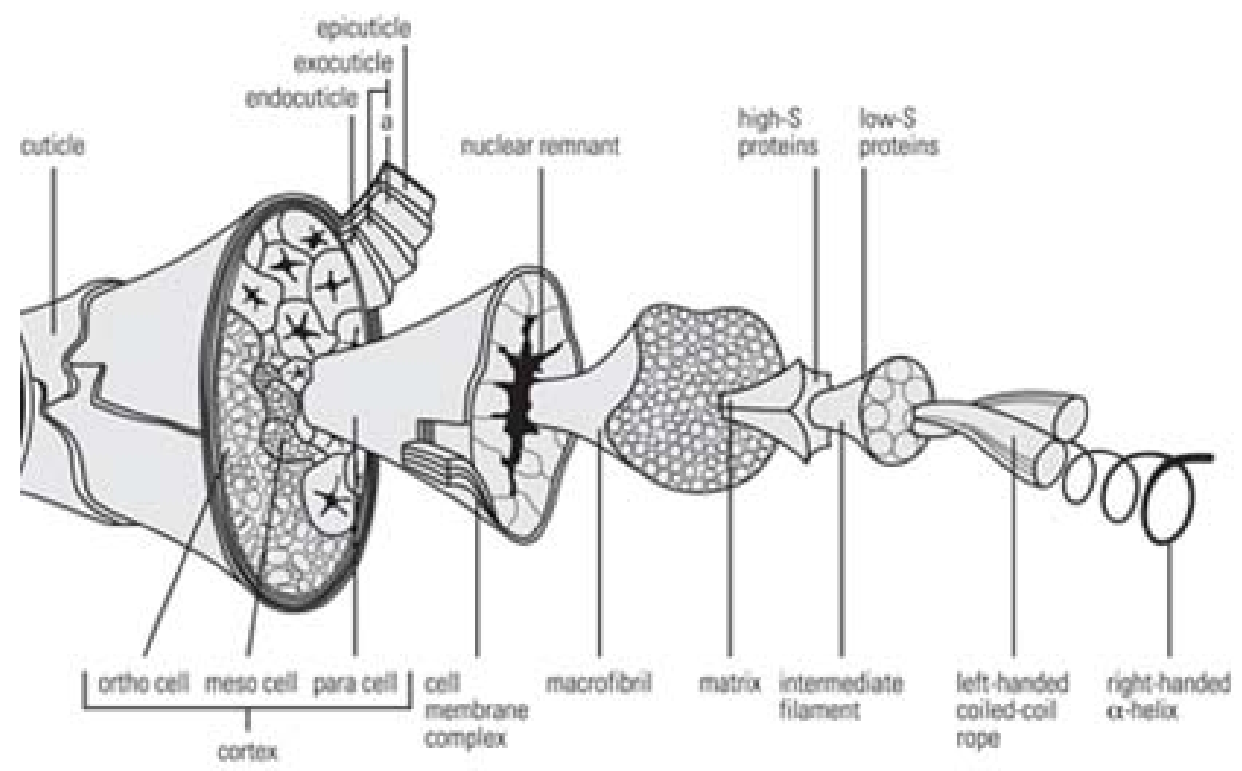

Figure 1.15: Physical structure of fine wool [53].

However, the physical structure of wool fibres can be simplified in two types of cells, the "internal cells of the cortex" and the "external cuticle cells" that form a sheath around the fibre (figure 1.16).

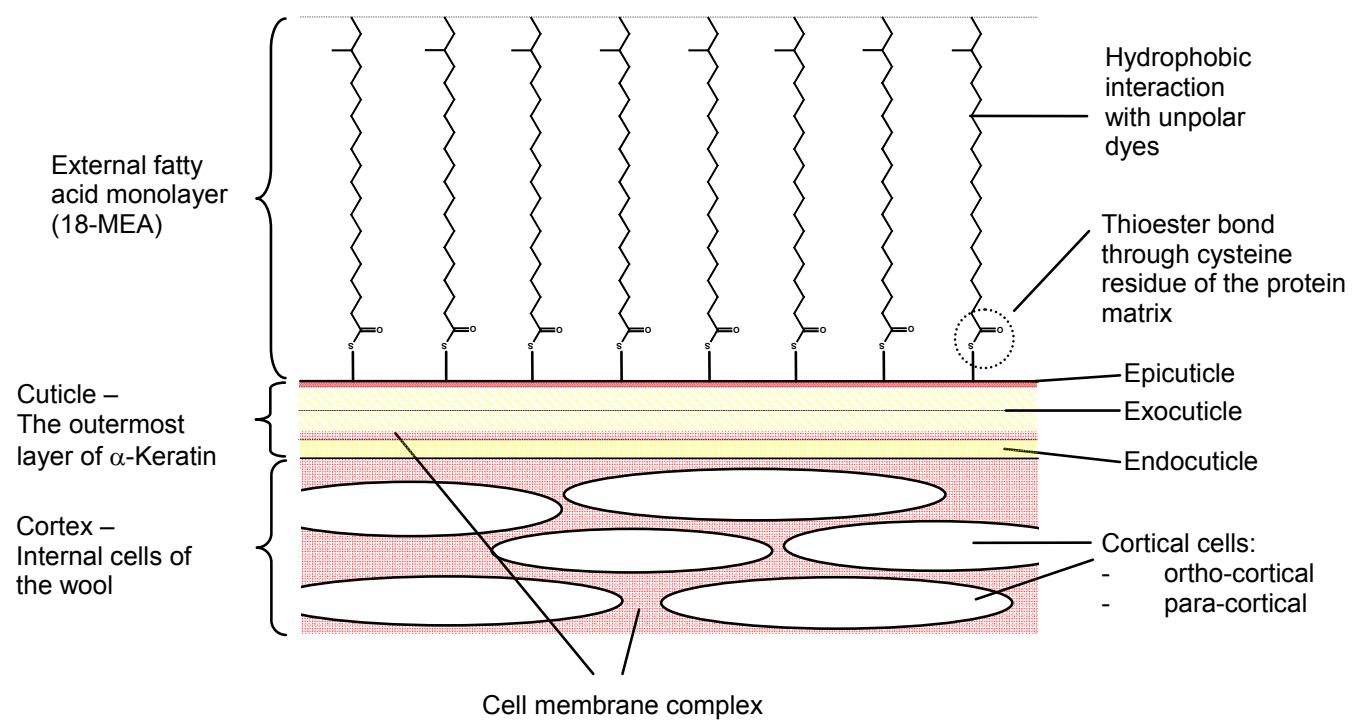

Figure 1.16: Schematic model of wool fibre composition. 
The composition of the cortex is quite complex as the illustration in figure 1.14 shows. The fibre cortex (internal cells) makes up to $90 \%$ of the keratin fibres and consists of overlapping cortical cells and cell membrane complex. There are two types of cortical cells inside the wool fibre; ortho-cortical and para-cortical cells [57-59]. They have different chemical composition and they expand differently when they adsorb moisture. The cortical cells are surrounded and held together by the cell membrane complex, which contains proteins and waxy lipids. The cell membrane complex runs all along the wool fibre. The cortical cells contain macrofibrils which are made of bundles of finer filaments knows as microfibrils. The microfibrils are surrounded by intermacrofibrillar materials [60]. These intermacrofibrillar materials are also known as matrix region. The matrix region is responsible for the uptake of moisture inside the wool fibre. The matrix contains proteins, which are rich in sulphur. The sulphur atoms of the protein attract the water molecules.

The cuticle is the outermost layer that protects the cortex. It is made up of three layers, the epicuticle, exocuticle and endocuticle. These layers are separated from each other and the cortex by a cell membrane complex consisting of non-keratinous proteins and lipids [60-63]. The epicuticle is the outermost membrane of the cuticle, and is resistant to acids, alkalis, oxidizing agents and enzymatic attack [63]. The epicuticle consists of approximately $75 \%$ protein and $25 \%$ lipid [62-64]. The protein component contains approximately $12 \%$ cystine [64] and the lipid component a mixture of fatty acids, predominantly 18-MEA [64, 65].

The surface of the cuticle cell (outermost layer of the wool fibre) is heavily acylated with fatty acids, predominantly 18-methyleicosanoic acid (18-MEA), which forms a hydrophobic surface layer around the wool fibre (see figure 1.16). The 18-MEA is bound covalently to the protein surface by means of a thioester bond through cysteine residues [65]. The presence of the external fatty acid monolayer imparts a hydrophobic nature to the fibre surface. The cuticle cell, however, is not a continuous layer around the wool fibre. The cuticle cell form scales which surround the wool fibre by overlapping like tiles on a roof. By doing this, small gaps between the cuticle layers allow intercellular diffusion of dye molecules and metal ions to the fibre cortex as shown in figure 1.17. 


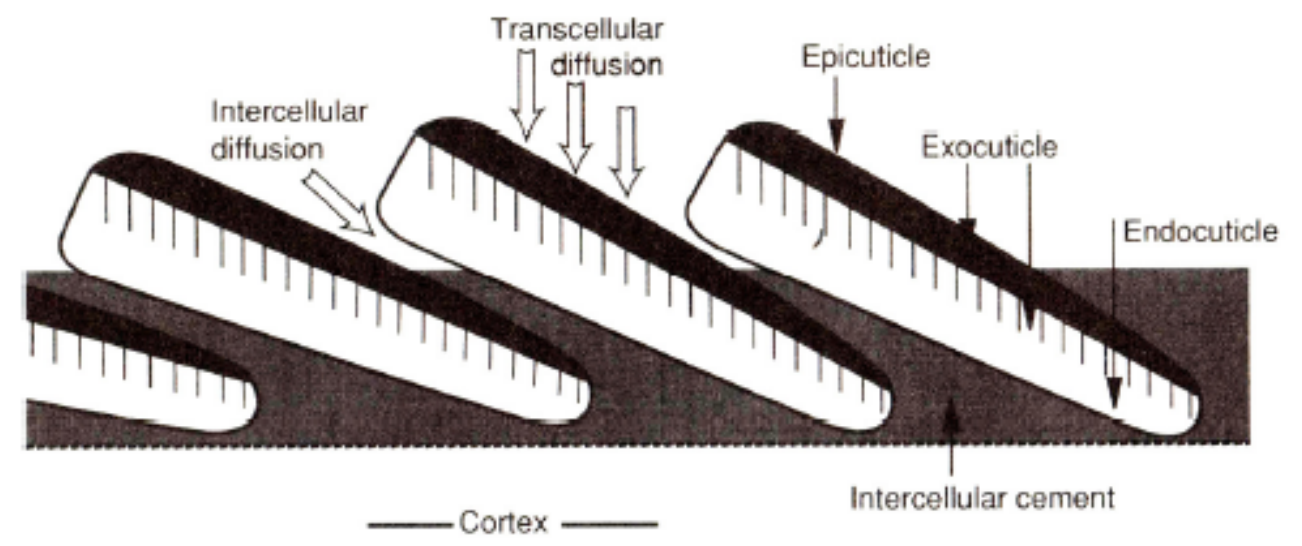

Figure 1.17: Illustration of wool fibre surface showing overlapping scales allowing intercellular diffusion for dyes [66].

To obtain a higher quality appearance, some wool fibres undergo further processing, which can be either an oxidative or reductive bleaching process. However, the most widely used surface treatment of wool is the Chlorine Hercosett process. It is an oxidative process, whereby wool fibre is first exposed in chlorine gas. The chlorine gas breaks up disulphide bonds in cysteine moieties and hydrolyses peptide bonds within the exocuticle $[67,68]$. After this treatment, the surface of the wool is coated with a polymer, Hercosett 125, which is capable to undergo cross-links with the primary and secondary amino groups at the surface of the wool fibre protein (exocuticle). Due to the Hercosett surface layer, wool fibre becomes shrink resistant [69]. The polymer is capable to absorb a high amount of water and swells up. The swelling prevents the interaction between scale edge of adjacent fibres and by doing so prevent the shrinkage of the fibres when washed.

The final step of the wool processing is the conversion of the fibres into yarn. This can be conducted according to the woollen, semi-worsted or worsted systems [66]. Each system uses lubricants to reduce fibre damage and breakage. In the worsted processing routes, these lubricants are commonly poly alkylene glycols, ethoxylated fatty acids and fatty acid esters combined with oxidation stabilisers. The woollen and semi-worsted routes often use only one lubricant, typically ethylene oxide polymers or ethylene oxide/propylene oxide block co-polymers [66]. The lubricants usually remain onto the wool after processing, and hence influence the surface properties and chemistry of the processed wool. 


\subsubsection{Paper fibre}

Paper is largely made of wood fibre. Wood consists of four separate components; cellulose, hemicellulose, lignin and extractives, as shown in figure 1.18.

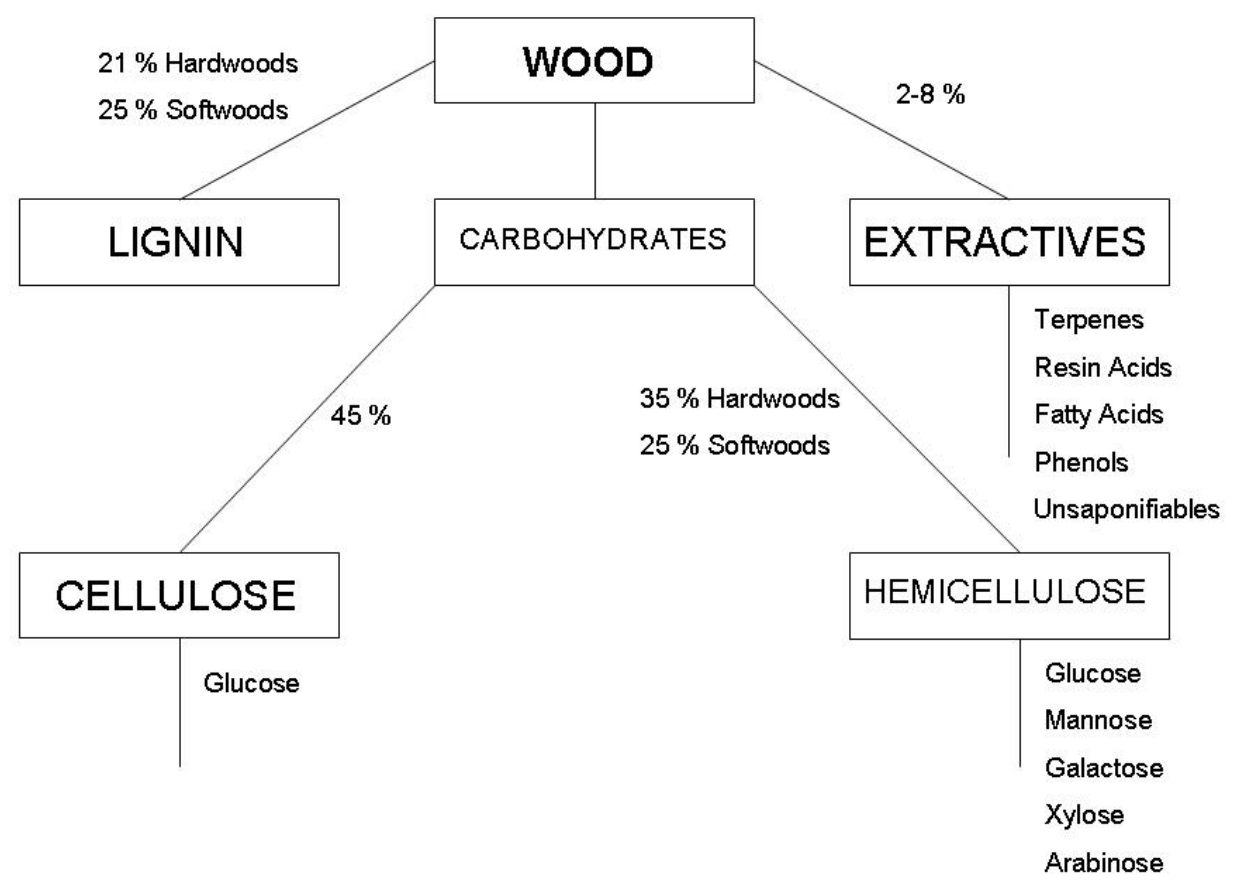

Figure 1.18: Chemical composition of wood [55].

In the papermaking process, cellulose and hemicellulose are the most important components. Cellulose, $\left(\mathrm{C}_{6} \mathrm{H}_{10} \mathrm{O}_{5}\right)_{\mathrm{n}}$, is a long-chain polymeric carbohydrate exclusively made up of $\beta$-glucose monomers. Each monomer is oriented $180^{\circ}$ to the next, as seen in figure 1.19, and the chain is built up two units at a time [55].

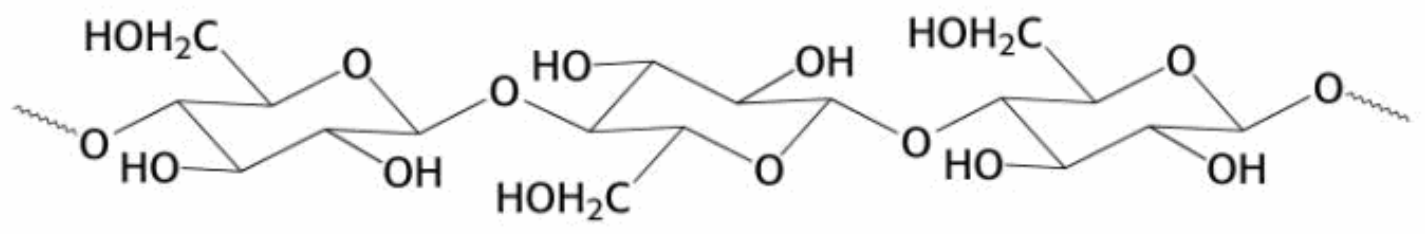

\section{$\beta-1,4$ linkages of glucose monomers}

Figure 1.19: Chemical structure of cellulose $\left(\mathrm{C}_{6} \mathrm{H}_{10} \mathrm{O}_{5}\right)_{\mathrm{n}}$.

Hemicellulose contains a mixture of five different sugars; glucose, mannose, galactose, xylose and arabinose. Hemicellulose is more easily degraded than cellulose and 
therefore is present in smaller quantities in the wood [55]. Lignin is a complex and highly polymerised material consisting of propenyl units linked together to a three dimensional polymer. The main role of lignin is to act as the "glue" which holds the fibres together in the wood matrix [70]. The amounts of lignin, along with cellulose and hemicellulose, vary between different wood types. Wood is classified into two main categories: soft wood (gymnosperms) and hard wood (angiosperms). Soft woods are obtained mostly from coniferous trees, while hardwoods are obtained from deciduous trees and evergreens such as eucalypt. The major differences between the two types are the fibre content and the fibre dimensions (see figure 1.20).

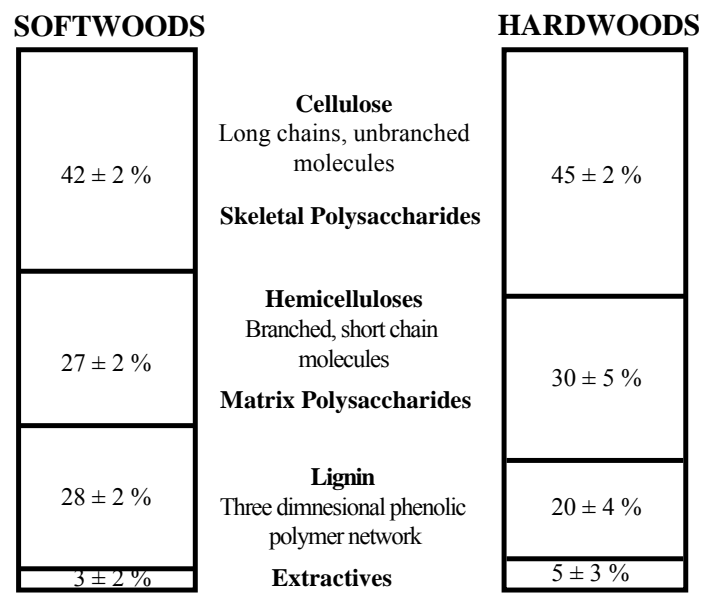

Figure 1.20: Average composition of softwoods and hardwoods [55].

The difference of the commercially available papers is found in the original fibre feedstock (hardwood or softwood) and in the papermaking process. For the papermaking process, wood has to be converted in a pulp form. Pulp is the fibrous raw material of wood residuals for the papermaking processes, which is obtained by pulping. In the pulping process wood residuals are reduced to a fibrous mass by the breaking of the bonds between the fibres. Pulping is necessary, because some pulps are unusable in their raw state due to their relative non-conformability and non-bonding ability.

There are several papermaking processes to separate fibres into pulp. Paper pulp can be produced either by a mechanical or chemical pulping process or a combination of both (CTMP, NSSC, etc.). The differences between mechanical and chemical pulping are evident in the fibres they produce (see figure 1.21). 


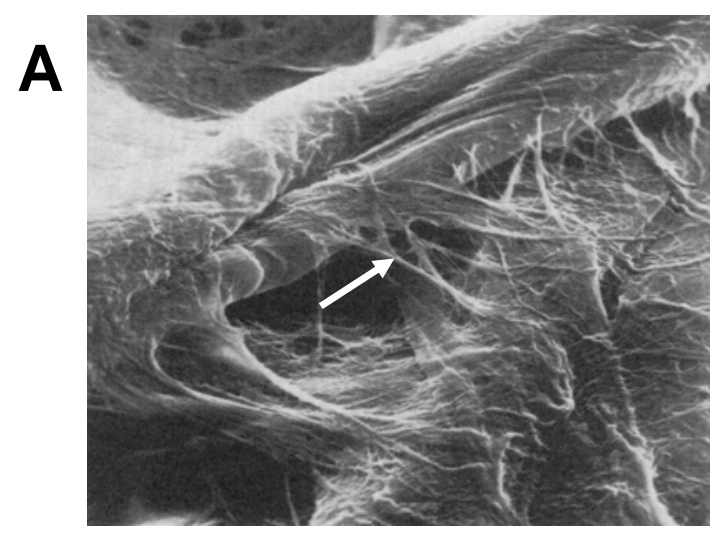

Mechanical pulping

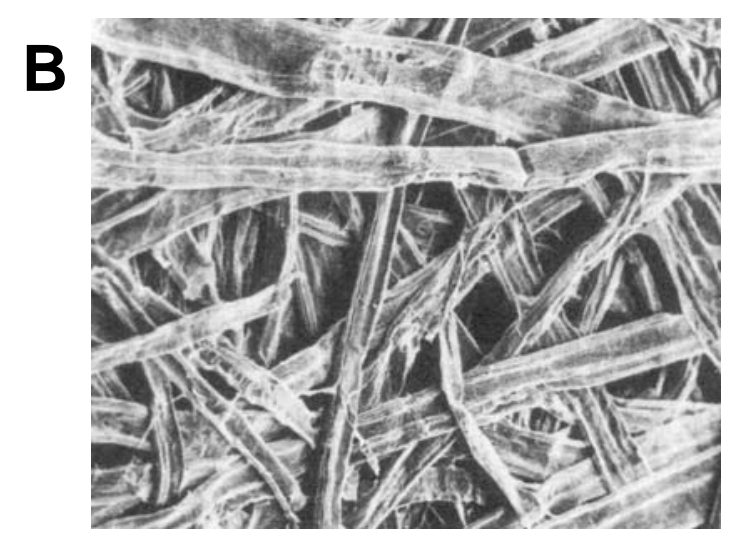

Chemical pulping

Figure 1.21: SEM image of paper fibres with fines (A, white arrow) produced by the RMP method and SEM image of “clean” fibres (B) produced by the Kraft method [55].

The current method of mechanical pulping involves shredding wood chips between the rotating discs of a machine known as a refiner. The pulp produced by this process is called refined mechanical pulp and typically contains shorter fibres when compared with chemical pulping. During mechanical pulping, cellulose fibres tend to unwind to a certain extent, producing fibrils and micro-fibrils. These fibrils and micro-fibrils or fines (figure 1.21; A) help to fill the network voids of paper and as a result enhance optical and print properties. There are a number of disadvantages associated with mechanical pulping. These include the high energy consumption involved in the pulping process, the weakening of fibres due to fibre damage during the pulping process, and that papers made of mechanical pulp darken on exposure to light as the lignin is not removed in the mechanical pulping process.

In contrast, the chemical pulping process dissolves away the lignin and leaves only the cellulose fibres. This is achieved by cooking the wood at elevated temperatures along with the appropriate chemicals. Chemical pulping produces undamaged fibres (figure 1.21 ; B) that are stronger and therefore produce a stronger paper. There are various methods of chemical pulping, but the most common is the "Kraft process". Here the wood is heated in a solution of sodium hydroxide $(\mathrm{NaOH})$ and sodium sulphide $\left(\mathrm{Na}_{2} \mathrm{~S}\right)$ at elevated temperature $\left(\sim 170{ }^{\circ} \mathrm{C}\right)$ and pressure. The alkaline $\mathrm{NaOH}$ causes the lignin to break down into smaller molecules whose sodium salt is soluble in the solution. Due to the lower content of lignin, pulp produced according the Kraft process does not darken on exposure to light. 


\subsubsection{Theoretical background for the development of quantum dot - fibre composites}

For the development of quantum dot - fibre composites, it is important to identify the best processing strategy to obtain intense, stable fluorescent colours which can be stimulated by UV light. In principle, there are two different synthetic strategies for the formation of hybrid materials comprising quantum dots and organic substrate material. These can be either the building block approach or the in-situ approach [52].

\subsubsection{Building block approach}

In the building block approach, quantum dots and organic substrate material react with each other and form the final nanocomposite material. The principle behind the building block approach is illustrated in figure 1.22.

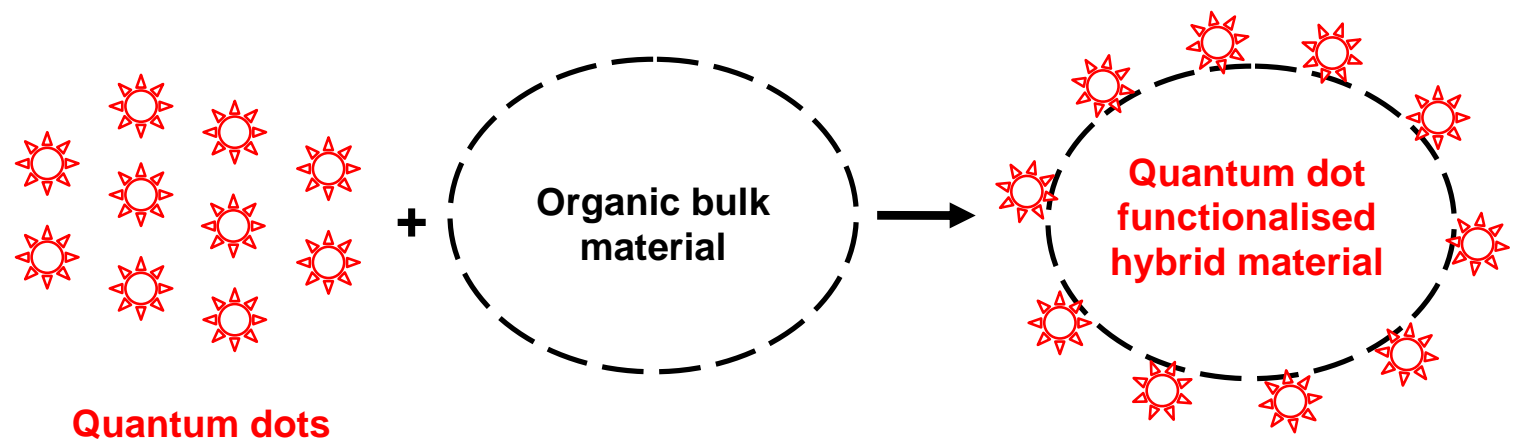

Figure 1.22: Schematic illustration of the development of new hybrid materials using the building block approach.

The attachment of the quantum dots depends on the presence of functional groups that allows the formation of chemical bonds between the quantum dot material and substrate material. When the quantum dots are incorporated or embedded in the organic substrate material, it is important to maintain the structure and size of both components and to keep, or improve, the properties of interest of the combined components.

The stability and optical properties of the new composite material are dependent on the chemical bonding between the quantum dots and the organic component connecting them together. A successful attachment of the quantum dots to an organic substrate material is only achieved, when the chemical composition at the interface of both 
components is attractive to each other. Therefore, the functional groups at the surface of both components will determine whether a chemical bonding is created or not.

If a chemical bonding is not achieved directly, then basically two processing strategies are available to create quantum dot - fibre composites. This can be either the modification of the quantum dot surface area by tailoring the surface with linker molecules that contain the desired functional groups for the interaction with the substrate material or the functionalisation of the network structure of the substrate material with linker molecules that contain specific anchor groups for the substrate and the quantum dot material.

Quantum dots are usually functionalised with capping agents to ensure a dispersion of mono-dispersed particles. Quantum dots functionalised with capping agents can be used in the synthesis of hybrid materials as long as the surface coverage of the particles is compatible with the system the quantum dots will be incorporated to. The compatibility is usually achieved by capping agents that contain additional functional groups which enable some kind of interaction with the structure of the second component used for the preparation of the hybrid material. If the interaction between quantum dots and substrate material is not attractive, the surface area of the inorganic semiconductor nanomaterial can be further functionalised either by an exchange of the capping agent (ligandexchange) or by tailoring the surface area with linker molecules that can undergo a chemical reaction with the particle's surface.

By modifying the surface area of the quantum dots with a different capping agent or linker molecule that contains the desired functionalities for an interaction with the network structure, a stable chemical interaction can be created between quantum dots and the substrate material. However, this is only possible, when the new capping agent show a better bonding capability than the one used during the particle preparation. In this way, quantum dots can be functionalised with linker molecules that contain additional reactive functional groups which do not interact with the particle surface, but with the substrate material. 
Another approach to increase the compatibility between quantum dots and substrate material is the modification of the network structure (substrate material). By functionalising the network structure with linker molecules or coupling agents, which provide anchor groups for both components - substrate material and quantum dots - a better uptake of the quantum dots to the substrate material is guaranteed. The linker molecule has to contain at least two segments with functional groups. One segment undergoes interaction with the network structure of the substrate material, for example by covalent or electrostatic bonding, and the other segment interacts with the surface functionalised quantum dots.

However, the chemical attachment of quantum dots to organic substrate materials is strongly dependent on the processing parameters. Changes in the process parameters can have a big influence on the surface coverage of the quantum dots and may result in the loss of capping agents at the particle surface. Without a strong surface coverage, quantum dots will aggregate/agglomerate in the dispersion medium and a homogeneous distribution of the quantum dots on the substrate material can not be achieved. Furthermore, when quantum dots aggregate/agglomerate at the interface of the substrate material the optical properties of the quantum dots are negatively affected. Processing parameters such as high temperature can also have a negative effect on the preparation of hybrid materials, since organic materials can degrade or even decompose at high temperature. The same applies for highly acid solutions which can dissolve the quantum dot material or damage the organic substrate material. Therefore, it is important that all parameters are optimized in order to obtain attractive interaction between both components as well as with the solvent.

\subsubsection{In-situ approach}

The in-situ approach is based on the chemical transformation of the quantum dot precursor material inside the network structure of the organic substrate material. For example, the chemical transformation can be induced by a sol-gel process which forms the inorganic phase by hydrolysis and condensation reactions. The network structure of the organic substrate material usually operates as a natural barrier for the particle's growth and thereby controls the size of the quantum dots. Figure 1.23 shows a simplified illustration of the principle of the in-situ approach. 


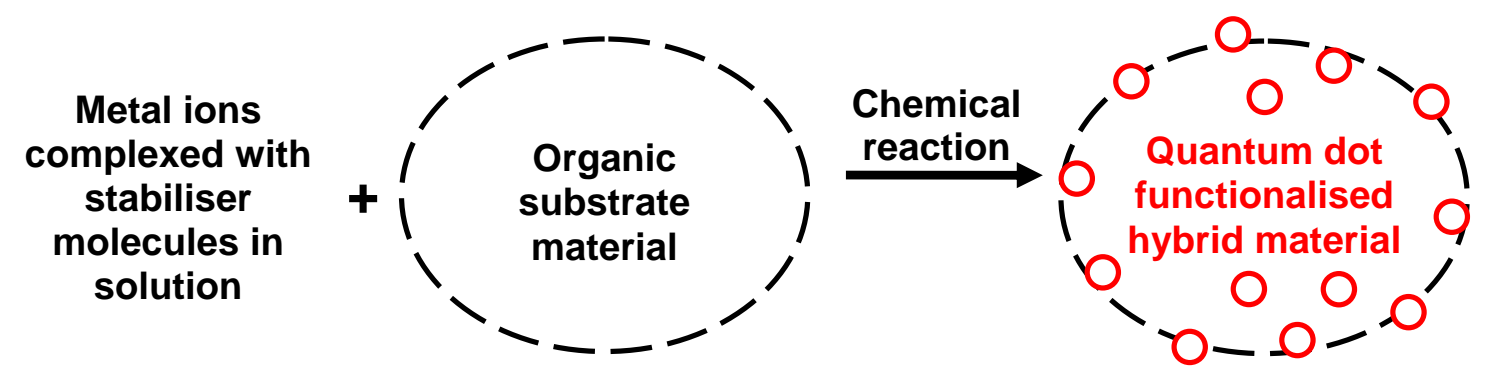

Figure 1.23: Schematic illustration of the development of new hybrid materials using the in-situ approach.

In general, the in-situ approach for the formation of quantum dot - fibre composites can be divided in two steps. The first step is the uptake of the quantum dot precursor material onto the substrate material before a chemical transformation takes place and forms the quantum dots. By diffusing inside the network structure, the metal ions will bind to functional groups of the substrate material. These chemical bondings will also trap the precursor material inside the network structure of the substrate material. Then, the chemical transformation is induced by a chemical reaction which is either based on the concept of a wet chemical method such as sol-gel method, chemical reduction or precipitation method.

The network structure can be viewed as nano-chambers which confine the growth of the quantum dots. During the chemical transformation, chemical linkages can also be formed between the quantum dots and the network structure of the organic substrate material. This is possible when the organic component contains functional group which allows it to participate in the chemical reaction of the quantum dot formation. The network forming reaction between the quantum dots and the functional groups of the organic substrate material will also control the size and shape of the quantum dots in the new hybrid material. A typical example for network forming reactions, or cross-linking reactions, is the sol-gel process used for the preparation of metal oxide particles.

In a sol-gel process, covalent linkages are formed between hydroxyl groups $(\mathrm{OH}-$ groups) via a condensation reaction. If the organic substrate material also contains $\mathrm{OH}-$ groups that react with the hydroxyl groups of the quantum dot material during hydrolysis and condensation reaction, then a covalent bonding between the inorganic and organic component is achieved. Strong chemical interaction can also be created by 
forming hydrogen bridges (H-bonding) between the metal oxide surface of the quantum dots and the OH-groups of the organic substrate material. The cross-link reactions, however, will lead to structural changes of the organic substrate material during the chemical reaction. Here, the formation of a highly cross-linked network structure of the organic substrate material with the quantum dot material is desired, because this ensures a higher stability of the final hybrid material.

It is important to note that the network forming reactions are either based on kinetically or thermodynamically controlled processes. Hence, the growth of the quantum dots inside the substrate material is strongly dependent on process parameters such as $\mathrm{pH}$, temperature and concentration used for the formation of the composite material. Furthermore, the crystallites formed in the chemical surrounding of the organic substrate material can also adopt different morphologies such as rods, wires, ribbons, sheets and many others. Since the properties of nanometric semiconductor materials are size- and shape dependent, the formation of particles with different sizes and shapes will result in composite materials with unpredictable properties. Therefore, the in-situ process parameters have to be optimised for the chemical transformation of the quantum dot material inside the organic substrate material to obtain uniform composite materials.

\subsection{Research goals}

The main goal of this project is to synthesise and bind quantum dots to wool and paper fibres to produce new materials that exhibit tuneable optical fluorescence, for potential use in textiles, packaging and labelling papers. It is desired that these new fluorescent composite materials show the properties of both precursor materials, and that the quantum dots show intense, stable, spectroscopically pure fluorescent colours under UV light stimulation.

The quantum dots should be made up of non-toxic or low toxicity materials, so they are compatible with the human skin and are suitable for the use in textiles and other everyday products. The synthesis of quantum dots should be based on wet chemical methods. In a wet chemical method, the quantum dots are also homogeneously dispersed in solution which is useful in further processing. Furthermore, by using wet chemical methods, the fluorescence colour of the quantum dots can be made tuneable in 
the visible range by controlling their size or by incorporating small quantities of impurities into their lattice structure.

For this reason, the first part of the thesis, chapter 3 and 4, explores wet chemical synthesis methods to produce size- and shape controlled nanocrystals consisting of the metal oxide semiconductors $\mathrm{Cu}_{2} \mathrm{O}$ and $\mathrm{ZnO}$. The selection of these quantum dot materials is based on the non-toxicity in their bulk form and the simplicity of their wet chemical synthesis methods. Chapter 5 explores the coating of metal (oxide) nanocrystals with ZnS. Chapter 6 deals with the development and characterisation of new hybrid nano-materials comprising $\mathrm{ZnO}$ quantum dots and NZ wool and paper fibres, respectively. The fluorescence properties of the Zinc(II)-Bis(8hydroxyquinoline) complex $\left(\mathrm{Zn}(8-\mathrm{Q})_{2} \cdot 2 \mathrm{H}_{2} \mathrm{O}\right)$ is also investigated and its use to functionalise wool with optical fluorescence is explored and characterised in chapter 7 .

To provide guidance in this thesis, the following section presents an outline of each chapter and their research goals.

\section{Chapter 2}

An outline of the experimental procedure for the synthesis of the quantum dots and development of fluorescent composite materials is presented in the beginning of chapter 2 (see flow chart in figure 2.1). Chapter 2 also list the different characterisation method used to provide an understanding of the new hybrid materials created in this project.

\section{Chapter 3}

The synthesis and characterisation of $\mathrm{Cu}_{2} \mathrm{O}$ quantum dots produced by a wet chemical reduction method is investigated in chapter 3 . In the wet chemical reduction method, $\mathrm{Cu}_{2} \mathrm{O}$ quantum dots are formed by the reduction of the corresponding metal precursor material to produce a suspension of colloidal particles. Here, the size and shape of the particles depends strongly on the growth kinetics, which are based on nucleation and condensation reactions. The growth kinetics, can however, be controlled during the chemical reaction by the use of capping agents. The passivation of the semiconductor surface area with capping agents after or during particle synthesis will also decrease the 
surface energy of the crystals and reduce the reactivity of the surface atoms towards agglomeration and oxidation. This will ensure a stable suspension of quantum dots. For this reason, charged and bulkier surfactants are used to obtain a dispersion of homogenously arranged nanocrystals with a narrow size distribution. Furthermore, different combinations of charged and spatial capping agents with their different strength of adsorption on the particle surface are investigated to obtain a well-defined nanosized structure and a uniform size-distribution of the quantum dots. The effect of different surface coatings of these semiconductor nanocrystals using ionic and polymeric stabilizers as well as the incorporation of dopants into the final lattice structure on the optical properties of the nanocrystals, are also investigated and their applicability in the production of new hybrid materials evaluated.

\section{Chapter 4}

Chapter 4 deals with the synthesis and characterisation of $\mathrm{ZnO}$ quantum dots synthesised using a combination of sol-gel and wet chemical reduction methods. Sol-gel methods are widely used because of the inexpensive equipment required, mild reaction conditions and the potential to upgrade into a large-scale production. The sol-gel method was selected because it allows the incorporation of small quantities of dopants into the chemical composition of the final product where the nature and level of dopant can be used to control the fluorescence wavelength (colour in the visible range). The sol-gel method is based on hydrolysis and condensation reaction of hydrolysed metal cations. The size and size distribution of the $\mathrm{ZnO}$ quantum dots can be controlled by changing the reaction conditions in the sol-gel methods, such as the concentration of the precursor material(s), temperature, time, $\mathrm{pH}$ and concentration of capping agents. For example, by tailoring the semiconductor surface with a polymeric dispersant, the particle growth is confined through steric hindrance ensuring the production of size and shaped controlled nanocrystals. Different techniques to control the size and shape of the quantum dot material as well as the doping of the quantum dot material with a variety of metal ions is explored to obtain fluorescence at different wavelengths and hence colours in the visible range. The methodology and the effect of surface modifications with different surfactants on nanocrystals particle growth, particle shape, size and size distribution and photoluminescence properties are investigated and the synthesis is optimised to produce $\mathrm{ZnO}$ nanocrystals showing stable fluorescent emission intensities. 


\section{Chapter 5}

Chapter 5 explores the incorporation of different metal nanoclusters within the lattice structure of $\mathrm{ZnS}$ quantum dots. Metal nanoclusters (or metal oxide nanoclusters) formed by a wet chemical reduction method are co-precipitated with $\mathrm{ZnS}$ to obtain fluorescence in the visible range. The wet chemical precipitation method of $\mathrm{ZnS}$ quantum dots is based on the slow addition of sodium sulphide $\left(\mathrm{Na}_{2} \mathrm{~S}\right)$ solution into a zinc chloride $\left(\mathrm{ZnCl}_{2}\right)$ solution and and allows the incorporation of different impurities. By incorporating metal (oxide) nanoclusters into the crystal lattice of $\mathrm{ZnS}$ quantum dots, it is hoped to change the fluorescence colour and increase the stability of the quantum dots for long-term storages.

\section{Chapter 6}

The synthetic strategies to produce $\mathrm{ZnO}$ quantum dot - fibre composites is presented in chapter 6. Two strategies are used to combine the properties of $\mathrm{ZnO}$ quantum dots with those of wool and paper; the building block and in-situ approach. The building block approach relies on strong chemical interactions between quantum dots and substrate material, e.g. covalent bonds, to ensure stable and long-lived composite materials. Hence, chemical methods to attach or exchange functional groups at the surface of the quantum dot are often required to create a chemical interaction between quantum dots and wool and paper fibres. The surface area of the substrate material, however, can also be tailored with coupling agents or linker molecules that contains at least two segments; one reacts with the surface of the substrate material and the other one with the surface of the quantum dots. Thereby, different binding modes such as disulphide, peptide and hydrogen bonds can be created between the quantum dot surface, linker and functional groups of the substrate material. In the in-situ method for the preparation of quantum dot - fibre composites, the chemical transformation of the $\mathrm{ZnO}$ quantum dot material will be conducted inside the organic substrate material by using sol-gel reactions. This method involves the adsorption of the precursor material onto the organic substrate material, before a chemical transformation takes place to form the quantum dot material. The crystal formation inside the substrate material is strongly dependent on the reaction conditions used such as temperature, time and $\mathrm{pH}$. Furthermore, the process parameters have to be optimised to ensure cross-link reaction between the $\mathrm{ZnO}$ quantum dot material and the fibrous substrate material during nucleation and condensation reaction. 


\section{Chapter 7}

Besides quantum dots, the bright fluorescence properties of chelates can also be used in the creation of unique hybrid materials. In chapter 7, an alternative procedure to develop fluorescent wool fibre composites using the fluorescent chelate $\mathrm{Zn}(8-\mathrm{Q})_{2} \cdot 2 \mathrm{H}_{2} \mathrm{O}$ is explored. This procedure involves the uptake of zinc and 8-HQ onto wool in a two-step batch process. This method ensures that the complex formation takes place in the chemical surrounding of the wool resulting in an in-situ complex formation of the fluorescent chelate. The fluorescence of chelates is determined by the fluorescence of the monomer chelate and fluorescence of two chelates interacting with each other. Hence, the wavelength of the emitted light is determined by the spatial distances between adjacent chelates. The spatial distances between the chelates can be controlled by the uptake parameters of the precursor materials. Therefore, the fluorescent characteristics of the final product can be regulated by modifying the different uptake parameters and the influence of different process parameters such as concentration of 8Hydroxyquinoline (8-HQ), choice of solvent, choice of zinc precursor material, soaking temperature and time on the fluorescence properties is here investigated and characterised.

\section{Chapter 8}

Final conclusions, recommendations and future directions are presented in chapter 8 . 


\section{Chapter 2}

\section{Experimental procedure}

This chapter presents the different methods and materials used for the synthesis of quantum dots and creation of composite materials. The strategy followed for the development of new fluorescent hybrid materials is illustrated in a flow chart (figure 2.1).

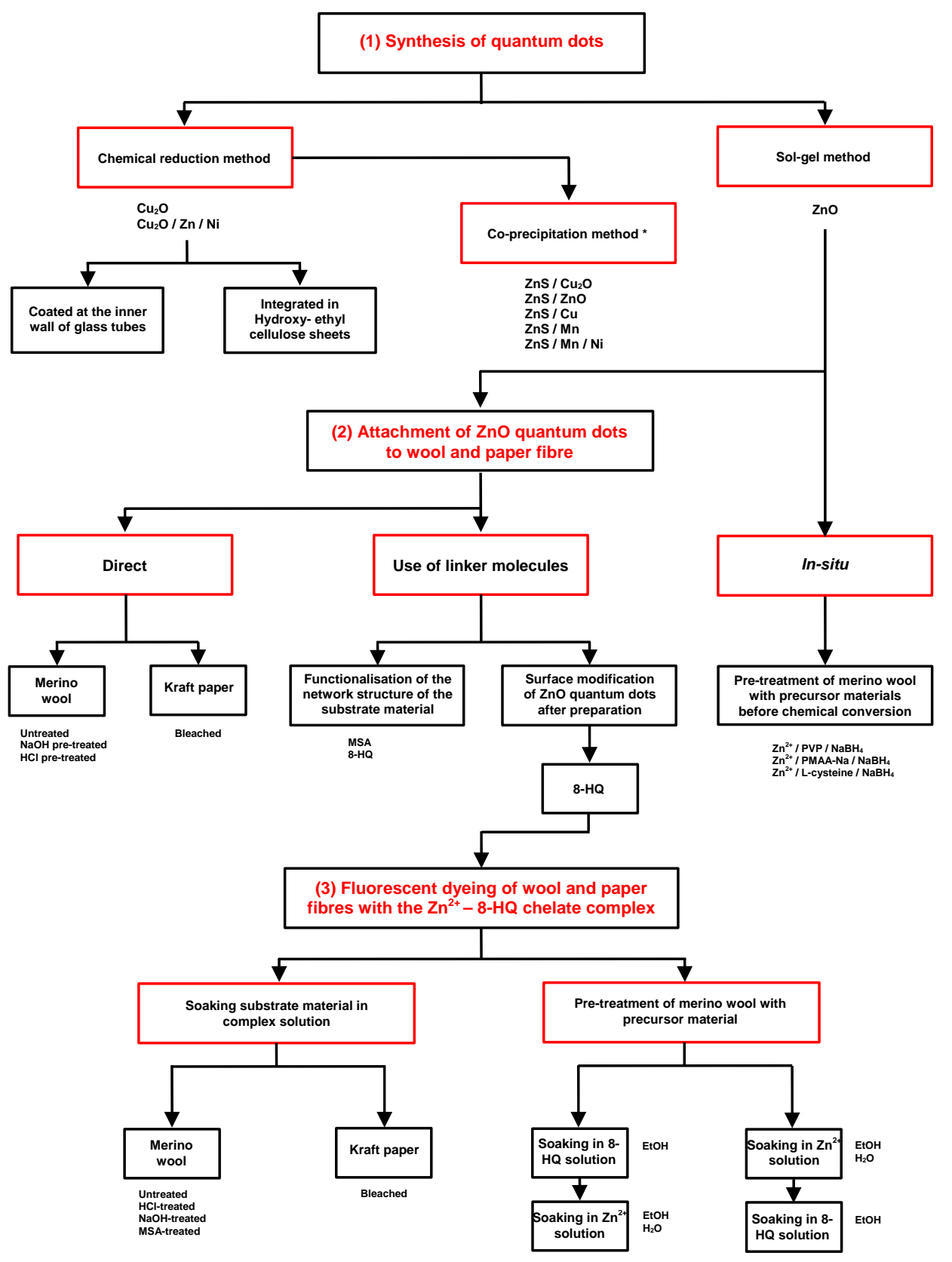

Figure 2.1: Flow chart for the synthesis of quantum dots and development of fluorescent composite materials. 


\subsection{Synthesis of quantum dots}

It is expected that the combination of fluorescent quantum dots and fibrous substrate material such as wool and paper will create new composite materials that exhibit the inherent the properties of both precursors.

In this project, copper (I) oxide $\left(\mathrm{Cu}_{2} \mathrm{O}\right)$ and zinc oxide $(\mathrm{ZnO})$ have been selected as possible quantum dot material to develop quantum dot - fibre composites. The synthesis of $\mathrm{Cu}_{2} \mathrm{O}$ and $\mathrm{ZnO}$ quantum dots was carried out predominately in an aqueous environment and in the presence of capping agents. Charged and bulkier surfactants were used as capping agents to control the particle growth and ensure a stable suspension in water. The combination of different surfactants with different strengths of adsorption (chemical interaction) on the particle surface was used to control the structure, shape, size and size distribution of the nanocrystals. Also, the effect of the solvent type and the ratio of different solvents mixed together were considered on the morphology and dispersion of the nanocrystals.

\subsubsection{Experimental setup}

Quantum dots were formed in a $150 \mathrm{~mL}$ three-neck round bottom flask equipped with a refluxing device. The reflux device was used to avoid shifts in the concentration of the reaction medium at higher temperatures through the loss of solvent or reagents by vapour emission. Different temperatures were obtained by using a silicone oil bath positioned on top of a hot plate with an integrated magnetic stirrer. The entire process was carried out under vigorous stirring using the magnet stirrer. The illustration in figure 2.2 shows the typical setup of the glassware and electrical equipment used for the synthesis of quantum dots. 


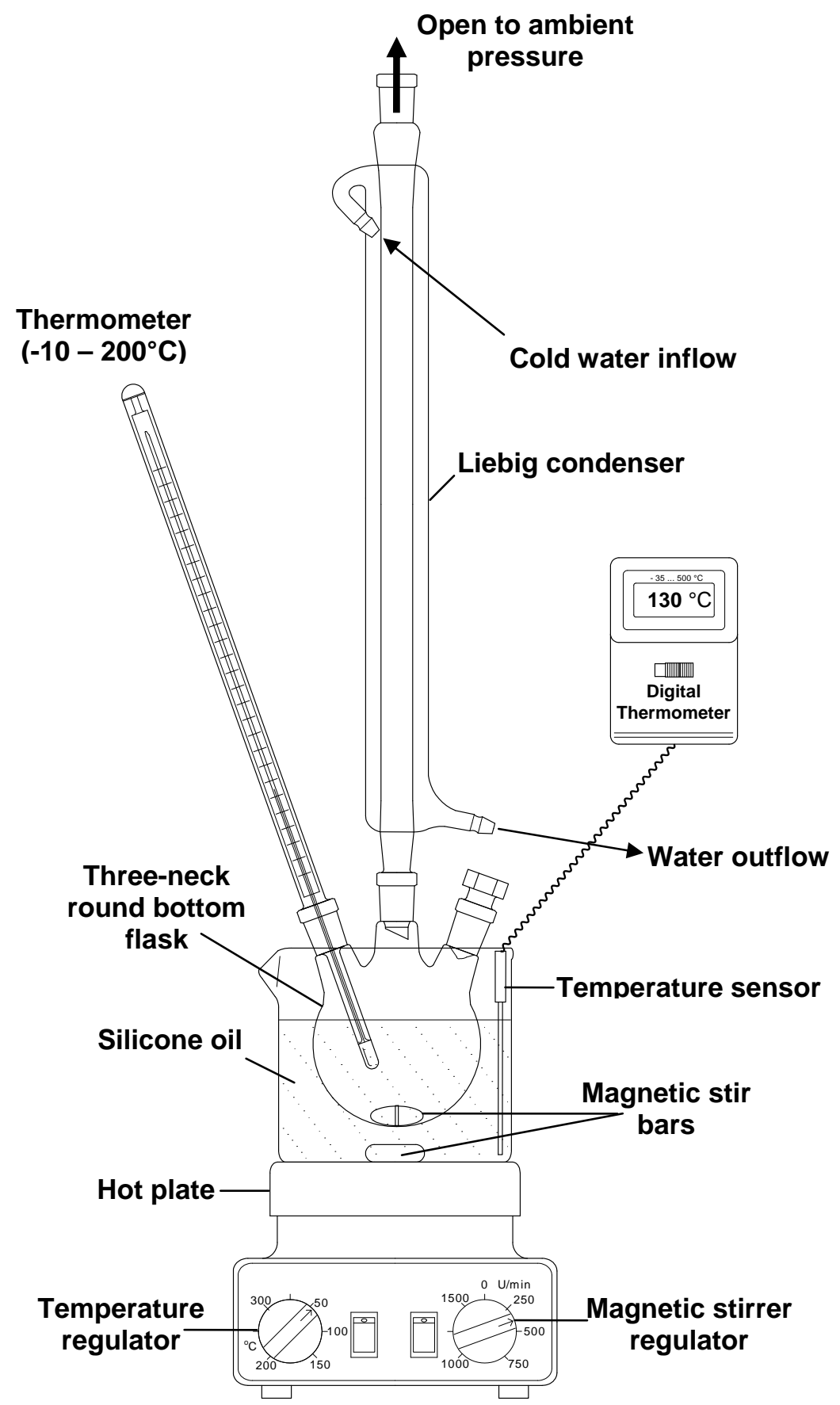

Figure 2.2 Experimental setup for the synthesis of quantum dots carried out in a fume hood.

For the purification of the quantum dot material, the suspensions were centrifuged at $6000 \mathrm{rpm}$ for at least $15 \mathrm{~min}$. After centrifugation, the supernatant solution was discarded, but only if all material had precipitated, and the precipitate of nanoparticles was further re-dispersed in $25 \mathrm{~mL}$ water. The separation and washing by centrifugation and re-dispersion in $25 \mathrm{~mL}$ water was repeated twice to ensure that all unwanted byproducts and unreacted chemicals were removed from the precipitate. The separation and washing procedure of the quantum dot material is described in table 2.1. 
Table 2.1: Separation and washing procedure for synthesised quantum dot materials.

\begin{tabular}{|c|c|c|c|}
\hline \multirow[b]{2}{*}{ Final suspension $\rightarrow$} & \multicolumn{2}{|c|}{$50 \mathrm{~mL}$ centrifuge glass tube } & \multirow[t]{2}{*}{$\begin{array}{l}\text { Equipment: } \\
\text { Centrifuge, ultrasonic bath }\end{array}$} \\
\hline & & transfer & \\
\hline \multirow{5}{*}{$25 \mathrm{~mL}$ distilled $\mathrm{H}_{2} \mathrm{O} \rightarrow$} & & centrifuge & $\begin{array}{l}\rightarrow \text { Separation by centrifugation at } 6000 \mathrm{rpm} \\
\text { for } 15 \mathrm{~min}\end{array}$ \\
\hline & & decant & $\begin{array}{l}\rightarrow \text { save supernatant for further separation if } \\
\text { necessary }\end{array}$ \\
\hline & & $\begin{array}{l}\text { add to the } \\
\text { precipitate }\end{array}$ & \\
\hline & & disperse & $\begin{array}{l}\rightarrow \text { using ultrasonic bath to ensure that by- } \\
\text { product impurities are completely washed out }\end{array}$ \\
\hline & & $\begin{array}{l}\text { repeat steps } 2 \text { to } 5 \\
\text { twice }\end{array}$ & \\
\hline
\end{tabular}

If desired, nanocrystals can also be transformed into powder by freeze-drying. For the freeze-drying procedure, a small amount of the precipitation $(2-5 \mathrm{~mL})$ was transferred in a $50 \mathrm{~mL}$ round bottom flask and cooled down by spinning the flask in liquid nitrogen until the precipitate froze at the inner wall of the flask. Then, the flask was attached to the freeze-dryer. The freeze-drying process removes the water from the solid material by reducing the surrounding pressure. The frozen water then sublimates and passes from the solid to the gas phase. The dry precipitate remains inside the wall of the round bottom flask and can be later scratched off.

\subsubsection{Materials used for the synthesis of quantum dots}

\subsubsection{Capping agents}

The following ionic capping agents were used after or during the particle formation to electronically passivate the semiconductor surface and obtain a homogeneous suspension with a narrow particle size distribution:

- Sodium citrate tribasic dihydrate $\left(\mathrm{Na}_{3} \mathrm{C}_{6} \mathrm{H}_{5} \mathrm{O}_{7} \cdot 2 \mathrm{H}_{2} \mathrm{O}\right)_{\text {solid; }}$;

Formula Weight $(\mathrm{FW})=294.1 \mathrm{~g} \mathrm{~mol}^{-1}$; ACS reagents; minimum assay $99.0 \%$

- L-cysteine $\left(\mathrm{C}_{3} \mathrm{H}_{7} \mathrm{NO}_{2} \mathrm{~S}\right)_{\text {solid; }}$;

$\mathrm{FW}=121.16 \mathrm{~g} \mathrm{~mol}^{-1}$; Aldrich reagent; minimum assay $98 \%$

- Glycine $\left(\mathrm{C}_{2} \mathrm{H}_{5} \mathrm{NO}_{2}\right)_{\text {solid }}$

$\mathrm{FW}=75.07 \mathrm{~g} \mathrm{~mol}^{-1}$; ACS reagent; minimum assay $99 \%$

- Sodium thiosulfate $\left(\mathrm{Na}_{2} \mathrm{~S}_{2} \mathrm{O}_{3}\right)_{\text {solid; }}$;

$\mathrm{FW}=158.11 \mathrm{~g} \mathrm{~mol}^{-1}$; minimum assay $99 \%$ 
- Urea $\left(\mathrm{CH}_{4} \mathrm{~N}_{2} \mathrm{O}\right)_{\text {solid; }}$;

$\mathrm{FW}=60.06 \mathrm{~g} \mathrm{~mol}^{-1}$; minimum assay $99 \%$

- Mercaptosuccinic acid $\left(\mathrm{C}_{4} \mathrm{H}_{6} \mathrm{O}_{4} \mathrm{~S}\right)_{\text {solid; }}$;

$\mathrm{FW}=150.5 \mathrm{~g} \mathrm{~mol}^{-1}$; Sigma Aldrich; minimum assay $97 \%$

- 8-Hydroxylquinoline $\left(\mathrm{C}_{9} \mathrm{H}_{7} \mathrm{NO}\right)_{\text {solid; }}$;

$\mathrm{FW}=145.16 \mathrm{~g} \mathrm{~mol}^{-1}$; minimum assay $99 \%$

The following bulkier dispersants were used to passivate the surface area during particle synthesis and create greater steric hindrance between nanoparticles:

- Poly(vinyl-pyrrolidone) (PVP) $)_{\text {solid; }}$

$\mathrm{FW} \approx 55000 \mathrm{~g} \mathrm{~mol}^{-1}$; Sigma Aldrich

- Poly(methacrylic acid, sodium salt) (30 wt \% PMAA-Na solution in water);

$\mathrm{FW} \approx 9500 \mathrm{~g} \mathrm{~mol}^{-1}$; Sigma Aldrich

\subsubsection{Copper (I) oxide}

$\mathrm{Cu}_{2} \mathrm{O}$ nanocrystals were synthesised by a chemical reduction method using the following analytically pure reagents:

- Sodium citrate tribasic dihydrate $\left(\mathrm{Na}_{3} \mathrm{C}_{6} \mathrm{H}_{5} \mathrm{O}_{7} \cdot 2 \mathrm{H}_{2} \mathrm{O}\right)_{\text {solid; }}$;

Formula Weight $(\mathrm{FW})=294.1 \mathrm{~g} \mathrm{~mol}^{-1}$; ACS reagents $\geq 99.0 \%$

- Copper nitrate $\left(\mathrm{Cu}\left(\mathrm{NO}_{3}\right)_{2} \cdot 3 \mathrm{H}_{2} \mathrm{O}\right)_{\text {solid; }}$

$\mathrm{FW}=241.6 \mathrm{~g} \mathrm{~mol}^{-1}$; Riedle-De Haën AG Seelze-Hannover; minimum assay $99 \%$

- Copper chloride $\left(\mathrm{CuCl}_{2} \cdot 2 \mathrm{H}_{2} \mathrm{O}\right)_{\text {solid; }}$;

$\mathrm{FW}=170.48 \mathrm{~g} \mathrm{~mol}^{-1} ;$ Ajax Fiechem, Technical $\geq 96.0 \%$

- Sodium borohydride $\left(\mathrm{NaBH}_{4}\right)_{\text {solid; }}$;

$\mathrm{FW}=37.83 \mathrm{~g} \mathrm{~mol}^{-1}$; Sigma-Aldrich, minimum assay $98.5 \%$

- Sodium borohydride in triethylene glycol dimethyl ether (2 $\left.\mathrm{M} \mathrm{NaBH}_{4}\right)$;

$\mathrm{FW}=37.83 \mathrm{~g} \mathrm{~mol}^{-1}$; Sigma-Aldrich, density: 0.998 at $25^{\circ} \mathrm{C}$

- Poly(vinyl-pyrrolidone) (PVP) $)_{\text {solid; }}$

$\mathrm{FW} \approx 55000 \mathrm{~g} \mathrm{~mol}^{-1}$; Sigma Aldrich

- Poly(methacrylic acid, sodium salt) (30 wt\% PMAA-Na solution in water);

$\mathrm{FW} \approx 9500 \mathrm{~g} \mathrm{~mol}^{-1}$; Sigma Aldrich

- Distilled water 


\subsubsection{Zinc oxide}

The following reagents were used for the preparations of zinc oxide nanocrystals via a sol-gel method:

- Zinc chloride $\left(\mathrm{ZnCl}_{2}\right)_{\text {solid; }}$;

$\mathrm{FW}=136.29 \mathrm{~g} \mathrm{~mol}^{-1}$; minimum assay $98 \%$

- Zinc acetate $\left(\mathrm{Zn}\left(\mathrm{CH}_{3} \mathrm{COO}\right)_{2} \cdot 2 \mathrm{H}_{2} \mathrm{O}\right)_{\text {solid; }}$;

$\mathrm{FW}=219.15 \mathrm{~g} \mathrm{~mol}^{-1}$; minimum assay $99 \%$

- Sodium borohydride in triethylene glycol dimethyl ether (2 $\left.\mathrm{M} \mathrm{NaBH}_{4}\right)$;

$\mathrm{FW}=37.83 \mathrm{~g} \mathrm{~mol}^{-1}$; Sigma-Aldrich, density: 0.998 at $25^{\circ} \mathrm{C}$

- Lithium borohydride in THF (2 $\left.\mathrm{M} \mathrm{LiBH}_{4}\right)$;

$\mathrm{FW}=21.78 \mathrm{~g} \mathrm{~mol}^{-1}$; Sigma-Aldrich, density: 0.896 at $25^{\circ} \mathrm{C}$

- Poly(vinyl-pyrrolidone) (PVP) $)_{\text {solid; }}$

$\mathrm{FW} \approx 55000 \mathrm{~g} \mathrm{~mol}^{-1}$; Sigma Aldrich

- Poly(methacrylic acid, sodium salt) (30 wt $\%$ PMAA-Na solution in water);

$\mathrm{FW} \approx 9500 \mathrm{~g} \mathrm{~mol}^{-1}$; Sigma Aldrich

- Distilled water

\subsubsection{Zinc sulphide}

For the preparation of zinc sulphide nanocrystals following reagents were used:

- $\quad$ Sodium sulphide $\left(\mathrm{Na}_{2} \mathrm{~S}\right)$

$\mathrm{FW}=78.04 \mathrm{~g} \mathrm{~mol}^{-1}$; minimum assay $99 \%$

- Zinc chloride $\left(\mathrm{ZnCl}_{2}\right)_{\text {solid; }}$

$\mathrm{FW}=136.29 \mathrm{~g} \mathrm{~mol}^{-1}$; minimum assay $98 \%$

- Poly(vinyl-pyrrolidone) (PVP) $)_{\text {solid; }}$

$\mathrm{FW} \approx 55000 \mathrm{~g} \mathrm{~mol}^{-1} ;$ Sigma Aldrich

- Poly(methacrylic acid, sodium salt) (30 wt $\%$ PMAA-Na solution in water);

$\mathrm{FW} \approx 9500 \mathrm{~g} \mathrm{~mol}^{-1}$; Sigma Aldrich

- Distilled water 


\subsubsection{Dopants used for doping of quantum dots}

The incorporation of dopant materials (impurities) in the lattice structure of the quantum dot materials can result in a shift of the fluorescence colour in the visible range of the quantum dot material. The following reagents were used to dope the nanocrystals:

- Zinc chloride $\left(\mathrm{ZnCl}_{2}\right)_{\text {solid; }}$

$\mathrm{FW}=136.29 \mathrm{~g} \mathrm{~mol}^{-1}$; minimum assay $98 \%$

- $\quad$ Nickel (II) chloride hexahydrate $\left(\mathrm{NiCl}_{2} \cdot 6 \mathrm{H}_{2} \mathrm{O}\right)_{\text {solid; }}$;

$\mathrm{FW}=237.71 \mathrm{~g} \mathrm{~mol}^{-1}$; BDH Chemicals, minimum assay $97 \%$

- Cerium (III) chloride anhydrous, beads $\left(\mathrm{CeCl}_{3}\right)$;

$\mathrm{FW}=246.48 \mathrm{~g} \mathrm{~mol}^{-1}$; minimum assay $99.99 \%$

- Manganese chloride $\left(\mathrm{MnCl}_{2} \cdot 4 \mathrm{H}_{2} \mathrm{O}\right)_{\text {solid; }}$;

$\mathrm{FW}=197.91 \mathrm{~g} \mathrm{~mol}^{-1}$; minimum assay $98 \%$

- Cobalt (II) chloride $\left(\mathrm{CoCl}_{2} \cdot 6 \mathrm{H}_{2} \mathrm{O}\right)_{\text {solid; }}$;

$\mathrm{FW}=237.93 \mathrm{~g} \mathrm{~mol}^{-1}$; minimum assay $97 \%$

\subsubsection{Methods used to produce quantum dots}

\subsubsection{Chemical reduction method - Copper (I) oxide nanocrystals}

In the chemical reduction method, quantum dots are formed by the reduction of their corresponding metal ions in solution in the presence of a particle stabilizer. This process is based on a chemical transformation of the precursor materials, whereby the particle growth takes place between nucleation and seed growth [71-75].

Copper (I) oxide $\left(\mathrm{Cu}_{2} \mathrm{O}\right)$ nanocrystals were formed in water by reducing aqueous copper nitrate $\left(\mathrm{Cu}\left(\mathrm{NO}_{3}\right)_{2}\right)$ solution with sodium borohydride $\left(\mathrm{NaBH}_{4}\right)$ in the presence of sodium citrate $\left(\mathrm{Na}_{3} \mathrm{C}_{6} \mathrm{H}_{5} \mathrm{O}_{7}\right)$ (ionic capping agent) and poly(methacrylic acid sodium salt) (PMAA-Na) (polymeric stabilizer) at a reaction temperature of $99-100{ }^{\circ} \mathrm{C}$ using silicone oil bath.

First $46 \mathrm{~mL}$ distilled water was transferred into the $150 \mathrm{~mL}$ round bottom flask and heated up to $99-100{ }^{\circ} \mathrm{C}$. Then, $2.5 \mathrm{~mL}$ aliquots of $0.01 \mathrm{M}$ copper nitrate aqueous solution, $0.5 \mathrm{~mL} 0.03 \mathrm{M}$ trisodium citrate aqueous solution and $0.5 \mathrm{~mL} 5 \mathrm{mg} \cdot \mathrm{mL}^{-1}$ PMAA-Na aqueous solutions were added to the refluxing water. Trisodium citrate and 
PMAA-Na act here as stabilizing agents for the copper ions by forming a surfactant complex, where copper ions are bound to the functional groups of citrate and PMAA$\mathrm{Na}$. The next step was the quick addition of $0.5 \mathrm{~mL}$ of $1 \mathrm{M}$ sodium borohydride stock solution injected by a $1 \mathrm{~mL}$ syringe at the top of the cooling device. After running down into the solution, the colour of the solution turned immediately from transparent into a coffee brown colour and then to a dark green-blue colour. After 30 min stirring the suspension was let to cool down slowly by removing the silicone oil bath. At around $80^{\circ} \mathrm{C}$ the suspension turned bright yellow-green. After cooling down to room temperature, the suspension was centrifuged for $15 \mathrm{~min}$ at $6000 \mathrm{rpm}$. Finally, a green precipitate was separated from the supernatant and re-dispersed in $50 \mathrm{~mL}$ water. Any further washing of the precipitate after centrifugation may result in particle loss. It is important to note that the supernatant contains small amounts of $\mathrm{Cu}_{2} \mathrm{O}$ nanoparticles, which can be precipitated by further centrifugation.

The procedure for the synthesis of pure copper (I) oxide nanocrystals is described in table 2.2.

Table 2.2: Instructions for the synthesis of copper (I) oxide nanocrystals.

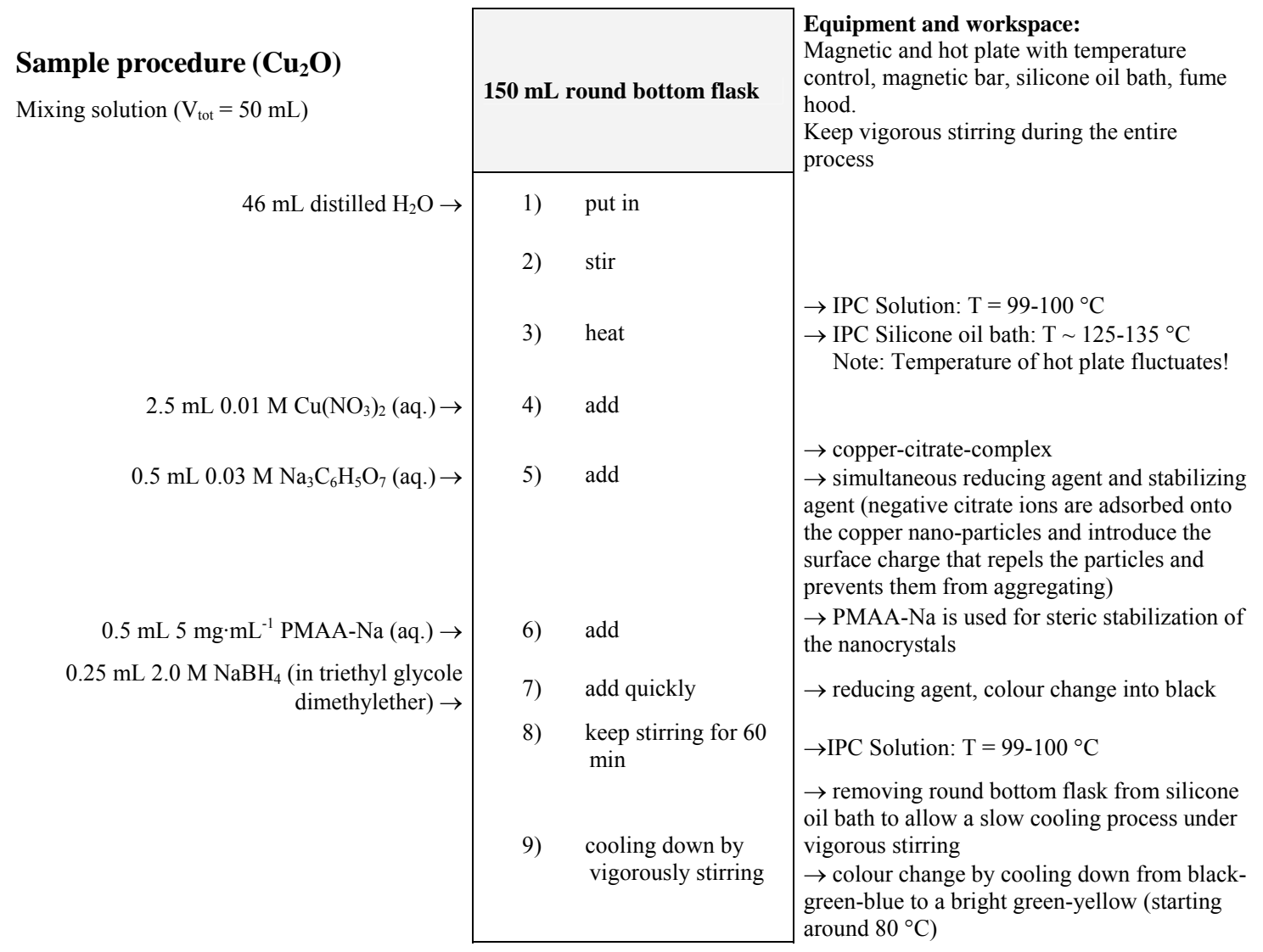


This reduction method can also be used to obtain nanocrystals containing a mixture of different elements. An example is given in table 2.3 which shows the steps to follow for the synthesis of $\mathrm{Cu}_{2} \mathrm{O}$ nanocrystals doped with zinc and nickel ions.

Table 2.3: Instructions for the synthesis of $\mathrm{Zn}^{2+}$ and $\mathrm{Ni}^{2+}$ doped copper (I) oxide nanocrystals.

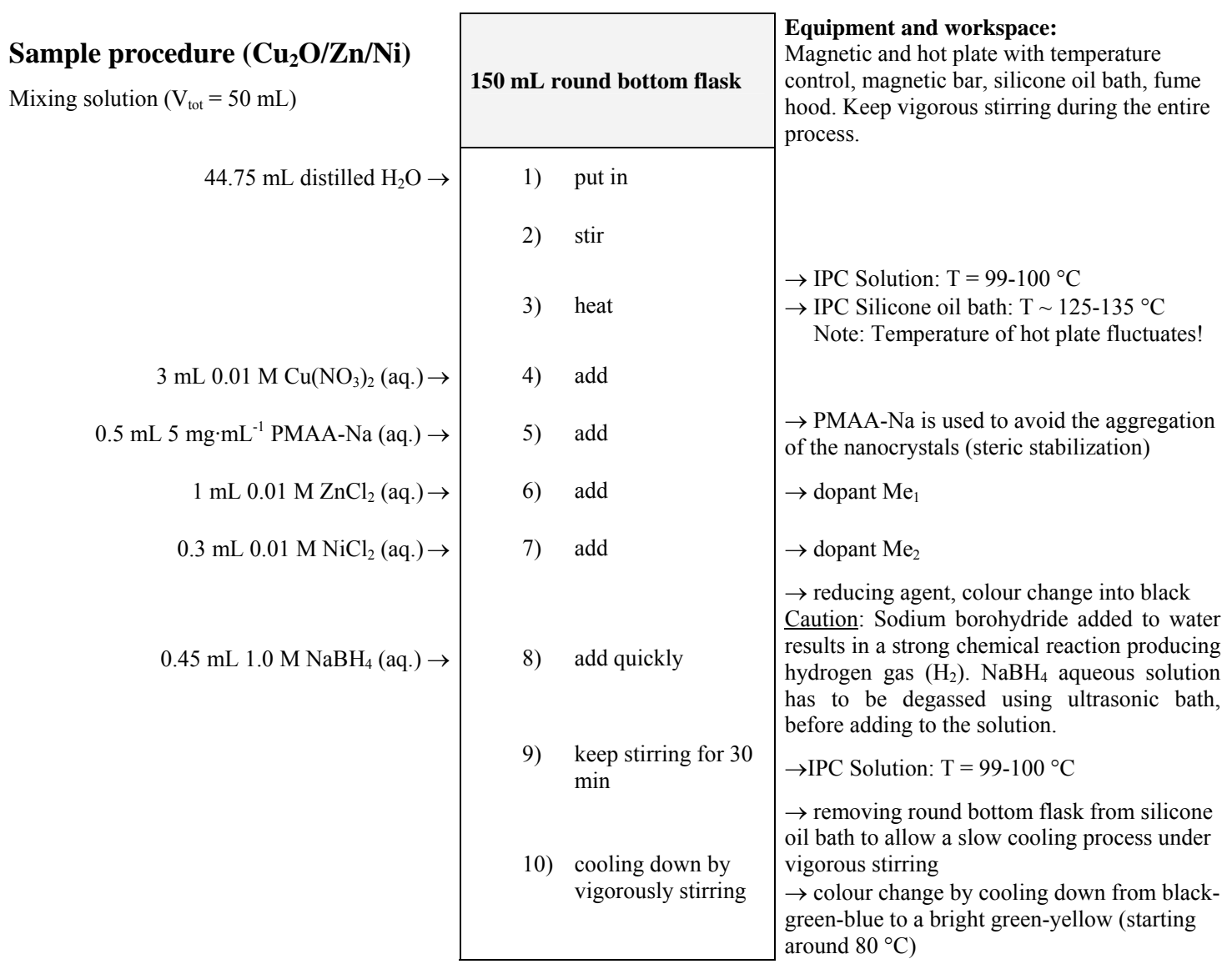

The methods used to synthesise $\mathrm{Cu}_{2} \mathrm{O}$ nanocrystals (table 2.2) and doped $\mathrm{Cu}_{2} \mathrm{O} / \mathrm{Zn} / \mathrm{Ni}$ nanocrystals (table 2.3) were standardised by selecting those process parameters that produced nanocrystals with the most interesting optical properties. The main process parameters evaluated were reaction time and temperature, $\mathrm{pH}$, solvent, choice of copper precursor material and capping agent, concentration of copper precursor material, concentration of capping agent (ionic and polymeric), copper ion to capping agent ratio, concentration of the reducing agent and presence of dopants (see table 3.1).

The effect of the these process parameters on the particle growth, particle shape and optical properties were studied by scanning electron microscopy (SEM), transmission electron microscopy (TEM), particle size analysis (Zetasizer), fluorescence and UV-vis spectroscopy. The crystal structure identification and composition of the $\mathrm{Cu}_{2} \mathrm{O}$ quantum 
dots were examined by using X-ray diffraction (XRD), X-ray photoelectron spectroscopy (XPS) and Energy dispersive X-ray spectroscopy (EDS).

\subsubsection{Sol-gel method - Zinc oxide nanocrystals}

The sol-gel method makes use of the aqueous chemistry of zinc salts, which is dominated by the complexation with water molecules and their subsequent deprotonation - hydrolysis [38-40, 76-79].

Zinc oxide $(\mathrm{ZnO})$ nanocrystals were prepared by a sol-gel method using $\mathrm{NaBH}_{4}$ as an alkali reducing agent in the presence of poly(vinyl-pyrrolidone) (PVP) in a zinc chloride aqueous solution at a reaction temperature of $70{ }^{\circ} \mathrm{C}$. In this sol-gel method, $\mathrm{NaBH}_{4}$ was used as an alkali agent for the forced hydrolysis reaction of the water complexed zinc ions.

The synthesis was carried out in a $150 \mathrm{~mL}$ three-neck round bottom flask equipped with a refluxing device under vigorous stirring using a magnetic stirrer during the entire process at $70{ }^{\circ} \mathrm{C}$ using silicone oil bath. First, $71.5 \mathrm{~mL}$ of highly purified water is transferred into a $150 \mathrm{~mL}$ round bottom flask. Then, aliquots of $1 \mathrm{~mL}$ of $1 \mathrm{M}$ zinc chloride aqueous solution, $2 \mathrm{~mL} 5 \mathrm{mg} \cdot \mathrm{mL}^{-1} \mathrm{PVP}$ aqueous solution were added to the 70 ${ }^{\circ} \mathrm{C}$ refluxing water. This step was followed by the quick addition of $0.5 \mathrm{~mL}$ of $2 \mathrm{M}$ sodium borohydride triethylene glycol dimethyl ether stock solution injected with a 1 $\mathrm{mL}$ syringe at the top of the cooling device. After running down along the wall inside of the Liebig condenser into the solution, the zinc/polymer complex solution turned immediately into a white milky suspension. The suspension was refluxed for another 30 min under vigorous stirring at $70{ }^{\circ} \mathrm{C}$ and then cooled down to room temperature. The precipitate was then centrifuged and washed with distilled water to ensure that all byproduct impurities and unreacted chemicals were removed from the suspension. In the end, the precipitate was re-dispersed in $10 \mathrm{~mL}$ water. The steps followed for the synthesis of zinc oxide nanocrystals are summarised in table 2.4. 
Table 2.4: Instructions for the synthesis of zinc oxide nanocrystals.

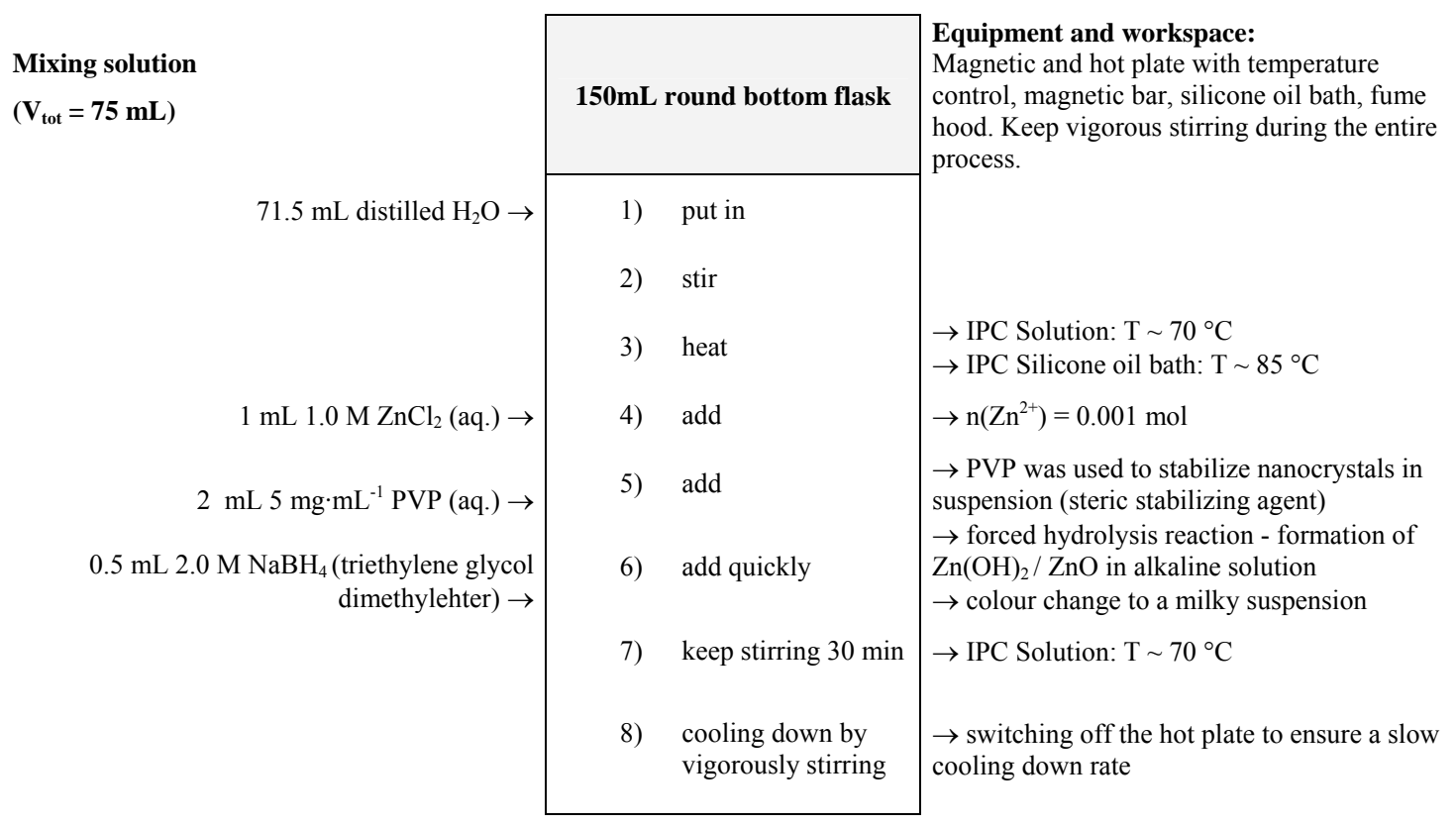

The standardised method for the synthesis of $\mathrm{ZnO}$ quantum dots presented in table 2.4 was designed by choosing those process parameters which produced $\mathrm{ZnO}$ quantum dots with the highest fluorescence intensity in the visible range. This involved the variation of process parameters such as reaction time and temperature, concentration of the zinc precursor material, concentration of polymeric stabilizer and concentration of the alkali reducing agent.

The effect of the surface coverage of the $\mathrm{ZnO}$ quantum dots was also investigated by changing the polymeric surfactant from PVP to PMAA-Na. The influence of this modification on the nanocrystal growth, shape and fluorescence properties were studied by transmission electron microscopy (TEM), scanning electron microscopy (SEM), particle size analysis (Zetasizer), fluorescence and UV-vis spectroscopy.

Furthermore, the introduction of dopant ions $\left(\mathrm{Me}^{2+/ 3+}\right)$ in the lattice structure of the $\mathrm{ZnO}$ quantum dots by using the standardised synthesis method described above was attempted. Here, small amounts of dopant aqua solution $\left(0.1-1 \mathrm{~mL} 0.01 \mathrm{M} \mathrm{CeCl}_{3}\right.$, $\mathrm{MnCl}_{2}, \mathrm{CoCl}_{2}$ and $\mathrm{NiCl}_{2}$ ) were added to the reaction medium between step 5 and 6 in table 2.4. 


\subsubsection{Precipitation method - Zinc sulphide nanocrystals}

The wet chemical precipitation method is based on dynamic equilibrium and allows the incorporation of small quantities of impurities (dopants) into the chemical compositon of the final product. The method involves first the formation of very small particles (seeds) which will subsequent grow to larger once. By controlling the kinetic of the subsequent particle growth, e.g. using complexing reagents for the precursor materials, it is possible to obtain a narrow size distribution [46, 80-84].

Zinc sulphide $(\mathrm{ZnS})$ quantum dots were formed by the slow addition of sodium sulphide $\left(\mathrm{Na}_{2} \mathrm{~S}\right)$ solution into a zinc chloride $\left(\mathrm{ZnCl}_{2}\right)$ solution in the presence of metallic nanoclusters formed during a chemical reduction method at $99-100{ }^{\circ} \mathrm{C}$. The precipitation reaction is completed under continuous stirring of the reaction mixture using a magnetic stirrer throughout the entire reaction process.

The general procedure for the wet chemical reduction and arrested precipitation method is carried out in a $150 \mathrm{~mL}$ three-necked round bottom flask equipped with a refluxing device. First, $36 \mathrm{~mL}$ Millipore water is transferred along with $3 \mathrm{~mL} 0.01 \mathrm{M} \mathrm{MnCl}_{2}$ (or $\mathrm{CuCl}_{2}$ ) aqueous solution and $0.5 \mathrm{~mL} 5 \mathrm{mg} \cdot \mathrm{mL}^{-1} \mathrm{PMMA}-\mathrm{Na}$ aqueous solution into a 150 $\mathrm{mL}$ round bottom flask and refluxed together under vigorous stirring at $99-100{ }^{\circ} \mathrm{C}$ using a silicone oil bath. The next step is the quick addition of $0.2 \mathrm{~mL} 2 \mathrm{M}$ sodium borohydride in triethylene glycol dimethyl ether to the solution. The colour of the solution will turn immediately from transparent into a light red colour. After 5 min stirring, $5 \mathrm{~mL} 1 \mathrm{M} \mathrm{ZnCl}_{2}$ aqueous solution is gradually added to the solution under rigorous mixing. Thereupon the suspension turns transparent.

For the basic precipitation reaction, $5 \mathrm{~mL} 1 \mathrm{M} \mathrm{Na}_{2} \mathrm{~S}$ aqueous solution is added dropwise using a dropping funnel to the reaction medium with the highest stirring speed possible. A white precipitate is formed immediately in the round bottom flask. It is important to keep the reaction mixture vigorously stirred in order to obtain a homogenous mixture of all components. After $5 \mathrm{~min}$ of stirring the hot plate is switched off and the white suspension is slowly cooled down under vigorous stirring. The precipitate is then centrifuged at $6000 \mathrm{rpm}$ for $15 \mathrm{~min}$ and re-dispersed in distilled 
water. This washing procedure is repeated several times in order to remove all unreacted chemicals from the final product.

The yield of $\mathrm{ZnS}$ quantum dots obtained by this method is high and can be transformed into powder by drying in a muffle furnace. To do this, the wet precipitate is transferred to an ashless filter paper inside a funnel filter, whereby the excess of the remaining water in the precipitate is extracted using a conical flask connected to a water-jet vacuum pump. The precipitate on the filter paper is then transferred into a crucible and heated up in a muffle furnace for 6 hours at $1000{ }^{\circ} \mathrm{C}$. Afterwards the crucible with the dry powder is cooled down in a desiccator.

The synthesis of zinc sulfide quantum dots precipitated in the presence of $\mathrm{Zn}, \mathrm{Mn}$ or $\mathrm{Cu}$-nanoclusters using this reduction method is described in table 2.5.

Table 2.5: Instruction for the synthesis of zinc sulfide nanocrystals containing Mn-nanocluster

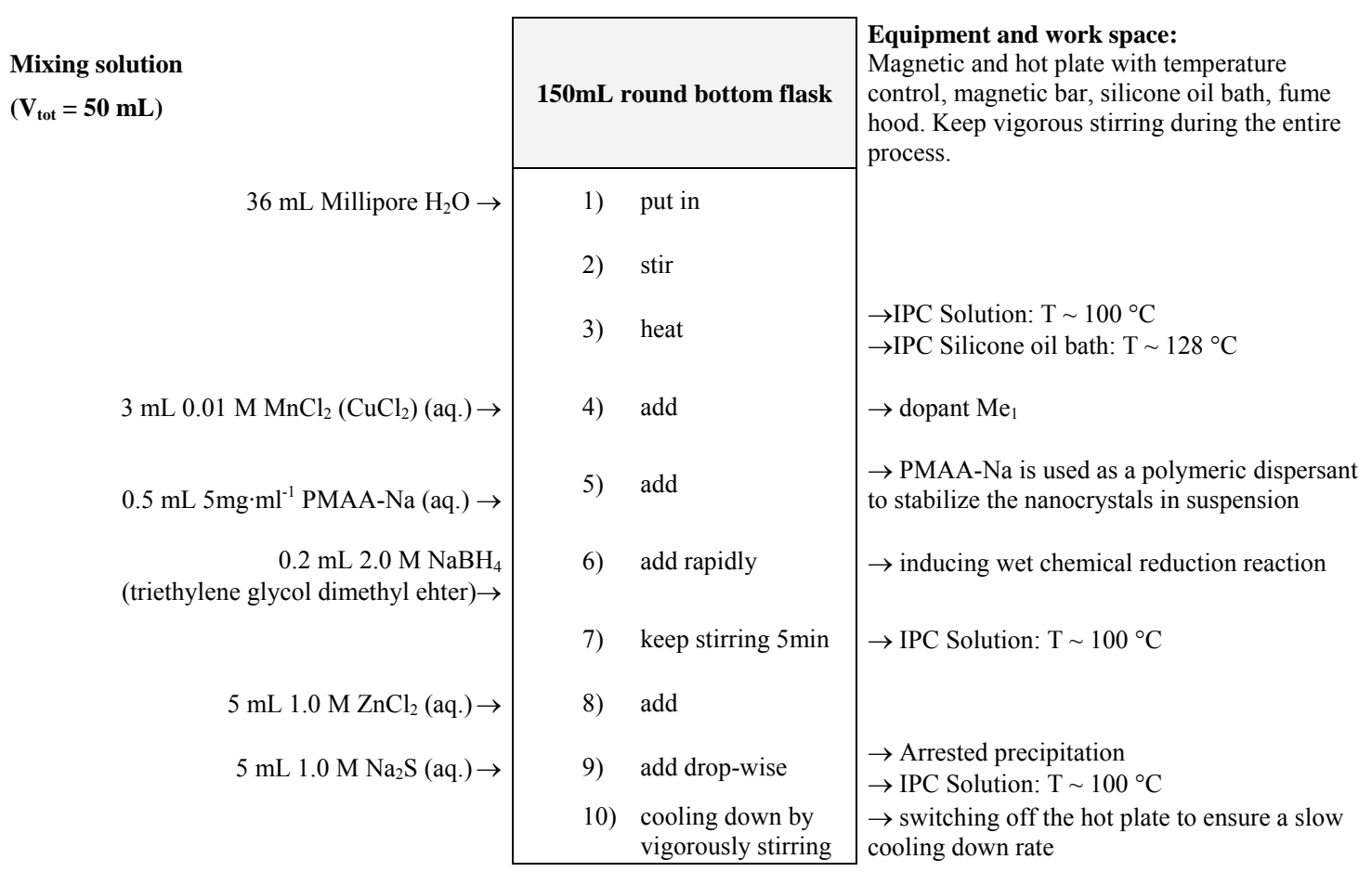

Nanoclusters of metals in mixture with other elements $\left(\mathrm{Me}_{1}, \mathrm{Me}_{2}\right)$ were also formed and co-precipitated during the formation of $\mathrm{ZnS}$ to produce $\mathrm{ZnS} / \mathrm{Me}_{1} / \mathrm{Me}_{2}$ quantum dots. An example is given in table 2.6. 
Table 2.6: Instruction for the synthesis of zinc sulfide nanocrystals containing $\mathrm{Mn} / \mathrm{Ni}$-nanoclusters

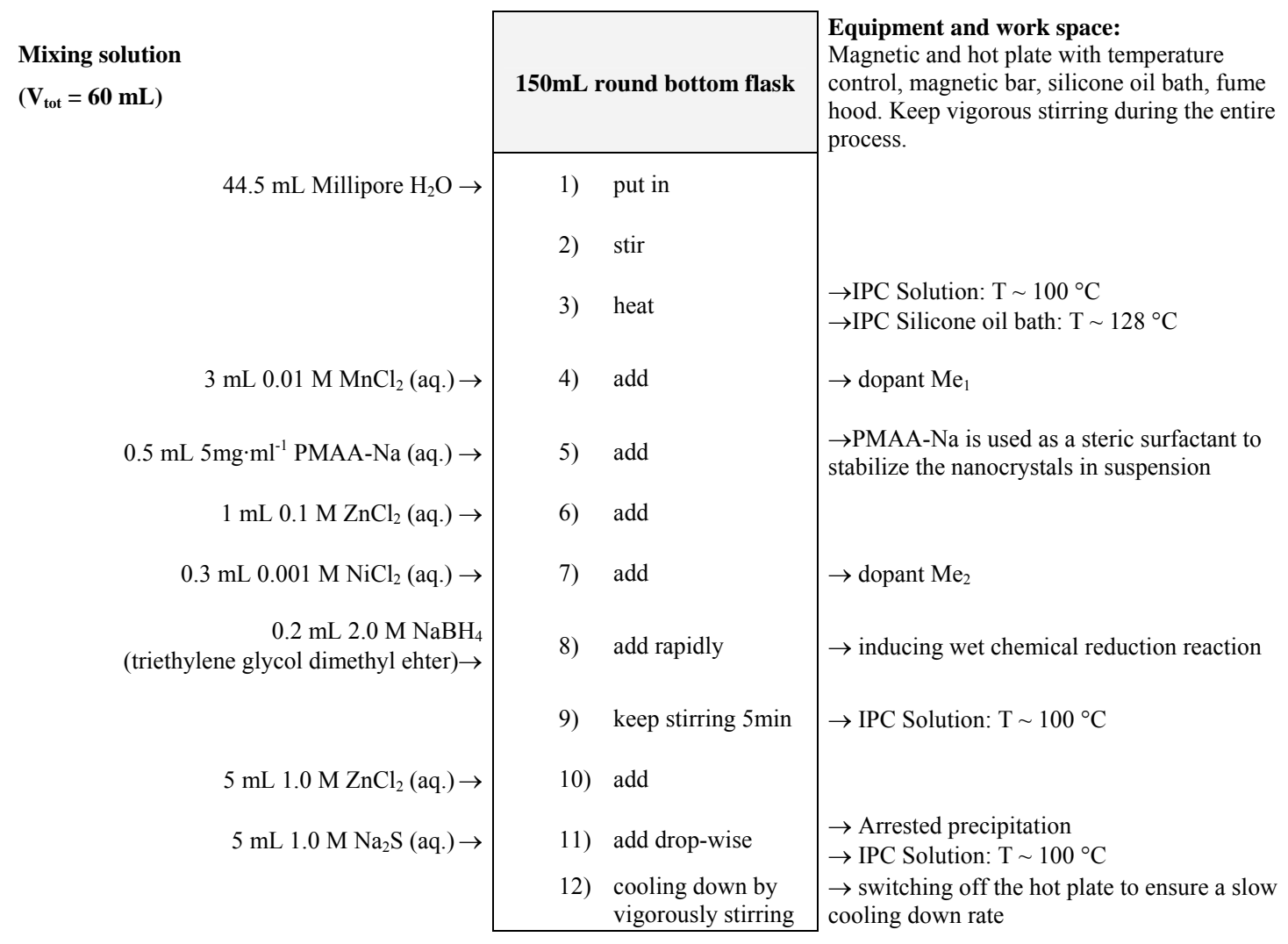

\subsection{Methods used to prepare quantum dot - fibre composites}

For the development of quantum dot - fibre composites, $\mathrm{ZnO}$ quantum dots have been combined with wool and paper using the in-situ and building block approach.

\subsubsection{In-situ binding of $\mathrm{ZnO}$ quantum dots to wool}

In the in-situ approach, $\mathrm{ZnO}$ quantum dots were formed in the presence of wool by using the sol-gel method described in 2.1.3.2 with minor modifications. This method relies on cross-link reactions between the quantum dots and wool fibre during nucleation and particle growth.

The in-situ approach involved first 3 hours soaking of $0.2 \mathrm{~g}$ merino wool in a $50 \mathrm{~mL}$ $0.02 \mathrm{M}$ zinc chloride $\left(\mathrm{ZnCl}_{2}\right)$ aqueous solution at $70{ }^{\circ} \mathrm{C}$. The $\mathrm{ZnCl}_{2}$ aqueous solution also contained a complex reagent for zinc cations such as PVP, PMAA-Na and Lcysteine. The amount of each complexing agent added and its concentration are indicated in table 2.7 . 
Table 2.7: List of capping agents used to modify the surface area of wool fibres.

\begin{tabular}{ccc}
\hline Capping agent & c (capping agent) & V (capping agent) / mL \\
\hline PVP $\left(\mathrm{H}_{2} \mathrm{O}\right)$ & $5 \mathrm{ppm}$ & 2 \\
PMAA- $\mathrm{Na}\left(\mathrm{H}_{2} \mathrm{O}\right)$ & $5 \mathrm{ppm}$ & 2 \\
L-cysteine $\left(\mathrm{H}_{2} \mathrm{O}\right)$ & $0.005 \mathrm{M}$ & 0.5 \\
\hline
\end{tabular}

The chemical transformation of the hydrolysed zinc cation into $\mathrm{ZnO}$ was then induced

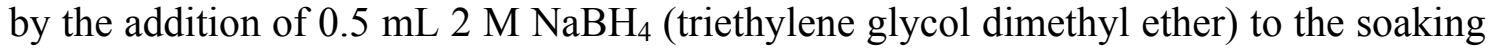
solution at $70{ }^{\circ} \mathrm{C}$. The reaction medium containing wool was well stirred to ensure homogeneity. The wool fibre was kept in the reaction medium for 24 hours at $70{ }^{\circ} \mathrm{C}$ under gentle agitation using water shake bath and then kept in solution for another week at room temperature. The final composite material was washed with distilled water and dried at room temperature.

\subsubsection{Building block approach for ZnO quantum dot - fibre composites}

In the building block approach, $0.1 \mathrm{~g}$ paper and wool were submerged inside $20 \mathrm{~mL}$ $\mathrm{ZnO}$ quantum dot suspension using different soaking parameters. Wool and paper fibres are excellent organic substrate materials for the uptake of nano-sized materials. These fibrous materials contain functional groups along their framework structure which provide anchor sites for the attachment of the quantum dots to the substrate material.

The building block approach can also make use of different chemical techniques to increase the uptake of quantum dots to the substrate materials. These techniques are based on chemical modification or functionalisation of the surface area of the substrate or quantum dot material.

\subsubsection{Merino wool and surface treatment methods}

Merino wool fibre consists of over $90 \%$ of protein, whereby the main component is keratin - a structured protein made up of different amino acids. The nitrogen, oxygen and sulphur atoms of the amino acids are the sites targeted for the attachment of the quantum dots. 
The uptake of quantum dots, however, can be further enhanced by removing the fatty acid monolayer around the wool, which may hinder the adsorption of quantum dots. Furthermore, the wool fibre surface can also be functionalised with linker molecules containing free functional groups that show a higher tendency to form a chemical bonding with the quantum dot surface. For this reason, merino wool was subject of different surface pre-treatment methods such as alcoholic alkali and acid treatment. Merino wool was also functionalised with Mercaptosuccinic acid (MSA) and 8Hydroxyquinoline (8-HQ) as linker molecules. The pre-treatment processes of merino wool are given below.

\subsection{Alcoholic alkali treatment}

For this treatment, $0.1 \mathrm{~g}(10 \mathrm{~g})$ untreated merino wool fibre was soaked in $5 \mathrm{~mL}(500$ $\mathrm{mL}) 0.001 \mathrm{M} \mathrm{NaOH} / \mathrm{KOH}$ aqueous solution. The glass jar containing wool fibre and $\mathrm{NaOH}$ solution was sealed and placed in a shaking water bath at $50{ }^{\circ} \mathrm{C}$ for 1 hour. The wool fibre was then rinsed with distilled water and dried at room temperature.

\subsection{Acid treatment}

Here, $0.1 \mathrm{~g}(10 \mathrm{~g})$ untreated merino wool fibre was soaked in $5 \mathrm{~mL}(500 \mathrm{~mL}) 0.01 \mathrm{M}$ $\mathrm{HCl}$ aqueous solution for 1 hour at a temperature of $50{ }^{\circ} \mathrm{C}$ in shaking water bath. The wool fibre was then washed with distilled water and dried at room temperature.

\subsection{Mercaptosuccinic acid treatment}

In this treatment, $0.1 \mathrm{~g}(10 \mathrm{~g})$ untreated wool was soaked in $5 \mathrm{~mL}(500 \mathrm{~mL}) 0.01 \mathrm{M}$ MSA aqueous solution. The wool was kept in the reaction medium for 1 hour at $50{ }^{\circ} \mathrm{C}$ using a water shake bath to allow the formation of chemical bonds between the functional groups of MSA and the functional groups of the substrate material. Finally, the wool fibre was washed with distilled water and dried at room temperature.

\subsection{8-Hydroxyquinoline treatment}

Here, $0.1 \mathrm{~g}$ untreated merino wool was immersed in $10 \mathrm{~mL} 10^{-4} \mathrm{M} 8$-HQ ethanol solution at different temperatures (room temperature, $50{ }^{\circ} \mathrm{C}, 70{ }^{\circ} \mathrm{C}, 80{ }^{\circ} \mathrm{C}$ ) using a 
shaking water bath for 1 day. The wool fibre was then washed with hot water $\left(70{ }^{\circ} \mathrm{C}\right)$ and ethanol to ensure that 8-HQ molecules loosely attached at the wool are removed from the fibres. The final 8-HQ fibre composite material was dried at room temperature. This procedure is summarised in table 2.8:

Table 2.8: Functionalisation of wool fibre using 8-HQ as linker molecule in EtOH solution.

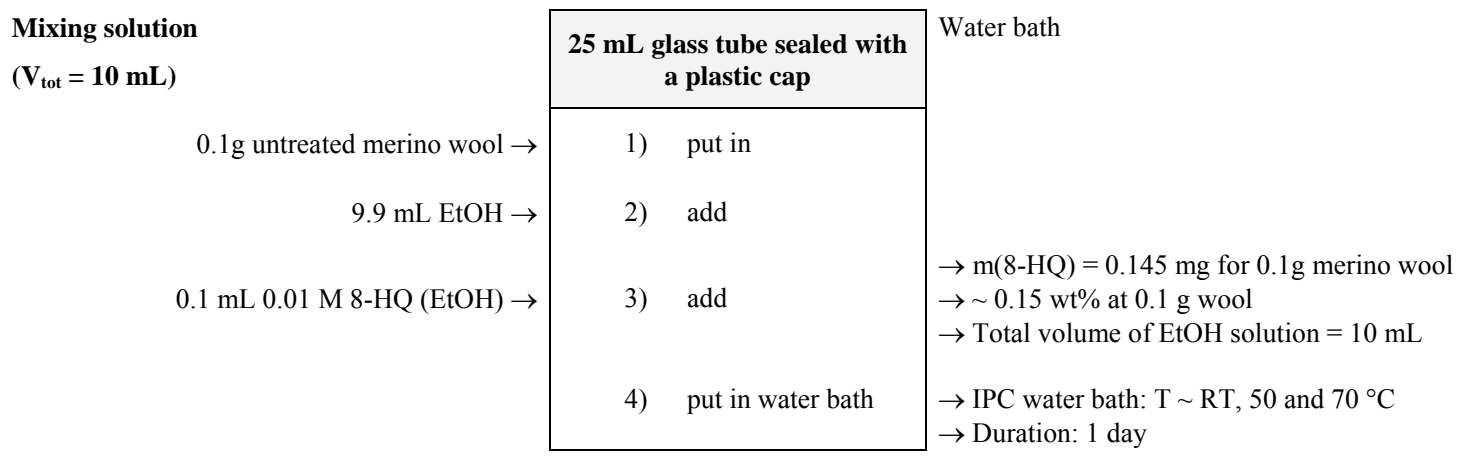

\subsubsection{Kraft paper fibres and pre-treatment methods}

For the development of quantum dot - paper fibre composites, bleached pulp paper fabricated by the Tasman Pulp \& Paper Company was used. Paper fibres are largely made of wood fibres, whereby cellulose is the target component for the attachment of quantum dots.

The bleached pulp paper is pressed forming thick square sheets. To use it in this study, the sheets have to be converted again into a pulp form. This was realised by shredding $0.1 \mathrm{~g}$ paper samples into a $5 \mathrm{~mL}$ aqueous solution using a Turrax mixer. The hydration of the fibres is promoted by the polar attraction of the water molecules for the hydroxyl groups covering the cellulose surface. The hydroxyl groups are also the anchor sites for the attachment of quantum dots to the cellulose fibres by forming hydrogen bonds. The attachment of quantum dots on paper fibres can also be enhanced by functionalising the cellulose chains with linker molecules. Therefore, paper fibre was pre-treated with Mercaptosuccinic acid (MSA) and 8-Hydroxyquinoline (8-HQ) as linker molecules.

\subsection{Mercaptosuccinic acid treatment}

For the functionalisation of paper fibre with MSA, $0.1 \mathrm{~g}$ bleached paper was shredded in $15 \mathrm{~mL}$ water using Turrax mixer and $5 \mathrm{~mL} 0.001 \mathrm{M}$ MSA aqueous solution were 
added to the paper pulp. The fibres were soaked in the reaction medium for one day at $50{ }^{\circ} \mathrm{C}$ to obtain a chemical bonding between the functional groups of MSA and the fibrous substrate. The paper pulp was then washed several times with water during filter suction according to the washing procedure in table 2.8. The wet paper pulp was then used for further processing.

\subsection{Washing procedure of paper composite materials}

After processing, paper fibres were separated and washed from reaction medium by vacuum filtration using a suction device. The suction device consists of a funnel filter with a filter paper, which is tightly attached to a conical flask, which in turn is connected to a water-jet vacuum pump. The paper fibres were washed three times with $25 \mathrm{~mL}$ distilled water under vacuum suction. The sheet thickness of the final paper composite material was controlled by spreading the fibres on a large or small area over the filter paper. After vacuum filtration, the paper filter and paper fibres were dried at room temperature on a watch glass of suitable size. After the remaining water is evaporated, the hydroxyl groups on opposing fibre surfaces will ultimately link together through hydrogen bonds and form a paper sheet [55]. The general procedure for washing and drying of paper fibres is given in table 2.9.

Table 2.9: Washing and drying procedure for paper fibre.

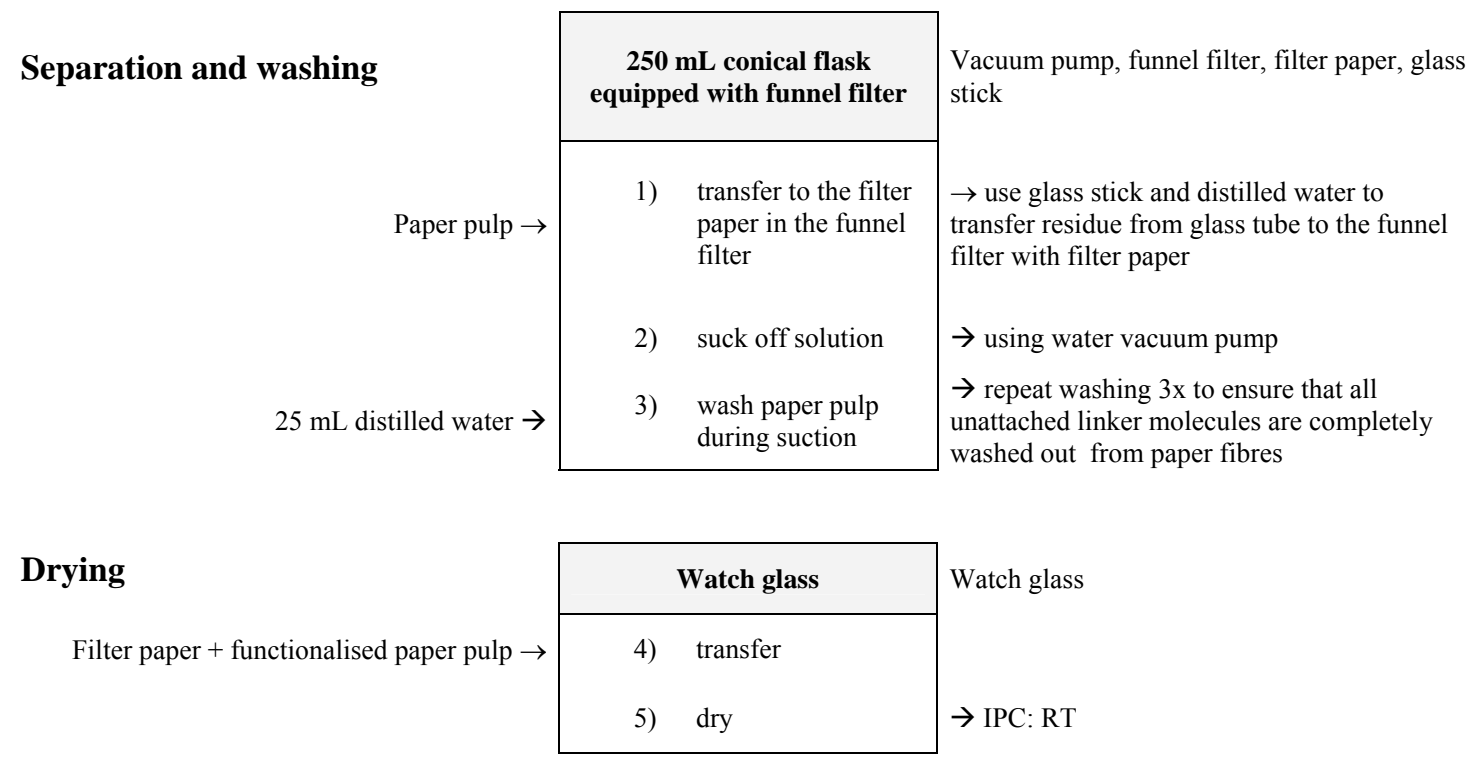




\subsubsection{Attachment of $\mathrm{ZnO}$ quantum dots onto wool}

For the attachment of $\mathrm{ZnO}$ quantum dots to wool, $0.1 \mathrm{~g}$ merino wool were soaked in 20 $\mathrm{mL} \mathrm{ZnO}$ quantum dot suspension (see procedure in 2.1.3.2) immediately after preparation and kept in a water shake bath at $70^{\circ} \mathrm{C}$ for 3 days. After soaking, the wool fibre was rinsed with distilled water and dried at room temperature. This procedure was repeated using different soaking times ( $3-9$ days) and temperatures $\left(25-90{ }^{\circ} \mathrm{C}\right)$ and the effect of these process parameters were evaluated by photoluminescence spectroscopy.

The uptake of $\mathrm{ZnO}$ quantum dots onto wool with 8-HQ as a fluorescent coordination ligand was carried out following the same procedure described above, except small amounts of 8-HQ (EtOH) $(0.1-1 \mathrm{~mL} 0.025 \mathrm{M} 8$-HQ (EtOH)) were added to the $20 \mathrm{~mL}$ $\mathrm{ZnO}$ quantum dot - wool suspension at the beginning. 8-HQ reacts as a bidentate ligand with the zinc cations at the particle surface and changes the surface coverage of the $\mathrm{ZnO}$ quantum dots.

Table 2.10 summarises the reaction partners and parameters of the batch operated procedure for the formation of $\mathrm{ZnO}$ quantum dot - wool composites with and without 8HQ.

Table 2.10: List of precursor materials and reaction conditions for the development of ZnO quantum dot - wool composite.

\begin{tabular}{|c|c|c|}
\hline Merino wool & Solutions & Reaction conditions \\
\hline Untreated & $\mathrm{ZnO} / \mathrm{PVP}\left(\mathrm{H}_{2} \mathrm{O}\right)$ & \multirow{5}{*}{$\begin{array}{l}\text { Soaking wool fibre in } 20 \mathrm{~mL} \mathrm{ZnO} \\
\text { suspension (freshly prepared) at } 70 \\
{ }^{\circ} \mathrm{C} \text { for } 3-9 \text { days using water shake } \\
\text { bath. For the functionalisation with 8- } \\
\mathrm{HQ}, 0.1-1 \mathrm{~mL} 0.025 \mathrm{M} 8-\mathrm{HQ} \text { (EtOH) } \\
\text { is added to the } \mathrm{ZnO}-\text { wool fibre } \\
\text { suspension. }\end{array}$} \\
\hline Pre-treated with $\mathrm{NaOH}$ & ZnO/PMAA-Na $\left(\mathrm{H}_{2} \mathrm{O}\right)$ & \\
\hline Pre-treated with $\mathrm{HCl}$ & $\mathrm{ZnO} / \mathrm{PVP} / 8-\mathrm{HQ}\left(\mathrm{H}_{2} \mathrm{O}\right)$ & \\
\hline Pre-treated with 8-HQ & & \\
\hline Pre-treated with MSA & & \\
\hline
\end{tabular}

\subsubsection{Attachment of $\mathrm{ZnO}$ quantum dots to paper fibre}

To attach $\mathrm{ZnO}$ quantum dots to paper, $0.1 \mathrm{~g}$ bleached Kraft paper (shredded in $5 \mathrm{~mL}$ aqueous solution) was mixed in $75 \mathrm{~mL} \mathrm{ZnO}$ quantum dot suspension (directly after preparation) in a $100 \mathrm{~mL}$ glass beaker. The $\mathrm{ZnO}$ quantum dot - paper fibre suspension was mixed at $70-100{ }^{\circ} \mathrm{C}$ under vigorous stirring between 30 and $60 \mathrm{~min}$. Afterwards, 
the suspension containing the paper pulp and $\mathrm{ZnO}$ quantum dots were cooled down to room temperature, under vigorous stirring, and transferred to a vial for a further ageing period of $3-7$ days at room temperature and under gentle agitation. After ageing, paper fibres functionalised with $\mathrm{ZnO}$ quantum dots were separated from the reaction medium and washed with distilled water several times by vacuum filtration. The wet $\mathrm{ZnO}$ quantum dot - paper pulp was then placed with the filter paper on a watch glass and dried at room temperature.

The general procedure for the development of $\mathrm{ZnO}$ quantum dot - paper composites was modified with the addition of 8-HQ (EtOH). Shredded paper pulp $(0.1 \mathrm{~g}$ in $5 \mathrm{~mL}$ aqueous solution) was mixed in $75 \mathrm{~mL} \mathrm{ZnO}$ suspension in a $100 \mathrm{~mL}$ beaker glass. Then, $0.1-1 \mathrm{~mL} 0.025 \mathrm{M} 8$-HQ (EtOH) was added drop-wise to the $\mathrm{ZnO}$ paper pulp suspension under vigorous stirring between $70{ }^{\circ} \mathrm{C}$ and $100^{\circ} \mathrm{C}$. This mixture was further stirred between $30 \mathrm{~min}$ and $60 \mathrm{~min}$ at $70-100{ }^{\circ} \mathrm{C}$ and then washed with distilled hot water $\left(70^{\circ} \mathrm{C}\right)$ using filter suction. The wet paper fibre composite was then dried at room temperature on the filter paper used for the filter suction. Reaction parameters of the batch operated procedure are summarized in table 2.11 .

Table 2.11: List of precursor materials and reaction conditions for the development of $\mathrm{ZnO}$ quantum dot - paper composite materials.

\begin{tabular}{lll}
\hline Kraft paper & Solutions & Reaction conditions \\
\hline Bleached & ZnO/PVP $\left(\mathrm{H}_{2} \mathrm{O}\right)$ & Mixing $0.1 \mathrm{~g}$ paper fibre in $75 \mathrm{~mL} \mathrm{ZnO}$ \\
& $\mathrm{ZnO} / \mathrm{PVP} / 8-\mathrm{HQ}\left(\mathrm{H}_{2} \mathrm{O}\right)$ & quantum dot suspension (freshly prepared) \\
& with or without the addition of $8-\mathrm{HQ}(0.1-1$ \\
& $\mathrm{mL} 0.025 \mathrm{M} 8-\mathrm{HQ}(\mathrm{EtOH}))$ at $70-100^{\circ} \mathrm{C}$ for \\
& $30-60 \mathrm{~min}$ using hot plate with magnet \\
& stirrer. Keep $\mathrm{ZnO}$ paper pulp soaking in the \\
& reaction medium at room temperature for 3- \\
& 7 days \\
\hline
\end{tabular}

\subsection{In-situ dyeing of wool using the fluorescent chelate $\mathrm{Zn}(8-\mathrm{Q})_{2}$}

Wool and paper fibre functionalised with $\mathrm{ZnO}$ and 8-HQ show strong fluorescence. However, a large amount of fluorescent particles covering the fibres are easily removed by friction. Therefore, a procedure to produce high fluorescent fibrous materials without the leaching of the fluorescent component was developed. 


\subsubsection{Materials used for the complex formation of $\mathrm{Zn}(8-\mathrm{Q})_{2}$ chelate}

Zinc - 8-Hydroxyquinoline chelate was used to dye wool and paper fibre in aqueous and non-aqueous condition. The following reagents were used for the formation of the $\mathrm{Zn}(8-\mathrm{Q})_{2}$ :

- Zinc chloride $\left(\mathrm{ZnCl}_{2}\right)_{\text {solid; }}$

$\mathrm{FW}=136.29 \mathrm{~g} \mathrm{~mol}^{-1}$; minimum assay $98 \%$

- Zinc acetate $\left(\mathrm{Zn}\left(\mathrm{CH}_{3} \mathrm{COO}\right)_{2} \cdot 2 \mathrm{H}_{2} \mathrm{O}\right)_{\text {solid; }}$;

$\mathrm{FW}=219.15 \mathrm{~g} \mathrm{~mol}^{-1}$; minimum assay $99 \%$

- 8-Hydroxylquinoline $\left(\mathrm{C}_{9} \mathrm{H}_{7} \mathrm{NO}\right)_{\text {solid; }}$

$\mathrm{FW}=145.16 \mathrm{~g} \mathrm{~mol}^{-1}$; minimum assay $99 \%$

- Drum ethanol $\left(95 \%\right.$ EtOH and $\left.5 \% \mathrm{H}_{2} \mathrm{O}\right)$

\subsubsection{Preparation route}

Initially, it was attempted to dye wool and paper fibre directly with a $\mathrm{Zn}(8-\mathrm{Q})_{2}(\mathrm{EtOH})$ complex solution. However, it was difficult to suppress the crystallisation of the fluorescent compound of $\mathrm{Zn}(8-\mathrm{Q})_{2}(\mathrm{EtOH})$ over longer time periods. It is possible that the introduction of surfactant molecules may suppress crystallisation in solution and improve emission efficiency and colour tuning, this, however, should be investigated in detail in future studies.

Instead, an in-situ dyeing method was developed for wool fibre. This method involved the uptake of the precursors of the fluorescent chelate by the wool in separated batch processes. It is expected that crystals / agglomerates formed by the complexation of zinc with 8-HQ molecules will bind to the functional groups of the wool fibres and leaching of the fluorescent compound from the fibrous material will be prevented. This in-situ dyeing method can be easily divided in two reactions steps illustrated in equations (8) and (9), where wool fibre reacts with reactant $A$ in the first batch process and then with reactant B after washing and drying of the wool composite "A - wool".

\begin{tabular}{|c|c|c|c|c|}
\hline Wool & + & A & EtOH & A - wool \\
\hline A - wool & + & B & EtOH & $A B-w 00$ \\
\hline
\end{tabular}


This dying procedure of wool by using a two-step operated batch process can involve first the adsorption of zinc ions onto wool (equation (8)) followed by the uptake of 8HQ in the second batch process (equation (9)), or wool is functionalised first with 8-HQ molecules and then with zinc ions in the second batch process. In either case, the uptake of the reactants by the wool has to be optimised. Table 2.12 lists the different precursor materials used for the development of $\mathrm{Zn}(8-\mathrm{Q})_{2}$ - wool composites.

Table 2.12: List of precursor materials and solutions used for the in-situ dyeing of wool with $\mathrm{Zn}(8$ Q) 2 .

\begin{tabular}{ll}
\hline Merino wool & Solutions \\
\hline Untreated & $\mathrm{ZnCl}_{2}\left(\mathrm{H}_{2} \mathrm{O}\right)$ \\
Pre-treated with $\mathrm{NaOH}$ (see 2.2.2.1.1) & $\mathrm{ZnCl}_{2}(\mathrm{EtOH})$ \\
Pre-treated with $\mathrm{HCl}$ (see 2.2.2.1.2) & $\mathrm{Zn}(\mathrm{Ac})_{2}\left(\mathrm{H}_{2} \mathrm{O}\right)$ \\
Pre-treated with $\mathrm{MSA}$ (see 2.2.2.1.3) & 8- $\mathrm{HQ}(\mathrm{EtOH})$ \\
Pre-treated with 8-HQ (see 2.2.2.1.4) & 8- $\mathrm{HQ}\left(\mathrm{H}_{2} \mathrm{O}\right)$ \\
\hline
\end{tabular}

For the in-situ dyeing procedure, it is essential to shield the soaking solutions containing 8-HQ from sun light exposure. Free 8-HQ molecules exhibit a high sensitivity towards sun light and can undergo oxidation and degradation reactions and the resulting products will darken the wool fibre. For this reason, a thorough washing of the wool fibre composite with $\mathrm{EtOH}$ is required to remove all 8-HQ molecules loosely bound to the substrate material. The EtOH solution was also used to dehydrate the wool fibres. In the presence of water molecules, free 8-HQ monomers can form a "one water complex" and this complex, as well as the degradation products through photo-bleaching or oxidation processes, has quenching properties.

\subsubsection{1 $\quad \mathrm{Zn}^{2+}$ uptake on wool}

For the uptake of $\mathrm{Zn}^{2+}$ ions onto wool fibre, $0.1 \mathrm{~g}$ merino wool samples (see table 2.12) were soaked in a total volume of $10 \mathrm{~mL}$ of different zinc precursor solutions $-0.01 \mathrm{M}$ $\mathrm{ZnCl}_{2}(\mathrm{EtOH}) / 0.01 \mathrm{M} \mathrm{ZnCl}_{2}\left(\mathrm{H}_{2} \mathrm{O}\right) / 0.01 \mathrm{M} \mathrm{Zn}(\mathrm{Ac})_{2}\left(\mathrm{H}_{2} \mathrm{O}\right)$. To maximise the uptake of zinc ions, the substrate material was kept in the zinc solution for 48 hours at $50{ }^{\circ} \mathrm{C}$ in water shake bath. The fibrous substrate material was washed with distilled water and ethanol and dried at room temperature. The standard operating procedure for the uptake of zinc ions onto wool is shown in table 2.13 . 
The uptake of zinc ions onto wool is small and a greater uptake can be achieved by functionalising the substrate material with linker molecules such as MSA and Lcysteine. The uptake of zinc ions onto wool fibre, however, can also be increased by removing the lipid fatty acid monolayer around the wool fibre surface through alcoholic alkali and acid treatment. A greater uptake of zinc ions onto wool will favour a greater formation of the fluorescent chelate onto wool, which in turn will increase the fluorescence intensity of the composite material.

Table 2.13: Instructions for the uptake of $\mathrm{Zn}^{2+}$ ions onto wool fibre.

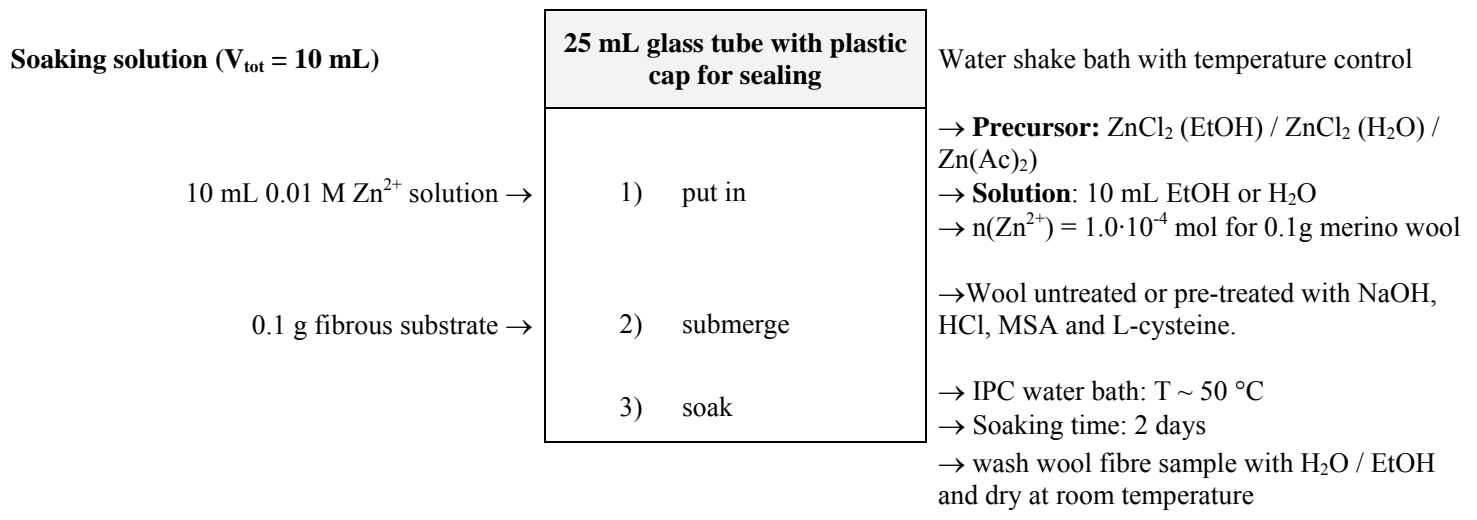

Table 2.14 shows the processing parameters which were considered for the uptake of $\mathrm{Zn}^{2+}$ ions onto wool.

Table 2.14: Process parameters used for the uptake of $\mathrm{Zn}^{2+}$ ions onto wool fibre.

\begin{tabular}{ll}
\hline Process Parameters & \\
\hline Precursor & $\mathrm{ZnCl}_{2}(\mathrm{EtOH}), \mathrm{ZnCl}_{2}\left(\mathrm{H}_{2} \mathrm{O}\right), \mathrm{Zn}(\mathrm{Ac})_{2}\left(\mathrm{H}_{2} \mathrm{O}\right)$ \\
Wool & Untreated, $\mathrm{NaOH}-, \mathrm{HCl}$ - and MSA pre-treated \\
{$\left[\mathrm{Zn}^{2+}\right] / \mathrm{M}$} & $0.01-0.1$ \\
Total soaking volume $/ \mathrm{mL}$ & $5,10-20$ \\
Soaking temperature $/{ }^{\circ} \mathrm{C}$ & $\sim 25,50,70,80,90$ \\
Soaking time / days & $1-3$ \\
Solvent & $\mathrm{H}_{2} \mathrm{O}, \mathrm{EtOH}$ \\
\hline
\end{tabular}

\subsubsection{8-HQ uptake on wool}

Here, $0.1 \mathrm{~g}$ wool was soaked in different concentration of 8-HQ ethanol solution ([8$\mathrm{HQ}]=1.0 \cdot 10^{-4}-4.0 \cdot 10^{-3} \mathrm{M}$ with a total solution of $10 \mathrm{~mL}$ ) for 3 days at $50{ }^{\circ} \mathrm{C}$ in a water bath. After ageing in solution, the 8-HQ - fibre composites were washed with ethanol and dried at $40{ }^{\circ} \mathrm{C}$ for 3 hours to remove unattached 8-HQ monomers from the 
wool fibre. The procedure for the uptake of 8-HQ onto wool fibre is shown in table 2.15 .

Table 2.15: Instructions for the uptake of 8-HQ onto wool fibre.

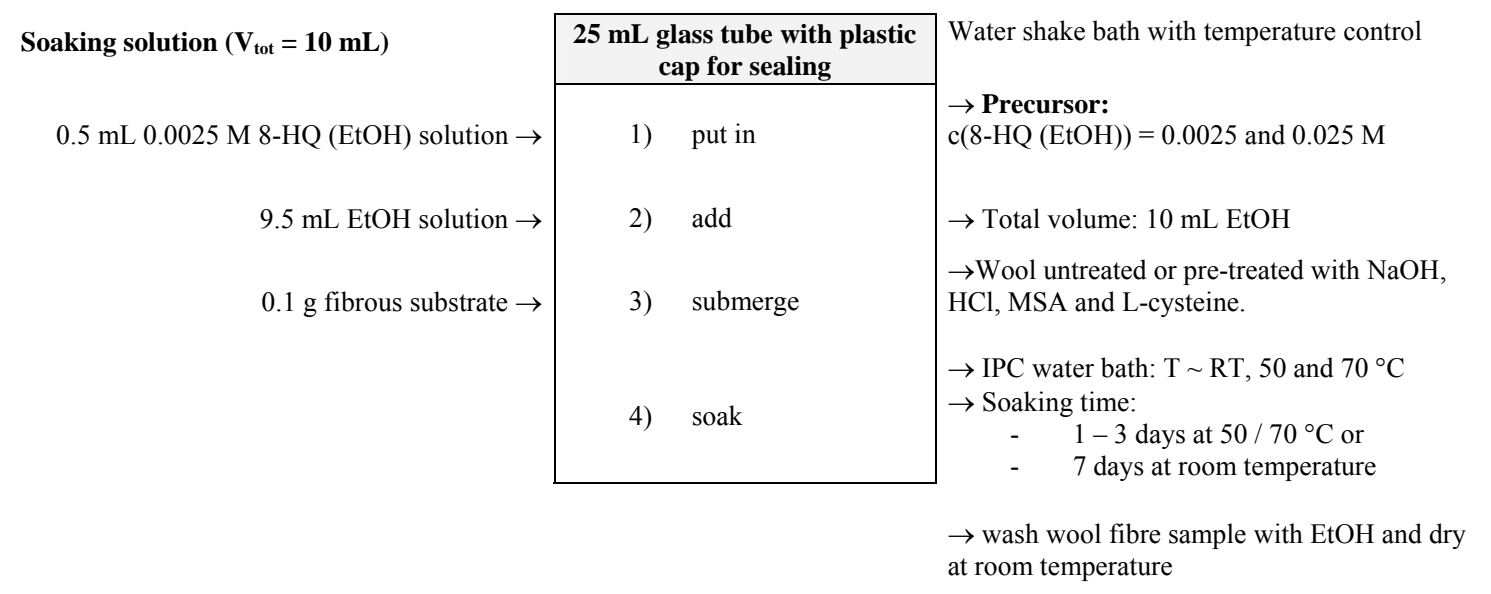

8-HQ is highly volatile in ethanol solution, in particular at high temperature. Therefore, it is important that the glass tube is sealed tightly during the soaking process. This is necessary, because minor deviation in the uptake of 8-HQ onto wool will have a strong effect on the emission wavelength of the final composite material.

It is important to mention that wool fibres soaked in 8-HQ ethanol solution obtain a shiny appearance, which however turns greyish at high 8-HQ concentrations. This change of colour may of several reasons such as a polymerisation reaction of 8-HQ monomers onto wool. The uptake parameters for 8-HQ molecules onto wool fibres are listed in table 2.16 .

Table 2.16: Process parameters investigated for the uptake of 8-HQ onto wool fibre.

\begin{tabular}{ll}
\hline Process Parameters & \\
\hline Precursor & $\begin{array}{l}\text { 8- } \mathrm{HQ}(\mathrm{EtOH}), 8-\mathrm{HQ}\left(\mathrm{H}_{2} \mathrm{O}\right) \\
\text { untreated }\end{array}$ \\
Wool & EtOH, $\mathrm{H}_{2} \mathrm{O}$ \\
Solvent & $1.0 \cdot 10^{-4}-4.0 \cdot 10^{-3}$ \\
{$[8-\mathrm{HQ}] / \mathrm{M}$} & 10,20 \\
Total soaking volume $/ \mathrm{mL}$ & $\sim 25,50,70$ \\
Soaking temperature $/{ }^{\circ} \mathrm{C}$ & $3-7$ \\
Soaking time / days & $1-5 \mathrm{~mL}$ distilled $\mathrm{H}_{2} \mathrm{O}$ \\
Presence of water &
\end{tabular}




\subsubsection{8-HQ uptake on zinc functionalised wool}

For the fluorescent dyeing of $\mathrm{Zn}^{2+}$ - wool composites with 8-HQ molecules, $0.1 \mathrm{~g}$ of zinc doped wool (see procedure in table 2.13) was submerged in $10 \mathrm{~mL} \mathrm{8-HQ} \mathrm{EtOH}$ solution with different concentration varying from $6.25 \cdot 10^{-5}-6.25 \cdot 10^{-3} \mathrm{M}$ at room temperature $\left(50,70^{\circ} \mathrm{C}\right)$ using a water shake bath. To ensure that 8 -HQ diffuse across the wool fibre and complex with zinc ions bound to the wool fibre, the fibres were soaked in the reaction medium for three days. Finally, the composite material was washed several times with EtOH to ensure that all loosely attached 8-HQ molecules were rinsed off the wool fibre and then air-dried. A standard procedure is represented in table 2.17.

Table 2.17: Instructions for the uptake of 8-HQ onto wool functionalised with $\mathrm{Zn}^{2+}$.

\begin{tabular}{|c|c|c|}
\hline Soaking solution $\left(\mathrm{V}_{\text {tot }}=10 \mathrm{~mL}\right)$ & $\begin{array}{l}25 \text { mL glass tube with plastic } \\
\text { cap for sealing }\end{array}$ & \multirow[t]{2}{*}{ Water shake bath with temperature control } \\
\hline $0.5 \mathrm{~mL} 0.0025 \mathrm{M} 8$-HQ (EtOH) solution $\rightarrow$ & 1) put in & \\
\hline $9.5 \mathrm{~mL} \mathrm{EtOH} \rightarrow$ & 2) add & $\begin{array}{l}\rightarrow \text { Total volume: } 10 \mathrm{~mL} \mathrm{EtOH} \\
\rightarrow[8-\mathrm{HQ}]=1.25 \cdot 10^{-4} \mathrm{M}\end{array}$ \\
\hline \multirow[t]{3}{*}{$0.1 \mathrm{~g}$ fibrous substrate with $\mathrm{Zn}^{2+}$ uptake $\rightarrow$} & 3) submerge & \\
\hline & soak & $\begin{array}{l}\rightarrow \text { IPC water bath: } \mathrm{T} \sim \mathrm{RT}, 50 \text { and } 70{ }^{\circ} \mathrm{C} \\
\rightarrow \text { Soaking time: } 3-7 \text { days } \\
\rightarrow \mathrm{wt}(8-\mathrm{HQ})=0.18 \% \text { onto } 0.1 \mathrm{~g} \text { wool }\end{array}$ \\
\hline & & $\begin{array}{l}\rightarrow \text { wash wool fibre sample with EtOH and dry } \\
\text { at room temperature }\end{array}$ \\
\hline
\end{tabular}

The presence of water, absorbed by the wool during the zinc uptake or added water during the soaking process of zinc-wool in 8-HQ EtOH solution, will result in the formation of fluorescent particles around the wool fibre. Therefore, it is important to ensure that wool fibre (functionalised in zinc aqueous solution) is dehydrated in $\mathrm{EtOH}$ solution before the uptake of 8-HQ in EtOH solution. The uptake parameters for 8-HQ molecules onto zinc functionalised wool fibres are summarised in table 2.18.

Table 2.18: Process parameters used for the uptake of 8-HQ onto wool functionalised with $\mathrm{Zn}^{2+}$.

\begin{tabular}{ll}
\hline Process Parameters & \\
\hline 8-HQ precursor & 8- $\mathrm{HQ}(\mathrm{EtOH})$ \\
Zinc precursor & $\mathrm{ZnCl}_{2}(\mathrm{EtOH}), \mathrm{ZnCl}_{2}\left(\mathrm{H}_{2} \mathrm{O}\right), \mathrm{Zn}(\mathrm{Ac})_{2}\left(\mathrm{H}_{2} \mathrm{O}\right)$ \\
Wool & Untreated, $\mathrm{NaOH}-, \mathrm{HCl}$ - and MSA pre-treated \\
Solvent & $\mathrm{EtOH}, \mathrm{H}_{2} \mathrm{O}$ \\
{$[8-\mathrm{HQ}] / \mathrm{M}$} & $6.25 \cdot 10^{-5}-2.5 \cdot 10^{-3}$ \\
Total soaking volume / $\mathrm{mL}$ & 10,20 \\
Soaking temperature / ${ }^{\circ} \mathrm{C}$ & $\sim 25,50,70$ \\
Soaking time / days & $3-7$ \\
Addition of water & $1-5 \mathrm{~mL}$ distilled $\mathrm{H}_{2} \mathrm{O}$ \\
\hline
\end{tabular}




\subsubsection{Zinc uptake on 8-HQ functionalised wool}

In this method, $0.1 \mathrm{~g}$ wool functionalised with different uptakes of 8-HQ (see procedure in table 2.15) was soaked into $10 \mathrm{~mL} 0.01 \mathrm{M} \mathrm{ZnCl}_{2} \mathrm{EtOH}$ solution for 3 days at $50{ }^{\circ} \mathrm{C}$ in water bath. The final $\mathrm{Zn}(8-\mathrm{Q})_{2}$ - fibre composites were washed with $\mathrm{EtOH}$ and dried at room temperature. The standard operating procedure for the uptake of zinc ions onto 8-HQ wool is shown in table 2.19 .

Table 2.19: Instructions for the uptake of $\mathrm{Zn}^{2+}$ ions onto wool treated with 8-HQ.

\begin{tabular}{|c|c|c|}
\hline \multirow[t]{2}{*}{ Soaking solution $\left(V_{\text {tot }}=5 \mathrm{~mL}\right)$} & $\begin{array}{l}25 \mathrm{~mL} \text { glass tube with plastic } \\
\text { cap for sealing }\end{array}$ & \multirow{2}{*}{$\begin{array}{l}\text { Water shake bath with temperature control } \\
\rightarrow \text { Precursor: } \mathrm{ZnCl}_{2}(\mathrm{EtOH}) / \mathrm{ZnCl}_{2}\left(\mathrm{H}_{2} \mathrm{O}\right) / \\
\left.\mathrm{Zn}(\mathrm{Ac})_{2}\right) \\
\rightarrow \text { Solution: } 10 \mathrm{~mL} \text { EtOH } \\
\rightarrow \mathrm{n}\left(\mathrm{Zn}^{2+}\right)=1.0 \cdot 10^{-4} \mathrm{~mol} \text { for } 0.1 \mathrm{~g} \text { merino woo }\end{array}$} \\
\hline & put in & \\
\hline \multirow[t]{3}{*}{$0.1 \mathrm{~g} 8$-HQ fibrous substrate $\rightarrow$} & submerge & \\
\hline & soak & $\begin{array}{l}\rightarrow \text { IPC water bath: } \mathrm{T} \sim 50^{\circ} \mathrm{C} \\
\rightarrow \text { Soaking time: } 1-3 \text { days }\end{array}$ \\
\hline & & $\begin{array}{l}\rightarrow \text { wash wool fibre sample with } \mathrm{H}_{2} \mathrm{O} / \mathrm{EtOH} \\
\text { and dry at room temperature }\end{array}$ \\
\hline
\end{tabular}

The zinc uptake onto 8-HQ treated wool will result in a bright white fluorescence colour of the wool with emission wavelengths below $490 \mathrm{~nm}$. The presence of water during the soaking process, however, has a strong influence on the final emission wavelength and colour of the wool fibre composite. For example, the uptake of 8-HQ onto $\mathrm{ZnCl}_{2}\left(\mathrm{H}_{2} \mathrm{O}\right)$ - wool resulted in a patchy colouration of the wool and emission wavelength above 490 $\mathrm{nm}$. For this reason, only $\mathrm{ZnCl}_{2}$ dissolved in $\mathrm{EtOH}$ solution was studied for this dyeing procedure. Table 2.20 shows the different process parameters considered for the uptake of $\mathrm{ZnCl}_{2}(\mathrm{EtOH})$ onto 8-HQ wool.

Table 2.20 Process parameters used for the uptake of $\mathrm{Zn}^{2+}$ ions onto wool treated with 8-HQ.

\begin{tabular}{ll}
\hline Process Parameters & \\
\hline Zinc precursor & $\mathrm{ZnCl}_{2}(\mathrm{EtOH}), \mathrm{ZnCl}_{2}\left(\mathrm{H}_{2} \mathrm{O}\right), \mathrm{Zn}(\mathrm{Ac})_{2}\left(\mathrm{H}_{2} \mathrm{O}\right)$ \\
8-HQ precursor & 8- $\mathrm{HQ}(\mathrm{EtOH})$ \\
Wool & Untreated \\
Solvent & $\mathrm{EtOH}$ \\
{$\left[\mathrm{Zn}^{2+}\right] / \mathrm{M}$} & $0.01-0.1$ \\
{$[8-\mathrm{HQ}] / \mathrm{M}$} & $6.25 \cdot 10^{-5}-6.25 \cdot 10^{-3}$ \\
Total soaking volume $/ \mathrm{mL}$ & $5,10-20$ \\
Soaking temperature $/{ }^{\circ} \mathrm{C}$ & $\sim 25,50,70$ \\
Soaking time $/$ days & $3-7$ \\
\hline
\end{tabular}




\subsection{Characterisation methods}

\subsubsection{Particle size and morphology analysis}

The characterisation of the size, structure and surface morphology of the nanocrystals and hybrid materials were carried out by Zetasizer measurements, scanning electron microscopy (SEM) and transmission electron microscopy (TEM).

\subsubsection{Zetasizer}

Particle size and particle size distribution were determined by using the Zetasizer apparatus (Malvern Instruments). Zetasizer use the dynamic light scattering (DLS) technique, in which a beam of monochromatic light is directed through a solution and the fluctuation of intensity of scattered light is analyzed. The DLS method determines particle size in a colloidal suspension by measuring the rate of diffusion of particles through a fluid. The rate of diffusion depends on three parameters: temperature, viscosity of fluid and particle size. If temperature and viscosity are known, the particle size as well as molecular weight of scattering species can be determined. The Zetasizer calculate the average size of the particle sizes obtained by number, volume and intensity. The detection system is self-optimizing and the software adjusts the optics, attenuation and measurement duration in order to produce consistent and reliable data. Expert advice in the software also provides information about how good or poor the data is. Samples were inserted in a disposal plastic cuvette and placed inside the Zetasizer apparatus. Before adding the sample in the cuvette, each sample was filtered using syringe-driven filter units with pore diameter of $200 \mathrm{~nm}$. This was done to remove dust particles. The sample suspension was then heated up to $25^{\circ} \mathrm{C}$ and the measurement started. To improve accuracy in the results, the viscosity of the colloidal aqueous suspension was determined at $25{ }^{\circ} \mathrm{C}$ and the viscosity value was manually inserted in the settings before the measurement was made.

\subsubsection{Scanning Electron Microscope (SEM)}

SEM images were taken with a JEOL $6500 \mathrm{~F}$ field-emission gun scanning electron microscope. Samples of nanocrystal were prepared by drying a drop of the aqueous nanocrystal dispersion on a piece of Si (100) wafer and then mounted on aluminium 
stubs with double sided carbon adhesive tape. Samples were then sputter coated with a carbon layer in order to reduce a build up of charges on their surface. Wool fibre composites were also mounted on stepped aluminium stubs using double sided carbon adhesive tape. Samples were coated twice with $7 \mathrm{~nm}$ of carbon by a JEOL JEC-560 carbon coater. Images were taken at different magnification and from different parts of the samples so that the samples can be thoroughly examined. Backscatter images were also taken to obtain a better contrast from materials with a higher molecular weight from the surrounding.

\subsubsection{Transmission Electron Microscopy (TEM)}

TEM measurements were performed on a JEOL 2010 high-resolution instrument with a $\mathrm{LaB}_{6}$ filament operated at $200 \mathrm{kV}$, by depositing a drop of sample dispersion onto a 200 mesh carbon coated $\mathrm{Cu}$ grid. The dilution of the samples was necessary to avoid overloading. The excess of solvent was evaporated by placing the sample under a heat lamp. By doing this, a thin film of the nanocrystals deposited across the grid was obtained which was later dried further in a vacuum chamber. For the characterisation of $\mathrm{Cu}_{2} \mathrm{O}$ nanocrystals an $\mathrm{Au}$ grid was used instead.

\subsubsection{Chemical composition}

Energy dispersive spectroscopy (EDS) and X-ray photoelectron spectroscopy (XPS) were used to determine the chemical composition of the surface of the nanocrystals and the fibrous composite materials. X-ray diffraction (XRD) was used to obtain information on the crystallographic structure and chemical composition.

\subsubsection{Energy dispersive X-ray spectroscopy (EDS)}

SEM and TEM equipped with Energy dispersive X-ray spectroscopy (EDS) were used to show the distribution of various elements on the surface of the nanocrystal and composite material by using elemental mapping. Also, EDS will produce a spectrum showing the analysis / concentration of the various elements on the surface in a histogram of electron energy versus count. The diffusion of zinc ions and location inside the wool fibre were also determined using EDS by examining fibre composites in cross sections. To do this, wool fibres were aligned horizontally and enclosed with a 
double sided carbon tape. This was then frozen in liquid nitrogen and a cross-section was obtained using a scalpel. The section was then mounted on the vertical face of a stepped aluminium stub. Special care was taken to align the upper surface of the cross section to the top horizontal face of the aluminium stub, so the electron beam is perpendicular to the centre of the fibre and the detector can collect the electrons / X-rays ejected from the sample.

\subsubsection{X-ray photoelectron spectroscopy (XPS)}

XPS produces a spectrum of emission intensity versus electron binding energy. Since each element has a unique set of binding energies, XPS can be used to identify the elements on the surface of the nanocrystals. Also, peak areas at nominal binding energies can be used to quantify the concentration of these elements. Small shifts in these binding energies provide useful information about valence state, the nature of the chemical bonding and short-range chemistry. XPS measurements were performed on a Kratos XSAM800 photoelectron spectrometer available at the University of Auckland. The binding energies were calibrated with the corresponding software Casa XPS by referencing the $\mathrm{C}-\mathrm{C} 1 \mathrm{~s}$ photoelectron peak at $285.0 \mathrm{eV}$.

\subsubsection{X-ray diffraction (XRD)}

Nanocrystals were characterised using a Philips X'Pert X-ray powder diffractometer with a copper anode glass X-ray source. Nanocrystal samples were prepared by depositing the dispersion in a Perspex sample holder with a sample area of $10 \mathrm{~mm} \times 20$ $\mathrm{mm} \times 0.2 \mathrm{~mm}$. After vaporising the solvent, the dry thin layer of nanocrystals were analyzed and characterized by XRD using the Bragg-Brentano operation, whereby the sample is placed in a horizontal position and both X-ray tube and detector rotate while scanning the sample. For the excitation of the samples a $\mathrm{Cu} \mathrm{K}_{\alpha}$ radiation source was used and diffraction patterns were recorded between $25-80^{\circ} 2$ Theta. Automatic identification of the diffraction pattern was done by using the search and match facility of the X'pert Highscore software which accesses the Powder Diffraction File database of known crystal structures published by the International Centre for Diffraction Data. 


\subsubsection{Spectroscopy}

The size and shape dependent optical properties of quantum dots were characterised using Ultra violet visible (UV/Vis) spectroscopy and fluorescence spectroscopy. Both methods provide an easy method for optical characterisation of colloidal suspensions. Fluorescence and reflectance spectroscopy were also used to characterise hybrid materials produced by attaching quantum dots onto fibrous substrate material or by insitu dyeing with zinc and 8-HQ. Infrared (IR) spectroscopy was used to examine differences between the untreated wool and wool fibre dyed with the in-situ complexation of zinc and 8-HQ.

\subsubsection{Fluorescence spectroscopy}

Emission spectra were recorded with a Fluorolog ${ }^{\circledR}-3$ spectrofluorometer from Horiba. The Fluorolog ${ }^{\circledR}-3$ is equipped with a $450 \mathrm{~W}$ xenon arc lamp, a double excitation monochromator, a sample compartment with a cuvette holder, a double emission monochromator, and a photomultiplier tube. The excitation and emission monochromator were both set on the same slit width between 3 and $10 \mathrm{~nm}$. For each scan from $300 \mathrm{~nm}$ to $900 \mathrm{~nm}$, corresponding optical filters were manually inserted into the instrument to remove the excitation wavelength. All four sides of the cuvette (disposal plastic cuvette) were thoroughly cleaned using paper tissue before taking the measurements.

\subsubsection{UV-Visible spectroscopy}

UV-absorption spectra of colloidal suspensions were obtained in the wavelength range of 200-900 nm using a Varian Cary 100 Scan spectrometer. This spectrometer is a double-beam spectrophotometer that uses a double chopper to send light along the blank and sample path, simultaneously. The light source is emitted by a tungsten and deuterium lamp which covers the wavelength range between $190-900 \mathrm{~nm}$. The light signal is detected by a R928 photomultiplier tube. The slit width was set to achieve a Spectral Bandwidth of $1 \mathrm{~nm}$. Zero correction and baseline measurement of blank solution were always carried out using a matched quartz cell when conducting UV-Vis measurements. 
The reflectance spectra of fibrous hybrid material were also measured using the Varian Cary 100 Scan spectrometer. For this, the instrument was modified with the required technical accessories supplied to the spectrometer.

\subsubsection{Anti-microbial testing}

The anti-microbial action of the quantum dots itself and their composites were tested at the Microbiology Laboratory of Wellington Hospital. Fragment of the composite materials were placed on agar plates from the staff of the Microbiology Laboratory after the plates were inoculated with a bacterial suspension of the pathogen Staphylococcus aureus (ATCC 25923). After 24 hours cultivating in an incubator at $37{ }^{\circ} \mathrm{C}$, the agar plates were sterilised in an autoclave to stop the bacteria colony growth. For assessment the Petri dishes were photographed on a bench top and under an optical microscope. 


\section{Chapter 3}

\section{Synthesis and characterisation of $\mathrm{Cu}_{2} \mathrm{O}$ quantum dots}

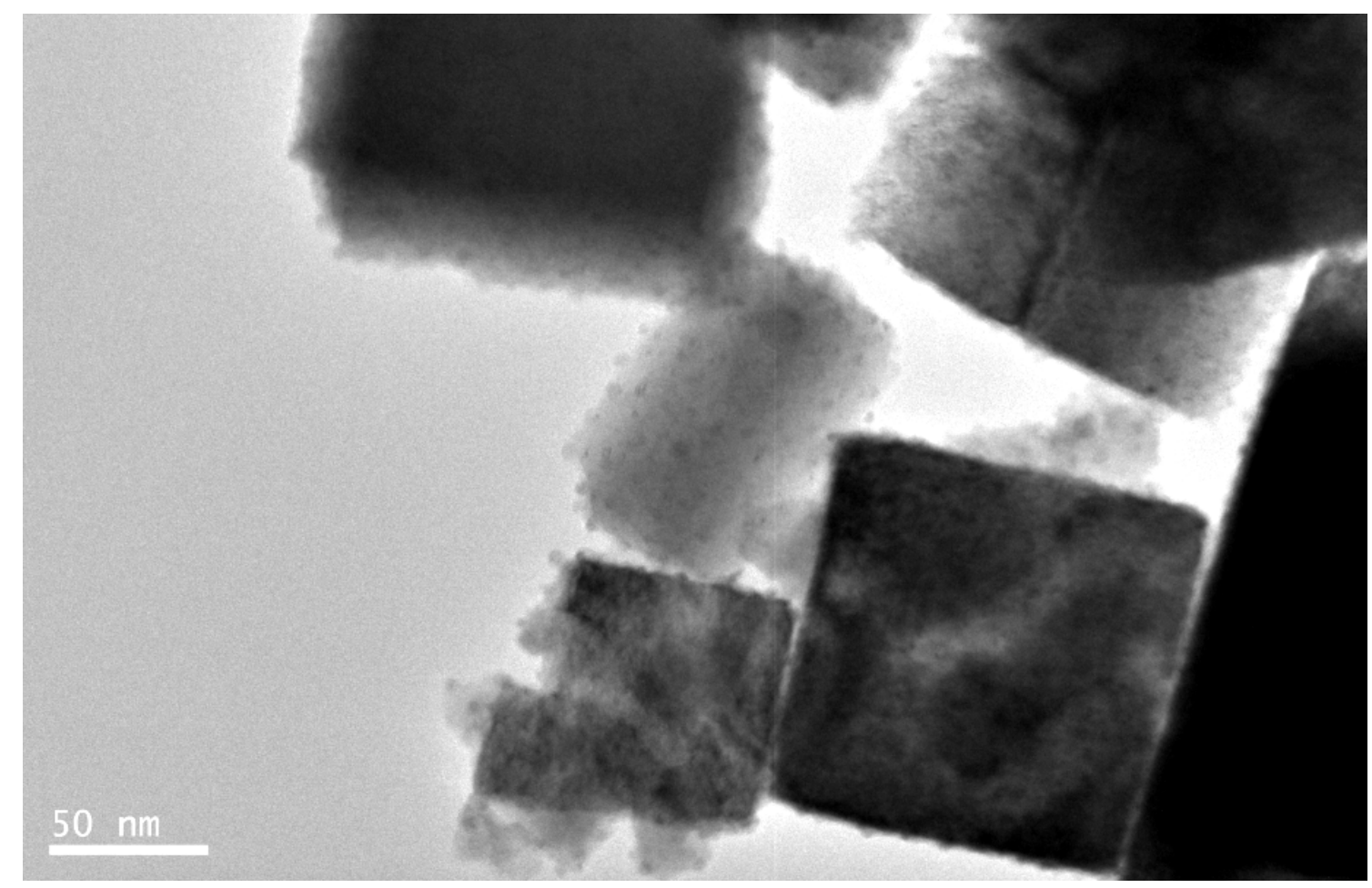

This chapter deals with the preparation of copper (I) oxide $\left(\mathrm{Cu}_{2} \mathrm{O}\right)$ as a potential quantum dot material for the development of new composite materials. The main goal of this chapter is to develop a wet chemical reduction method to synthesise $\mathrm{Cu}_{2} \mathrm{O}$ quantum dots that bind to wool and paper fibre and functionalise it with fluorescence. Also, the effect of quantum dots size and the addition of impurities / dopants to the lattice crystal structure on the optical fluorescence, and its use to tune the optical properties, are explored.

$\mathrm{Cu}_{2} \mathrm{O}$ nanocrystals have been produced by reducing aqueous copper nitrate $\left(\mathrm{Cu}\left(\mathrm{NO}_{3}\right)_{2}\right)$ solution with sodium borohydride $\left(\mathrm{NaBH}_{4}\right)$ in the presence of tri-sodium citrate $\left(\mathrm{Na}_{3} \mathrm{C}_{6} \mathrm{H}_{5} \mathrm{O}_{7}\right)$ and poly(methacrylic acid sodium salt) (PMAA-Na) at $99-100{ }^{\circ} \mathrm{C}$. 
This wet chemical method allows the controlled formation of cubic shaped $\mathrm{Cu}_{2} \mathrm{O}$ nanocrystals which are assembled of small spherical nanocrystals of $5 \mathrm{~nm}$. TEM images obtained in this project revealed that the small spherical nanocrystals show a super lattice structure. The arrangement of these nanocrystals into nanocubes following a selfassembled stabilization mechanism has a strong effect on the light absorption and scattering properties of the $\mathrm{Cu}_{2} \mathrm{O}$ suspension. Suspensions containing mainly large polycrystalline nanocubes, which show a high tendency to agglomerate, reveal a dichroic effect. The suspension is yellow-green in reflected light, whereas in transmitted light the suspension is red-purple and transparent. This phenomenon is caused by Surface Plasmon Resonance (SPR). Thereby, the size of the nanocubes and intermolecular interaction between the nanocubes determines the wavelength (range) of the adsorbed and scattered light and the ratio of light absorption and light scattering.

The influences of different process parameters such as stirring time, reaction temperature, concentration and choice of copper precursor material $\left(\mathrm{Cu}\left(\mathrm{NO}_{3}\right)_{2}, \mathrm{CuCl}_{2}\right)$, concentration and choice of polymeric dispersant (PVP, PMAA-Na), concentration of reducing agents $\left(\mathrm{NaBH}_{4}\right.$ and $\left.\mathrm{TSC}\right)$ were examined on the formation of the selfassembled nanocubes. Also, the effect of different dopants such as $\mathrm{Zn}^{2+}, \mathrm{Mn}^{2+}, \mathrm{Ni}^{2+}$, $\mathrm{Co}^{2+}, \mathrm{Mg}^{2+}$ and $\mathrm{In}^{3+}$ on the chemical reaction and light absorption and scattering properties of the nanocubes was evaluated. Results were characterised using absorbance spectroscopy, reflectance spectroscopy, Zetasizer and electron microscopy. Furthermore, the chemical composition of the nanocrystals were characterised by EDS, XPS and XRD.

\subsection{Introduction; $\mathrm{Cu}_{2} \mathrm{O}$ quantum dots}

Cuprous oxide $\left(\mathrm{Cu}_{2} \mathrm{O}\right)$ is a metal oxide semiconductor and shows high stability at nanoscale in comparison to the bulk form. It has received much attention recently for its potential applications in solar cells, pigments, and catalysts [85-88]. $\mathrm{Cu}_{2} \mathrm{O}$ is a metal oxide p-type semiconductor with a high optical absorption coefficient and relatively low direct band gap energy in bulk $(2.2 \mathrm{eV})$ and with very high exciton binding energy (140 $\mathrm{meV}$ ) [89-91]. It has many advantages in comparison to other inorganic semiconductor materials such as simplicity of preparation, scalability and non-toxicity. It is expected that the synthesis of $\mathrm{Cu}_{2} \mathrm{O}$ quantum dots using a wet chemical reduction method will 
result in optical fluorescence. Furthermore, it is expected that these $\mathrm{Cu}_{2} \mathrm{O}$ quantum dots can be used to functionalised fibrous material such as wool and paper with optical fluorescence.

\subsubsection{Chemical reduction method}

For the synthesis of $\mathrm{Cu}_{2} \mathrm{O}$ quantum dots, the chemical reduction method was applied using stable, non-toxic reactants under mild reaction conditions $\left(\mathrm{T} \leq 100{ }^{\circ} \mathrm{C}\right)$. The chemical reduction method provides an easy and safe method to produce $\mathrm{Cu}_{2} \mathrm{O}$ quantum dots at bench top conditions by using simple glassware.

The general principle of the chemical reduction method is the formation of very small particles (seeds) which will subsequently grow larger in solution. The formation of the small particles is induced by a chemical reaction which transforms solvated metal ions into a solid phase. Thereby, the particle growth of the solid phase is controlled by changing different processing parameters such as concentration of the raw materials, reaction temperature, $\mathrm{pH}$, titration rate, stirring rate and use of capping agents. Changes in process parameters can often lead to very different materials.

Chemical reduction methods rely on the use of capping agent for the synthesis of quantum dots. Very small particles exhibit a strong affinity to undergo agglomeration processes due to the high surface energy. However, this affinity can be reduced by coating the surface area with capping agents. By shielding the semiconductor surface with an ionic capping agent (electrostatic stabilization) or by tailoring the particle surface with polymeric dispersants (steric stabilization), aggregation processes are inhibited and the stability of the particle dispersion is enhanced over longer time. The passivation of the surface area with charged and bulkier surfactants also hinders surface atoms towards oxidation reactions. Along with other reaction parameters, capping agents determine the final shape and size of the quantum dots as well as the size distribution. Since the optical properties of quantum dots are size- and shape-dependent, the adsorption of capping agent onto the particle surface during the chemical reaction can be used to control the fluorescence colour in the visible range. 
Chemical reduction methods also allow the introduction of small quantities of dopants into the chemical composition of the final product. By doing so, the fluorescence colour of the quantum dot material can be tuned in the visible range by the additional energy levels of dopant materials within the band gap.

\subsubsection{Effect of capping agents on particle growth}

In the chemical reduction method, the particle growth is induced by a rapid nucleation reaction of reduced metal ions, which form small nucleation centres (seeds) in solution. These nucleation centres combine and form small nanoclusters, which are aggregates of atoms in a given shape. These nanoclusters undergo a further particle growth and form either polycrystalline particles or coalesce to larger nanocrystals. Here, the final product is strongly dependent on the process parameters used and its optimisation. Thereby, the right choice of capping agents plays an important factor in the chemical reduction method.

For this reason, to obtain a better understanding of the principle of the chemical reduction method, the role of the capping agents used for the synthesis of $\mathrm{Cu}_{2} \mathrm{O}$ quantum dots are briefly discussed. Here, $\mathrm{Cu}_{2} \mathrm{O}$ nanocrystals were formed in aqueous solution by reducing copper nitrate $\left(\mathrm{Cu}\left(\mathrm{NO}_{3}\right)_{2}\right)$ with sodium borohydride $\left(\mathrm{NaBH}_{4}\right)$ in the presence of trisodium citrate $\left(\mathrm{Na}_{3} \mathrm{C}_{6} \mathrm{H}_{5} \mathrm{O}_{7}\right)$, as an ionic capping agent (figure 3.1; A), and poly(methacrylic acid sodium salt) (PMAA-Na), as a polymeric stabilizer (figure 3.1 ; B), at a reaction temperature of $99-100^{\circ} \mathrm{C}$ using silicone oil bath.

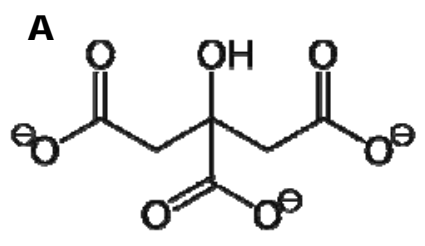

$3 \mathrm{Na}^{\oplus}$

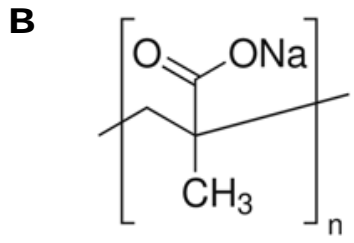

Figure 3.1: Formula of trisodium citrate - ionic capping agent (A) and PMAA-Na - polymeric dispersant (B).

The combination of ionic capping agents and polymeric stabilizers with their different strength of adsorption on the particle surface is called electrosteric stabilization and it is used in this project to control the size and shape of the particles. 
Citrate is a mild chelating agent and an excellent ionic capping agent which has three carboxyl groups and one hydroxyl group (figure 3.1; A). Before the chemical transformation is induced by the addition of a reducing agent, citrate ions undergo a complex formation with metal ions in solution. Then, the reduction of the metal precursor material form small nucleation centres (seeds) which aggregate to nanoclusters. The growth of the nanoclusters is confined by the absorption of citrate ions onto their surface. Citrate forms an electrically charged layer at the particle interface creating repulsive forces between approaching particles with similar electric charge. These repulsive forces stabilise the nanoclusters in the dispersion medium, however, these forces are strongly dependent on the thickness of the layer. For instance, a thin electrostatic layer will not be strong enough to counterbalance attractive van der Waals forces, which are always present between particles of similar composition. For this reason, cluster growth via nucleation and condensation continues until a strong repelling layer of citrate ions is formed at the interface of the nanoclusters. At this point, the positive surface charge of the nanoclusters is electronically passivated by the citrate's carboxylate groups.

However, nanoclusters with an electrically charged layer of citrate ions at their surface can still undergo further reactions. For example, nanoclusters can group together and form aggregates, agglomerates (figure $3.2 ; \mathrm{A}$ ) or they can coalesce into larger crystalline particles by reducing metal ions on their surface (figure 3.2; B) [51].

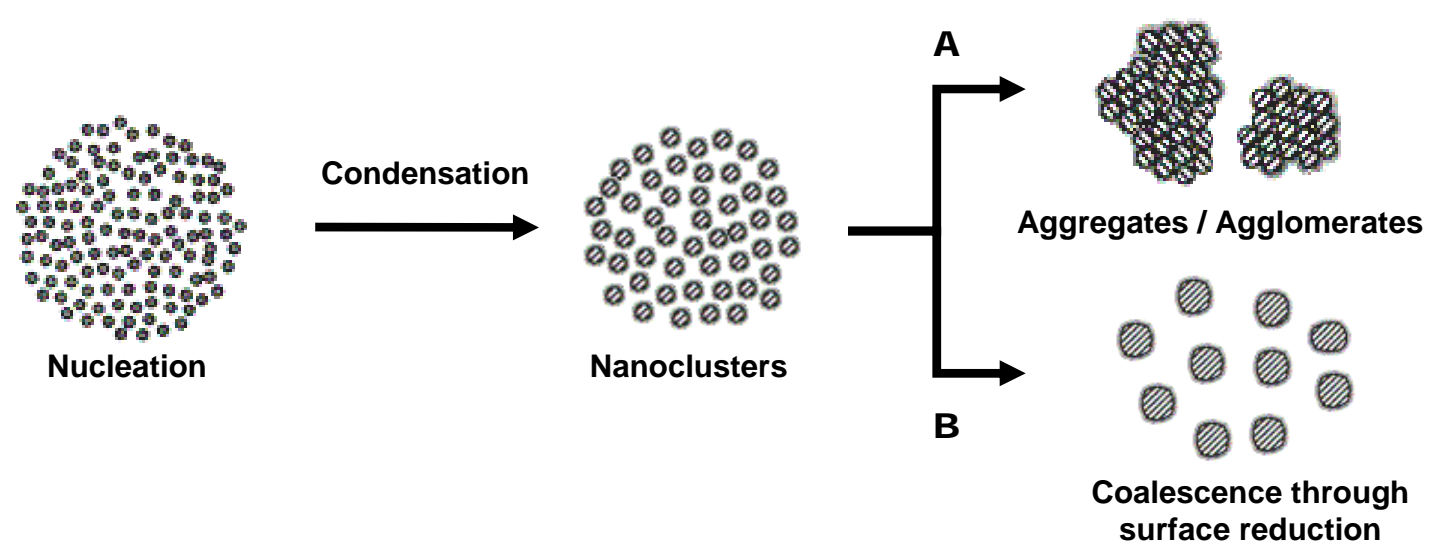

Figure 3.2: Schematic representation of particle growth by coalescence of small nanoclusters (A) and by surface reduction after nucleation and condensation (B). 
Here, it is important to distinguish between the formation of aggregates, agglomerates and crystalline particles. The formation of aggregates (figure 3.2; A) is an irreversible process, where particles are hold together by strong physical forces. In an agglomeration processes, particles are bonded by weak physical forces, which are stable enough not to be broken by Brownian motion, but may disperse again under an externally applied force such as vigorous stirring or ultra-sonic treatment. The driving force for aggregation / agglomeration processes is the surface energy of the nanoclusters. By grouping together and forming polycrystalline particles, nanoclusters decrease their exposed surface area and, as a result, they receive a lower surface energy which increases the stability of the particles in the dispersion medium. This kind of particle growth, however, has negative effects on the homogeneity of the suspension, since the uncontrolled formation of aggregates or agglomerates may trigger a precipitation reaction.

Besides the electrostatic stabilizing effects, citrate also has reducing properties and it can reduce positively charged metal ions at the surface of the clusters. This surface reduction will promote the coalescence of nanoclusters into typical colloidal particles with a narrow size distribution (see illustration in figure 3.2; B). Particles formed by the coalescence of the nanoclusters are thermodynamically more stable due to the crystalline character. Furthermore, the reduced surface-to-volume ratio caused by the coalescence allows the formation of a strong citrate layer around the particles. The oxidation/decarboxylation products of citrate may also remain chemisorbed on the surface of the colloidal particles and prevent the particles from further growth.

Here, the particle growth via aggregation, agglomeration or coalescence through surface reduction is most likely determined by the amount of citrate adsorbed on the surface of the nanoclusters. For example, at low concentration, the citrate layer may not be effectively built-up around the nanoclusters and they can further interact with each other due to the van der Waals forces. Hence, nanoclusters without a strong repelling layer are more likely to form polycrystalline particles with a wide size distribution (figure 3.2; A).

It is important to note that the same effect can occur, when the concentration of citrate is too high in the dispersion medium. High concentration of citrate will increase the ionic 
strength of the dispersion medium, which in turn has a negative effect on the thickness of the citrate layer around the particles. The ionic strength of the dispersion medium is determined by the polarity of the solvent and the presence of metal ions and / or ionic molecules in it. By increasing the ionic strength of the dispersion medium, the adsorption of ionic capping agents onto the particle surface will be reduced. Consequently, only a thin electrostatic layer is formed around the nanoclusters, which is not strong enough to counterbalance the attractive van der Waals forces between approaching particles. Hence, the nanoclusters show a higher chemical reactivity due to their thinner repelling layer and are more likely to undergo aggregation / agglomeration processes. On the contrary, nanoclusters surrounded by a strong (thick) repelling layer of citrate ions are less likely to form agglomerates. That is because the Coulombic repulsion between the charged surface layers is stronger. Neverthless, a second particle growth can occur by the coalescence of nanoclusters through surface reduction forming larger crystalline particles.

In this wet chemical reduction method, $\mathrm{Cu}_{2} \mathrm{O}$ quantum dots were also stabilized in the dispersion medium by steric hindrance. Here, the polymer PMAA-Na with an average molecular weight of 15000 (figure 3.1; B) was used for the polymeric stabilization of $\mathrm{Cu}_{2} \mathrm{O}$ quantum dots in aqueous solution. PMAA-Na, as well as PVP, is a polymeric stabilizer which binds to polar molecules exceptionally well and because of its polarity it is water-soluble. In the preparation, PMAA-Na forms complex compounds with the metal ions and the quantum dots. Metal ions and quantum dots coordinate with the oxygen atoms in PMAA-Na to form a protection layer around the surface of the nanocrystals. This layer exhibits steric stabilizing effects and inhibits the aggregation of the quantum dots into larger size particles. Usually, high concentrations of polymeric dispersants increase the shielding effect of the particles and a homogenous suspension with a narrow size distribution is obtained [92].

Beside the steric stabilization, polymeric stabilization can be achieved by another mechanism; depletion stabilization [93-95]. Here, the polymeric dispersant is not attached to the surface of the particles but situated between the particles in the dispersion medium. A combination of depletion and steric stabilization is possible by increasing the concentration of the polymeric dispersant in the dispersion medium. 


\subsection{Preparation of $\mathrm{Cu}_{2} \mathrm{O}$}

Well-dispersed cube-shaped $\mathrm{Cu}_{2} \mathrm{O}$ nanocrystals were formed in aqueous solution by reducing aqueous copper nitrate $\left(\mathrm{Cu}\left(\mathrm{NO}_{3}\right)_{2}\right)$ solution with sodium borohydride $\left(\mathrm{NaBH}_{4}\right)$ in the presence of sodium citrate $\left(\mathrm{Na}_{3} \mathrm{C}_{6} \mathrm{H}_{5} \mathrm{O}_{7}\right)$ and poly(methacrylic acid sodium salt) (PMAA-Na) at a reaction temperature of $99-100{ }^{\circ} \mathrm{C}$.

The synthesis procedures of $\mathrm{Cu}_{2} \mathrm{O}$ quantum dots described in chapter 2 (2.1.3.1) were standardised to optimise the optical properties of interest of the $\mathrm{Cu}_{2} \mathrm{O}$ nanocrystal suspensions. Therefore, different process parameters have been varied, which are summarised in the following table.

Table 3.1: List and details of process parameters investigated for the synthesis of doped and undoped $\mathrm{Cu}_{2} \mathrm{O}$ nanoparticles.

\begin{tabular}{ll}
\hline Process Parameter & \\
\hline Precursor & $\mathrm{Cu}\left(\mathrm{NO}_{3}\right)_{2} ; \mathrm{CuCl}_{2}$ \\
Reducing agent & $\mathrm{NaBH}_{4}\left(\mathrm{H}_{2} \mathrm{O}\right), \mathrm{NaBH}_{4}$ in triethyl glycole \\
dimethylether) & $\mathrm{Na}_{3} \mathrm{C}_{65} \mathrm{O}_{7}, \mathrm{C}_{2} \mathrm{H}_{5} \mathrm{NO}_{2}, \mathrm{Na}_{2} \mathrm{~S}_{2} \mathrm{O}_{3}$ \\
Charged capping agent & $\mathrm{PMAA}-\mathrm{Na}, \mathrm{PVP}$ \\
Polymeric capping agent & $0.0002-0.0008$ \\
{$\left[\mathrm{Cu}^{2+}\right] / \mathrm{M}$} & $0.004-0.013$ \\
{$\left[\mathrm{NaBH}_{4}\right] / \mathrm{M}$} & $0.0003-0.0006$ \\
{$[$ Citrate] / M } & {$[\mathrm{PVP}] \rightarrow 0.25,0.5,0.75 \mathrm{~mL} 5 \mathrm{mg} \mathrm{mL}^{-1}$} \\
{$[\mathrm{PVP}]$ resp. $[\mathrm{PMAA}-\mathrm{Na}] / \mathrm{mg} \mathrm{mL}^{-1}$} & $25,50,90-100$ \\
Reaction temperature / ${ }^{\circ} \mathrm{C}$ & $10-120$ \\
Reaction time / min & $0.25-1$ \\
Addition of $0.01 \mathrm{M} \mathrm{NaOH}\left(\mathrm{H}_{2} \mathrm{O}\right) / \mathrm{mL}$ & distilled and Millipore $\mathrm{H}_{2} \mathrm{O} ; \mathrm{H}_{2} \mathrm{O}: \mathrm{Et}(\mathrm{OH})_{2}$ \\
Solvent & $\mathrm{Zn}^{2+}, \mathrm{Ni}^{2+}, \mathrm{Mn}^{2+}, \mathrm{Co}^{2+}$ \\
Doping &
\end{tabular}




\subsection{Results}

The preparation of $\mathrm{Cu}_{2} \mathrm{O}$ nanocrystals undergoes several colour changes during the synthesis. The reaction mechanism is based on the reduction and oxidation of the copper ions to copper (I) oxide. The reaction starts with the addition of $\mathrm{NaBH}_{4}$ to the transparent aqueous solution containing $\mathrm{Cu}\left(\mathrm{NO}_{3}\right)_{2}$, trisodium citrate and PMAA-Na. Thereby, the solution turns into a coffee brown suspension, which turns again, after 10 min stirring, into a dark green-blue colour. The final colour change is observed after cooling down the reaction medium at around $80{ }^{\circ} \mathrm{C}$. The final $\mathrm{Cu}_{2} \mathrm{O}$ nanocrystals suspension exhibit a strong yellow-green appearance in visible light (figure 3.3).

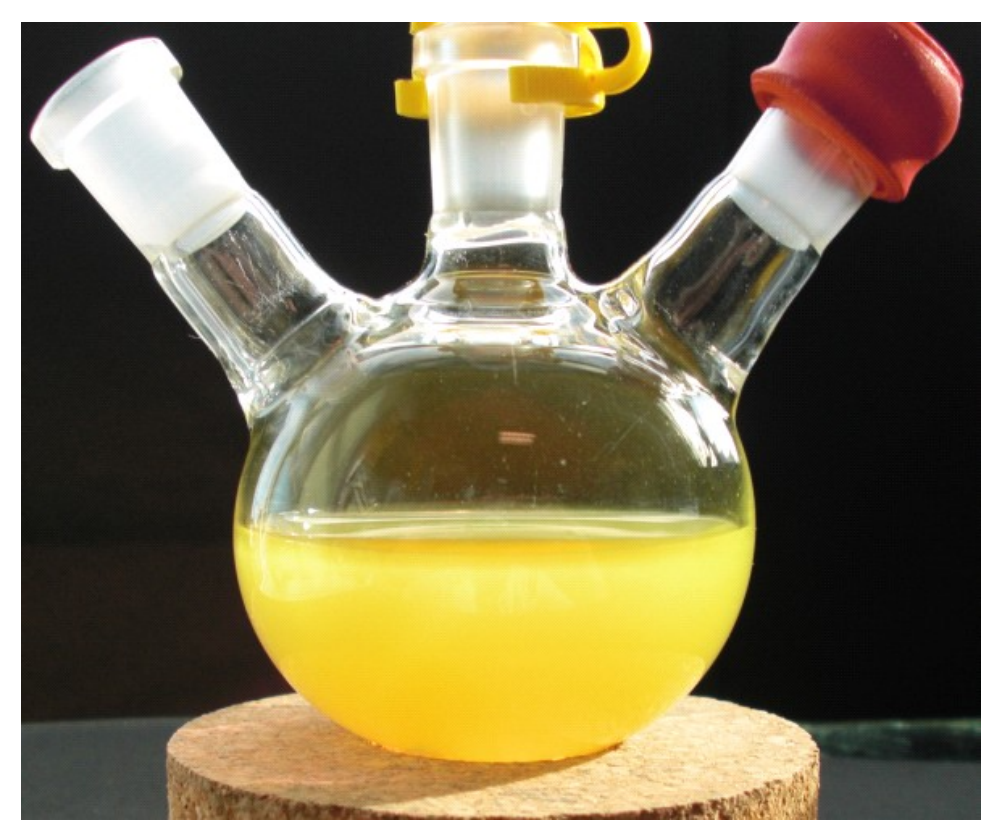

Figure 3.3: Final colour of the $\mathrm{Cu}_{2} \mathrm{O}$ nanocrystal suspension.

$\mathrm{Cu}_{2} \mathrm{O}$ nanoparticles in suspension or coated on the inner side of glass tubes show extraordinary dichroic effect (opalescence effect) depending on the angle of observation. It shows red colour in transmitted light and yellow green colour in reflected light (figure 3.4). The colour in transmitted light corresponds to the complementary colour of the absorbed light and the colour in reflected light corresponds to the light scattered at the particle surface. 


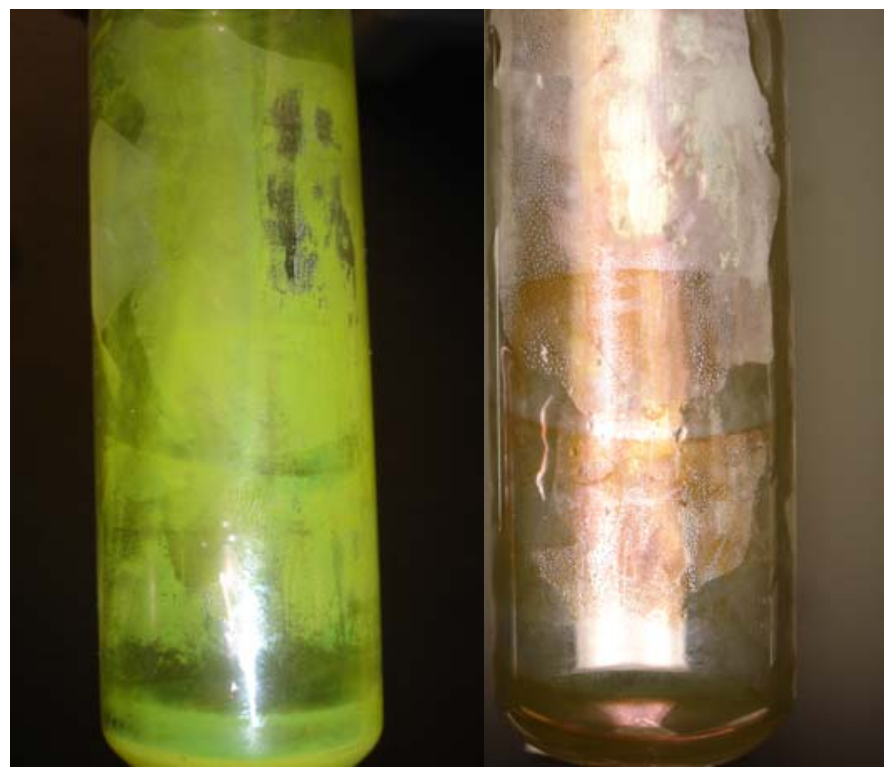

Figure 3.4: Dichroic effect of $\mathrm{Cu}_{2} \mathrm{O}$ nanocrystals in transmitted light (right) and reflected light (left).

This dichroic effect is also seen in the Lycurgus cup from $4^{\text {th }}$ century AD, which shows a green colour in reflected light and a red colour if illuminated with light from inside the cup (see figure 3.5). By closer examinations, it was shown that the cup contain small colloids of gold and copper.

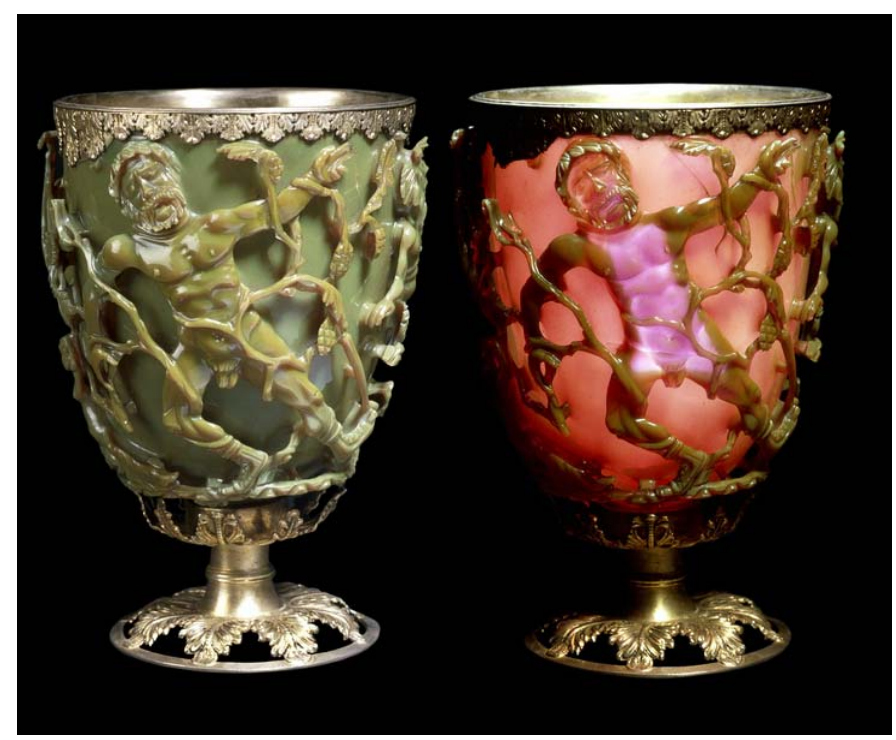

Figure 3.5: Lycurgus cup from $4^{\text {th }}$ century AD in reflected light (left) and illuminated with light from inside the cup (right) [Copy rights of British Museum].

The dichroic effect was also achieved by doping $\mathrm{Cu}_{2} \mathrm{O}$ nanocrystals with $\mathrm{Zn}^{2+}$ ions in the presence of nickel. The modification of the procedure by adding dopants resulted in suspension showing a yellow orange colour in reflected light and a purple colour in transmitted light (figure 3.6). 

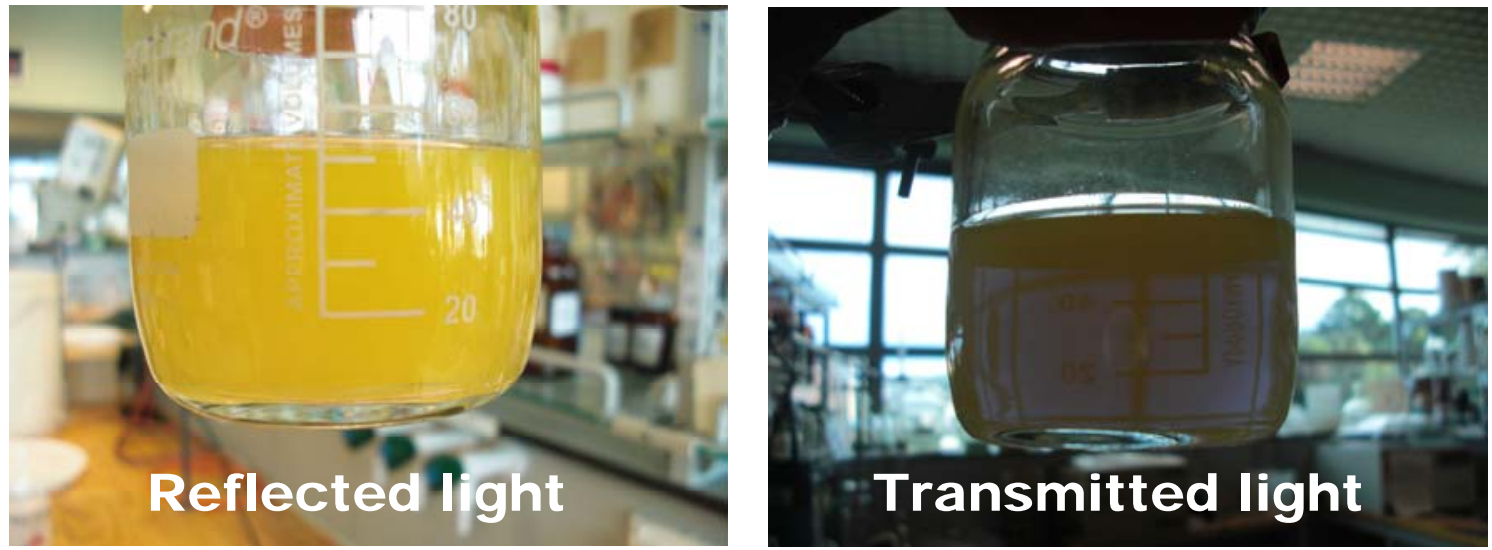

Figure 3.6: Aqueous suspension of $\mathrm{Cu}_{2} \mathrm{O}$ nanocrystals doped with $\mathrm{Zn}^{2+}$ ions in reflected (left) and transmitted light (right).

$\mathrm{Cu}_{2} \mathrm{O}$ nanocrystals were also integrated in Hydroxyethyl cellulose (HEC) sheets. HEC is water based gelling and thickening agent and forms a clear viscous solution mixed with water. The evaporation of water results in the hardening of the gelling agent and forms sheets with $\mathrm{Cu}_{2} \mathrm{O}$ nanocrystals homogeneously dispersed (see figure 3.7).

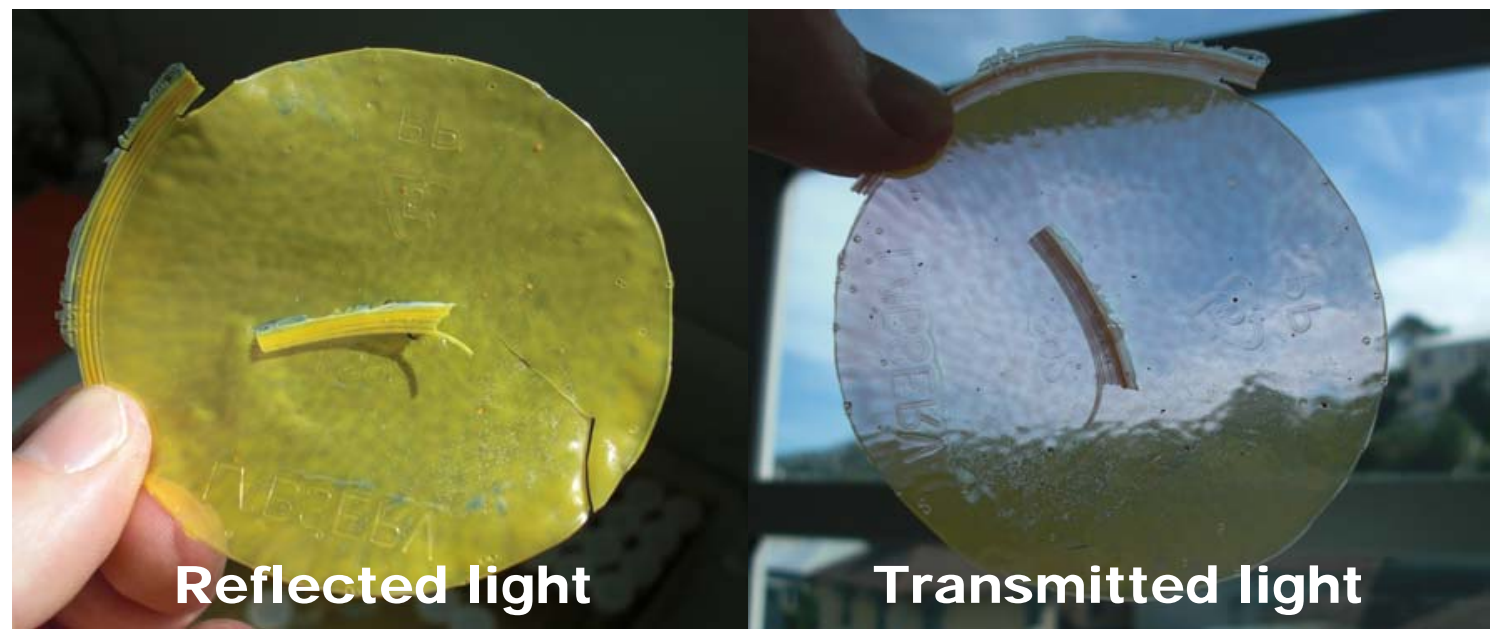

Figure 3.7: $\mathrm{Cu}_{2} \mathrm{O}$ nanocrystals doped with $\mathrm{Zn}^{2+}$ ions incorporated in hydroxyl-ethyl cellulose sheets. The sheets show an orange colour in reflected (left) and purple colour in transmitted light (right).

In general, the colour of a material is created by absorption-, reflection- and fluorescence effects. These effects are usually determined by the physical properties of the nanoparticles such as shape and size. Hence, changes in size and structure will have a strong influence on the optical properties of the $\mathrm{Cu}_{2} \mathrm{O}$ suspensions. Therefore, the influence of the different process parameters, listed in table 3.1, on the particle growth and their effect on the optical properties (opalescence effect) was investigated by diverse characterisation methods. 


\subsubsection{Chemical composition}

The crystal structure identification and elemental purity (composition) of the $\mathrm{Cu}_{2} \mathrm{O}$ nanocrystals were determined by Energy dispersive X-ray spectroscopy (EDS), X-ray diffraction (XRD) and X-ray photoelectron spectroscopy (XPS) examination.

\subsubsection{EDS mapping}

The chemical reduction method result in the formation of cube shaped nanocrystals varying in different sizes as shown in the TEM image figure 3.8. The composition of the lattice structure was characterised by energy dispersive X-ray analysis (EDS) as shown in figure 3.9. Images were obtained by a transmission electron microscope equipped with EDS.

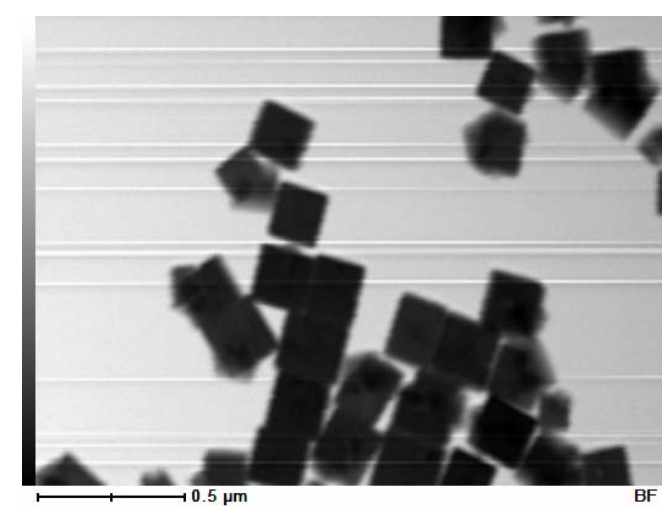

Figure 3.8: $\mathrm{Cu}_{2} \mathrm{O}$ nanocubes of $70-250 \mathrm{~nm}$. TEM image shows these are regular and welldispersed.

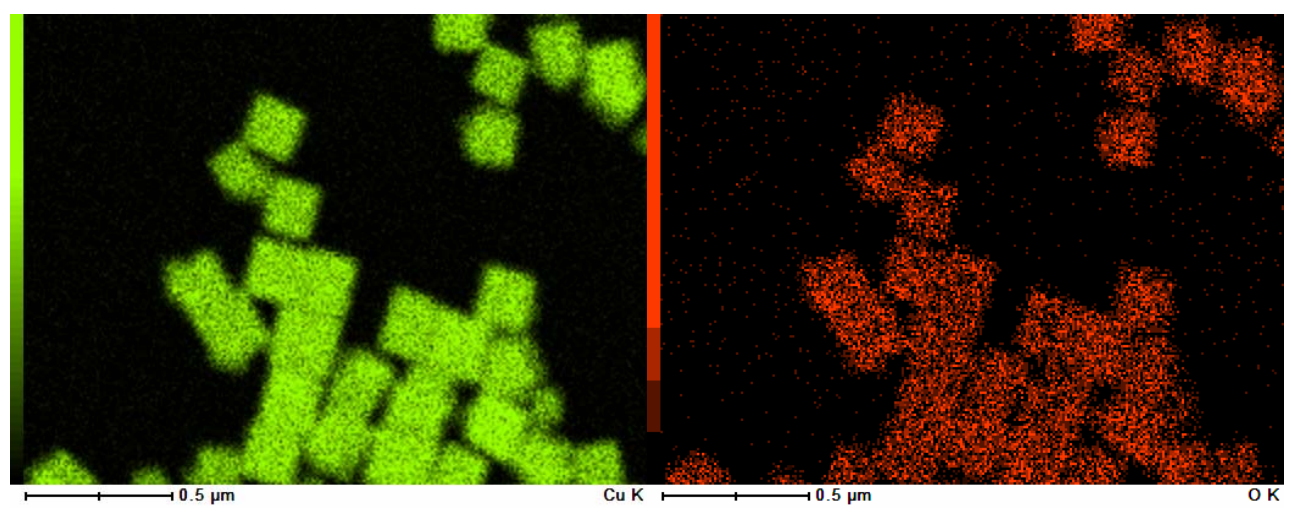

Figure 3.9: Energy dispersive X-ray spectroscopy (EDS) mapping of copper (left) and oxygen (right) of the nanocubes.

The EDS mapping of copper (left) and oxygen atoms (right) in figure 3.9 show a homogenous distribution on the cube shape crystals. 


\subsubsection{X-ray diffraction spectroscopy (XRD)}

XRD measurement of the nanocrystals synthesised by following the procedure described in chapter 2 reveals diffraction peaks corresponding to the cubic crystal structure of $\mathrm{Cu}_{2} \mathrm{O}$ (see figure 3.10).

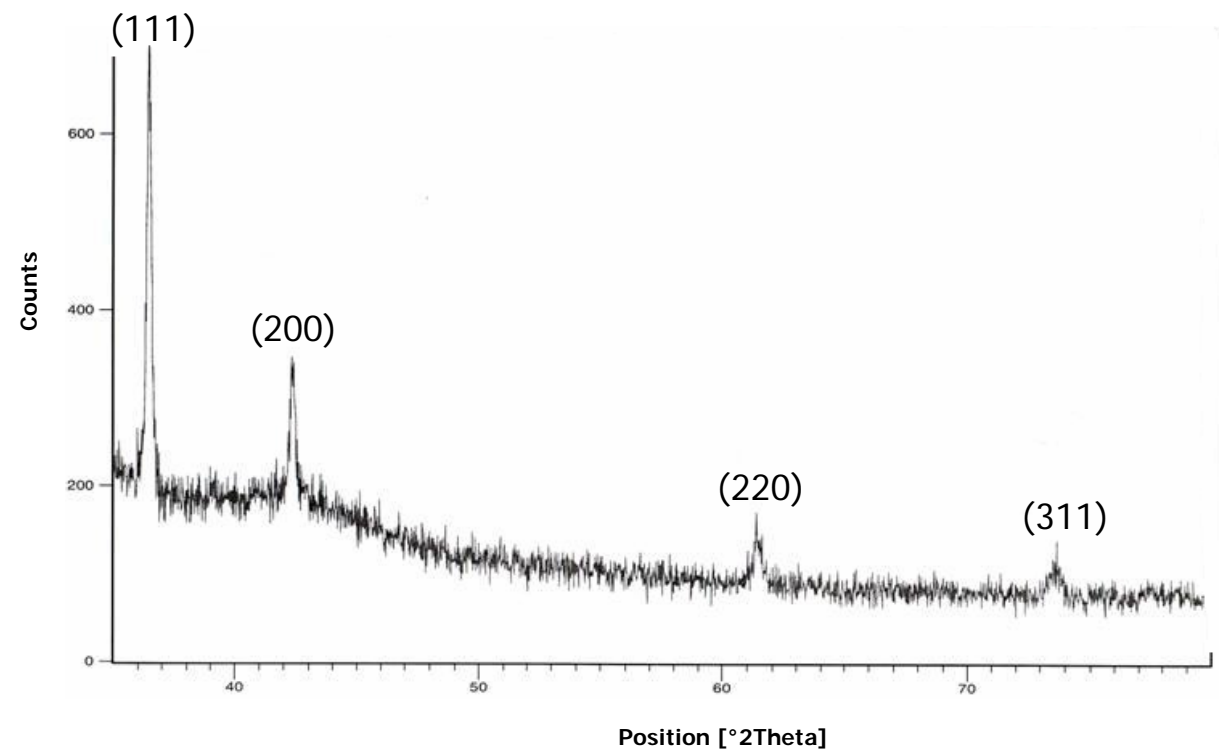

Figure 3.10: $\mathrm{X}$-ray powder diffraction of $\mathrm{Cu}_{2} \mathrm{O}$ nanocrystals synthesised following the wet chemical reduction method.

The cuprite crystal structure is composed of eight cubes, whereby the fcc positions of each cube is occupied by copper atoms and two oxygen atoms occupying two diagonal tetrahedral interstices.

\subsubsection{X-ray photoelectron spectroscopy}

XPS measurements also reveal the binding energies copper and oxygen according to the cuprite crystal structure. The graph A in figure 3.11 shows the X-ray photoelectron spectrum of $\mathrm{Cu}_{2} \mathrm{O}$ nanocrystals, with $\mathrm{Cu} 2 \mathrm{p}_{3 / 2}$ at $932.148 \mathrm{eV}$ and $\mathrm{Cu} 2 \mathrm{p}_{1 / 2}$ at $952.078 \mathrm{eV}$. However, it was found out that changes in the sample preparation such as different concentration of trisodium citrate, $\mathrm{Cu}\left(\mathrm{NO}_{3}\right)_{2}$ or $\mathrm{NaBH}_{4}$ affects the binding energy of the $\mathrm{Cu} 2 \mathrm{p}_{3 / 2}$ peak. For example, sample preparation without trisodium citrate resulted in the shift of the $\mathrm{Cu} 2 \mathrm{p}_{3 / 2}$ peak towards $934.3 \mathrm{eV}$ and in the shift of $\mathrm{Cu} 2 \mathrm{p}_{1 / 2}$ peak towards $953.54 \mathrm{eV}$. On the other hand, when the concentration of trisodium citrate is increased $\left(\left[\mathrm{Na}_{3} \mathrm{C}_{6} \mathrm{H}_{5} \mathrm{O}_{7}\right]>0.3 \cdot 10^{-3} \mathrm{M}\right)$ in the preparation procedure a shift of the $\mathrm{Cu} 2 \mathrm{p}_{3 / 2}$ peak towards $934 \mathrm{eV}$ is also observed (table 3.2). For example, the sample prepared with 
$\left[\mathrm{Na}_{3} \mathrm{C}_{6} \mathrm{H}_{5} \mathrm{O}_{7}\right]=0.48 \cdot 10^{-3} \mathrm{M}$ resulted in $\mathrm{Cu} 2 \mathrm{p}_{3 / 2}$ at $933.885 \mathrm{eV}$ and $\mathrm{Cu} 2 \mathrm{p}_{1 / 2}$ at 953.291 $\mathrm{eV}$ (see figure 3.11 ; B). These binding energies correspond to the monoclinic structure of cupric oxide $\mathrm{CuO}$.

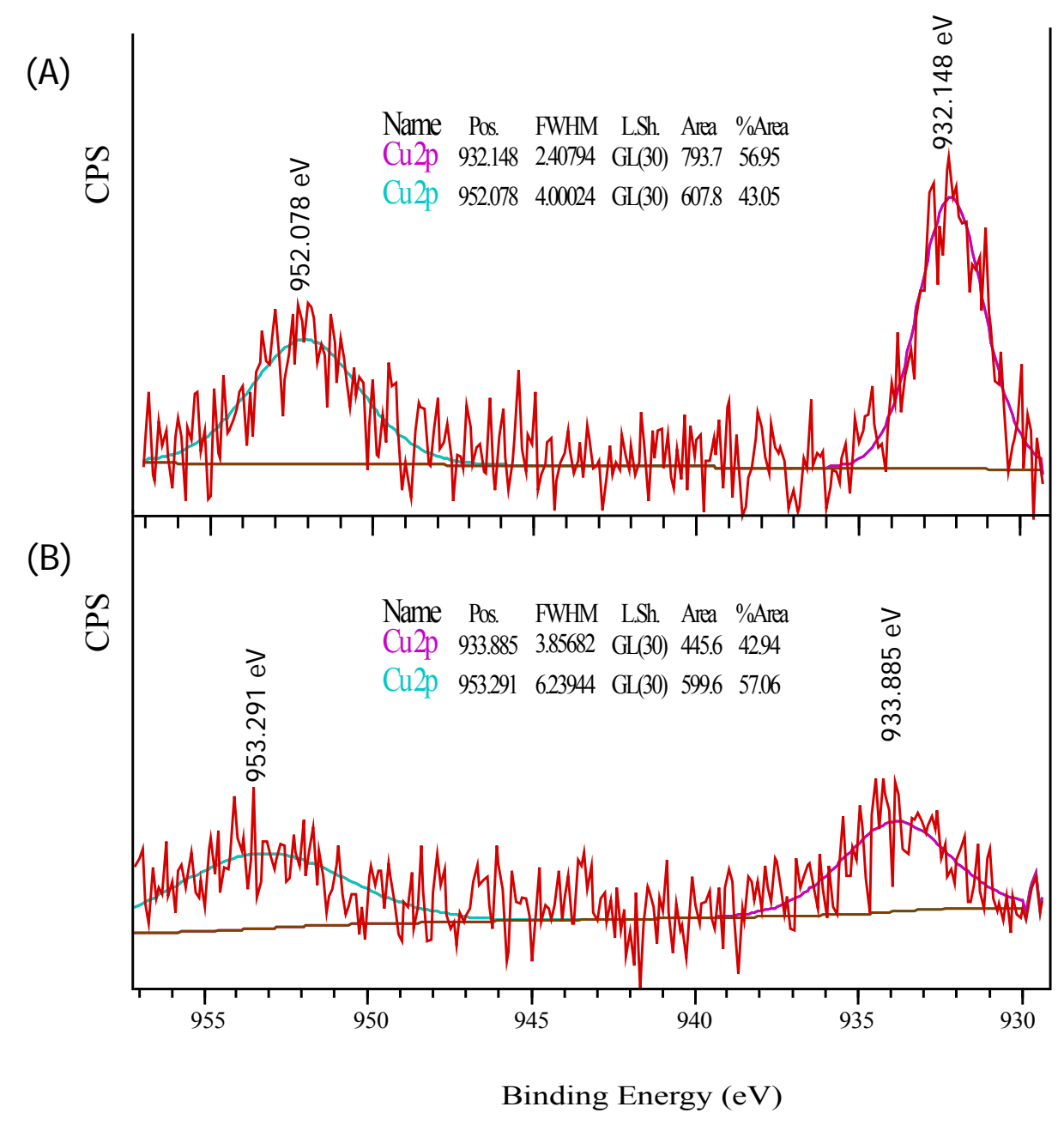

Figure 3.11: Binding energy of copper atoms in the cuprite crystal structure of $\mathrm{Cu}_{2} \mathrm{O}$ nanocrystals (A) formed with $0.3 \cdot 10^{-3} \mathrm{M}$ TSC and binding energy of copper atoms in a cupric oxide crystal structure of $\mathrm{CuO}$ nanocrystals (B) formed with $0.48 \cdot 10^{-3} \mathrm{M}$ TSC.

Table 3.2: Binding energy of copper atoms obtained after adding different concentrations of TSC to the reaction medium. Details of sample preparation are listed in the appendix in A.1; appendix.

\begin{tabular}{cc}
\hline$\left[\mathrm{Na}_{3} \mathrm{C}_{6} \mathrm{H}_{5} \mathrm{O}_{7}\right] / \mathrm{M}$ & $\mathrm{Cu} 2 \mathrm{p} 1 / 2 / \mathrm{eV}$ \\
\hline $0.3 \cdot 10^{-3}$ & 932.148 \\
$0.33 \cdot 10^{-3}$ & 932.452 \\
$0.36 \cdot 10^{-3}$ & 932.742 \\
$0.39 \cdot 10^{-3}$ & 932.997 \\
$0.48 \cdot 10^{-3}$ & 933.885 \\
\hline
\end{tabular}




\subsubsection{Morpholgy and size}

The effect of diverse process parameters on the structure, size and size distribution of the nanocrystals was investigated by using transmission and scanning electron microscopy (TEM and SEM) and findings are shown below.

\subsubsection{Transmission Electron Microscopy}

Figure 3.12 and 3.13 show TEM images with different magnifications of the standard preparation of $\mathrm{Cu}_{2} \mathrm{O}$ nanocrystals. The images show a mixture of regular shaped and well dispersed nanocubes in different sizes (70, 100, 150, 190 and $220 \mathrm{~nm})$.

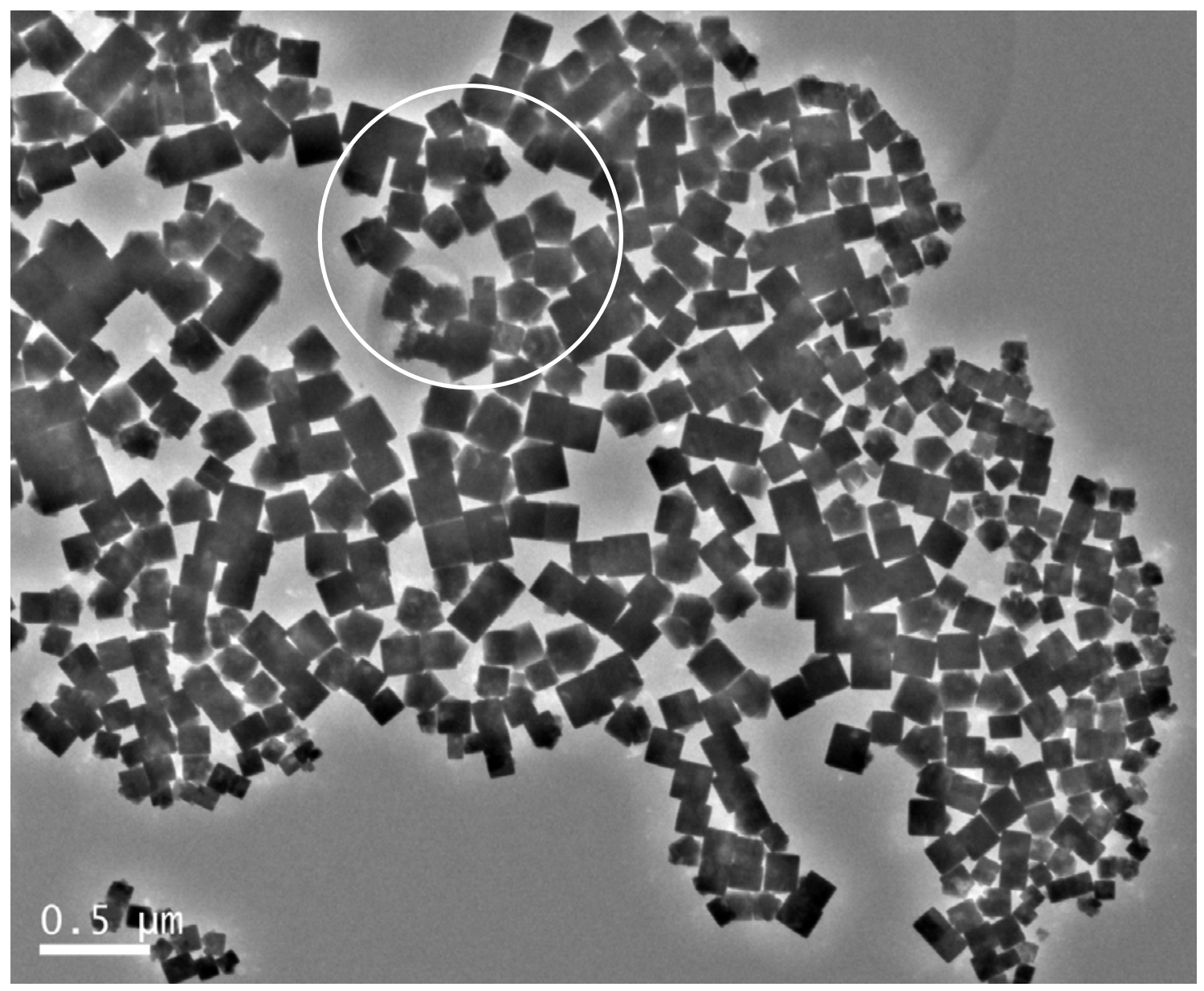

Figure 3.12: TEM image of regular shaped nanocubes obtained by the reduction of $\mathrm{Cu}\left(\mathrm{NO}_{3}\right)_{2}$ with $\mathrm{NaBH}_{4}$ in the presence of PMAA-Na.

However, not all cubes are perfectly shaped and of same length. In figure 3.13, irregular cubes with a length of $190 \mathrm{~nm}$ and width of $150 \mathrm{~nm}$ are highlighted by a white circle. 


\section{$\mathrm{Cu}_{2} \mathrm{O}$ quantum dots}
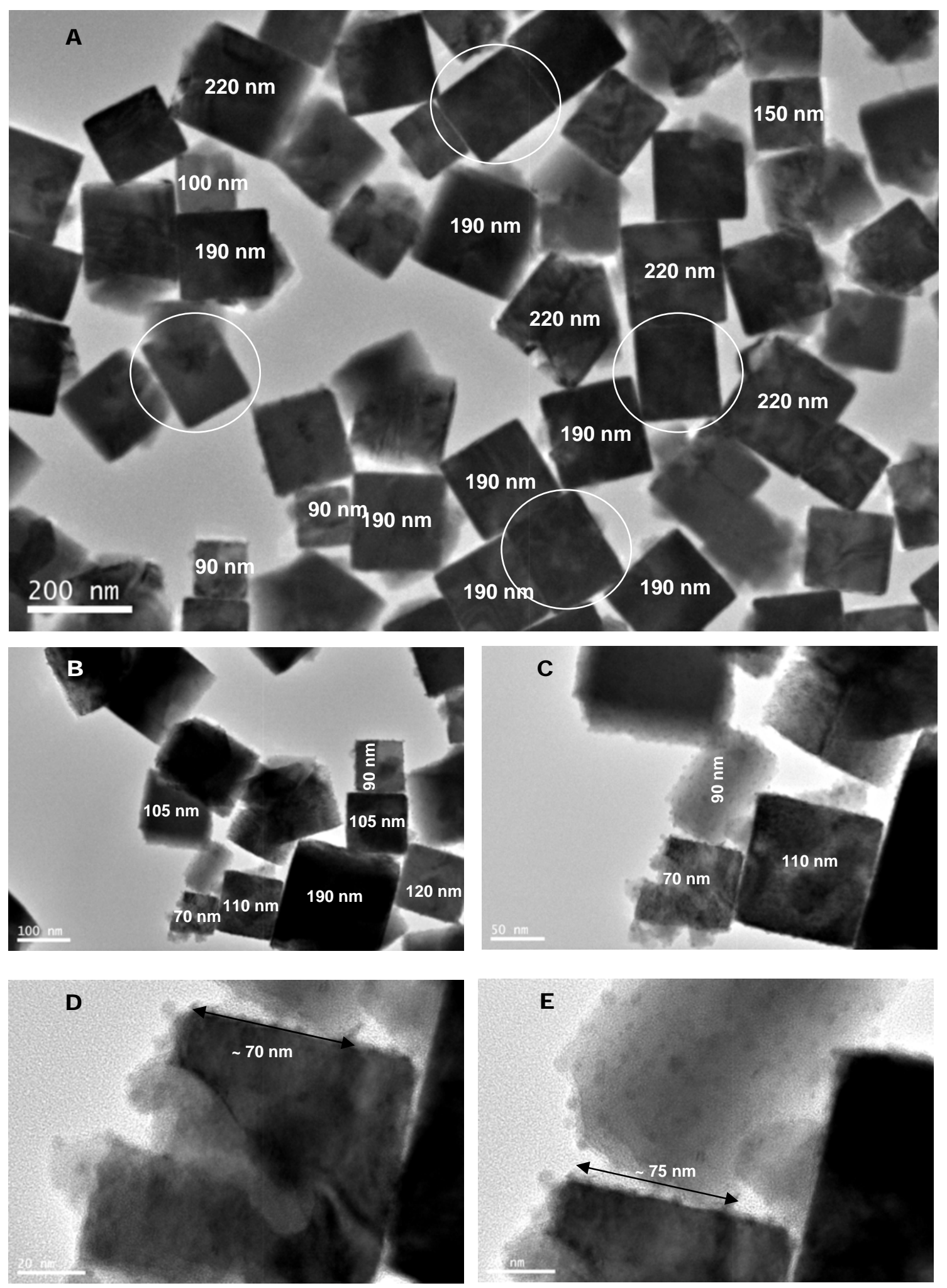

Figure 3.13: TEM images of small colloidal $\mathrm{Cu}_{2} \mathrm{O}$ nanocrystals forming regular shaped nanocubes.

The TEM images in figure 3.13 with a higher magnification $(C, D$ and $E)$ reveal that the cubes consist of very small nanocrystals in diameter of $5-10 \mathrm{~nm}$, which are almost spherical in shape. 
These small $\mathrm{Cu}_{2} \mathrm{O}$ nanocrystals (quantum dots) are embedded in a gel-like host matrix which forms the nanocubes in the size of $70-220 \mathrm{~nm}$ (figure 3.13; E). XPS measurements revealed a high content of carbon and boron atoms with the $\mathrm{Cu}_{2} \mathrm{O}$ nanocrystals. Therefore, it can be assumed that the matrix correspond to a polymer matrix consisting of the capping agents PMAA-Na and trisodium citrate (and the oxidation product of citrate) and $\mathrm{Na}_{2} \mathrm{BO}_{2}$ (oxidation product of $\mathrm{NaBH}_{4}$ ). Furthermore, the TEM image $\mathrm{E}$ in figure 3.13 shows that the cubes are covered by a thin dark layer which may correspond to a deposit layer of oxidation products.

At higher magnification, the TEM images revealed that the nanocubes consist of spherical nanocrystals showing a periodic structure of two layers. This structure is formed by $\mathrm{Cu}_{2} \mathrm{O}$ (dark) and template nano-layers (light) as seen in figure 3.14. The spherical nanocrystals show a wide size distribution in diameter varying from 5-10 nm and even larger structures of $15-25 \mathrm{~nm}$.
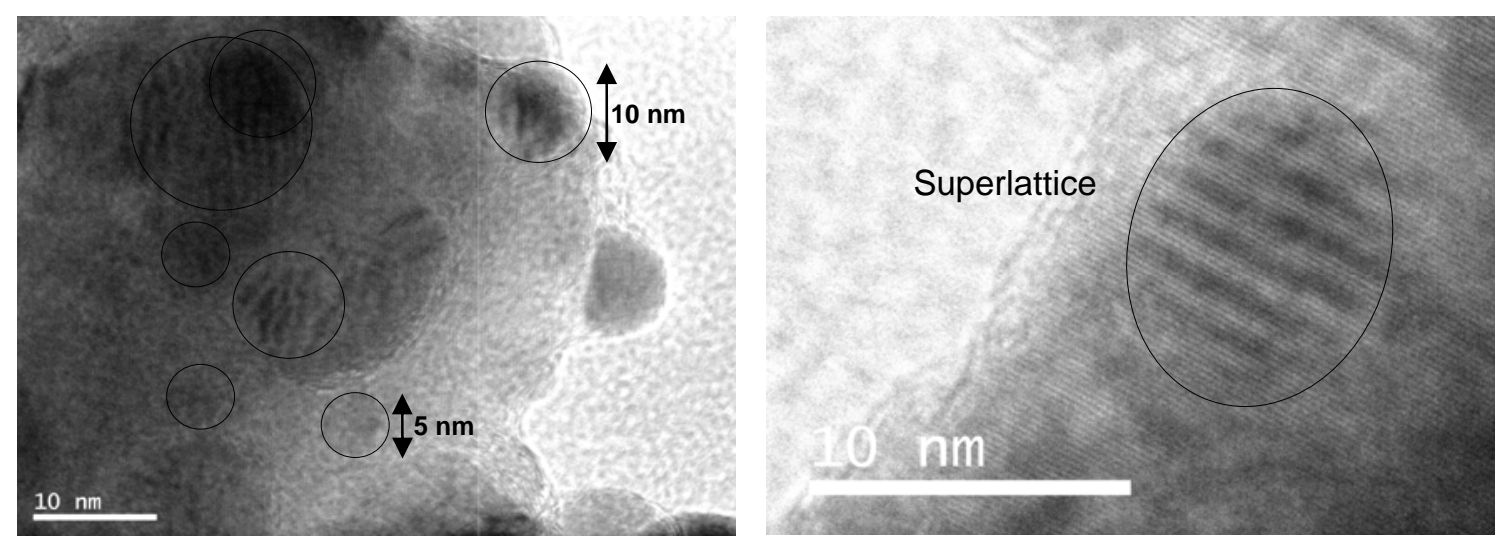

Figure 3.14: TEM images of colloidal $\mathrm{Cu}_{2} \mathrm{O}$ nano-crystals of $5-10 \mathrm{~nm}$ in diameter. Note superlattice structure encircled.

\subsubsection{Scanning Electron Microscopy}

Figure 3.15 shows the SEM (left) and back-scatter (right) images of the standard preparation of the $\mathrm{Cu}_{2} \mathrm{O}$ nanocrystals with the variation of one process parameter (AReaction temperature is adjusted at $96{ }^{\circ} \mathrm{C}, \mathrm{B}-\mathrm{CuCl}_{2}$ as copper precursor material, $\mathrm{C}$ PVP as polymeric surfactant) 


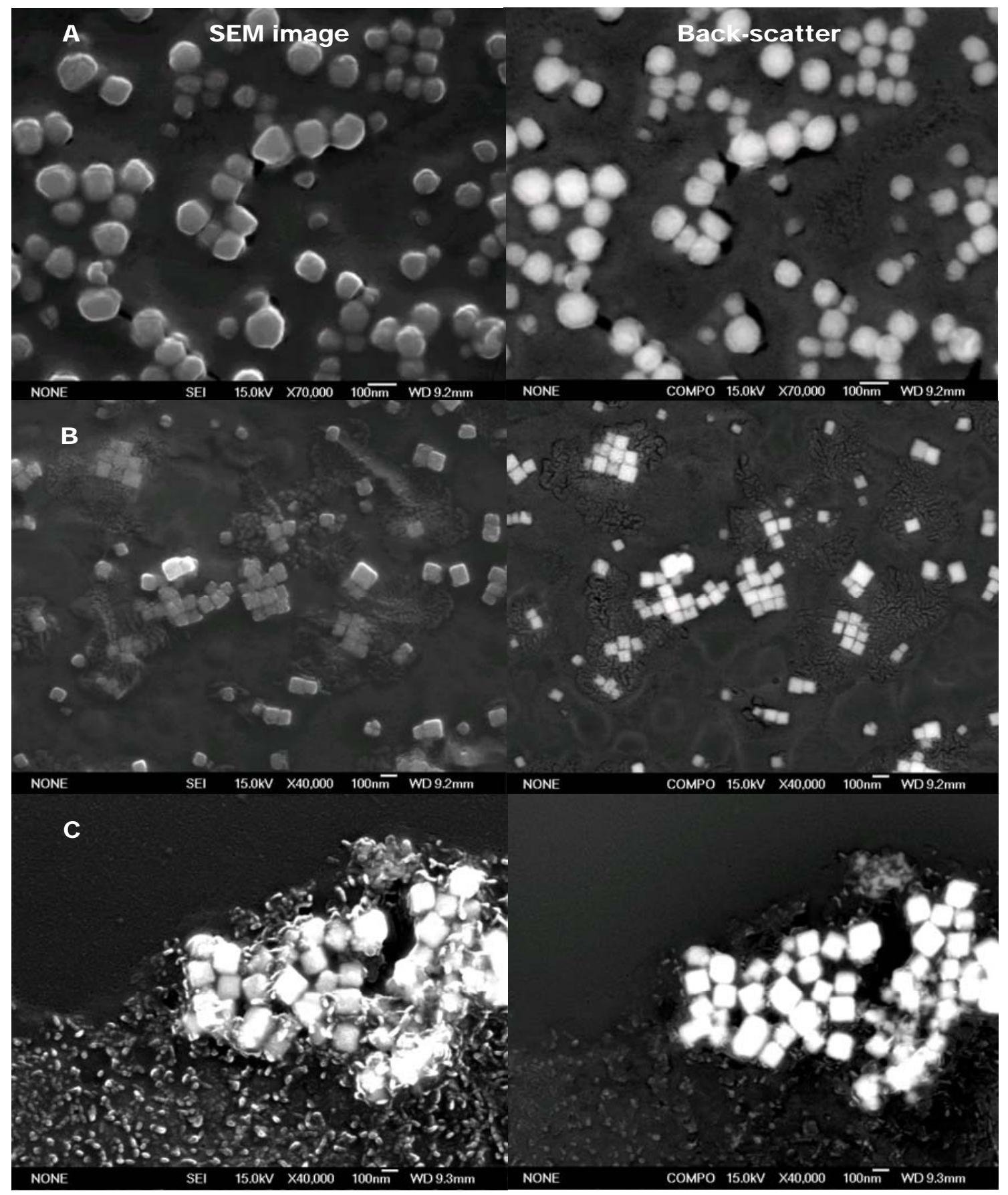

Figure 3.15: SEM and backs-scatter images of the preparation of $\mathrm{Cu}_{2} \mathrm{O}$ nanocrystals at $96{ }^{\circ} \mathrm{C}$ (A), with $\mathrm{CuCl}_{2}$ as copper precursor (B) and PVP as polymeric surfactant (C). Details of sample preparation are listed in the appendix in table A.2.

The standard procedure carried out at $96^{\circ} \mathrm{C}$ result in the formation of clumps (see figure 3.15; A). The shape of the clumps suggests that the transformation process of the nanocrystals into regular shaped cubes is uncompleted. Here, the nano-crystals which are not incorporated in the cubes are dispersed as a layer covering the surface and cubes half-way formed. The average size of the larger particles is around $110 \mathrm{~nm}$. The SEM 
and back-scatter image of $\mathrm{A}$ in figure 3.15 indicates that the reaction temperature has a strong effect on the formation of regular shaped cubes.

The standard procedure carried out with $\mathrm{CuCl}_{2}$ as copper precursor material also result in the half-way formation of cubes (figure 3.15; B). The SEM and back-scatter image of $\mathrm{B}$ in figure 1.15 shows a mixture of nano-cubes and nanocrystals covering the surface. Here, however, the cubes reveal a regular shape with sizes ranging from $70-200 \mathrm{~nm}$, but cubes of $150 \mathrm{~nm}$ dominate. At a closer look, the images also show random agglomerations smaller than $70 \mathrm{~nm}$. From these images, it can be observed that the growth of the particle occurs in stages, starting from small agglomerates below $70 \mathrm{~nm}$ to the formation of nano-cubes with constant sizes ranging of 70, 100 and $150 \mathrm{~nm}$.

The standard procedure carried out with PVP as polymeric dispersant instead of PMAA-Na result in the formation of larger cubes ranging from $100-220 \mathrm{~nm}$ (see figure 3.15 ; C). When compared with PMAA-Na cubes, PVP cubes are less regular shaped. The images of $\mathrm{C}$ in figure 3.15 also show a higher number of random agglomerates below $70 \mathrm{~nm}$ covering the cubes.

The $\mathrm{Cu}_{2} \mathrm{O}$ nanocrystal preparation with the incorporation of the metal ions $\mathrm{Zn}^{2+}$ and $\mathrm{Ni}^{2+}$ described in chapter 2 (2.1.3.1) also resulted in the agglomeration of nanocrystals to larger particles (see figure 3.16).

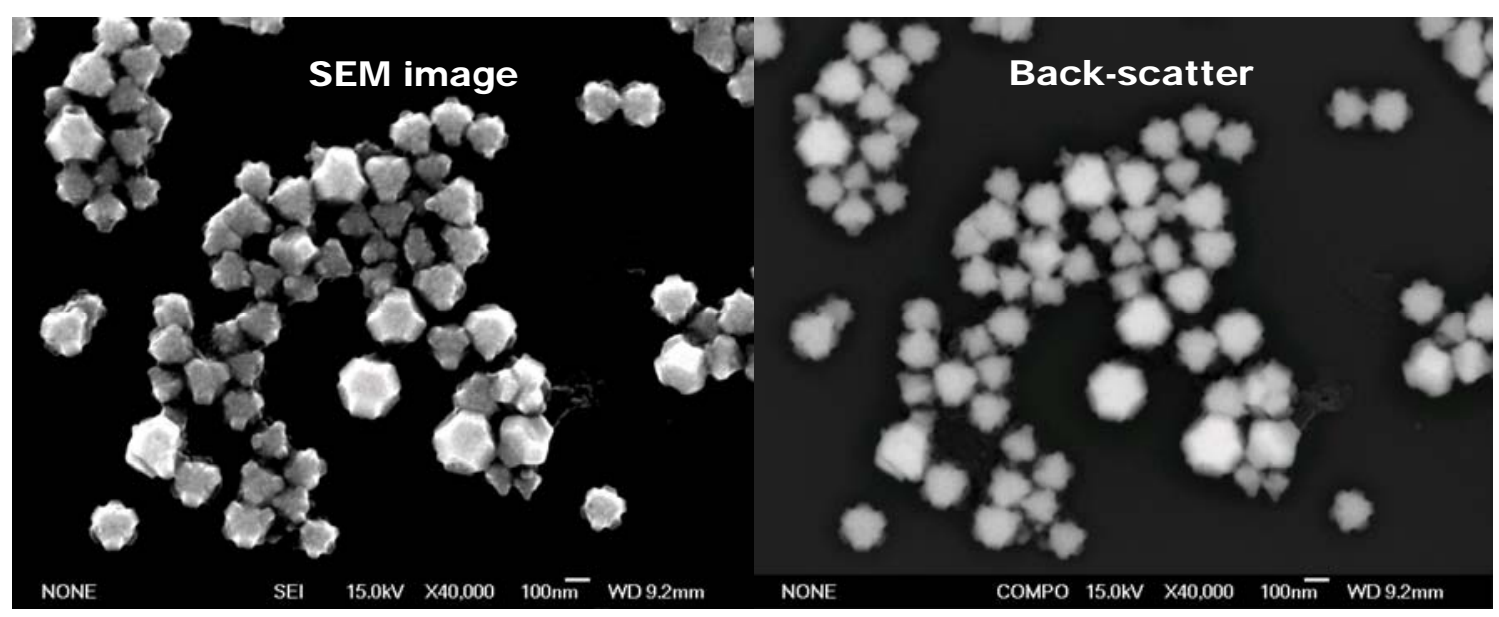

Figure 3.16: SEM and back-scatter image of $\mathrm{Cu}_{2} \mathrm{O}$ nanocrystals doped with zinc and nickel ions. Note that the doped $\mathrm{Cu}_{2} \mathrm{O}$ nanocrystals form larger pseudo-dodecahedron shapes. 
The formed particles vary between 100 to $250 \mathrm{~nm}$ in diameter and show small square shaped particles on the surface of roughly $50-70 \mathrm{~nm}$ in length. The colour of the suspension in reflected and transmitted light is seen in figure 3.6.

The process parameters used for the doping procedure of $\mathrm{Cu}_{2} \mathrm{O}$ nanocrystals with zinc and nickel, also show a strong effect on the size and shape of the particles. For example, the doping procedure carried out without the addition of zinc form regular shaped cubes (figure $3.17 ; \mathrm{A}$ ). On the other side, refluxing of the reaction medium using high temperature of the silicone oil bath $\left(\mathrm{T}_{\text {oil bath }}>140{ }^{\circ} \mathrm{C}\right)$ during the synthesis of doped $\mathrm{Cu}_{2} \mathrm{O}$ nanocrystals results in a mixture of cubes in different sizes and particles in different shapes and sizes (figure 3.17; B).

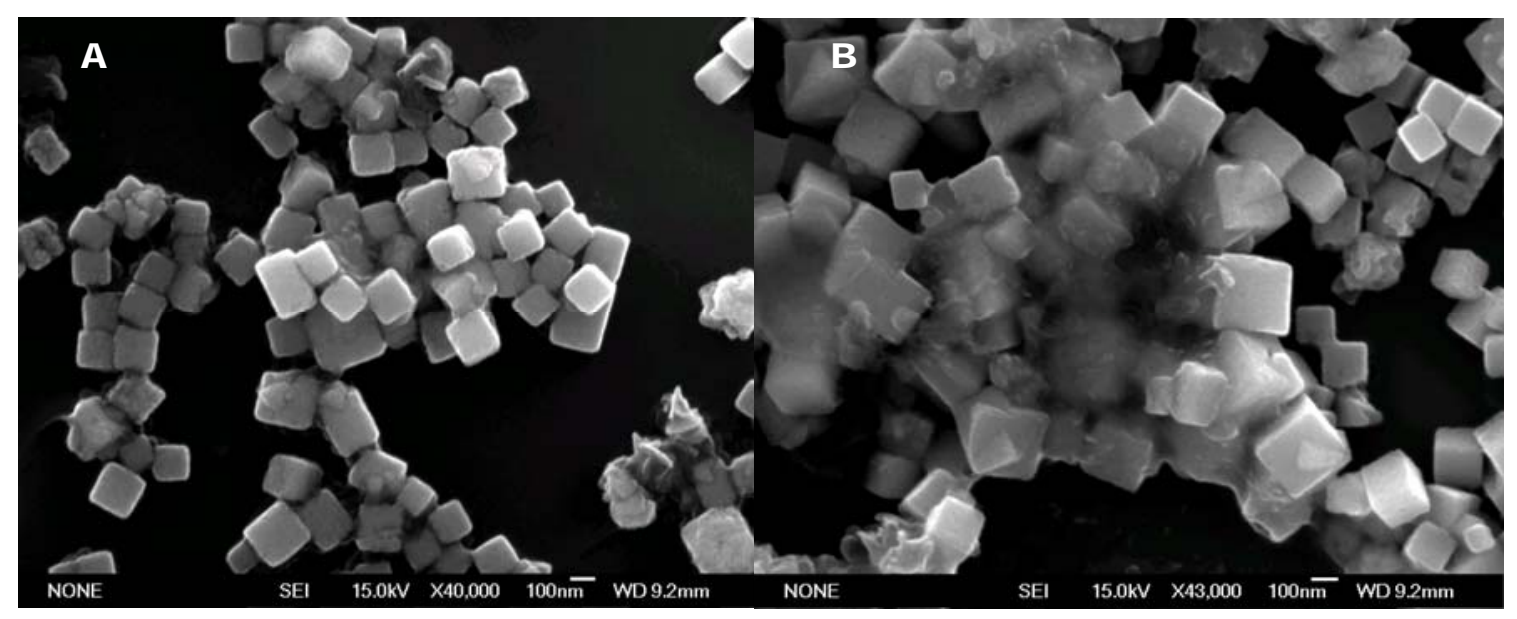

Figure 3.17: SEM images of $\mathrm{Cu}_{2} \mathrm{O}$ nanocrystals prepared according to the doping procedure without zinc (A) and with the addition of $\mathrm{Zn}^{2+}$ and $\mathrm{Ni}^{2+}$ and overheated in a silicone oil bath at $~$ $140{ }^{\circ} \mathrm{C}(\mathrm{B})$. Details of sample preparation are listed in the appendix in table A.3.

The size distribution of the cubes without the addition of zinc (figure 3.17; A) is similar to the size distribution of un-doped $\mathrm{Cu}_{2} \mathrm{O}$ nano-cubes obtained by the standard procedure (see figure 3.12). The SEM image B in figure 3.17 shows that the doping procedure with the addition of zinc results in the formation of larger cubes and particles with different shapes. The layer in the centre of the image covering the particles may be nickel oxide according to the EDS mapping. Here, it is important to mention that nickel used in the doping procedure for $\mathrm{Cu}_{2} \mathrm{O}$ nanocrystals is not detectable in the cubes using EDS mapping. However, EDS mappings of $\mathrm{Cu}_{2} \mathrm{O}$ crystals doped with zinc show zinc atoms homogeneously distributed in the particles. 


\subsubsection{Zetasizer}

The size and size distribution of the $\mathrm{Cu}_{2} \mathrm{O}$ nanocrystal suspensions were characterised by Zetasizer measurements. For the standard procedure of the synthesis of $\mathrm{Cu}_{2} \mathrm{O}$ nanocrystals (un-doped), Zetasizer measurements showed narrow size distributions of the $\mathrm{Cu}_{2} \mathrm{O}$ nanocrystal suspension at 5, 30, 70, 100 and $220 \mathrm{~nm}$ (see figure 3.18).

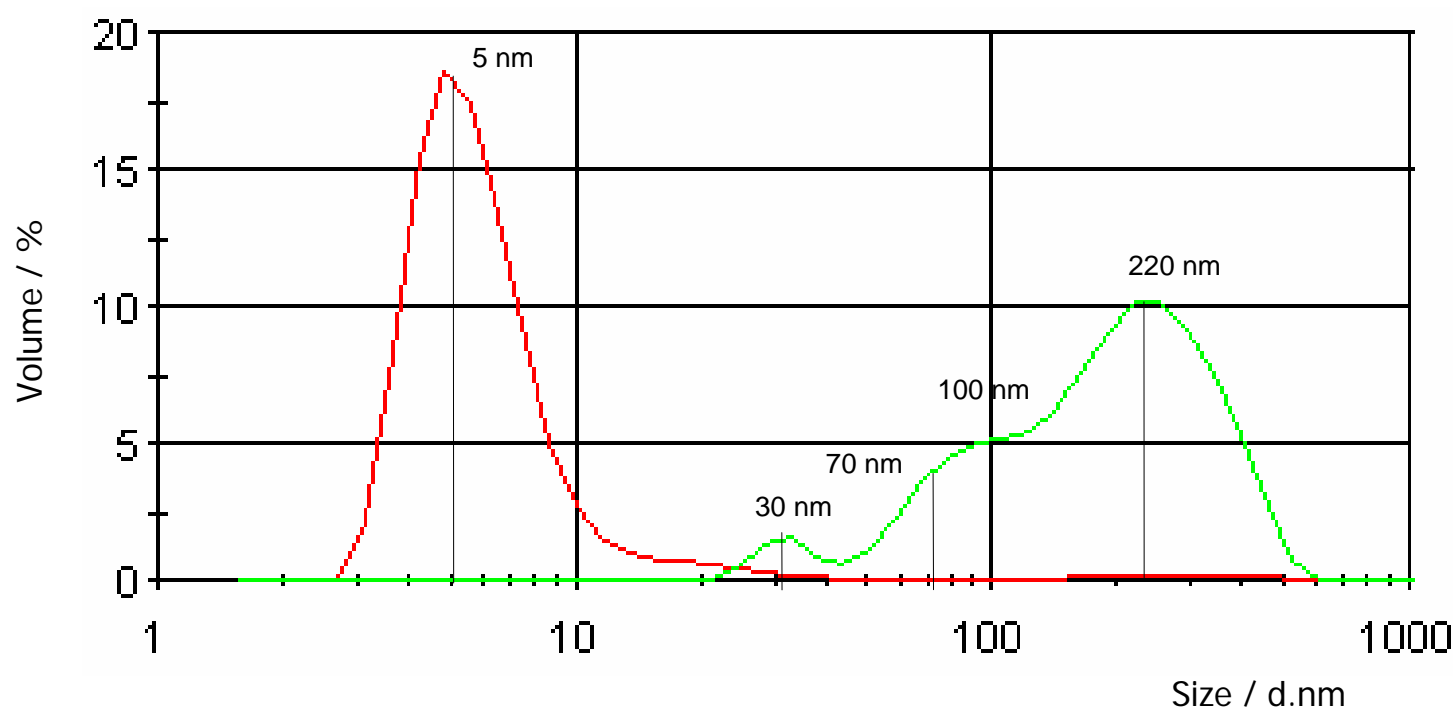

Figure 3.18: Size distribution of the $\mathrm{Cu}_{2} \mathrm{O}$ suspension (given in volume \%) prepared according to the standard procedure (green line) and diluted with water in a ratio of 1:5 (red line).

The size and size-distribution obtained with Zetasizer (in volume \%) match with the size of the small spherical nanocrystals and nanocubes shown in the TEM images in figure 3.13. The green curve corresponds to size distribution of the $\mathrm{Cu}_{2} \mathrm{O}$ nanocrystal suspension, whereas the red curve was obtained by diluting the $\mathrm{Cu}_{2} \mathrm{O}$ nanocrystal suspension with water in a ratio of 1:5 and aged for three days until the suspension turned transparent. This curve confirms that the dilution of the $\mathrm{Cu}_{2} \mathrm{O}$ suspension with water breaks up the self-assembled structures formed by the nanocrystals and nanocrystals become homogenously distributed in solution. The peak at $30 \mathrm{~nm}$ corresponds to random agglomeration of the nanocrystals, which can be also seen for example in the SEM images in figure 3.15. The peaks at 100 and $220 \mathrm{~nm}$ correspond to the cube shaped particles. A distinct hump at $70 \mathrm{~nm}$ is also visible at closer examination of the curve and this coincides with observation using TEM. The TEM images C, D and E in figure 3.13 reveal the presence of small cubes of around $70 \mathrm{~nm}$ in length. 
Figure 3.19 shows the size distribution by number (given in \%) of $\mathrm{Cu}_{2} \mathrm{O}$ nanocrystals doped with zinc and nickel. The different peaks (colours) were obtained at several steps of dilution and centrifugation of the suspension. With each dilution step (Dil. steps $=1-$ 5 ), the size-distribution of the crystal suspension decreases and finally reveal only particle sizes of around $2 \mathrm{~nm}$.

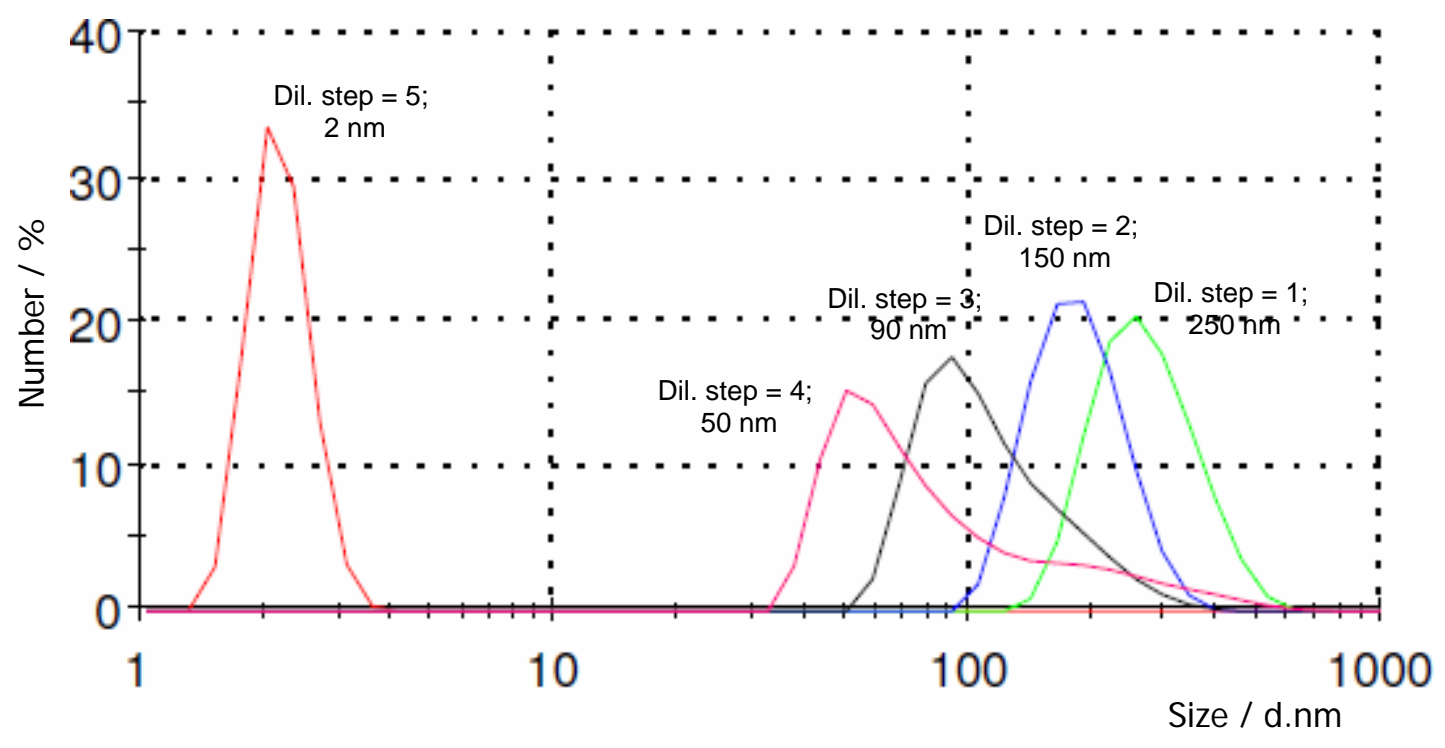

Figure 3.19: Size distribution of $\mathrm{Cu}_{2} \mathrm{O}$ nanocrystals doped with zinc and nickel (given in number $\%)$ after several steps of dilution with water and centrifugations.

The Zetasizer measurement showed that nanocrystals doped with zinc and nickel are smaller $(2 \mathrm{~nm})$ than un-doped nanocrystals $(5 \mathrm{~nm})$. The size distribution of the agglomerates varies between 50 and $250 \mathrm{~nm}$ (see figure 3.19). Similar sizes are observed in the SEM image in figure 3.16.

Results of Zetasizer showed that the particle size and size distribution of $\mathrm{Cu}_{2} \mathrm{O}$ nanocrystal suspensions can be controlled by the sodium borohydride concentration used. Figure 3.20 shows the size distribution of $\mathrm{Cu}_{2} \mathrm{O}$ samples by number, volume and intensity (given in \%) prepared according the standard procedure with the addition of different amounts of $\mathrm{NaBH}_{4}$ to the reaction medium and table 3.3 lists the particle sizes of the corresponding $\mathrm{Cu}_{2} \mathrm{O}$ samples. The size distribution of the standard procedure with $\mathrm{CuCl}_{2}$ is also shown here $\left(\mathrm{E}^{*}\right)$ to illustrate the effect of the copper precursor material on the particle growth. 
Table 3.3: Size of the particles (d.nm) in figure 3.19 obtained by adding $\mathrm{NaBH}_{4}$ in different concentration of to the standard reaction. ${ }^{*} \mathrm{Cu}_{2} \mathrm{O}$ suspension prepared with $\mathrm{CuCl}_{2}$ as copper precursor material. Details of sample preparation are listed in the appendix in table A.4.

\begin{tabular}{ccc}
\hline Sample label & {$\left[\mathrm{NaBH}_{4}\right] / \mathrm{M}$} & Size $/ \mathrm{d} . \mathrm{nm}$ \\
\hline $\mathrm{A}$ & 0.004 & 5.5 and 100 \\
$\mathrm{~B}$ & 0.005 & 150 \\
$\mathrm{C}$ & 0.006 & 25 and 220 \\
$\mathrm{D}$ & 0.008 & 190 \\
$\mathrm{E}$ & 0.01 & 220 \\
$\mathrm{E}^{*}$ & 0.01 & 150 \\
\hline
\end{tabular}

Zetasizer measurement revealed that with the increment of the $\mathrm{NaBH}_{4}$ concentration in the reaction medium the size of the particle gradually increases starting from 5.5 to 220 $\mathrm{nm}$ (see figure 3.20; A and table 3.3). However, the size distribution of the samples by number (A), volume (B) and intensity (C) as seen in figure 3.20 does not always show the same size/peak. For example, sample A and $\mathrm{C}$ show two distinct peaks when presented in the size-distribution by intensity (figure 3.20 ; C). However, when presented in the size-distribution by number (figure 3.11 ; A), both samples only show one peak. Here, the addition of low $\mathrm{NaBH}_{4}$ concentration $\left(\left[\mathrm{NaBH}_{4}\right]=0.004-0.006 \mathrm{M}\right)$ result in a mixture of particles with different sizes from small spherical nanocrystals $(5.5 \mathrm{~nm})$, small agglomerates $(25 \mathrm{~nm})$ and small cubes $(100-150 \mathrm{~nm})$ which can not be isolated from each other. Zetasizer calculate the average size of the particle sizes obtained by number, volume and intensity. However, when the suspension contains a mixture of particles of very different sizes, as seen by sample A and $C$, the average size calculation is not possible. Nevertheless, the different particle-size distribution peaks correspond well with the size of the spherical nanocrystals, agglomerates and cubes observed with electron microscopy images. This suggests that the size distribution obtained by Zetasizer could be used as a good estimate of particle size. 

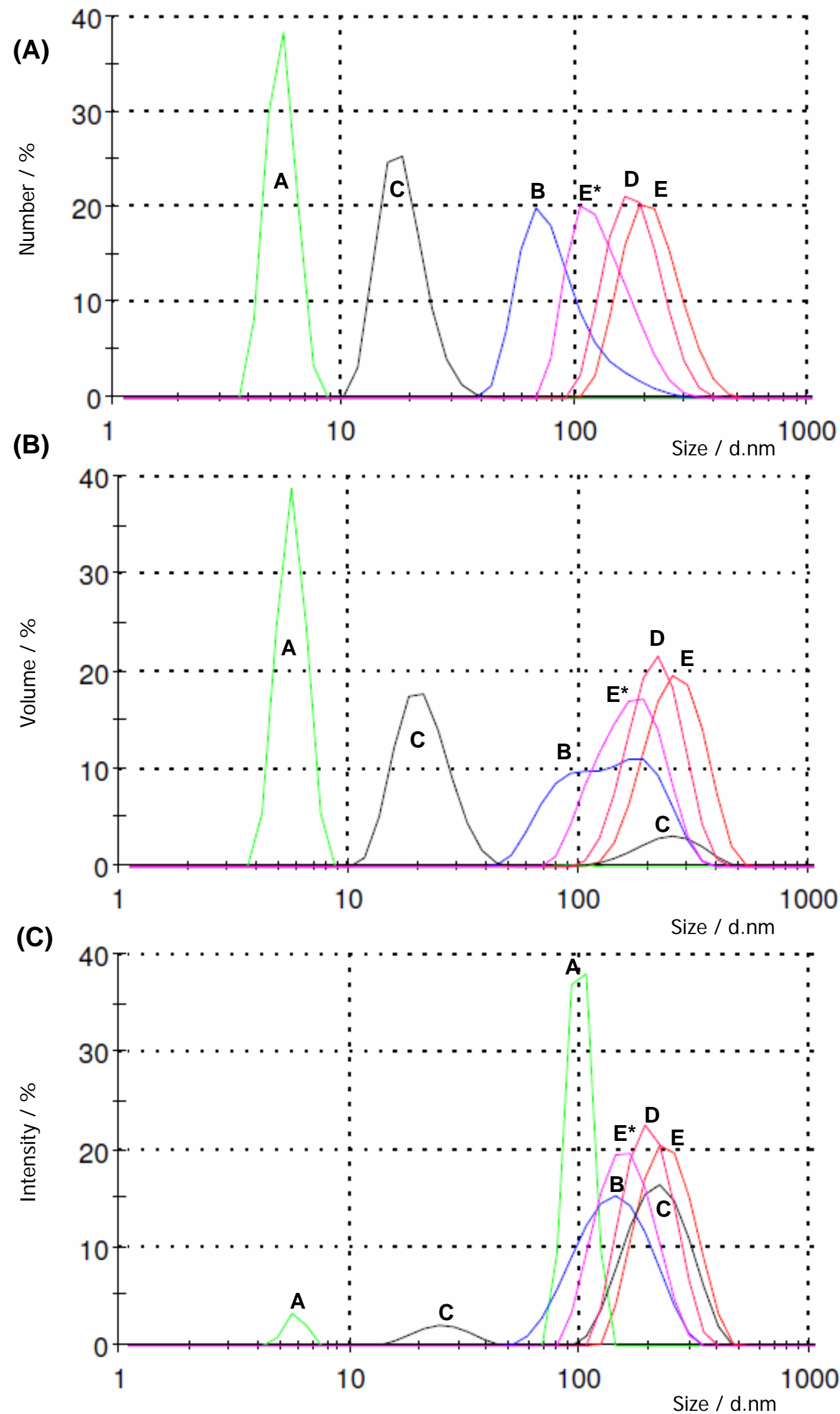

Figure 3.20: Size distribution of $\mathrm{Cu}_{2} \mathrm{O}$ suspension by number (A), volume (B) and intensity (C) given in \% prepared following the standard procedure with different concentration of $\mathrm{NaBH}_{4}(\mathrm{see}$ table 3.3). 


\subsubsection{Spectroscopy}

\subsubsection{UV-vis spectroscopy of $\mathrm{Cu}_{2} \mathrm{O}$}

The absorption spectroscopy can provide helpful information's about the size and sizedistribution of the particles in solution. For instance, sharp peaks in UV-vis spectra indicate the presence of particles with a narrow size-distribution and broad absorbance peaks indicate the presence of particles with a wide size distribution. Thereby, changes observed by a shift or broadening of the absorption peak/band refers to a change of the size and size distribution of the particles in solution. Figure 3.21 shows the absorbance peaks and bands of the $\mathrm{Cu}_{2} \mathrm{O}$ samples listed in table 3.3.

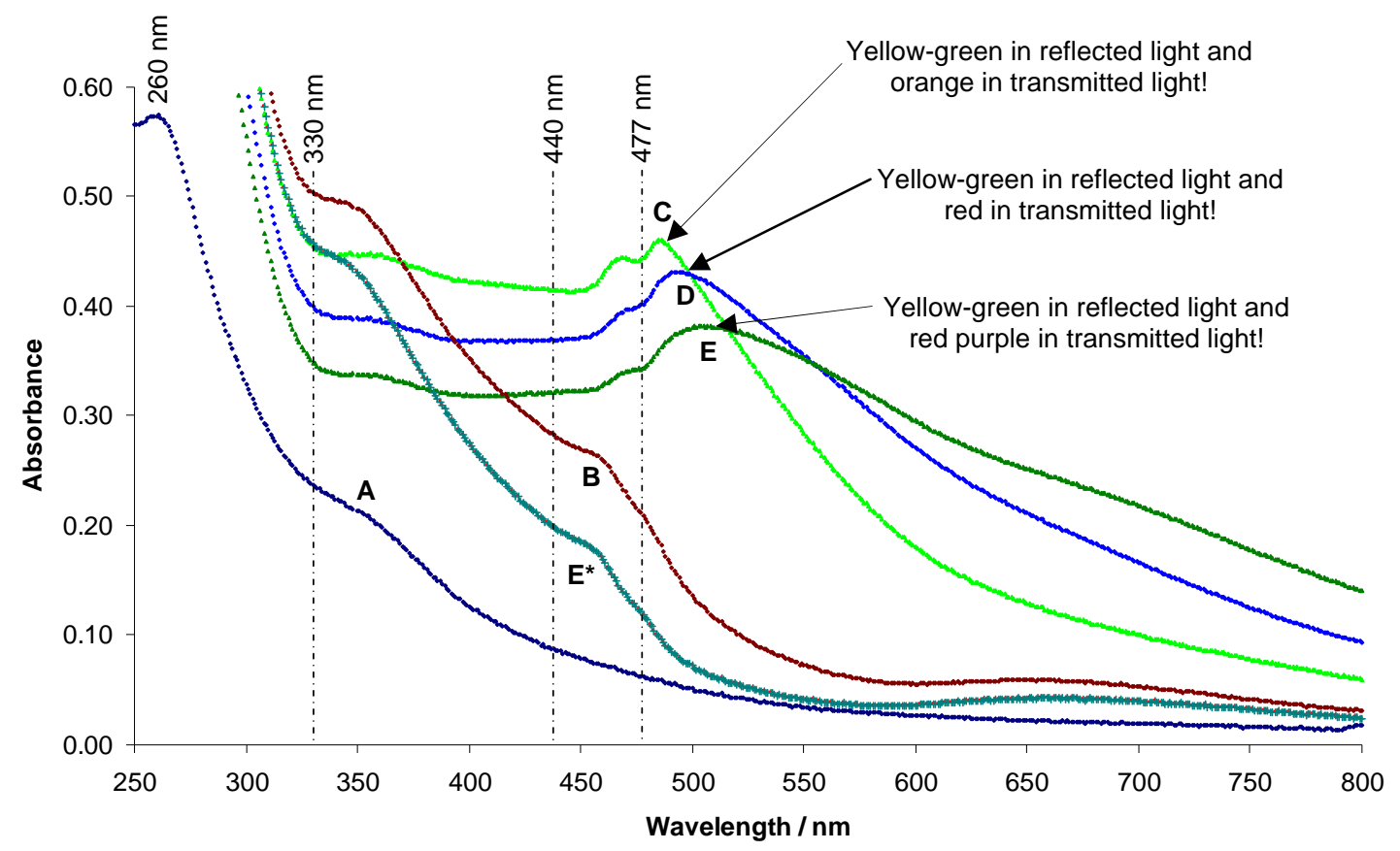

Figure 3.21: Absorbance scan of $\mathrm{Cu}_{2} \mathrm{O}$ samples with different concentration of $\mathrm{NaBH}_{4}$ listed in table 3.3. ${ }^{*} \mathrm{Cu}_{2} \mathrm{O}$ suspension prepared with $\mathrm{CuCl}_{2}$ as copper precursor material.

The absorbance wavelengths of each sample shown in figure 3.21 and the resulting colour in reflected and transmitted light are displayed in table 3.4 with the average size obtained by Zetasizer. 
Table 3.4: List of $\mathrm{Cu}_{2} \mathrm{O}$ samples showing particle size, absorbance wavelengths and their colour in reflected and transmitted light. ${ }^{*} \mathrm{Cu}_{2} \mathrm{O}$ suspension prepared with $\mathrm{CuCl}_{2}$ as copper precursor material.

\begin{tabular}{cccccc}
\hline Sample label & {$\left[\mathrm{NaBH}_{4}\right] / \mathrm{M}$} & Size / d.nm & $\lambda_{\text {abs }} / \mathrm{nm}$ & $\begin{array}{c}\text { Colour in } \\
\text { reflected light }\end{array}$ & $\begin{array}{c}\text { Colour in } \\
\text { transmitted light }\end{array}$ \\
\hline A & 0.004 & 5.5 and 100 & 345 & Transparent & Transparent \\
B & 0.005 & 150 & $345 / 455$ & Yellow & Yellow \\
C & 0.006 & 25 and 220 & $355 / 467 / 485$ & Yellow-green & Orange \\
D & 0.008 & 190 & $355 / 470 / 492$ & Yellow-green & Red \\
E & 0.01 & 220 & $355 / 470 / 503$ & Yellow-green & Red-purple \\
E* $^{\star}$ & 0.01 & 150 & $345 / 455$ & Yellow & Yellow \\
\hline
\end{tabular}

The $\mathrm{Cu}_{2} \mathrm{O}$ suspensions listed in table 3.4 (sample $\mathrm{A}-\mathrm{E}$ ) contain different sizes and size-distributions of spherical nanocrystals and nanocubes. These samples show characteristic absorbance peaks and distinctive colours in reflected and transmitted light. Table 3.5 displays the wavelength ranges of the visible light (blue, greenish blue, blue green and green) and their corresponding complementary colours which determine to the colour of the $\mathrm{Cu}_{2} \mathrm{O}$ suspension in transmitted light.

Table 3.5: Absorbance range of visible light with the corresponding complementary colour.

\begin{tabular}{ccc}
\hline$\lambda_{\text {abs }} / \mathrm{nm}$ & $\begin{array}{c}\text { Absorption colour / } \\
\text { Reflection colour }\end{array}$ & $\begin{array}{c}\text { Complementary colour / } \\
\text { Transmission light }\end{array}$ \\
\hline $435-480$ & Blue & Yellow \\
$480-490$ & Green-blue & Orange \\
$490-500$ & Blue-green & Red \\
$500-560$ & Green & Red-purple \\
\hline
\end{tabular}

Sample A $\left(\left[\mathrm{NaBH}_{4}\right]=0.004 \mathrm{M}\right)$ contains predominantly small spherical nanocrystals $(\sim$ $5 \mathrm{~nm}$ ). The UV-vis scan show absorbance peaks at 220, 260 and $350 \mathrm{~nm}$, but no peaks in the visible range (figure 3.21; sample A). For that reason, the final suspension is transparent with a slightly bluish appearance due to the absorbance peak at $350 \mathrm{~nm}$.

Sample B $\left(\left[\mathrm{NaBH}_{4}\right]=0.005 \mathrm{M}\right)$ and $\mathrm{E}^{*}\left(\left[\mathrm{NaBH}_{4}\right]=0.01 \mathrm{M}, \mathrm{CuCl}_{2}\right)$ contain small spherical nanocrystals and small cubes varying in the size between 70 and $150 \mathrm{~nm}$. Both samples show identical absorbance scans with an absorbance peak at $457 \mathrm{~nm}$ and small hump at $477 \mathrm{~nm}$ in the visible range. This wavelength corresponds to the absorption of blue light, which result in the complementary colour yellow of the suspension. For this reason, both samples show a bright yellow colour. 
Sample C $\left(\left[\mathrm{NaBH}_{4}\right]=0.006 \mathrm{M}\right), \mathrm{D}\left(\left[\mathrm{NaBH}_{4}\right]=0.008 \mathrm{M}\right)$ and $\mathrm{E}\left(\left[\mathrm{NaBH}_{4}\right]=0.01 \mathrm{M}\right)$ contain cubes with a wider size distribution varying between 70 and $220 \mathrm{~nm}$. These samples show two absorbance peaks in the visible range of light. The first absorbance peak is located between 450 and $480 \mathrm{~nm}$, which refer to the absorbance of blue light resulting in the complementary colour yellow. The second absorbance peak is located above $480 \mathrm{~nm}$ and gradually broadens with the increment of the $\mathrm{NaBH}_{4}$ concentration in the reaction medium (figure 3.21 ).

The broadening and shift of the second absorbance peak to higher wavelengths with higher $\mathrm{NaBH}_{4}$ concentration $\left(\left[\mathrm{NaBH}_{4}\right]=0.006-0.01 \mathrm{M}\right)$ refer to the formation of larger cubes in solution. With the formation of larger cubes, however, the suspensions obtain stronger light scattering properties. Here, cubes of certain sizes absorb and scatter light in the visible range. Due to the light absorption and scattering properties of the cubes, the $\mathrm{Cu}_{2} \mathrm{O}$ suspensions show a dichroic effect. Figure 3.22 shows the colour of the samples $\mathrm{C}, \mathrm{D}$ and $\mathrm{E}$ in reflected light (top row) and transmitted light (bottom row).

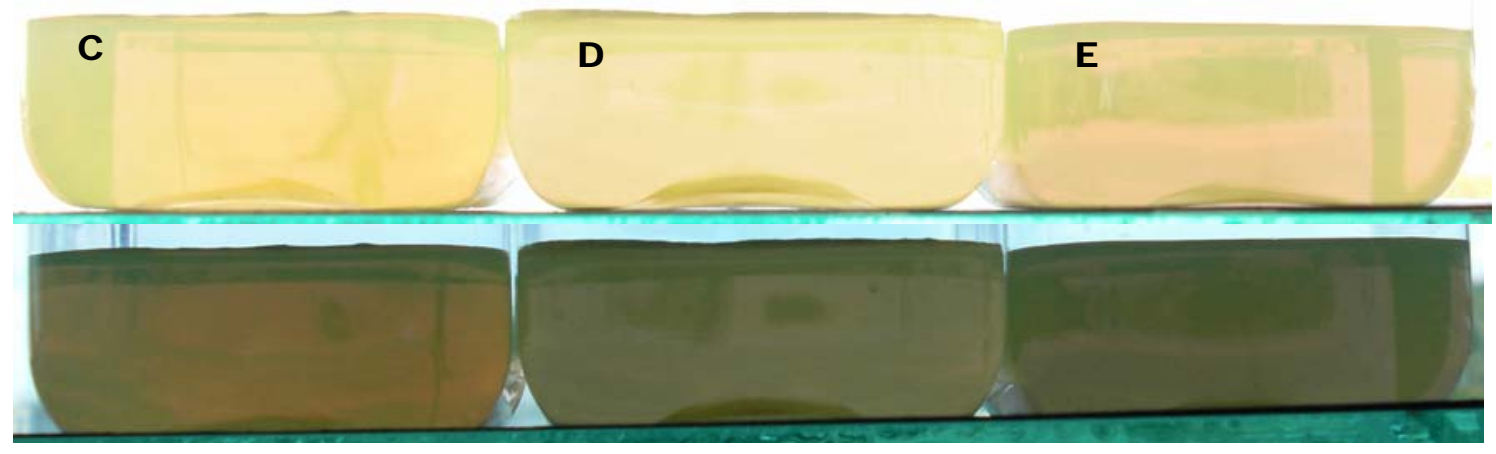

Figure 3.22: $\mathrm{Cu}_{2} \mathrm{O}$ samples $\mathrm{C}, \mathrm{D}$ and $\mathrm{E}$ (listed in table 3.4) observed in transmitted light. Pictures were taken in a different angle to the transmitted light. Note reflection of the light is higher at the top photographs.

Depending on the wavelength of the absorbed light (see table 3.4), the suspensions show the colour of the absorbed light in reflected/scattered light and the complementary colour of the absorbed light in transmitted light. For instance, sample $\mathrm{C}$ is yellow-green in reflected light and orange-red in transmitted light due to the absorption of blue light $(467 \mathrm{~nm})$ and green-blue light $(485 \mathrm{~nm})$. The complementary colour of green-bluish light $(\lambda=480-490 \mathrm{~nm})$ is orange as shown in transmitted light.

A similar situation is observed in sample $\mathrm{D}$ and $\mathrm{E}$, where a shift of the absorbance peak resulted in the absorbance of light with a higher wavelength. Therefore, sample D and E 
show different colours in the transmitted light depending on the wavelength of the absorbed light. Here, sample D show a red colour in transmitted light, which is the complementary colour of blue-green light $(490-500 \mathrm{~nm})$. Sample E show a red-purple colour in transmitted light, which is the complementary colour of green light (500-560 $\mathrm{nm})$. In reflected light, however, the samples shows the colour of the absorbed light, which is scattered on the surface of the cubes.

It was discovered that the addition of $\mathrm{NaOH}$ to the reaction medium result in a further shift and broadening of the absorbance peak in the visible range. Figure 3.23 shows the effect of the $\mathrm{pH}$ by adding $0.25 \mathrm{~mL} 0.01 \mathrm{M} \mathrm{NaOH}$ to reaction medium for the standard procedure of $\mathrm{Cu}_{2} \mathrm{O}$ suspension (sample E) after the addition of $\mathrm{NaBH}_{4}$.

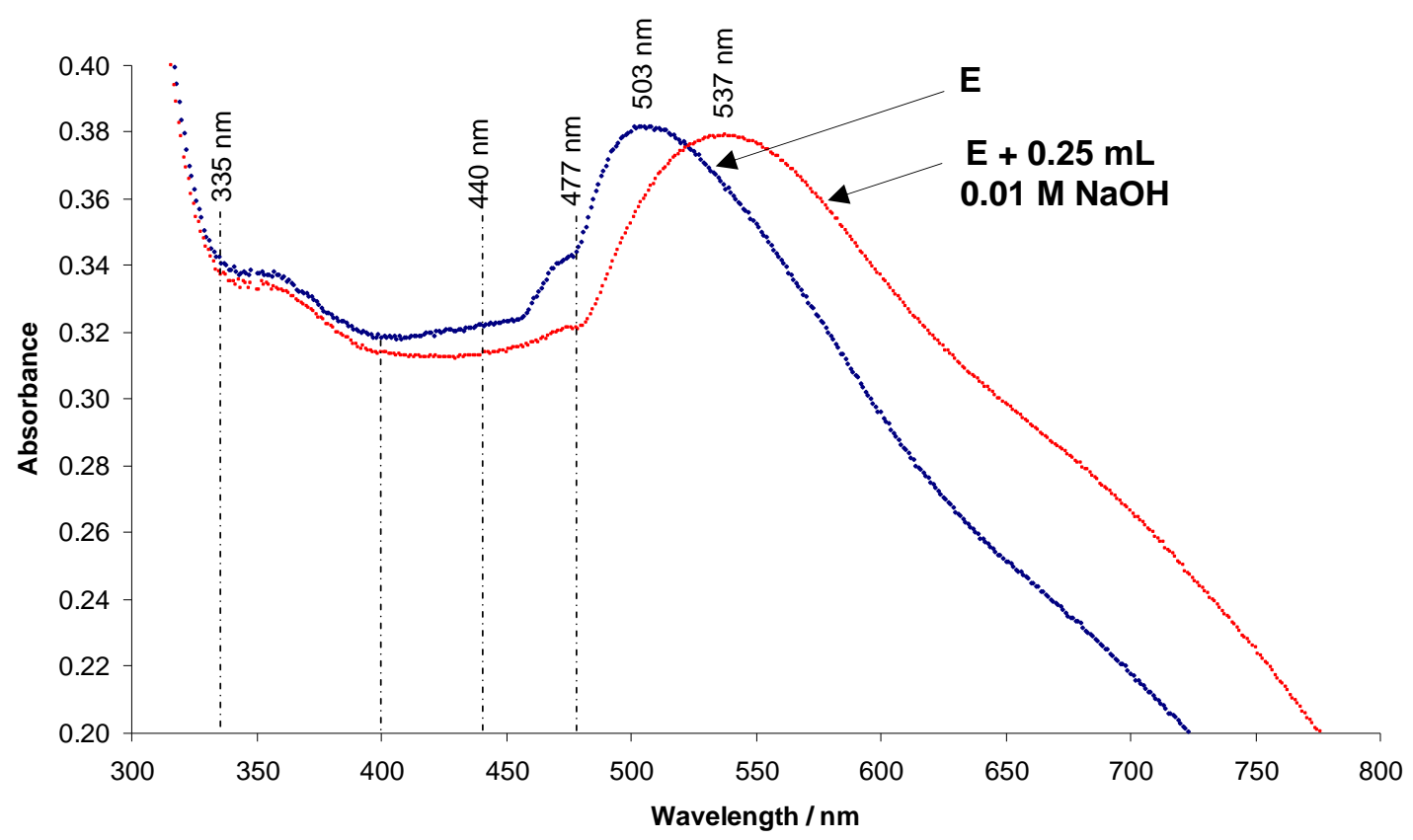

Figure 3.23: $\mathrm{UV}$-vis scans of $\mathrm{Cu}_{2} \mathrm{O}$ prepared following the standard procedure without and with the addition of $0.25 \mathrm{~mL} 0.01 \mathrm{M} \mathrm{NaOH}$ after $\mathrm{NaBH}_{4}$ (blue line and red line, respectively).

The broadening and strong red shift of the absorbance peak from 503 to $537 \mathrm{~nm}$ refers to the formation of larger structures in suspension which are most likely formed by the cube-shaped particles. The agglomeration of the cubes in suspension with the increment of the $\mathrm{pH}$ may also explain the vanishing of the absorbance peak at $470 \mathrm{~nm}$ which corresponds to the absorption of blue light resulting in the complementary colour yellow. With the loss of this absorbance peak, the colour of the suspension changes from yellow-green to green. Here, the suspension only absorbs green light with a 
wavelength around $537 \mathrm{~nm}$ resulting in the complementary colour red purple in transmitted light and green in reflected light. The formation of larger structures by adding $\mathrm{NaOH}$ to the reaction medium is also indicated by a stronger precipitation reaction after preparation and higher transparency in reflected light.

It can be suggested that the absorbance peaks in the visible range between 450 and 480 and above $480 \mathrm{~nm}$ stand for two distinctive size distributions of the nanocubes. Zetasizer measurements, TEM and SEM images showed nanocubes of constant sizedistributions of about 100 and $220 \mathrm{~nm}$ for sample $\mathrm{E}$ (standard procedure of $\mathrm{Cu}_{2} \mathrm{O}$ ). For this reason, the first absorbance peak can be related to cubes sizes between 70 and 150 $\mathrm{nm}$, whereas the second absorbance peak stands for cubes sizes larger than $180 \mathrm{~nm}$. Here table 3.6 correlates the sizes of the nanocrystals and nanocubes with the corresponding wavelength (in $\mathrm{nm}$ ) of the absorbance peaks and bands.

Table 3.6: Correlation between particle sizes and absorbance peaks.

\begin{tabular}{ccc}
\hline & $\lambda_{\mathrm{abs}} / \mathrm{nm}$ & Particle size $/ \mathrm{nm}$ \\
\hline $\mathrm{Cu}_{2} \mathrm{O}$ quantum dots & $350-390$ & $5-15$ \\
\hline \multirow{2}{*}{ Cube-shaped } & 477 & 150 \\
particles & 487 & 190 \\
& 495 & 220 \\
& 503 & 255 \\
\hline
\end{tabular}

By plotting the particle sizes of the nanocubes obtained by Zetasizer and electron microscopy images with the corresponding absorbance peaks / bands, a linear function was obtained (see figure 3.24). 


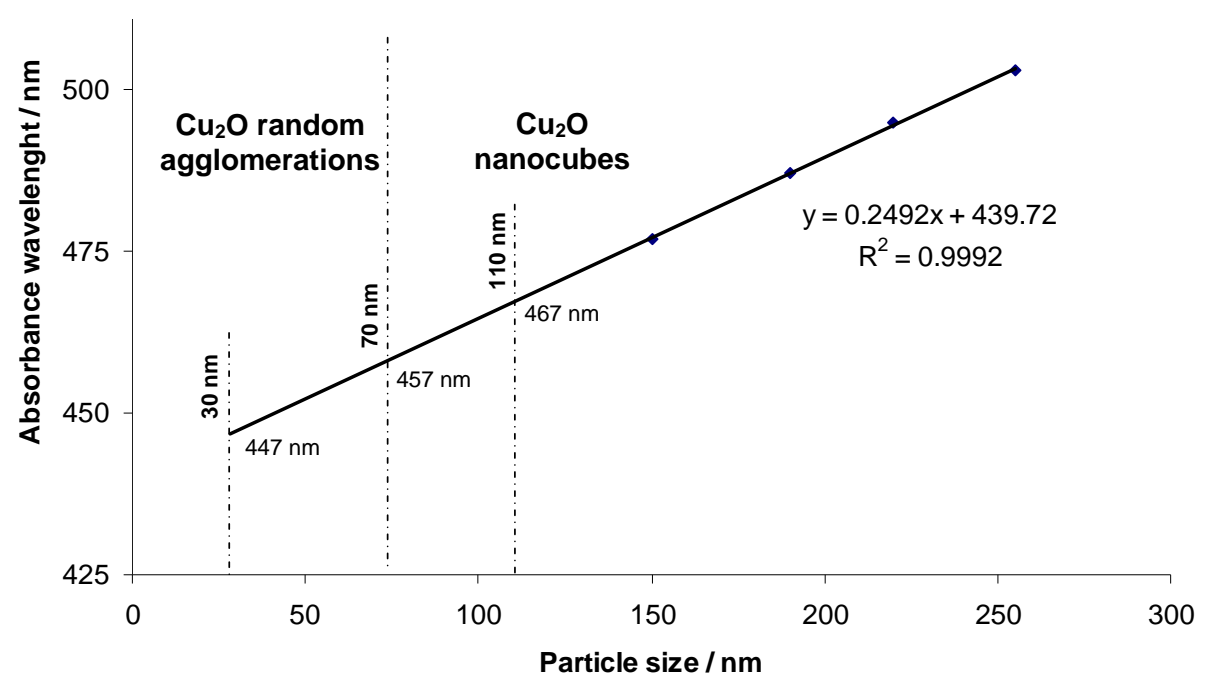

Figure 3.24: Correlation between nanocubes size and their corresponding absorbance peak.

The directly relationship between particle size and absorbance as shown in figure 3.24, enables the correlation of the distinctive absorbance peaks to certain sizes of the nanocubes. For example, it was found out that the sharp absorbance peak at $457 \mathrm{~nm}$ of sample $B$ and $E^{*}$ in figure 3.21 refers to a particle size of $70 \mathrm{~nm}$. Electron microscopy images (figure 3.13) confirmed that the size of the smallest cubes was around $70 \mathrm{~nm}$. Particle sizes below $70 \mathrm{~nm}$ obtained by Zetasizer belong to random agglomerations of spherical nanocrystals, which can be seen in the SEM images in figure 3.15. With the correlation of particles sizes and absorbance wavelength, a borderline between spherical nanocrystals and self-assembled structures was also obtained around $440 \mathrm{~nm}$. Here, absorbance peaks below $440 \mathrm{~nm}$ are related to spherical nanocrystals, whereas absorbance peaks above $440 \mathrm{~nm}$ are related to random agglomerates and regular cubeshaped particles.

Due to the relation of particle size and absorbance wavlenght, the effect of different process parameters on the particle size and size-distribution can be easily assessed with UV-vis spectroscopy. For example, UV-vis spectroscopy has determined that the size and size distribution of the cubes in solution is strongly dependent on the stirring time and reaction temperature. When the reaction was stirred shorter than $20 \mathrm{~min}$, the absorbance spectrums showed narrow absorbance peaks in the visible range. However, when the stirring times were above $20 \mathrm{~min}$, broad absorbance bands in the visible range were obtained indicating the dichroic effect of the suspension. UV-vis spectroscopy also 
showed that the broad absorbance peak in the visible range is only observed when the reaction temperature was kept constant at $99{ }^{\circ} \mathrm{C}$.

\subsubsection{UV-vis spectroscopy of $\mathrm{Cu}_{2} \mathrm{O} / \mathrm{Zn} / \mathrm{Ni}$}

$\mathrm{Cu}_{2} \mathrm{O}$ nanocrystals prepared in the presence of zinc and nickel (see procedure in chapter $2 ; 2.1 .3 .1)$ show similar absorbance spectrums such as undoped $\mathrm{Cu}_{2} \mathrm{O}$ suspensions. The absorbance peaks and bands are almost identical, apart from a shift of the absorbance peak from 260 to $290 \mathrm{~nm}$. This shift is attributed to the incorporation of zinc atoms into the lattice structure of $\mathrm{Cu}_{2} \mathrm{O}$, since the doping procedure without nickel also reveal the peak shift.

A major difference between the doping procedure and undoped procedure is that only one absorbance peak is observed above $440 \mathrm{~nm}$ instead of two absorbance peaks, when the reaction was carried out below $99{ }^{\circ} \mathrm{C}$. Here, the optical properties of $\mathrm{Cu}_{2} \mathrm{O}$ nanoparticles prepared in the presence of zinc and nickel are strongly dependent on the reaction temperature. When the reaction temperature was kept between 92 and $98{ }^{\circ} \mathrm{C}$, the doping procedure resulted in a bright yellow suspension. This colour is due to the absorbance of blue light in the visible wavelength range between 440 and $477 \mathrm{~nm}$ as seen figure 3.25 .

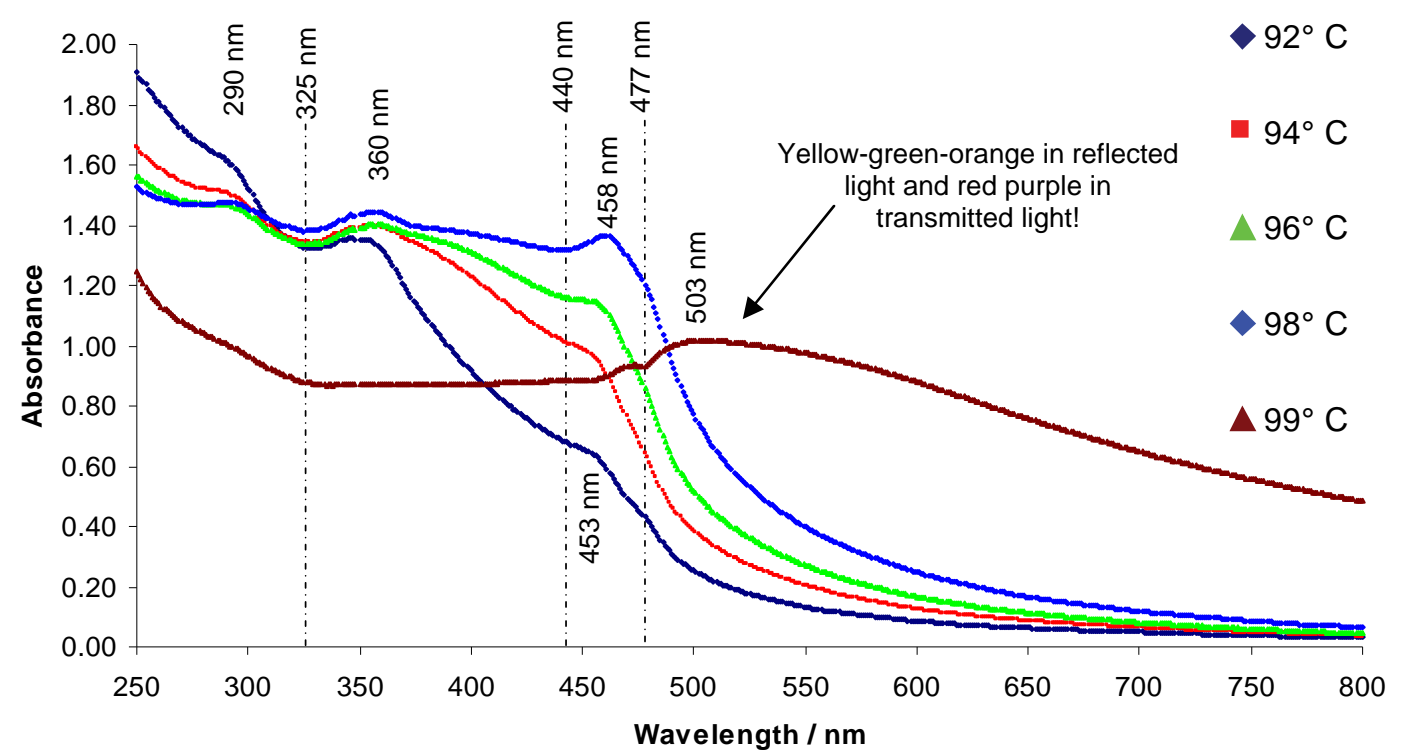

Figure 3.25: Absorbance scan of $\mathrm{Cu}_{2} \mathrm{O}$ samples doped with $\mathrm{Zn}^{2+}$ and $\mathrm{Ni}^{2+}$ carried out at 92, 94, 96, 98 and $99^{\circ} \mathrm{C}$. 
However, suspensions prepared at $99{ }^{\circ} \mathrm{C}$ show an extreme change in their absorbance spectrum, when the reaction temperature was kept constant during $20 \mathrm{~min}$ stirring. Thereby, the sharp absorbance peak between 440 and $477 \mathrm{~nm}$ is transformed into a broad absorbance band starting above $477 \mathrm{~nm}$ (figure 3.25). Due to the formation of the broad absorbance band, the suspension shows a dichroic effect, red purple colour in transmitted light and a yellow-orange-green colour in reflected light (figure 3.6). Furthermore, figure 3.25 shows that the narrow absorbance peak between 440 and 477 $\mathrm{nm}$ gradually increases in magnitude with the increment of the reaction temperature (92 $-98{ }^{\circ} \mathrm{C}$ ). Also, a small shift of the absorbance from 453 to $458 \mathrm{~nm}$ occurred, when the reaction temperature was gradually increased from 92 to $98{ }^{\circ} \mathrm{C}$. The increment of the absorbance peak with higher reaction temperature suggests that more particles are formed in solution, while the shift indicates the formation of larger particles. The increase in magnitude of the absorbance peak between 440 and $477 \mathrm{~nm}$ is also shown by a lower transparency of the suspension. Figure 3.26 shows the reflectance scans of the doped $\mathrm{Cu}_{2} \mathrm{O}$ suspensions prepared between 92 and $99{ }^{\circ} \mathrm{C}$.

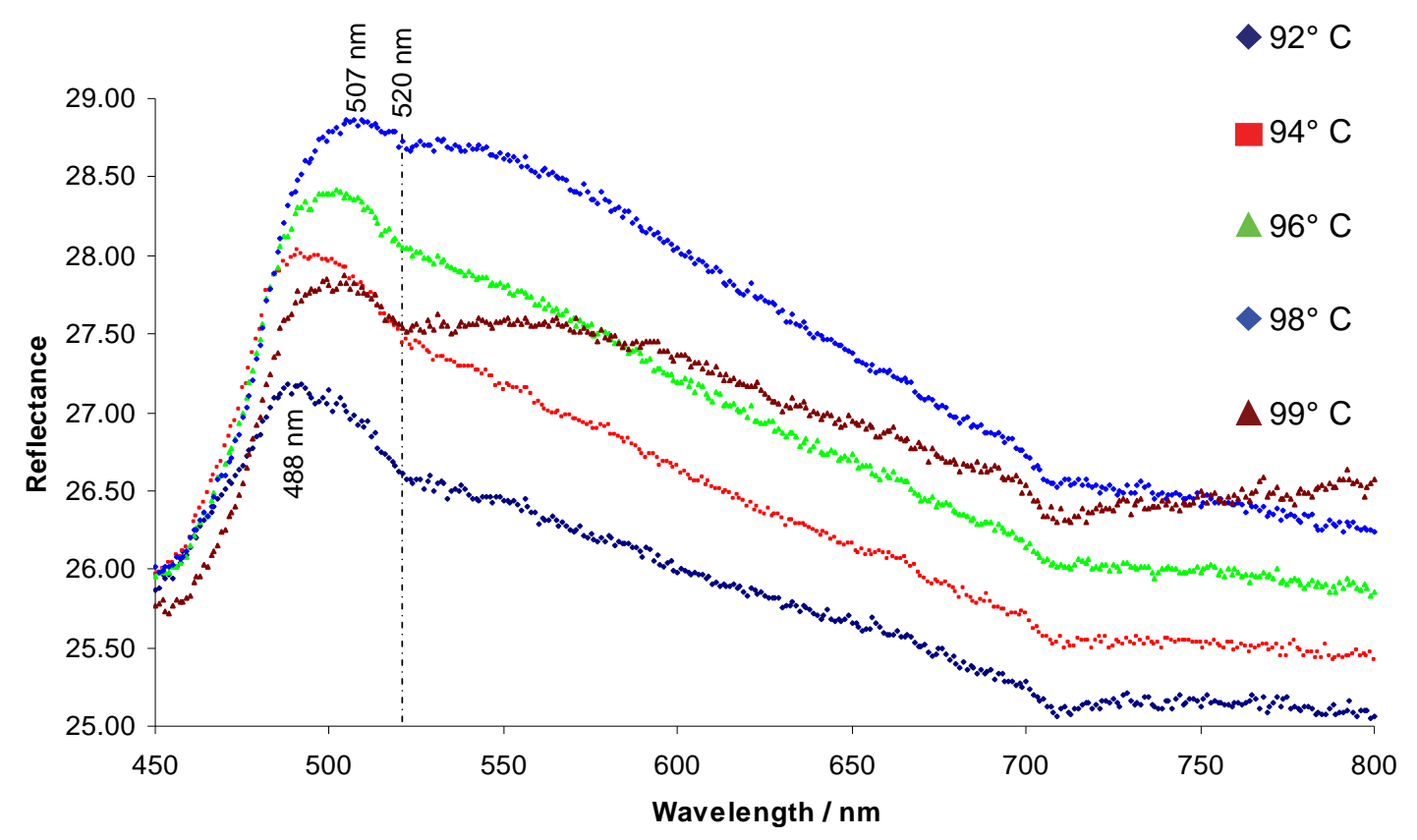

Figure 3.26: Reflectance scan of $\mathrm{Cu}_{2} \mathrm{O}$ samples doped with $\mathrm{Zn}^{2+}$ and $\mathrm{Ni}^{2+}$ carried out at 92, 94, 96, 98 and $99^{\circ} \mathrm{C}$.

The reflectance scans of doped $\mathrm{Cu}_{2} \mathrm{O}$ suspensions show that the light scattering properties of the particles formed in suspension increase with the increment of the reaction temperature from $92-98^{\circ} \mathrm{C}$. Furthermore, a shift of the maximum wavelength 
of the reflected/scattered light from $488-507 \mathrm{~nm}$ was observed, when the reaction temperature was increased from 92 to $99{ }^{\circ} \mathrm{C}$. Here, the wavelengths of the scattered light correspond to green-blue $(480-90 \mathrm{~nm})$, blue-green $(490-500 \mathrm{~nm})$ and green $(500-560 \mathrm{~nm})$ light. In general, larger particles have stronger light scattering abilities than smaller particles. Therefore, the increment and shift of the reflectance from $92-98$ ${ }^{\circ} \mathrm{C}$ can be referred to an increased formation of larger particles in solution.

The reflectance scan of doped $\mathrm{Cu}_{2} \mathrm{O}$ suspension prepared at $99{ }^{\circ} \mathrm{C}$, however, show a lower reflectance than the suspension prepared at $98^{\circ} \mathrm{C}$ (figure 3.26). The decrease of the light scattering properties is connected with a reduction of the surface-to-volume ratio of $\mathrm{Cu}_{2} \mathrm{O}$ nanocubes in the suspension. Here, nanocubes represented by the absorbance peak between 440 and $477 \mathrm{~nm}$ agglomerate and form larger structures. The agglomeration of the nanocubes also allows the adsorption of light of higher wavelengths and over a wider wavelength range, which explains the shift and broadening of the absorbance peak in the visible range (figure 3.25).

The doping procedure of $\mathrm{Cu}_{2} \mathrm{O}$ nanocrystals was also carried out with different dopants such as cobalt and manganese or only with zinc as dopant ion. Independent on the dopants, the $\mathrm{Cu}_{2} \mathrm{O}$ suspensions showed identical absorbance spectra with an absorbance peak around $457 \mathrm{~nm}$ and a small hump at $477 \mathrm{~nm}$ as seen in figure 3.27 .

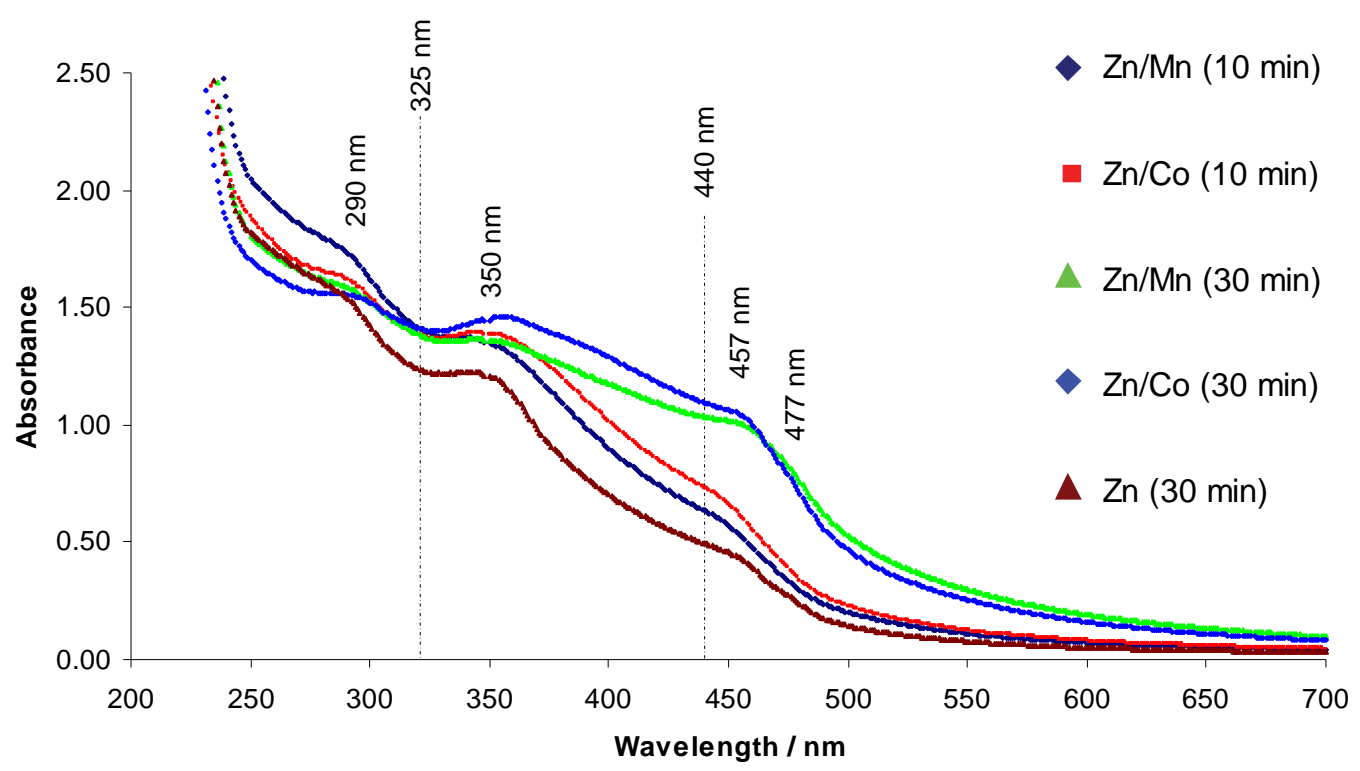

Figure 3.27: Absorbance scan of $\mathrm{Cu}_{2} \mathrm{O}$ samples doped with $\mathrm{Zn}^{2+}, \mathrm{Zn}^{2+}$ and $\mathrm{Mn}^{2+}, \mathrm{Zn}^{2+}$ and $\mathrm{Co}^{2+}$ and using different stirring times. Details of sample preparation are listed in the appendix in table A.5. 
Here, it is important to point out that a broad absorbance band above $477 \mathrm{~nm}$ was not obtained in the doping procedure, when nickel was replaced by cobalt or manganese or only zinc was used. However, figure 3.27 shows the effect of stirring time on the absorbance peak between 440 and $477 \mathrm{~nm}$. $\mathrm{Cu}_{2} \mathrm{O}$ suspensions stirred for less than 20 min show an absorbance peak at around $455 \mathrm{~nm}$. In contrary, stirring times above 20 min result in an increment and shift of the absorbance peak to $457 \mathrm{~nm}$. The increment in magnitude and shift of the absorbance peak to higher wavelengths indicate the formation of larger particles in suspension as well as in larger quantities. This is also reflected by a lower transparency of the suspension in reflected light, when the suspension was stirred longer than 20 min.

$\mathrm{Cu}_{2} \mathrm{O}$ nanocrystals doped only with zinc ions also show an absorbance peak between 440 and $477 \mathrm{~nm}$ (figure 3.27). However, prolonged stirring times does not result in an increment or shift of the peak. By increasing the zinc concentration $\left(\left[\mathrm{Zn}^{2+}\right]=0.6 \cdot 10^{-3}\right.$ $\mathrm{M})$ and adding additional trisodium citrate $\left(\left[\mathrm{Na}_{3} \mathrm{C}_{6} \mathrm{H}_{5} \mathrm{O}_{7} \cdot 2 \mathrm{H}_{2} \mathrm{O}\right]=0.3 \cdot 10^{-3} \mathrm{M}\right)$ to the reaction medium, an increment and shift of the absorbance peak to $457 \mathrm{~nm}$ was observed (figure 3.28).

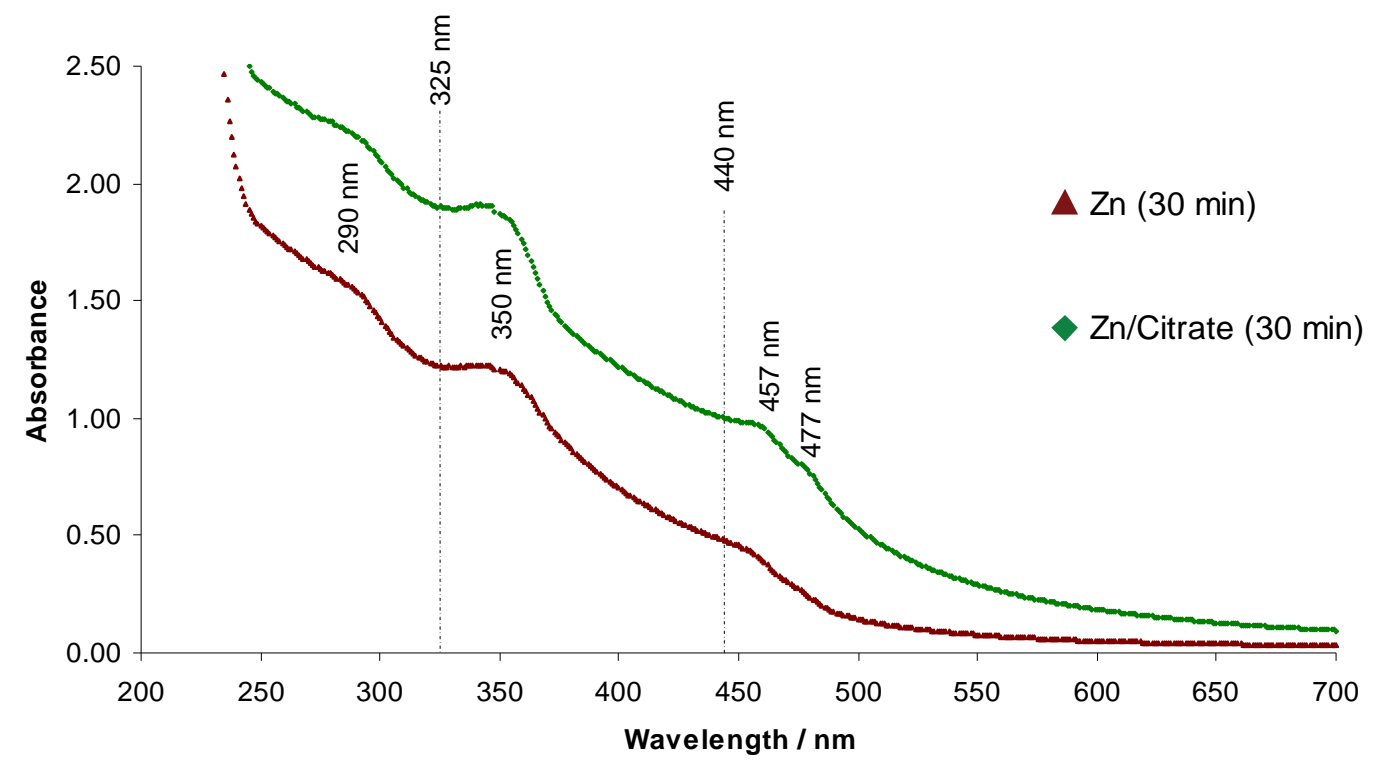

Figure 3.28: Absorbance scan of $\mathrm{Cu}_{2} \mathrm{O}$ samples doped with $\mathrm{Zn}^{2+}$, and $\mathrm{Zn}^{2+} /$ Citrate, stirred for 30 min. Details of sample preparation are listed in the appendix in table A.5. 
The increment in magnitude and shift of the absorbance peak (440 to $477 \mathrm{~nm}$ ) refer to the increased formation of larger particles. The particle growth may be supported by trisodium citrate (TSC) due to its reducing properties. It can be assumed that TSC progressively reduce copper ions in the reaction medium causing the increased formation of zinc doped $\mathrm{Cu}_{2} \mathrm{O}$ nanoparticles. The doping procedure carried out using TSC; however, has a negative effect on the optical properties of the nanocrystal suspensions with ageing. Here, the bright yellow suspensions of zinc doped $\mathrm{Cu}_{2} \mathrm{O}$ nanocrystals faded after $3-5$ days and turned transparent. The absorbance spectrum showed then only a strong absorbance peak at $350 \mathrm{~nm}$.

The same effect was also observed, when TSC or other ionic surfactants were added to $\mathrm{Cu}_{2} \mathrm{O}$ suspension after sample preparation. For example, the effect of $2 \mathrm{~mL} 0.001 \mathrm{M}$ $\mathrm{NaS}_{2} \mathrm{O}_{3}$ aqua solution (ionic stabilizer), added drop wise to a $\mathrm{Cu}_{2} \mathrm{O}$ nanocrystal suspension is shown in figure 3.29. The $\mathrm{Cu}_{2} \mathrm{O}$ nanocrystal suspension was prepared in the presence of zinc and nickel at $98{ }^{\circ} \mathrm{C}$ (see figure 3.25).

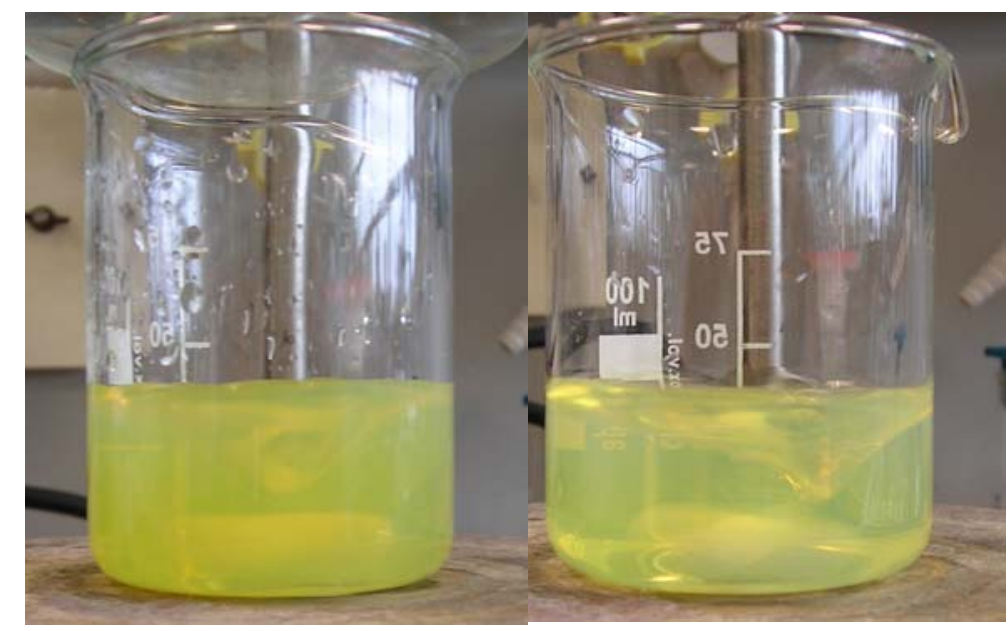

Figure 3.29: Change of the optical properties of $\mathrm{Cu}_{2} \mathrm{O}$ nanocrystal suspension doped with zinc before and after the addition of $2 \mathrm{~mL} 0.001 \mathrm{M} \mathrm{Na}_{2} \mathrm{SO}_{3}$.

With the addition of $\mathrm{NaS}_{2} \mathrm{O}_{3}$ the colour of the suspension turned gradually brighter yellow and also the transparency of the suspension increased. The addition of more $\mathrm{NaS}_{2} \mathrm{O}_{3}$ solution $\left(\mathrm{V}\left(0.001 \mathrm{M} \mathrm{NaS}_{2} \mathrm{O}_{3}\right)>2 \mathrm{~mL}\right)$ resulted in the fading of the suspension. These changes are shown by a gradually decrease of the absorbance peak between 440 and $477 \mathrm{~nm}$ in the absorbance spectrum. Transparent suspensions revealed a broad absorbance band at $350 \mathrm{~nm}$. The same effect was also observed by diluting the suspensions with $\mathrm{H}_{2} \mathrm{O}$. Thereby, Zetasizer detected a gradually decrease of the particle 
size in suspension depending on the dilution ratio (figure 3.19). Here, a dilution ratio of 1:5 only detected small particles in the size of $2 \mathrm{~nm}$ in solution.

\subsubsection{Photoluminescence spectroscopy}

Aqueous suspension of $\mathrm{Cu}_{2} \mathrm{O}$ nanocrystals and $\mathrm{Cu}_{2} \mathrm{O}$ nanocrystals doped with zinc and nickel show weak fluorescence. Figure 3.30 shows the PL scan of undoped $\mathrm{Cu}_{2} \mathrm{O}$ nanocrystals (sample $\mathrm{A}$ ) and the PL scans of $\mathrm{Cu}_{2} \mathrm{O}$ nanocrystals doped with zinc and nickel $(\mathrm{B}, \mathrm{C})$, when excited with $300 \mathrm{~nm}$. It is important to point out that the $\mathrm{Cu}_{2} \mathrm{O}$ aqueous suspension in $\mathrm{A}, \mathrm{B}$ and $\mathrm{C}$ contained particles with different size distributions. Sample A contained small $\mathrm{Cu}_{2} \mathrm{O}$ nanocrystals with an average size of $5 \mathrm{~nm}$ shown by a transparent suspension and a broad absorbance band around $360 \mathrm{~nm}$ (see figure 3.21; A). Sample $\mathrm{C}$ consisted of a mixture of small $\mathrm{Cu}_{2} \mathrm{O}$ nanocrystals and cube-shaped particles in solution. This is indicated by a broad absorbance band around $360 \mathrm{~nm}$ and a narrow absorbance peak between 440 and $477 \mathrm{~nm}$ (see figure 3.25; $98{ }^{\circ} \mathrm{C}$ ). In contrary, sample B contained only cube-shaped particles in different sizes shown by a broad absorbance band above $477 \mathrm{~nm}$ (see figure 3.25; $99^{\circ} \mathrm{C}$ ). Furthermore, sample B and C are different in their optical properties in reflected and transmitted light. Sample B shows a distinct two colour effect in reflected and transmitted light, whereas sample C shows the same colour in reflected and transmitted colour.

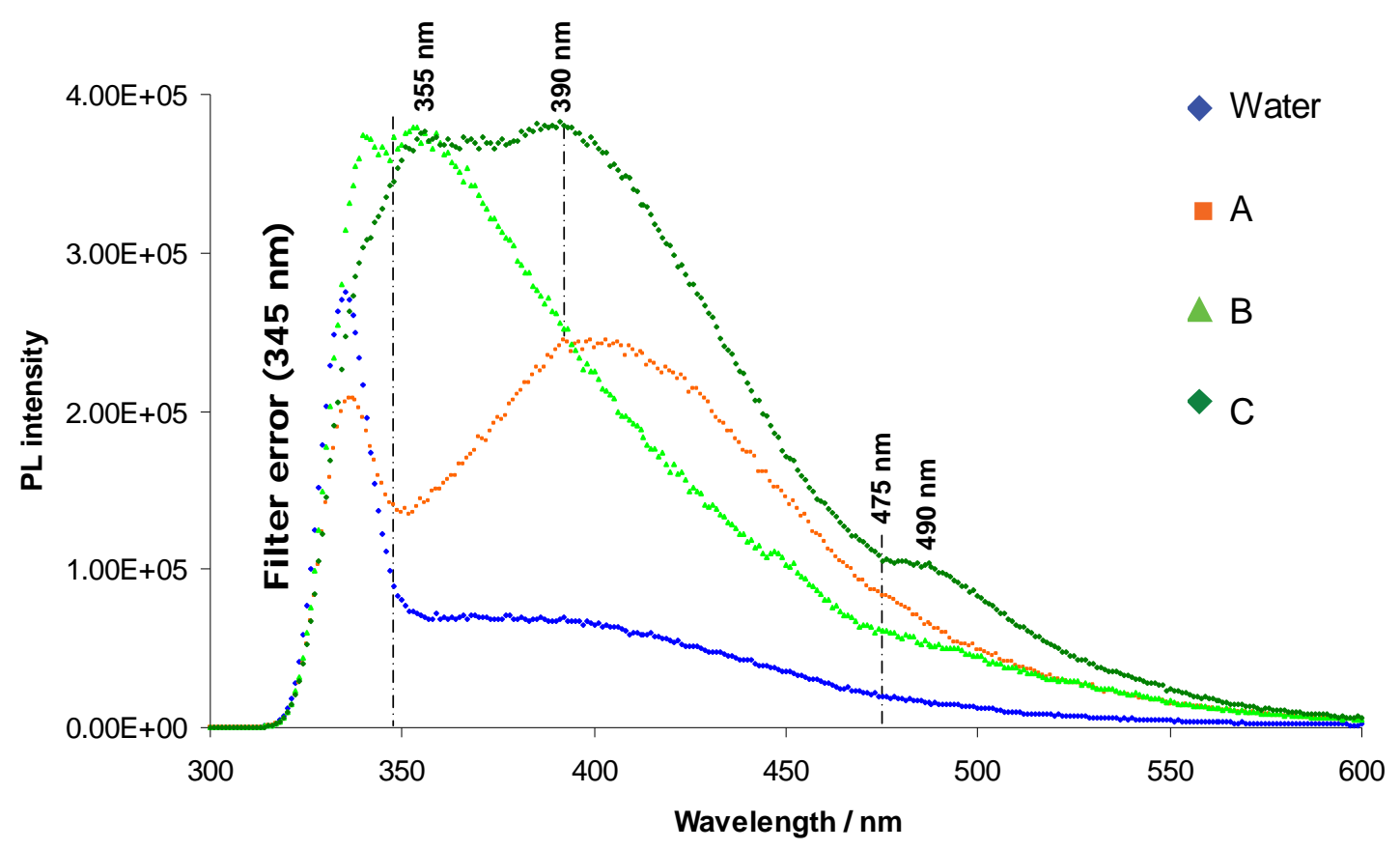

Figure 3.30: $\mathrm{PL}$ scans of $\mathrm{Cu}_{2} \mathrm{O}$ aqueous suspensions undoped (A) and doped with $\mathrm{Zn}^{2+}$ and $\mathrm{Ni}^{2+}(\mathrm{B}$ and $C$; $B$ shows the dichroic effect $)\left(\lambda_{\text {exc }}=300 \mathrm{~nm}\right.$; exc. $/ \mathrm{em}$. slit $\left.=10 \mathrm{~nm}\right)$. 
It is interesting that the different samples with their individual optical properties in reflected and transmitted light can be distinguished by their PL scan. The dichroic effect in reflected and transmitted light of zinc and nickel doped $\mathrm{Cu}_{2} \mathrm{O}$ nanocrystals (B) reveals only one emission peak around $355 \mathrm{~nm}$. Aqueous suspension of zinc and nickel doped $\mathrm{Cu}_{2} \mathrm{O}$ nanocrystals without showing a distinct two colour effect in transmitted and reflected light $(\mathrm{C})$ reveals several emission peaks at 355, 390 and $490 \mathrm{~nm}$. In contrast, aqueous suspension of undoped $\mathrm{Cu}_{2} \mathrm{O}$ nanocrystals (A) reveals a broad peak around $390-400 \mathrm{~nm}$.

The emission peak at $355 \mathrm{~nm}$ in sample B and C matches with the absorbance peak observed at $355-360 \mathrm{~nm}$ in the doped and undoped $\mathrm{Cu}_{2} \mathrm{O}$ samples. It is likely that this emission corresponds to the band-edge transition between the LUMO and HOMO level of the band gap of the $\mathrm{Cu}_{2} \mathrm{O}$ nanocrystals. However, when mono-dispersed in solution, the nanocrystals show a broad emission band at $390 \mathrm{~nm}$ and higher. This red-shifted and broad emission peak may refer to a trap emission caused by defects at the surface of the $\mathrm{Cu}_{2} \mathrm{O}$ nanocrystals. The literature suggests that the trap emission is enhanced by surface modification such as the loss or exchange of capping ligands [93, 94].

\subsection{Discussion}

The spontaneous organisation of the spherical nanocrystals into self-assembled structures is predominantly determined by the chemical interaction of the surfactants, adsorbed onto the surface of the nanocrystals, with the surrounding. Here, these specific interactions based on attractive and repulsive forces promote the arrangement of the spherical nanocrystals into regular cube-shaped particles.

The size and size-distribution of the polycrystalline nanocubes, however, is affected by different parameters such as the choice of copper precursor material, choice of polymeric stabilizer, concentration of the stabilizer, temperature, reaction time, solvent (dielectric constant) and $\mathrm{pH}$ of the wet chemistry method. In addition to it, the stability of the self-assembled cubes is also strongly affected by dilution and/or the addition of ionic surfactants after the preparation of the $\mathrm{Cu}_{2} \mathrm{O}$ suspension. 
Here, it is important to understand the mechanism of the particle growth of selfassembled structures, because the size and shape of the structures affect the wavelength of the absorbed and scattered light and hence colour. In general, the colour of the $\mathrm{Cu}_{2} \mathrm{O}$ suspension can be determined by the absorbance peak in the visible range. Depending on the absorption wavelength of the visible light, the suspension will show the complementary colour of the absorbed light. However, when the scattering effect of the particles dominates, then the suspension will show the colour of the scattered light which correspond to the wavelength of the absorbed light. The amount of light absorbed or scattered by nanoparticles is determined by the physical properties of the nanoparticles. While small nanoparticles are predominately absorptive, the absorption to scattering ratio decreases with the increment of the particle size. Therefore, larger particles are better light scatters than light absorbers. For this reason, the optical properties of the $\mathrm{Cu}_{2} \mathrm{O}$ suspensions are strongly dependent on the size and size distribution of the nanocubes in solution due to the size-dependence of light absorption and scattering effects.

\subsubsection{Mechanism of particle growth - Wet chemical reduction method}

The whole process occurs through a series of intermediate reaction which is indicated by a continuous change in colour of the solution from clear to coffee black, dark greenblue and finally yellow-green. These colour changes correspond to the different stages of the particle growth starting from the formation of nucleation centres and nanoclusters to the formation of spherical nanocrystals and finally the agglomeration of the spherical nanocrystals into cube-shaped particles. Furthermore, it can be expected that the cubeshaped particles also undergo a further particle growth and form agglomerates.

\subsubsection{Nucleation and Condensation}

Initially, the particle growth is based on the mechanism of nucleation and condensation of small nanoclusters formed by the reduction of copper ions to $\mathrm{Cu}_{2} \mathrm{O}$ with $\mathrm{NaBH}_{4}$ in aqueous solution carried out at $99^{\circ} \mathrm{C}$. 
The redox reaction is given by the following equations.

$\begin{array}{lllll}\text { Oxidation: } & & \mathrm{BH}_{4}^{-} & \mathrm{BH}_{3}+1 / 2 \mathrm{H}_{2}+\mathrm{e}^{-} \\ \text {Reduction: } & \mathrm{Cu}^{2+}+\mathrm{e}^{-} & \rightarrow & \mathrm{Cu}^{+} \\ \text {Redox reaction: } & \text { (a) } 2 \mathrm{Cu}^{2+}+2 \mathrm{BH}_{4} & \rightarrow & 2 \mathrm{Cu}^{+}+2 \mathrm{BH}_{3}+\mathrm{H}_{2} \\ & \text { (b) } \mathrm{Cu}^{+}+\mathrm{OH}^{-} & \rightarrow & \mathrm{Cu}(\mathrm{OH}) \\ & \text { (c) } 2 \mathrm{Cu}(\mathrm{OH}) & \rightarrow & \mathrm{Cu}_{2} \mathrm{O}+\mathrm{H}_{2} \mathrm{O}\end{array}$

$\mathrm{NaBH}_{4}$ is a strong alkali reducing agent and produces instantaneously a large number of nucleation centres (nuclei). This boost of $\mathrm{Cu}_{2} \mathrm{O}$ nucleation centres will result in a supersaturation of the solution. Supersaturation is the driving forces for crystal nucleation and growth. Here, the formed $\mathrm{Cu}_{2} \mathrm{O}$ nucleation centres condense due to the high surface energy and form small crystals. These small crystals are acting as seed particles and grow to larger nanoclusters by the deposition of more and more nucleation centres on the surface. Due to the fast nucleation rate, the concentration of the nucleation centres will drop quickly in the dispersion medium. This, however, will affect the supersaturation of the solution and hence stop the crystal growth of the nanoclusters. The nuclei remaining in the dispersion medium are relative unstable because of the large surface area-to-volume ratio. For these reason a large number of the nucleation centres will tend to dissociate before they can grow to larger sizes. The dissolved ions, however, can re-crystallise on larger crystallites, which are thermodynamically more stable.

\subsubsection{Coalescence}

The nanoclusters formed by the boost of nucleation centres have a strong tendency to form clumps ("agglomerates"). As mentioned in the introduction chapter (1.2.4), the reactivity of the particles in the dispersion medium is determined by the surface area. The higher the surface-to-volume ratio, the higher is the chemical reactivity of the particles to form aggregates / agglomerates. By decreasing their exposed surface area, the nanoclusters obtain a lower surface energy and hence higher chemical stability in solution. 
By shielding the surface area with PMAA-Na as a polymeric dispersant and trisodium citrate as ionic capping agent, the growth of the nanoclusters in solution is confined. The polymer chains confine the nanoclusters growth through steric hindrance, while the citrate ions stabilize the nanoclusters by electrostatic repulsion in the dispersion medium. Here, the capping agents coordinate as organic ligands to the surface atoms and thereby reduce the chemical reactivity of the surface area of the nanocrystals. However, the adsorption of these capping agents on the surface induces the transformation of the nanoclusters into spherical nanocrystals with a superlattice structure (see TEM images, figure 3.14). The superlattice is formed by the periodic separation of $\mathrm{Cu}_{2} \mathrm{O}$ and template layers according to a self-assembly mechanism. This speparation restrict the growth of the $\mathrm{Cu}_{2} \mathrm{O}$ layer between the template layers which consist most like of PMAA and citrate.

In general, the spontaneous organisation of self-assembled structures is determined by the mechanism of the particle growth. As mentioned before, the formation of a lamellar structure promotes the growth of crystalline $\mathrm{Cu}_{2} \mathrm{O}$ layer between the arranged template layers of the surfactant. Here, the growth of the $\mathrm{Cu}_{2} \mathrm{O}$ layer is enhanced by the reductive properties of citrate ions, which progressively reduce copper ions at the surface of nanoclusters. This causes the coalescence of the nanoclusters between the template layers of the polymeric dispersant resulting in the progressively growth of crystalline $\mathrm{Cu}_{2} \mathrm{O}$ layers in the spherical nanocrystals. Thereby, the oxidation / decarboxylation products of citrate remain chemisorbed on the surface and affect further the particle growth. For a better visualisation, the different stages of the particle growth are illustrated in figure 3.31, starting with the formation of nucleation centres to spherical nanocrystals.

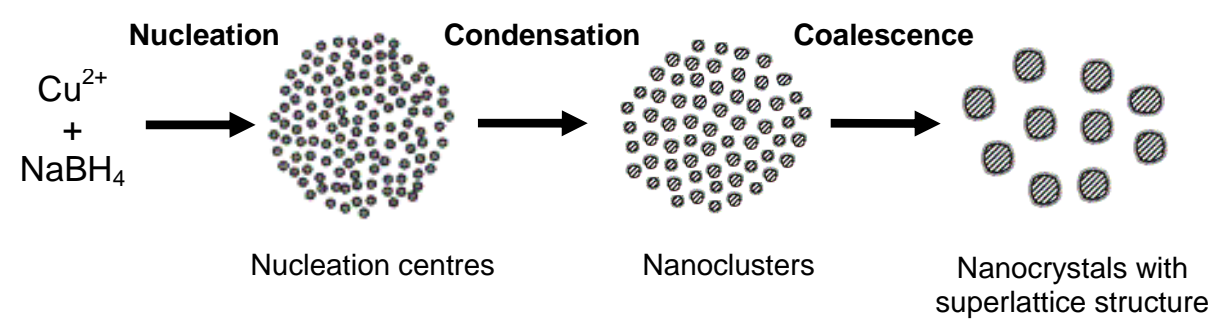

Figure 3.31: Illustration of particle growth of spherical $\mathrm{Cu}_{2} \mathrm{O}$ nanocrystals with a superlattice structure. 
Optimal reaction conditions will promote the formation of spherical nanocrystals with a superlattice structures. However, the progressive reduction of $\mathrm{Cu}^{2+}$ ions at the surface of the nanoclusters will reduce the concentration of citrate ions. With the progressive loss of citrate ions, the electrostatic repulsion forces between the spherical nanocrystals decreases. This means that the repelling layer around the crystals is not strong enough to repulse approaching particles and hence show a higher tendency to form agglomerates. As seen from the TEM images in figure 3.12 the spherical nanocrystals agglomerate into cube-shaped particles. This transformation, however, is strongly dependent on the reaction conditions applied as well as on the precursor materials used.

\subsubsection{Self-assembled agglomeration}

When the reaction was stirred over $20 \mathrm{~min}$ at a constant reaction temperature of $99{ }^{\circ} \mathrm{C}$, the sample preparation resulted in the agglomeration of the small spherical $\mathrm{Cu}_{2} \mathrm{O}$ nanocrystals into larger polycrystalline nanocubes varying in sizes of $70-250 \mathrm{~nm}$ (figure 3.32).

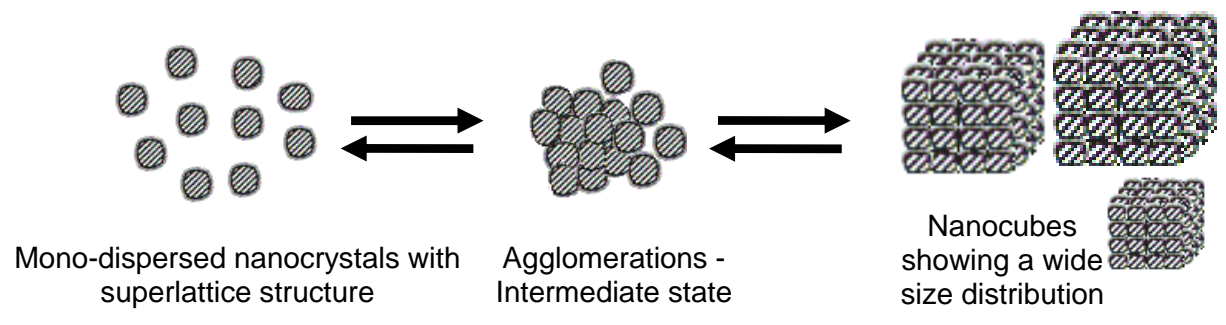

Figure 3.32: Illustration of the $\mathrm{Cu}_{2} \mathrm{O}$ particle growth starting from mono-dispersed nanocrystals with a superlattice structure followed by an agglomeration processes to the transformation in regular cube-shaped particles.

The agglomeration of the spherical particles follows a self-assembled stabilization mechanism. By forming self-assembled structures, the spherical nanocrystals obtain a higher stability in the dispersion medium. As seen in the TEM and SEM images, the cubic structure corresponds to the most stable configuration, which is the driving force of the spherical nanocrystals to arrange themselves into regular shaped cubes. The cubeshape of the self-assembled structures may be associated with the crystal lattice structure of $\mathrm{Cu}_{2} \mathrm{O}$. XRD and XPS measurments (figure 3.10 and 3.11) revealed that the $\mathrm{Cu}_{2} \mathrm{O}$ lattice structure is cubic. Hence, the association of the spherical nanocrystals into regular shaped cubes may follow the crystal lattice structure of $\mathrm{Cu}_{2} \mathrm{O}$. The association of the self-assembled structures, however, is based on hydrogen bonds or weak van der 
Waals interactions, since subsequent dilution of the suspension with water leads to the collapse of the self-assembled structures (see Zetasizer measurements in figure 3.18 and 3.19).

The agglomeration of the spherical nanocrystals into larger polycrystalline cubes is indicated by a colour change of the suspension from dark green-blue to yellow-green. This colour change is generally induced by the drop of the reaction temperature. However, the agglomeration may also be promoted by the high dielectric constant of water. In general, solvents with a high dielectric constant interrupt the growth of crystallites by forming aggregates / agglomerates [95].

In general, the alignment of the mono-dispersed nanocrystals in small agglomerates or cube-shaped particles, as illustrated in figure 3.32, is dependent on the repulsive forces between the particles. Usually, small spherical nanocrystals remain mono-dispersed (isolated) in suspension, when their specific surface area is covered by a strong repelling layer of capping agents. Depending on the capping agent, ionic or steric, the repelling layer stabilizes the particles in the dispersion medium by electrostatic or steric repulsion.

In the electrostatic stabilization, the repulsive forces are dependent on the uptake of the electrolyte (citrate ions) on the surface of the particles. The higher the uptake, the greater is the electrostatic repelling effect between the charged layers of the particles in the dispersion medium.

In the steric stabilization, the repulsive forces are determined by the length of the bulky organic ligands (PMAA chains) adsorbed onto the particle surface. To achieve optimal steric stabilization, the chains need to be long enough to overcome attractive van der Waals forces between the particles and they need to be compatible with the solvent during the reaction. Here, an appropriate solvation will stretch individual polymer chains in an aligned position and in that way increases the length of the polymer chains from the surface of the particles into the dispersion medium. In general, the longer the length of the chains into the dispersion medium, the larger is the repelling layer around the crystals and the better is the steric stabilization. However, when the solvent compatibility is negatively affected during the reaction, the polymer chains can collapse 
on the particle surface allowing the particles to agglomerates. In general, the solvent compatibility of the polymer chains depends on the dielectric constant of the solvent and temperature applied.

The stability of the particles in the dispersion medium, however, is also strongly dependent on the chemical bonding of the capping agents to the atoms at the surface of the particles. These bonds can range from covalent to ionic and hydrogen bonds. Covalent bonds between the surface atoms and capping agents are preferred, since ionic and hydrogen bonds can undergo ligand exchange reactions with solvent molecules. Ligand exchange reaction will decrease the surface coverage of the nanocrystals and hence increase the chemical reactivity of the particles.

As mentioned before, the agglomeration of the spherical nanocrystals into larger polycrystalline cubes is triggered by the drop of temperature. Here, it is possible that the drop of temperature reduces the chemical interaction between solvent molecules and polymer chains inside the dispersion medium resulting in the entanglement of the chains on the particle surface. This reduces the repulsive forces of the repelling layer allowing the chemical interaction between the particles. By getting close together, the repelling layer around the crystals can react with each and induce a gel-formation. This, however, is only possible when the polymer chains are very long and the affinity between the polymer chains is large enough to overcome the repulsive forces caused by the steric stabilization. In general, the gel-formation is the basis for the formation of a gel-like host matrix which encloses the nanocrystals in the self-assembled structures as seen in the TEM images (figure 3.13).

The size of the self-assembled structure is determined by the gel-formation, which involves cross-link reactions between the particles and selective adsorption and desorption processes of the polymer on the surface of particles. The gel-formation, in turn, is dependent on the chemical interaction between the particles. Strong chemical interaction between the particles will result in the formation of large self-assembled structures; while weak interactions will lead to the formation of agglomerates and/or small self-assembled structures. 
Here, the surface coverage of the nanocrystals will determine the extent of the interaction between the particles and the formation of smaller or larger self-assembled structures. The surface coverage of the particles is composed of PMAA polymer chains and citrate ions. However, it can also include the oxidation / decarboxylation products of citrate and the decomposition products of sodium borohydride. The mixture of the different compounds adsorbed onto the particle surface may certainly have a strong influence on the repulsive forces between the particles.

Strong repulsive forces between the repelling layers around the particles will hinder agglomeration and cross-link reaction (forming bridges) between the particles. These repulsive forces, however, can be reduced by the loss of surfactants at the particle surface through ligand exchange reaction or selective desorption processes. These processes reduce the surface coverage of the particles, which enhance the chemical interaction between the particles. Consequently, particles with a weak repelling layer will form larger self-assembled structures.

\subsubsection{Effect of process parameters on particle growth}

It was demonstrated that the growth of the spherical nanocrystals and cube-shaped particles can be controlled by changing different process parameters such as concentration of reducing agent $\left(\mathrm{NaBH}_{4}\right), \mathrm{pH}$, solvent, reaction temperature, reaction time, choice of the copper precursor material $\left(\mathrm{Cu}\left(\mathrm{NO}_{3}\right)_{2}, \mathrm{CuCl}_{2}\right)$, choice of the polymeric dispersant (PVP, PMAA-Na), ratio of copper concentration and capping agent concentration, presence of doping materials and so on.

All these parameters influence the stability of the spherical nanocrystals in dispersion medium. In general, particles lose their stability in dispersion medium, when their repelling layer is not strong enough to counterbalance attractive van der Waals forces between the particles. Here, the thickness of the repelling layer around the particles determines the stability of the nanocrystals in the dispersion medium. A thin (weak) repelling layer promotes the interaction of the particles due to the attractive van der Waals forces, which will lead to the formation of larger structures. On the contrary, a thick repelling layer exhibits stronger repulsion effects, which will prevent particles from coming close to each other. 


\subsubsection{1 $\quad \mathrm{NaBH}_{4}$ concentration}

In this wet chemical reduction method, small nucleation centres are initially formed by the reduction of $\mathrm{Cu}^{2+}$ with $\mathrm{NaBH}_{4}$. These nucleation centres condense and form nanoclusters, which in turn transform into spherical nanocrystals (see figure 3.31). The amount of nanoclusters formed in solution depends on the saturation point of the solution with $\mathrm{Cu}_{2} \mathrm{O}$ nucleation centres, which in turn depends on the concentration of the reducing agent, concentration of the metal ion, surfactants, temperature, dilution and pressure of the solution. Here, the saturation point is easily controlled by the concentration of the reducing agent $\mathrm{NaBH}_{4}$ added to the reaction medium.

Low concentration of $\mathrm{NaBH}_{4}$ result in small boost of nucleation centres in solution, whereas higher $\mathrm{NaBH}_{4}$ concentrations lead to a supersaturation of the solution. A supersaturation promotes the formation of a larger number of crystallites in solution. However, a larger number of crystallites reduce the amount of surfactants available for the stabilization. Without a strong repelling layer, particles lose their stability in solution (water) and subsequently agglomerate to larger structures following the selfassembled mechanism described above.

For this reason, the addition of low $\mathrm{NaBH}_{4}$ concentration $\left(\left[\mathrm{NaBH}_{4}\right]=0.002-0.004 \mathrm{M}\right)$ results in the formation of spherical nanocrystals with an average size of $5 \mathrm{~nm}$, which are homogeneously dispersed in solution (figure 3.20; A). Here, the concentrations of the surfactants are high enough to form a strong repelling layer around the nanocrystals. With higher $\mathrm{NaBH}_{4}$ concentration (e.g. $\left[\mathrm{NaBH}_{4}\right]=0.005-0.001 \mathrm{M}$ ), more nucleation centres are produced which favour the growth of a larger number of nanoclusters via condensation. This in turn promotes the formation of a greater number of spherical nanocrystals. A larger amount of nanocrystals in dispersion medium, however, show a higher tendency to transform in a self-assembled mechanism to cube-shaped particles in different sizes (see TEM image in figure 3.12 and Zetasizer measurement in figure $3.20)$.

An excess of the reducing agent $\left(\left[\mathrm{NaBH}_{4}\right]>0.001 \mathrm{M}\right)$ results in a total precipitation of the particles in solution. An excess of the reducing agent may destroy the micelle structures in the early stages of the particle growth. This, however, will result in the 
formation of larger particles, which have a higher affinity to undergo oxidation reaction, since the atomic electron configuration of $\mathrm{Cu}_{2} \mathrm{O}$ is only stable at nano-scale. The oxidation to $\mathrm{CuO}$ is usually shown by a black colour of the precipitate.

With the addition of higher $\mathrm{NaBH}_{4}$ concentration, side reactions can also occur during the redox reaction. For instance, the cuprous ions can be further reduced and form copper metal atoms (equation (15)), which then oxidise to $\mathrm{CuO}$ (equation (16)), or undergo a disproportionation reaction as shown in equation (17).

$$
\begin{array}{llll}
\text { By-products } & \mathrm{Cu}^{+}+\mathrm{e}^{-} & \rightarrow & \mathrm{Cu}^{0} \\
\mathrm{Cu}^{0}+1 / 2 \mathrm{O}_{2} & \rightarrow & \mathrm{CuO} \\
2 \mathrm{Cu}^{+} & \rightarrow & \mathrm{Cu}^{0}+\mathrm{Cu}^{2+}
\end{array}
$$

\subsubsection{Trisodium citrate concentration}

The use of TSC in this wet chemical reduction method is essential for the formation of size-controlled $\mathrm{Cu}_{2} \mathrm{O}$ nanocubes. As illustrated in figure 3.31, the formation of spherical $\mathrm{Cu}_{2} \mathrm{O}$ nanocrystals with a superlattice structure undergoes different stages of particle growth and each stage is highly affected by the citrate concentration used. Initially, citrate ions form a chelate composition with $\mathrm{Cu}^{2+}$ ions in aqueous solution before the chemical transformation occurs. Then, the growth of the nanoclusters, via nucleation and condensation after the addition of $\mathrm{NaBH}_{4}$, is controlled by the adsorption of citrate ions and polymeric dispersant on the surface of the nanoclusters.

Low concentration or the absence of trisodium citrate in the reaction medium (e.g. $\left.\left[\mathrm{Na}_{3} \mathrm{C}_{6} \mathrm{H}_{5} \mathrm{O}_{7}\right]<0.24 \cdot 10^{-3} \mathrm{M}\right)$ will cause an uncontrolled formation of agglomerates / aggregates of the $\mathrm{Cu}_{2} \mathrm{O}$ nanoclusters formed by the boost of nucleation centres with the addition of $\mathrm{NaBH}_{4}$ to the reaction medium. Here, the concentration of citrate ([Citrate]: $\left[\mathrm{Cu}^{2+}\right]<0.5$ ) is not high enough to form a strong repelling layer around the nanoclusters and the nanoclusters will agglomerate / aggregate to larger structures as illustrated in figure 3.2; A. This kind of particle growth, however, can reach sizes exceeding the nano-level, which finally precipitate from solution. The product is a green precipitate with black spots. The formation of larger particles can lead to the oxidation 
of $\mathrm{Cu}_{2} \mathrm{O}$ to $\mathrm{CuO}$, shown by the black spots, since the atomic bonding structure of $\mathrm{Cu}_{2} \mathrm{O}$ is only stable at nanoscale size.

Higher concentrations of citrate (e.g. $\left.\left[\mathrm{Na}_{3} \mathrm{C}_{6} \mathrm{H}_{5} \mathrm{O}_{7}\right]>0.24 \cdot 10^{-3} \mathrm{M}\right)$, together with the polymeric dispersant, stabilize the nanoclusters better after nucleation and condensation reactions. Thereby, the polymeric dispersant can induce the next stage of the particle growth. Here, the adsorption of polymer macromolecules induces the arrangement of the nanoclusters in a lamellar structure. This arrangement induces the coalescence of the nanoclusters between template layers of the polymer and hence the growth of crystalline $\mathrm{Cu}_{2} \mathrm{O}$ layers.

The coalescence of the nanoclusters and growth of the crystalline $\mathrm{Cu}_{2} \mathrm{O}$ layers is caused by the reduction of $\mathrm{Cu}^{2+}$ ions at the surface of the nanoclusters with citrate ions (see mechanism of surface reduction in figure 3.2; B). This process is most likely to be kinetically controlled and strongly dependent on the process parameters such as concentration of citrate, reaction time and temperature.

Here, the concentration of citrate used by the synthesis of $\mathrm{Cu}_{2} \mathrm{O}$ quantum dots has a big influence whether the spherical nanocrystals remain mono-dispersed in suspension or transform into polycrystalline cubes. The formation of agglomerates and cube-shaped particles can be principally avoided by adding higher citrate concentration $\left(\left[\mathrm{Na}_{3} \mathrm{C}_{6} \mathrm{H}_{5} \mathrm{O}_{7}\right]>0.39 \cdot 10^{-3} \mathrm{M}\right)$ to the reaction medium. A higher citrate ion - to - copper ion ratio ([Citrate]: $\left[\mathrm{Cu}^{2+}\right]>0.75$ ) favours the formation mono-dispersed nanocrystals in the dispersion medium. Here, the nanocrystals / nanoclusters are stabilized by a strong ionic repelling layer of citrate around the particle surface. The negative charge on the surface prevents the particles from coming into close contact and protects them from aggregating. These suspensions are transparent with a light bluish colour.

However, $\mathrm{Na}_{3} \mathrm{C}_{6} \mathrm{H}_{5} \mathrm{O}_{7}\left(\mathrm{H}_{2} \mathrm{O}\right)$ concentration between $0.24 \cdot 10^{-3}$ and $0.39 \cdot 10^{-3} \mathrm{M}$ will favour the organisation of the spherical nanocrystals into polycrystalline nanocubes in the size of $70-250 \mathrm{~nm}$. Here, the size and size-distribution of the cubes is strongly dependent on the reaction time and temperature. For example, a complete transformation of the spherical nanocrystals into polycrystalline nanocubes of $70-250$ 
$\mathrm{nm}$ was not obtained when the reaction temperature was not constant $99{ }^{\circ} \mathrm{C}$ during 20 min stirring (see SEM image A in figure 3.15).

\subsubsection{Reaction time}

Depending on the reaction time, before or after $20 \mathrm{~min}$ stirring, two different dispersions are obtained. When the colour change was induced before 20 min stirring, the suspension contains a mixture of mono-dispersed nanocrystals, small agglomerates and small cube-shaped particles. This suspension has a yellow-green colour and shows a higher transparency in reflected light. On the contrary, when the colour change was induced after $20 \mathrm{~min}$, the dispersion only contains cube-shaped particles (see TEM image in figure 3.12). This suspension is darker yellow-green and shows a lower transparency in reflected light.

As mentioned before, the size and size-distribution of the nanocubes is determined by the repelling layer around the nanocrystal. The repelling layer in turn is dependent on the particle growth of the spherical nanocrystals in dispersion medium. Here, short stirring times (below $20 \mathrm{~min}$ ) will result in the formation of fewer spherical nanocrystals in the dispersion medium. Short stirring times also involves a lower consumption of citrate ions and therefore, the concentration of citrate and polymer molecules in the dispersion medium is high enough to form a strong repelling layer around individual nanocrystals. A strong repelling layer, however, will hinder the interaction between the nanocrystals and so the formation of larger self-assembled structures. Hence, $\mathrm{Cu}_{2} \mathrm{O}$ aqueous suspension prepared under 20 min stirring contain a mixture of mono-dispersed nanocrystals, small agglomerates and small cube-shaped particles.

On the contrary, a subsequent reduction of $\mathrm{Cu}^{2+}$ at the surface of the nanoclusters with citrate over a longer period ( $\geq 20 \mathrm{~min}$ ), enhance the growth of crystalline $\mathrm{Cu}_{2} \mathrm{O}$ layers and hence the growth of the nanocrystals in dispersion medium. This, however, reduce the amount of citrate ions adsorbed on the surface of the nanocrystals, which in turn weakens the electrostatic stabilization of the crystals in dispersion medium. Hence, nanocrystals agglomerate and form larger polycrystalline particles by following a selfassembled mechanism. 


\subsubsection{Reaction temperature}

The self-assembled stabilization mechanism is initiated by the drop of temperature which induces intermolecular interaction between the polymer chains of adjacent nanocrystals. These chemical interactions, however, also interrupt the particle growth of the spherical nanocrystals during the reaction time.

To obtain regular cube-shaped particles, it is crucial to maintain the reaction temperature constant at $99{ }^{\circ} \mathrm{C}$ during 20 min stirring. Lower temperature or the drop of the temperature will result in a mixture of mono-dispersed nanocrystals, small agglomerates and cube-shaped particles (figure 3.15; A). It is also important to note that the overheating of the hot-plate also affects a successively organisation of spherical nanocrystals in cube-shaped particles.

The change in the temperature can affect the spontaneous organisation of the lamellar structure of the spherical nanocrystals. This, however, will affect the coalescence of the nanoclusters between the template layers and hence stop the $\mathrm{Cu}_{2} \mathrm{O}$ layer growth of the spherical nanocrystals. Depending on the repelling layer around the nanocrystals, the particles can either interact with each other and form larger self-assembled structure or remain mono-dispersed in the dispersion.

\subsubsection{Effect of $\mathrm{pH}$}

The $\mathrm{pH}$ value of the reaction medium plays an important role for the formation of cuprous oxide in an aqueous solution. Here, the formation of $\mathrm{Cu}_{2} \mathrm{O}$ takes place under alkaline reaction condition, since the addition of $\mathrm{NaBH}_{4}$ as an alkali reducing agent increases the neutral $\mathrm{pH}$ value of the Polymer/Citrate/Cu aqueous complex solution. The cuprous ion $\left(\mathrm{Cu}^{+}\right)$formed by the reduction of $\mathrm{Cu}^{2+}$ with $\mathrm{BH}_{4}^{-}$combines with hydroxyl groups $\left(\mathrm{OH}^{-}\right)$in aqueous solution (equation (13)) before forming $\mathrm{Cu}_{2} \mathrm{O}$ under the elimination of $\mathrm{H}_{2} \mathrm{O}$ (equation (14)).

It was discovered that the addition of $\mathrm{NaOH}(\mathrm{V}(0.01 \mathrm{M} \mathrm{NaOH})=0.1-1.0 \mathrm{~mL})$ to the reaction medium, directly after the addition of $\mathrm{NaBH}_{4}$, promotes the agglomeration of the spherical nanocrystals into larger cube-shaped particles. Furthermore, it was noted that the addition of $\mathrm{NaOH}$ in large quantities $(\mathrm{V}(0.01 \mathrm{M} \mathrm{NaOH})>1.0 \mathrm{~mL})$ resulted in a 
complete precipitation of the $\mathrm{Cu}_{2} \mathrm{O}$ nanoparticles in solution. As mentioned before, the formation of spherical nanocrystals is dependent on several process parameters. Here, the addition of $\mathrm{NaOH}$ to the reaction medium may enhance the formation of $\mathrm{Cu}_{2} \mathrm{O}$ according to equation (13). The addition of $\mathrm{NaOH}$, however, will also result in the functionalisation of the surface area of the nanoclusters with hydroxyl groups. These $\mathrm{OH}^{-}$groups, however, may have a strong influence on the chemical reactivity of the nanoclusters. The nucleophilic properties of the $\mathrm{OH}^{-}$ligands attached at the surface of the nanoclusters may promote the coalescence of the nanoclusters between the template layers of the spherical nanocrystals. Consequently, the increase of the $\mathrm{pH}$ will enhance the particle growth of the spherical nanocrystals in the dispersion medium.

An increased formation of spherical nanocrystals, however, will result in a lack of citrate ions used for the coalescence of nanoclusters. Hence, nanocrystals formed in the dispersion medium have only a weak repelling layer, which enhances the chemical interaction between the particles and so the formation of larger self-assembled structures.

Furthermore, a $\mathrm{pH}$ increment in the reaction medium may promote the agglomeration of the cube-shaped particles. Cube-shaped particles may interact with each by forming hydrogen bridge bondings, which will result in the formation of larger structures. These structures will precipitate out of the solution after reaching a certain size. The formation of hydrogen bonds may be caused by $\mathrm{OH}^{-}$ligands attached at the surface of the cubeshaped particles.

\subsubsection{The choice of the $\mathrm{Cu}$ precursor material}

The choice of the copper precursor material $\left(\mathrm{CuCl}_{2}, \mathrm{Cu}\left(\mathrm{NO}_{3}\right)_{2}\right)$ has also an effect on the size distribution of the cube-shaped particles and optical properties of the $\mathrm{Cu}_{2} \mathrm{O}$ suspension. The use of $\mathrm{CuCl}_{2}$ results in a bright yellow transparent suspension and SEM images (figure 3.15; B) and Zetasizer measurement (figure 3.20; $\mathrm{E}^{*}$ ) revealed cube sizes with a maximum size of $150 \mathrm{~nm}$. Here, the counter ions $\mathrm{Cl}^{-}$affect the particle growth of the spherical nanocrystals in the dispersion medium. In general, the presence of charged counter-ions in the liquid solution will affect the ionic strength of the dispersion medium. The ionic strength of the dispersion medium, however, determines the 
thickness of the electrolyte layer around the particles. In general, the higher the ionic strength of the medium the lower the uptake of the electrolyte on the surface of the particles is $[96,97]$.

Because of the presence of $\mathrm{Cl}^{-}$causing the increment of the ionic strength of the dispersion medium, the uptake of citrate ions on the surface area of the $\mathrm{Cu}_{2} \mathrm{O}$ nanoclusters is reduced. The growth of spherical nanocrystals with a superlattice structure, however, relies on a strong citrate layer around the nanoclusters. Here, the citrate layer reduces $\mathrm{Cu}^{2+}$ ions at the surface of the nanoclusters and by doing so promotes the coalescence of the nanoclusters between the template layers.

With a lower uptake of citrate, the particles growth will be restricted to a lower number of nanocrystals, which however are stabilized by a higher concentration of the polymer in dispersion medium. The steric stabilization of the particles with the polymer is strong enough to counterbalance attractive van der Waals forces between the particles. Hence, the final dispersion consists of mono-dispersed nanocrystals and a small number of small self-assembled structures (figure $3.15 ; \mathrm{B}$ ).

\subsubsection{The choice of the polymeric dispersant}

The choice of the polymeric dispersant has also a strong influence on the size distribution of the cubes. For example, the coating of the surface area with PVP results in the formation of larger self-assembled structures, while the use of PMAA-Na leads to smaller size distribution of the cubes (figure 3.15; C). The different size-distribution of the $\mathrm{Cu}_{2} \mathrm{O}$ nanocubes is also noticeable by the colour and turbidity of the $\mathrm{Cu}_{2} \mathrm{O}$ suspension. $\mathrm{Cu}_{2} \mathrm{O}$ suspension prepared with PVP are green turbid, while suspension prepared with PMAA-NA show a yellow green colour.

As mentioned before, the stability of the particles in the dispersion medium is determined by the repulsive forces of the repelling layer around the particles. The stability of the repelling layer, in turn, is affected by several factors such as ionic strength of the dispersion medium, solvent compatibility, $\mathrm{pH}$, temperature etc. However, the chemical bonding between surface atoms and anchor groups of the surfactants is the most important factor in the stability of the repelling layer. A weak 
chemical interaction can result in the loss of surfactants at the particle surface. This will consequently decrease the surface coverage of the particles and make it more reactive.

It is likely, that the chemical interaction between the anchoring groups of the PMAA chains and particle's surface is stronger than those with the PVP. In general, the chemical bonds between polymer chains and the surface area depend on the surface properties of the nanocrystals. The surface area of the $\mathrm{Cu}_{2} \mathrm{O}$ nanocrystals is positively charged due to the presence of $\mathrm{Cu}^{2+}$ ions at the particle surface. Based on the positive surface charge, the carboxylic groups at the side groups of the PMAA main chain can undergo an electrostatic interaction with the surface area and form in that way a strong repelling layer around the nanocrystals. On the contrary, PVP may undergo a different binding mode such a hydrogen bonding with the surface area of the particles.

Furthermore, the negative charge of the $\mathrm{COO}^{-}$groups at the side groups of the main chain of PMAA will form an electrically charged layer around the particles surface. The steric hindrance of the polymer chains and the electrically charged layer around the particles will enhance the repulsive forces between the repelling layers surrounding each particle. This, in turn, enhances the stability of the particles in the dispersion medium and will complicate the interaction between the particles. Hence, particles coated with PMAA chains will form smaller self-assembled structures.

However, it is important to mention that the presence of PMAA-Na will increase the ionic strength of the dispersion medium and, as mentioned before, an increment in the ionic strength may result in a lower uptake of citrate ions at the surface area of the nanoclusters. Furthermore, it is likely that the citrate ions and the carboxylic groups of the PMAA chains are competing for the chemical interaction with the surface atoms of the nanocrystals. This, however, will affect the growth of the spherical nanocrystals in the reaction medium, since the crystalline growth of the $\mathrm{Cu}_{2} \mathrm{O}$ layers in the lamellar structure of the spherical nanocrystal depends on a strong uptake of citrate ions around the nanoclusters. Therefore, it can be expected that the use of PMAA-Na will suppress the particle growth of the spherical nanocrystals in the dispersion medium, which will result in the formation of fewer particles, which in turn transform in smaller selfassembled structures. 


\subsubsection{Optical properties}

$\mathrm{Cu}_{2} \mathrm{O}$ suspensions containing nanocrystals and nanocubes of different size and size distribution show different optical properties. For example, suspensions containing mono-dispersed quantum dots with an average size of $5 \mathrm{~nm}$ are transparent and show a slightly bluish colour. However, when the quantum dots agglomerate in solution and form polycrystalline cubes the colour of the suspension changes to yellow. With an increment in size of the cubes, the colour of the suspension turns greener and become less transparent in reflected light. When the suspension contains mainly nanocubes of $190-220 \mathrm{~nm}$, a dichroic effect occurs depending on the angle of the incident light. Here, the suspension looks yellow-green in reflected light and red in transmitted light (figure 3.22). The dichroic effect occurs due to the change in the absorption to scattering ratio of the particles. Larger particles have a stronger tendency to scatter light, while smaller particles show stronger absorption characteristics. Hence, suspension containing predominantly larger polycrystalline nanocubes will show stronger light scattering properties.

The optical properties of the $\mathrm{Cu}_{2} \mathrm{O}$ suspensions can be determined by UV-vis spectroscopy, which show characteristic absorbance peaks or bands in the UV- and visible range of light depending on the size and size distribution of the nanocrystals and nanocubes in suspension. The absorbance peaks or bands display the wavelength range over which light is absorbed and scattered by the nanostructure. Depending on the wavelength of the absorbed light, the suspension will show the complementary colour (see table 3.5). For example, transparent $\mathrm{Cu}_{2} \mathrm{O}$ suspensions containing only small monodispersed spherical nanocrystals show a broad absorbance peak around $350 \mathrm{~nm}$ (figure 3.21; A). $\mathrm{Cu}_{2} \mathrm{O}$ suspension containing cubes in the size of $70-150 \mathrm{~nm}$ and showing a sharp absorbance peak between 440 and $477 \mathrm{~nm}$ (figure 3.21; B and $\mathrm{E}^{*}$ ) are a bright yellow due to the absorbance of blue light. $\mathrm{Cu}_{2} \mathrm{O}$ suspension containing cube-shaped particles of 190 - $250 \mathrm{~nm}$ absorb light in the wavelength range between 490 and $505 \mathrm{~nm}$ (figure 3.21; E), which correspond to the absorption of green light (see table 3.4). The complementary colour of green is red-purple. These larger nanocubes, however, show also enhanced scattering properties. Due to the light absorption and scattering properties of the cubes $(190-250 \mathrm{~nm})$, the suspension look green in reflected light and red-purple in transmitted light. Furthermore, due to the stronger light scattering properties of the 
polycrystalline cubes $(190-250 \mathrm{~nm})$, the suspension is non-transparent in reflected light, whereas in transmitted light it is transparent.

Figure 3.33 illustrates the light absorption and scattering effect of the polycrystalline cubes varying in size between 190 and $250 \mathrm{~nm}$.

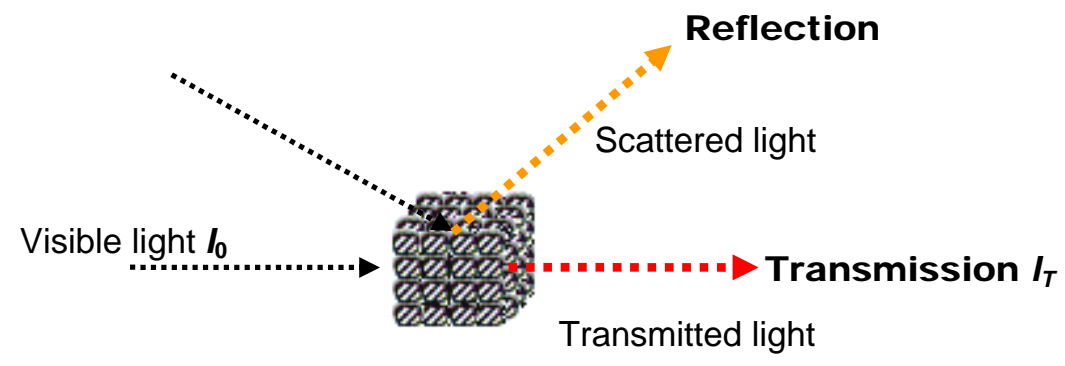

Figure 3.33: Absorption and scattering of incident light at the surface of cube-shaped $\mathrm{Cu}_{2} \mathrm{O}$ nanocrystals.

When the suspension is observed in transmitted light, the complementary colour of the light absorbed by the cubes is observed. Under reflected light; however, it is the colour of the light scattered by the cubes what is observed.

In general, the light absorption and scattering properties of the $\mathrm{Cu}_{2} \mathrm{O}$ suspension is dependent on the size and size-distribution of the nanocubes. Here, the size of the nanocubes determines the wavelength of the absorbed light and the amount of light scattered onto the surface of the particles. The arrangement of the spherical nanocrystals in larger polycrystalline nanocubes results in the absorbance of light with higher wavelengths. Therefore, an increment in size of the cube-shaped particle is shown by a shift and broadening of the absorbance peak to higher wavelength (figure 3.21; C and D). Furthermore, the light scattering behaviour increases with the formation of larger self-assembled structures. This is indicated by a lower transparency of the suspension in reflected light.

By controlling the size and size-distribution of the $\mathrm{Cu}_{2} \mathrm{O}$ nanocubes with simple process parameters such as reaction temperature, stirring time and $\mathrm{NaBH}_{4}$ concentration, it is possible to tune the optical properties of $\mathrm{Cu}_{2} \mathrm{O}$ suspension. These process parameters have a stong influence on the agglomeration of the small spherical nanocrystals into larger cube-shaped particles. 
It was found that the increment of the $\mathrm{pH}$ promote the agglomeration of the cubes in the reaction medium. Here, the addition of $0.25 \mathrm{~mL} 0.01 \mathrm{M} \mathrm{NaOH}$ to the reaction medium resulted in a strong right shift of the broad absorbance band in the visible range (figure 3.23). With the agglomeration of the cubes, larger structures are formed, which in turn can absorb light with a higher wavelength and over a wider wavelength range. The agglomeration of the cubes with the increment of the $\mathrm{pH}$ may be promoted by $\mathrm{OH}^{-}-$ ligands attached at surface of the nanocubes. The agglomeration of the cubes, however, affects the light scattering properties of the suspension due to the reduction of the surface-to-volume ratio. $\mathrm{Cu}_{2} \mathrm{O}$ suspension prepared with the addition of $\mathrm{NaOH}$ show a higher transparency in reflected light than suspensions prepared without the additon of $\mathrm{NaOH}$.

The association of the cubes as well as the agglomeration products of the cubes are based on weak chemical bondings such as hydrogen bonds. For this reason, $\mathrm{Cu}_{2} \mathrm{O}$ suspensions are highly sensitive to dilution and post-treatment with ionic surfactants. For example, dilution with water and the additon of ionic capping agents (such as TSC and $\mathrm{Na}_{2} \mathrm{~S}_{2} \mathrm{O}_{3}$ ) result in in the collapse of the self-assembled structure. With the collapse of the self-assembled structure, however, the light absorption and scattering properties of the suspension changes and only the absorption characteristics of the small spherical nanocrystals remain.

\subsubsection{Surface Plasmon Resonance}

The different optical properties of the $\mathrm{Cu}_{2} \mathrm{O}$ suspensions may be caused by Surface Plasmon Resonance (SPR). SPR improves the radiative properties of the metal nanoparticle such as scattering and absorbing properties. SPR is a well-known phenomenon for many metallic nanocrystals. For example, small gold and silver nanocrystals show intense colours due to the SPR effect when light passes through their colloidal solution $[98,100]$.

SPR is related to the collective oscillation of conduction band electrons at the surface of metallic nanoparticles, induced by a resonant incident electric field of an electromagnetic wave, Photons. The oscillation of these electrons can be visualised as a free electron cloud oscillating against the restoring forces of a positive nucleus. The 
oscillation of the free electron cloud results in a periodic charge separation at the interface of spherical nanoparticles. This periodic charge separation, upon excitation by an electromagnetic wave, is termed dipolar oscillation. The dipolar oscillation increases the charge density at the surface of spherical nanoparticles, which in turn leads to the enhancement of the electromagnetic field along the particle interface (metal-dielectric interface). Due to the dipolar oscillation at the surface of the particle, the charge density at the surface of the particle moves along the metal-dielectric interface in a wave pattern. This charge-density wave is known as Surface plasmon and the effect is termed as SPR. The charge-density oscillation of spherical metal nanoparticles induced by an oscillating electric field of a photon is illustrated in figure 3.34 .

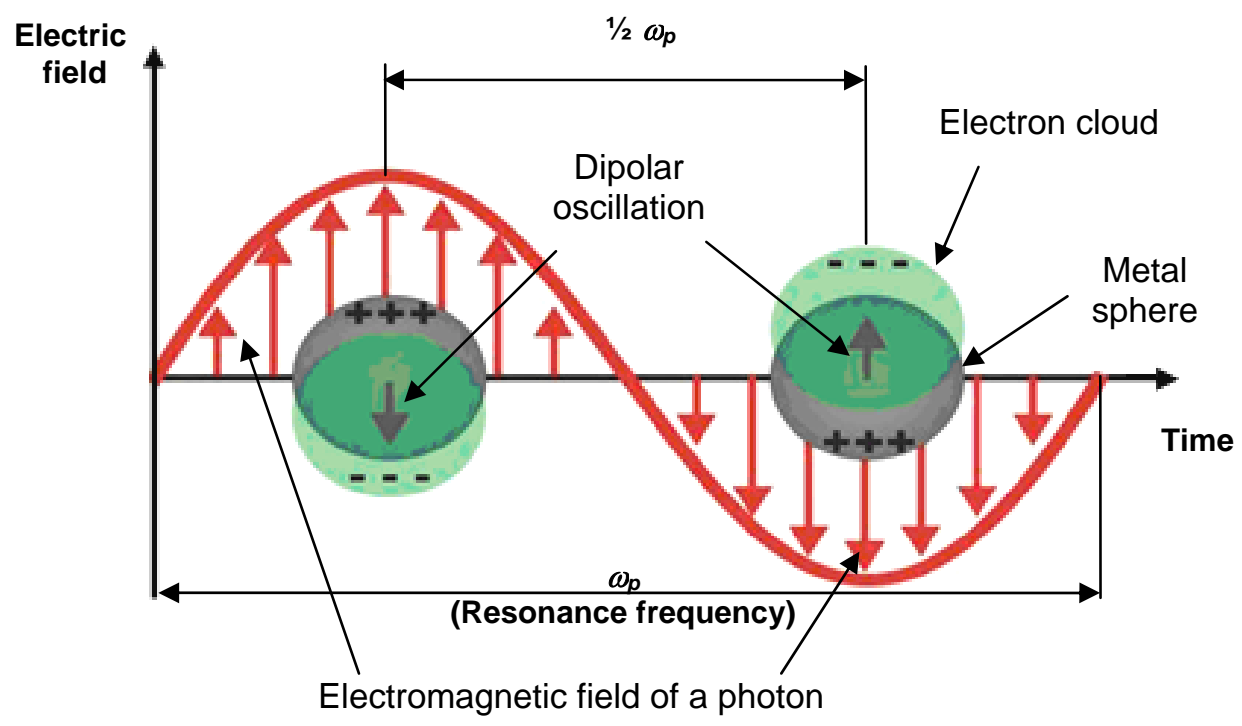

Figure 3.34: Graphic illustration of the periodical displacement of the surface conduction band's electrons in spherical metal nanoparticles induced by a resonant incident electric field of incoming light [98].

The SPR effect is typically shown as a sharp and intense band in the visible range of the absorbance spectrum. This band defines the range of light, which stimulates the SPR and it is know as the Surface Plasmon absorption band.

The occurrence of Surface plasmon is dependent on the dimension of the nanoparticles itself. Surface Plasmons only occur, when the size of the spherical metal nanoparticle approaches the magnitude of the de Broglie wavelength of the surface conduction band electrons. Under this condition, the collective motion of the surface conduction band electrons is confined by the restoring forces of the positive nucleus and the dimensional limits of the particle. This is comparable with the confinement of an electron in a one- 
dimensional box. As a consequence, the wave propagation of Surface Plasmons along the metal-dielectric interface in such small particles can be visualised as a harmonic wave defined by a resonance frequency:

\section{Resonance frequency}

$$
\omega_{p}=2 \pi f
$$

The resonance frequency $\omega_{p}$ of Surface Plasmons is determined by the wavelength of the incident light at the interface between the metal nanoparticles and the surrounding medium. However, the resonance frequency $\omega_{p}$ of Surface Plasmons in metallic nanostructures is also dependent on the composition of the material, shape, and nature of the surrounding medium (dielectric constant of the medium) [99-101].

Inorganic semiconductors are characterised by an empty conduction band in ground state and for this reason is less likely that SPR will occur in semiconductor materials. $\mathrm{Cu}_{2} \mathrm{O}$ suspensions, however, show two sharp absorbance peaks in the visible range between $440-477 \mathrm{~nm}$ and $477-505 \mathrm{~nm}$, when nanocubes in the size of $70-150 \mathrm{~nm}$ and $190-220 \mathrm{~nm}$ dominates. These sharp peaks in the visible absorbance spectrum correspond to nanocubes with a narrow size distribution. A change in size and sizedistribution of the nanocubes was observed by a shift of these narrow absorption peaks in the visible range of light.

Due to the enhanced light absorption and scattering properties of the $\mathrm{Cu}_{2} \mathrm{O}$ nanocubes, these sharp absorbance peaks most likely refer to SPR. SPR, however, occurs at the interface of metallic nanoparticles, where surface conduction band electrons absorb and scatter incident light with certain wavelengths depending on the size and shape of the nanocrystals and their arrangement in the surrounding medium. Hence, it can be assumed that the spherical $\mathrm{Cu}_{2} \mathrm{O}$ nanocrystals agglomerated into nanocubes are affected by a further reduction reaction. The formation of a thin layer of elementary copper around the $\mathrm{Cu}_{2} \mathrm{O}$ nanocrystals would give rise to SPR [102]. This is most likely, since the nanocrystals inside the cubes are surrounded by TSC. The surface of the nanocrystals is usually positively charged due to the presence of metal cations. This positive charge attracts negatively charged TSC ions and forms an electrostatic layer around the particles. TSC, however, has also reducing properties, which can cause the 
reduction of copper cations to elementary copper. By doing so, a thin layer of elementary $\mathrm{Cu}^{0}$ is formed at the interface of the nanocrystals.

According to this, the SPR effect is determined by the size and size-distribution of the self-assembled cubes and on the dielectric constant of the surrounding medium. The surrounding medium corresponds to a mixture of PVP, TSC and the oxidation products of TSC and $\mathrm{NaBH}_{4}$, which enclose the nanocrystals inside the cubes. Therefore, light scattering and absorption effects are only observed, when the small spherical $\mathrm{Cu}_{2} \mathrm{O}$ nanocrystals are fully incorporated into larger polycrystalline particles.

Intermolecular interactions between mono-dispersed cubes and the formation of agglomerates, however, will have also a big influence on the SPR and hence on the wavelengths range of the absorbed and scattered light. For example, enhanced chemical interaction between the cubes-shaped particles and formation of larger structures was shown by a gradually broadening of the absorbance peaks in the visible range (figure 3.21 ; $)$

Since Surface Plasmons occur at the interface between the nanoparticles and the surrounding medium, any changes at the interface, such as the exchange of the surrounding medium or the adsorption of molecules onto the surface, will affect the electromagnetic field at the surface of the nanoparticles and hence the resonance frequency $\omega_{p}$ of the metal nanoparticle. For this reason, the collapse of the selfassembled structure (through dilution with water or addition of ionic capping agents) will change the medium around the nanocrystals and hence the light absorption and scattering properties. Spherical nanocrystals mono-dispersed in solution are characterised by a transparent suspension and a broad absorbance peak around $350 \mathrm{~nm}$ in the UV-vis scan.

The formation of a thin $\mathrm{Cu}^{0}$ layer around the nanocrystals may also suppress the formation of defect states at the particle surface. $\mathrm{Cu}_{2} \mathrm{O}$ nanocrystals incorporated into nanocubes and mono-dispersed in solution revealed different PL scans. It was found that the arrangement of the spherical nanocrystals into larger polycrystalline particles cause the emission of one peak at $355 \mathrm{~nm}$ (figure 3.30; B), whereas $\mathrm{Cu}_{2} \mathrm{O}$ nanocrystals mono-dispersed in solution showed a broad emission peak at $400 \mathrm{~nm}$ (figure 3.30; A). 
This broad emission peak may correspond to a trap emission caused by surface defects. However, when incorporated into larger self-assembled structures, the trap emission is suppressed and only the band-edge emission $(\sim 355 \mathrm{~nm})$ of the $\mathrm{Cu}_{2} \mathrm{O}$ nanocrystals is observed.

In order to confirm the formation of elementary copper at the surface of the nanocrystals, XRD and XPS measurements have to be repeated which focus on the surface of the nanocrystals. In these studies, a thin layer of copper was not detected by XRD and XPS.

\subsubsection{Doping procedure}

The formation of $\mathrm{Cu}_{2} \mathrm{O}$ in the presence of zinc and nickel also results in the formation of self-assembled structures of different sizes. Here, the shape of the self-assembled structures, however, can vary from cubes to more complicated structures such as pseudo-dodecahedron structures and other structures (see figure 3.16-3.17).

Zetasizer revealed that the doping procedure forms nanocrystals smaller than the standard procedure of undoped $\mathrm{Cu}_{2} \mathrm{O}$ nanocrystals with an average size of $2 \mathrm{~nm}$ (see figure 3.19). These nanocrystals also organise themselves into large self-assembled structures within the same size range of those undoped $\mathrm{Cu}_{2} \mathrm{O}$ suspensions (see figure 3.18 and 3.19); however, the size-distribution of the self-assembled structures of doped $\mathrm{Cu}_{2} \mathrm{O}$ nanocrystals are slightly larger.

EDS mappings of doped $\mathrm{Cu}_{2} \mathrm{O}$ nanoparticles show zinc atoms homogeneously distributed across the cubes; however nickel could not be detected in the cubes. Instead, nickel was homogeneously distributed in a layer covering the cubes (see figure 3.15). EDS mapping suggest that this layer may be related to $\mathrm{NiO}$ embedded by the polymeric dispersant. It is also interesting that $\mathrm{Ni}^{2+}$ could be replaced by $\mathrm{Co}^{2+}$ and $\mathrm{Mn}^{2+}$ ions without changing the absorbance spectra of the $\mathrm{Cu}_{2} \mathrm{O}$ suspension (see figure 3.27).

UV-vis scans of $\mathrm{Cu}_{2} \mathrm{O}$ nanocrystals prepared in the presence of zinc and other dopants such as nickel, cobalt and manganese were practically identical to the scans of undoped $\mathrm{Cu}_{2} \mathrm{O}$ nanocrystal, except from a peak shift from 260 to $290 \mathrm{~nm}$ (compare figure 3.21 
with figure 3.25). This peak shift indicates a new band transition in the electronic band structure of $\mathrm{Cu}_{2} \mathrm{O}$ nanocrystals and is attributed to the incorporation of zinc atoms into the lattice structure of $\mathrm{Cu}_{2} \mathrm{O}$. This was confirmed by the UV-vis scans of $\mathrm{Cu}_{2} \mathrm{O}$ suspension prepared in the presence of zinc only (figure 3.28).

In contrast, the dopants nickel, cobalt and manganese are not incorporated into the lattice structure; however, play an important role on the particle growth of the spherical nanocrystals in suspension. Here, it is important to point out that the doping procedure was carried out without the addition of TSC. Trisodium citrate, however, is required for the preparation of un-doped $\mathrm{Cu}_{2} \mathrm{O}$ nanocrystals due to the stabilizing and reducing properties. Without TSC in the reaction medium, the reaction results in a totally precipitation reaction. However, when nickel was added to the reaction medium instead of TSC, $\mathrm{Cu}_{2} \mathrm{O}$ nanocubes with a similar size-distribution was obtained (see figure 3.17; A).

To understand the mechanism of the particle growth of $\mathrm{Cu}_{2} \mathrm{O}$ crystals in the presence of dopants, it is important to understand the electrochemical behaviour of the dopants used. The dopants zinc, nickel, cobalt and manganese have a negative standard reduction potential (redox potential), whereas copper has a positive standard reduction potential. The standard reduction potential indicates the affinity of an element towards electrons. The higher the reduction potential, the greater is the tendency to be reduced. The addition of $\mathrm{NaBH}_{4}$ to the reaction medium will result in the reduction of copper ions as well as of the doping ions.

Due to the higher standard reduction potential of copper ions, zinc and nickel ions (respectively cobalt and manganese) initially reduced by the addition of $\mathrm{NaBH}_{4}$ will subsequently oxidise and by doing so reduce copper ions in reaction medium. These redox reactions promote the formation of small spherical $\mathrm{Cu}_{2} \mathrm{O}$ nanocrystals which then organise themselves into larger self-assembled structures. Hence, the dopants zinc and nickel as well as cobalt and manganese fulfil the function of TSC in the standard procedure of $\mathrm{Cu}_{2} \mathrm{O}$.

For this reason, $\mathrm{Cu}_{2} \mathrm{O} / \mathrm{Zn}$ nanocrystals prepared in the presence of nickel, cobalt or manganese show identical absorbance spectra with a narrow absorbance peak in the 
visible range between 440 and $477 \mathrm{~nm}$. This absorbance peak, however, is strongly dependent on the stirring time and reaction temperature. For example, with higher reaction temperature (figure 3.25) and longer stirring times (figure 3.27) the absorbance peak between 440 and $477 \mathrm{~nm}$ gradually increases and slightly shifts to higher wavelength.

Similar to undoped $\mathrm{Cu}_{2} \mathrm{O}$ suspension, the absorbance peaks and bands in the UV-vis scans represent the size and size distribution of nanocrystals and their self-assembled structures. Thereby, the increment of the narrow absorbance peak between 440 and 477 $\mathrm{nm}$ with a slightly shift to higher wavelength $(\max .460 \mathrm{~nm})$ reflects a greater formation of spherical nanocrystals which in turn organise themselves into larger self-assembled structures.

However, $\mathrm{Cu}_{2} \mathrm{O} / \mathrm{Zn}$ nanocrystals prepared in the presence of nickel undergo a further particle growth, when the reaction was carried out at $99{ }^{\circ} \mathrm{C}$ and stirred longer than 20 min. Here, the narrow absorbance peak between 440 and $477 \mathrm{~nm}$ transforms to a broad absorbance band starting at $477 \mathrm{~nm}$ to the end of the visible spectrum. As well the absorbance peak around $350 \mathrm{~nm}$ flattens with the transition to a broad absorbance band (see figure 3.25).

The particle size and size-distribution of the doped $\mathrm{Cu}_{2} \mathrm{O}$ suspension prepared at $99{ }^{\circ} \mathrm{C}$ is shown in the SEM images in figure 3.16. It shows particles with a wide sizedistribution between 100 and $250 \mathrm{~nm}$. At closer look it can be seen that these particles were assembled by the agglomeration of small nanocubes varying in the size from 50 to $70 \mathrm{~nm}$.

Here, the strong absorbance peak at $458 \mathrm{~nm}$ corresponds to nanocubes in the size of around $70 \mathrm{~nm}$, which agglomerate to larger particles when the reaction was carried out at $99{ }^{\circ} \mathrm{C}$ and stirred for longer than $20 \mathrm{~min}$. The cube-size correlation with the absorbance peak at $458 \mathrm{~nm}$ matches perfectly with the linear function obtained in figure 3.24. Furthermore, according to the linear function, the absorbance peak at $453 \mathrm{~nm}$ obtained at $92{ }^{\circ} \mathrm{C}$ (figure 3.25 ) corresponds to nanocubes in the size of $50 \mathrm{~nm}$, which are also seen in the agglomeration products (figure 3.16). 
By grouping together, the small nanocubes obtain a higher chemical stability by reducing their surface area. However, the light scattering properties of the suspension decreases with the agglomeration of the cubes due to the increase of the surface-tovolume ratio (figure 3.26). The formation of larger structures by sticking together also allows the absorption of light with a higher wavelength and over a wider wavelength range. This results in a shift and broadening of the absorbance peak in the visible range (figure 3.25). Due to the broad absorbance peak, the $\mathrm{Cu}_{2} \mathrm{O}$ suspension shows a dichroic effect depending on the angle of the incident light (figure 3.6).

\subsection{Conclusions}

The novel preparation used for the synthesis of $\mathrm{Cu}_{2} \mathrm{O}$ nanocrystals allows the controlled formation of $\mathrm{Cu}_{2} \mathrm{O}$ nanocubes with unique optical properties. Initially, the sample preparation results in the formation of small spherical nanoparticles $(5 \mathrm{~nm})$ which then successively transform, by a self-assembled stabilizing mechanism, into cube-shaped particles of about $70-250 \mathrm{~nm}$; depending upon the synthesis conditions. TEM images show that the spherical nanocrystals consist of an ordered arrangement of $\mathrm{Cu}_{2} \mathrm{O}$ and surfactant layers resulting in a superlattice structure. Here, the formation of selfassembled structures is connected with the destabilization of the nanocrystals in aqueous solution by the drop of the reaction temperature. By sticking loosely together, the nanocrystals reduce their surface energy and obtain thereby a higher stability in solution. Thereby, the agglomeration of the nanocrystals is regulated by specific interactions between the chains of the polymeric dispersant and the surface of the $\mathrm{Cu}_{2} \mathrm{O}$ nanocrystals. By selective adsorption and desorption of the polymeric dispersant on the surface of the $\mathrm{Cu}_{2} \mathrm{O}$ nanocrystals, the particles arrange themselves into a polymer matrix and forming cube-shaped particles.

The size distribution of the nanocubes depends on several process parameters. In general, the formation of cubic $\mathrm{Cu}_{2} \mathrm{O}$ nanoparticles of different sizes depends on the ratio of copper concentration and capping agent concentration, sodium borohydride concentration, reaction temperature and stirring time. These process parameters have a strong effect on the repelling layer around the spherical nanocrystals. The formation of a weak repelling layer will result in a stronger chemical interaction between the nanocrystals which in turn lead to the formation of larger self-assembled structures. 
Here, the organisation of the quantum dot into nanocubes leads to unique light absorption and scattering effects. For example, $\mathrm{Cu}_{2} \mathrm{O}$ suspension containing only large nanocubes show a dichroic effect. Depending on the angle of the incident light, two distinctive colours are observed. Here, the colour in reflected light corresponds to the light scattered by the nanocubes and the colour in transmitted light correspond to the complementary colour of the light absorbed by the nanocubes.

The enhanced light absorption and scattering properties of the cubes may be based on a surface plasmon resonance (SPR) effect. SPR occurs at the interface of metallic nanoparticles. It can be assumed that the surface of the spherical $\mathrm{Cu}_{2} \mathrm{O}$ nanocrystals inside the nanocubes is reduced to elementary copper by trisodium citrate. The SPR effect is usually shown by narrow and broad absorbance peaks in the visible range of the spectrum. The absorbance peaks obtained by the $\mathrm{Cu}_{2} \mathrm{O}$ suspension can be correlated with the physical dimension of the nanocubes. Thereby, the wavelength of the light absorbed or scattered by the nanocubes is determined by the size of the nanocubes and on the chemical interaction with the surrounding. Therefore, the optical properties of the $\mathrm{Cu}_{2} \mathrm{O}$ suspension can be tuned by controlling the size of the nanocubes in suspension.

However, the association of the cubes are based on weak electrostatic interaction and hydrogen bondings. These bondings can easily be broken by diluting the suspension with water or adding ionic surfactants to the suspension. However, when the selfassembled structures collapse the light absorption and scattering properties disappear. For this reason, further investigation is needed to reinforce the cube-shaped structure of $\mathrm{Cu}_{2} \mathrm{O}$ particles in order to obtain a higher chemical stability. By doing so, $\mathrm{Cu}_{2} \mathrm{O}$ nanocubes can be incorporated in polymers to create composite materials with a dichroic behaviour. This material can be used, for example, in the preparation of anticounterfeiting bank notes. 


\section{Chapter 4}

\section{Synthesis and characterisation of $\mathrm{ZnO}$ quantum dots}

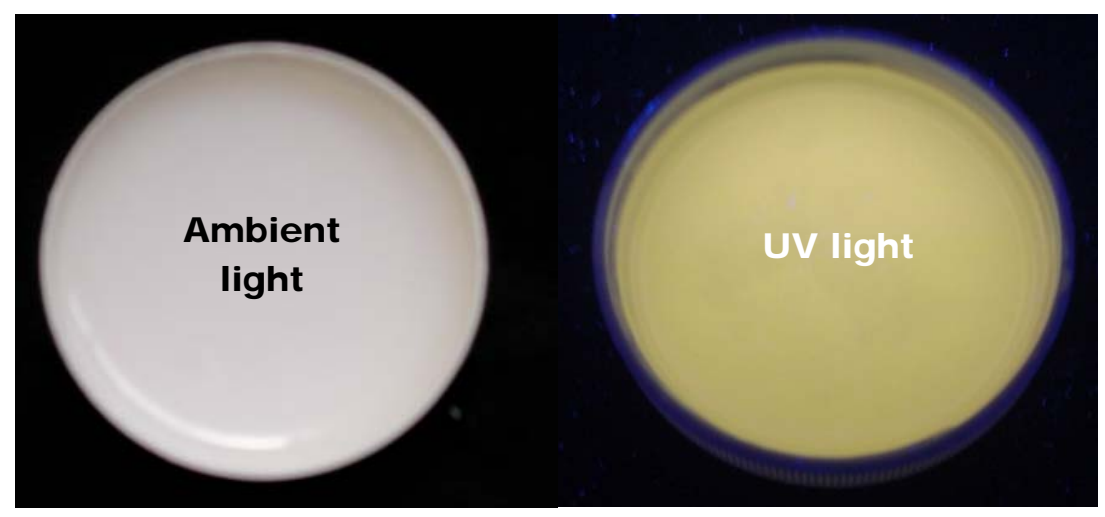

This chapter deals with the synthesis and characterisation of zinc oxide $(\mathrm{ZnO})$ quantum dots, which have the potential to be used in the development of new hybrid composite materials. The main goal is to develop a sol-gel method to synthesis $\mathrm{ZnO}$ quantum dots that show strong fluorescence in the visible range. Furthermore, the influences of various parameters on particle size and size distribution, particle growth and shape, and fluorescence properties are investigated.

$\mathrm{ZnO}$ quantum dots have been prepared by a sol-gel method adding an alkali reducing agent, $\mathrm{NaBH}_{4}$, to a zinc chloride aqueous solution complexed with polyvinylpyrrolidone (PVP) at a reaction temperature of $70^{\circ} \mathrm{C}$. $\mathrm{ZnO}$ quantum dots formed by this sol-gel method are spherical and ellipsoidal in shape with an average size of $10-12 \mathrm{~nm}$. However, the $\mathrm{ZnO}$ quantum dots underwent a further particle growth forming agglomerates under the terms of a self-assembled mechanism. The agglomerates have the shape of round rice grains varying between $100 \mathrm{~nm}$ and $1 \mu \mathrm{m}$. The final $\mathrm{ZnO}$ suspension is white under ambient light and yellow-orange fluorescence under UV light. 
When excited with UV-light $\left(\lambda_{\text {exc }}=260 \mathrm{~nm}\right)$, the $\mathrm{ZnO}$ suspension showed two distinct emission peaks in the UV and visible range. The emission peak in the UV range $(\sim 380$ $\mathrm{nm}$ ) is directly related to the radiative recombination of the electron-hole pair (exciton) in spherical zinc oxide quantum dots and corresponds to the band-edge emission of the quantum dots. The broad emission peak in the visible range $(\sim 560 \mathrm{~nm})$ is caused by surface defects of the quantum dots. These defects incorporated surface related defect states within the band gap, which progressively quenched the band-edge emission. This was confirmed by PL scans of the $\mathrm{ZnO}$ quantum dot suspension conducted over time. Ageing ( $2-3$ days after preparation) increased the fluorescence intensity of the defect emission which in turn caused the reduction of the band-edge emission. The maximum intensity of the defect emission was obtained when the band-edge emission was reduced to a small hump around $390 \mathrm{~nm}$. The defect emission, however, was further affected by ageing shown by a progressively reduction in intensity. This reduction indicated nonradiative relaxation processes which may occur simultaneously with the defect emission. It can be assumed that the agglomeration of the quantum dots and formation of polycrystalline particles may also contribute to the non-radiative relaxation processes.

The band-edge emission of the $\mathrm{ZnO}$ quantum dots is size-dependent, whereas the defect emission depends on the surface state of the quantum dots. The sol-gel method was optimised to obtain maxium fluorescence intensity of the defect emission. Therefore, the influence of different process parameters such as reaction temperature, choice of polymeric dispersant (PVP, PMAA-Na), concentration of polymeric dispersant and concentration of sodium borohydride on the defect emission were investigated by fluorescence and UV-vis spectroscopy and optimised. Furthermore, the effect of these process paramertes on the formation of the self-assembled polycrystalline particles were studied by scanning electron microscopy (SEM), transmission electron microscopy (TEM) and particle size analysis. The effect of doping on the fluorescence properties of the zinc oxide nanocrystals was also investigated.

\subsection{Introduction; $\mathrm{ZnO}$ quantum dots}

Bulk zinc oxide $(\mathrm{ZnO})$ is a well known II-VI semiconductor material with a wide direct band gap at room temperature $(3.37 \mathrm{eV})$ and high exciton binding energy $(\sim 60 \mathrm{meV})$ 
$[79,103]$. It can be readily synthesised via a wet chemical method and exhibit fluorescence in the UV and visible range of light. Other useful features of $\mathrm{ZnO}$ are its biocompatible properties and good optical transparency in the visible range at nanoscale, high electron mobility and insolubility in water. Furthermore, zinc oxide prepared at the nano-scale size shows anti-bacterial, anti-fungal, anti-corrosion, catalytic, and UV filtering properties. It also shows long-term photostability in comparison to organic fluorophores. Because of the numerous properties, $\mathrm{ZnO}$ is an excellent candidate to be used as a fluorescent agent to produce new composite materials comprising quantum dots and wool and paper fibres.

\subsubsection{Sol-gel method}

$\mathrm{ZnO}$ quantum dots are easy to obtain through a sol-gel method which production can also readily be scaled up to a large scale at mild reaction conditions by using aqueous solution and cheap raw materials [76-79]. The sol-gel method is widely used for the synthesis of metal oxides materials. It is a cheap and easy technique that also allows the introduction of small quantities of dopants into the chemical composition of the final product. Here, the metal oxide composition, nature and level of dopants and the particle size collectively control the band gap and hence the fluorescence of the metal oxide quantum dots.

The principle of the sol-gel method is the formation of a solid phase from solution during a chemical reaction. Here, the injection of an alkaline agent into a neutral aqueous solution of zinc ions induces a forced alkaline hydrolysis which results in the chemical transformation of water complexed zinc cations into colloidal $\mathrm{ZnO}$ particles. The final material is determined by the composition of the precursors but also on the reaction parameters. Changes in process parameters can often lead to very different materials /oxide phases such as amorphous gel or crystalline solids with different crystal structures. For example, sol-gel techniques carried out at low temperature provide quantum dots with poor degree of crystallinity and surface defects. It is also difficult to obtain a narrow size-distribution of quantum dots using sol-gel methods.

For this reason, the reaction parameters have to be optimised to produce colloidal particles with crystalline character and to control the sizes and shapes of the crystal 
during the nucleation and condensation growth stages. Here, the forced alkaline hydrolysis can be controlled by different processing parameters such as concentration of the raw materials, reaction temperature, choice of solution, $\mathrm{pH}$, titration rate of the reactants, stirring rate and use of capping agents. All these parameters have a strong effect on the final structure of the solid material and on the growth of quantum dots during the chemical reaction.

\subsubsection{Forched alkaline hydrolysis}

Most of the sol-gel techniques make use of the aqueous chemistry of metal salts, typically metal chlorides. In general, metal ions in aqueous solution form an aqua coordination complex. This complex, however, is affected by hydrolysis and condensation reaction which give rise to the formation of hydrolysed monomers to larger polycation species.

Hydrolysis is caused by the subsequent deprotonation of the water ligands attached to the metal ions in an aqueous solution. The aqua ligands exhibits more acidic properties (dissociation) and for this reason, many metal ions, in particular rare earth- and transition metal ions, are acidic when dissolved in aqueous solution. Condensation reaction can be defined as a reaction between two hydrolysed ion species with the elimination of one water molecule. The condensation reaction result in the formation of polymer cations starting from dimer to larger polycation species. For example, zinc ions hydrated in an aqueous solution will have the chemical formula $\left[\mathrm{Zn}\left(\mathrm{H}_{2} \mathrm{O}\right)_{6}\right]^{2+}$ before any reaction will occur. Then, the zinc ion in the aqueous solution will experience hydrolysis reactions which give rise to the formation of a reactive hydrolysed monomer. Equation (19) shows the first hydrolysis reaction of the zinc hexaaqua complex:

First hydrolysis step of water complexed zinc ions $(h=1)$ :

$$
\left[\mathrm{Zn}\left(\mathrm{H}_{2} \mathrm{O}\right)_{6}\right]^{2+}+\mathrm{H}_{2} \mathrm{O} \quad \rightleftarrows \quad\left[\mathrm{Zn}(\mathrm{OH})\left(\mathrm{H}_{2} \mathrm{O}\right)_{5}\right]^{+}+\mathrm{H}_{3} \mathrm{O}^{+}
$$

The hydrolysed monomer in equation (19) is more likely to be involved in condensation reactions due to the higher nucleophilic properties of the $\mathrm{OH}^{-}$ligand [38-40]. Because of this property, both condensation and hydrolysis reactions can occur simultaneously. 


$$
2\left[\mathrm{Zn}(\mathrm{OH})\left(\mathrm{H}_{2} \mathrm{O}\right)_{5}\right]^{+} \quad \rightleftarrows \quad\left[\left(\mathrm{H}_{2} \mathrm{O}\right)_{4} \mathrm{Zn}(\mathrm{OH})_{2} \mathrm{Zn}\left(\mathrm{H}_{2} \mathrm{O}\right)_{4}\right]^{2+}+2 \mathrm{H}_{2} \mathrm{O}
$$

These intermediates formed by hydrolysis and condensation in equation (19) and (20) can react further with each other and form larger polycation species under the elimination of water.

$$
\left[\left(\mathrm{H}_{2} \mathrm{O}\right)_{4} \mathrm{Zn}(\mathrm{OH})_{2} \mathrm{Zn}\left(\mathrm{H}_{2} \mathrm{O}\right)_{4}\right]^{2+} \rightleftarrows \quad \begin{gathered}
\text { Polyoxo-cations } \\
\mathrm{ZnO} \times \mathrm{H}_{2} \mathrm{O}
\end{gathered}
$$

The polycation species (equation (21)) are also affected by hydrolysis and can form reactive intermediates. These intermediates can then undergo further poly-condensation reactions and form either an elastic solid, also known as gel, or a colloidal suspension.

Figure 4.1 shows two types of condensation reactions, oxolation or olation, which can occur between hydrolysed zinc cations (monomers or polycations). In the condensation reactions, a ligand bridge is formed between two metal ions under the elimination of water. Depending on the condensation reaction, the metal cations are connected together either by an oxo or hydroxyl ligand bridge.

A
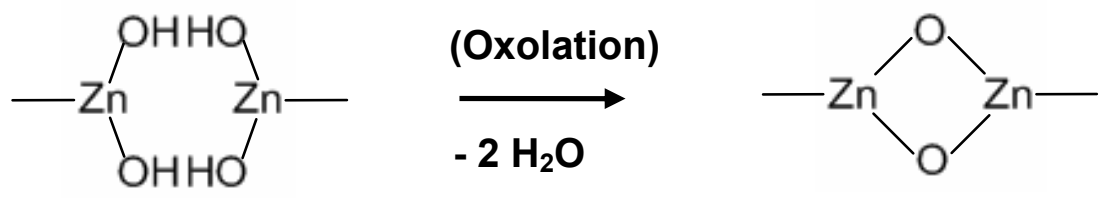

B
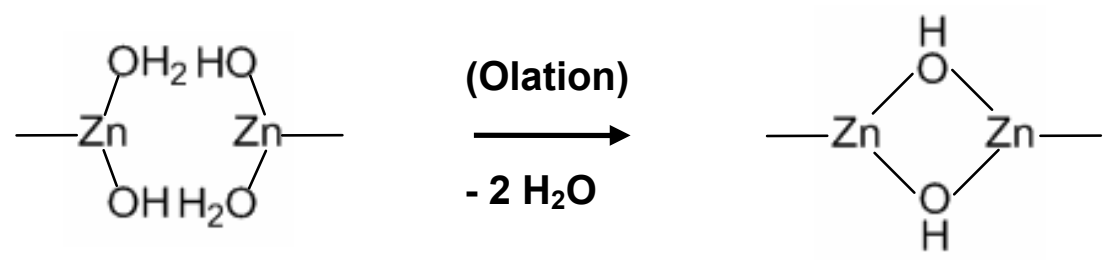

Figure 4.1: Schematic illustration of the oxalation (A) and olation (B) condensation reactions for hydrolysed zinc ions.

In the oxolation reaction (figure $4.1 ; \mathrm{A})$ an oxo bridge (-O-) is formed between two hydrolysed metal centres. The elimination of water between both metal centres can be attributed to a nucleophilic substitution $\left(\mathrm{S}_{\mathrm{N}}\right)$ or nucleophilic addition $\left(\mathrm{A}_{\mathrm{N}}\right)$ mechanism [43]. Here, the mechanism of condensation reaction is determined by the coordination of the metal ion centre. If the coordination is 'satisfied' then the mechanism that occurs 
is nucleophilic substitution. If the coordination is 'not satisfied' then the mechanism is nucleophilic addition.

In the olation reaction (figure 4.1; B) a hydroxyl bridge (-OH-) is formed between two metal centres. Initially, two metal centres interact with each other through their ligands, hydroxyl and aqua respectively, and form a semi stable intermediate. Their interaction is based on a hydrogen-bridge bonding between the hydroxyl and aqua ligand. The second stage of the mechanism is the loss of water (condensation), which left behind a single hydroxyl bridge.

The rate and extent of hydrolysis and condensation reactions depends on many parameters such as temperature, ionic strength of the solvent, concentration of the metal ions, presence of different anions etc. However, the most important parameter is the $\mathrm{pH}$ of the solution. With the increase of $\mathrm{pH}$, the strength of bonding between the oxygen and hydrogen atom of the aqua ligands decreases. Hence, a hydrolysis and condensation reaction can be easily induced by the addition of an alkali reagent to an acidic metal salt aqueous solution. This method is known as a forced alkaline hydrolysis and commonly used for sol-gel methods [39].

The size of particles formed by a forced alkaline hydrolysis, however, is difficult to control. Hydrolysis results in a rapid neutralisation reaction of the aqua complexed metal cation whereby hydroxyl groups are formed around the solvated metal ions ("sol"), followed by a subsequent poly-condensation of reactive intermediates which form either a network structure ("gel”) or a colloidal suspension. Thereby, the formation of the final solid product is mainly dependent on the reaction conditions applied such as $\mathrm{pH}$, temperature, concentration of the metal ion in aqueous solution, etc. However, mixing is also an essential part of this reaction. The introduction of alkalis to the metal ion solution can result in areas of local concentration and local perturbation. In these local areas, some metal ions are surrounded by more alkali which can cause the rapid formation of larger particles as well as the precipitation of the metal cations as metal hydroxide such as shown in figure 4.2. 


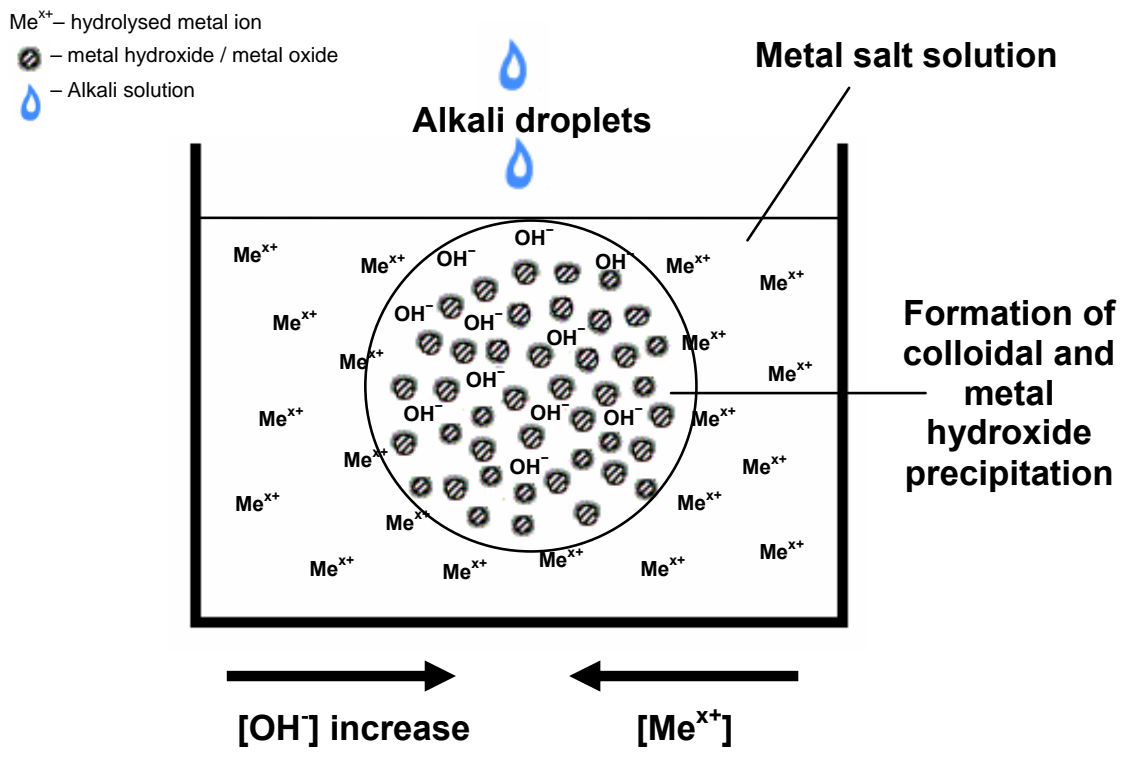

Figure 4.2: Schematic illustration of the addition of a droplet of alkali to a metal salt solution causing a concentration gradient.

Fast vigorous mixing of the reagents ensures a higher hydrolysis rate of the water complexed metal cations and it also allows a homogeneous particle growth of the small nucleation centres formed instantaneously by the addition of alkali to the reaction medium. These nucleation centres, hydrolysed zinc cation monomers and polymers will polycondensate and form larger particles in the nanometre range with a narrow sizedistribution.

The sol-gel method also allows the incorporation of small quantities of impurities (dopants) into the chemical composition of the final product during the hydrolysis and condensation reaction. Thereby, the optical fluorescence of the $\mathrm{ZnO}$ quantum dots can be tuned in the visible range by the additional energy levels of dopant materials within the band gap. Fast vigorous mixing is also important for the incorporation of dopant ions into the lattice structure of the final nanocrystals. Many transition metals used as dopant ions can precipitate as metal hydroxide in aqueous solutions by the addition of an alkali. Some examples are given below: 


$$
\begin{aligned}
& \mathrm{Cu}^{2+}+2 \mathrm{OH}^{-} \stackrel{\mathrm{H}_{2} \mathrm{O}}{\longrightarrow} \quad \mathrm{Cu}(\mathrm{OH})_{2(\mathrm{~s})} \\
& \text { bluish precipitation } \\
& \mathrm{Ni}^{2+}+2 \mathrm{OH}^{-} \stackrel{\mathrm{H}_{2} \mathrm{O}}{\longrightarrow} \quad \mathrm{Ni}(\mathrm{OH})_{2(\mathrm{~s})} \\
& \text { green precipitation } \\
& \mathrm{Co}^{2+}+2 \mathrm{OH}^{-} \stackrel{\mathrm{H}_{2} \mathrm{O}}{\longrightarrow} \quad \mathrm{Co}(\mathrm{OH})_{2}(\mathrm{~s}) \\
& \text { blue precipitation } \\
& \mathrm{Mn}^{2+}+2 \mathrm{OH}^{-} \stackrel{\mathrm{H}_{2} \mathrm{O}}{\longrightarrow} \quad \mathrm{Mn}(\mathrm{OH})_{2}(\mathrm{~s}) \\
& \text { pinkish precipitation }
\end{aligned}
$$

Most metal hydroxides are very stable which means being insoluble except for $\mathrm{Zn}(\mathrm{OH})_{2}$. Zinc hydroxide can re-dissolve by further addition of $\mathrm{OH}^{-}$-ions and convert to a colourless complex $\left[\mathrm{Zn}(\mathrm{OH})_{4}\right]^{2-}$.

$$
\mathrm{Zn}(\mathrm{OH})_{2(\mathrm{~s})}+2 \mathrm{OH}^{-} \quad \stackrel{\mathrm{H}_{2} \mathrm{O}}{\longrightarrow} \quad\left[\mathrm{Zn}(\mathrm{OH})_{4}\right]^{2-}
$$

\subsection{Preparation of $\mathrm{ZnO}$}

For the synthesis of $\mathrm{ZnO}$ quantum dots, the sol-gel method described in chapter 2 was used. In this sol-gel method, $\mathrm{ZnO}$ nanocrystals were formed by adding $\mathrm{NaBH}_{4}$ in a $\mathrm{ZnCl}_{2} / \mathrm{PVP}$ aqueous complex solution at a reaction temperature of $70{ }^{\circ} \mathrm{C}$. To ensure crystallinity the suspension was stirred for at least $30 \mathrm{~min}$.

The sol-gel method used for the synthesis of $\mathrm{ZnO}$ quantum dots was standardised to obtain maximum fluorescence intensity in the visible range. This was conducted by changing different process parameters, which are listed in table 4.1. Also, the stability of the fluorescence intensity and dispersion behaviour of the $\mathrm{ZnO}$ nanocrystals was tested by ageing the solution. 
Table 4.1: List and details of process parameters investigated for the synthesis of $\mathrm{ZnO}$ nanoparticles.

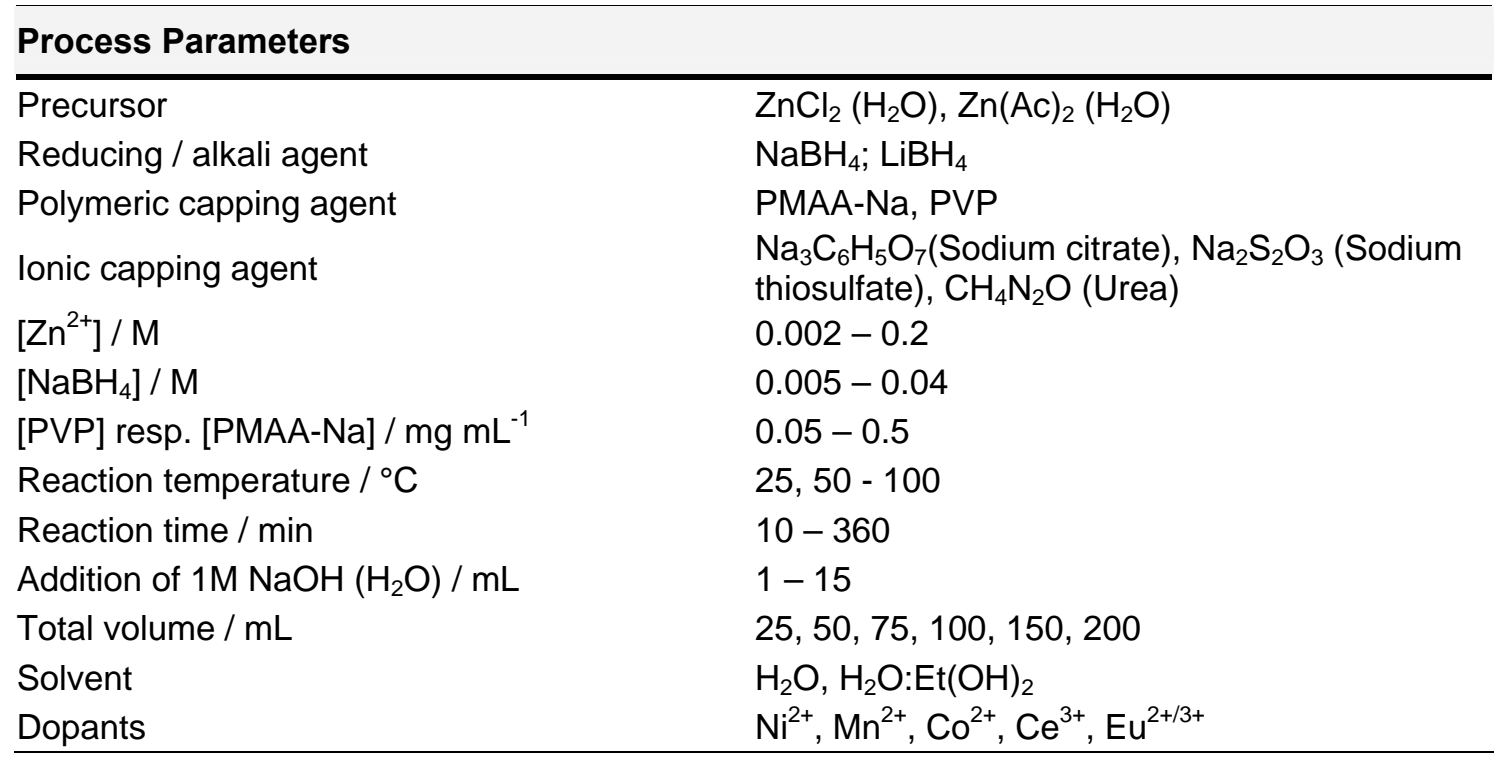

\subsection{Results}

The preparation of $\mathrm{ZnO}$ quantum dots is based on a forced alkaline hydrolysis, which transform aqueous complexed zinc cations into colloidal $\mathrm{ZnO}$ particles at mild reaction conditions and using a polymeric stabilizer to control the size of the quantum dots. The forced alkaline hydrolysis reaction is very quick and shows a rapid colour change to white after the addition of $\mathrm{NaBH}_{4}$. The preparation results in the precipitation of the $\mathrm{ZnO}$ quantum dots, however, the precipitate can be easily re-dispersed. The final zinc oxide suspension appears white under ambient light and shows a yellow-orange fluorescence under UV light $\left(\lambda_{\text {exc }}=254 \mathrm{~nm}-360 \mathrm{~nm}\right.$ ) (figure 4.3). Formed particles show a long fluorescence life-time and also show a high affinity to attach to polymer surfaces (figure 4.3; right) and fibrous material.

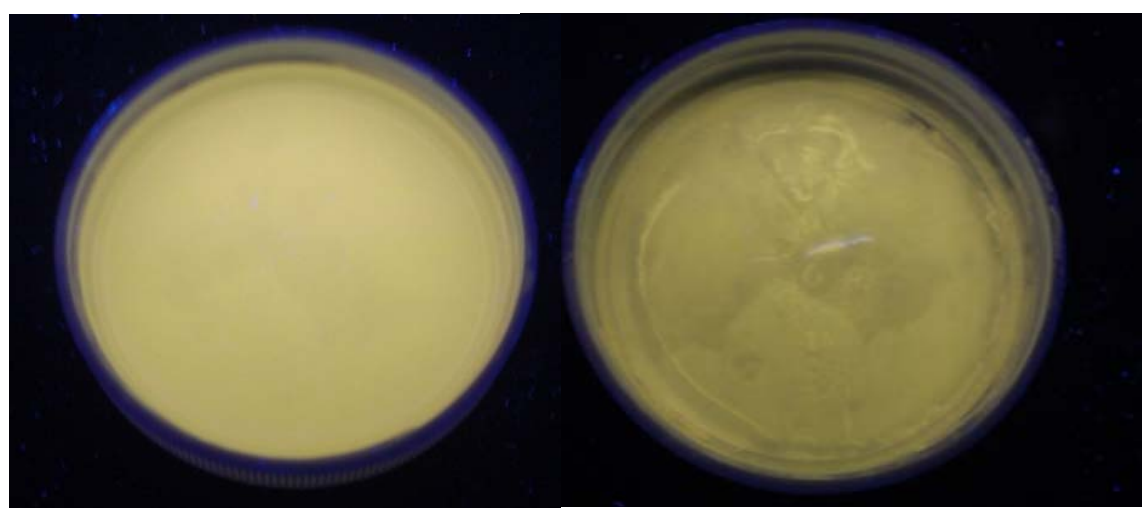

Figure 4.3: Optical fluorescence of $\mathrm{ZnO}$ nanocrystals suspended in water (left) and attached to the base of the plastic receptacle (right) $\left(\lambda_{\text {exc }}=254 \mathrm{~nm}\right)$. 


\subsubsection{Size and morphology}

The effect of the polymeric dispersant as well as the choice of the alkali / reducing agent (listed in table 2.4) on the size, size-distribution and morphology of the final $\mathrm{ZnO}$ nanocrystals were investigated by using transmission and scanning electron microscopy.

\subsubsection{Electron microscopy - TEM}

Figure 4.4 shows the TEM images of $\mathrm{ZnO}$ nanocrystals prepared according to the standard procedure described in chapter 2.

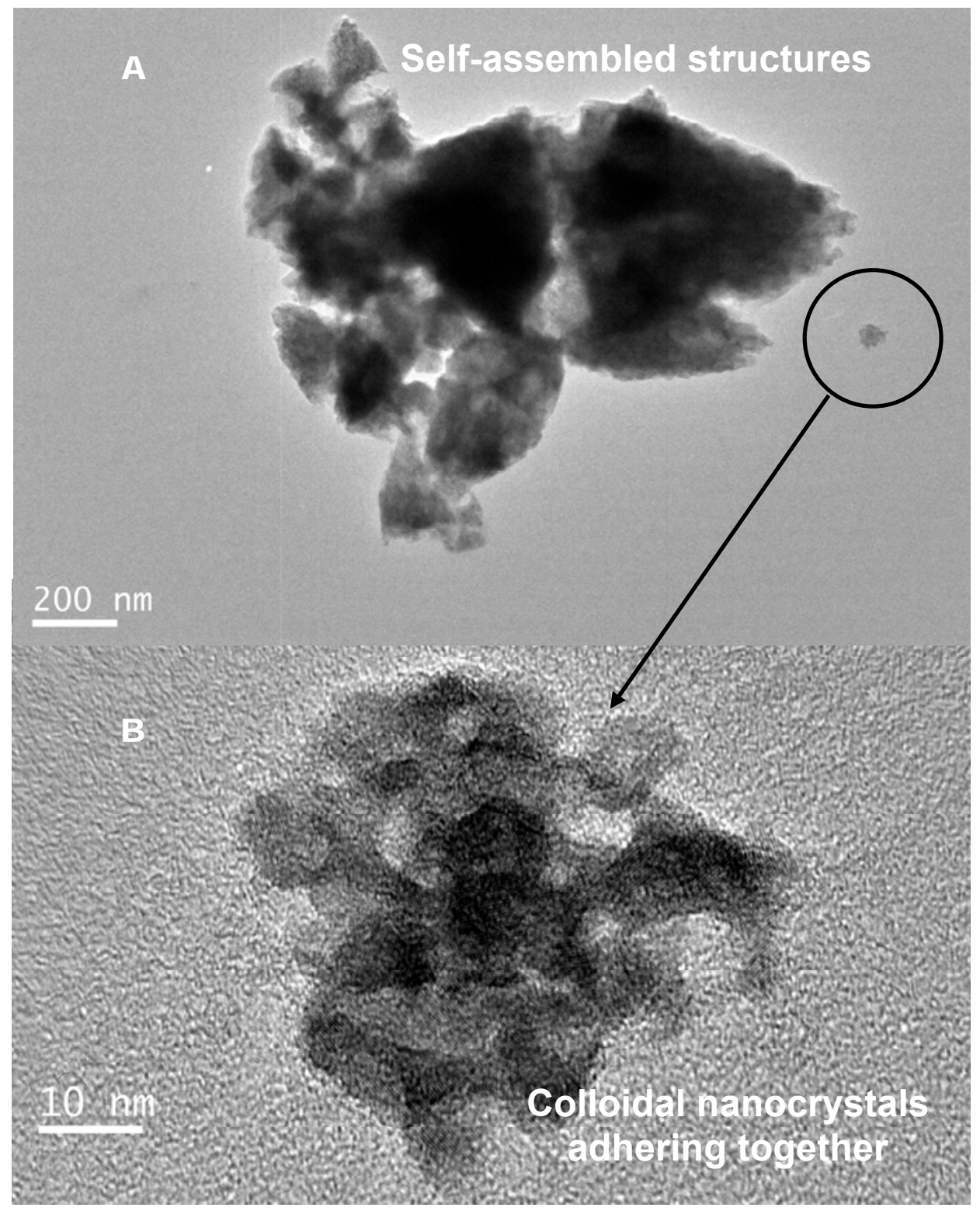

Figure 4.4: TEM image of $\mathrm{ZnO}$ self-assembled structures (A) and detailed view of the small colloidal nanoclusters (B) that form them. 
The TEM image (A) in figure 4.4 shows polycrystalline particles in the form of rice grains varying in the size between 200 and $800 \mathrm{~nm}$. The TEM image (B) in figure 4.4 shows individual $\mathrm{ZnO}$ nanoclusters with wide size distributions from 5 to $10 \mathrm{~nm}$ which are adhering loosely together by a surrounding layer consisting most likely of the polymeric dispersant PVP. At a higher magnification, these nanoclusters show a crystalline lattice structure (see figure 4.5).

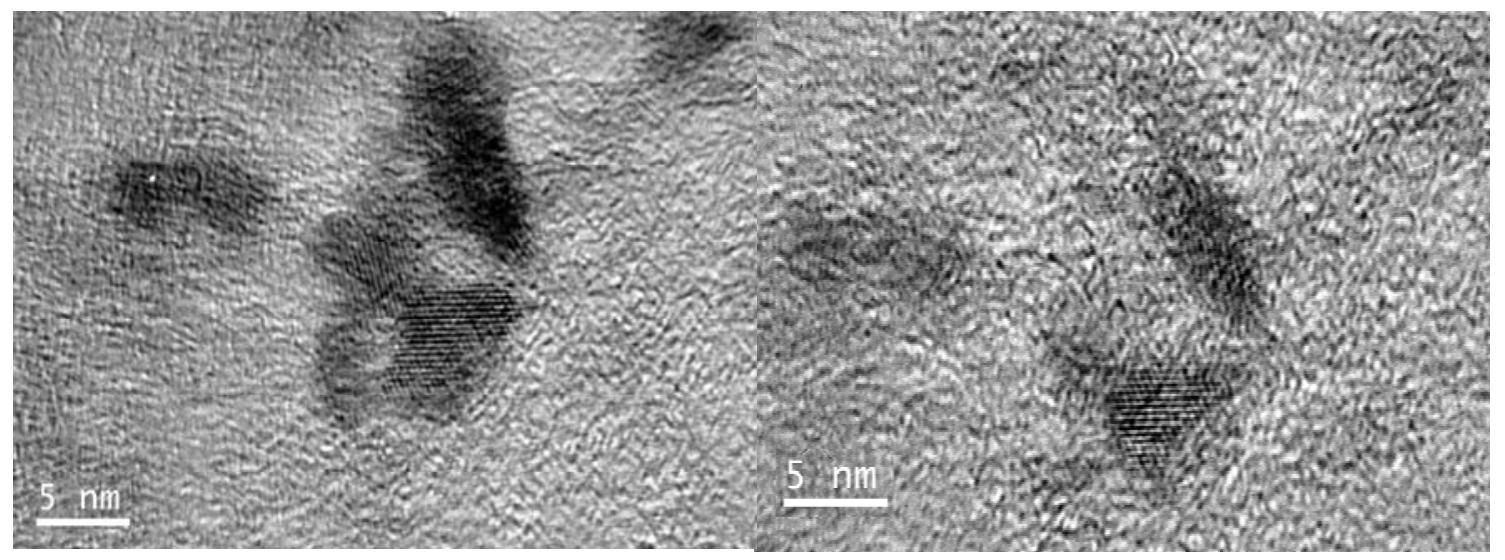

Figure 4.5: TEM images of ellipsoidal ZnO nanoclusters adhering loosely together.

The TEM images show that the colloidal $\mathrm{ZnO}$ crystals show a strong affinity to form agglomerates. However, the self-assembled structures of the polycrystalline particles seem to be unstable when dried under ambient conditions. These structures break in halves as observed in the TEM image in figure 4.4; A.

\subsubsection{Electron microscopy - SEM}

It was found out that the choice of the reducing agent $\mathrm{NaBH}_{4}$ in triethylene glycol dimethylether and $\mathrm{LiBH}_{4}$ in tetrahydrofuran (THF) has a strong effect on the size and size distribution of the self-assembled rice grain structures (see figure 4.6, following page).

The SEM images in figure 4.6 show a wide size-distribution of $\mathrm{ZnO}$ nanocrystals in the shape of rice grains formed by a self-assembled mechanism. $\mathrm{ZnO}$ nanocrystals formed after the addition of $\mathrm{NaBH}_{4}$ (figure 4.6; A) reveal particle sizes between $100 \mathrm{~nm}$ and 1 $\mu \mathrm{m}$. Here, particle sizes around $800 \mathrm{~nm}-1 \mu \mathrm{m}$ predominate. $\mathrm{ZnO}$ nanocrystals prepared with $\mathrm{LiBH}_{4}$ (figure 4.6; B) show identical structures, however the sizedistributions are distinctive smaller in comparison to the procedures with $\mathrm{NaBH}_{4}$. The 
figure B also shows particle sizes between $100 \mathrm{~nm}$ and $1 \mu \mathrm{m}$, however here smaller sizes around 200 and $500 \mathrm{~nm}$ dominate.

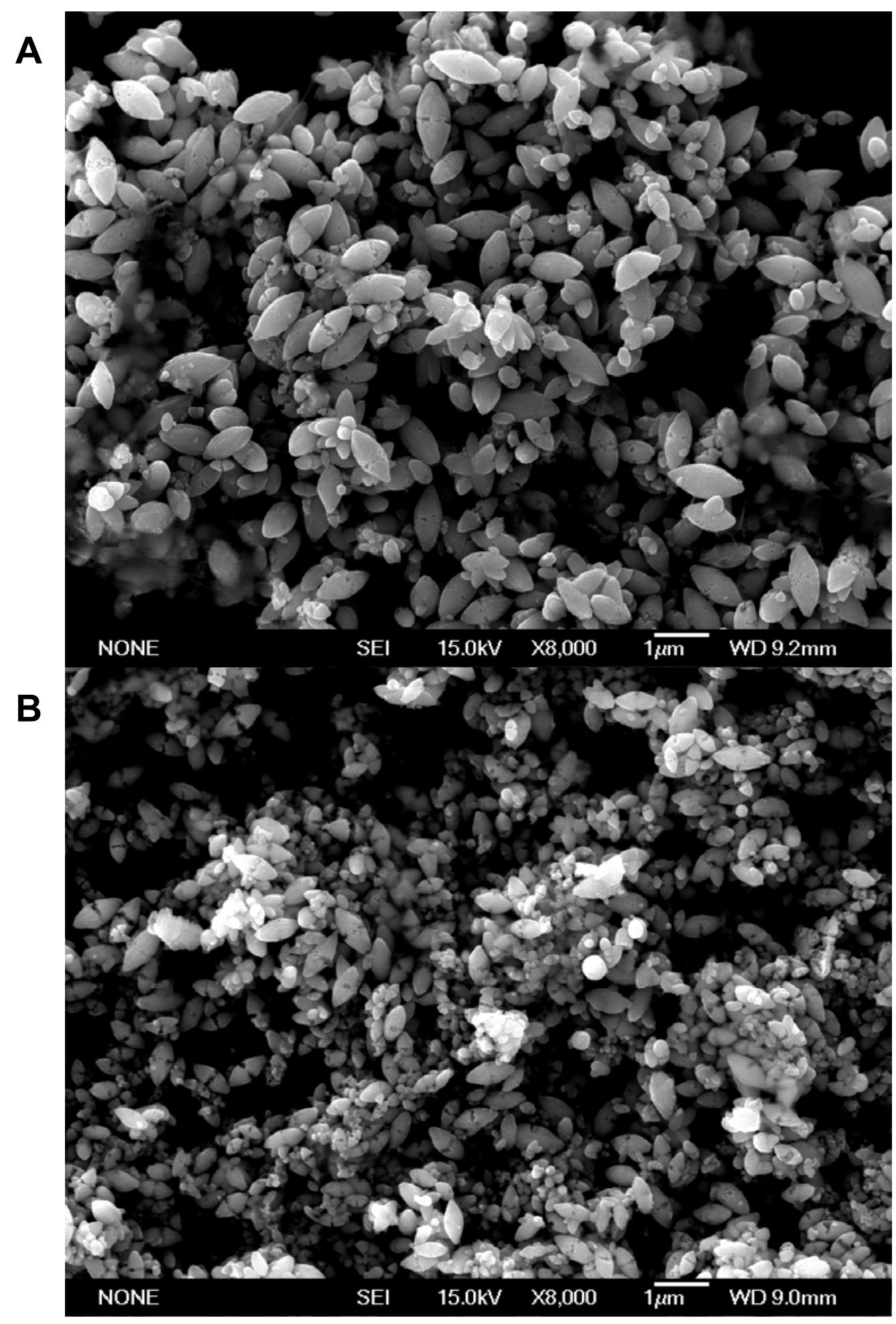

Figure 4.6: SEM images of $\mathrm{ZnO}$ nanoparticles formed with $\mathrm{NaBH}_{4}(\mathrm{~A})$ and $\mathrm{LiBH}_{4}(\mathrm{~B})$ as reducing and alkali agent, respectively. Details of sample preparation are listed in the appendix in table A.6. 
The effect of the polymeric dispersant PVP and PMAA-Na as well their combination on the structure, size and size distribution of the $\mathrm{ZnO}$ nanocrystals was also investigated by SEM. Figure 4.7 show SEM images of $\mathrm{ZnO}$ nanocrystals at a higher magnification ( $\mathrm{x}$ $45 \mathrm{k}$ and $\mathrm{x} 120 \mathrm{k}$ ) prepared according to the standard procedure with PVP (A), PMAA$\mathrm{Na}(\mathrm{B})$ and the combination of PVP and PMAA-Na in a ratio of 1:1 (C).

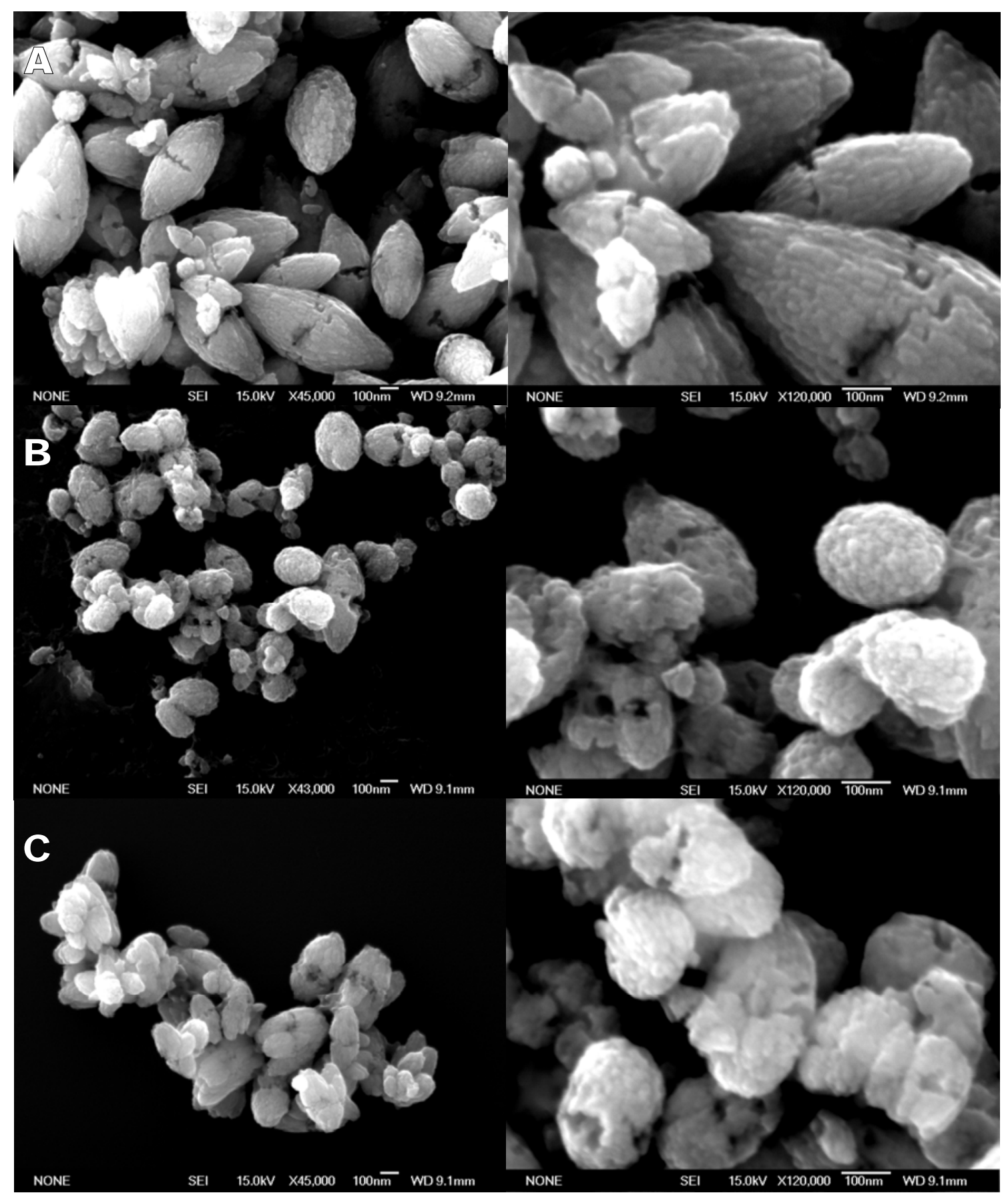

Figure 4.7: High magnification SEM images of ZnO nanoparticles prepared with PVP (A), PMAANa (B) and 1:1 PVP / PMAA-NA (C). Details of sample preparation are listed in the appendix in table A.7. 
The SEM images show that the choice of the polymeric dispersant also affects the size distribution of the nanocrystals. The use of PVP results in the formation of larger structures, whereas PMAA-Na results in particles with a smaller size distribution. As well, the choice of the polymeric dispersant influences the morphology of the formed structures. The agglomerations of colloidal $\mathrm{ZnO}$ nanocrystals with PMAA-Na as capping agent are rounder than those of $\mathrm{ZnO}$ nanocrystals with PVP. The SEM images in figure 4.7 also indicate the porous character of the self-assembled structures.

\subsubsection{Zetasizer}

The size distribution of the $\mathrm{ZnO}$ nanocrystals was also investigated by Zetasizer measurements. The preparation results in mixture of particles with different sizes varying from 0.1 to $1 \mu \mathrm{m}$ as observed in the SEM images (figure 4.6; A). To obtain the smallest particle size, the $\mathrm{ZnO}$ suspension were diluted with water and centrifuged in several steps. Figure 4.8 illustrated the size distribution by intensity of the standard procedure of $\mathrm{ZnO}$ nanocrystals.

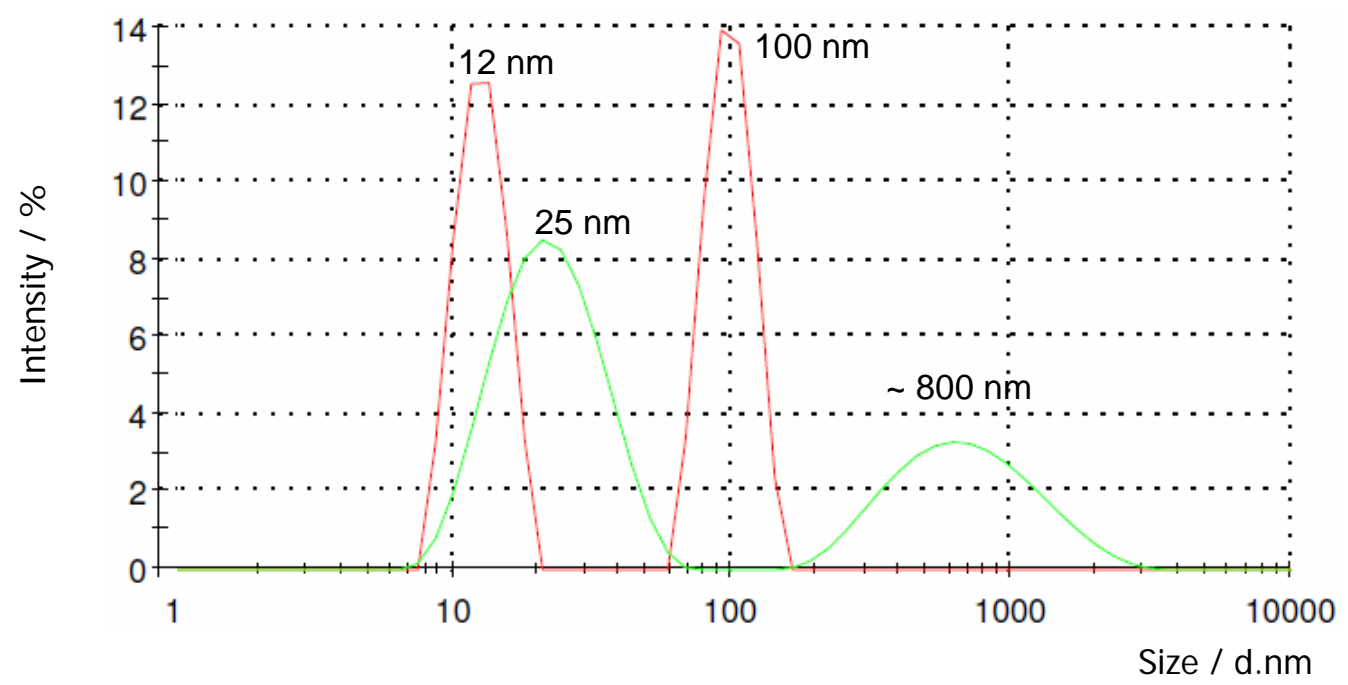

Figure 4.8: Size distribution of ZnO nanocrystals (given in intensity / \%) after several steps of dilution with water and centrifugation; green curve corresponds to a dilution ratio of 1:5 and red curve to 1:10.

The different dilutions of the $\mathrm{ZnO}$ nanocrystals in figure 4.8, 1:5 (green) and 1:10 (red), revealed two distinctive size distributions, respectively. The dilution represented by the red curve correspond to the highest dilution and show two narrow peaks around 10 and $100 \mathrm{~nm}$. The dilution represented by the green curve correspond to an intermediate dilution and show two broad peaks around 25 and $800 \mathrm{~nm}$. 
The size distributions obtained by the different dilution steps agree well with the particle sizes observed by SEM and TEM images. The smallest size obtained by Zetaseizer was circa of $10 \mathrm{~nm}$ and corresponds to colloidal $\mathrm{ZnO}$ nanocrystals (figure 4.4; B). The broader size distribution of circa $25 \mathrm{~nm}$ corresponds to the agglomeration of the colloidal $\mathrm{ZnO}$ nanocrystals (figure 4.4; B). The narrow size distribution around $100 \mathrm{~nm}$ reveals the smallest particle size of the self-assembled structure in the shape of rice grains. The broad size-distribution around $800 \mathrm{~nm}$ agrees with the larger particles of the self-assembled structures seen at the SEM images in figure 4.6 and 4.7.

\subsubsection{Optical properties}

\subsubsection{UV-Visible spectroscopy}

Figure 4.9 shows the absorption spectra of $\mathrm{ZnO}$ aqueous suspensions containing selfassembled structures in the shape of rice grains as well as the absorption spectrum of $\mathrm{ZnO}$ nanocrystals prepared in the presence of cerium $\left(1 \mathrm{~mL} 0.01 \mathrm{M} \mathrm{CeCl}_{3}\left(\mathrm{H}_{2} \mathrm{O}\right)\right)$

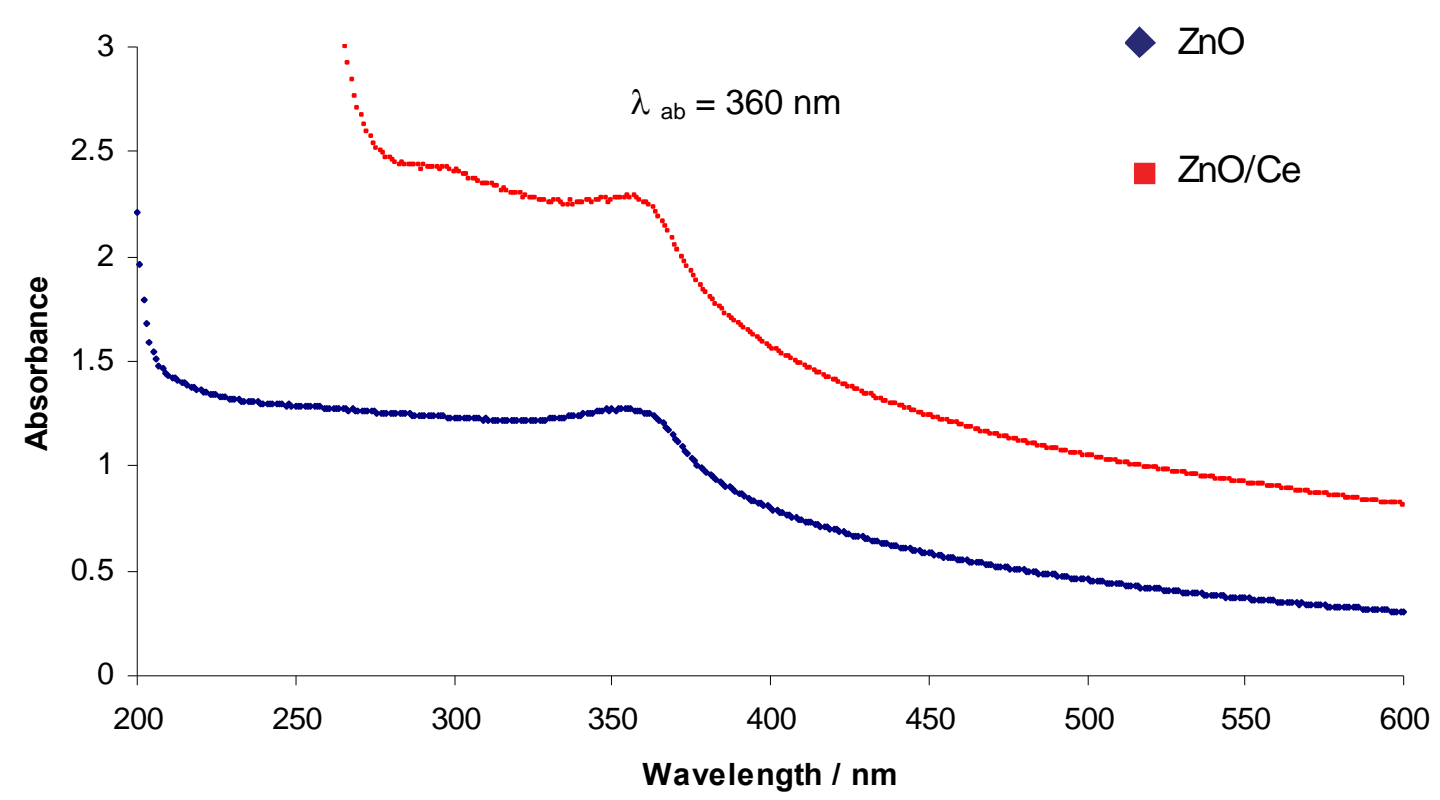

Figure 4.9: Absorbance scan of $\mathrm{ZnO}$ nanocrystals undoped (blue line) and doped with $\mathrm{Ce}^{3+}$ (red line) in aqueous solution. Details of sample preparation are listed in the appendix in table A.8.

For both suspensions, UV-vis spectroscopy revealed an absorbance peak at $360 \mathrm{~nm}$. This suggests that both procedures result in the formation of $\mathrm{ZnO}$ nanoparticles with similar sizes and size-distributions. The position of the absorbance maxima did not 
change significantly after ageing processes. A shift of the absorbance maxima to longer wavelengths would indicate a further growth of the particles in suspension.

\subsubsection{Fluorescence spectroscopy}

$\mathrm{ZnO}$ suspensions show characteristic fluorescence peaks in the UV and visible wavelength range depending on the exciting wavelength used and time of measurement. Figure 4.10 shows the PL scans $\left(\lambda_{\text {exc }}=260 \mathrm{~nm}\right)$ of $\mathrm{ZnO}$ aqueous suspension at different ageing stages and the PL scan of $\mathrm{ZnO}$ suspension doped with $\mathrm{CeCl}_{3}$.

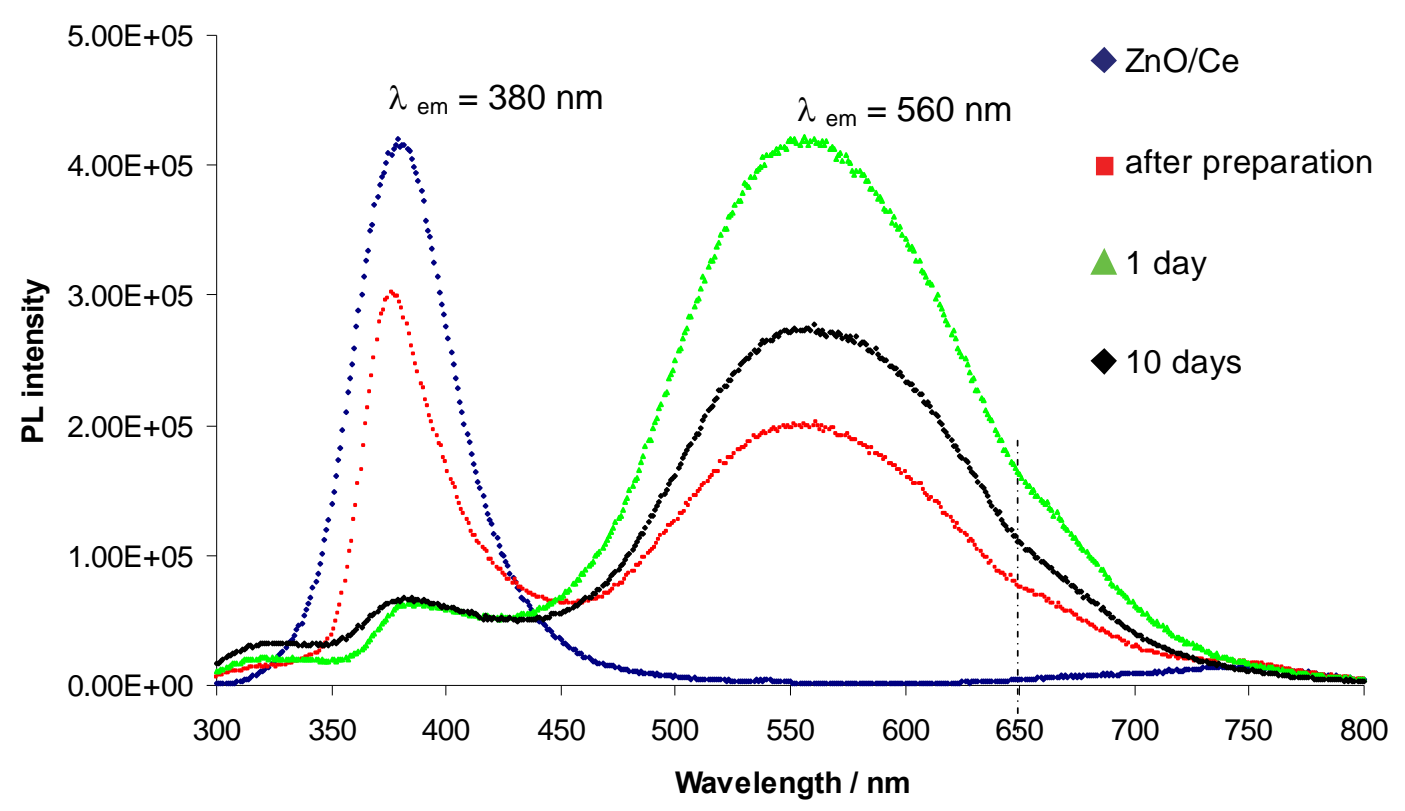

Figure 4.10: $\mathrm{PL}$ scan of $\mathrm{ZnO}$ suspension doped with $\mathrm{CeCl}_{3}$ (blue line) and undoped, measured right after preparation, 1 and 10 days of ageing $\left(\lambda_{\text {exc }}=260 \mathrm{~nm}\right.$, exc. $/ \mathrm{em}$. slit $\left.=10 \mathrm{~nm}\right)$.

When excited with $260 \mathrm{~nm}$, a freshly prepared $\mathrm{ZnO}$ suspension shows two distinct fluorescence peaks, one at $380 \mathrm{~nm}$ and the other at $560 \mathrm{~nm}$ (see figure 4.10, red line). However, ageing of the suspensions results in the reduction of the narrow peak at 380 $\mathrm{nm}$ and increment of the broad emission peak at $560 \mathrm{~nm}$. On the contrary, ZnO suspension doped with $\mathrm{CeCl}_{3}$ shows only one fluorescence peak around $375-380 \mathrm{~nm}$ (see figure 4.10; blue line). Here, ageing does not affect the PL peak, but shift the emission to higher wavelengths $(385 \mathrm{~nm})$. 
When excited with $350 \mathrm{~nm}$, the $\mathrm{ZnO}$ suspension doped with $\mathrm{CeCl}_{3}$ shows a broad emission peak at $550 \mathrm{~nm}$. The PL intensity of the broad emission peak, however, is significantly lower than that of un-doped $\mathrm{ZnO}$ nanocrystals (see figure 4.11).

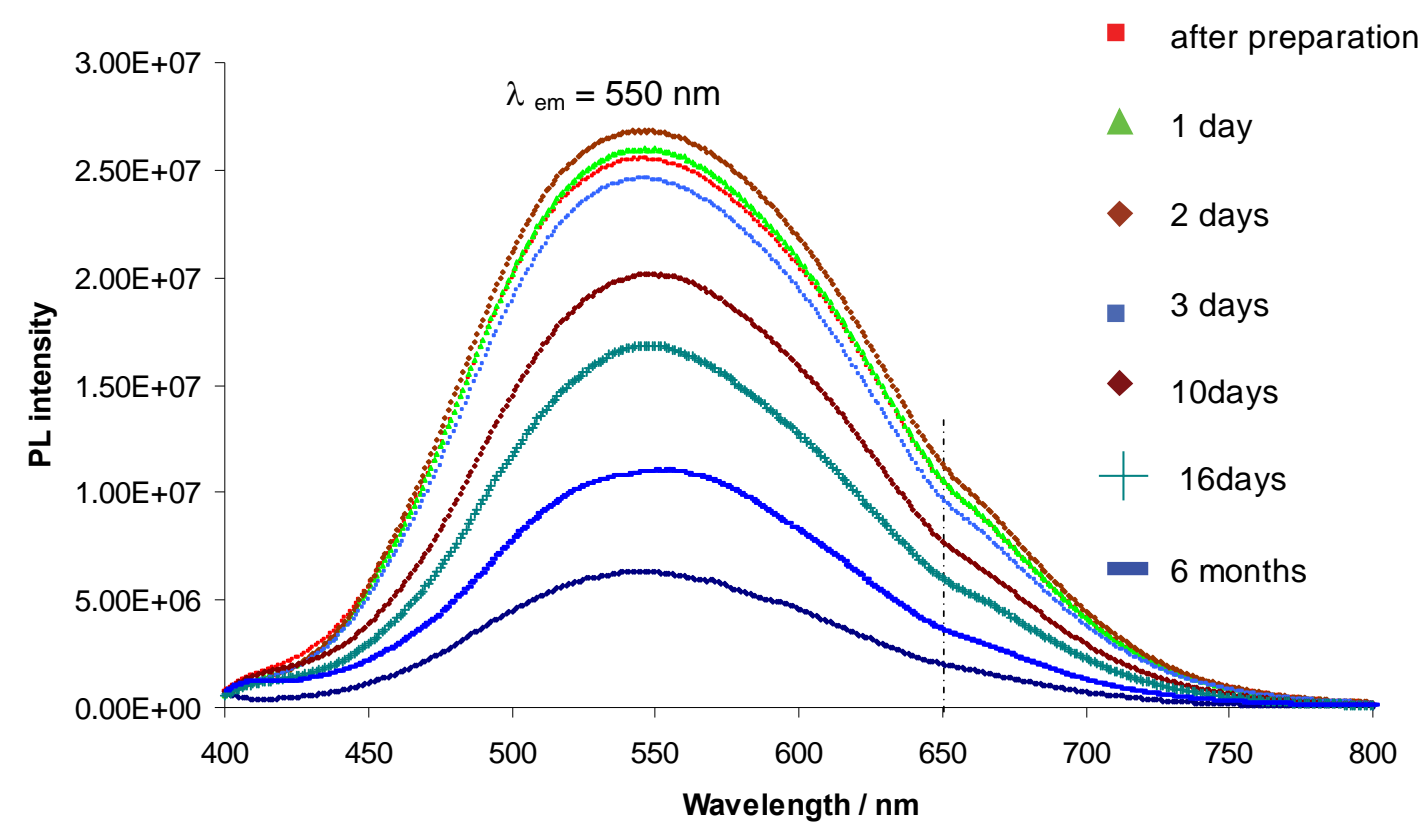

Figure 4.11: Emission spectra of rice grain shaped $\mathrm{ZnO}$ nanocrystals in aqueous solution at different ageing stages and $\mathrm{ZnO}$ nanocrystals doped with $\mathrm{Ce}^{3+}$ (blue line) $\left(\lambda_{\text {exc }}=350 \mathrm{~nm}\right.$ (exc. $/ \mathrm{em}$. slit $=10 \mathrm{~nm})$ ).

Figure 4.11 shows that the PL intensity of the $\mathrm{ZnO}$ suspension remains nearly unchanged in the first three days after preparation. However with ageing, the PL intensity of the suspension progressively decreases. Nevertheless, fluorescence is still observed after two years of ageing. It is also interesting that all $\mathrm{ZnO}$ suspensions, doped and undoped, show a hump around $675 \mathrm{~nm}$, when excited with $350 \mathrm{~nm}$ (see figure 4.11).

Ageing, the intensity of the broad emission peak at $550 \mathrm{~nm}\left(\lambda_{\text {exc }}=350 \mathrm{~nm}\right)$ is also affected by PVP and $\mathrm{NaBH}_{4}$ concentration and reaction temperature. The effect of these different process parameters on the PL intensity of the emission peak is graphically illustrated in figures $4.12-4.14$. 


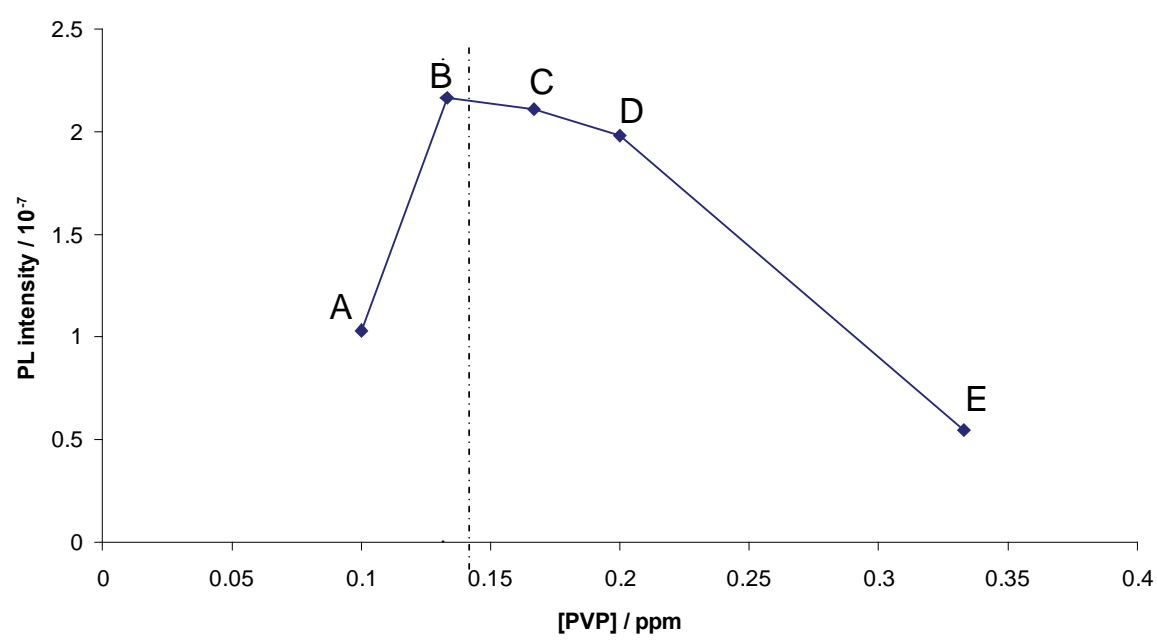

Figure 4.12: Effect of PVP concentration on the PL intensity of the maxima emission wavelength in the visible range $\left(T=70{ }^{\circ} \mathrm{C}\right)\left(\lambda_{\text {exc }}=350 \mathrm{~nm}\right.$; exc. / em. slit $\left.=10 \mathrm{~nm}\right)$. Details of sample preparation are listed in the appendix in table A.9.

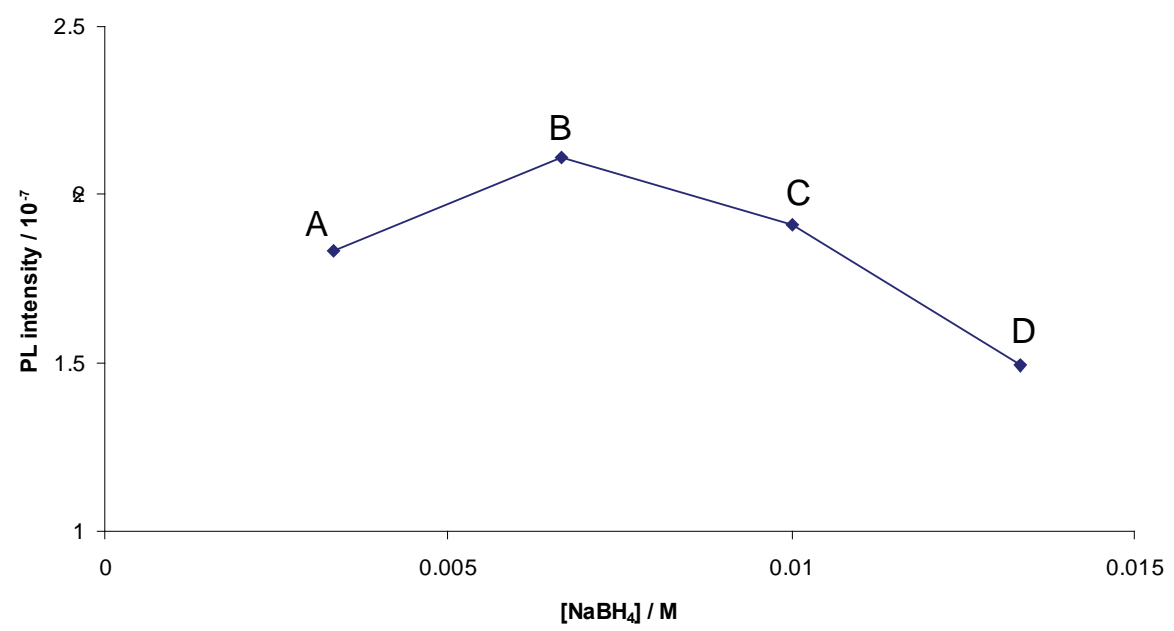

Figure 4.13: Effect of $\mathrm{NaBH}_{4}$ concentration on the $\mathrm{PL}$ intensity of the maxima emission wavelength in the visible range $\left(T=70{ }^{\circ} \mathrm{C}\right)\left(\lambda_{\text {exc }}=350 \mathrm{~nm}\right.$; exc. $/$ em. slit $\left.=10 \mathrm{~nm}\right)$. Details of sample preparation are listed in the appendix in table A.10.

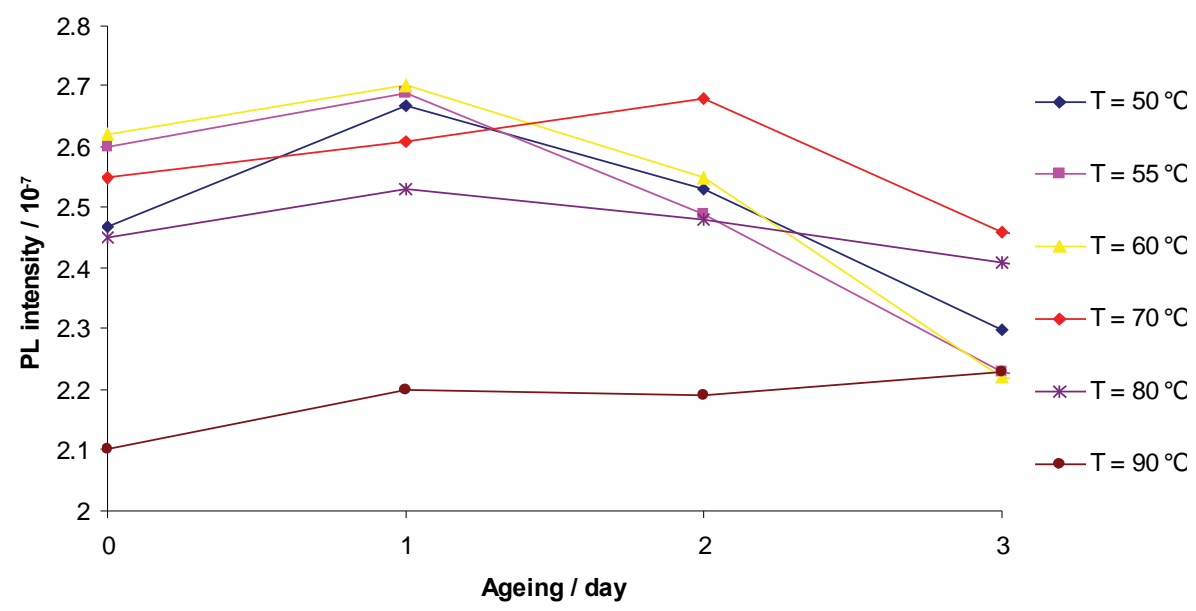

Figure 4.14: Effect of temperature on the PL intensity of the maxima emission wavelength in the visible range $\left(\lambda_{\text {exc }}=350 \mathrm{~nm}\right.$; exc. $/$ em. slit $\left.=10 \mathrm{~nm}\right)$. 
Changing the process parameters shown above did not affect the maxima wavelength of the broad emission peak $\left(\lambda_{\text {exc }}=350 \mathrm{~nm}\right)$. However, when the $\mathrm{ZnO}$ particles were dispersed in EtOH solution, a blue shift of the maxima emission wavelength from 550 to $530 \mathrm{~nm}$ was observed in the visible range. Furthermore, a slightly blue shift was observed when the total volume of the $\mathrm{ZnO}$ suspension was reduced. Here, the blue shift of the broad emission peak is attributed to the ionic strength of dispersion medium.

\subsection{Discussion}

$\mathrm{ZnO}$ quantum dots showing green yellow fluorescence were formed in aqueous solution by a sol-gel method using $\mathrm{ZnCl}_{2}$ as zinc precursor material, PVP as polymeric dispersant and $\mathrm{NaBH}_{4}$ as reducing and alkali reagent. The sol-gel method produces small colloidal $\mathrm{ZnO}$ nanocrystals in the size of $5-12 \mathrm{~nm}$. These nanocrystals are spherical and ellipsoidal in shape and show a high tendency to form agglomerates. By adhering loosely together, the colloidal nanocrystals form polycrystalline particles in the shape of rice grains varying in the size of $100-1000 \mathrm{~nm}$.

\subsubsection{Different steps of the particle growth}

$\mathrm{ZnO}$ quantum dots are formed by a sol-gel method induced by a forced alkaline hydrolysis. The size and size distribution of quantum dots using sol-gel techniques are difficult to control. Here, the growth of the quantum dots is confined by the adsorption of polymeric chains on the surface of the particles during the hydrolysis and condensation reaction. However, the nanocrystals show a strong affinity to agglomerate and form self-assembled structures in the shape of rice grains in different sizes (see figure $4.4 ; \mathrm{A})$. The presence of hydroxyl ligands $\left(\mathrm{OH}^{-}\right)$on the surface of the nanocrystals may support the formation of a porous host matrix consisting of the polymeric dispersant PVP and the oxidation products of the reducing (alkali) agent $\mathrm{NaBH}_{4}$. The nanocrystalline particles are embedded in the gel-like host matrix by forming cross-links.

The formation of $\mathrm{ZnO}$ quantum dots using forced alkaline hydrolysis can be divided in the following steps:

- Formation of a dispersal complex compounds; Polymer/metal salt complex 
- Addition of alkali reagent induce forced alkaline hydrolysis and condensation reactions

- Confinement of the particle growth by the adsorption of polymer chains on the surface of the colloidal nanocrystals

- Formation of a polymeric network structure (gel) reinforced by cross links between $\mathrm{ZnO}$ quantum dots

- Further particle growth with ageing - Ostwald ripening

\subsubsection{Forced alkaline hydrolysis}

The addition of sodium borohydride (alkali) to the acidic zinc chloride / PVP aqueous solution at a reaction temperature of $70^{\circ} \mathrm{C}$ induces a forced alkaline hydrolysis reaction, which produce small colloidal $\mathrm{ZnO}$ particles in the size of $5-12 \mathrm{~nm}$. The overall chemical reaction can be simplified with the following equation:

$$
\mathrm{Zn}^{2+} \text { (aqua) } \quad+2 \mathrm{OH}^{-} \quad \rightleftarrows \quad \mathrm{ZnO}_{\text {(solid) }}+\mathrm{H}_{2} \mathrm{O}
$$

Despite the apparent simplicity of this synthesis process, in reality it can be very complex and result in various reaction products. Here, $\mathrm{NaBH}_{4}$ was used as an alkali and reducing agent for the sol-gel method. $\mathrm{NaBH}_{4}$ added to water create a sodium borate buffer solution $(\mathrm{pH} \sim 8)$ turning the solution alkaline and leading to the precipitation of zinc oxide.

$$
\begin{array}{llll}
\text { Oxidation: } & \mathrm{NaBH}_{4} & \rightarrow & \mathrm{Na}^{+}+\mathrm{BH}_{3}+1 / 2 \mathrm{H}_{2}+\mathrm{e}^{-} \\
\text {Reduction: } & \mathrm{H}_{2} \mathrm{O}+\mathrm{e}^{-} & \rightarrow & \mathrm{OH}^{-}+1 / 2 \mathrm{H}_{2} \\
\text { Redox reaction: } & \mathrm{NaBH}_{4}+\mathrm{H}_{2} \mathrm{O} & \rightarrow & \mathrm{BH}_{3}+\mathrm{H}_{2}+\mathrm{NaOH}
\end{array}
$$

The hydrolysis of $\mathrm{BH}_{3}$ results in the formation of hydrogen and boric acid $\mathrm{B}(\mathrm{OH})_{3}$ a weak lewis acid in aqueous solution.

$$
\begin{array}{llll}
\text { Hydrolysis: } & \mathrm{BH}_{3}+3 \mathrm{H}_{2} \mathrm{O} & \rightarrow & \mathrm{B}(\mathrm{OH})_{3}+3 \mathrm{H}_{2} \\
\mathrm{~B}(\mathrm{OH})_{3}+\mathrm{H}_{2} \mathrm{O} & \rightarrow & \mathrm{B}(\mathrm{OH})_{4}{ }^{-}+\mathrm{H}^{+} \\
\mathrm{B}(\mathrm{OH})_{4}{ }^{-}+\mathrm{H}^{+}+\mathrm{NaOH} & \rightarrow & \mathrm{NaB}(\mathrm{OH})_{4}+\mathrm{H}_{2} \mathrm{O}
\end{array}
$$


A mixture of $\mathrm{B}(\mathrm{OH})_{3}$ and $\mathrm{NaB}(\mathrm{OH})_{4}$ creates a alkaline buffer solution of sodium borate $(\mathrm{pH} \sim 8)$.

$$
2 \mathrm{NaB}(\mathrm{OH})_{4}+2 \mathrm{~B}(\mathrm{OH})_{3} \quad \rightarrow \quad \mathrm{Na}_{2}\left[\mathrm{~B}_{4} \mathrm{O}_{5}(\mathrm{OH})_{4}\right]+5 \mathrm{H}_{2} \mathrm{O}
$$

It is important to note that the hydrolysis of $\mathrm{NaBH}_{4}$ and the formation of $\mathrm{OH}^{-}$and $\mathrm{H}_{2}$ molecules are strongly dependent on the $\mathrm{pH}$. At low $\mathrm{pH}$, the redox reaction and hydrolysis rection shown above will proceed very fast.

The precipitation reaction is induced by the formation of $\mathrm{NaOH}$ (equation (30)) leading to the increase of $\mathrm{pH}$ of the zinc salt / polymer complex aqueous solution and causing the hydrolysis reaction of the water complexed zinc cations. Hydrolysis leads to formation of reactive hydrolysed intermediates, zinc cation monomers and polymers (see equation (19) - (21)), which are metastable and react further to form a solid oxide phase through poly-condensation reactions.

However, hydrolysis can also result in the precipitation of the zinc cations as a hydroxide, which is insoluble in a wide range of $\mathrm{pH}$ values:

$$
\mathrm{Zn}^{2+} \text { (aqua) } \quad+2 \mathrm{OH}^{-} \quad \rightleftarrows \quad \mathrm{Zn}(\mathrm{OH})_{2} \text { (solid) }
$$

To form a stable suspension of colloidal $\mathrm{ZnO}$ nanocrystals, the reaction conditions have to be optimised. In general, the rate of hydrolysis and poly-condensation reactions is influenced by the reaction temperature and presence of complexing agents.

For example, high temperature enhances condensation reaction by the elimination of water. On the other hand, sol-gel processes carried out at low temperature will result in the formation of quantum dots with a poor degree of crystallinity and surface defects. To enhance the crystalline character of the particles, post-treatment methods at high temperature can be applied.

Hydrolysis and condensation are kinetically controlled reactions and can be influenced by complexing one of the reaction partners or intermediates states. In this sol-gel method, PVP initially stabilises zinc cations in aqueous solution by forming a complex compound, $\mathrm{ZnCl}_{2} / \mathrm{PVP}$ before the chemical reaction is induced. However, zinc cations 
and chloride anions can also form different complexes in aqueous solution. Depending on the complexation, less "free" cations are available for the hydrolysis reaction, which will affect the kinetic of the hydrolysis reaction. Furthermore, the intermediate formed by the hydrolysis will also be complexed by PVP. This in turn will affect the kinetic of the condensation reaction and hence particle growth. For this reason, complexing agents can be used to control the size as well as the morphology of the nanocrystals. Nevertheless, most of the time, hydrolysis and condensation reactions are so fast that they can not be controlled easily. That's why it is difficult to obtain a narrow sizedistribution of colloidal nanocrystals using forced alkaline hydrolysis.

It can be assumed that the crystal lattice structure of the $\mathrm{ZnO}$ nanocrystals is also affected by the reducing properties of $\mathrm{NaBH}_{4}$ and the immediate formation of $\mathrm{H}_{2}$ by the addition of $\mathrm{NaBH}_{4}$ to water. $\mathrm{NaBH}_{4}$ is a strong reducing agent, which may react with the formed precipitate and create defects such as interstitial zinc, reduced zinc lattice ions, in the solid. Furthermore, hydrogen released by the hydrolysis of $\mathrm{NaBH}_{4}$ can also be incorporated in the solid during hydrolysis and condensation reactions. Both, interstitial zinc and hydrogen would cause a defect structure of $\mathrm{ZnO}$.

$\mathrm{ZnO}$ nanocrystals have been also formed by the addition of $1 \mathrm{~mL} 1 \mathrm{M} \mathrm{NaOH}\left(\mathrm{H}_{2} \mathrm{O}\right)$ solution to the $\mathrm{ZnCl}_{2}$ / PVP aqueous complex solution at constant reaction conditions. However, the $\mathrm{ZnO}$ suspension shows a lower fluorescence intensity in the visible range and a stronger repelling effect on plastic surfaces which can be attributed to the adsorption of $\mathrm{OH}^{-}$ligands around the particle surface. This surface layer results in the electrostatic stabilization of the nanocrystals due to the electrostatic repulsion of similar charged particles.

On the contrary, $\mathrm{ZnO}$ quantum dots formed by the addition of $\mathrm{NaBH}_{4}$ show high fluorescence intensity in the visible range and high affinity to attach on surfaces such as plastic (see figure 4.3; right). Based on this behaviour, it can be used to functionalise different materials such as wool and paper fibres. 


\subsubsection{Confinement of particle growth}

To ensure a stable colloidal suspension, the particles need to be stabilized in the dispersion medium. Due to the hydrolysis reaction, the surface area of the $\mathrm{ZnO}$ quantum dots is functionalised with hydroxide groups. These $\mathrm{OH}^{-}$ligands increase the chemical reactivity of the particles and promote condensation and agglomeration processes.

The polymeric dispersant PVP, initially used to stabilize the zinc ions in aqueous solution, will form a bulky surface layer around the particles during the chemical reaction. This organic bulky surface layer confines the particle growth via nucleation and condensation reactions and also counterbalances attractive van der Waals forces between particles due to the steric hindrance of the polymer chains. Based on the surface modification of the quantum dots with a polymeric dispersant, the final properties are determined not only by the inorganic semiconductor material, but also by the properties of the organic surface layer around the particles.

In general, polymers can be attached to the particle surface through anchor groups such as hydrogen-bonding groups (esters, ketones, ethers), polarizing groups, ionic or acidic/basic groups (carboxylates, sulfonates, phosphonates). The hydroxyl groups at the surface area of the $\mathrm{ZnO}$ quantum dots most likely undergo hydrogen bridge bonds with functional groups of organic macromolecules. The polymeric dispersants PVP contain oxygen and nitrogen atoms which can form a hydrogen bond with an $\mathrm{OH}^{-}-$ ligands at the surface of the $\mathrm{ZnO}$ quantum dots. Consequently, functional groups of PVP can coordinate with the $\mathrm{OH}^{-}$- ligands at the surface and form a protection sphere around the particles. The effectiveness of the protection sphere, steric repulsion between the polymer layers around the particles, depends on the optimum length of the polymer chains and optimum uptake of the polymer on the particle surface. Aside from these, the polymer must be compatible with the solvent.

To suppress chemical interaction between the particles caused by attractive van der Waals forces, the polymer chains need a minimum length to overcome these forces. As well, a low uptake of polymer chains is not able counterbalance attractive van der Waals forces between the particles. On the contrary, a large uptake can result in the collapse of the chains on the surface of the particles resulting in a limited performance. For an 
optimal steric stabilization effect, the polymer chains have to be absorbed in an aligned position without sticking together. If the chains interact with each other, the protection sphere can collapse resulting in the instability of the particle dispersion. The interaction between the chains is usually prevented by a proper solvation of the polymer chains. If the interaction between polymer and solvent molecules is favoured, the length of the chains can also be extended from the particle surface into the dispersion medium resulting in an increment of the protection sphere around the particles. For this reason, the solvent affinity of the polymer chains is a very important factor in steric stabilization.

The stability of the protection sphere is usually dependent on the chemical bonding between surface atoms and functional groups of the polymer chains. In order to be effective, the chemical bonding between polymer chain and particle surface have to be strong and enduring. Thereby, the surface properties of the particles play an important role. Metal oxides have a heterogeneous surface. The charge of metal oxides is also known to be frequently delocalised over the whole particle surface and can change over a wide area depending on the metal and $\mathrm{pH}$. Therefore, mostly weak chemical bondings such as ionic or hydrogen bonds are formed between polymer chains and metal oxides. Weak chemical bonds, however, can lead to desorption and loss of polymer chains at the particle surface. This will reduce the surface coverage of the particles and hence increase the chemical reactivity of the particles towards agglomeration processes.

Furthermore, it can be assumed that besides the polymeric dispersant, the oxidation / degradation products of $\mathrm{NaBH}_{4}$ may also be adsorbed on the particle surface and influence the particle growth of the $\mathrm{ZnO}$ quantum dots as well as their optical properties.

\subsubsection{Formation of self-assembled structures}

$\mathrm{ZnO}$ quantum dots formed by the addition of $\mathrm{NaBH}_{4}$ to the $\mathrm{ZnCl}_{2} / \mathrm{PVP}$ aqueous complex solution undergo further particle growth. They combine with each other during hydrolysis and condensation reactions and form larger self-assembled structures in the shape of rice grains. The smallest size of the self-assembled structure is around $100 \mathrm{~nm}$, whereas larger structure can obtain a length of $1 \mu \mathrm{m}$ (see Zetasizer measurements in 
figure 4.8 and SEM image in figure 4.6; A). However, the self-assembled structures are fragile and break in halves independent on the size of the particles (see TEM image 4.4 and SEM images in figures 4.6 and 4.7).

The agglomeration of the $\mathrm{ZnO}$ quantum dots during the chemical reaction is shown by the instant colour change from transparent to white after the addition of $\mathrm{NaBH}_{4}$. The white colour of the colloidal suspension is attributed to the light scattering properties of polycrystalline particles. This kind of particle growth in which the particles adhere together by van der Waals forces, however, is difficult to control and often result in the precipitation of the $\mathrm{ZnO}$ quantum dots from solution.

TEM images of these self-assembled structures revealed that the $\mathrm{ZnO}$ quantum dots are most likely hold together by the polymeric dispersant. Furthermore, Zetasizer revealed that only small particles of $10-12 \mathrm{~nm}$ are detected, when the $\mathrm{ZnO}$ suspension was diluted in several steps with water (see figure 4.8). This suggests that the association of the quantum dots in the self-assembled structures is based on non-covalent bondings such as hydrogen bridge bondings, weak ionic bondings or van der Waals interactions.

The size and shape of the self-assembled structures is also dependent on the choice of the polymeric dispersant. In this method, PVP and PMAA-NA as polymeric dispersant were used for the synthesis of $\mathrm{ZnO}$ quantum dots, since these polymers are known to coordinate well to metal cations and absorb well on oxide surfaces. The use of PVP, a non-ionic polyelectrolyte, results in the formation of larger and more robust (compact) self-assembled structures; whereas PMAA-Na, a ionic polyelectrolyte, forms smaller and more porous sol-gel agglomerates (see SEM images in figure 4.7). A combination of both polymers, PVP and PMAA-Na in different ratios, can be used to control the size and shape of the nanostructure of the polymer - quantum dot composite (see figure 4.7; C).

It can be assumed that the spontaneously organisation of the quantum dots in larger selfassembled structures is induced by a stabilization mechanism. In general, when the organic surface layer around the particles is not strong enough to counterbalance attractive van der Waals forces, particles will agglomerate. By sticking together, the colloidal suspension obtains a higher stability through the reduction of the surface-to- 
volume ratio. Furthermore, particles coated with a steric stabilizer can be linked to one another by cross-link reactions. Cross-link reactions may occur simultaneously with hydrolysis and condensation reaction. Thereby, polymer chains adsorbed on the surface of the quantum dots interact with each other through attractive and repulsive forces and form a network structure, polymer matrix, which immobilise the $\mathrm{ZnO}$ quantum dots in the dispersion medium. The formation of the polymeric network structure may be stabilised by selective adsorption and desorption processes of the polymer chains on the surface of the crystals.

The formation of a polymeric nanostructure, however, is only possible when the polymer chains are very long and the affinity between the polymer chains is large enough to overcome the repulsive forces caused by the steric stabilization. As mentioned previously, the effectiveness of the steric stabilization using polymer chains is dependent on several factors such as the optimum length of the chains, optimum uptake of the polymer chains on the particle surface and solvent affinity of the polymer chains. Furthermore, the stability of the protection sphere around the particles is determined by the chemical bonding between polymer chains and particle surface. The chemical bonding, in turn, is dependent on the type of anchor groups of the polymer chains, charge distribution of the particle surface and $\mathrm{pH}$, which determines the degree of dissociation of the hydroxyl groups at the particle surface.

However, the steric protection sphere around the particles may also be affected by the presence of counter ions and temperature during the chemical reaction. These factors can interrupt the chemical interaction between polymer chains and solvent molecules resulting in the collapse of the polymer chains at the particle surface. This, however, will allow intermolecular interaction between the quantum dots. Thereby, the quantum dots can form large and small polycrystalline particles, depending on the extent of intermolecular interaction between the particles. The extent of intermolecular interaction between the particles is determined by the shielding / repelling effect of the polymer chains at the particle surface.

For example, PMAA-Na - is an anionic surfactant which contains negatively charged carboxylic groups in the main chain. These negative groups impart additionally a negative charge to the particle surface, which increase the repulsive effect of the 
surfactant layer around the particles through electrostatic repulsion. For this reason, it can be assumed that particles coated with PMAA are better stabilized in the dispersion medium than particles coated with PVP. Consequently, the intermolecular interaction between PMAA functionalised $\mathrm{ZnO}$ quantum dots are complicated by the electrostatic charge around the particles resulting in the formation of smaller and less robust selfassembled structures.

The morphology, size and size distribution of the polycrystalline particles is basically determined by the nanostructure of the polymer matrix formed by the intermolecular interaction between $\mathrm{ZnO}$ quantum dots. However, the size distribution of the selfassembled structures is also influenced by the stirring time. Longer stirring times $(>60$ min) result in the formation of larger self-assembled structures shown by a quicker settlement of the particles after preparation $(10 \mathrm{~min})$. It can be assumed that longer stirring times promote the re-dispersion of small agglomerates. In general, small polycrystalline structures are less stable and tend to dissolve into their respective crystals. These crystals will then attach to larger particles, which are thermodynamically more stable. This process can be compared with the Ostwald ripening process which promotes the growth of particles with ageing.

Ageing processes may also lead to the loss of polymer chains at the particle surface through ligand exchange reaction with solvent molecules and / or through selective desorption processes by the formation of larger self-assembled structures. Without a strong surface coverage, however, the particles can come closer together by van der Waals forces resulting in an irreversible process where the particles remain strongly adhered. In general, the extent, to which the particles adhere together in the selfassembled structures, is mainly determined by the repulsive forces between the polymer chains adsorbed onto the particle surface. A high uptake of polymers will increase the thickness of the coating around the particles, which prevent the particles from coming too close together due to the greater steric barriers around the particles. Thereby, the self-assembled structures can be broken by dilution processes. 


\subsubsection{Optical properties}

$\mathrm{ZnO}$ quantum dots show optical fluorescence in the UV-vis and visible range of the spectrum when excited with UV light $\left(\lambda_{\text {exc }}=260 \mathrm{~nm}\right)$. This property, however, is not permanent and the fluorescence intensities of the peaks change with ageing. For instance, the sharp fluorescence peak observed at ca. $380 \mathrm{~nm}$ decreases with ageing, while the broad fluorescence peak at ca. $560 \mathrm{~nm}$ increases. After 3 days of ageing, the peak at ca. $380 \mathrm{~nm}$ is reduced two small bumps in the scan, one at $325 \mathrm{~nm}$ and another at $390 \mathrm{~nm}$, respectively. Ageing of the suspension for $>3$ days results in the progressively decrease of the fluorescence intensity of the broad emission peak in the visible range.

The emission peak at ca. $380 \mathrm{~nm}\left(\lambda_{\mathrm{exc}}=260 \mathrm{~nm}\right)$ is directly related to the band-edge emission of the spherical zinc oxide quantum dots, while the broad emission band in the visible range $\left(\lambda_{\mathrm{em}}=540-560 \mathrm{~nm}\right)$ is caused by surface defects. Surface defects introduce energy levels within the band gap of the inorganic semiconductor, which then act as charge carrier traps for the radiative recombination of the electron-hole pair between the LUMO and HOMO energy state. By trapping the excited electron or hole within the band gap area, the band-edge emission is quenched.

An increment of the defect emission and a reduction of the band-edge emission within the first three days of preparation indicate ageing processes are affecting the surface state of the $\mathrm{ZnO}$ quantum dots and likely also the size of the $\mathrm{ZnO}$ quantum dots. The size, however, plays an important role in the band-edge emission of quantum dots.

\subsubsection{Band-edge emission of $\mathrm{ZnO}$ quantum dots}

$\mathrm{ZnO}$ is a typical semiconductor material which shows size-dependent optical properties due to the change of the band gap energy at nanoscale. The size of the quantum dots determines the band gap energy and hence the wavelength of the emitted light (figure 1.7). The band-gap energy of $\mathrm{ZnO}$ quantum dots is shown by a sharp emission peak between 375 and $385 \mathrm{~nm}$ (figure 4.10), which corresponds to the band-edge emission. The band-gap energy of the $\mathrm{ZnO}$ quantum dots is also characterised by an absorbance 
peak between 355 and $360 \mathrm{~nm}$. The wavelength of the light absorbed is always shorter than the wavelength of the light emitted.

Quatnum dot suspensions are usually affected by a further particle growth occurring over a longer period of time. Small crystals are generally less stable and tend to dissolve into their respective ions/atoms. The dissolved ions/atom attach then to larger crystals, which are thermodynamically more stable. This process is known as Ostwald ripening and result in the subsequently growth of the particles in dispersion medium. The increment in size is usually shown by a shift of the band-edge emission to longer wavelength. A shift of the band-edge emission to longer wavelengths could not be observed since the intensity of the band-edge emission progressively decreased after three days of ageing. However, the formation of larger larger quantum dots was determined by UV-vis spectroscopy shown by a shift of the absorbance maxima to longer wavelengths $\left(\lambda_{\mathrm{abs}}=360-365 \mathrm{~nm}\right)$.

\subsubsection{Defect emission of $\mathrm{ZnO}$ quantum dots}

The reduction of the band-edge emission with ageing is due to the incorporation of surface defect energy states within the band gap energy. Thereby, an excited electron can be trapped by these donor and acceptor defect states during the radiative recombination of the electron-hole pair (exciton) between the LUMO and HOMO energy states. This consequently quenches the intensity of the band-edge emission. It is important to note that the band-edge emission depends on the size of the quantum dots, whereas the the defect emission depends on the surface state of the qantum dots.

The surface state of the $\mathrm{ZnO}$ quantum dots plays an important role in the optical fluorescence. In general, crystals in a colloidal suspension with a high surface-tovolume ratio show a higher number of surface defects. The broad emission band in the visible range $\left(\lambda_{\mathrm{em}}=540-560 \mathrm{~nm}\right)$ is caused by surface defects and it is unusually large red shifted to the band-edge emission. For this reason, the fluorescence in the visible range is also referred to as a defect emission.

Although the exact mechanism for the broad defect emission band in the visible range is still debated, involvement of singly ionized oxygen vacancies in radiative 
recombination is the most widely accepted explanation at this time [76; 103-109]. It is assumed that a shallowly trapped electron close to the band edge of the conduction band recombines with the oxygen vacancy within the band gap and causes the "trap emission".

By passivating the surface area with capping agents, surface defects can be suppressed. The passivation effect, however, is dependent on the uptake of the capping agent on the particle surface and chemical bonding between surface atoms and functional groups of the capping agents. Since the surface area of $\mathrm{ZnO}$ quantum dots is covered by hydroxyl groups due to the hydrolysis reaction, it can be assumed that the functional groups of the polymer molecules will only form hydrogen bondings with the particle surface. The strength of the hydrogen bonding between anchoring groups of the polymeric dispersant and particle surface, however, is dependent on several factors such as type of anchor groups, surface charge of the particles, $\mathrm{pH}$ and so on.

A strong chemical interaction will suppress the formation of surface defects, because it may reduce the loss of polymer chains at the surface through ligand exchange reactions or desorption processes. The number of surface defects, however, increased in the first 3 days of ageing as shown by the reduction of the band-edge emission around $380 \mathrm{~nm}$ and the increment of the defect emission around $560 \mathrm{~nm}$. This can be attributed to several processes occurring over time such as ligand exchange reaction with solvent molecules and selective desorption processes at the particle surface by the formation of larger polycrystalline particles.

Ageing processes usually promote the formation of larger self-assembled structures by selective desorption and adsorption processes of polymeric chains at the surface of the quantum dots. These processes follow the terms of a self-assembled stabilization mechanism, by which quantum dots organise themselves into polycrystalline particles. Desorption processes are generally promoted by weak chemical bondings between the functional groups of the polymer and surface area of the quantum dots and can result in the loss of surfactants $[110,111]$.

With the loss of chemical bondings between surface atoms and polymer, free energy will accumulate at the particle surface (surface energy), which will increase the 
chemical reactivity of the quantum dots towards agglomeration processes. Furthermore, defects states at the particle surface are formed due to the loss of chemical bondings with the polymer chains, which result in the incorporation of energy levels within the band gap. These surface related defect states within the band gap, however, will quench the band-edge emission of the quantum dots. The higher the number of defects states at the particle surface, the more charge carries can be trapped and the higher the fluorescence intensity in the visible range. Thereby, the band-edge emission can be completely quenched.

Consequently, the slight increment of the intensity of the defect emission observed during the first three days of ageing can be attributed to the loss of chemical bonds at the quantum dot surface through the modification / reconstruction of the surface area by the formation of larger self-assembled structures.

As mentioned before, with the loss of surfactants around the particles, the chemical reactivity increases due to the higher surface energy. Thereby, the intermolecular interaction between the quantum dots are enhanced which can result in stronger bonds between adjacent particles inside the self-assembled structures. In general, strong chemical interaction will stabilize the particles in the dispersion medium by reducing their surface energy. By sticking together, however, energy can be exchanged between the particles through vibration. Thereby, the excess energy of an excited electron trapped by a donor or acceptor defect state can be converted into vibration energy through the chemical interaction between the particles. Therefore, non-radiative recombination processes can occur simultaneously with the defect emission.

It can be assumed that the intermolecular interaction between the particles increases with ageing due to the loss of surfactants at the particle surface. Stronger chemical interaction, however, will favour non-radiative recombination processes between charge carriers, which will result in the reduction of the fluorescence intensity of the defect emission, quenching. Hence, the subsequently reduction of the defect emission after three days of ageing (see figure 4.11) can be attributed to stronger van der Waals interaction between the particles inside the self-assembled structures. As long as the association of the polycrystalline particles is based on weak chemical interaction, 
dilution of the suspension can interrupt these intermolecular interactions between the particles resulting again in an increment of the defect emission.

In summary, the change of the fluorescence properties of the $\mathrm{ZnO}$ quantum dots is attributed to the surface modification of the quantum dots taking place with ageing. Initially, ageing processes promote the gradually loss of surfactants at the particle surface, which increase the number of surface defects. This, however, affect the bandedge emission, which will be progressively quenched by the increase of the defect emission. With the loss of surfactants, however, quantum dots can stronger interact with each other in the self-assembled structures. Strong chemical interaction favour nonradiative recombination processes of the charge carries, which in turn will quench the defect emission of the quantum dots.

Besides ageing processes, the loss of surfactants can also be promoted by longer stirring times at high temperature. It was found out that prolonged stirring times $(>60 \mathrm{~min}, 70$ ${ }^{\circ} \mathrm{C}$ ) resulted in a stronger precipitation reaction of the $\mathrm{ZnO}$ particles and lower fluorescence intensity of the defect emission. Prolonged stirring times at high temperatures may promote desorption processes of the polymer chains at the particle surface through ligand exchange reaction with solvent molecules resulting in the formation of larger polycrystalline particles and stronger chemical interaction between the $\mathrm{ZnO}$ quantum dots inside the self-assembled structures.

\subsubsection{Effect of process parameters on optical properties}

To increase the defect emission of colloidal $\mathrm{ZnO}$ nanocrystals, the influence of different process parameters such as choice of polymeric dispersant (PVP and PMAA-Na respectively), concentration of polymeric dispersant PVP, concentration of $\mathrm{NaBH}_{4}$, temperature, solvent $\left(\mathrm{H}_{2} \mathrm{O}, \mathrm{EtOH}\right)$ and total volume of the reaction medium were investigated.

Furthermore, experiments were carried out to incorporate different dopants such as $\mathrm{Mn}^{2+}, \mathrm{Ni}^{2+}, \mathrm{Co}^{2+}, \mathrm{In}^{3+}$ and $\mathrm{Ce}^{3+}$ into the lattice structure of $\mathrm{ZnO}$ nanocrystals and their effect on the optical properties of the semiconductor material were evaluated. 


\subsubsection{PVP and PMAA-Na}

ZnO nanocrystals prepared with PVP and PMAA-Na have identical PL scans. However, the intensity of the PL scans of $\mathrm{ZnO}$ nanocrystals prepared with PVP is higher than those of PMAA-Na. Furthermore, $\mathrm{ZnO}$ quantum dots coated with PMAA-Na formed small and porous polycrystalline particles, whereas quantum dots coated with PVP formed large polycrystalline particles.

Differences in the PL intensity can be attributed to the number of defect states on the particle surface. As explained before, the adsorption of polymer chains on the particle surface will passivate the particle surface and reduce the number of surface defects. However, weak chemical bondings between surface atoms and functional groups of the polymer can be broken, which will increase the number of defects states at the particle surface resulting in an increase of the defect emission. Hence, the strength of the chemical bonding between surface atoms and anchor groups of the polymeric surfactant has a big influence on the defect emission.

It is most likely that the negatively charged carboxylic groups $\left(\mathrm{COO}^{-}\right)$of PMAA-Na underwent a stronger chemical bonding with the particle surface than the functional groups of PVP resulting in a higher surface coverage of the $\mathrm{ZnO}$ quantum dots and therefore a lower PL intensity of the defect emission. Yet, the carboxylate groups of PMAA-Na are not strong enough to neutralise all surface related defect states. As mentioned earlier, the strength of the chemical bonding between anchor groups of the surfactant and particle surface is also dependent on the charge distribution at the particle surface, which is know to be frequently delocalised in metal oxides. Furthermore, the $\mathrm{pH}$ of the dispersion medium will also affect the chemical bonding, since it determines the dissociation of the carboxylic groups.

\subsubsection{Uptake of polymeric surfactant}

The intensity of the defect emission was strongly dependent on the uptake of the polymeric stabilizer on the particle surface. The highest intensity of the defect emission was obtained, when $2 \mathrm{~mL} 5 \mathrm{ppm}$ PVP was added to the reaction medium. The addition of higher concentration of PVP ( $>2 \mathrm{~mL} 5 \mathrm{ppm}$ PVP) to the reaction medium resulted in a progressive reduction of the PL intensity of the defect emission. However, the PL 
intensity of the defect emission significantly dropped, when the concentration of PVP in the dispersion medium was below 0.133 ppm PVP $(<2 \mathrm{~mL} 5 \mathrm{ppm}$ PVP) (see figure 4.12; A).

The size of the quantum dots was controlled by the uptake of polymer chains on the particle surface. The size has a strong influence on the surface state of the quantum dot and the number of surface related defect states within the band gap. Smaller quantum dots usually exhibit a larger number of surface defects; however with the increment in size, the number of surface defect decreases. This was also shown by the PL scans of $\mathrm{ZnO}$ suspensions, when the quantum dots underwent a further particle growth in the dispersion medium.

For instance, the uptake of PVP below $0.133 \mathrm{ppm}(<2 \mathrm{~mL} 5 \mathrm{ppm}$ PVP) resulted in an incomplete surface coverage of the nanocrystals. Therefore, nanocrystals exhibited a higher chemical reactivity due to the van der Waals forces, which caused a further particle growth via aggregation and condensation reactions. Thereby, the surface-tovolume ratio of the colloidal suspension decreased. With the decrease of the surface area, the number of surface defects also decreased resulting in lower intensities of the defect emission.

The increment of the polymer concentration ( $\geq 2 \mathrm{~mL} 5 \mathrm{ppm}$ PVP) resulted in a higher uptake of polymer chains at the particle surface. Thereby, quantum dots obtained a stronger surface coverage which inhibited a further growth of the quantum dots. The plot of the defect PL intensity against the polymer concentration in figure 4.12 illustrates the minimum concentration of PVP $(0.133 \mathrm{ppm})$, which is necessary to suppress condensation and aggregation reactions of the $\mathrm{ZnO}$ quantum dot in the dispersion medium. The gradual decrease of the intensity with higher concentration $(>2$ $\mathrm{mL} 5 \mathrm{ppm}$ PVP) as shown in this plot is attributed to an enhanced passivation effect of the particle surface with the polymer chains. A higher uptake of polymer chains reduces the number of surface related defect states by forming chemical bonds with the particle surface. Furthermore, a larger uptake of polymer chains results in the formation of a stronger repelling layer around the particles. Thereby, intermolecular interaction between the quantum dots is inhibited and the quantum dots remain mono-dispersed in solution. For example, the $\mathrm{ZnO}$ suspension prepared with the addition of $5 \mathrm{~mL} 5 \mathrm{ppm}$ 
PVP to the zinc chloride solution (figure 4.12; E) had a transparent slightly milky appearance.

It is important to note that gel lumps can be formed in the dispersion medium, when the polymer concentration is too high. Nevertheless, a high uptake of polymer chains at the particle surface results in a strong surface coverage which will reduce the surface energy of the quantum dots and the number of surface defects resulting in a lower fluorescence intensity of the defect emission.

\subsubsection{Effect of $\mathrm{NaBH}_{4}$ concentration on the defect emission}

The effect of $\mathrm{NaBH}_{4}$ on the intensity of the defect emission was examined by varying the amount of $2 \mathrm{M} \mathrm{NaBH}_{4}$ in triethylene glycol dimethylether from 0.25 to $1 \mathrm{~mL}$ added to the reaction medium. The highest fluorescence was obtained, when $0.5 \mathrm{~mL} 2 \mathrm{M}$ $\mathrm{NaBH}_{4}$ was added to the reaction medium (see figure 4.13; B). Below and above this concentration the fluorescence intensity decreased noticeably. The addition of $\mathrm{NaBH}_{4}$ to the reaction medium leads to the formation of $\mathrm{NaOH}$ molecules according to the redox reaction in (30), which will increase the $\mathrm{pH}$ value of the aqueous solution. The higher the amount of $\mathrm{NaBH}_{4}$ added to the reaction medium, the higher the change of the $\mathrm{pH}$ value of the reaction medium. The increment of the $\mathrm{pH}$ induces the hydrolysis reaction of the water complexed zinc cations. Hydrolysis can result either in the precipitation of the metal ion as metal hydroxide or in the formation of a solid phase through gelation polymerisation (polycondensation).

The change in $\mathrm{pH}$ caused by the addition of $\mathrm{NaBH}_{4}$ will define the hydrolysis ratio of the water complexed zinc cation monomers and polymers. The hydrolysis ratio indicates the rate and extent of the deprotonation of aqua ligands coordinated to zinc cations in aqueous solution. The deprotonation of two aqua ligands of the zinc hexaaqua complex (hydrolysis ratio is " 2 ") will result in the in the precipitation of the zinc cation as zinc hydroxide $\left(\mathrm{Zn}(\mathrm{OH})_{2}\right)$. This usually happens, when the number of alkali is twice to the number of moles of the zinc cations. The deprotonation of one aqua ligand will result in the formation of a reactive hydrolysed zinc cation monomer (see equation (19)), which is more likely to be involved in condensation reaction due to the higher nucleophilic properties of the $\mathrm{OH}^{-}$- ligand. Thereby, different zinc cation species such 
as dimers (equation (20)) and polymers can be formed either by the olation or oxolation mechanism (see figure 4.1).

These zinc cation intermediate species are the precursors of the solid zinc oxide phase, which polycondensate and from either a crystalline or amorphous structure. In general, the crystallinity of the solid oxide phase is strongly dependent on the ratio of ol-bridges (-OH-) and oxo-bridges (-O-) in the polymerised structure. Hence, the final structure of the solid phase is determined by the zinc cation intermediate species and their polycondensation mechanism via olation or oxolation.

The kinetics of the oxolation mechanism is faster than the kinetics of olation and result in the formation of oxo-bridges (-O-) between two zinc cation centres instead of olbridges (-OH-). It is most certainly that with the increment of $\mathrm{pH}$, the oxolation mechanism is favoured due to the fast neutralisation reaction of the water complexed zinc cations. In general, particles formed by oxolation feature a higher crystallinity than particles formed by the olation mechanisms. The higher the crystallinity of the particles, the lower is the number of ol-bridges (-OH-) in the final structure of the solid oxide phase and the lower is the number of defect states in the lattice structure. Consequently, the addition of $\mathrm{NaBH}_{4}$ in higher concentration promotes the formation of colloidal particles with a higher crystallinity characterised by a progressive decrease of the fluorescence intensity in figure 4.13 .

However, $\mathrm{NaBH}_{4}$ is also a strong reducing agent. High concentration of $\mathrm{NaBH}_{4}$ can destroy the organic surfactant layer around the particles causing a further particle growth. Thereby, the surface-to-volume ratio decreases and also the number of surface defects resulting in lower PL intensity of the defect emission. At this stage, further research is needed to confirm which of these explanations is valid.

The type of the zinc cation species formed by the addition of alkali to the reaction medium as well as their polycondensation reaction (olation or oxolation) is not only dependent on the $\mathrm{pH}$ change of the reaction medium but also on the process parameters used during the chemical reaction. For example, the formation of large polycation species in the reaction medium may be suppressed by the complexation of the zinc 
cation monomers, dimers and small poylmers with the polymeric dispersant used in this sol-gel method.

\subsubsection{Effect of temperature on the defect emission}

The fluorescence intensity of the defect emission as well as their increment and reduction with ageing is strongly affected by the temperature used during the synthesis of the $\mathrm{ZnO}$ quantum dots.

When, the sol-gel method was carried out at room temperature a precipitation reaction of $\mathrm{ZnO}$ occurred. The solid phase showed no optical fluorescence when excited with $\lambda_{\text {exc }}=350$ or $256 \mathrm{~nm}$. Conversely, $\mathrm{ZnO}$ suspensions formed between $50-70{ }^{\circ} \mathrm{C}$ showed similar fluorescence intensities of the defect emission after preparation; however, suspensions prepared below $70{ }^{\circ} \mathrm{C}$ were affected by a strong decrease of the fluorescence intensity after one day ageing (see figure 4.14). $\mathrm{ZnO}$ suspensions prepared between 70 and $90{ }^{\circ} \mathrm{C}$ showed a progressive decrease of the defect emission with the increase of the reaction temperature; however, the fluorescence intensity of these suspensions remained nearly constant in the first three days of ageing.

As mentioned earlier, a stable colloidal suspension was formed by gelation polymerisation, whereby zinc cation monomers and polymers polycondensate and form a solid phase, which can be either crystalline or amorphous. Low temperature promotes the formation of an amorphous phase with a poorly defined structure, whereas high temperature favours the dehydration of the solid oxide phase and hence the formation of crystalline particles. Furthermore, high temperature can break down larger polycation species increasing the number of smaller polycation species in the dispersion medium which can then condensate to crystalline particles via oxolation. It is likely that colloidal particles with a higher crystalline character have a lower number of surface defects and hence show a lower intensity of the defect emission. To confirm this statement, XRD measurements should be done for each sample prepared at different temperature.

Therefore, the gradual reduction of the defect emission by increasing the reaction temperature from 70 to $90{ }^{\circ} \mathrm{C}$ can be attributed to the formation of colloidal $\mathrm{ZnO}$ particles featuring higher crystallinity. The reduction of the defect emission of 
crystalline colloidal $\mathrm{ZnO}$ particles may also be attributed to a lower number of $\mathrm{OH}$ ligands at the particle surface. This may reduce the number of hydrogen bondings between particle surface and anchor groups of the polymeric dispersant. Thereby, particle can agglomerate to form larger polycrystalline particles. This, however, promote non-radiative recombination processes through the enhanced chemical interaction between the particles by forming cross-links resulting in the reduction of the defect emission.

$\mathrm{ZnO}$ suspension prepared at $70{ }^{\circ} \mathrm{C}$ showed the highest intensity of the defect emission. It is likely that the solid oxide phase of $\mathrm{ZnO}$ particles prepared at $70{ }^{\circ} \mathrm{C}$ has a higher number of ol-bridges (-OH-) in the lattice structure caused by olation and hence a less crystalline character. Also, $\mathrm{ZnO}$ suspension prepared at 50 and $60{ }^{\circ} \mathrm{C}$ show similar high intensities of the defect emission, however, it dropped significantly at the second day of ageing. This change can be explained through stronger chemical interaction between the quantum dots inside the polycrystalline particles, which enables non-radiative recombination processes of the exciton.

Colloidal $\mathrm{ZnO}$ particles with a high number of ol-bridges in their lattice structure are metastable and will subsequently dehydrate by the oxolation mechanism. This process enhances the crystallinity of the particles with ageing; however, it may also affect the surface state of the particles and chemical bondings between the surface atoms and anchor groups of the polymeric dispersant. For example, oxolation can lead to desorption processes and loss of polymer chains at the particle surface, which consequently will lead to an increment of the intensity of the defect emission (see figure 4.14). With the loss of polymer chains at the particle surface, however, quantum dots can stronger interact with each other inside the polycrystalline particles. This, however, will activate non-radiative recombination processes of the exciton resulting in a reduction of the fluorescence intensity of the defect emission.

In summary, the intermediate zinc cation species, formed by olation and oxolation respectively, define the final structure of the solid oxide phase of the quantum dots and also determine the chemical interaction inside the growing agglomerates with ageing. 


\subsubsection{Doping of ZnO quantum dots}

This sol-gel method was also used to dope $\mathrm{ZnO}$ quantum dots with transition metals $(\mathrm{Cu}, \mathrm{Ni}, \mathrm{Mn}$ and $\mathrm{Co})$ and rare earth metals (Ce and Er). It was expected that the use of $\mathrm{NaBH}_{4}$ will enhance the incorporation of dopants into the lattice structure of $\mathrm{ZnO}$ nanocrystals. By doing so, the dopants will introduce energy states within the band gap of $\mathrm{ZnO}$, which can shift the fluorescence to the visible range.

The incorporation of metal ions into the lattice structure of $\mathrm{ZnO}$ using sol-gel techniques is complicated by the formation of hydroxides (see examples in equation (22) to (25)). However, the formation of hydroxides can be suppressed by reducing the dopants with an alkali reducing agent. The reduced dopants may form a complex compound with the polymeric dispersant, which may enhance the incorporation of the dopants into the lattice structure of the $\mathrm{ZnO}$ nanocrystals.

The optical properties of the colloidal $\mathrm{ZnO}$ suspensions did not change with the addition of small amounts of dopants $\left(0.1-1 \mathrm{~mL} 0.01 \mathrm{M} \mathrm{MeCl}_{2 / 3}\left(\mathrm{H}_{2} \mathrm{O}\right)\right.$, listed in table 4.1) to the reaction medium. However, $\mathrm{ZnO}$ suspension synthesised with $1.0 \mathrm{ml} 0.01 \mathrm{M} \mathrm{CeCl}_{3}$ $\left(\mathrm{H}_{2} \mathrm{O}\right)$ showed only one sharp emission peak at $375 \mathrm{~nm}$ when excited with $\lambda_{\text {exc }}=260$ $\mathrm{nm}$. Here, the band-edge emission at $375 \mathrm{~nm}$ was not quenched by a defect emission at $560 \mathrm{~nm}$. Nevertheless, the $\mathrm{ZnO} / \mathrm{Ce}$ nanocrystal suspension still showed the characteristic broad emission peak around $550 \mathrm{~nm}$, when excited with $\lambda_{\text {exc }}=350 \mathrm{~nm}$. Ageing of the $\mathrm{ZnO} / \mathrm{Ce}$ nanocrystals in aqueous solution revealed a shift of the bandedge emission to higher wavelength $(380-385 \mathrm{~nm}$ ) (see figure 4.10). This suspension also showed a slight shift of the absorbance peak $(\sim 360 \mathrm{~nm})$ to higher wavelengths with ageing (figure 4.9). These peak shifts in the PL and UV-vis scans can be attributed to a further particle growth of the quantum dots by Ostwald ripening process. However, further studies are still required to fully understand the suppression of the defect emission of $\mathrm{ZnO} / \mathrm{Ce}$ nanocrystals. 


\subsection{Conclusions}

The synthesis of $\mathrm{ZnO}$ nanocrystals using the sol-gel method resulted in the formation of polycrystalline particles in the shape of round rice grains in different sizes varying from 0.1 to $1 \mu \mathrm{m}$. The polycrystalline particles are assembled by small nanocrystals of $\mathrm{ZnO}$ in the size of $10-12 \mathrm{~nm}$.

$\mathrm{ZnO}$ suspensions show two emission peaks at 380 and $560 \mathrm{~nm}$, when excited with 260 $\mathrm{nm}$. The emission peak at $380 \mathrm{~nm}$ refers to the band-edge emission which correspond to the radiative recombination of the electron-hole pair, exciton. The broad emission band in the visible range $\left(\lambda_{\mathrm{em}}=540-560 \mathrm{~nm}\right)$ is attributed to a trap or defect emission caused by surface defects of the nanocrystals. These surface defects incorporate surface defect energy levels within the band gap of the $\mathrm{ZnO}$ nanocrystals. The radiative recombination of the exciton (band-edge emission) is interfered by these additional energy states by trapping the excited electron or hole with the surface related defect states inside the band gap causing the defect emission.

The defect emission is strongly dependent on the surface coverage of the $\mathrm{ZnO}$ nanocrystals. The surface coverage also determines the stability of the colloidal crystals in suspension. By coating the surface area with PVP, surface related defect states are passivated and the growth of the quantum dots is confined. The stability of the surface coverage, however, is strongly dependent on the bonding character between functional groups of the polymer and surface atoms. The charge of metal oxides, however, is known to be frequently delocalised over the whole particle surface resulting in the formation of weak chemical bonds such as hydrogen bond. Weak chemical bondings, however, support the loss of polymer chains giving rise of surface defects. However, with the loss of polymer chains, particles lose their stability in suspension and agglomerate to larger self-assembled structures.

The agglomeration into larger polycrystalline particles follows the terms of a selfassembled stabilization mechanism in aqueous solution and it is regulated through specific intermolecular interactions between the chains of the polymeric surface layers around the $\mathrm{ZnO}$ nanocrystals. By sticking together, the nanocrystals reduce their surface-to-volume ratio and obtain so a higher stability in suspension. 
The formation of the self-assembled structures may also involve selective adsorption and desorption processes of the polymeric dispersant at the surface of the nanocrystals. These processes support network forming reaction between the nanocrystals leading to the arrangement of the particles into a polymer host matrix with the characteristic shape of rice grains. These processes, however, may also reduce the number of chemical bonds between polymer and surface atoms of the quantum dots. With the loss of chemical bondings at the particle surface, more surface related defects states are incorporated within the band gap leading to the increment of the fluorescence intensity of the defect emission. The increase of the defect emission, however, results in the quenching of the band-edge emission.

The agglomeration of the $\mathrm{ZnO}$ quantum dots into larger polycrystalline particles enhances the fluorescence intensity of the defect emission. However, it is important to note that with the loss of capping agents, the attractive van der Waals forces can pull the nanocrystal into closer contact, where they adhere strongly and irreversible together. This, however, enables non-radiative recombination processes resulting in the reduction of the defect emission (quenching). However, being enclosed inside the polymer host matrix, particles are largely inhibited by further condensation processes.

The self-assembled structures, however, are not stable and split in halves. Furthermore, the stability of the polycrystalline particles is negatively affected by dilution. When diluted in water, the self-assembled structures broke apart and small nanocrystals prevailed mono-dispersed in solution. Here, the self-assembled structures are held together by van der Waals forces such as weak electrostatic interaction and hydrogen bondings.

Interestingly, $\mathrm{ZnO}$ prepared in the presence of $\mathrm{CeCl}_{3}$ showed only one sharp and intense band-edge emission around $380 \mathrm{~nm}$, when excited with $\lambda_{\text {exc }}=260 \mathrm{~nm}$. Here, ageing did not lead to the reduction of the band-edge emission; however a shift of the fluorescence peak to longer wavelength was observed $(\sim 385 \mathrm{~nm})$. This shift is caused by an Ostwald ripening process leading to the growth of the $\mathrm{ZnO}$ quantum dots in suspension. 


\section{Chapter 5}

\section{Co-precipitation of metallic nanoclusters with zinc sulfide}

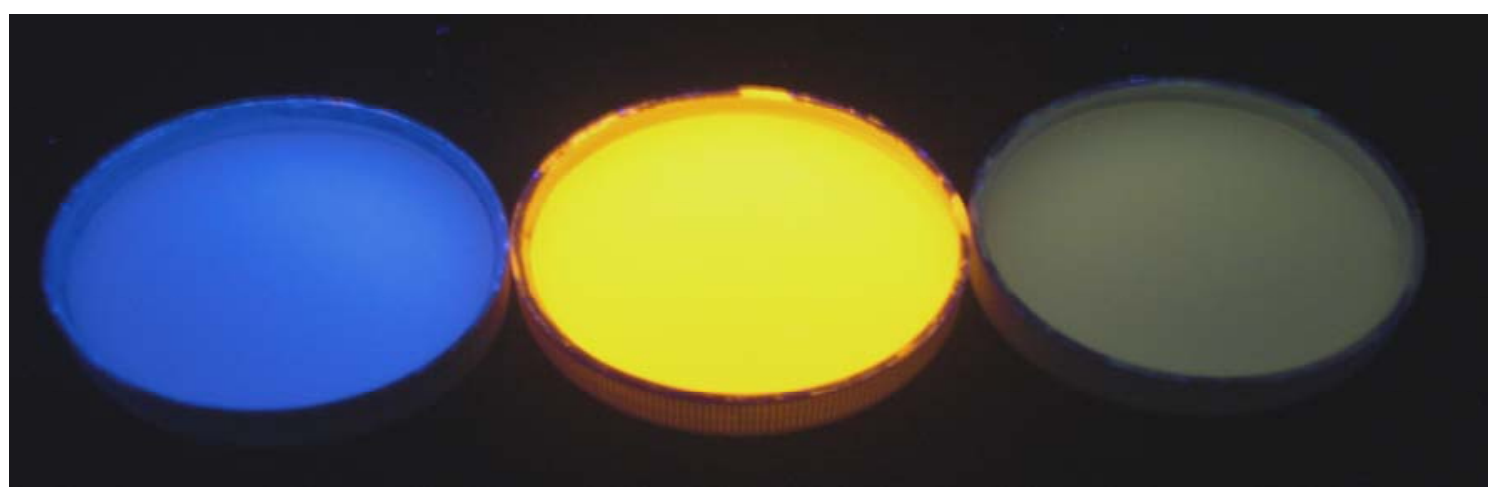

Chapter 3 reported the development of a wet chemical reduction method to produce $\mathrm{Cu}_{2} \mathrm{O}$ quantum dots. The production of $\mathrm{Cu}_{2} \mathrm{O}$ quantum dots, however, did not exhibit optical fluorescence. This can be attributed to the lack of crystallinity of the nanocrystals and / or the formation of defects states at the surface of the nanocrystals. The electronic structure of semiconductor nanocrystals is very sensitive to surface defects. In general, surface defects can be considered as "trap sites" for an electron-hole pair (exciton) generated in the core of the crystal. Once an electron-hole pair is trapped by a surface related defect state (lower energy state), non-radiative pathways for the recombination of the electron-hole pair are possible. This, however, result in the quenching of the fluorescence intensity of the quantum dot material.

Surface related trap states can usually be suppressed by a strong surface coverage of capping agents. The surface charge of metal oxide nanocrystals, however, is known to be frequently delocalised over the whole particle surface causing the loss of capping agents and giving rise to the formation of surface defects. Surface defects can be suppressed permanently by coating the surface area of metallic nanoclusters with inorganic semiconductor materials producing core-shell particles [136]. To restrict the exciton to the core of the metal nanoclusters, however, the band gap of the shell has to 
be wider than the band gap of the core. This prevents non-radiative pathways for the relaxation of the exciton at the surface of the core semiconductor material. This chapter deals with the coating of metal oxide nanocrystals produced by a wet chemical reduction method with $\mathrm{ZnS}$ to obtain strong bright fluorescence colours in the visible range.

\subsection{Introduction; Co-precipitation method}

Zinc sulfide $(\mathrm{ZnS})$ as II-VI semiconductor material has the largest energy band gap $(\sim 3.6 \mathrm{eV})$ among the II-V compound semiconductors of direct transition type and it can be used to passivate the surface area of metal nanoclusters. However, coating nanocrystals with $\mathrm{ZnS}$ is only possible when the lattice structure of the nanocrystals match with the lattice structure of $\mathrm{ZnS}$. This facilitates the epitaxial growth of the $\mathrm{ZnS}$ shell on the surface of the nanocrystals. ZnS can adopt either a wurtzite or zinc blende structure. $\mathrm{ZnS}$ quantum dots are easy to obtain by a wet chemical precipitation method using inexpensive raw materials and simple glassware [46, 80-84].

The wet chemical reduction method evaluated in chapter 3 can be used to form a variety of metallic nanoclusters, which can be then (theoretically) coated with $\mathrm{ZnS}$ or arrested in the chemical composition of $\mathrm{ZnS}$ using a wet chemical precipitation method. By doing this, nanocrystals with bright fluorescence colours can be obtained by eliminating / passivating surface defects. The methodology is graphically displayed in figure 5.1:

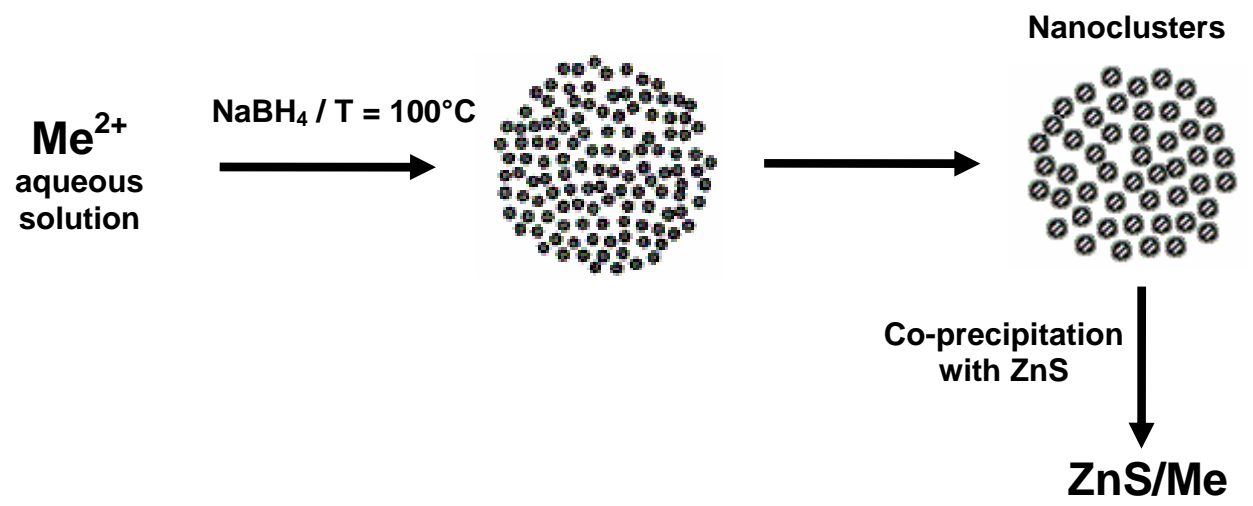

Figure 5.1: Nucleation centres (seeds) formed instantaneously after the addition of $\mathrm{NaBH}_{4}$. These can form nanoclusters which is promoted by stirring time or arrested into the chemical composition of ZnS quantum dots. 


\subsection{Method}

In the wet chemical reduction method, small metallic nucleation centres are formed instantaneously by the addition of $\mathrm{NaBH}_{4}$ to the reaction medium containing metal ions and stabilizer. The nucleation centres condensate due to their high surface energy and grow forming larger nanoclusters (see figure 5.1). The growth of the nanoclusters is kinetically controlled by the use of a stabilizer. The nanoclusters can then be coprecipitated with $\mathrm{ZnS}$ using a wet chemical precipitation method. The precipitation reaction of the $\mathrm{ZnS}$ nanocrystals is represented by the following chemical equilibrium:

$$
\mathrm{S}^{2-}{ }_{\text {(solvent) }}+\mathrm{Zn}^{2+} \text { (solvent) } \quad \rightleftarrows \quad \mathrm{ZnS}_{\text {(solid) }} \downarrow
$$

This chemical equation is reversible and can be easily controlled by different processing parameters such as concentration of the raw materials, reaction temperature, choice of solution, $\mathrm{pH}$, titration rate of the reactants, stirring rate and use of capping agents. All these parameters have effects on the crystal growth and final size and shape of the nanocrystals, as well as on the size distribution [80]. Several transition and rare-earth metal ions such as $\mathrm{Cu}^{2+}, \mathrm{Mn}^{2+}, \mathrm{Ni}^{2+}, \mathrm{Zn}^{2+}, \mathrm{Co}^{2+}, \mathrm{In}^{3+}, \mathrm{Ce}^{3+}$ and $\mathrm{Eu}^{2+/ 3+}$ were reduced using the wet chemical reduction method described in chapter 3 and co-precipitated with $\mathrm{ZnS}$. However, this procedure was only successful with the transition metal ions $\mathrm{Cu}^{2+}, \mathrm{Mn}^{2+}, \mathrm{Ni}^{2+}$ and $\mathrm{Zn}^{2+}$. The experimental procedures used for these transition metals are presented in chapter $2 ; 2.1 .3 .3$.

\subsection{Results}

The arrested precipitation of $\mathrm{ZnO}-(\mathrm{A}), \mathrm{Cu}-(\mathrm{B}), \mathrm{Mn}(\mathrm{C})$ - and $\mathrm{Mn} / \mathrm{Ni}$ (D) - nanoclusters with $\mathrm{ZnS}$ resulted in the formation of a white suspension. Under UV-light, however, the suspension glows in bright fluorescence colours as shown in figure 5.2.

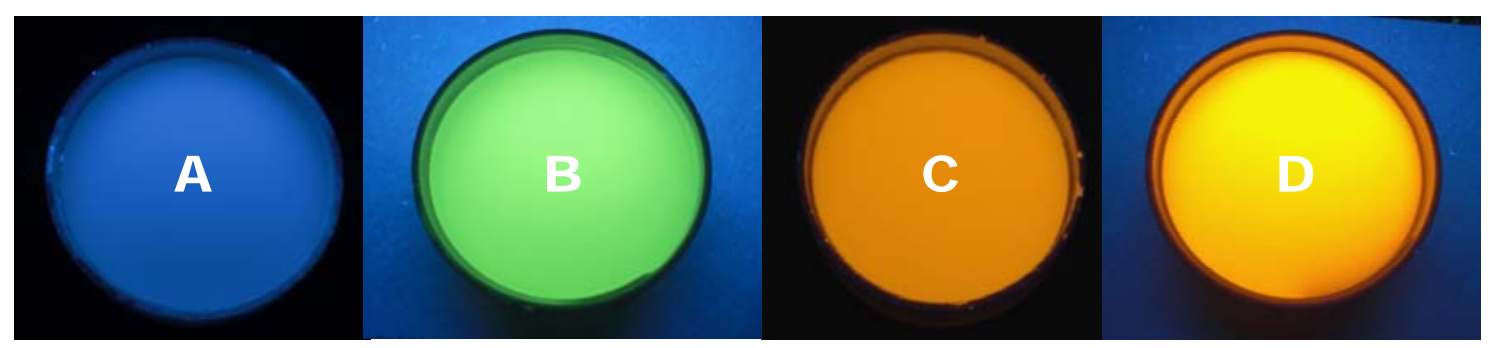

Figure 5.2: Fluorescence colours of ZnS quantum dot suspension obtained by the co-precipitation of $\mathrm{ZnO}$ (A), Cu (B), Mn (C) and $\mathrm{Mn} / \mathrm{Ni}$ (D) nanoclusters $\left(\lambda_{\text {exc }}=256 \mathrm{~nm}\right)$. Details of sample preparation are listed in the appendix in table A.11. 


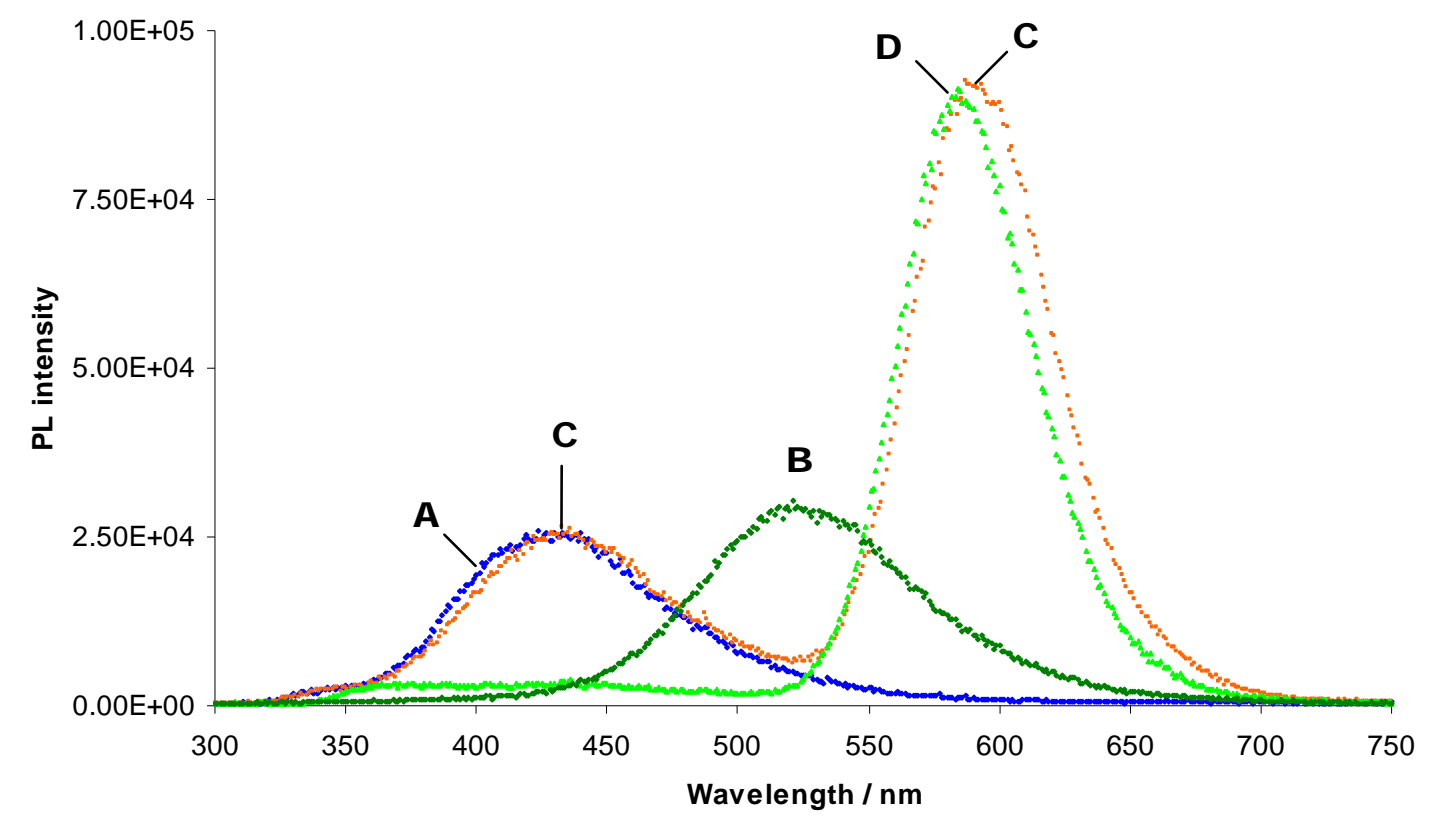

Figure 5.3: PL scans of ZnS quantum dot suspensions precipitated from solution in the presence of ZnO (A), Cu (B), Mn (C) and Mn/Ni (D) nanoclusters ( $\lambda_{\text {exc }}=260 \mathrm{~nm}$, slit $\left.=5 \mathrm{~nm}\right)$.

Table 5.1: List of ZnS quantum dot suspension precipitated from solution in the presence of $\mathrm{ZnO}$, $\mathrm{Cu}, \mathrm{Mn}$ and $\mathrm{Mn} / \mathrm{Ni}$ nanoclusters with corresponding maximum emission wavelength obtained with exciting wavelengths $\lambda_{\text {exc }}=260$ and $350 \mathrm{~nm}$.

\begin{tabular}{cccc}
\hline Sample label & Compounds & $\begin{array}{c}\lambda_{\text {em }} / \mathrm{nm} \\
\left(\lambda_{\text {exc }}=260 \mathrm{~m}\right)\end{array}$ & $\begin{array}{c}\lambda_{\text {em }} / \mathrm{nm} \\
\left(\lambda_{\text {exc }}=350 \mathrm{~m}\right)\end{array}$ \\
\hline $\mathrm{A}$ & ZnS / ZnO(PMAA-Na) & 435 & 444 \\
$\mathrm{~B}$ & ZnS / (Zn/Cu) & 523 & 526 \\
$\mathrm{C}$ & ZnS / Mn (PMAA-Na) & 435 and 590 & 445 and 595 \\
$\mathrm{D}$ & ZnS / (Zn/Mn/Ni) & 584 & 587 \\
\hline
\end{tabular}

$\mathrm{ZnS}$ quantum dots with $\mathrm{ZnO}$ impurities (figure 5.2; A) show a strong blue fluorescence under UV light $\left(\sim \lambda_{\mathrm{em}}=445 \mathrm{~nm}\right)$. $\mathrm{ZnS}$ quantum dots doped with $\mathrm{Cu}$ (figure 5.2; B) exhibit green fluorescence $\left(\sim \lambda_{\mathrm{em}}=525 \mathrm{~nm}\right)$ and $\mathrm{Mn}$-doped $\mathrm{ZnS}$ quantum dots (figure 5.2; C) show orange fluorescence $\left(\sim \lambda_{\mathrm{em}}=595 \mathrm{~nm}\right)$. $\mathrm{ZnS}$ quantum dots doped with $\mathrm{Mn}$ and $\mathrm{Ni}$ (figure 5.2; D) show a bright yellow-orange colour $\left(\sim \lambda_{\mathrm{em}}=585 \mathrm{~nm}\right)$. When the $\mathrm{PL}$ scans of $\mathrm{ZnS} / \mathrm{Mn}(\mathrm{C})$ and $\mathrm{ZnS} / \mathrm{Mn} / \mathrm{Ni}$ (D) are compared (see figure 5.3), it can be seen that $\mathrm{ZnS} / \mathrm{Mn} / \mathrm{Ni}$ only show one strong emission peak at $584 \mathrm{~nm}$. This is about $5 \mathrm{~nm}$ blue-shifted from the emission peak of $\mathrm{ZnS} / \mathrm{Mn}$. 


\subsection{Discussion}

The wet chemical precipitation method allows the incorporation of small quantities of impurities (dopants) into the chemical composition of the final product during the precipitation method. The incorporation of dopant materials into the crystal lattice of $\mathrm{ZnS}$ quantum dots interferes with the electron-hole recombination mechanism of an exciton. In general, the excited electron from the conduction band will return to the hole on the top of the valence band. However the electron can be trapped on the return pathway due to additional energy levels introduced by dopant ions in the band gap. Thereby the electron will release energy by emitting light during the transition between the energy levels. Because of this, the nature and level of the dopant ion(s) can be used to control the fluorescent wavelength(s) (see illustration in figure 5.4 and figure 5.5).

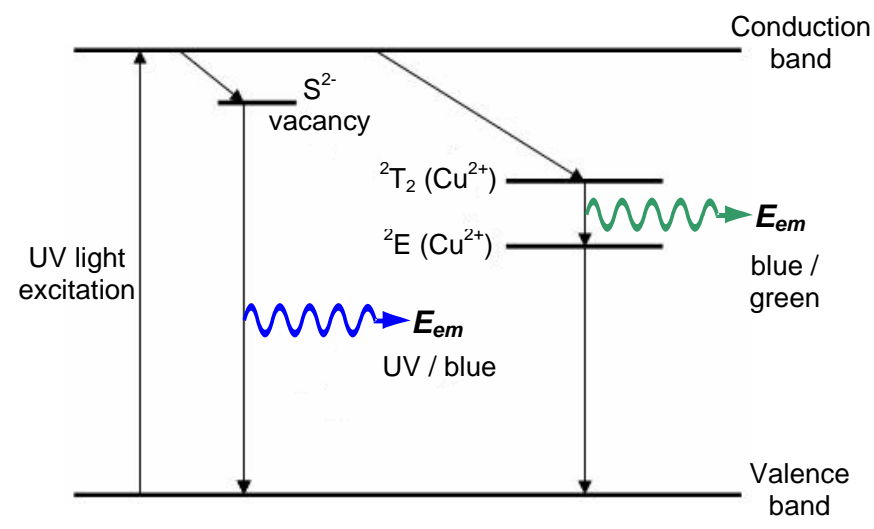

Figure 5.4: Electron-hole recombination in $\mathrm{ZnS}: \mathrm{Cu}^{2+}$ quantum dots with intermediate green fluorescence.

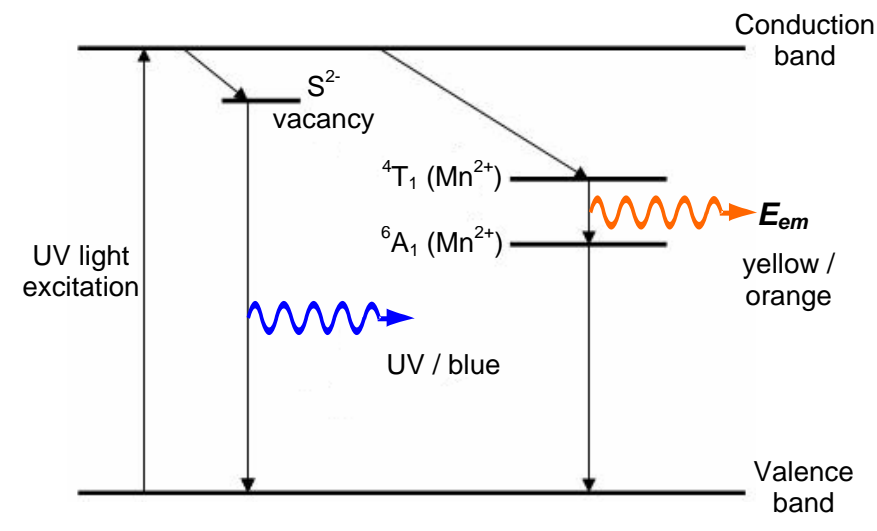

Figure 5.5: Electron-hole recombination in $\mathrm{ZnS}: \mathrm{Mn}^{2+}$ quantum dots with intermediate orange fluorescence.

As seen in the figures 5.4 and 5.5, the strong blue fluorescence of $\mathrm{ZnS}$ under UV light is linked to $\mathrm{S}^{2-}$ vacancies in the $\mathrm{ZnS}$ lattice [80]. The green and orange fluorescent colour 
is caused by introduced energy levels of the respective dopant material. The green emission is created by the transition of the excited electron between the intermediate energy level ${ }^{2} \mathrm{~T}_{2}$ to the ${ }^{2} \mathrm{E}$ of the $\mathrm{Cu}^{2+}$ ion (figure A.4) and the orange emission colour by the intermediate energy level ${ }^{4} \mathrm{~T}_{1}$ to ${ }^{6} \mathrm{~A}_{1}$ of the $\mathrm{Mn}^{2+}$ ion (figure 5.4).

It was also observed that the fluorescence efficiency of the $\mathrm{ZnS}$ suspensions gradually increased with ageing. The cause of this change can be explained as follow. Small nanocrystals / nanoclusters are less stable due to their high surface energy. For this reason, they are affected by a second growth stage, Ostwald ripening. In this process small nanocrystals / nanoclusters tend to dissolve into their respective ions and recrystallise on larger crystals, which are thermodynamically more stable. It can be assumed that the metallic nanoclusters dissolve and the dissolved metal atoms are incorporated into the chemical composition of $\mathrm{ZnS}$. It is important to note that the optical properties of $\mathrm{ZnS}$ quantum dots are not affected by the size, but by the type of dopant incorporated in the lattice structure of the $\mathrm{ZnS}$ nanocrystals. It is interesting to note that fluorescence efficiency of the $\mathrm{ZnS}$ suspensions is unchanged over a long period of time ( 3 years). This could be attributed to the larger size of the nanocrystals which are thermodynamically more stable.

\subsection{Conclusions}

The method presented here is suitable to create doped and undoped $\mathrm{ZnS}$ quantum dots in an inexpensive, straight forward and reproducible way. The $\mathrm{ZnS}$ suspensions produced also show a long lasting bright fluorescence. However, more characterisation studies are needed to fully understand the particle growth and to fine-tune the fluorescence colour. 


\section{Chapter 6}

\section{Development of $\mathrm{ZnO}$ quantum dot - fibre composites}

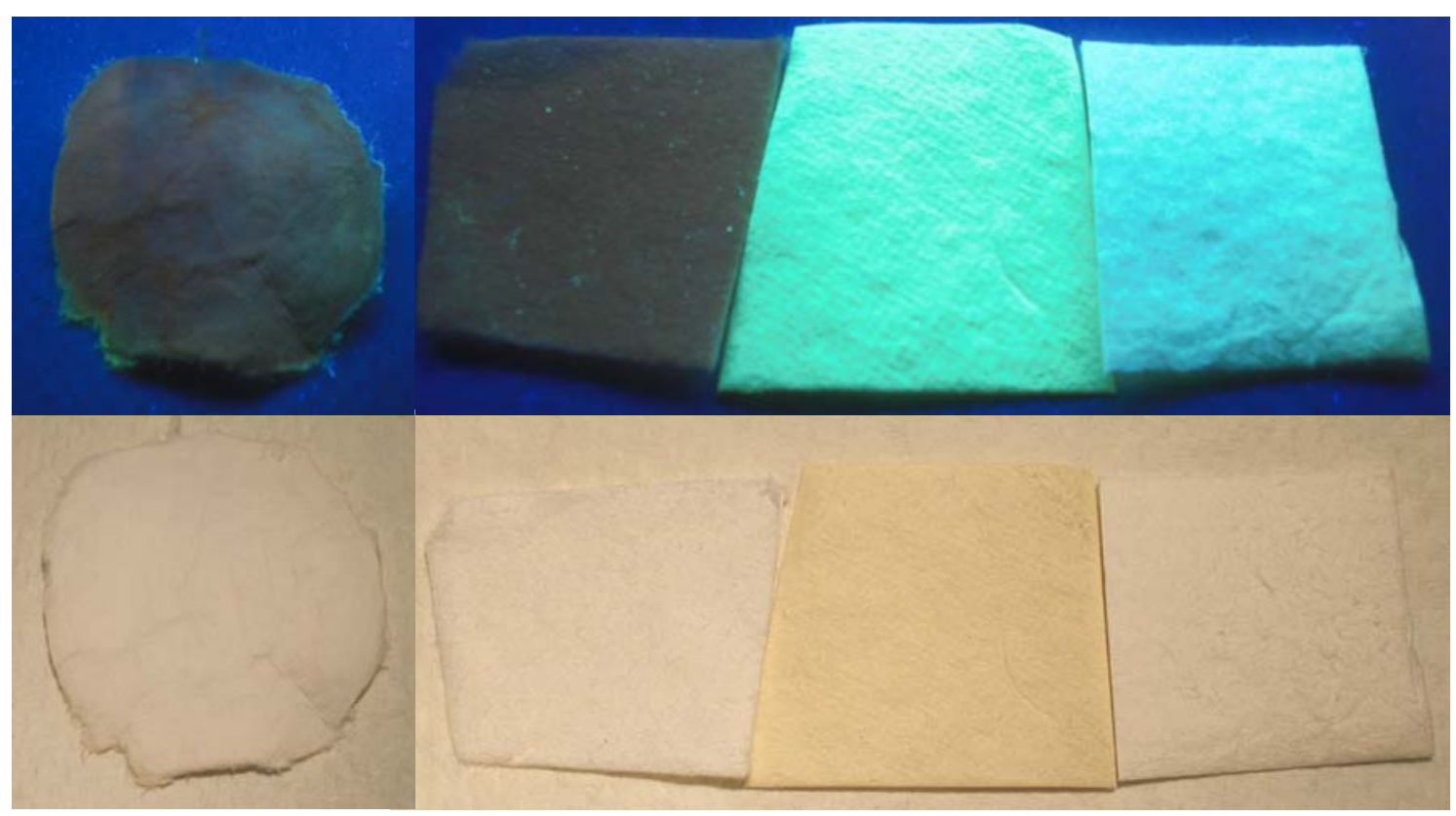

This chapter explores different methods to create quantum dot - composite materials that combines the properties of quantum dots with the bulk properties of wool and paper. The inorganic semiconductor $\mathrm{ZnO}$ is chosen as a non-toxic quantum dot material. It shows two distinct emission peaks in the UV-vis range $(\sim 380 \mathrm{~nm})$ and visible range $(\sim 560 \mathrm{~nm})$, when excited with $260 \mathrm{~nm}$. The goals are to bind $\mathrm{ZnO}$ quantum dots to wool and paper fibre and to functionalise it with fluorescence in the visible range. The different approaches are evaluated according to their chemical bonding between the quantum dots and fibrous material and their fluorescence properties.

For the development of multifunctional hybrid materials comprising $\mathrm{ZnO}$ quantum dots and wool and paper fibres, the in-situ and building block approach were applied. In the in-situ approach, quantum dots are formed in the chemical surrounding of the substrate material. This method relies on cross-link reactions between the quantum dots and wool 
fibre during nucleation and particle growth. In the building block approach, quantum dots are attached to the substrate material through a chemical bonding. This approach can make use of different processing strategies to improve the chemical bonding between the inorganic and organic phase. This is achieved by modifying the interface between both components either by functionalising the surface area of the substrate material or quantum dots.

The building block and in-situ approach produced $\mathrm{ZnO}$ quantum dot - wool composites with a sharp emission peak in the UV range. This emission peak around $380 \mathrm{~nm}$ refers to the band-edge emission of the $\mathrm{ZnO}$ quantum dots. The attachment of $\mathrm{ZnO}$ quantum dots to bleach Kraft paper did not result in the functionalisation of the paper fibre with fluorescence; however, the fibres were covered with a surface layer that exhibit UV filtering properties.

The use of 8-Hydroxyquinoline (8-HQ) as a fluorescent chelate agent for zinc cations at the $\mathrm{ZnO}$ quantum dot surface was also explored. $\mathrm{ZnO}$ quantum dots functionalised with 8-HQ provided wool and paper fibre with strong fluorescence in the visible range. Depending on the concentration of 8-HQ and other parameters such as choice of solvent, soaking temperature and time, the fluorescence of $\mathrm{ZnO}$ quantum dot - paper and wool composites were tuned from white, light green to yellow-green. The optical properties of the quantum dot - fibre composites have been characterised using photoluminescence spectroscopy.

\subsection{Introduction; Quantum dot - fibre composites}

New Zealand wool and paper industry can benefit from the use of nanotechnology in their product finishing. Wool and paper are available in abundant supply and inexpensive. When combined with nano-seized materials, however, new multifunctional hybrid materials can be created which add value to the starter material. Hence, research involving nanotechnology to create new hybrid materials with new properties is of a great industrial interest.

Quantum dots showing fluorescence have a great potential for the production of new hybrid materials. Quantum dots are more stable to external influences such as sun light 
exposure and have a longer lifespan unlike organic fluorophores. Quantum dots have also a great potential to replace many commercial organic fluorophores which are used in a wide field of applications. The adsorption of quantum dots onto wool and paper fibres should be supported by the large surface area of the quantum dots and of these fibrous substrate materials. When the chemical interaction between both components is attractive, the fibres obtain a surface layer of the nano-sized semiconductor material, functionalising it with optical fluorescence. By controlling/tuning the fluorescence colour of the quantum dots in the visible range, e.g. by varying the size and/or incorporation of metal ion impurities into the quantum dot material, it is possible to produce fibres with different fluorescence colours. Apart from optical fluorescence, quantum dots can also have properties such as UV absorption properties and antimicrobial activity. For example, quantum dots with a large band gap can absorb UVlight, which otherwise can cause damage to the fibres. Also, some nano-materials exhibit biological activity based on the high surface area as well as specific crystal morphologies such as edges and corners. Therefore, quantum dots have also the potential to be used as an anti-microbial agent. Feature like these will add extra functionality to these fibrous materials $[45 ; 112-115]$.

These new fibrous composite materials can be further processed and used in the fabrication of fabrics and packing materials. For example, the fashion industry has a high demand for new multifunctional textiles with high wearing comfort and various safety aspects. In particular, the sport and outdoor sector is constantly looking for new textiles with unique properties. For this reason, research involving nanotechnology to create new functional textiles is highly welcomed in the textile industry. The paper industry could also be affected quite significantly by nanotechnology. Quantum dots attached to paper fibres have the potential to be used in security packaging and labels for brand protections and papers for security documents. In general, quantum dots have a great potential to be used for brand protecting against product counterfeiting. Year after year, companies suffer a great loss of sales due to product counterfeiting. To guarantee product verification, brands may, and need to, protect themselves against illegal duplication by using anti-counterfeiting technologies. By anti-counterfeiting labelling the package or the product itself with quantum dots, items can be identified as authentic or fake. For example in fabrics, the embedding of fluorescent textile fibres into the product can be used as a method to demonstrate the authenticity of the product. 
Therefore, the fabrication of new hybrid materials comprising quantum dots and fibrous material such as wool and paper can contribute significantly to the economical growth of New Zealand.

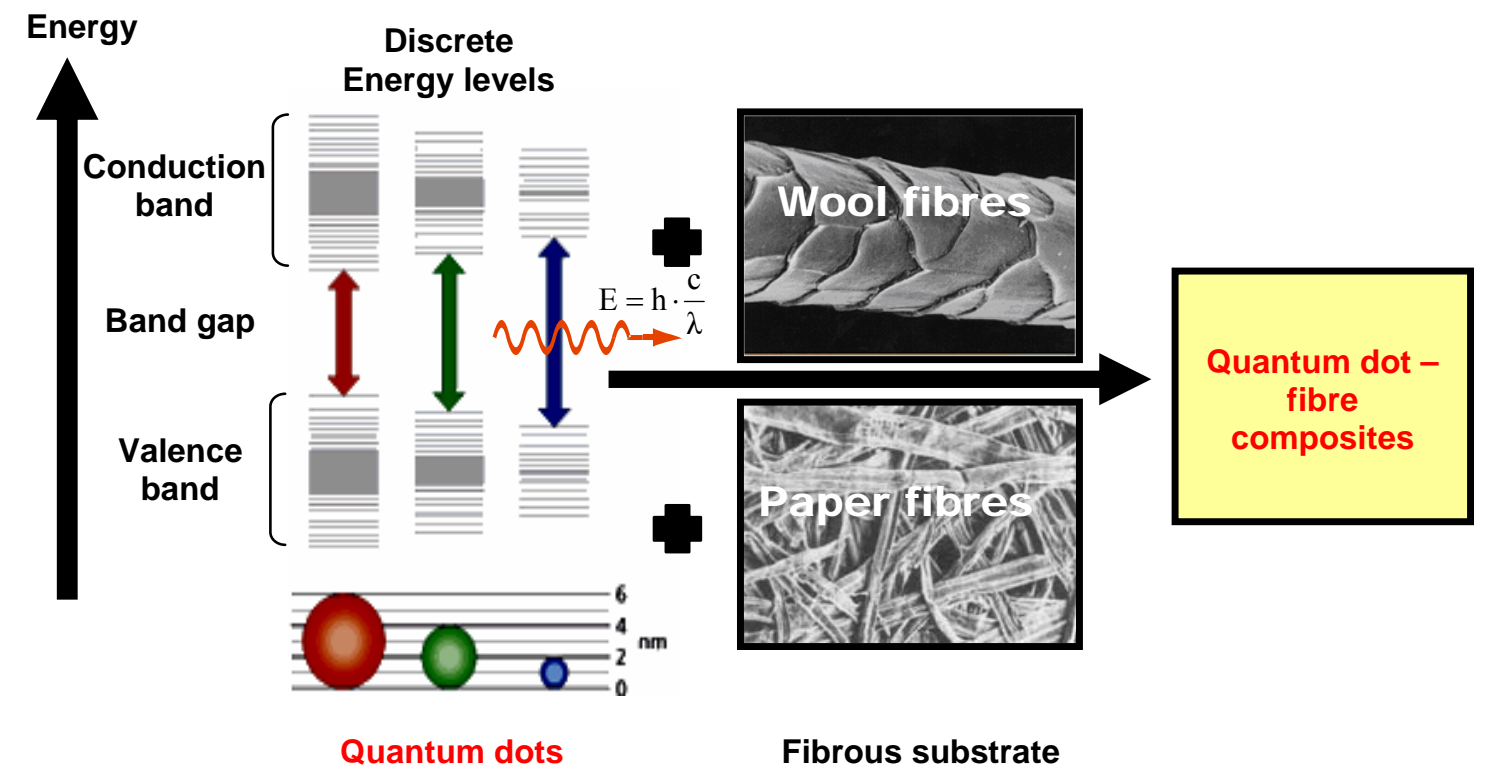

Figure 6.1: Schematic illustration of the development of new hybrid materials comprising quantum dots and wool and paper fibres.

The stability and optical properties of the quantum dot - fibre composite material is determined by the chemical bonding between the inorganic and organic component connecting them together. Here, the chemical bonding can be divided in two classes dependent on the strength of chemical interaction. This interaction can be either strong (e.g. covalent bonding, coordinated complex formation) or weak (e.g. H-bonding, weak electrostatic interaction through van der Waals forces). The type of interaction has an important role in the stability of the hybrid material. For example, weak chemical interactions have negative effects on the long-term stability of the material and may lead to leaching of the quantum dots from the substrate material [52]. Furthermore, if only a weak interaction prevails between the quantum dots and the organic substrate material, aggregation processes of the quantum dots can occur and change the homogeneity of the material. Therefore, chemical bondings with strong characteristics are preferred for the formation of quantum dot functionalised hybrid materials. The stronger the interaction between the components, the more stable is the final material. The type of interaction between quantum dot and substrate material can also affect the optical properties of the quantum dots. Similar to capping agents, the formation of an energy transfer mechanism 
between quantum dot and organic substrate material can result in quenching of the fluorescence intensity.

\subsubsection{Choice of quantum dot material}

Quantum dots attached to the fibrous substrate material should show intense, stable, spectroscopically pure fluorescent colours under UV light stimulation. Furthermore, the chemical composition of the quantum dot material plays also an important role, which should be compatible with human skin to avoid skin irritation. The toxicity of many common available quantum dot materials and the danger of leaching restrict their use in every day products and therefore should be avoided for the development of hybrid materials.

Zinc oxide $(\mathrm{ZnO})$ is a suitable reaction partner for wool and paper to produce quantum dot - fibre composite. Zinc oxide $(\mathrm{ZnO})$ can be readily synthesized via a wet chemistry method (see chapter 4) and show two primary emission bands in its photoluminescence spectra; around $380 \mathrm{~nm}$ and a maximum between 500 and $550 \mathrm{~nm}$ (see figure 4.10). Furthermore, the increased band gap of $\mathrm{ZnO}$ nanocrystals makes it to attractive entities in UV-protections films [112]. Also, the bacteriostatic and fungistatic properties of $\mathrm{ZnO}$ nanoparticles [113] are well studied and because of the compatibility with skin, it is already used as an environmentally friendly, non-toxic inorganic component in various personal care products such as baby powder and sun screen lotion. The sol-gel method used for the preparation of $\mathrm{ZnO}$ may also be able to be extended on relative large scale.

By combining the properties of the $\mathrm{ZnO}$ quantum dots with the bulk properties of paper and wool, new hybrid materials are formed which have great potential applications in the paper and fashion industry for product authentication, labelling and many more applications. There are two approaches which can be used for the production of quantum dot - fibre composites, the building block approach and in-situ approach. The theoretical background of these approaches is described in the introduction chapter 1; 1.3.4. 


\subsection{Methods}

The properties of $\mathrm{ZnO}$ quantum dots were combined with the bulk properties of paper and wool using in-situ approach and building block approach. Both approaches were based on wet chemical techniques to produce $\mathrm{ZnO}$ quantum dot - fibre composites and to obtain a strong chemical bonding between the substrate and quantum dot material.

\subsubsection{In-situ binding of $\mathrm{ZnO}$ quantum dots to wool}

The formation of $\mathrm{ZnO}$ quantum dots and in-situ binding to wool is based on a sol-gel process. This process relies on cross links between the quantum dot material and organic substrate material and will lead to structural changes of the substrate material.

The sol-gel process is an easy and convenient method for the synthesis of metal oxides. It makes use of the aqueous chemistry of water complexed metal ions, which is dominated by hydrolysis and condensation reactions (see equations (19)-(21)). Sol-gel methods involve a long reaction time and it is hoped that $\mathrm{ZnO}$ quantum dots will form cross-links with the protein structure of the wool during the hydrolysis and condensation reactions. This, however, is only possible, when the organic substrate material contains functional groups that can react with the surface of the solid inorganic phase. The formation of cross links would provide the chemical bonding of the quantum dots to the substrate material and may also confine the particle growth.

The in-situ method involves first the adsorption of the precursor material of the $\mathrm{ZnO}$ quantum dots by the organic substrate material, before a chemical transformation is induced. Here it is important to note that the adsorption of the quantum dot precursor material depends on diffusion processes inside the substrate material and selective binding and nonbinding processes to the functional groups of the substrate material. The transformation of the precursor materials into a solid phase is then induced by a sol-gel method. The crystal growth as well as the network forming reactions in the chemical surrounding of the organic substrate material can proceed under kinetically or thermodynamically controlled reactions. Hence, simple changes in the process parameters such as temperature, time, solvent, $\mathrm{pH}$ or concentration of precursor material can often result in the formation of particles with different sizes, shapes, compositions and phase structures. For this reason, the adjustment and optimisation of the process 
parameters during the formation of the composite material is important to control the nucleation and crystal growth inside the substrate material.

Therefore, different precursor solutions with a total volume of $50 \mathrm{~mL}$ were prepared beforehand. Each solution contained $1 \mathrm{~mL} 1 \mathrm{M} \mathrm{ZnCl}_{2}\left(\mathrm{H}_{2} \mathrm{O}\right)$ and a complexing agent. The amount of each complexing agent added and its concentration are given in table 2.5. Then, $0.2 \mathrm{~g}$ merino wool was soaked in each precursor solution for 3 hours at $70{ }^{\circ} \mathrm{C}$ using a water shake bath to ensure a uniform uptake of the zinc cations by the wool. It is expected that the zinc ions are easily absorbed in the framework structure of wool fibres, because of the electrostatic interaction between the positively charged metal ions and functional groups of wool's keratin located on the surface of the fibres. Zinc cations remaining in solution are complexed with the complexing agent. The sol-gel process

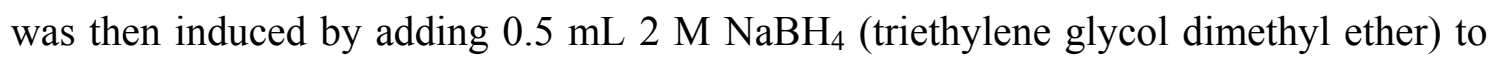
the soaking solution containing the wool fibre.

The addition of $\mathrm{NaBH}_{4}$ as an alkali reducing agent reduces the $\mathrm{pH}$ of the soaking medium and promotes the deprotonation of aqua ligands around the zinc cations leading to the formation of reactive intermediates which polycondensate and form a solid phase. The composition of the solid phase in the course of hydrolysis can vary between amorphous and crystalline and it is strongly dependent on the process parameters used. Furthermore, the adsorption of the zinc cations by wool and the complexation of zinc cations with a complexing agent in aqueous solution affect the kinetic of the hydrolysis and condensation reaction. For this reason, it is important that the process parameters of the sol-gel process are adjusted to precision so that the size and shape of the crystals inside and onto the substrate material can be controlled. The different growth stages of the crystals during nucleation and condensation are strongly dependent on the process parameters such as temperature, time, $\mathrm{pH}$, solvent, etc. Also, the presence of counter ions in solution and absorbed by the substrate can influence the particle formation and growth. Reaction parameters used for the in-situ formation of $\mathrm{ZnO}$ quantum dot - wool fibre composites are presented in table 6.1. 
Table 6.1: Reaction parameters used for the in-situ formation of $\mathrm{ZnO}$ quantum dot - wool fibre composites.

\begin{tabular}{|c|c|}
\hline Process Parameters & \\
\hline Wool fibre substrate & Untreated, $\mathrm{HCl}$-, $\mathrm{NaOH}$ - treated \\
\hline Precursor $\mathrm{Zn}^{2+}$ & $\mathrm{ZnCl}_{2}\left(\mathrm{H}_{2} \mathrm{O}\right), \mathrm{Zn}(\mathrm{Ac})_{2}\left(\mathrm{H}_{2} \mathrm{O}\right)$ \\
\hline Complexing agents & $\begin{array}{l}\text { PVP, PMAA-Na, } \mathrm{Na}_{2} \mathrm{~S}_{2} \mathrm{O}_{3}, \mathrm{TSC} \\
\text { Glycine, L-cysteine and MSA }\end{array}$ \\
\hline Total soaking volume / mL & $20-50$ \\
\hline Solvent & $\mathrm{H}_{2} \mathrm{O}$ \\
\hline $\mathrm{m}$ (Merino wool) / g & $0.1-0.5$ \\
\hline$\left[\mathrm{Zn}^{2+}\right] / \mathrm{M}$ & 0.0025 \\
\hline $\mathrm{V}\left(2 \mathrm{M} \mathrm{NaBH}_{4}\right) / \mathrm{mL}$ & 0.5 \\
\hline Reaction temperature $/{ }^{\circ} \mathrm{C}$ & $-25,50-70$ \\
\hline Soaking time after $\mathrm{NaBH}_{4}$ / days & $3-7$ \\
\hline
\end{tabular}

\subsubsection{Building block approach}

The building block approach is the simplest methods to produce quantum dot -fibre composites. This method relies on the formation of a chemical bonding between the surface area of the quantum dots and the functional groups of the substrate material. For this reason, quantum dots should consist of a well-defined nanosized structure with a tailored surface structure. The fibrous substrate should provide the network structure of the final composite material and functional groups for a chemical bonding. Wool and paper are excellent organic substrate materials for the uptake of nanosized materials because of the different functional groups they have along their framework structure. These functional groups should provide anchor sites for the attachment of quantum dots. Furthermore, the adsorption of quantum dots onto the fibrous substrate material should be supported by the large surface area of the quantum dots and substrate material.

The uptake of $\mathrm{ZnO}$ quantum dots onto wool and paper fibres was carried out by submerging $0.1 \mathrm{~g}$ of the substrate material into $20 \mathrm{~mL} \mathrm{ZnO}$ quantum dot suspension. For this, $\mathrm{ZnO}$ quantum dots suspension prepared according to the procedure developed in chapter 4 were directly used after preparation. To ensure a uniform attachment of the $\mathrm{ZnO}$ quantum dots to the substrate material, the soaking medium was gentle agitated using a water shake bath. Here, it is important to note that a uniform composite material is only obtained, when the quantum dots are evenly distributed at the surface of the 
substrate material and agglomeration of the quantum dots was prevented during the formation of the hybrid material.

The attachment of the quantum dots to substrate material is also strongly dependent on the process parameters used during the soaking process. Therefore, the influence of process parameters, such as soaking volume $(20-50 \mathrm{~mL})$, soaking time $(3-7$ days $)$ and temperature $\left(25-90{ }^{\circ} \mathrm{C}\right)$, were examined for the attachment of $\mathrm{ZnO}$ quantum dots onto the network structure of the fibrous substrate material. The strength of the chemical bonding between both components will determine the life-time stability of the new composite material. Thereby, the quantum dots should be preferable covalently bound to functional groups of the substrate material. In that way, quantum dots do not easily leached out, providing long term effectiveness of the fluorescent activity.

However, when a chemical interaction between the inorganic and organic components is not achieved or weak, the building block approach can make use of different chemical techniques to increase the compatibility between them such as modifying the surface area of the substrate or quantum dot material by attaching or exchanging functional group. These chemical techniques can also be used to increase the uptake of quantum dots onto the substrate material and reinforce the chemical bonding between quantum dots and organic substrate material.

Therefore, surface modification of quantum dots and fibrous substrate material was also conducted and their influence on the process parameters (e.g. soaking time and temperature) were examined. Results were compared and the method producing the best possible optical properties for the new hybrid materials identified. 


\subsubsection{Surface modification of $\mathrm{ZnO}$ quantum dots}

Surface modification of the quantum dot material by an exchange of the organic surfactant layer around the quantum dots can increase the compatibility between the quantum dot material and fibrous substrate material leading to stronger chemical bondings and a higher uptake of the quantum dots onto the substrate material. Furthermore, the exchange of the organic surface layer around the quantum dots can improve or tune the fluorescence colour of the quantum dots.

Quantum dots are usually functionalised with capping agents to control the particle growth and ensure a stable dispersion in solution. They also determine along with other reaction parameters the final shape and size of the nanocrystals as well as the size distribution. If the capping agents of the quantum dot material contain additional functional groups which are not reacting with the particle surface, but with the surrounding phase, then a chemical interaction between quantum dot and fibrous substrate material can be created. A chemical interaction is achieved, if the interaction between both components is attractive. If the interaction between both systems is not attractive, then the surface of the quantum dot can be further modified either by an exchange of the capping agent or by tailoring the surface area with linker molecules that can undergo a chemical reaction with functional groups at the particle's surface. By doing so, the modified particle surface should exhibit greater compatibility with the functional groups of the organic substrate. However, the reconstruction of the surface area with different surfactants will not only influence the interaction with the surrounding phases (solvent and substrate material), but may also affect the size, shape and optical properties of the quantum dots. For this reason, the process parameters for the ligand exchange reaction have to be optimised to obtain optical fluorescence and avoid agglomeration reaction of the quantum dot material in solution or substrate material.

The functionalisation of the quantum dots surface was conducted by using two different methods. The first method consisted in the mixture of the capping agent with $\mathrm{ZnO}$ suspension, hoping that the capping agent will exchange the surface coverage of the quantum dot. In the second method, the ionic capping agent was added to the $\mathrm{ZnO}$ suspension containing the fibre material. 
For the modification of the quantum dot surface area, the ionic capping agents listed in table 6.2 were used for $20 \mathrm{~mL} \mathrm{ZnO}$ suspension.

Table 6.2: List of ionic surfactants and linker molecules used to modify the surface area of quantum dots.

\begin{tabular}{ccc}
\hline Capping agent & c (capping agent) & V (capping agent) $/ \mathbf{~ m L}$ \\
\hline $\mathrm{Na}_{2} \mathrm{~S}_{2} \mathrm{O}_{3}\left(\mathrm{H}_{2} \mathrm{O}\right)$ & $0.001-0.05 \mathrm{M}$ & $0.5-5$ \\
$\mathrm{TSC}\left(\mathrm{H}_{2} \mathrm{O}\right)$ & $0.001-0.05 \mathrm{M}$ & $0.5-5$ \\
Glycine $\left(\mathrm{H}_{2} \mathrm{O}\right)$ & $0.001-0.05 \mathrm{M}$ & $0.5-5$ \\
L-cysteine $\left(\mathrm{H}_{2} \mathrm{O}\right)$ & $10^{-4}-0.005 \mathrm{M}$ & $0.5-5$ \\
$\mathrm{MSA}\left(\mathrm{H}_{2} \mathrm{O}\right)$ & $10^{-4}-0.001 \mathrm{M}$ & $0.5-5$ \\
8- $\mathrm{HQ}(\mathrm{EtOH})$ & $10^{-4}-0.0025 \mathrm{M}$ & $0.1-1$ \\
\hline
\end{tabular}

The effect of surface modification on the fluorescence properties of the new $\mathrm{ZnO}$ suspension was investigated as well as the influence of process parameters such as concentration of ionic capping agent (listed in table 6.2), stirring time (1 - $48 \mathrm{hrs})$ and temperature $\left(25-80{ }^{\circ} \mathrm{C}\right)$. Furthermore, the fluorescence properties of the fibrous substrate material, after soaking in the modified $\mathrm{ZnO}$ quantum dot suspension at different temperature $\left(25-70{ }^{\circ} \mathrm{C}\right)$ and soaking times $(3-7$ days $)$, were investigated.

\subsubsection{Merino wool and pre-treatment methods}

The wool fibre surface is coated with an external fatty acid monolayer, which may hinder the adsorption of quantum dots. Pre-treatment methods such as alcoholic alkali $(\mathrm{NaOH})$ and acid $(\mathrm{HCl})$ treatments remove parts of the fatty lipid acid monolayer and expose underlying amino acids [116-118]. Thereby the quantum dot can react with the nitrogen, oxygen and sulphur atoms on the surface proteins of the wool fibres.

However, alcoholic alkali and acid treatment of the wool fibre, however, can cause fibre damage if carried out carelessly and several properties of the wool fibre can change for the worse. For example, wool fibre will shrink and lose elasticity if soaked in a strong acid solution. High temperature $\left(\mathrm{T}>80{ }^{\circ} \mathrm{C}\right)$ and strong alkali solution $\left(\mathrm{OH}^{-}\right)$will also result in the degradation of the wool fibre and cause a hydrothermal yellowing. Therefore, the processing of the wool fibre with alcoholic alkali and acid solutions 
hadto be carried out with caution. The pre-treatment procedure of merino wool with $\mathrm{NaOH}$ and $\mathrm{HCl}$ is described in chapter 2; 2.2.2.1.1 and 2.2.2.1.2.

The uptake of quantum dots onto the substrate material can be significantly increased, when the surface area of the substrate material is functionalised with linker molecules. Linker molecules have to consist of at least two segments. One segment of the linker molecule has to undergo a chemical bonding with the surface of the organic fibrous material, and the second segment has to contain free functional groups that can interact with the surface area of the quantum dots. Depending on the linker molecule, different binding modes such as disulfide, peptide and hydrogen bonds can be created between the quantum dot surface and linker and functional groups of the substrate material.

Mercaptosuccinic acid (MSA), L-cysteine and 8-Hydroxyquinoline (8-HQ) were chosen as linker molecules to create a chemical linkage between the surface of the fibres and quantum dots. The pre-treatment of merino wool and paper with these linker molecules are described in chapter $2 ; 2.2 .2 .1 .3,2.2 .2 .1 .4$ and 2.2.2.2.1.

Mercaptosuccinic acid (MSA) contain three functional groups - two carboxyl and one thiol-group (see figure 6.2), which makes it an optimal linker or coupling agent. It can react in two different ways with wool fibre. One of the possibilities is the formation of a disulphide bond between the cysteine (R-SH) or the cystine (R-S-S-R) present in the wool's keratin and the mercapto group of MSA or the formation of a peptide bond between the carboxylic group in the MSA and the amino group of the wool's keratin. The quantum dots can then interact with the negatively charged carboxyl group or the mercapto group of the MSA. It also can form H-bondings with the oxygen atoms of the cellulose chains of paper fibres.<smiles>O=C(O)CC(S)C(=O)O</smiles>

Figure 6.2: Chemical structure of mercaptosuccinic acid.

L-cysteine is one of the 22 natural existing amino acids. It is also a complexing agent that contains a carboxyl-, thiol- and amino group (figure 6.3). The presence of these 
three different functional groups promises the possibility of different binding modes such as disulfide, peptide and hydrogen bonds between the linker molecule and the functional groups of the fibrous substrate material. The functional groups which do not react with the fibrous substrate material can react with the particle surface of the quantum dots.

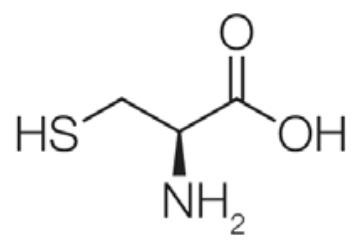

Figure 6.3: Chemical structure of L-cysteine.

8-Hydroxyquinoline (8-HQ) is a bidentate chelating agent and forms fluorescent complexes with zinc and a variety of other metal ions. The hydrogen atom bound to the oxygen atom can be relocated to the nitrogen atom due to the acid-base behaviour of 8HQ and forms a tautomer as shown in figure 6.4. When incorporated onto the network structure of the wool fibre, it can act as linker molecule / complex binder for $\mathrm{ZnO}$ quantum dots and by doing so increase the uptake of $\mathrm{ZnO}$ onto wool.

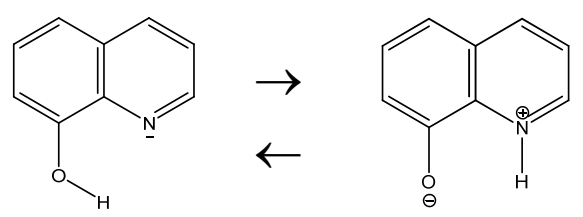

Figure 6.4: Tautomerization of 8-Hydroxyquinoline.

It is important to note that the use of linker molecules between the organic and inorganic phase can influence the fluorescence properties of the quantum dot material. For example, if a coordinated complex is formed or an electron transfer process is enabled by the interaction between the substrate material, linker molecule and quantum dot surface, the fluorescence intensity of a quantum dot can be enhanced or quenched. Furthermore, MSA and L-cysteine are acid reagents and can cause fibre damage to wool if not handled with care. Paper fibre, however, shows a higher chemical stability against acid and alkali solutions. Also, high temperatures $\left(\mathrm{T} \sim 100{ }^{\circ} \mathrm{C}\right)$ do not affect the properties of cellulose fibres. Therefore, the processing of the substrate material had to be individualised to maintain the unique natural properties of the fibrous material. 


\subsubsection{Kraft paper fibres and pre-treatment methods}

The bleached Kraft paper used in the project contains anthraquinone, an aromatic compound which is commonly used as an additive in the Kraft paper process. The production of Kraft paper is carried out in alkaline conditions, which result in the degradation of the cellulose chains through alkaline hydrolysis. The addition of anthraquinone, however, prevents the alkaline degradation process by oxidising the cellulose chains. Apart from the hydroxyl groups of the cellulose chains, quantum dots can also react with anthraquinone which may improve the uptake of the quantum dots to the paper fibres.

However, in EtOH solution, bleached paper pulp turns grey due to the degradation of anthraquinone. Therefore, the uptake of quantum dots as well as the functionalisation with linker molecules was conducted in aqueous solutions.

\subsection{Results}

\subsubsection{In-situ binding of $\mathrm{ZnO}$ quantum dot onto wool}

Wool fibre composites functionalised with $\mathrm{ZnO}$ quantum dots using the in-situ approach showed in their PL scans a characteristic peak round $380 \mathrm{~nm}$ (figure 6.5). Depending on the capping agent used during the soaking process, different intensities for this emission peak were obtained. The highest intensity was obtained with L-cysteine which suggests a better binding mode / cross link of the quantum dots to the protein structure of wool. The complex binding of the zinc cations with PMAA-Na and PVP before inducing the chemical reaction also resulted in an emission peak around $380 \mathrm{~nm}$. The intensity of this peak obtained by using PMAA-NA, however, is higher than that with PVP. When the in-situ approach / binding was carried out using different linker molecules such as MSA, $\mathrm{Na}_{2} \mathrm{~S}_{2} \mathrm{O}_{3}$, TSC, and glycine for the zinc cations in aqueous solution, no emission peak between 375 and $385 \mathrm{~nm}$ was observed in the PL scans of the wool.

The emission peak with a maximum wavelength between 375 and $385 \mathrm{~nm}$ corresponds to the band-edge emission of $\mathrm{ZnO}$ quantum dots. The in-situ formation of $\mathrm{ZnO}$ quantum dots, however, did not result in a defect emission. The binding of $\mathrm{ZnO}$ quantum dots resulted in the quenching of the natural fluorescence of the wool. 


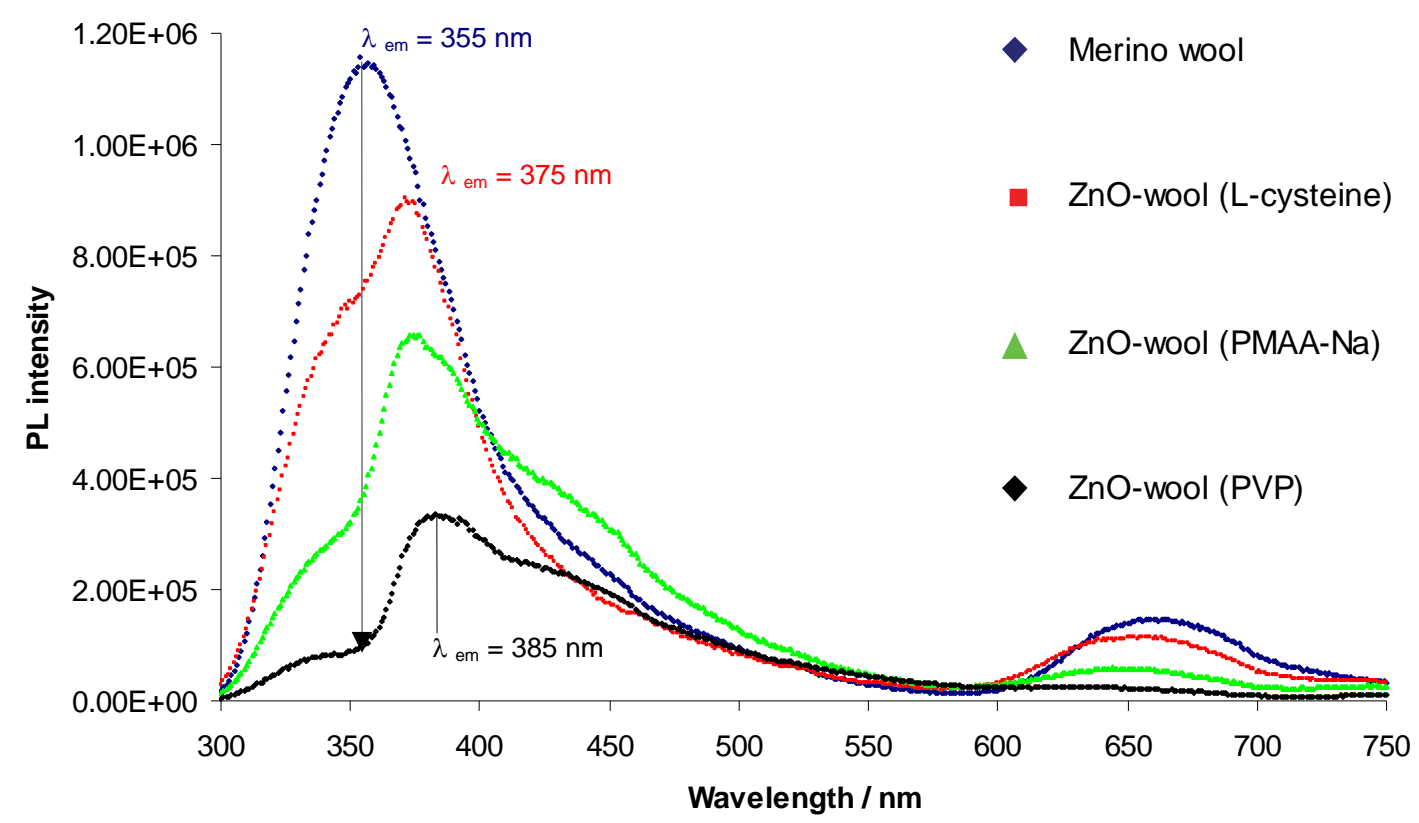

Figure 6.5: Fluorescence scan of $\mathrm{ZnO}$ quantum dot - wool composite prepared using the in-situ approach and different capping agents $\left(\lambda_{\text {exc }}=260 \mathrm{~nm}\right.$, exc. $/$ em. slit $\left.=5 \mathrm{~nm}\right)$. Details of sample preparation are listed in the appendix in table A.12.

\subsubsection{Building block approach}

\subsubsection{Attachment of $\mathrm{ZnO}$ quantum dot onto wool and pre-treated with $\mathrm{NaOH}$ and $\mathrm{HCl}$}

The uptake of $\mathrm{ZnO}$ quantum dots onto merino wool, untreated and pre-treated with $\mathrm{NaOH}$ and $\mathrm{HCl}$, was conducted by immersing the fibrous substrate material inside the $\mathrm{ZnO}$ quantum dot suspension directly after preparation. A successful attachment of $\mathrm{ZnO}$ quantum dots onto merino wool was confirmed by the formation of an emission peak between 375 and $385 \mathrm{~nm}$ (figure 6.6) after being soaked for three days at $70{ }^{\circ} \mathrm{C}$. It was found out that $\mathrm{NaOH}$ treated wool revealed slightly higher fluorescence intensity of the emission peak around $380 \mathrm{~nm}$. The emission peak was highly affected by ageing processes leading to the decrease of the emission peak and shift to higher wavelength. The lowest PL intensity was obtained after 9 days ageing with a maximum emission wavelength at $390 \mathrm{~nm}$. When compared with $\mathrm{ZnO}$ quantum dot suspension, the emission peak around $380 \mathrm{~nm}$ also decreased with ageing; however, by doing so the intensity of the defect emission increased. Here, wool fibre composites do not show a defect emission caused by defect states at the quantum dot surface. 
Instead of showing fluorescence, $\mathrm{ZnO}$ quantum dots attached to wool exhibit UV filtering properties. This is shown by the quenching of the naturally fluorescence of the wool (figure 6.6).

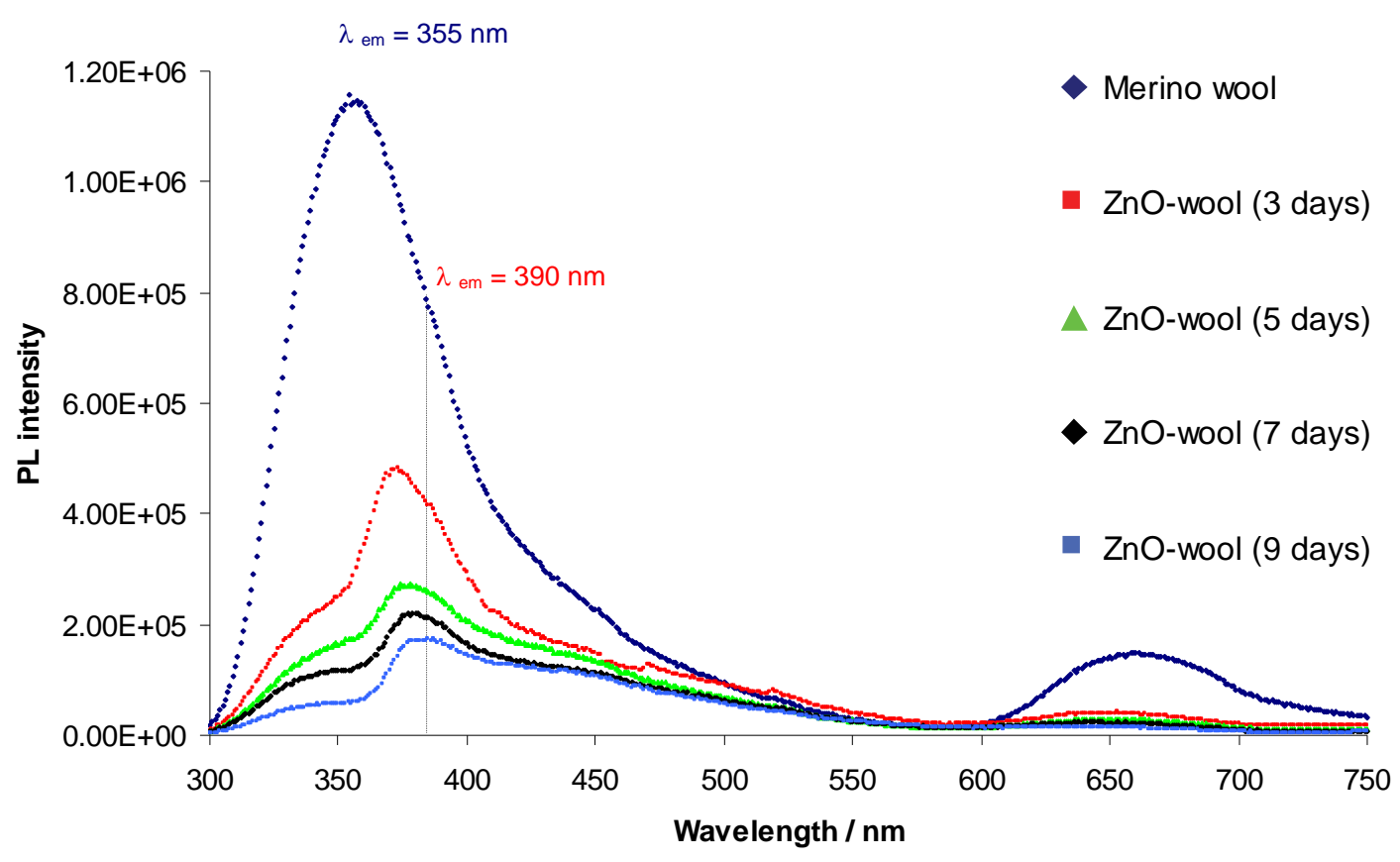

Figure 6.6: Fluorescence scans of ZnO quantum dot - wool composited prepared using the building block approach and the effect of $3,5,7$ and 9 days ageing on the fluorescence scans $\left(\lambda_{\text {exc }}=260 \mathrm{~nm}\right.$, exc. $/$ em. slit $=5 \mathrm{~nm})$. Details of the sample preparation are listed in the appendix in table A.13.

\subsubsection{Attachment of $\mathrm{ZnO}$ quantum dot onto wool functionalised with linker molecules}

The uptake of $\mathrm{ZnO}$ quantum dots onto merino wool functionalised with different capping and linker molecules such as $\mathrm{Na}_{2} \mathrm{~S}_{2} \mathrm{O}_{3}$, TSC, Glycine, L-cysteine and MSA was conducted. However, these procedures did not result in the development of new fluorescent composite material. PL scans of these wool fibres did not show difference in their fluorescence properties after the uptake of $\mathrm{ZnO}$ quantum dots.

The uptake of $\mathrm{ZnO}$ quantum dots by wool treated with 8-HQ (EtOH), however, was successful (see pre-treatment of wool with 8-HQ in chapter 2; 2.2.2.1.4). These wool fibre composites showed in their PL scans an emission peak between 375 and $385 \mathrm{~nm}$, which gradually decreased with ageing. The intensity of this peak was higher than the peak observed in samples where $\mathrm{ZnO}$ quantum dots were attached to untreated wool and wool pre-treated with $\mathrm{HCl}$ and $\mathrm{NaOH}$ (figure 6.7). The higher intensity indicates that 
the use of 8-HQ as fluorescent complex binder and linker molecules increased the uptake of $\mathrm{ZnO}$ quantum dots onto wool.

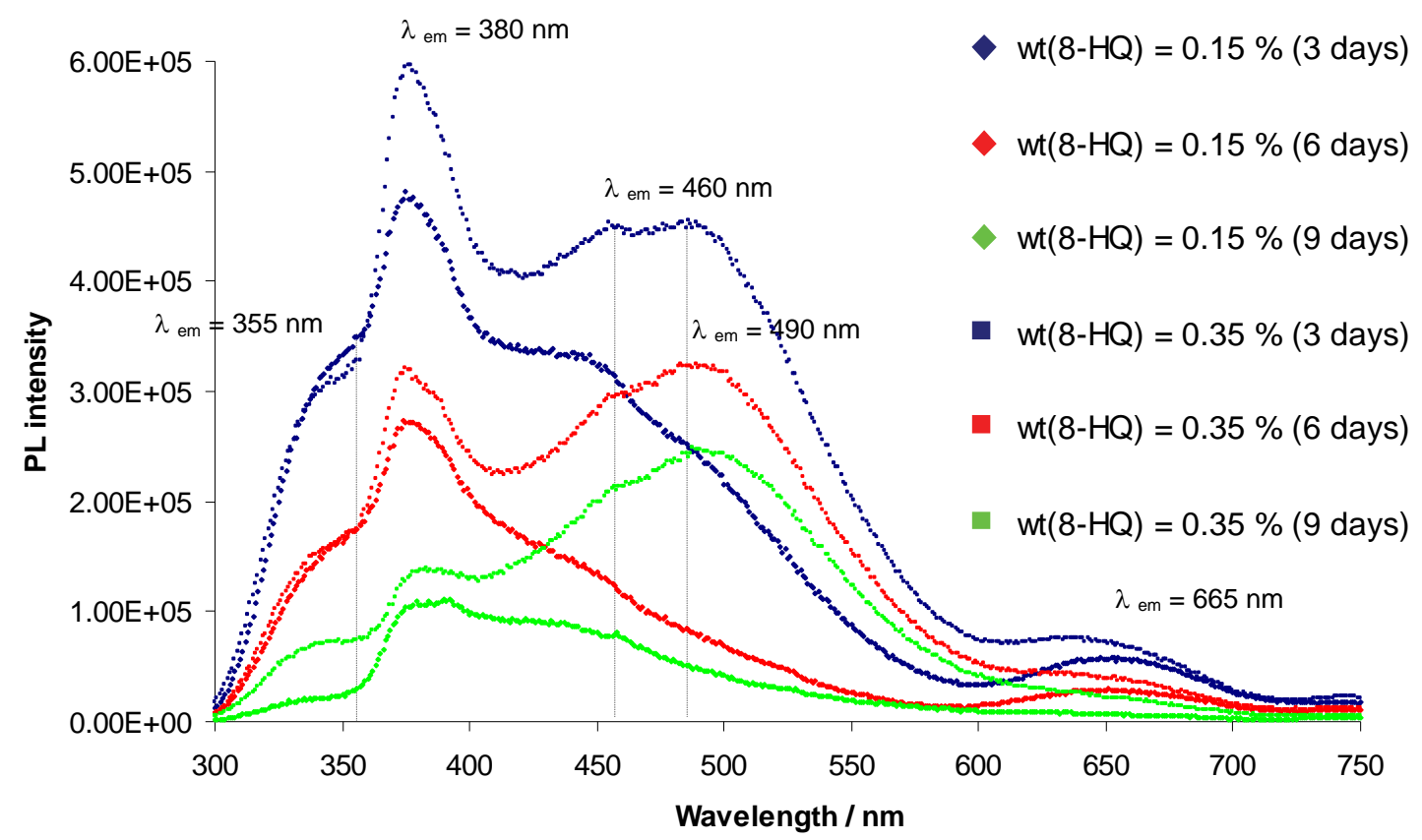

Figure 6.7: Attachment of $\mathrm{ZnO}$ quantum dots to wool using 8-HQ as linker molecule and fluorescent coordination ligand $(\lambda$ exc $=260 \mathrm{~nm}$; exc. $/$ em. slit $=5 \mathrm{~nm})$. Details of sample preparation are listed in the appendix in table A.14.

Figure 6.7 also shows that the natural fluorescence of the wool is quenched by the uptake of $\mathrm{ZnO}$ onto 8-HQ treated wool. Furthermore, two peaks at 460 and around 490 $\mathrm{nm}$ were formed which overlapped to a broad emission peak. These two peaks are strongly dependent on the uptake of 8-HQ onto wool (see figure 6.7). With the formation of the broad peak, the wool fibres obtained a brighter appearance under UVlight and showed a white colour instead of the characteristic bluish colour.

It is important to note that the use of wool with a high uptake of 8-HQ (wt(8-HQ) > $0.35 \%$ ) resulted in the colouration of the wool with different patches of greenish and yellow-greenish colours depending on the 8-HQ uptake by the wool. 


\subsubsection{Surface modification of $\mathrm{ZnO}$ quantum dots and attachment on wool}

The different fluorescence colours of $\mathrm{ZnO}$ suspension created in this project are shown below in figure 6.8 and the corresponding emission peaks are portrayed in figure 6.9.

The surface modification of $\mathrm{ZnO}$ quantum dots by exchanging the polymeric surfactant layer around the $\mathrm{ZnO}$ quantum dots was only successful when 8-HQ was used as a bidentate chelating agent. The addition of $0.1-1 \mathrm{~mL} 0.025 \mathrm{M} \mathrm{8-HQ}(\mathrm{EtOH})$ to $75 \mathrm{~mL}$ $\mathrm{ZnO}$ quantum dot suspension changed the fluorescence of the suspension into a bright white-greenish colour (see figure 6.8; B). The maximum emission wavelength of the suspension was between 500 and $505 \mathrm{~nm}$ (figure 6.9; B) and it is strongly dependent on the 8-HQ concentration.

The addition of MSA to $\mathrm{ZnO}$ quantum dot suspension did not result in optical fluorescence. However, when MSA (0.5 mL 0.5 M MSA) was added to $75 \mathrm{~mL} \mathrm{ZnO}$ quantum dot suspension containing $0.5 \mathrm{~g}$ wool, the optical fluorescence of the $\mathrm{ZnO}$ suspension did change. Here, $\mathrm{ZnO}$ quantum dots were first completely absorbed by the wool, however, some of them were only loosely attached to wool and shaking of the wool resulted in their dispersion back to the suspension. When $0.5 \mathrm{~mL} 0.5 \mathrm{M}$ MSA was added and left at room temperature for 7 days under constant gentle agitation, the $\mathrm{ZnO}$ suspension without the wool showed a strong blue emission (see figure 6.8; A). The maximum emission wavelength of this suspension is in the same wavelength range of the first emission peak of the $\mathrm{ZnO}$ quantum dots suspension (see figure 6.9; $\mathrm{A}$ and $\mathrm{C}$ ). However, this procedure did not improve or change of the fluorescence of the wool soaked inside the suspension.

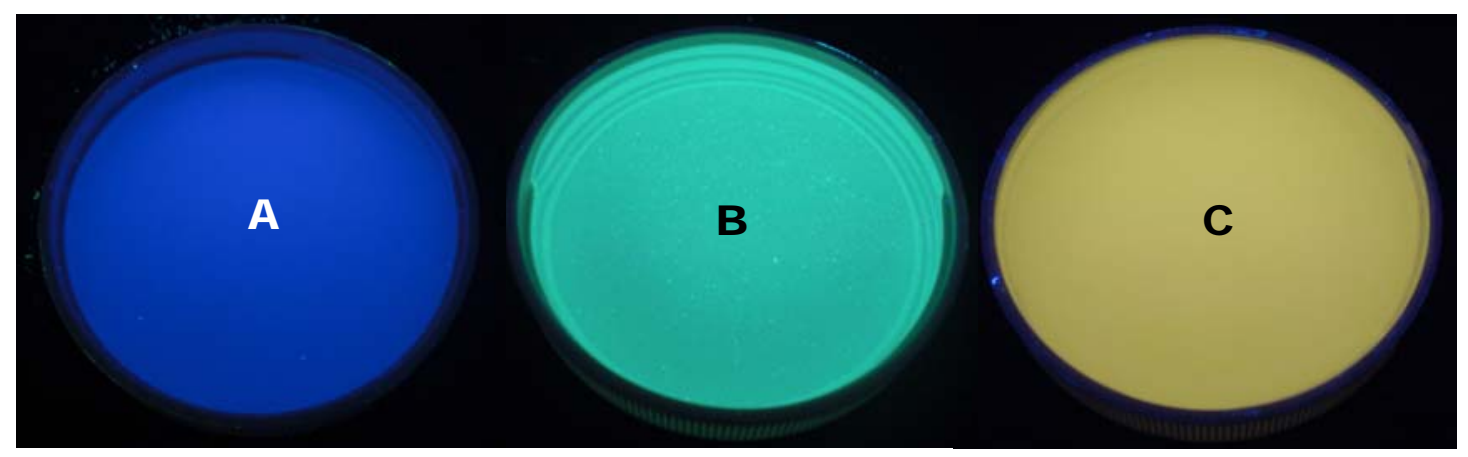

Figure 6.8: Different fluorescence colours of $\mathrm{ZnO}$ quantum dots under $\mathrm{UV}$ light $\left(\lambda_{\text {exc }}=366 \mathrm{~nm}\right)$. A: ZnO quantum dots functionalised with 18-MEA (fatty acid monolayer of the wool fibre); B: ZnO quantum dots functionalised with 8-HQ and C: ZnO quantum dots coated with PVP. 


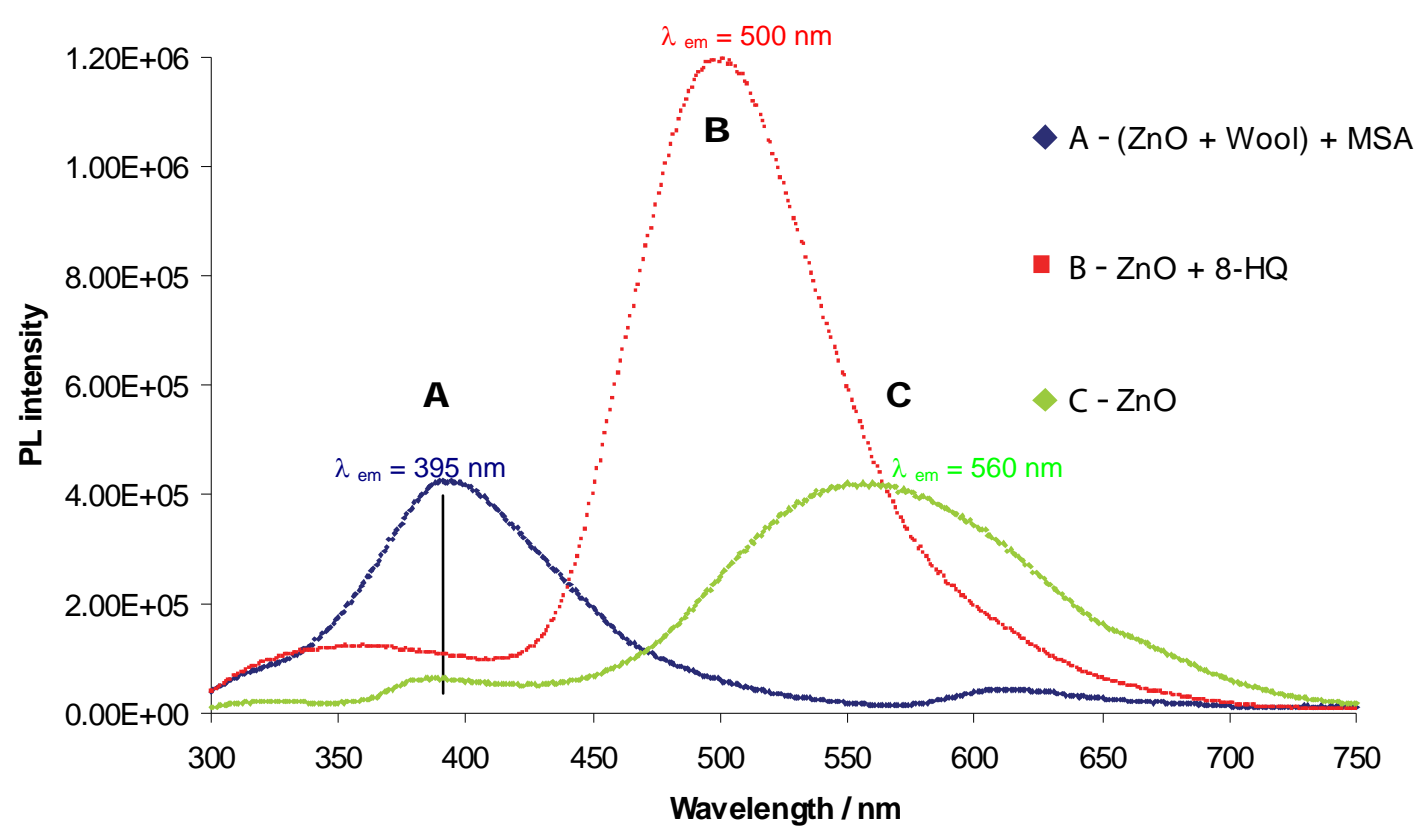

Figure 6.9: Fluorescence scan of $\mathrm{ZnO}$ quantum dot suspensions functionalised with MEA (A), 8-HQ (B) and PVP (C). A: $0.5 \mathrm{~g}$ merino soaked into $75 \mathrm{~mL} \mathrm{ZnO}$ aqueous suspension $+0.5 \mathrm{~mL} 0.5 \mathrm{M}$ MSA. B: $75 \mathrm{~mL} \mathrm{ZnO}+0.5 \mathrm{~mL} 0.025 \mathrm{M}$ 8-HQ (EtOH). C: $\mathrm{ZnO}$ quantum dot suspension. ( $\lambda_{\text {exc }}=260$ $\mathrm{nm}$, exc $/ \mathrm{em}$. slit $=10 \mathrm{~nm}$ ). Details of sample preparation are listed in the appendix in table A.15.

For the functionalisation of wool using $\mathrm{ZnO}$ quantum dots and 8-HQ, different procedures were conducted. One of these procedures involved the addition of $0.1-0.5$ $\mathrm{mL} 0.025 \mathrm{M} 8$-HQ (EtOH) to $75 \mathrm{~mL} \mathrm{ZnO}$ quantum dot aqueous suspension. Then, $0.1 \mathrm{~g}$ merino wool was soaked into $20 \mathrm{~mL}$ of this aqueous suspension for 3 days at $50{ }^{\circ} \mathrm{C}$ under gentle agitation using water shake bath. In another procedure, $0.1 \mathrm{~g}$ wool was soaked directly into $20 \mathrm{~mL} \mathrm{ZnO}$ quantum dot suspension at different soaking temperatures (room, temperature, 50 and $\left.70^{\circ} \mathrm{C}\right)$ and 8-HQ (EtOH) $(0.1-1 \mathrm{~mL} 0.025 \mathrm{M}$ 8-HQ (EtOH)) was added to the aqueous soaking solution. The wool was soaked in this suspension for three days under gentle agitation using water shake bath.

Independent of the procedure used, the final composite materials is covered by fluorescent particles and show PL scans similar to them as shown in figure 6.10; B. The PL scans of these composites materials are characterised by a strong emission peak whose maximum emission wavelength $\left(\lambda_{\mathrm{em}}, \max \right)$ varies between 500 and $505 \mathrm{~nm}$ depending on the 8-HQ concentration added to the $\mathrm{ZnO}$ suspension and soaking solution of wool and $\mathrm{ZnO}$, respectively. However, the fluorescence intensity of the emission peak between 500 and $505 \mathrm{~nm}$ was higher when 8-HQ was added to the $\mathrm{ZnO}$ quantum dot aqueous solution containing wool and soaked at $\mathrm{T}>50^{\circ} \mathrm{C}$. The emission 
wavelength between 500 and $505 \mathrm{~nm}$ refers to the fluorescent particles, which are formed by the addition of 8 -HQ to the $\mathrm{ZnO}$ quantum dot suspension. These particles, however, are not strongly attached to wool and come off by friction. Even so, a large amount remains on the wool fibre and functionalised it with optical fluorescence.

Instead of adding 8-HQ to the aqueous solution of $\mathrm{ZnO}$ quantum dot and wool, $\mathrm{ZnO}$ quantum dot - wool composite was also soaked in 8-HQ (EtOH) solution or exposed to 8-HQ EtOH gas atmosphere. Independently of the method used for the uptake of 8-HQ, the wool fibre composite also showed an emission peak between 500 and $505 \mathrm{~nm}$. The colour of the composite material, however, changed over time. It turned from white greenish to yellow greenish both under ambient and UV-light. This change was also reflected in a shift of the maximum emission to higher wavelength $\left(\lambda_{\mathrm{em}}=520-535\right.$ $\mathrm{nm})$ and lower fluorescence intensity. Figure 6.10 shows the changes in fluorescence of dried $0.1 \mathrm{~g} \mathrm{ZnO}$ quantum dot - wool composite soaked for 3 days in $20 \mathrm{~mL} 1.25 \cdot 10^{-3} \mathrm{M}$ 8-HQ EtOH solution at $50{ }^{\circ} \mathrm{C}$ and 15 days of ageing.

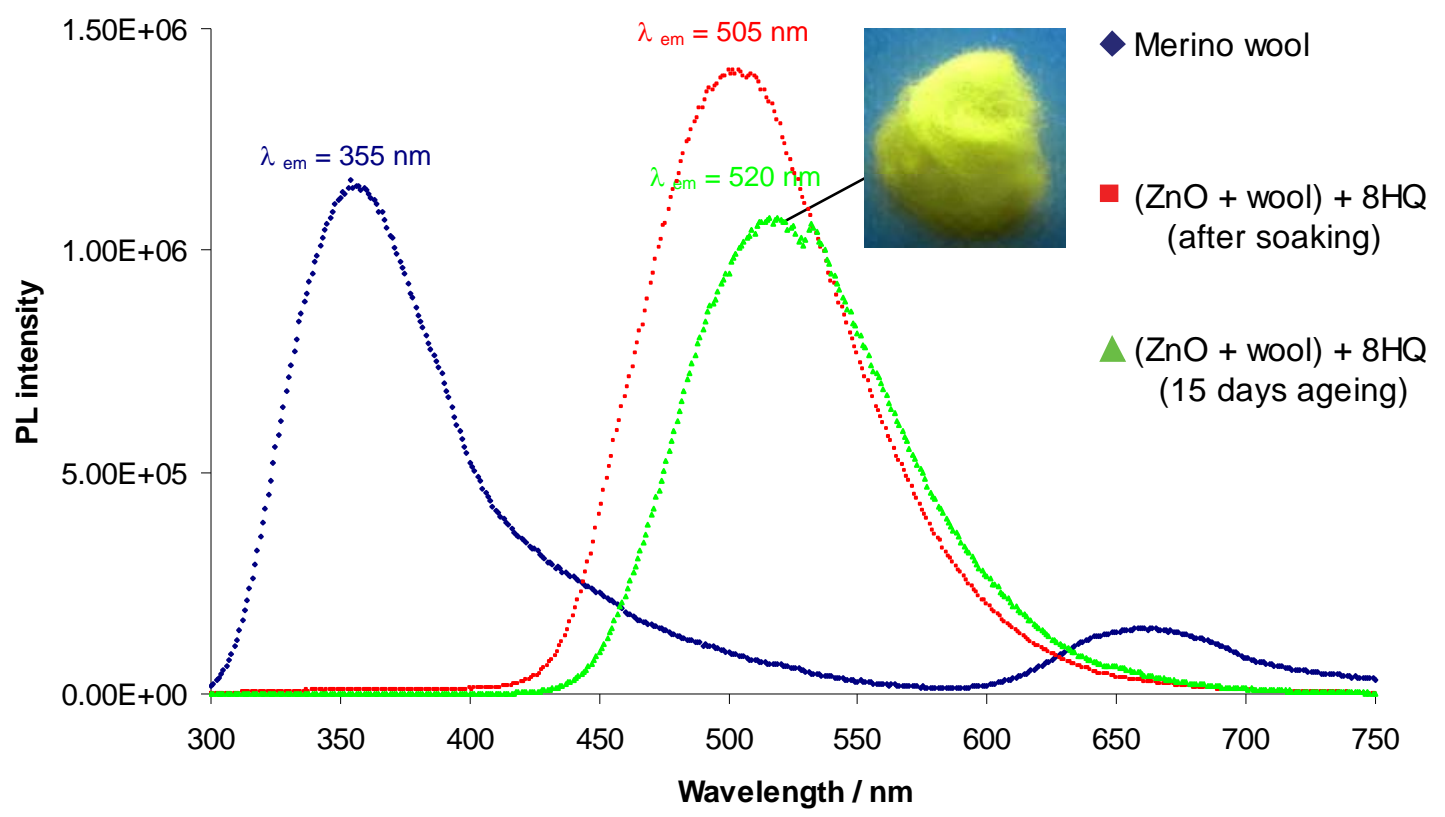

Figure 6.10: Fluorescence scan of $0.1 \mathrm{~g} \mathrm{ZnO}$ quantum dot wool fibre soaked in $20 \mathrm{~mL} 1.25 \cdot 10^{-3} \mathrm{M}$ 8-HQ (EtOH) at $50{ }^{\circ} \mathrm{C}$ for 3 days (red) and aged under ambient atmosphere at room temperature for 15 days (green) and untreated wool (blue) $\left(\lambda_{\text {exc }}=260 \mathrm{~nm}\right.$, exc $/ \mathrm{em}$. slit $=5 \mathrm{~nm}$.) Details of sample preparation are listed in the appendix in table A.16.

The emission shift to longer wavelength $\left(\lambda_{\text {em.max }}>505 \mathrm{~nm}\right)$ was strongly dependent on the uptake of 8-HQ onto $\mathrm{ZnO}$-wool. Soaking $\mathrm{ZnO}$-wool in low concentration of 8-HQ 
([8-HQ] $<1.25 \cdot 10^{-3} \mathrm{M}$ in $20 \mathrm{~mL}$ EtOH) principally suppressed a further emission shift to longer wavlenght with ageing. Even so, $\mathrm{ZnO}$-wool functionalised with 8-HQ may still appear with a pale green colour under ambient light.

\subsubsection{ZnO quantum dot - paper fibre composites}

$\mathrm{ZnO}$ quantum dots attached to bleached Kraft paper fibres by mixing paper pulp ( $0.1 \mathrm{~g}$ shredded paper fibres in $5 \mathrm{~mL}$ water) with $75 \mathrm{~mL} \mathrm{ZnO}$ quantum dot suspension for 7 days at room temperature did not functionalise the paper fibres with optical fluorescence. However, the fluorescence activity of bleached Kraft paper was completely quenched indicating to UV-filtering absorbance properties (see figure 6.12; A).

Fluorescence was obtained, when 8-HQ (1 mL $0.025 \mathrm{M})$ was added to the $\mathrm{ZnO}$ quantum dot paper fibre suspension. The suspension shows a bright white-greenish colour when illuminated with UV light (see figure 6.11). The maximum emission wavelength varies between 500 and $505 \mathrm{~nm}$ depending on the 8-HQ amount added to the $\mathrm{ZnO}$-paper fibre suspension (see figure 6.12; B, C and D).

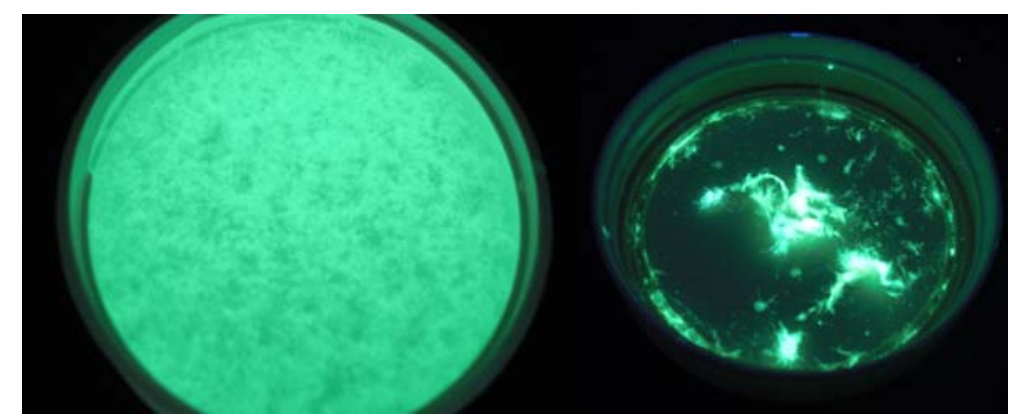

Figure 6.11: ZnO-paper fibre aqueous suspension functionalised with 8-HQ (+ $1 \mathrm{~mL} \mathrm{0.025} \mathrm{M} \mathrm{8-HQ}$ at $70^{\circ} \mathrm{C}$ ) in aqueous suspension (right) and dried (left) observed under $\mathrm{UV}$-light $\left(\lambda_{\text {exc }}=254 \mathrm{~nm}\right.$ ).

The addition of 8-HQ (EtOH) to the $\mathrm{ZnO}$ quantum dot - paper fibre aqueous suspension results in the instant formation of fluorescent particles covering the paper fibres. Some of the particles are loosely attached and come off by friction. For this reason, the washing process is essential to remove all loosely bound particles from the substrate material. The maximum emission wavelength and the fluorescence intensity of these particles are strongly dependent on the 8-HQ concentration added to the $\mathrm{ZnO}$ quantum 
dot - paper suspension as well on the soaking parameters such as $\mathrm{pH}$ and soaking temperature.

Figure 6.12 shows the different colours of paper fibre composites under UV light and ambient light obtained in this project. Sample A shows the final composite material obtained by mixing paper pulp ( $0.1 \mathrm{~g}$ shredded paper fibres in $5 \mathrm{~mL}$ water) with $75 \mathrm{~mL}$ $\mathrm{ZnO}$ quantum dot suspension. Sample $\mathrm{B}$ and $\mathrm{C}$ shows the change of fluorescence colour, when $0.1 \mathrm{~mL} 0.025 \mathrm{M} 8$-HQ (EtOH) and $1 \mathrm{~mL} 0.025 \mathrm{M}$ 8-HQ (EtOH), respectively, was added to the $\mathrm{ZnO}$ quantum dot - paper aqueous suspension during soaking at $70{ }^{\circ} \mathrm{C}$. Sample D shows the fluorescence colour of $\mathrm{ZnO}$ quantum dot - paper fibre when $1 \mathrm{~mL} 0.025 \mathrm{M}$ 8-HQ (EtOH) was added to the boiling $\mathrm{ZnO}$ quantum - dot paper fibre composite.

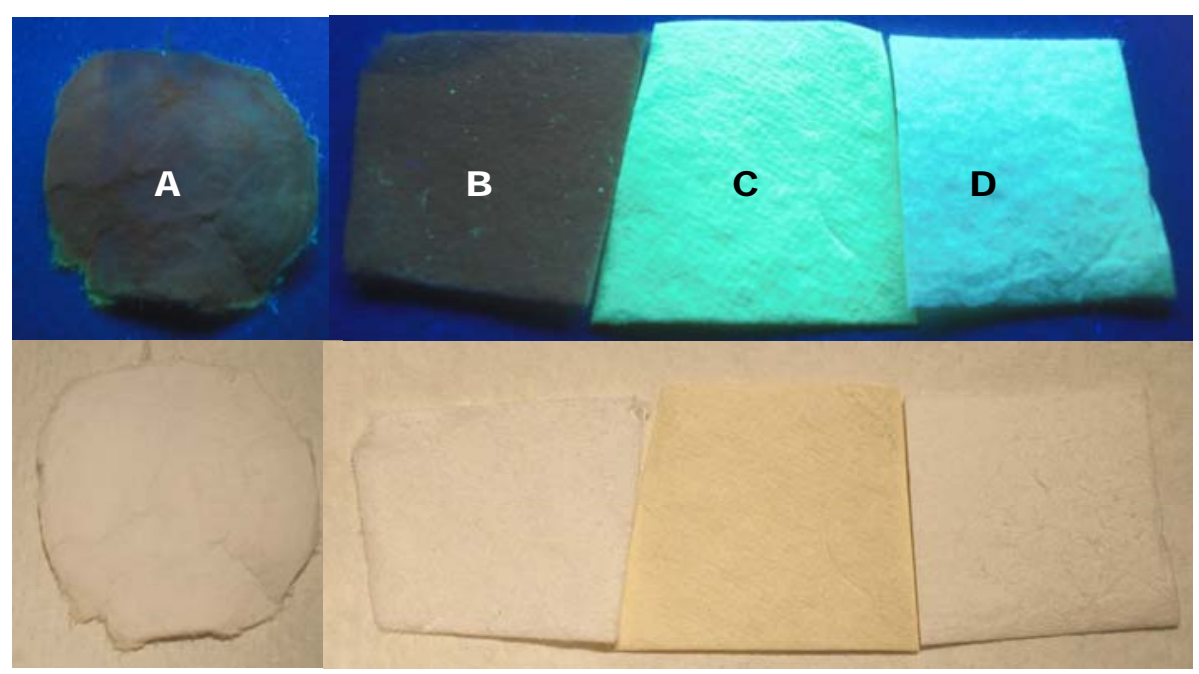

Figure 6.12: Paper fibre functionalised with $\mathrm{ZnO}$ quantum dots (A) and $\mathrm{ZnO}$-paper fibre composites functionalised with different concentration of 8-HQ (B, C and D) under UV light (top, $\lambda_{\text {exc }}=254 \mathrm{~nm}$ ) and ambient light (bottom). Details of sample preparation are listed in the appendix in table A.17.

Here, it is important to note that $\mathrm{ZnO}$ quantum dot - paper fibre suspensions were stirred vigorously, when 8-HQ was added to each sample to ensure homogeneity. The PL scans of the samples in figure 6.12 are shown in figure 6.13 . 


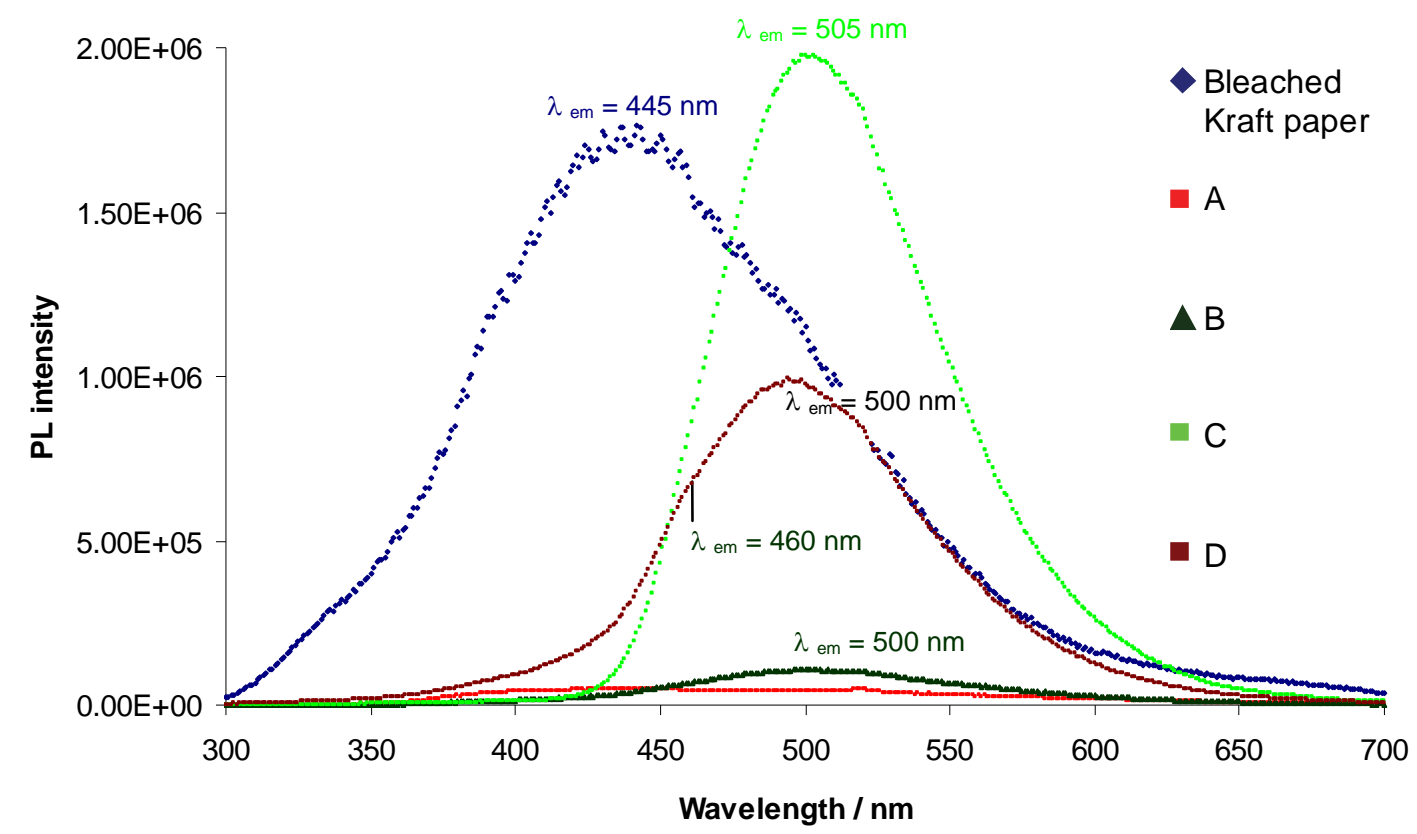

Figure 6.13: PL scan of bleached Kraft paper (blue line), ZnO quantum dot - paper composite (A, red line) and $\mathrm{ZnO}$ quantum dot - paper composites functionalised with different concentration of 8-HQ (B, black line: V(0.025 M 8-HQ $\left(\right.$ EtOH)) $=0.1 \mathrm{~mL}, 70{ }^{\circ} \mathrm{C}$; C, green line: V(0.025 M 8-HQ $($ EtOH $))=1 \mathrm{~mL}, 70{ }^{\circ} \mathrm{C}$; D, brown line: $\left.\mathrm{V}(0.025 \mathrm{M} \mathrm{8-HQ}(\mathrm{EtOH}))=1 \mathrm{~mL}, 100{ }^{\circ} \mathrm{C}\right)\left(\lambda_{\text {exc }}=260 \mathrm{~nm}\right.$; exc $/$ em. slit $=5 \mathrm{~nm})$.

The PL scans of the $\mathrm{ZnO}$ quantum dot - paper fibre composites shows that the addition of $\mathrm{ZnO}$ quantum dots to the paper fibres completely quenches the fluorescence of the bleached Kraft paper fibres shown by a broad emission peak with a maximum emission wavelength at $445 \mathrm{~nm}$ (figure 6.13; A).

The PL scans of $\mathrm{ZnO}$ quantum dot - paper fibres functionalised with 8-HQ (figure 6.13; B, C and D) also show that the fluorescence of the bleached Kraft paper fibres is completely quenched. The addition of small 8-HQ concentration $(\mathrm{V}(0.025 \mathrm{M} 8-\mathrm{HQ})=$ $0.1 \mathrm{~mL}$ ) to $80 \mathrm{~mL} \mathrm{ZnO}$-paper fibre suspension at $70{ }^{\circ} \mathrm{C}$ resulted in a small broad emission peak around $500 \mathrm{~nm}$ (figure 6.13; B), which however increased in magnitude with the addition of higher 8-HQ concentration (V(0.025 M 8-HQ) = $1 \mathrm{~mL}$; figure 6.13; C). Furthermore, the maximum emission shifted to higher wavelengths (max. $505 \mathrm{~nm}$ ) and the colour of the paper fibre turned green-yellowish under ambient and UV-light (figure 6.12 ; C). The addition of 8 -HQ to hot $\mathrm{ZnO}$-quantum dot - paper aqueous suspension $\left(\sim 100{ }^{\circ} \mathrm{C}\right)$ resulted in lower PL intensity and lower maximum emission wavelengths (figure 6.13; D). Furthermore, the emission peak is broader and shows a distinct hump at $460 \mathrm{~nm}$. The paper fibre composite, however, has a brighter and whiter 
appearance under UV-light and ambient light, when compared with sample C in figure 6.12 .

Ageing affects the chemical bonding between $\mathrm{ZnO}$ quantum dots and paper fibres causing the loss of $\mathrm{ZnO}$ at the edges of the paper fibre. Here, the fluorescence of the bleached Kraft paper is observed (figure 6.14).

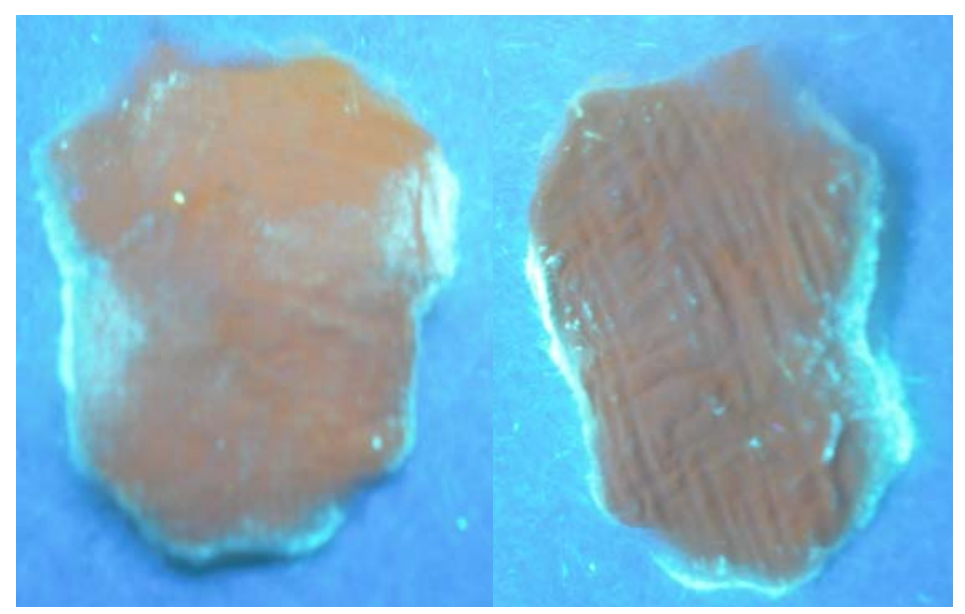

Figure 6.14: Upper and lower side of ZnO quantum dot - paper fibre obtained after ageing $(\sim 3$ months). Note the $\mathrm{ZnO}$ quantum dots leaching out at the fringes of the paper sheets and the fluorescence of the bleached Kraft paper $\left(\lambda_{\mathrm{exc}}=366 \mathrm{~nm}\right)$.

\subsection{Discussion}

The main focus of this chapter was to obtain composite materials comprising of $\mathrm{ZnO}$ quantum dots and natural fibrous substrate that show fluorescence in the visible range. Although this feature was not observed, the composite materials produced here revealed interesting UV-absorbance properties. $\mathrm{ZnO}$ quantum dots attached to wool and bleached Kraft paper quenched the natural fluorescence of the wool and the fluorescence of the bleached Kraft paper fibres, without changing the white appearance of the fibrous substrate under ambient light. This quenching property can be of interest for the preparation of new composite materials. Therefore, further characterisation of the composite materials using methods such as XPS to determine the chemical bonding between $\mathrm{ZnO}$ quantum dots and fibrous material is highly recommend in future studies. Determining the chemical bonding between both components is important to avoid leaching effects. 
Fluorescence in the visible range was achieved, when 8-Hydroxyquinoline (8-HQ) was used as fluorescent ligand for the $\mathrm{ZnO}$ quantum dot surface. Futhermore, the use of 8$\mathrm{HQ}$ resulted in a higher uptake of $\mathrm{ZnO}$ quantum dots onto the fibrous substrate material.

\subsubsection{ZnO quantum dot - wool fibre composites}

\subsubsection{In-situ binding of $\mathrm{ZnO}$ quantum dots to wool}

The in-situ preparation of $\mathrm{ZnO}$ quantum dot - wool composite was based on a sol-gel process, which makes use of hydrolysis and condensation reactions of water complexed metal ions. These are kinetic reactions which will be influenced by the complexation of the zinc cations with surfactants such PVP, PMAA-NA and L-cysteine in solution. This in-situ method involved first the adsorption of the quantum dot precursor material on the substrate material. Here, it is important to note that the adsorption of the zinc cations depends on steric diffusive kinetics and selective binding and non-binding processes to the functional groups of the wool fibre.

The chemical transformation of the water complexed zinc cation into a solid phase was then induced by the addition of $\mathrm{NaBH}_{4}$ leading to a forced alkaline hydrolysis. $\mathrm{NaBH}_{4}$ is an alkali reducing agent, which increase the $\mathrm{pH}$ of the soaking solution. Under alkaline conditions, water complexed zinc cation form a solid phase via hydrolysis and condensation reaction. The addition of $\mathrm{NaBH}_{4}$ to the soaking solution, however, did not result in the precipitation of $\mathrm{ZnO}$ particles in solution, as happened in the preparation of $\mathrm{ZnO}$ quantum dot aqueous suspension in chapter 4. Here, the hydrolysis and condensation reaction of water complexed zinc cations is affected by the electrostatic interaction between zinc cations and functional groups of the wool. Thereby, the reaction kinetic of hydrolysis and condensation is slowed down promoting a slow crystal growth. In general, a slow crystal growth favours the formation of thermodynamically stable particles with a crystalline character. Slow hydrolysis reactions also enable cross-link reactions between the inorganic and organic phase leading to structural changes of the wool. This, however, is only possible when the protein structure of wool contains functional groups that can react with the surface of the quantum dots. 
The in-situ binding of $\mathrm{ZnO}$ quantum dots onto wool resulted in the formation of an emission peak between 375 and $385 \mathrm{~nm}$ (figure 6.5). This emission peak corresponds to the band-edge emission of $\mathrm{ZnO}$ quantum dots. Furthermore, $\mathrm{ZnO}$ quantum dots attached to wool resulted in the quenching of the natural fluorescence of the wool fibre. Wool fibre contains fluorescent amino acids, phenylalanine, tyrosine and tryptophan. Each aromatic amino acid show distinct emission peaks, however, the emission peak of tryptophan or the photo-degradation product of tryptophan, is dominating the fluorescence spectra of the wool and gives an overall emission peak at $355 \mathrm{~nm}\left(\lambda_{\text {exc }}=\right.$ $260 \mathrm{~nm})$.

The use of $\mathrm{NaBH}_{4}$ as an alkali reducing agent in this method may enhance the chemical attachment of $\mathrm{ZnO}$ quantum dots to wool. The main component of wool is keratin, a fibrous structural protein. The most important cross-link of keratin is the disulphide bond of cysteine, which however is not stable in a reducing environment. The addition of $\mathrm{NaBH}_{4}$ to the soaking solution containing wool can break the disulphide bonds according to the following equation:<smiles>N[C@@H](CSSC[C@H](N)C(=O)O)C(=O)O</smiles>

Cystine
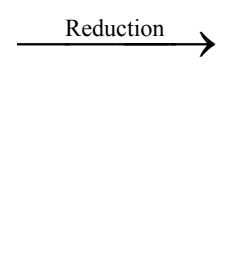

2 H<smiles>NC(CS)C(=O)O</smiles>

Cysteine

The thiol groups formed by the reductive splitting of the disulphide bonds in wool keratin are potential anchor groups for the attachment of quantum dots to wool by forming cross-links with the surface of the $\mathrm{ZnO}$ quantum dots.

The nucleation and growth of the quantum dots in solution was also controlled by the use of capping agents. Capping agents play an important role in the stabilization of the $\mathrm{ZnO}$ quantum dots in suspension. The in-situ binding of $\mathrm{ZnO}$ onto wool with L-cysteine as capping agent revealed a high and sharp emission peak. It is likely, that L-cysteine 
act as a linker molecule between the quantum dots and wool fibre, which also increase the uptake of the quantum dots onto wool.

The in-situ binding of $\mathrm{ZnO}$ onto wool with polymers revealed lower band-edge emission peaks and a stronger quenching effect of the wool fibre fluorescence. Furthermore, they showed small humps around $445 \mathrm{~nm}$ (figure 6.5). These humps may be related to specific chemical bondings between quantum dot surface and wool fibre. It was expected that the absorption of $\mathrm{ZnO}$ quantum dots to wool will show the fluorescence of the defect emission. However, $\mathrm{ZnO}$ wool fibre composites did not show a broad emission band in the wavelength range between 450 and $750 \mathrm{~nm}$ as shown in figure 6.9; C. It is assumed that this broad emission peak is caused by crystalline imperfections at the quantum dot surface. Since $\mathrm{ZnO}$ quantum dot - wool fibre composite only show the fluorescence peak of the band-edge emission $(380 \mathrm{~nm})$, it can be assumed that cross-link reactions between quantum dots and wool fibre formed during the in-situ growth has a passivation effect on the quantum dot surface.

\subsubsection{Building block approach}

\subsection{Attachment of $\mathrm{ZnO}$ quantum dot onto wool}

The attachment of $\mathrm{ZnO}$ quantum dots onto wool, untreated and pre-treated with $\mathrm{NaOH}$ or $\mathrm{HCl}$, also resulted in the quenching of the natural fluorescence of the wool. Furthermore, the $\mathrm{ZnO}$ quantum dot - wool fibre composite shows a fluorescence peak between 375 and $385 \mathrm{~nm}$ (figure 6.6), which can be referred to the band-edge emission of $\mathrm{ZnO}$ quantum dots.

The band-gap emission of the $\mathrm{ZnO}$ quantum dots, however, is affected by ageing of the fibre composite. With ageing, in solution or dried in ambient atmosphere, the emission peak gradually decreases. This age-related reduction of the band-edge emission is also observed by $\mathrm{ZnO}$ quantum dot aqueous suspensions (figure 4.10). Here, the decrease is caused by the incorporation of surface related defect states within the band gap causing a defect emission around $560 \mathrm{~nm}\left(\lambda_{\mathrm{exc}}=260 \mathrm{~nm}\right)$. With the rise of the defect emission, the band-edge emission is gradually quenched until a small hump around $390 \mathrm{~nm}$ remains. This hump is also seen in $\mathrm{ZnO}$ quantum dot - wool fibre composites. 
However, wool fibre functionalised with $\mathrm{ZnO}$ quantum dots does not show a defect emission in the visible range. This suggests that the band-edge emission is quenched by non-radiative recombination processes between quantum dots and wool fibre. Therefore, it is important to determine the chemical bonding between both components using XPS measurements.

It was observed that the intensity of the band-edge emission was slightly higher when $\mathrm{ZnO}$ quantum dots were absorbed onto $\mathrm{NaOH}$ treated wool. This slightly higher intensity may be attributed to a higher uptake of $\mathrm{ZnO}$ quantum dots onto wool. As mentioned before, the wool fibre surface is coated with a fatty acid monolayer, which may hinder the adsorption of the $\mathrm{ZnO}$ quantum dots onto wool. The pre-treatment of the wool with $\mathrm{NaOH}$, as well as $\mathrm{HCl}$, remove parts of the fatty acid monolayer around the wool fibre and exposes the underlying amino acids of the protein structure. However, the soaking of wool in strong alkali solutions $(\mathrm{NaOH})$ and at high temperature can result in a hydrothermal yellowing. This effect is caused by the oxidation of the disulphide bonds in keratin, which break apart as shown in the following equations.

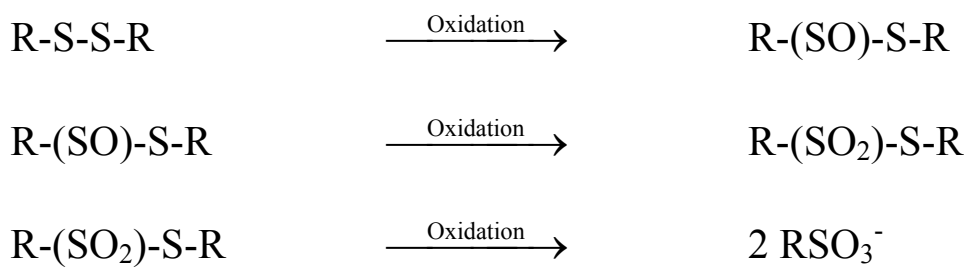

$\mathrm{ZnO}$ quantum dots may show a higher affinity to undergo a chemical binding to the oxidised disulphide bonds which finally increase the uptake of $\mathrm{ZnO}$ quantum dots to the wool fibre protein structure.

\subsection{Attachment of $\mathrm{ZnO}$ quantum dot onto wool using linker molecules}

Different chemical techniques such as surface modification of the wool fibre with linker molecules and surface modification of the quantum dots with different capping agents were explored to increase the uptake of $\mathrm{ZnO}$ quantum dots onto wool.

The surface modification of the wool fibre with MSA and L-cysteine as linker molecules and $\mathrm{Na}_{2} \mathrm{~S}_{2} \mathrm{O}_{3}$, TSC and glycine as ionic molecules did not improve the uptake 
of the $\mathrm{ZnO}$ quantum dots or changed the optical properties of the composite. The ionic capping agents may preferentially interact with the substrate material rather than with the quantum dots. For example, TSC may reduce the wool and become oxidised. Also, the pre-treatment of the wool fibre surface with MSA and L-cysteine resulted in the shrinking of the fibres. The functionalisation of wool fibre with L-cysteine also caused a yellowing of the wool. Because of these unwanted results, it was attempted to modify the surface area of the $\mathrm{ZnO}$ quantum dots to obtain a better compatibility with the organic substrate material.

$\mathrm{ZnO}$ quantum dot suspensions prepared following the method in chapter 2 (2.1.3.2) formed large polycrystalline particles between 100 and $10^{3} \mathrm{~nm}$. In order to enhance the attachment of the $\mathrm{ZnO}$ quantum dots onto the fibrous substrate material, the suspension was mixed with different capping agents such as $\mathrm{Na}_{2} \mathrm{~S}_{2} \mathrm{O}_{3}$, TSC, glycine, L-cysteine and MSA. By doing this, it was hoped to break up the agglomerations of $\mathrm{ZnO}$ quantum dots and increase the uptake of the quantum dots on the fibrous substrate material. Furthermore, it was hoped that the polymeric dispersant at the surface of the $\mathrm{ZnO}$ quantum dots can be exchanged by the different ionic capping agents through ligand exchange reaction. By doing this it was expected that the new surface coverage will obtain a higher compatibility to bind with the substrate material.

The mixing of $\mathrm{ZnO}$ quantum dots with these ionic agents resulted in the breaking up of the $\mathrm{ZnO}$ agglomerates. However, this had a negative effect on the fluorescence intensity of the defect emission. Also, none of the suspensions showed the band-edge emission of $\mathrm{ZnO}$ quantum dots. Furthermore, it was not possible to functionalise the wool fibre by immersing it in the different modified $\mathrm{ZnO}$ suspensions. For this reason, another approach to attach $\mathrm{ZnO}$ quantum dots to wool was attempted. In this approach, the ionic agents were added to the soaking solution containing wool and $\mathrm{ZnO}$ quantum dots.

$\mathrm{ZnO}$ quantum dots show a high affinity to absorb onto wool; however, particles are only loosely attached and they can be easily dispersed into solution again by shaking the wool. To create a strong chemical bonding between $\mathrm{ZnO}$ quantum dots and wool, MSA, L-cysteine, $\mathrm{Na}_{2} \mathrm{~S}_{2} \mathrm{O}_{3}$, TSC and glycine were added to the soaking solution of wool and $\mathrm{ZnO}$ quantum dots. It was expected that these agents will operate as linker molecules between quantum dots and wool and provide a strong chemical bonding between both 
components to avoid leaching of the quantum dots; however functionalisation was not obtained. Here, it is important to note that MSA and L-cysteine are acid reagents and dissolve $\mathrm{ZnO}$ quantum dots when used in high concentration.

Although, the wool fibre was not functionalised by this approach, the soaking of wool inside the $\mathrm{ZnO}$ quantum dot suspension and the addition of MSA to the soaking solution resulted in a strong blue emission of the $\mathrm{ZnO}$ suspension with a maximum emission wavelength at $395 \mathrm{~nm}$ (figure 6.9; A). This emission peak matches with the emission peak in the UV region of the initial $\mathrm{ZnO}$ quantum dots suspension obtained after ageing. However, the fluorescence intensity of PVP coated $\mathrm{ZnO}$ quantum dots is significantly lower (figure $6.9 ; \mathrm{C}$ ). The strong blue emission may arise from the surface modification of the $\mathrm{ZnO}$ quantum dots with 18-MEA chains from the wool fibre. During the soaking process, $\mathrm{ZnO}$ quantum dots are absorbed at the wool fibre surface which is coated by the external fatty acid monolayer 18-MEA (see figure 1.15). As mentioned before, MSA is an acid reagent and will remove parts of this fatty acid monolayer. The long chains of 18-MEA may then bind to the hydroxyl groups at the surface of the $\mathrm{ZnO}$ quantum dots via hydrogen bonds. The new surface coverage of the $\mathrm{ZnO}$ quantum dots with 18-MEA chains give rise to a strong blue emission. The literature also report about a stable aqueous suspension of $\mathrm{ZnO}$ quantum dots with strong blue emission, when the surface area of the quantum dots was functionalised with oleic acids [107]. Further research is needed to understand the chemical bonding between the fatty acid chains and $\mathrm{ZnO}$ surface.

\subsection{Attachment of $\mathrm{ZnO}$ quantum dot onto wool using 8-HQ}

It turned out that the uptake of $\mathrm{ZnO}$ quantum dots onto wool was enhanced when 8-HQ was used. This was shown by a higher intensity of the emission peak at $380 \mathrm{~nm}$. Furthermore, the uptake of $\mathrm{ZnO}$ quantum dots onto wool with 8-HQ resulted in brightening of the wool. Here, 8-HQ acts as a linker molecule for the chemical attachment of the $\mathrm{ZnO}$ quantum dots to the fibrous substrate material and it also forms a fluorescent coordination complex at the surface of the $\mathrm{ZnO}$ quantum dots. 8-HQ is known to form fluorescent chelates with zinc and other metal ions [119-122]. 
The uptake of $\mathrm{ZnO}$ quantum dots onto wool with 8-HQ can be carried out by functionalising the wool with small amounts of 8-HQ before the uptake of $\mathrm{ZnO}$ quantum dots or by adding very small concentrations of 8-HQ directly to the $\mathrm{ZnO}$ quantum dot aqueous suspension containing wool. In both procedures, $\mathrm{ZnO}$ quantum dot - wool composites showed the characteristic band-edge emission from $\mathrm{ZnO}$ quantum dots and two emission peaks at 460 and around $490 \mathrm{~nm}$ (see figure 6.7). The peak at $460 \mathrm{~nm}$ is constant, whereas the peak at 490 can vary depending on the 8-HQ concentration. These peaks are overlapping and form a broad emission peak which gives the $\mathrm{ZnO}$ quantum dot - wool composite a brighter and whiter appearance under UV-light.

The emission peaks at 460 and $490 \mathrm{~nm}$ refer to the fluorescence of a coordination complex formed by the absorption of 8-HQ onto the $\mathrm{ZnO}$ quantum dot surface. Here, 8HQ act as a bidentate ligand that bonds with zinc cations at the quantum dot surface and form a fluorescent coordination complex, $\mathrm{Zn}(8-\mathrm{Q})_{2}$ chelate. The fluorescence of the chelate originates by electronic transitions between the molecular orbitals of 8-HQ and the atomic orbitals of zinc of the fluorescent coordination complex. The fluorescence wavelength of the monomer chelate is indicated by the emission peak observed at 460 $\mathrm{nm}$. The fluorescence of the $\mathrm{Zn}(8-\mathrm{Q})_{2}$ chelate, however, is highly affected by intermolecular interaction between adjacent chelates at the particle surface. Intermolecular interaction such as dipole-dipole coupling enables non-radiative energy transfer processes between two adjacent chelates. This, however, result in the shift of the fluorescence to longer wavelength. Therefore, the second emission peak observed around $490 \mathrm{~nm}$ (figure 6.7) can be attributed to the fluorescence caused by intermolecular interaction between adjacent chelates.

The fluorescence properties of wool fibre composites functionalised with of $\mathrm{ZnO}$ quantum dots and 8-HQ is strongly dependent on the concentration of 8-HQ added to $\mathrm{ZnO}$ aqueous suspension. The light emission and fluorescence intensity of the $\mathrm{ZnO}$ quantum dot suspension can be significantly enhanced by the addition of higher 8-HQ concentration. By doing so, the suspension illuminates in a bright white-greenish fluorescence (figure 6.8; B) with a maximum emission wavelength between 500 and $505 \mathrm{~nm}$ under UV-light (see figure 6.9; B). With the addition of higher 8-HQ concentration, more 8-HQ molecules will attach on the $\mathrm{ZnO}$ quantum dot surface, 
which consequently increase the fluorescence intensity of the chelate in suspension. Furthermore, a higher uptake of 8-HQ onto the particle surface will reduce the distances between adjacent $\mathrm{Zn}(8-\mathrm{Q})_{2}$ chelates and a uniform surface layer of 8-HQ can be formed around the quantum dots. This enhances intermolecular interaction between adjacent chelates which is shown by the fluorescence shift from $490 \mathrm{~nm}$ to $505 \mathrm{~nm}$. Here, it is important to note that fluorescence at $490 \mathrm{~nm}$ is only observed by wool fibre composites functionalised with $\mathrm{ZnO}$ and $8-\mathrm{HQ}$ in small concentration. $\mathrm{ZnO}$ quantum dot suspension functionalised with 8-HQ does not show fluorescence between 490 and $500 \mathrm{~nm}$ and the characteristic emission peak at $460 \mathrm{~nm}$ is also not observed.

8-HQ is hydrophobic due to the aromatic ring structure and form clumps in the presence of water. With the uptake of 8-HQ onto the quantum dot surface, the surface area also impart a hydrophobic character. Therefore, quantum dots with a surface layer of 8-HQ show a higher affinity to stick together through van der Waals forces to reduce their overall energy in aqueous solution. By sticking together, however, larger particles are formed in suspension. Furthermore, the fluorescence of the monomer chelate is quenched in solid state and only the fluorescence caused by intermolecular interactions is obtained. This is shown by an emission peak with a maximum emission wavelength between 500 and $505 \mathrm{~nm}$, whereas the fluorescence of the monomer chelate is shown at $460 \mathrm{~nm}$. Hence, the soaking of wool inside these suspensions resulted in the coating of the wool fibre surface with fluorescent particles showing a bright white fluorescence with a maximum emission wavelength between 500 and $505 \mathrm{~nm}$. The chemical bonding between these particles and wool fibre surface, however, is based on weak chemical attraction and most of the fluorescent particles came easily off by friction.

To reduce the amount of particles coming off the fibre, wool fibres were first functionalised with $\mathrm{ZnO}$ quantum dots in a previous batch process and then soaked in 8HQ (EtOH) solution. Depending on the concentration of 8-HQ added to the EtOH soaking solution, wool showed a peak (hump) at $460 \mathrm{~nm}$ and a maximum emission wavelengths between 490 and $505 \mathrm{~nm}$. The latter one is strongly dependent on 8-HQ concentration. The addition of higher 8-HQ concentration leads to the shift of the emission to longer wavelength. This, however, is observed with a gradually decrease of the peak at $460 \mathrm{~nm}$ and the florescence changes colour from white to white-green, both under UV-light and ambient light. Here, it is important to note that the white 
fluorescence colour of the wool fibre is obtained by overlapping the emission peaks at 460 and between $500-505 \mathrm{~nm}$. Hence, the fluorescence colour of the wool fibre composites can be controlled with the addition of 8-HQ. However, the soaking of ZnOwool in very high 8-HQ concentration resulted in further emission shift from 505 to 535 $\mathrm{nm}$ (see figure 6.10). This shift involved a reduction of the fluorescence intensity. The shift of the peak matches with the changes in the colouration of the composite material from white-green to yellow; indicating that a new compound is formed. This observation, however, will be discussed more in detail in chapter 7 .

\subsubsection{ZnO quantum dot - paper fibre composites}

For the development of $\mathrm{ZnO}$ quantum dot - paper fibre, different chemical techniques to attach or exchange functional groups at the surface of the paper fibres or $\mathrm{ZnO}$ quantum dot surface were also conducted as it was done for the development of wool fibre composites. Apart from using 8-HQ as fluorescent ligand, none of the techniques resulted in the functionalisation of the paper fibre with optical fluorescence.

Bleached Kraft paper shows a broad emission peak with a maximum emission wavelength around $445 \mathrm{~nm}$ (figure 6.13). With the attachment of $\mathrm{ZnO}$ quantum dots to the paper fibre, the new composite material shows UV absorbance properties. Here, $\mathrm{ZnO}$ quantum absorbs UV-light causing the completely quenching of the fluorescence of the organic fluorophore used for the preparation of bleached Kraft paper (figure 6.13; A). The adsorption of the $\mathrm{ZnO}$ quantum dots to paper fibre, however, appeared unevenly and the chemical attachment seemed to be unstable with ageing (figure 6.14). The attachment of the $\mathrm{ZnO}$ quantum is most likely based on hydrogen bonds to the hydroxyl groups at the surface of the paper cellulose chains.

For the development of fluorescent $\mathrm{ZnO}$ quantum dot - paper fibres, 8-HQ was also used as a fluorescent coordination ligand for the quantum dot surface area. The soaking of the bleached Kraft paper fibre in $\mathrm{ZnO}$ quantum dots aqueous suspension with the addition of small amounts of 8-HQ resulted in the uniform coating of the paper fibres with $\mathrm{ZnO}$ quantum dots. The fluorescence of the bleached Kraft paper was homogeneously quenched and a slightly dark greenish colour under UV-light was observed (figure 6.20; B). Under ambient light, however, it showed the characteristic 
white colour of the bleached Kraft paper. The PL scans showed a small broad emission peak with a maximum emission wavelength around $500 \mathrm{~nm}$ (figure 6.13; B).

$\mathrm{ZnO}$ quantum dots, which surface area has been functionalised with 8-HQ showed a higher affinity to bind to the hydroxyl groups of the cellulose chains of paper fibres. However, the hydroxyl groups may also cause the agglomeration of the quantum dots due the hydrophobic character of 8-HQ. The agglomeration, in turn, enhances the intermolecular interaction between $\mathrm{Zn}(8-\mathrm{Q})_{2}$ chelates at the surface of the $\mathrm{ZnO}$ quantum dots shown by the emission wavelength at $500 \mathrm{~nm}$ (figure 6.13; B).

With the addition of higher 8-HQ concentration, the fluorescence intensity of the fluorescent $\mathrm{Zn}(8-\mathrm{Q})_{2}$ chelate can be significantly increased (see figure 6.13; C and D). The fluorescence obtained, is identical to the PL scans of $\mathrm{ZnO}$ quantum dot - wool fibre with emissions peaks at 460 and between 500 to $505 \mathrm{~nm}$ depending on the 8-HQ concentration added to the $\mathrm{ZnO}$ quantum dot suspension. At low 8-HQ concentration a broad emission peak with a distinct hump at $460 \mathrm{~nm}$ and a maximum emission wavelength around $500-505 \mathrm{~nm}$ was observed. At high 8-HQ concentration, a high and sharp emission peak without a hump at $460 \mathrm{~nm}$ was observed. The fluorescence colour of the paper composite material is determined by these emission peaks. For example, the overlap of the emission peaks at $460 \mathrm{~nm}$ (fluorescence of the monomer chelate) and between 500 and $505 \mathrm{~nm}$ (fluorescence obtained by intermolecular interaction between adjacent chelates) results in a white to white-greenish fluorescence colour (figure 6.12; D). Without the peak at $460 \mathrm{~nm}$, the composite material shows a green-yellowish colour under UV-light and ambient light (figure 6.12; C).

Consequently, the fluorescence colour of the paper fibre composite can be also tuned from white, white-greenish to green-yellowish with the addition of 8-HQ to the soaking solution. However, it is important to note that with the addition of higher 8-HQ concentration, the surface coverage of the $\mathrm{ZnO}$ quantum dots gradually become hydrophobic causing the agglomeration of the particles in aqueous solution and hence the formation of larger fluorescent particles. By forming clumps, $\mathrm{ZnO}$ quantum dots functionalised with 8-HQ ligands obtain a higher stability inside aqueous solutions, but also lose their attachment on the paper fibres. 


\subsubsection{Different factors affecting the fluorescence efficiency of fibre composites functionalised with ZnO quantum dots and 8-HQ}

Wool and Paper fibre functionalised with $\mathrm{ZnO}$ quantum dots and 8-HQ show strong optical fluorescence. The fluorescence is caused by the complexation of zinc cations at the quantum dot surface with 8-HQ as a bidentate ligand. This chemical bonding, however, is not stable in acid conditions due to the Lewis acid / base relationship between the organic ligand and metal ion. Furthermore, processes such as ligand exchange reactions with water can also lead to the reduction of the fluorescence activity [123]. Ligand exchange reaction may also be promoted at very high temperature. Very high processing temperatures may also cause the degradation of the 8-HQ ligand and form optically inactive degradation products which have quenching properties.

Sun light exposure can also have a negative effect on the fluorescence activity. Wool and paper fibre composites functionalised with $\mathrm{ZnO}$ quantum dots and 8-HQ in aqueous solution are generally resistant to sun light exposure. However, their PL intensity slightly decreased over time, which may be caused by photo-bleaching. 8-HQ is sensitive to sun light and may form degradation products which quench the fluorescence intensity of the composite materials. Therefore, a thorough washing is essential to remove all loosely bound 8 -HQ molecules from the substrate material to avoid the formation of quencher molecules.

The decrease of the PL intensity with ageing may also be attributed to the loss of fluorescent particles from the substrate material. In particular, wool fibre composites showed a stronger reduction in their PL intensity than paper fibre composites. In paper fibres, the fluorescent particles fill spaces and crevices between the fibres which are enclosed by micro-fibrils. This minimises the loss of the particles with ageing. On the contrary, in wool fibre composites fluorescent particles are attached to the wool fibre surface by weak van der Waals forces. Friction results in the removal of the particles from the fibres. For this reason, an in-situ dyeing method to avoid the loss of fluorescent particles was evaluated to dye wool fibre with zinc and 8-HQ. This is further discussed in chapter 7 . 


\subsubsection{Anti-microbial coating of wool with $\mathrm{ZnO}$}

$\mathrm{ZnO}$ prepared at the nano-scale size is known for its anti-microbial properties. Antimicrobial testing of $\mathrm{ZnO}$ quantum dot - wool fibre composites against Staphylococcus aureus was conducted and preliminary results showed bacterial growth was restricted around the fibre and no growth was observed on the fibres. The anti-microbial action of this new composite material should be further assessed as it possesses great potential in the fabrication of anti-microbial textiles.

\subsection{Conclusion}

This chapter dealt with the development of $\mathrm{ZnO}$ quantum dot - fibre composites. $\mathrm{ZnO}$ quantum dots aqueous suspension show two distinct emission peaks in the UV-vis range $(\sim 380 \mathrm{~nm})$ and visible range $(\sim 560 \mathrm{~nm})$, when excited with $260 \mathrm{~nm}$. It was hoped to functionalise the fibrous substrate material with the visible fluorescence of the $\mathrm{ZnO}$ quantum dots. Different procedures were explored to attach $\mathrm{ZnO}$ quantum dots onto wool and paper fibre. These involved the building block approach as well as the in-situ binding of the $\mathrm{ZnO}$ quantum dots to the fibres. Apart from the use of 8-HQ as fluorescent coordination ligand, none of the processes presented resulted in optical fluorescence in the visible range. The attachment of $\mathrm{ZnO}$ quantum dots to wool and paper fibre may have a passivation effect on the quantum dot surface, which quenches the quantum dots defect emission shown in the visible range. The attachment of the quantum dots onto wool and paper, however, resulted in other features of interest. For instance, $\mathrm{ZnO}$ quantum dots suspensions are white and show UV-absorbance properties, when attached onto wool and paper fibres.

Wool fibre functionalised with $\mathrm{ZnO}$ quantum dots via in-situ binding or simple soaking in the $\mathrm{ZnO}$ quantum dot suspension revealed the characteristic band-edge emission of $\mathrm{ZnO}$ quantum dots at $380 \mathrm{~nm}$. The uptake of $\mathrm{ZnO}$ quantum dots has also a quenching effect on the natural fluorescence of the wool. It can be assumed that $\mathrm{ZnO}$ quantum dots attached on the wool fibre surface act as UV-absorbers and shield wool from harmful UV radiation. This feature will prevent photo-degradation of the wool protein structure and photo-bleaching of dyed fabrics. 
This project has shown that $\mathrm{ZnO}$ quantum dots attached to bleached Kraft paper suppress the fluorescence of the organic fluorophore used in the paper preparation process. Hence, the printing of the $\mathrm{ZnO}$ quantum dots on white paper sheets will also suppress the fluorescence of bleached paper fibres, where the quantum dots were topically applied. Hence, $\mathrm{ZnO}$ quantum dot suspension could be potentially used for inject-printing confidential information's on paper.

The uptake of $\mathrm{ZnO}$ quantum dots onto wool and paper fibre can be increased using the aromatic compound 8-HQ. 8-HQ bonds as a bidentate ligand to zinc cations at the surface of the $\mathrm{ZnO}$ quantum dots and form a fluorescent coordination complex, $\mathrm{Zn}(8$ Q) 2 chelate. This chelate shows different emission peaks, depending on the spatial distances between them. $\mathrm{ZnO}$ quantum dot - wool fibre composites functionalised with small amounts of 8-HQ showed, besides the band-edge emission of $\mathrm{ZnO}$ quantum dots, two emission peaks at 460 and $490 \mathrm{~nm}$ which overlap and form a broad emission peak. The emission peak at $460 \mathrm{~nm}$ corresponds to the fluorescence of the monomer chelate, whereas the peak at $490 \mathrm{~nm}$ is caused by the intermolecular interaction between adjacent chelates. The overlap of these emission peaks functionalises the wool fibre with a bright white fluorescence under UV light. Consequently, the use of $\mathrm{ZnO}$ quantum dots and 8-HQ in small concentration can be used to dye fabrics instead of using organic fluorophores.

Higher fluorescence intensities with a maximum emission wavelength between 500 and $505 \mathrm{~nm}$ and a distinct emission peak at $460 \mathrm{~nm}$ can also be obtained by adding higher 8$\mathrm{HQ}$ concentration to the $\mathrm{ZnO}$ quantum dot aqueous solution containing wool. The shift of the emission wavelength from 490 to $505 \mathrm{~nm}$ is a result of enhanced intermolecular interaction between adjacent chelates. However, with the shift of the maximum emission wavelength to $505 \mathrm{~nm}$, the fluorescence intensity at $460 \mathrm{~nm}$ gradually decreases. This has a strong effect on the fluorescence colour of the wool fibre composites. The fluorescence colour of the composite material is determined by the overlap of the emission peaks at 460 and between 500 and $505 \mathrm{~nm}$. This overlap of blue and green light results in a bright white florescence colour. Hence, the decline of the fluorescence intensities at $460 \mathrm{~nm}$ changes the fluorescence to a bright white greenish colour. Composite materials without the fluorescence peak at 460 have a bright green to green-yellowish colour both under UV-light and ambient light. Consequently, different 
fluorescence colours of the $\mathrm{ZnO}$ quantum dot - wool composite can be obtained depending on the concentration of 8-HQ added to the aqueous suspension.

It important to note that the addition of highly concentrated 8-HQ to the $\mathrm{ZnO}$ quantum dot - wool suspension results in the agglomeration of the quantum dots. This agglomeration is due to the hydrophobic character of 8-HQ and promote the formation of larger particles, which lose their chemical attachment to the fibre and easily come off by friction. To avoid this, the wool fibre should be dyed following the different in-situ dyeing methods evaluated in chapter 7 .

$\mathrm{ZnO}$ quantum dot - wool fibre composites can also be dyed in 8-HQ EtOH solution. This procedure resulted in optical fluorescence of the wool fibre composite with emission peaks between 500 and $505 \mathrm{~nm}$. However, the fluorescence activity of these wool fibre composites were negatively affected by sun light exposure, when soaked in high concentration of 8-HQ (EtOH). This is most likely connected with the photodegradation of free 8-HQ molecules absorbed by the wool fibre composite.

In contrast, $\mathrm{ZnO}$ quantum dot - paper composites functionalised with 8-HQ in aqueous solution showed stable fluorescence colours and the PL intensity remained nearly unchanged. Bleached Kraft paper fibre soaked in $\mathrm{ZnO}$ quantum dots aqueous suspension with the addition of 8-HQ also showed fluorescence peaks at 460 and between 500 and $505 \mathrm{~nm}$ depending on the 8-HQ concentration. The fluorescence colour of the paper fibre composite is also determined by the overlap of the emission peaks at 460 and between 500 and $505 \mathrm{~nm}$. The use of small concentration of 8-HQ in the soaking process of bleached Kraft paper in $\mathrm{ZnO}$ quantum dot suspension resulted in an uniform coating of the fibres with $\mathrm{ZnO}$ quantum dots. Thereby, the fluorescence of the bleached Kraft paper was homogeneously quenched. The paper fibre obtained a slightly greenish colour under UV-light, whereas under ambient light it showed the characteristic white colour of the bleached Kraft paper. This colour is caused by the agglomeration of $\mathrm{ZnO}$ quantum dots with 8-HQ at the particle surface shown by a small broad emission peak with an emission wavelength around $500 \mathrm{~nm}$.

The use of 8-HQ can add additional functionality to the fibres composites beside the $\mathrm{UV}$-absorbance properties and optical fluorescence in the visible range between 350 and 
$650 \mathrm{~nm}$. 8-HQ has inherent anti-microbial properties and the attachment of $\mathrm{ZnO}$ quantum dots to wool and paper using 8-HQ as linker molecule can also impart antimicrobial properties to the fibre composites. 


\section{Chapter 7}

\section{Dyeing wool with $\mathrm{Zn}^{2+}$ and 8-HQ using in- situ approach}

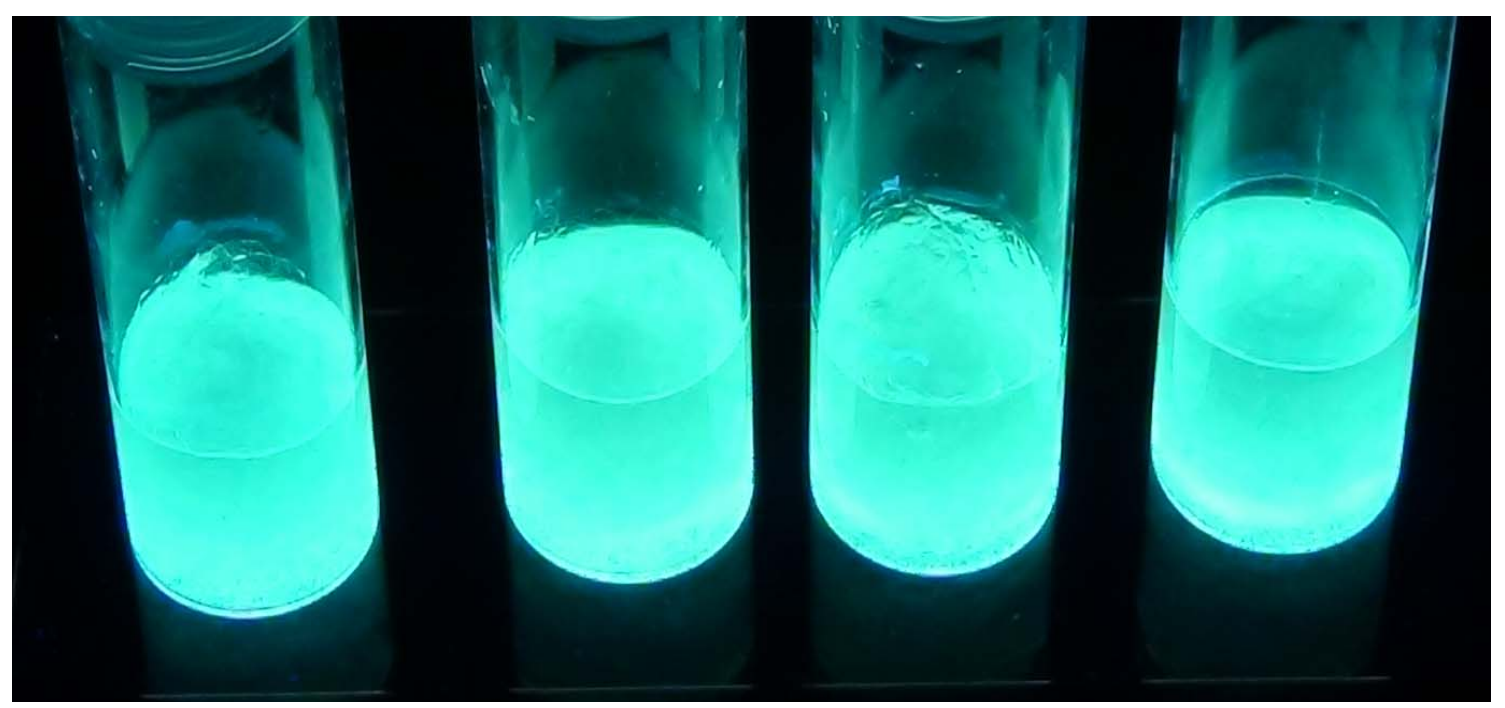

This chapter evaluates two in-situ approaches to dye wool fibre with the fluorescent chelate Zinc(II)-Bis(8-hydroxyquinoline) $\left(\mathrm{Zn}(8-\mathrm{Q})_{2} \cdot 2 \mathrm{H}_{2} \mathrm{O}\right)$. The effect of different process parameters used in these methods on the fluorescence of the new wool composite material is evaluated.

The aromatic ligand 8-Hydroxyquinoline (8-HQ) is a bi-dentate chelating agent and forms with zinc cation a planar coordination complex, chelate. This complex formation provides fluorescence in the blue and green region of the visible spectrum. The uptake of 8-HQ and zinc using two separated batch processes will result in the in-situ complex formation of $\mathrm{Zn}(8-\mathrm{Q})_{2}$ in the chemical surrounding of the wool and produce new fluorescent wool fibre composites. Wool is made up of hydrophilic and hydrophobic components which promote the uptake of organic molecules and metal cations. The dyeing methods developed in this chapter differ in the order of uptake of the precursor materials. The first dyeing method involves the uptake of zinc ions onto 8-HQ treated wool in EtOH solution. The second dyeing method involves the uptake of 8-HQ onto zinc doped wool in EtOH solution. Instead of absorbing 8-HQ onto zinc doped wool 
fibre in solution, the wool fibre can also be exposed into an 8-HQ gas atmosphere at different temperature. Depending on the dyeing bath conditions used, the optical fluorescence of the wool fibre was tuned between white-bluish, white, white-greenish and green-yellowish. The fluorescence is dependent on parameters such as the concentration of the precursor material, type of solvent, reaction temperature, reaction time, $\mathrm{pH}$, type of zinc precursor material, etc. However, the in-situ complex formation and hence optical fluorescence was largely controlled by the concentration of 8-HQ in the soaking solution and the type of zinc precursor material $\left(\mathrm{ZnCl}_{2}(\mathrm{EtOH}), \mathrm{ZnCl}_{2}\right.$ $\left(\mathrm{H}_{2} \mathrm{O}\right)$ and $\mathrm{Zn}(\mathrm{Ac})_{2}\left(\mathrm{H}_{2} \mathrm{O}\right)$ used. The results were mainly obtained by PL and UV-vis spectroscopy. The effect of different process parameters on the fluorescence properties of the wool fibre composite were compared and discussed. A final conclusion about the different dyeing processes is also presented in this chapter.

\subsection{Introduction; Fluorescent chelates}

It has been shown in the previous chapter 6 that the complexation of zinc with 8-HQ as a bidentate complex binder create optical fluorescence. 8-HQ is a chelating agent and forms fluorescent chelates (FCs) with several metal ions [119-125]. The most known fluorescent hydroxyquinoline complex is tris-(8-hydroxyquinolinato) aluminium $\left(\mathrm{Alq}_{3}\right)$ and it is used in organic light emitting diodes (OLED) [123].

In general, a chelate is formed by the complexation of a transition metal cation with bidentate or polydentate organic ligands that have a conjugated $\pi$ - electron system. Here, the fluorescence is generated by a charge transfer mechanism between the organic ligand and the transition metal ion. For example, the absorption of light can promote the transition of an electron from the $\sigma$ - or $\pi$ - $M O$ of the organic ligand to the d- orbital of the atom. This electronic transition converts the chelate in an excited state. The reverse transition of the electron in the ground state will release the excitation energy in form of light.

The bright fluorescence properties of hydroxyquinoline complexes make them to an interesting entity to combine with a wide range of different substrate materials and create new fluorescecnt hybrid materials. The fluorescence colour in coordination complexes or FCs is usually determined by several factors such as the geometrical 
structure of the chelate, chemical interaction with the surrounding medium (solvent molecules) and intermolecular interaction between adjacent chelates.

The geometrical structure of the chelate determines the arrangement of the d- orbitals $\left(\mathrm{d}_{\mathrm{xz}}, \mathrm{d}_{\mathrm{yz}}, \mathrm{d}_{\mathrm{xy}}, \mathrm{d}_{\mathrm{z}^{2}}, \mathrm{~d}_{\mathrm{x}^{2}-\mathrm{y}^{2}}\right)$ of the transition metal in a MO-diagram (see chapter $\left.1 ; 1.2 .2\right)$. Depending on the energy splitting of the five d- orbitals in a ligand field, different $\mathrm{d}$ orbital states are formed. The formation of $\mathrm{d}$ orbital states allows electronic transition between the d- orbitals of the transition metal and the $\pi$ - $M O(\sigma-M O)$ of the organic ligand. The fluorescence colour in coordination complexes is determined by the energy difference between the d- orbitals of the transition metal and $\pi$ - $M O(\sigma-M O)$ of the organic ligand.

The fluorescence colour, however, is also determined by the chemical environment of the chelate and its interaction. For example, the energy of the emitted light and hence fluorescence colour is strongly affected by solvation effects. The choice of solvent has a strong effect on the splitting of the energy levels of the atomic orbitals and MO of the chelate in an energy diagram. Depending on the chemical interaction between chelate and solvent molecules, the energy levels of the organic ligand can be stabilized or destabilized. Depending on attractive or repulsive interaction, the MOs are lowered or raised, respectively (see solvatochromic effect in figure 1.12). In general, non-polar solvents stabilize organic compounds, either with a conjugated $\pi$ - electron system or aromatic rings, by lowering the energy level of the MOs. Solvents with a high dielectric constant, however, have a destabilization effect on electronic structures with a conjugated $\pi$ - electron system. For example, water is a strong polar solvent and will cause the aggregation of organic compounds with a conjugated $\pi$ - electron system or aromatic rings. By sticking together, via $\pi-\pi$ stacking and / or hydrogen bonding, the organic compounds will obtain a higher chemical stability than when complexed with water molecules.

The optical properties of a FC are also affected by intermolecular interaction between adjacent chelates. Intermolecular forces such as $\pi$ - $\pi$ stacking and hydrogen bondings are commonly found between aromatic compounds or molecular structures with a conjugated $\pi$ - electron system. These forces are much weaker than that of covalent 
forces. Nevertheless, these forces will lead to the spontaneous arrangement of the molecules in solution and can also induce energy transfer processes between two chelates. There are different processes by which transfer of energy can occur. For example, energy can be transferred by the formation of charge transfer complexes (excimer / exciplex formation) and / or dipole-dipole interaction between induced dipole moments of adjacent molecular structures (coulombic interaction).

A typical example for a charge transfer complex is the formation of an excimer [126, 127]. An excimer is a complex between two identical molecules, one in excited state (donor) and one in ground state (acceptor). In the complex state, energy is transferred between the excited molecule (donor) and the molecule in ground state (acceptor). Energy can also be transferred by the complex formation of two different molecules. In this case, the charge transfer complex is called exciplex, which is derived from "excited complex" [128]. Here, energy is also transferred between an excited molecule (donor) and an acceptor molecule. In general, the formation of a charge transfer complex is strongly dependent on the intermolecular interaction between donor and acceptor molecule, which in turn depends on the temperature, concentration, type of solvent and the presence of impurities. Charge transfer complexes show usually broad absorption bands. The optical absorption bands are often referred to as charge transfer bands.

By the dipole-dipole interaction, the energy is transferred through a resonance effect between the transition dipoles of the donor and acceptor. Here, the energy transfer is extremely dependent on the donor-acceptor distance, donor-acceptor spectral band overlap, and the relative orientation of the donor and acceptor transition dipole moments [128]. When the overlap of the molecular orbitals of the donor and acceptor is enhanced by dipole-dipole interaction, electron exchange reactions between donor and acceptor can also occur [128].

Independent of the energy transfer process, formation of excimer / exciplexes states or dipole-dipole interaction, the transfer of energy between a donor (excited molecule) and an acceptor molecule will always result in the loss of excitation energy. For example, vibration motions can be easily passed on due to the weak internal forces between the molecules in the complex state. For that reason, the intermolecular interaction between adjacent molecular structures usually results in a lowering of the LUMO level of the 
organic ligand and consequently a wavelength shift to the red end of the spectra occurs. For example, the energy transfer in an excimer, two identical molecules, one in ground state and one in excited state, would usually result in the same complex state; however, during the energy migration a part of the excitation energy will get lost through vibration and / or rotation motion. Because of the energy loss, the wavelength of the emitted light released by the intermolecular interaction is always longer than the wavelength of the emitted light released by the monomer chelate.

The fluorescence activity and efficiency of chelates is dependent on their stability against external influences. The stability of chelates is often limited due to their sensitivity towards moisture and light. Water has a negative effect on most chelates. Their immersion in water often result in dye aggregation due to the hydrophobic nature of many bidentate or polydentate organic ligands that contains a conjugated $\pi$ - electron molecular structure. The aggregation in condensed phase can induce different electronic processes such as electron transfer, energy transfer, and excimer/exiplex formation $[126,128]$. As mentioned before, such intermolecular interactions usually affects the fluorescence properties of the monomer chelate. In some cases, dye aggregation can result in the complete loss of the fluorescence by creating non-radiative pathways for the recombination of the electron-hole pair. Here, the excitation energy is not released by radiative relaxation but converted into vibration and / or rotation energy.

Organic ligands with a conjugated $\pi$ - electron molecular structure are often highly toxic [129] and sensitive to sun exposure causing the degradation of the organic ligand. Furthermore, optical inactive degradation products formed after long exposure to light (photo-bleaching) can progressively reduce (quench) the fluorescence activity of adjacent chelates. Exposure to oxygen can have a similar fading effect [130]. Despite of these drawbacks, the fluorescence poperties of hydroxyquinoline chelates are promising and worth of further exploration.

\subsubsection{Use of fluorescent chelates for dyeing fibrous substrate materials}

Fluorescent chelates are interesting entities for the production of new hybrid materials due to their bright optical fluorescence colours. When evenly adsorbed on the surface of wool and paper fibre, chelates can impart optical fluorescence to these fibrous materials. 
The use of different chelates emitting light with different wavelengths can also tune the fluorescence colour of the fibrous substrate. By overlapping the emission wavelengths of the different chelate compounds, the fluorescence colour can be controlled. In general, fluorescent chelates have the potential to replace commercial available organic dyes used in paper and fabrics. The dyeing of wool or paper with fluorescent chelates can be conducted either by soaking the fibrous material inside the chelate solution or by forming the chelate compound inside the chemical surrounding of the substrate material.

\subsubsection{Soaking fibrous substrate in chelate solution}

In general, wool and paper fibre can be functionalised with fluorescent chelates by soaking / submerging the fibrous substrate material directly in the chelate solution (building block approach). The adsorption of the chelate should be supported by the large surface area of the fibres. For this dyeing approach, however, it is important to suppress the crystallisation of the chelate in solution and the aggregation of the chelate at the fibre surface. This, however, is difficult to achieve and usually result in an irregular colouration of the fibres.

Crystallisation and aggregation are often the result of intermolecular interaction between adjacent molecular structures promoted at higher concentration. Specific interactions between solvent molecules and chelate can also induce a crystallisation reaction in solution. In general, crystallisation is dependent on several parameters such as level of saturation, $\mathrm{pH}$, temperature, exposure in sunlight, polarity of the solvent, etc. Also, the uptake of fluorescent chelates in aqueous solution may be difficult to achieve due to the hydrophobic character of most chelates. The hydrophobic character originates from the conjugated $\pi$ - electron system of the organic ligands. In the presence of water, the chelate will form clumps to minimize the contact to water. By converting into a solid state, the chelate compounds obtain a lattice energy that exceeds the energy obtained by the solvation with water molecules. Thereby, the chelate obtain a higher chemical stability in aqueous solution. However, the conversion in a condensed phase will result in the precipitation of the chelate in solution. 
When the crystallisation or aggregation of the chelate is difficult to suppress, controlling the uptake of the fluorescent chelate onto the substrate material is also difficult. On the other side, the crystallisation of the chelate inside / onto the fibrous substrate material would be useful when formed crystals are chemical bound to the functional groups of the organic fibres. This can be achieved by using an in-situ approach.

\subsubsection{In-situ dyeing approach}

The in-situ dyeing method involves the adsorption of the reactants onto fibrous substrate material divided into two separated batch processes. The in-situ dyeing method is illustrated in chapter 2; equation (8) and (9). The first batch process involves the uptake of one of the precursor materials, transition metal (A) or organic coordination ligand (B), onto the fibrous substrate material, which is then followed by the uptake of the second component in a separated batch process. By doing so, the complex formation is confined in the chemical surrounding of the substrate material and the crystallisation or precipitation of the chelate in solution is avoided.

Each uptake can be operated at different process parameters such as temperatures and also in aqueous and non-aqueous dyeing bath conditions. By optimising the process parameters for each uptake of the reactants onto the substrate material, the fluorescence of the chelate can be controlled. However, the in-situ complex formation inside the substrate material is also dependent on the chemical environment, where the chemical reaction occurs. For example, the wool fibre structure consists of hydrophobic and hydrophilic regions and the interfaces between them. It can be assumed that the complex formation is influenced by attractive and repulsive forces in the different regions of the wool. Furthermore, the presence of counter ions absorbed by the substrate material during the uptake of the precursor material has to be considered. For example, counter ions such as chloride ions diffusing along with the precursor material throughout the wool can inhibit the complexation of the chelate by forming a sphere around the metal ions.

\subsubsection{Choice of fluorescent chelate $-\operatorname{Zn}(8-Q)_{2}$}

8-Hydroxyquinoline (8-HQ) is a mono-protic bidentate ligand, which form with zinc and many other metal ions fluorescent chelate complexes. The fluorescence occurs by 
electron transitions between the Fermi level of the metal atom and the HOMO and LUMO (conduction) level of 8-HQ [131-132].

A mixture of zinc ions and 8-HQ molecules in ethanol solution in a 1:2 ratio result in the formation of a planar yellow fluorescent chelate complex $\mathrm{Zn}(8-\mathrm{Q})_{2}$.

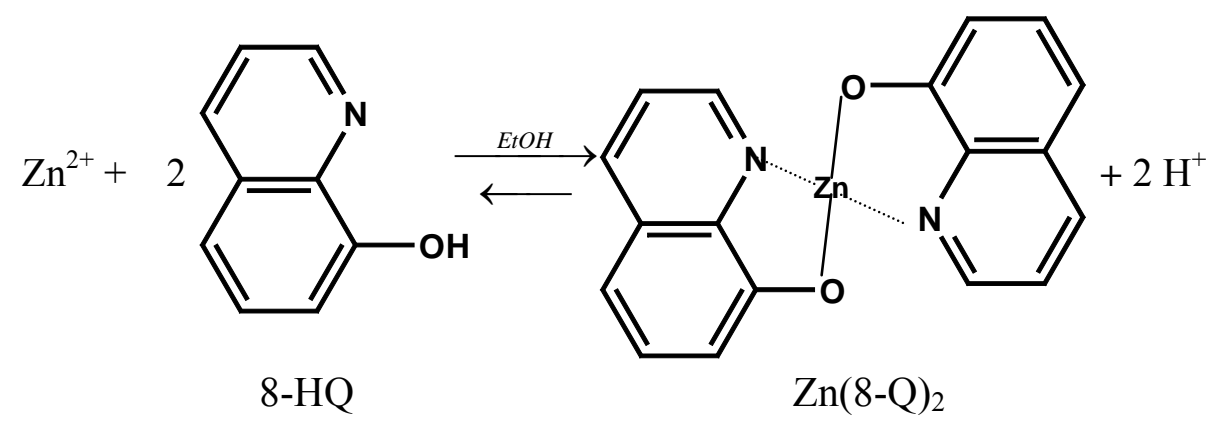

The yellow colour is caused by intermolecular interaction between the aromatic rings of adjacent chelates. In aqueous solution, zinc salts and 8-HQ as a organic ligand form the dihydrate chelate complex $\mathrm{Zn}(8-\mathrm{Q})_{2} \cdot 2 \mathrm{H}_{2} \mathrm{O}$.

$$
\mathrm{Zn}^{2+} \cdot 2 \mathrm{H}_{2} \mathrm{O}+28-\mathrm{HQ} \quad \rightleftarrows \quad \mathrm{Zn}(8-\mathrm{Q})_{2} \cdot 2 \mathrm{H}_{2} \mathrm{O}+2 \mathrm{H}^{+}
$$

The dihydrate complex in non-aqueous solutions is known to undergo a conversion process into an anhydrous tetramer complex as shown in equation (43) [125, 131].

$$
4\left[\mathrm{Zn}(8-\mathrm{Q})_{2} \cdot 2 \mathrm{H}_{2} \mathrm{O}\right] \quad \rightleftarrows \quad\left[\mathrm{Zn}(8-\mathrm{Q})_{2}\right]_{4}+8 \mathrm{H}_{2} \mathrm{O}
$$

The crystalline tetramer structure is an association formed by hydrophobic, electrostatic and steric forces between adjacent dihydrate complexes and under the elimination of water molecules. The conversion process, a chemical equilibrium, is enhanced by removing of water molecules and at higher temperatures.

Zinc and 8-HQ form electrically neutral chelates which show bright intense fluorescent colours depending on the chemical surrounding. These chelates can be used as fluorescent dyes for wool and paper fibres. The fluorescence colour of the composite material is determined by the molecular structure and / or crystalline structure of the chelate. Furthermore, each complex structure shown in equation (41) - (43) are supposed to have individual optical properties such as different PL intensities and

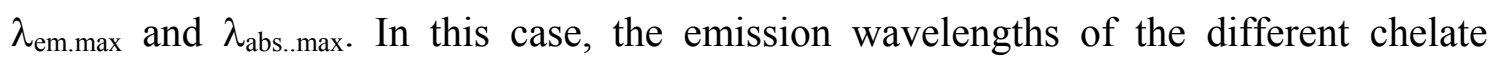
structures overlap and create new fluorescence colours. For example, the combination 
of blue, red and green fluorescence results in white fluorescence. Consequently, the formation of two or more chelate compounds with different composition can be used to fine-tune the fluorescence colour of the final composite material.

\subsection{Methods}

Wool can be functionalised with the fluorescent chelate $\mathrm{Zn}(8-\mathrm{Q})_{2}$ by soaking the fibrous substrate material directly in the fluorescent chelate solution (building block approach) or by a divided uptake of the chelate precursor materials onto the substrate material (insitu approach).

\subsubsection{Preparation of $\mathrm{Zn}(8-\mathrm{Q})_{2}$ chelate solution}

For the direct dyeing method, soaking wool inside the fluorescent chelate solution of zinc and 8-HQ, different concentration of $\mathrm{Zn}(8-\mathrm{Q})_{2}$ were prepared in EtOH solution. The fluorescence properties of the solutions were recorded with PL spectroscopy. In this dyeing method, however, it was difficult to suppress the crystallisation and aggregation of the chelate in the solution with or without wool fibre in it. For example, the dihydrate chelate structure of $\mathrm{Zn}(8-\mathrm{Q})_{2}$ in EtOH solution may convert into the crystalline anhydrous tetramer structure depending on the dyeing conditions. Because of the precipitation of the $\mathrm{Zn}(8-\mathrm{Q})_{2}$ chelate in $\mathrm{EtOH}$ solution and aqueous solution, this method was not further conducted. The introduction of surfactant molecules may suppress the precipitation / crystallisation in solution and give benefits such as improved emission efficiency and colour tuning. However, this requires further examinations.

\subsubsection{Dyeing of wool fibre with $\mathrm{Zn}(8-\mathrm{Q})_{2}$ using in-situ approach}

Three dyeing methods were elaborated, which are differ in the order of addition of the precursor material zinc and 8-HQ onto wool and dyeing conditions. The first dyeing method "1" involves the uptake of zinc ions onto 8-HQ treated wool in EtOH solution. The second dyeing method "2" involves the uptake of 8-HQ onto zinc doped wool. Here, the uptake of 8-HQ onto zinc doped wool was carried out in solution ("Method 2") and gas atmosphere ("Method 3"). These methods are further detailed blow. 


\subsubsection{In-situ method 1 - Zinc uptake onto 8-HQ-wool}

The wool fibres were dyed in the first dyeing method "1" with 8-HQ and $\mathrm{ZnCl}_{2}$ according to the following uptake of the precursor materials in EtOH solution.

$$
\begin{aligned}
& \text { Wool }+28-\mathrm{HQ}(\mathrm{EtOH}) \stackrel{\text { EtOH }}{\longrightarrow}(8-\mathrm{HQ})_{2}-\text { wool } \\
& (8-\mathrm{HQ})_{2}-\text { wool }+\quad \mathrm{ZnCl}_{2}(\mathrm{EtOH}) \stackrel{\text { EtOH }}{\longrightarrow} \mathrm{Zn}(8-\mathrm{Q})_{2}-\text { wool }+2 \mathrm{HCl}
\end{aligned}
$$

This method was carried out in non-aqueous dyeing bath conditions due to the hydrophobic character of 8-HQ. The adsorption of 8-HQ onto wool is a process of hydrophobic and electrostatic interaction in the different regions of the wool. As described in chapter $1,(1.3 .3 .1)$, the wool fibre structure can be simplified by a centre the cortex consisting of cortical cells (hydrophilic) and cell membrane complex (hydrophobic) and cuticle cells, which form a sheath around the cortex. The cuticle cells consist of three layers, endocuticle - the inner layer, exocuticle - the middle layer and epicuticle - the outermost layer. The epicuticle layer is heavily acylated with 18-MEA, which forms a hydrophobic surface [64, 65].

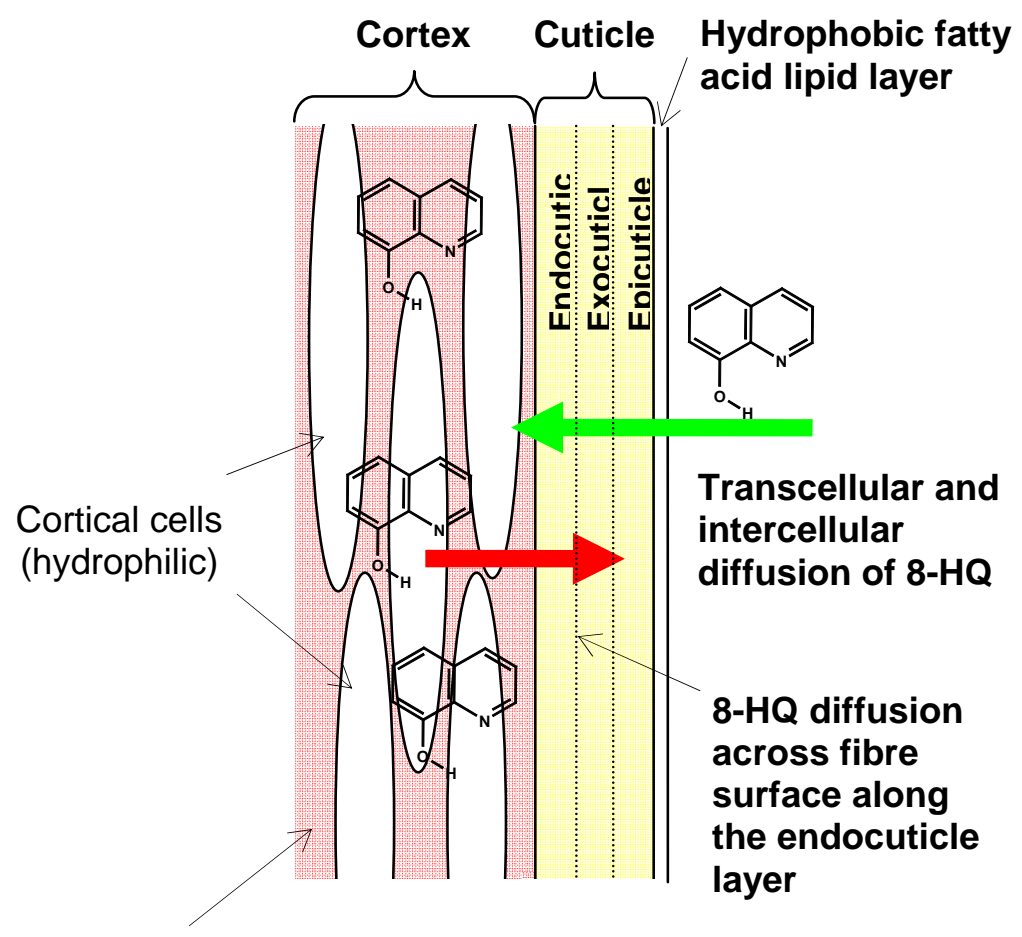

Dispersion of 8-HQ along the cell membrane complex (hydrophobic)

Figure 7.1: Graphic illustration of the uptake of 8-HQ onto wool through transcellular and intercellular diffusion. 
8-HQ has a hydrophobic character and will interact with the external fatty acid monolayer at the surface of the wool fibre. The epicuticle and exocuticle represent a natural barrier for dye penetration due to highly cross-linked protein matrix of $\alpha$ Keratin [116, 134-136]. For that reason, the diffusion from the surface (transcellular diffusion) throughout the wool fibre is mostly hindered. The diffusion of 8-HQ inside the fibre may take place preferred through intercellular regions (gaps between cuticle layers) providing a pathway of least resistance to the penetration of 8-HQ. Due to the hydrophobic character of 8-HQ, the distribution of 8-HQ is initially directed in the nonkeratinous region of the fibre such as the cell membrane complex, endocuticle and also nuclear remnants inside the cortical cells. It is assumed that nuclear remnants are connected by "membrane pores" with the endocuticle and the cell membrane complex [135]. At diffusion equilibrium, 8-HQ is progressively transferred from the nonkeratinous region into the sulphur rich matrix inside the cortical cells. 8-HQ is also transferred from the endocuticle layer along the inner side of the wool into the exocuticle layer across the fibre surface (see figure 7.1).

In the second batch process (equation 45), zinc ions are absorbed onto 8-HQ treated wool fibre in EtOH solution. The soaking of the 8-HQ treated wool in $\mathrm{Zn}^{2+}$ solution should result in the homogenous dispersion of zinc ions throughout the wool fibre by transcellular and intercellular diffusion. Here, zinc combine with 8-HQ in the chemical surrounding of the wool and form an in-situ complex in the different regions of the wool.

\subsubsection{In-situ method 2 - 8-HQ uptake onto zinc-wool}

The dyeing method 2 involves first the doping of wool fibre with zinc ions, before the uptake of 8-HQ was carried out in separated batch process. The procedure is given by the following order of addition.

$$
\begin{array}{lll}
\text { Wool }+\mathrm{Zn}^{2+} & \stackrel{\mathrm{EtOH} / \mathrm{H}_{2} \mathrm{O}}{\longrightarrow} & \mathrm{Zn}^{2+}-\text { wool } \\
\mathrm{Zn}^{2+}-\text { wool }+2 \text { 8-HQ }(\mathrm{EtOH}) \stackrel{\text { EtOH }}{\longrightarrow} & \mathrm{Zn}(8-\mathrm{Q})_{2}-\text { wool }+2 \mathrm{H}^{+}
\end{array}
$$


In the first batch process (equation (46)), wool was functionalised with zinc ions in EtOH or aqueous solution. The soaking of the wool in $\mathrm{Zn}^{2+}$ solution should result in a homogenous dispersion of zinc throughout the wool fibre by transcellular and intercellular diffusion. Thereby, the uptake of $\mathrm{Zn}^{2+}$ ions onto wool should be concentrated in the hydrophilic compounds of the wool such as the wool matrix inside the cortical cells and protein matrix of $\alpha$-Keratin at the fibre's surface. Wool fibres possess the chemical functionality to bind metal ions to the protein structure by electrostatic interaction between charged cations and functional groups of the amino acids. The disulfide and peptide groups are the main anchor groups for the attachment of zinc onto the wool. Here, $\mathrm{Zn}^{2+}$ coordinates to the nitrogen, oxygen and sulphur atoms by electrostatic interaction.

It is known that the adsorption of zinc ions onto wool fibre is generally low. A greater uptake can be achieved by functionalising the substrate material with linker molecules. For example Mercaptosuccinic acid (MSA) can react with wool fibre in two different ways. It can bind to wool fibre either by the mercapto group forming a disulfide bond with cysteine or cysteine of wool keratin or bound by the carboxylic group forming a peptide bond with the amino groups of wool keratin. $\mathrm{Zn}^{2+}$ ions can then interact with the functional group of MSA which is not bound to the wool fibre. By doing so, the uptake of zinc ions onto the protein matrix of the wool will be increased. Also, pre-treatment methods to remove the fatty acid monolayer around the wool fibre surface may increase the uptake of $\mathrm{Zn}^{2+}$ ions onto wool fibre. The lipid layer at the surface (see figure 7.1) may present an adsorption barrier for $\mathrm{Zn}^{2+}$ ions. By removing the fatty acid monolayer through alcoholic alkali $(\mathrm{NaOH})$ and acid $(\mathrm{HCl})$ treatment, underlying amino acids of the protein matrix of the wool $\alpha$-keratin are exposed, which then can undergo a chemical bonding with $\mathrm{Zn}^{2+}$ ions.

The second batch process (equation (47)) can be carried out in solution ("Method 2") or in an 8-HQ gas atmosphere ("Method 3"). The soaking of the zinc doped wool fibre in 8-HQ (EtOH) solution ("Method 2") should result in the uniform distribution of 8-HQ molecules across the wool fibre by hydrophobic and electrostatic interactions. The second batch process may result in the in-situ formation of all three chelate structures as shown in equation (41) - (43), when soaked in EtOH solution. However, the anhydrous chelate $\mathrm{Zn}(8-\mathrm{Q})_{2}$ is not reported in the literature. 
Instead of absorbing 8-HQ onto zinc doped wool fibre in solution, the wool fibre can also be exposed into 8-HQ gas at different temperature (Dyeing method "3"). 8-HQ has a highly volatile nature; in particular at higher temperature. The penetration of 8-HQ gas inside the wool, however, is limited due to the deficiency of the osmotic pressure existing in solution. For that reason, 8-HQ gas will principally interact with the surface of the wool. Free 8-HQ molecules, however, can easily degrade under sun light exposure and produce non-emissive degradation products that can act as luminescence quencher [124]. For that reason, the uptake should not be carried out in direct sun exposure.

\subsubsection{Process parameters}

To restrict or promote the formation of different chelate structures (see equation (41) (43)), the uptake process parameters in the two-step operated dyeing process have to be individualised and optimised for each precursor material. The uptake of zinc ions and 8HQ onto wool depends on several parameters. Soaking time, - temperature, type of solvent, presence of counter ions and $\mathrm{pH}$ value are the main parameters.

For example, the in-situ complex formation of the anhydrous and dihydrate chelate is strongly affected by the $\mathrm{pH}$ value of the surrounding medium. The formations of the different chelate structures of zinc and 8-HQ are balance reactions and reversible (see equation (41) - (43)). By lowering the $\mathrm{pH}$ of the soaking solution, the organic ligand 8HQ will act as proton acceptor. This, however, will interfere with the complex formation of $\mathrm{Zn}(8-\mathrm{Q})_{2}$ and the balance reactions are reversed to the reactant side.

Furthermore, 8-HQ metal complexes may be affected by further reaction processes inside the wool. For example, the literature reports ligand exchange reactions with water molecules at elevated temperature [123]. The result is the formation of free 8-HQ molecules and by-products. It has also been reported that free 8-HQ molecules react with water molecules and form one-water complexes. This one-water complex is nonemissive and can act as a luminescence quencher. Hence, the uptake of zinc and 8-HQ onto wool may also be affected by the type of solvent used for the dyeing bath. In addition, 8-HQ as organic ligand is sensitive to sunlight exposure and can form degradation products, which may also act as quencher molecules and reduce the 
fluorescence efficiency of the wool fibre composite. The process parameters used and varied for each batch process are given in the following sections.

\subsection{Uptake of 8-HQ onto wool}

For the uptake of 8-HQ onto wool in the first batch process of the dyeing method 1, 0.1 g merino wool was soaked in $10 \mathrm{~mL}$ 8-HQ (EtOH) solution varying in concentration from $6.25 \cdot 10^{-5}$ to $6.25 \cdot 10^{-3} \mathrm{M}$ at room temperature for 7 days and at $50{ }^{\circ} \mathrm{C}$ for 3 days. For the 8-HQ aqueous uptake, $0.1 \mathrm{~g}$ merino wool was soaked in $10 \mathrm{~mL} 8$-HQ aqueous solution varying in concentration from $7.5 \cdot 10^{-5}$ to $7.5 \cdot 10^{-4} \mathrm{M}$ at $50{ }^{\circ} \mathrm{C}$ for 7 days. The uptake parameters for 8-HQ molecules onto wool fibres are listed in table 7.1.

Table 7.1: Process parameters used for the uptake of 8-HQ molecules onto wool.

\begin{tabular}{ll}
\hline Process Parameters & \\
\hline 8-HQ precursor & 8- $\mathrm{HQ}(\mathrm{EtOH}), 8-\mathrm{HQ}\left(\mathrm{H}_{2} \mathrm{O}\right)$ \\
Wool & Untreated merino wool \\
$\mathrm{m}($ Wool $) / \mathrm{g}$ & $0.1-10$ \\
{$[8-\mathrm{HQ}] / \mathrm{M}$} & $6.25 \cdot 10^{-5}-6.25 \cdot 10^{-3}$ \\
Solvent & $\mathrm{EtOH}, \mathrm{H}_{2} \mathrm{O}$ \\
Soaking volume / $\mathrm{mL}$ & $10-20$ \\
Soaking temperature / ${ }^{\circ} \mathrm{C}$ & $\sim 25,50,70$ and 90 \\
Soaking time / days & $3-7$ \\
Addition of water / $\mathrm{mL}$ & $1-5$ \\
\hline
\end{tabular}

\subsection{Uptake of $\mathrm{Zn}^{2+}$ onto 8-HQ-wool}

The second batch process in the dyeing method 1 involves the soaking of 8-HQ treated wool $(0.1 \mathrm{~g})$ into $10 \mathrm{~mL} 0.01 \mathrm{M} \mathrm{ZnCl}_{2}(\mathrm{EtOH})$ solution at room temperature for 7 days and $50^{\circ} \mathrm{C}$ for 3 days, respectively. Before the uptake of zinc onto 8-HQ treated wool, the wool fibre composite was washed with EtOH to ensure that all loosely attached 8HQ molecules onto wool were rinsed off. It is important to note that the soaking at $50^{\circ} \mathrm{C}$ or higher can result in the loss of the EtOH solution through evaporation and adequate sealing of the glass tubes containing 8-HQ-wool and EtOH is required. Table 7.2 shows the different process parameters considered for the uptake of $\mathrm{ZnCl}_{2}(\mathrm{EtOH})$ onto 8-HQ wool. 
Table 7.2: Process parameters used for the uptake of $\mathrm{Zn}^{2+}$ ions onto 8-HQ treated wool.

\begin{tabular}{ll}
\hline Process Parameters & \\
\hline Zinc precursor & $\mathrm{ZnCl}(\mathrm{EtOH}), \mathrm{ZnCl}{ }_{2}\left(\mathrm{H}_{2} \mathrm{O}\right), \mathrm{Zn}(\mathrm{Ac})_{2}\left(\mathrm{H}_{2} \mathrm{O}\right)$ \\
Wool & $8-\mathrm{HQ}(\mathrm{EtOH})-$ wool, $8-\mathrm{HQ}\left(\mathrm{H}_{2} \mathrm{O}\right)-$ wool \\
{$\left[\mathrm{Zn}^{2+}\right] / \mathrm{M}$} & $0.01-0.1$ \\
Solvent & $\mathrm{EtOH}$ \\
Soaking volume $/ \mathrm{mL}$ & 5,10 and 20 \\
Soaking temperature $/{ }^{\circ} \mathrm{C}$ & $\sim 25,50,70$ and 90 \\
Soaking time / days & $3-7$ \\
\hline
\end{tabular}

\subsection{Uptake of $\mathrm{Zn}^{2+}$ onto wool}

The uptake of zinc ions onto wool was carried out using two different precursor materials, $\mathrm{ZnCl}_{2}$ and $\mathrm{Zn}(\mathrm{Ac})_{2}$, and at different dyeing bath conditions; aqueous and ethanol solutions. To obtain constant uptakes of zinc onto wool, $0.1 \mathrm{~g}$ merino wool fibres (untreated and pre-treated with $\mathrm{NaOH}, \mathrm{HCl}$ and $\mathrm{MSA}$ ) were soaked in $10 \mathrm{~mL}$ $0.01 \mathrm{M} \mathrm{Zn}^{2+}$ aqueous and ethanol solution, respectively, for 2 days at $50{ }^{\circ} \mathrm{C}$. Table 7.3 shows the processing parameters which were considered for the uptake of $\mathrm{Zn}^{2+}$ ions onto wool. The pre-treatment procedures of merino wool with $\mathrm{NaOH}, \mathrm{HCl}$ and $\mathrm{MSA}$ are described in chaper $2 ; 2.2 .2 .1 .1,2.2 .2 .1 .2$ and 2.2.2.1.3, respectively.

Table 7.3: Process parameters used for the uptake of $\mathrm{Zn}^{2+}$ ions onto wool.

\begin{tabular}{ll}
\hline Process Parameters & \\
\hline Zinc precursor & $\mathrm{ZnCl}_{2}(\mathrm{EtOH}), \mathrm{ZnCl}_{2}\left(\mathrm{H}_{2} \mathrm{O}\right), \mathrm{Zn}(\mathrm{Ac})_{2}\left(\mathrm{H}_{2} \mathrm{O}\right)$ \\
Wool & Untreated, $\mathrm{NaOH}-, \mathrm{HCl}$ - and $\mathrm{MSA}$ pre-treated \\
$\mathrm{m}($ Wool $) / \mathrm{g}$ & $0.1-10$ \\
{$\left[\mathrm{Zn}{ }^{2+}\right] / \mathrm{M}$} & $0.01-0.1$ \\
Solvent & $\mathrm{H}_{2} \mathrm{O}, \mathrm{EtOH}$ \\
Soaking volume $/ \mathrm{mL}$ & $5,10-20$ \\
Soaking temperature $/{ }^{\circ} \mathrm{C}$ & $\sim 25,50,70$ and 90 \\
Soaking time / days & $1-3$ \\
\hline
\end{tabular}




\subsection{Uptake of 8-HQ onto Zn-wool in EtOH solution}

For the 8-HQ uptake, $0.1 \mathrm{~g}$ zinc doped wool were soaked in $10 \mathrm{~mL}$ 8-HQ EtOH solution varying in concentration from $6.25 \cdot 10^{-5}$ to $3.75 \cdot 10^{-3} \mathrm{M}$ at room temperature for 7 days and at $50{ }^{\circ} \mathrm{C}$ for 3 days. A uniform diffusion of 8-HQ throughout the wool in the second batch process of the dyeing method 2 is only guaranteed, if the zinc doped wool is free of water. Therefore, zinc doped wool has to be washed with EtOH solution and then sufficient dried to ensure that all water absorbed is removed before soaking in 8-HQ EtOH solution. As well, washing the wool fibres with EtOH after soaking in 8-HQ solution is essential to remove unbounded 8-HQ molecules.

This dyeing method was also carried out using different process parameters:

- Wool doped with $\mathrm{ZnCl}_{2}(\mathrm{EtOH}), \mathrm{ZnCl}_{2}\left(\mathrm{H}_{2} \mathrm{O}\right)$ and $\mathrm{Zn}(\mathrm{Ac})_{2}\left(\mathrm{H}_{2} \mathrm{O}\right)$

- 8-HQ uptake onto zinc doped wool in EtOH and in aqueous solution

- Wool modified with MSA, $\mathrm{NaOH}$ and $\mathrm{HCl}$

The uptake parameters for 8-HQ molecules onto zinc functionalised wool fibres are summarised in table 7.4.

Table 7.4: Process parameters used for the uptake of 8-HQ onto zinc doped wool.

\begin{tabular}{ll}
\hline Process Parameters & \\
\hline 8-HQ precursor & 8- $\mathrm{HQ}(\mathrm{EtOH})$ \\
Zinc precursor & $\mathrm{ZnCl}_{2}(\mathrm{EtOH}), \mathrm{ZnCl}_{2}\left(\mathrm{H}_{2} \mathrm{O}\right), \mathrm{Zn}(\mathrm{Ac})_{2}\left(\mathrm{H}_{2} \mathrm{O}\right)$ \\
Wool & Untreated, $\mathrm{NaOH}-, \mathrm{HCl}$ - and MSA pre-treated \\
[8-HQ] / M & $0.25 \cdot 10^{-4}-0.25 \cdot 10^{-2}$ \\
Solvent & $\mathrm{EtOH}, \mathrm{H}_{2} \mathrm{O}$ \\
Soaking volume / $\mathrm{mL}$ & $10-20$ \\
Soaking temperature / ${ }^{\circ} \mathrm{C}$ & $\sim 25,50,70,80$ and 90 \\
Soaking time / days & $3-7$ \\
Addition of water / $\mathrm{mL}$ & $1-5$ \\
\hline
\end{tabular}

\subsection{Uptake of 8-HQ-onto Zn-wool in gas atmosphere}

The 8-HQ gas uptake was realised by sealing $0.2 \mathrm{~g} 8$-HQ treated wool $(\mathrm{wt}(8-\mathrm{HQ})=$ $8.31 \%$ ) together with $0.1 \mathrm{~g} \mathrm{Zn}$ doped wool fibre in a glass tube for 1 day at $80{ }^{\circ} \mathrm{C}$. By changing ambient temperature $\left(50-70{ }^{\circ} \mathrm{C}\right)$ and exposure time, the fluorescence colour of the composite material was controlled. 


\subsection{Results}

\subsubsection{Preparation of $\mathrm{Zn}(8-\mathrm{Q})_{2}$ chelate solution}

\subsubsection{1 $\mathrm{Zn}(8-\mathrm{Q})_{2}$ in EtOH solution}

The mixture of $\mathrm{Zn}^{2+}(\mathrm{EtOH})$ and 8-HQ $(\mathrm{EtOH})$ in a 1:2 ratio results in the complexation of the zinc ions with 8-HQ as a bidentate complex binder. The complexation was followed by a gradually crystallisation of the chelate in EtOH solution. The chelate in EtOH solution and the crystallisation product of the chelate has fluorescence properties and showed different fluorescence scans depending on the exciting wavelength used.

Figure 7.2 shows the fluorescence scans obtained from a $10 \mathrm{~mL} 0.001 \mathrm{M} \mathrm{Zn}(8-\mathrm{Q})_{2}$ $(\mathrm{EtOH})$ complex solution with crystals homogeneously distributed in solution. The exciting wavelengths were varied from 315 to $370 \mathrm{~nm}$. When excited with 315 and 330 $\mathrm{nm}$, a flat emission peak with a maximum emission peak around $460 \mathrm{~nm}$ and hump at $490 \mathrm{~nm}$ was obtained. When excited with $350 \mathrm{~nm}$, a maximum emission peak at $510 \mathrm{~nm}$ was shaped. This peak also showed a distinct hump at $460 \mathrm{~nm}$. When excited with 370 $\mathrm{nm}$, the hump at $460 \mathrm{~nm}$ disappeared and a maximum wavelength at $520 \mathrm{~nm}$ is created.

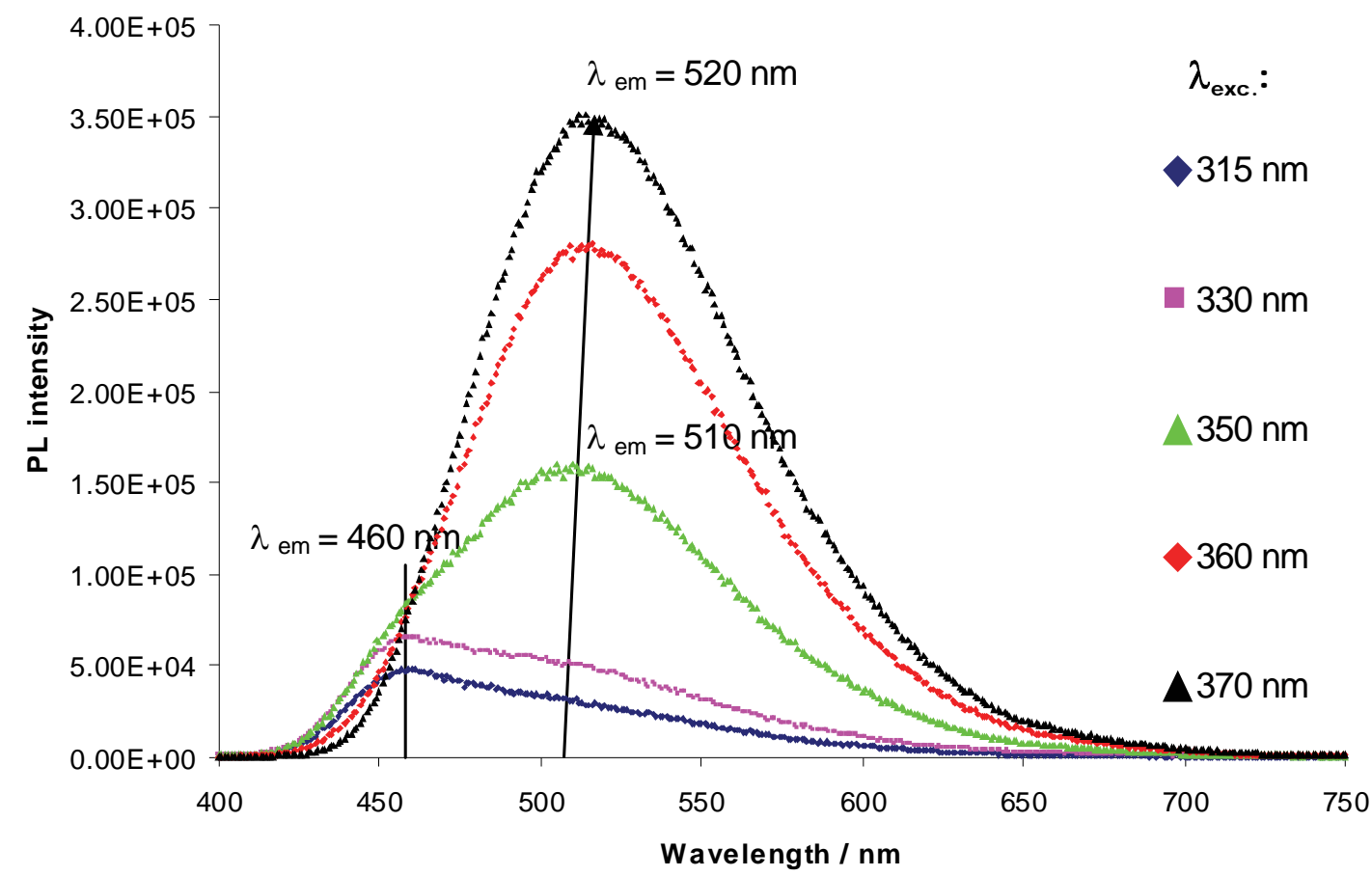

Figure 7.2: PL scan of homogeneously distributed crystals of the $\mathrm{Zn}(8-\mathrm{Q})_{2}$ chelate $(0.001 \mathrm{M})$ in EtOH solution excited with wavelengths in the range of $\lambda_{\mathrm{exc}}=315-370 \mathrm{~nm}$; (exciting / emission slit width $=5 \mathrm{~nm}$ ). Details of sample preparation are listed in the appendix in table A.18; A. 
Over time, the crystals progressively settled down in solution. Thereby, differences in the fluorescence scans were observed. Figure 7.3 shows the change in PL intensity and emission wavelength with the settlement of the crystals in solution / suspension, when excited with 330 and $370 \mathrm{~nm}$.

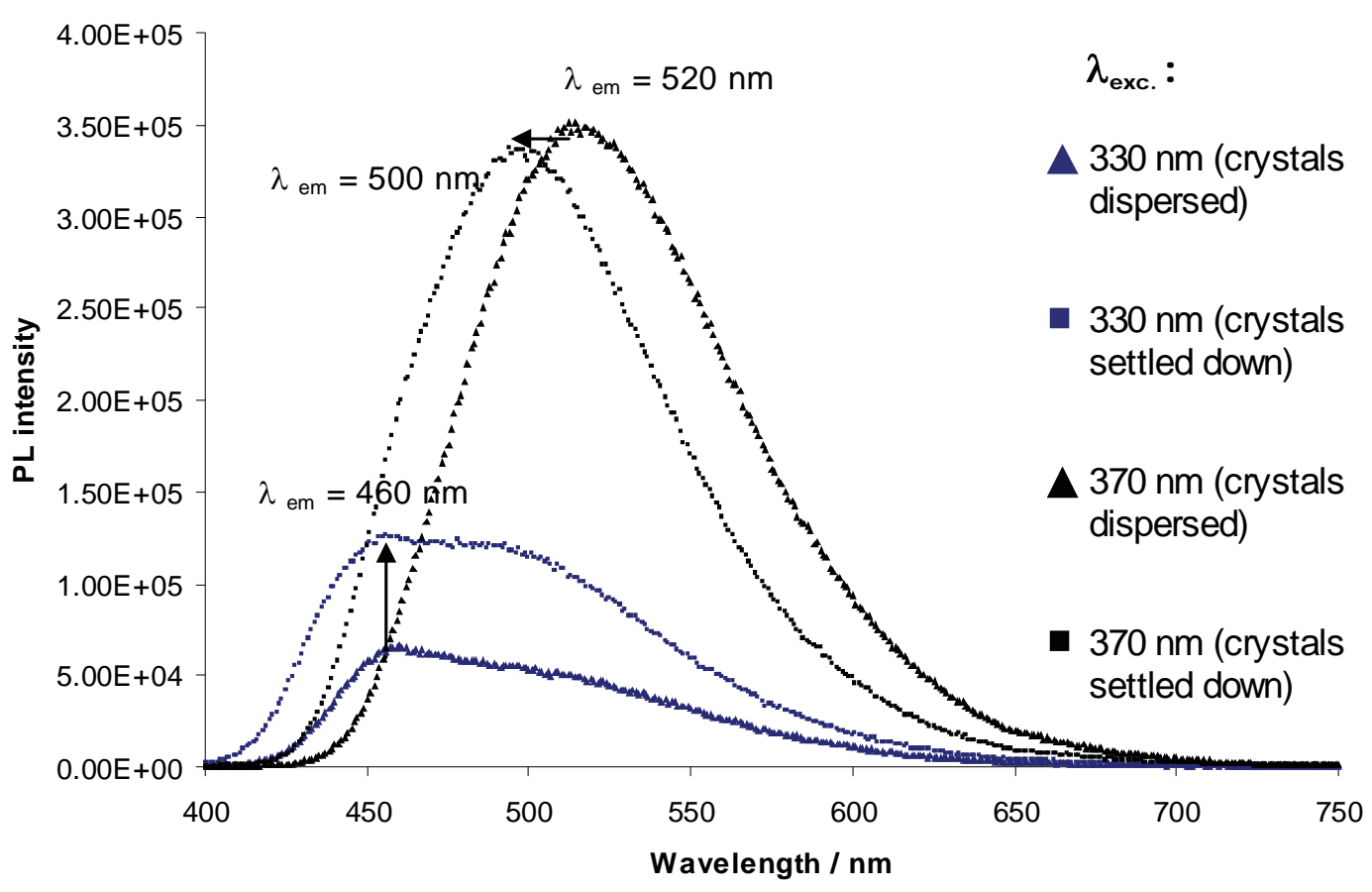

Figure 7.3: PL scan of Zn(8-Q) $)_{2}$ EtOH suspension (0.001 M) before (solid square) and after (solid triangle) precipitation of the crystals in solution. Exciting wavelength: 330 and $370 \mathrm{~nm}$; (exciting / emission slit width $=5 \mathrm{~nm}$ ).

The settlement of the crystals in the chelate solution caused an increment in the PL intensity of the maximum emission wavelength at $460 \mathrm{~nm}$, when excited with $330 \mathrm{~nm}$. Also, the hump at $490 \mathrm{~nm}$ increased with the precipitation of the crystals from solution. The same trend was observed, when excited with $315 \mathrm{~nm}$. When excited with $370 \mathrm{~nm}$, the precipitation of the crystals resulted in a blue shift of the maximum emission wavelength from 520 to $500 \mathrm{~nm}$. A blue shift of $10 \mathrm{~nm}$ was also observed, when excited with $350 \mathrm{~nm}$.

The chelate solution without crystals and diluted with EtOH reveals further changes in their fluorescence scans depending on the exciting wavelength. When excited with 315 and $330 \mathrm{~nm}$, a single emission peak around $430 \mathrm{~nm}$ is visible. When excited with $350-$ $370 \mathrm{~nm}$, two emission peaks around 430 and $555 \mathrm{~nm}$ are shown. Here, the intensity of 
the second emission peak around $555 \mathrm{~nm}$ increases with higher exciting wavelength, whereas the emission peak around $430 \mathrm{~nm}$ decreases (see figure 7.4).

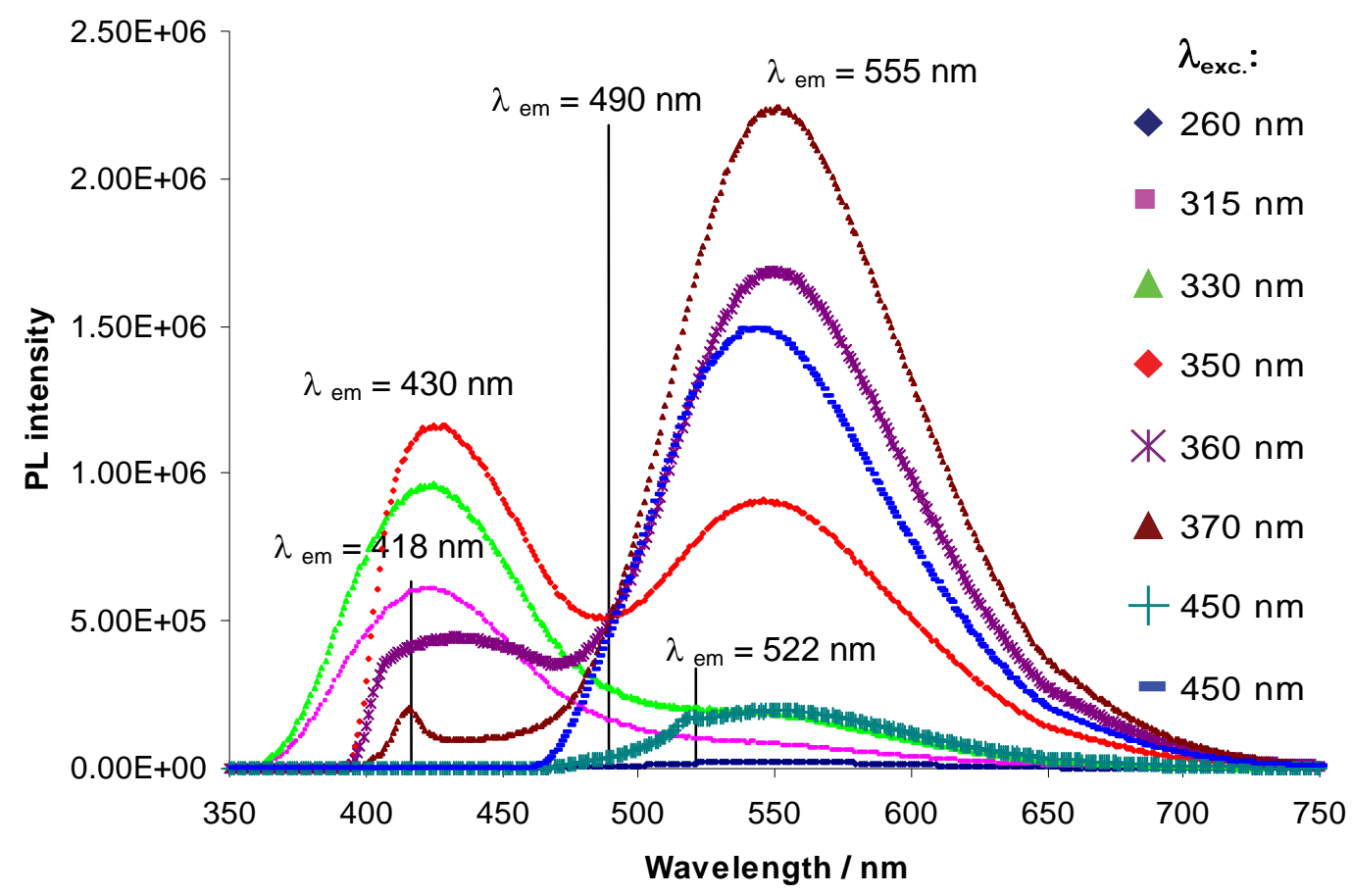

Figure 7.4: PL scans of diluted $\mathrm{Zn}(8-\mathrm{Q})_{2}$ EtOH solution without crystals at different exciting wavelengths $\lambda_{\text {exc }}$ ( (exciting / emission slit width $=5 \mathbf{~ n m}$ ).

There are two noteworthy differences between the PL scans of chelate solutions containing crystals (figure 7.2 and 7.3) and that of highly diluted chelate solution in $\mathrm{EtOH}$ (figure 7.4). The first difference is that the fluorescence intensity of the highly diluted chelate solution is higher than the intensity of the chelate solution containing crystals. The second difference is that the peaks observed at 460 and $490-520 \mathrm{~nm}$ in chelate solution with crystals are not present in highly diluted chelate solutions. Moreover, a gap at $492 \mathrm{~nm}$ is observed in highly diluted chelate solutions, when excited with $\lambda_{\text {exc }}=350 \mathrm{~nm}$.

Ethanol solutions of 8-HQ show only one emission peak around $420 \mathrm{~nm}$, when excited with wavelengths above $300 \mathrm{~nm}$. In figure 7.5, the different PL scans of $0.25 \mathrm{M} 8-\mathrm{HQ}$ EtOH solution are plotted according to their exciting wavelength. In the exciting wavelength range between 240 and $300 \mathrm{~nm}$, the PL scans show a light scattering effect with peaks at $408,437,545,587$ and $612 \mathrm{~nm}$. With the increase of the exciting 
wavelength $\left(\lambda_{\text {exc }}=315-350 \mathrm{~nm}\right)$, the PL intensity of the emission peak around $420 \mathrm{~nm}$ gradually increases.

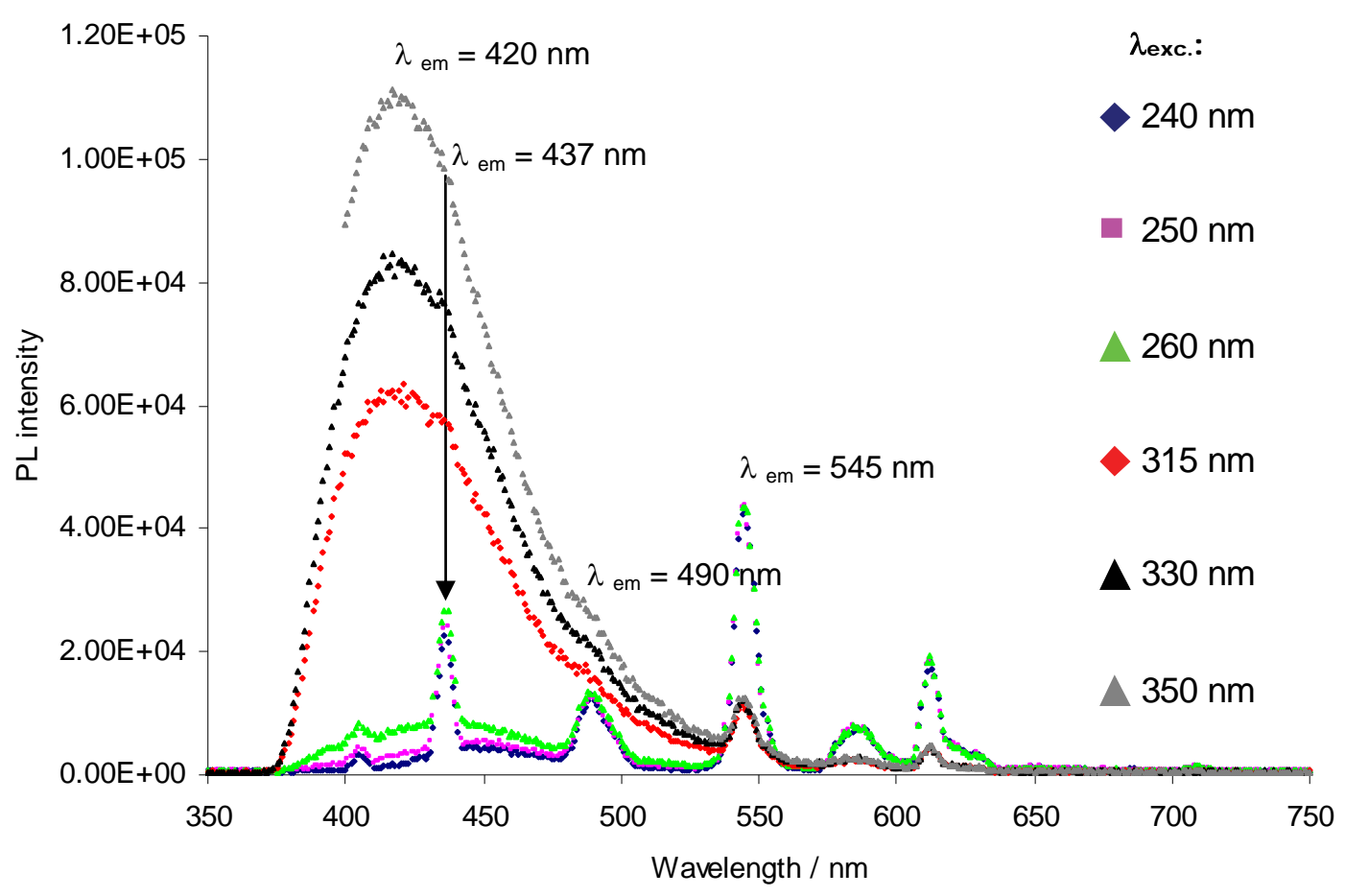

Figure 7.5: PL scans of 8-HQ EtOH solution (0.25 M) excited with wavelengths in the range of 240 $-350 \mathrm{~nm}$ (exciting / emission slit width $=5 \mathrm{~nm}$ ).

In EtOH solution, 8-HQ shows an absorbance band between 270 and $375 \mathrm{~nm}$ with a maximum absorbance around $320 \mathrm{~nm}$ (figure 7.6, red curve). The PL scan of the corresponding solution $\left(\lambda_{\text {exc }}=315-350 \mathrm{~nm}\right)$ shows an emission peak around $420 \mathrm{~nm}$ (figure 7.6, black curve). Thereby, a Stoke shift of $100 \mathrm{~nm}$ is obtained $(\Delta \lambda=100 \mathrm{~nm})$ (see figure 7.6). 


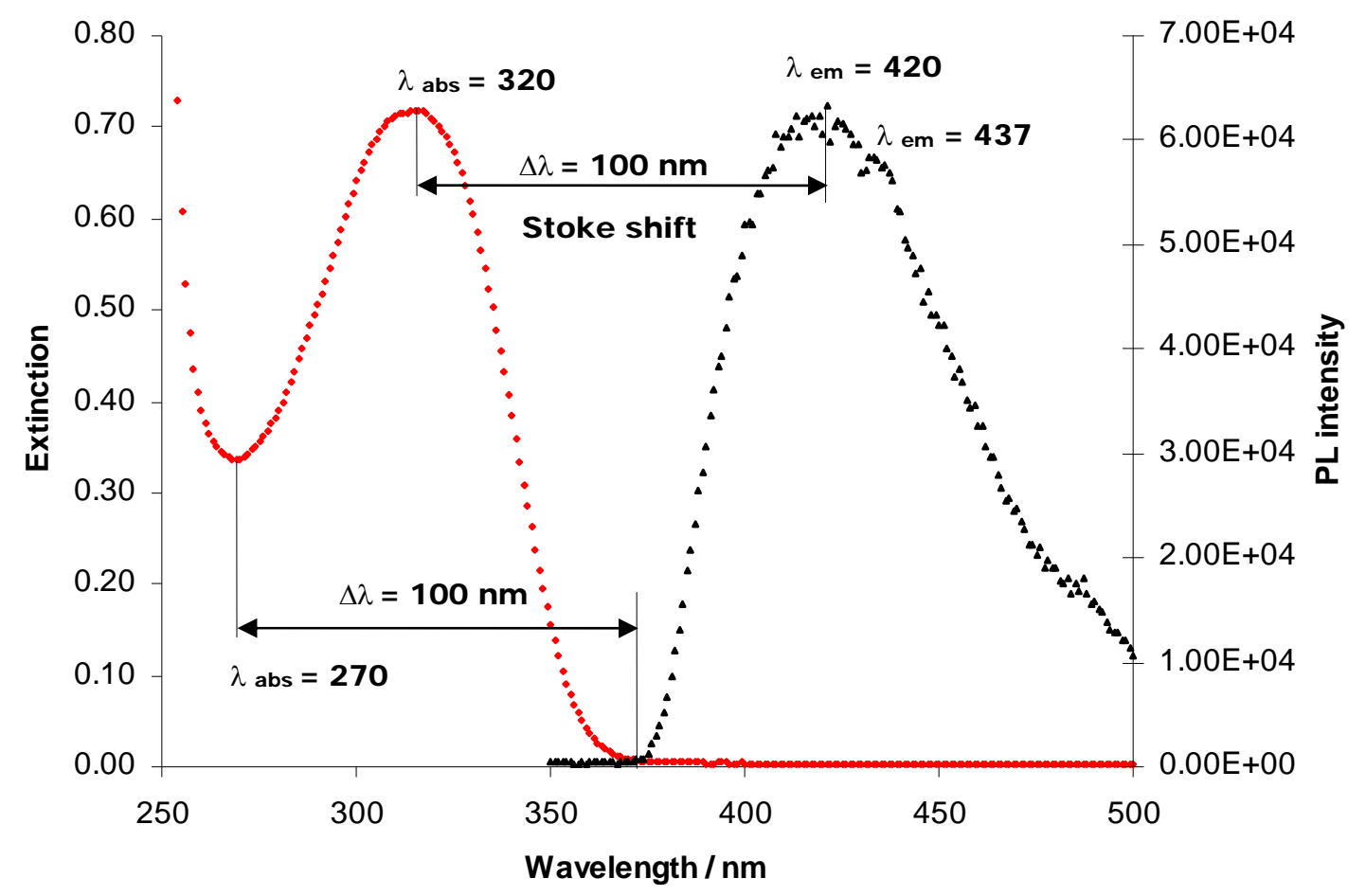

Figure 7.6: Stoke shift of 8-HQ (EtOH) (red curve - absorbance curve; black curve - PL scan). The PL scan of 8-HQ was recorded with an excitation wavelength of $315 \mathrm{~nm}$ and the excitation / emission slit was set up with $5 \mathrm{~nm}$.

\subsubsection{2 $\quad \mathrm{Zn}(8-\mathrm{Q})_{2}$ in $\mathrm{H}_{2} \mathrm{O}$ solution}

In aqueous solution, zinc cations and the bidentate complex binder 8-HQ formed an insoluble chelate, which precipitated from the aqueous solution. The precipitate was yellowish and showed a strong fluorescence with a white to white-greenish colour. Depending on the ratio of zinc and 8-HQ, the maximum emission wavelength of the aqueous chelate suspension showed a shift from 490 to $505 \mathrm{~nm}$, when excited with 350 nm. This red-shift is observed in figure 7.7, which shows the PL scans of the combination of $\mathrm{ZnCl}_{2}\left(\mathrm{H}_{2} \mathrm{O}\right)$ and 8-HQ $(\mathrm{EtOH})$ in a ratio of 1:2 and 1:4. These complex solutions were obtained in aqueous solution by mixing $1 \mathrm{~mL} 0.01 \mathrm{M} \mathrm{ZnCl}_{2}\left(\mathrm{H}_{2} \mathrm{O}\right)$ with $1 \mathrm{~mL} 0.025 \mathrm{M} 8$-HQ (EtOH) and $2 \mathrm{~mL} \mathrm{0.025} \mathrm{M} \mathrm{8-HQ} \mathrm{(EtOH),} \mathrm{respectively.} \mathrm{The} \mathrm{total}$ volume of each complex solution was adjusted to $10 \mathrm{~mL}$. 


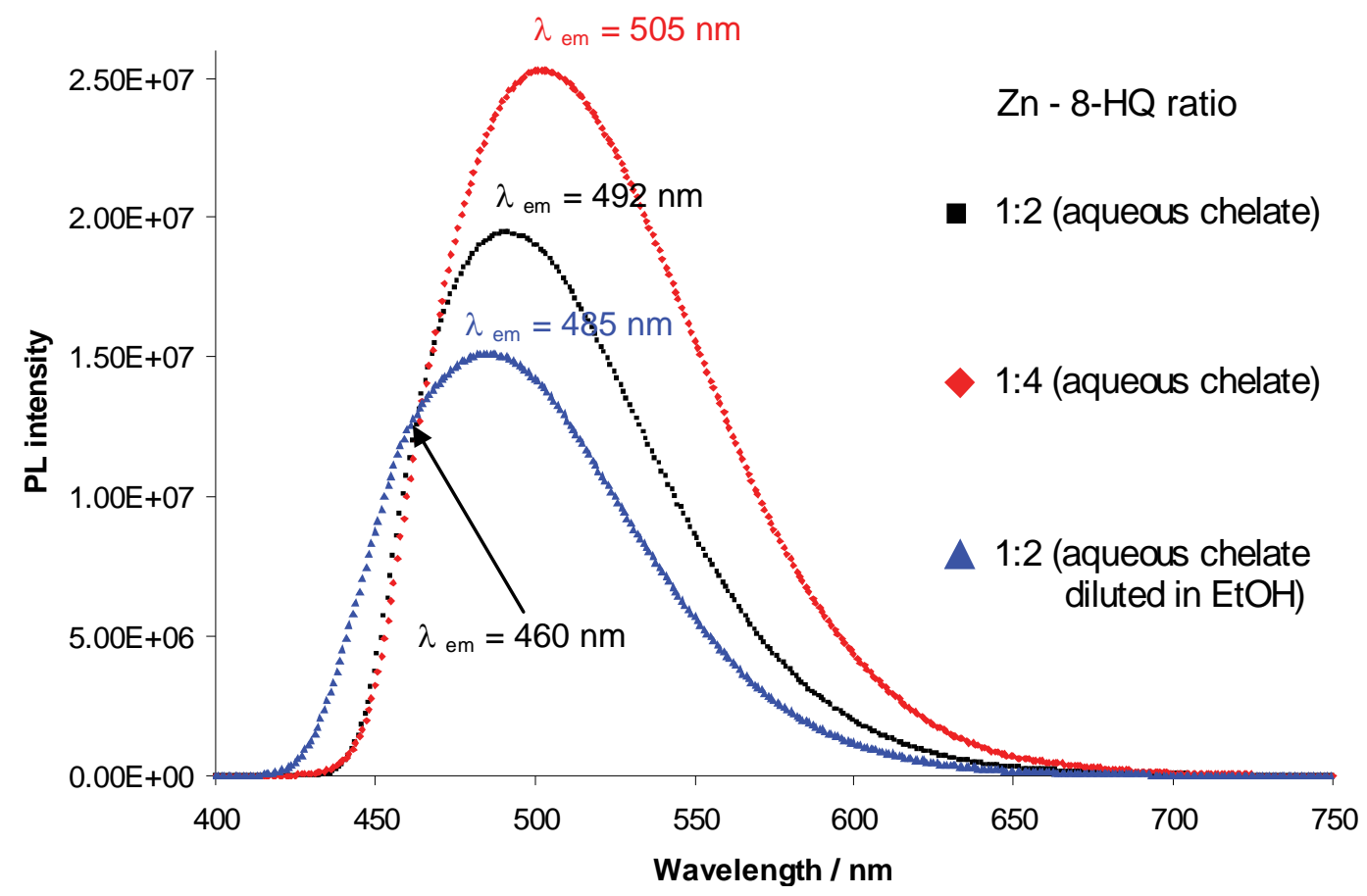

Figure 7.7: PL scans of $\mathrm{Zn}(8-\mathrm{Q})_{2} \mathrm{H}_{2} \mathrm{O}$ suspension in different zinc to 8-HQ ratio; 1:2 (black line); 1:4 (red line) and 1:2 diluted in EtOH solution (blue line) (exciting / emission slit width $=5 \mathbf{~ n m}$ ). Details of sample preparation are shown in the appendix in table A.18; B.

The $0.001 \mathrm{M} \mathrm{Zn}(8-\mathrm{Q})_{2}$ aqueous chelate suspension, prepared by mixing zinc and 8-HQ in a 1:2 ratio, shows a maximum emission wavelength at $492 \mathrm{~nm}$. The addition of $8-\mathrm{HQ}$ $(\mathrm{EtOH})$ in a higher concentration to the zinc aqueous solution resulted in a broadening and increment of the emission peak towards $505 \mathrm{~nm}$. A maximum red-shift at $505 \mathrm{~nm}$ was achieved with a ratio of 1:4 of zinc and 8-HQ. However, a blue shift of the emission peak at $492 \mathrm{~nm}$ was also achieved by diluting the $0.001 \mathrm{M} \mathrm{Zn}(8-\mathrm{Q})_{2}$ aqueous chelate suspension in EtOH. For example, a dilution ratio of 1:3 caused a blue shift from $492 \mathrm{~nm}$ to $485 \mathrm{~nm}$ and a distinct hump at $460 \mathrm{~nm}$ also occurred (see figure 7.7).

\subsubsection{Dyeing of wool fibre using in-situ method 1}

The in-situ dyeing method linvolves first the uptake of 8-HQ onto wool followed by the uptake of zinc in a separated batch process.

The uptake of the precursor materials onto wool was conducted at different soaking temperatures from room temperature to $90{ }^{\circ} \mathrm{C}$. It was found that the soaking temperature does not affect the optical properties of the wool fibre composites, however; higher soaking temperature $\left(50-70{ }^{\circ} \mathrm{C}\right)$ quickened the diffusion processes of 
8-HQ and zinc throughout the wool without damaging the wool fibre. For example, the uptake of 8-HQ onto wool needed a soaking period of seven days, when carried out at room temperature. By increasing the temperature $\left(50-70{ }^{\circ} \mathrm{C}\right)$, the soaking time can be significantly shortened to three days. Also, the complex formation of $\mathrm{Zn}(8-\mathrm{Q})_{2}$ inside the wool was shortened to three days, when 8-HQ-wool was soaked in $\mathrm{ZnCl}_{2}(\mathrm{EtOH})$ solution using a soaking temperature between 50 and $70{ }^{\circ} \mathrm{C}$. However, at very high processing temperature $\left(>70{ }^{\circ} \mathrm{C}\right)$, wool fibres lose their elastic properties and shrink. Furthermore, at high temperature, the wool protein keratin can degrade causing a hydrothermal yellowing effect of the wool. Also, the organic ligand 8-HQ is highly volatile at high temperature resulting in the loss of 8-HQ through evaporation.

The uptake of the precursor materials 8-HQ and zinc onto wool was carried out in EtOH solution. The effect of water was also assessed as soaking solution. Here, it is important to note that 8-HQ is easily soluble in EtOH solutions, whereas only small amounts are dissolvable in aqueous solution under gentle heating $([8-\mathrm{HQ}]=0.003 \mathrm{M})$. 8-HQ aqueous solutions are bright yellow and gradually re-crystallise with ageing.

\subsubsection{Uptake of 8-HQ (EtOH) onto wool}

Figure 7.8 shows the PL scans of the different uptakes of 8-HQ onto $0.1 \mathrm{~g}$ merino wool in $10 \mathrm{~mL}$ EtOH solution carried out at $50^{\circ} \mathrm{C}$. Scans in (A) and (B) were obtained with an exciting wavelength of $260 \mathrm{~nm}$ and (C) and (D) with $360 \mathrm{~nm}$.

Table 7.5 shows the content of 8-HQ in wool after being soaked in EtOH solution of 8HQ with different concentration $\left(1.0 \cdot 10^{-4}-4.0 \cdot 10^{-3} \mathrm{M}\right)$. Here, the uptake of 8-HQ is given as mass percentage, which represents the amount of 8-HQ as a percentage on the weight of the wool. These values are based on the assumption that all 8-HQ was absorbed by the wool during the soaking process. 


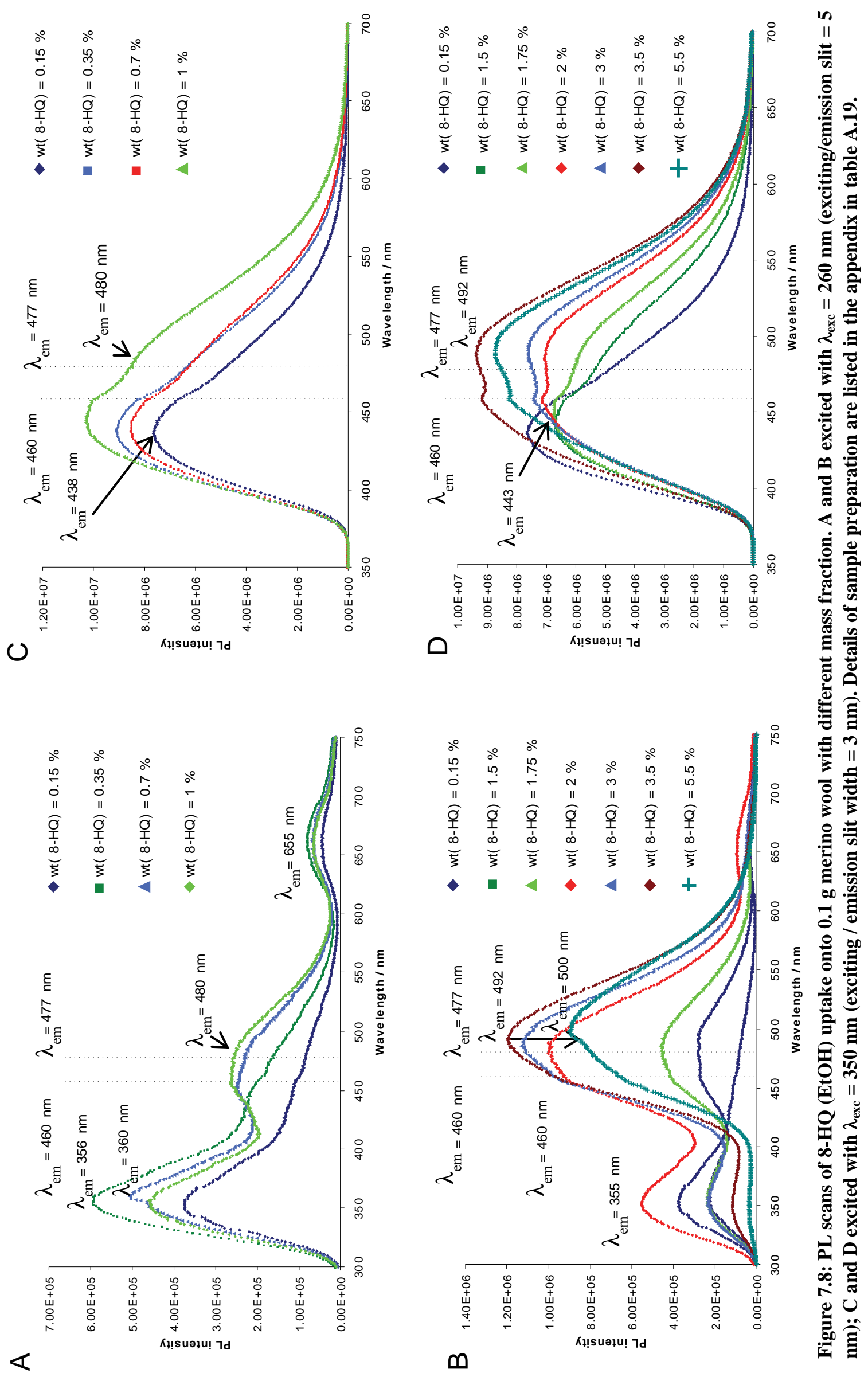


Table 7.5: Volume and concentration of 8-HQ (EtOH) used for the uptake of 8-HQ onto wool and 8-HQ mass percentage on weight of wool (wt \%), assuming 8-HQ was completely absorbed by the wool.

\begin{tabular}{ccc}
\hline $\mathrm{V}(\mathbf{0 . 0 1} \mathbf{M}$ 8-HQ (EtOH) $) / \mathbf{~ m L}$ & {$[\mathbf{8}-\mathrm{HQ}] / \mathbf{M}$} & $\mathbf{w t}(\mathbf{8}-\mathrm{HQ}) / \%$ \\
\hline 0.1 & $1.0 \cdot 10^{-4}$ & 0.15 \\
0.25 & $2.5 \cdot 10^{-4}$ & 0.35 \\
0.5 & $5.0 \cdot 10^{-4}$ & 0.7 \\
0.75 & $7.5 \cdot 10^{-4}$ & 1.0 \\
1 & $1.0 \cdot 10^{-3}$ & 1.5 \\
1.25 & $1.25 \cdot 10^{-3}$ & 1.75 \\
1.5 & $1.5 \cdot 10^{-3}$ & 2.0 \\
2 & $2.0 \cdot 10^{-3}$ & 3 \\
2.5 & $2.5 \cdot 10^{-3}$ & 3.5 \\
4 & $4.0 \cdot 10^{-3}$ & 5.5 \\
\hline
\end{tabular}

Wool fibre itself shows a natural fluorescence peak at $355 \mathrm{~nm}$, when excited with 260 $\mathrm{nm}$. This peak, however, decreases with the uptake of 8-HQ. 8-HQ is known to act as a quencher molecule. The reduction of the emission peak is strongly dependent on the content of 8-HQ absorbed onto wool as seen in (A) and (B) in figure 7.8.

The soaking of wool in high concentrated 8-HQ ethanol solution ([8-HQ] $=0.004 \mathrm{M} \rightarrow$ $\operatorname{wt}(8-\mathrm{HQ})=5.48 \%)$ results in total quenching of the emission wavelength at $355 \mathrm{~nm}$ (see figure 7.8, B). With the uptake of 8-HQ onto wool, however, a new broad emission peak is formed between 400 and $650 \mathrm{~nm}$.

Wool fibre composites with a mass fraction of wt $(8-\mathrm{HQ})=1.5 \%$ show that the broad emission peak is made up of two peaks overlapping at $460 \mathrm{~nm}$ and around $480 \mathrm{~nm}$ (see figure 7.8; B). Both peaks increase their PL intensity with a higher uptake of 8-HQ wool. With the uptake of 8-HQ onto wool, the position of the peak at $460 \mathrm{~nm}$ remains constant, meanwhile the second emission peak shifts to higher wavelengths.

The soaking of $0.1 \mathrm{~g}$ wool fibre in $10 \mathrm{~mL} 0.0025 \mathrm{M}$ 8-HQ EtOH solution (wt $(8-\mathrm{HQ})=$ $3.5 \%$ ) reveals the highest PL intensity with an emission wavelength at $490-492 \mathrm{~nm}$. The soaking of the wool fibre in higher 8-HQ concentration leads to a decrease of the $\mathrm{PL}$ intensity and to a further maximum emission wavelength shifts to maximal $500 \mathrm{~nm}$. 
When excited with $350 \mathrm{~nm}$, PL scans in (C) and (D) (figure 7.8) shows also peaks at exactly $460 \mathrm{~nm}$ and a peak shift between 480 to $490 \mathrm{~nm}$ depending on the 8 -HQ adsorption onto wool.

When wool is soaked in low concentration of 8-HQ $(\mathrm{EtOH})$ as shown in $(\mathrm{C})([8-\mathrm{HQ}]=$ $\left.0.1 \cdot 10^{-3}-0.75 \cdot 10^{-3} \mathrm{M}\right)$, the maxima wavelength of the wool fibre $\lambda_{\mathrm{em}, \max }$ shifts from 435 to $442 \mathrm{~nm}$ and a small hump around $480 \mathrm{~nm}$ is formed.

By increasing the uptake of 8-HQ onto wool (soaking wool in [8-HQ] $>1.0 \cdot 10^{-3} \mathrm{M}$ ), the emission peak at $460 \mathrm{~nm}$ is formed and the initially hump at $480 \mathrm{~nm}$ successively transforms to a peak, whose $\lambda_{\text {em max }}$ shift to longer wavelength around $492 \mathrm{~nm}$. Further uptake of 8-HQ molecules onto wool (wt $(8-\mathrm{HQ})>3.5 \%)$ involves a decrease of the PL intensity and wavelength shift from $492 \mathrm{~nm}$ to $500 \mathrm{~nm}$ maximal.

\subsubsection{Uptake of 8-HQ $\left(\mathrm{H}_{2} \mathrm{O}\right)$ onto wool}

Figure 7.9 shows the PL scans of $0.1 \mathrm{~g}$ wool fibre soaked in $10 \mathrm{~mL}$ 8-HQ aqueous solution at $50{ }^{\circ} \mathrm{C}$ for 7 days. The scans in (A) and (B) were obtained with an exciting wavelength of $260 \mathrm{~nm}$ and (C) and (D) with $350 \mathrm{~nm}$.

The concentration of the 8-HQ aqueous solution used for functionalising merino wool ranged between $0.75 \cdot 10^{-4}$ and $7.5 \cdot 10^{-4} \mathrm{M}$. Table 7.6 shows the uptake of 8-HQ onto wool in mass percentage after being soaked in the different 8-HQ concentrations.

Table 7.6: Volume and concentration of 8-HQ $\left(\mathrm{H}_{2} \mathrm{O}\right)$ used for the uptake of 8-HQ onto wool and 8HQ mass percentage on weight of wool (wt \%), assuming 8-HQ was completely absorbed by the wool.

\begin{tabular}{ccc}
\hline V (0.003 M 8-HQ ( $\left.\left.\mathbf{H}_{2} \mathbf{O}\right)\right) / \mathbf{m L}$ & {$[8-\mathrm{HQ}] / \mathbf{M}$} & $\mathbf{w t}(\mathbf{8}-\mathrm{HQ}) / \%$ \\
\hline 0.25 & $0.75 \cdot 10^{-4}$ & 0.1 \\
0.5 & $1.5 \cdot 10^{-4}$ & 0.2 \\
0.75 & $2.25 \cdot 10^{-4}$ & 0.3 \\
1.0 & $3.0 \cdot 10^{-4}$ & 0.4 \\
1.5 & $4.5 \cdot 10^{-4}$ & 0.6 \\
2.0 & $6.0 \cdot 10^{-4}$ & 0.8 \\
2.5 & $7.5 \cdot 10^{-4}$ & 1.0 \\
\hline
\end{tabular}


When excited with $260 \mathrm{~nm}$, the PL intensity of the natural fluorescence of the wool fibres is reduced almost tenfold in comparison to the uptake of 8-HQ in EtOH solution.

The uptake of 8-HQ onto wool in low concentration as shown in (A) ([8-HQ $\left.\left(\mathrm{H}_{2} \mathrm{O}\right)\right]=$ $\left.7.5 \cdot 10^{-5}-3.0 \cdot 10^{-4} \mathrm{M}\right)$ also result in the formation of two small peaks at $460 \mathrm{~nm}$ and $480 \mathrm{~nm}$ which are overlapping and forming a broader peak. Initially the peak at $460 \mathrm{~nm}$ dominates over the peak at $480 \mathrm{~nm}$, however decreases again with the uptake of higher concentration of 8-HQ $\left(w t\left(8-\mathrm{HQ}\left(\mathrm{H}_{2} \mathrm{O}\right)\right)>0.3 \%\right)$.

The PL intensity of the second peak remain nearly unchanged, however shifts from 480 to $490 \mathrm{~nm}$. Wool fibre soaked in higher 8-HQ aqueous solution (B) show strong fluctuation in the intensity of the PL scans. Nevertheless, the scans show a shift of the maximum wavelength towards $505 \mathrm{~nm}$.

The PL intensity of the wool fibre soaked in 8-HQ aqueous solution is also reduced, when excited with $350 \mathrm{~nm}$ (figure 7.9; C and D). In addition, the PL scan of wool containing mass fractions of 8 - $\mathrm{HQ}\left(\mathrm{H}_{2} \mathrm{O}\right)$ over $0.2 \%$ show a strong fluctuation. Here, the PL curves are not smooth. However, it can still be seen that with the uptake of 8-HQ $\left(\mathrm{H}_{2} \mathrm{O}\right)$ onto wool a second peak is formed between 470 and $520 \mathrm{~nm}$. 


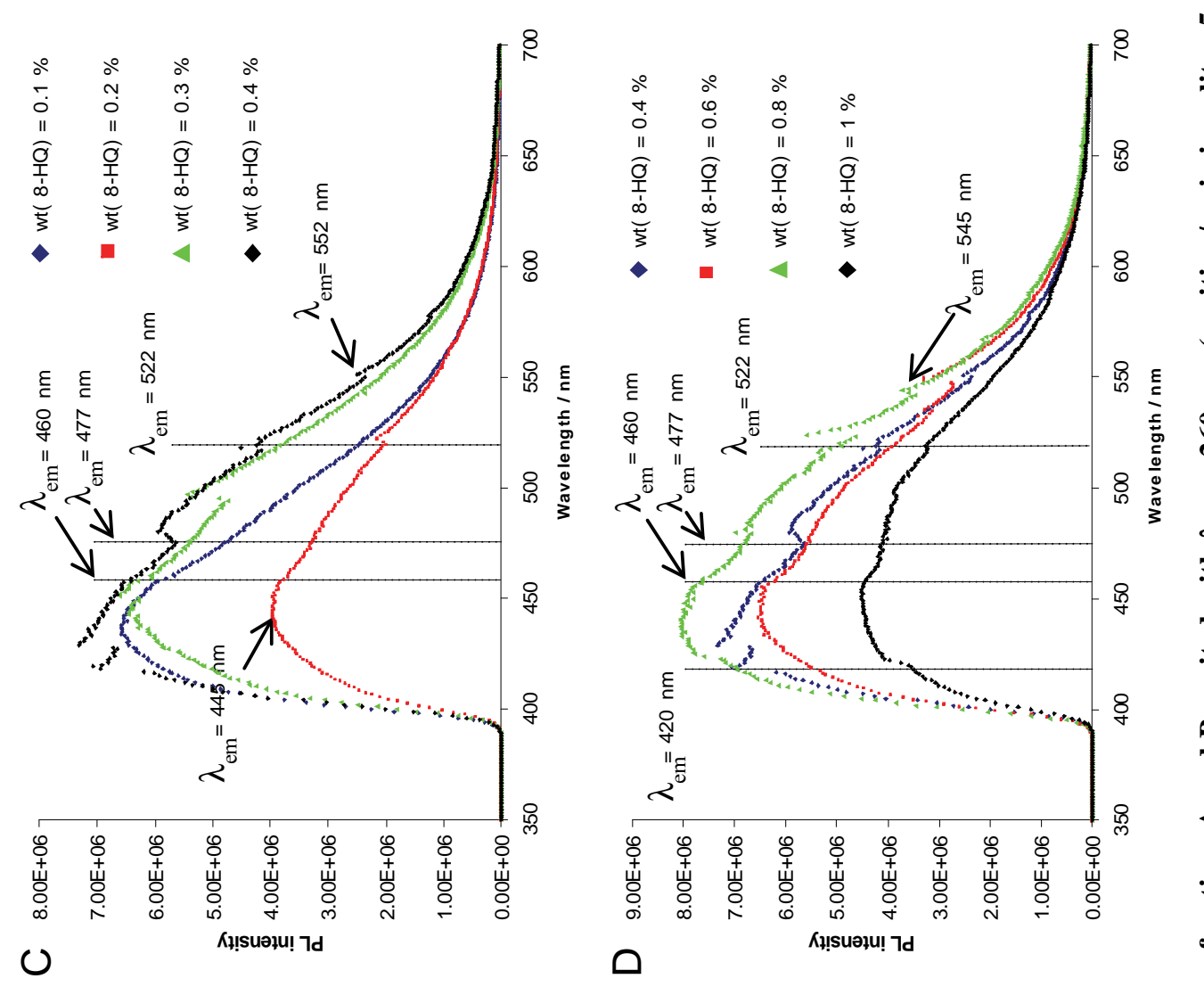

告

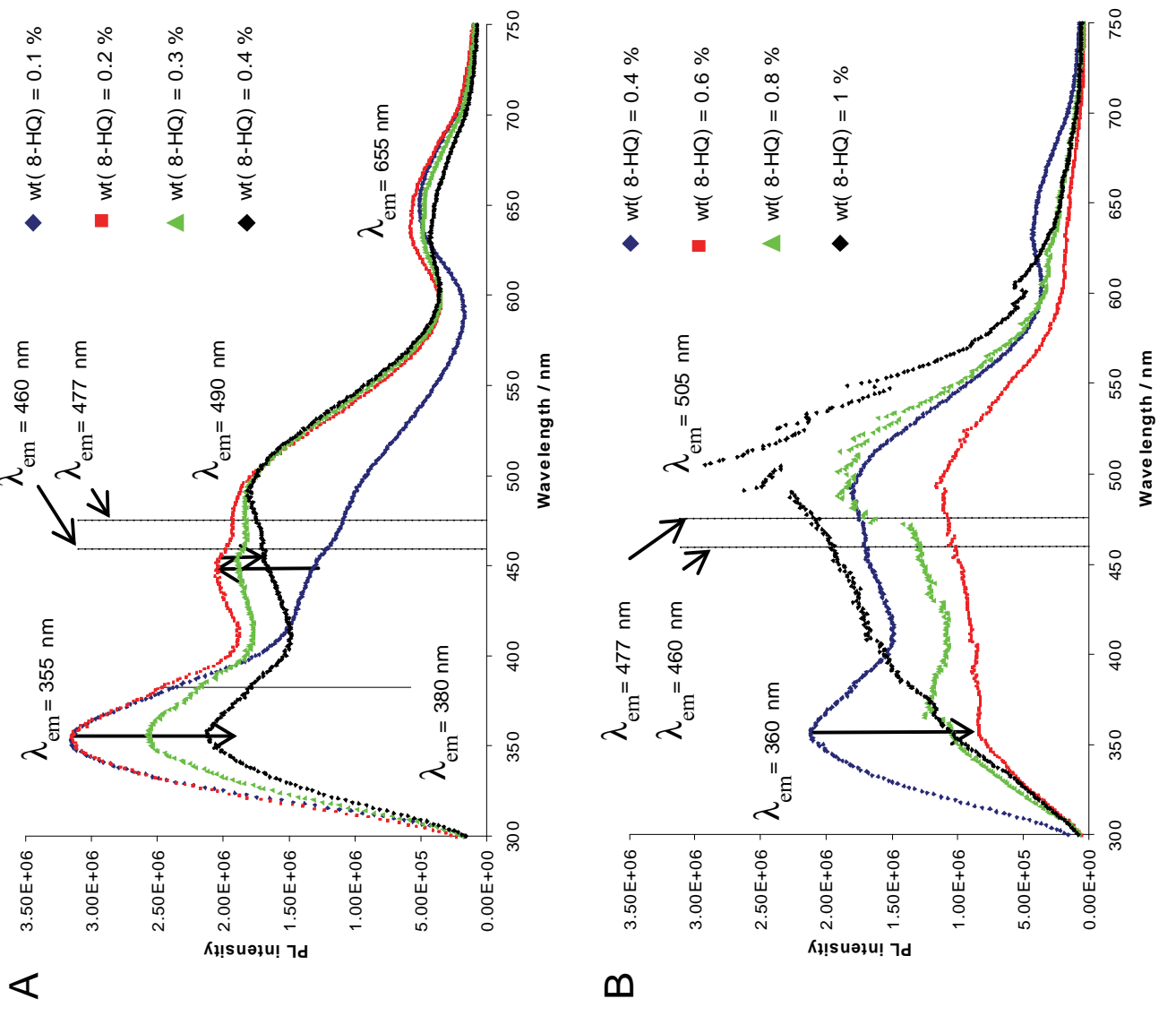

突

ㅇํㅇ

تี 


\subsubsection{Uptake of $\mathrm{ZnCl}_{2}(\mathrm{EtOH})$ onto 8-HQ-wool}

For the uptake of $\mathrm{ZnCl}_{2}(\mathrm{EtOH})$ onto 8-HQ-wool, merino wool was first soaked in different 8-HQ EtOH concentration with a total volume of $10 \mathrm{~mL}$ at $50{ }^{\circ} \mathrm{C}$ for 3 days. Table 7.7 shows the 8-HQ content in $0.1 \mathrm{~g}$ merino wool after being soaked in $10 \mathrm{~mL} \mathrm{8-}$ $\mathrm{HQ}(\mathrm{EtOH})$ solution varying in concentration from $6.25 \cdot 10^{-5}$ to $6.25 \cdot 10^{-3} \mathrm{M}$ used for this dyeing procedure.

Table 7.7: Volume and concentration of 8-HQ (EtOH) used for the in-situ dyeing method, and 8HQ mass percentage on weight of wool (wt \%), assuming 8-HQ was completely absorbed by the wool.

\begin{tabular}{ccc}
\hline $\mathbf{V}$ (0.0025 M 8-HQ (EtOH)) / mL & {$[\mathbf{8 - H Q ] ~ / ~ M ~}$} & wt(8-HQ) / \% \\
\hline 0.25 & $6.25 \cdot 10^{-5}$ & 0.09 \\
0.5 & $1.25 \cdot 10^{-4}$ & 0.18 \\
0.75 & $1.875 \cdot 10^{-4}$ & 0.27 \\
1 & $2.5 \cdot 10^{-4}$ & 0.36 \\
\hline $\mathbf{V}$ (0.025 M 8-HQ (EtOH)) / mL & {$[\mathbf{8 - H Q}] / \mathbf{M}$} & $\mathbf{w t}(\mathbf{8}-\mathrm{HQ}) / \%$ \\
\hline 0.25 & $6.25 \cdot 10^{-4}$ & 0.9 \\
0.5 & $1.25 \cdot 10^{-3}$ & 1.8 \\
0.75 & $1.875 \cdot 10^{-3}$ & 2.7 \\
1 & $2.5 \cdot 10^{-3}$ & 3.5 \\
2.5 & $6.25 \cdot 10^{-3}$ & 8.3 \\
\hline
\end{tabular}

After soaking in 8-HQ EtOH solution, 8-HQ treated wool was washed with EtOH and soaked into $10 \mathrm{~mL} 0.01 \mathrm{M} \mathrm{ZnCl}_{2}(\mathrm{EtOH})$ solution at $50{ }^{\circ} \mathrm{C}$ for 3 days. The uptake of $\mathrm{Zn}^{2+}$ ions onto 8-HQ treated wool changed the optical properties of the composite material significantly as shown in figure 7.10 and 7.11 .

Figure 7.10 shows the PL scans of the wool fibre composites, when excited with 260 $\mathrm{nm}$. It shows emission peaks with constantly straight slope until $460 \mathrm{~nm}$ after the quenching of the emission peak of the wool $\left(\lambda_{\mathrm{em}}=355 \mathrm{~nm}\right)$. The angle, and consequently, the PL intensity increases with the mass friction of 8-HQ onto the wool fibre. In addition, the emission peaks are broader with the increased uptake of 8-HQ onto wool. Thereby, the emission peak is mainly dominated by two peaks located at 460 $\mathrm{nm}$ and between $480-490 \mathrm{~nm}$. The emission peak at $460 \mathrm{~nm}$ remains constant independent of the uptake of 8-HQ onto wool. However, the wavelength of the second 
emission peak shift between 480 and maximal $490 \mathrm{~nm}$ dependent on the mass friction of 8-HQ onto wool.

When excited with $350 \mathrm{~nm}$ (figure 7.11), the wool fibre composites show broad PL peaks starting from around 400 to $650 \mathrm{~nm}$. At low mass friction of 8 -HQ (wt $(8-\mathrm{HQ})=$ $0.1-1 \%$ ), the emission peak at $460 \mathrm{~nm}$ dominate. However, with higher content of 8HQ absorbed onto wool (wt(8-HQ) $\geq 2 \%$ ), the peak at $460 \mathrm{~nm}$ is gradually displaced by a second emission peak around $480 \mathrm{~nm}$. This is also represented by a change of the curve slope between 400 and $460 \mathrm{~nm}$; the curve shape changes from a bend to a straight slope until reaching $460 \mathrm{~nm}$. Soaking in higher concentration of 8-HQ EtOH solution $\left([8-\mathrm{HQ}(\mathrm{EtOH})]=6.25 \cdot 10^{-3} \mathrm{M}\right)$ also leads to a broadening of the emission peak with a maximum PL intensity at $490 \mathrm{~nm}$.

Similar to the 8-HQ uptake, the soaking temperature has no effects on optical properties of the final wool fibre composites, however on the uptake time.

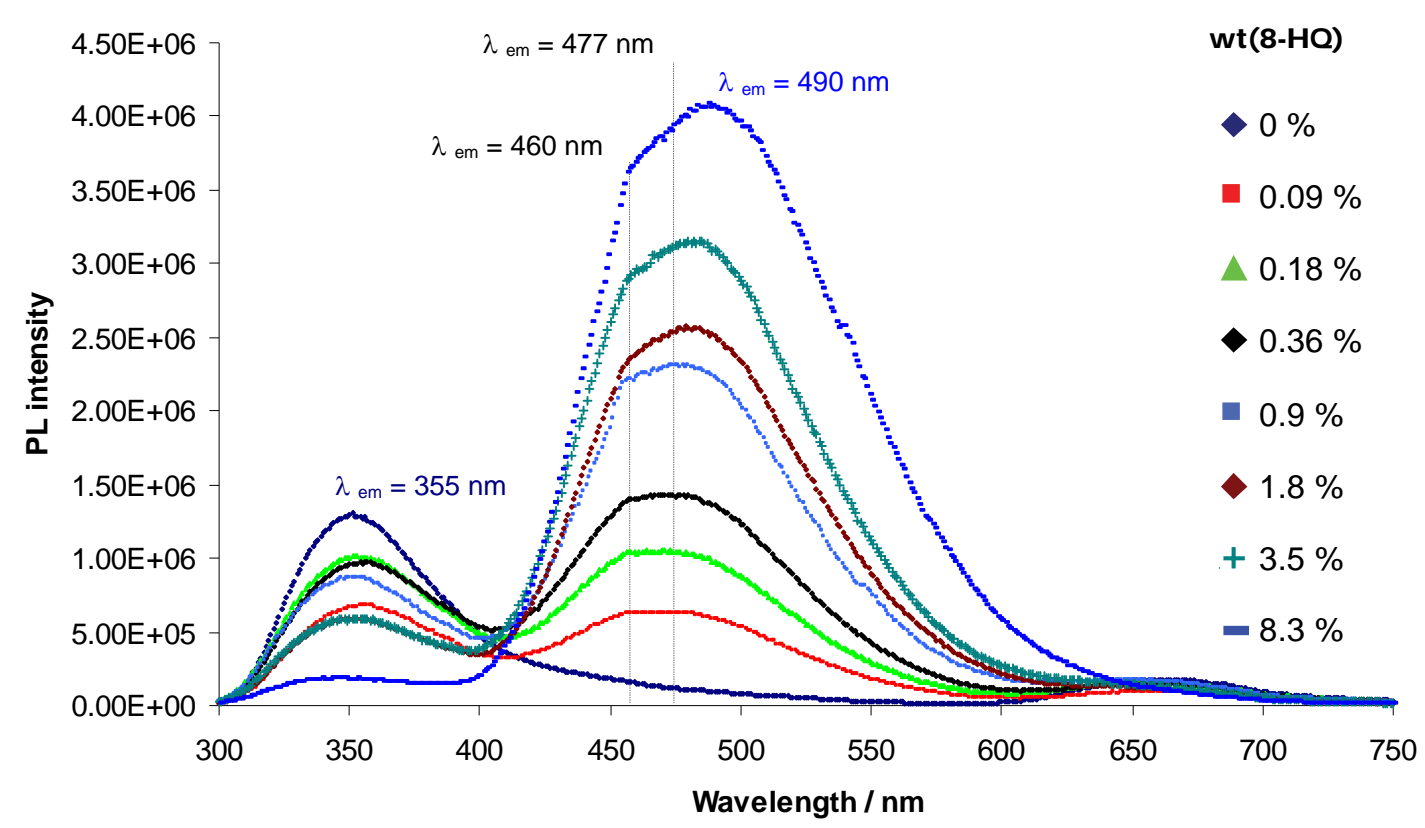

Figure 7.10: PL scans of wool fibre composites produced by the uptake of $\mathrm{ZnCl}_{2}$ (EtOH) onto 8-HQ treated wool $(\mathrm{wt}(8-\mathrm{HQ})=0.1-8 \%)\left(\lambda_{\text {exc }}=260 \mathrm{~nm}\right.$; exciting $/$ emission slit width $\left.=5 \mathrm{~nm}\right)$. Details of sample preparation are listed in appendix in table A.21. 


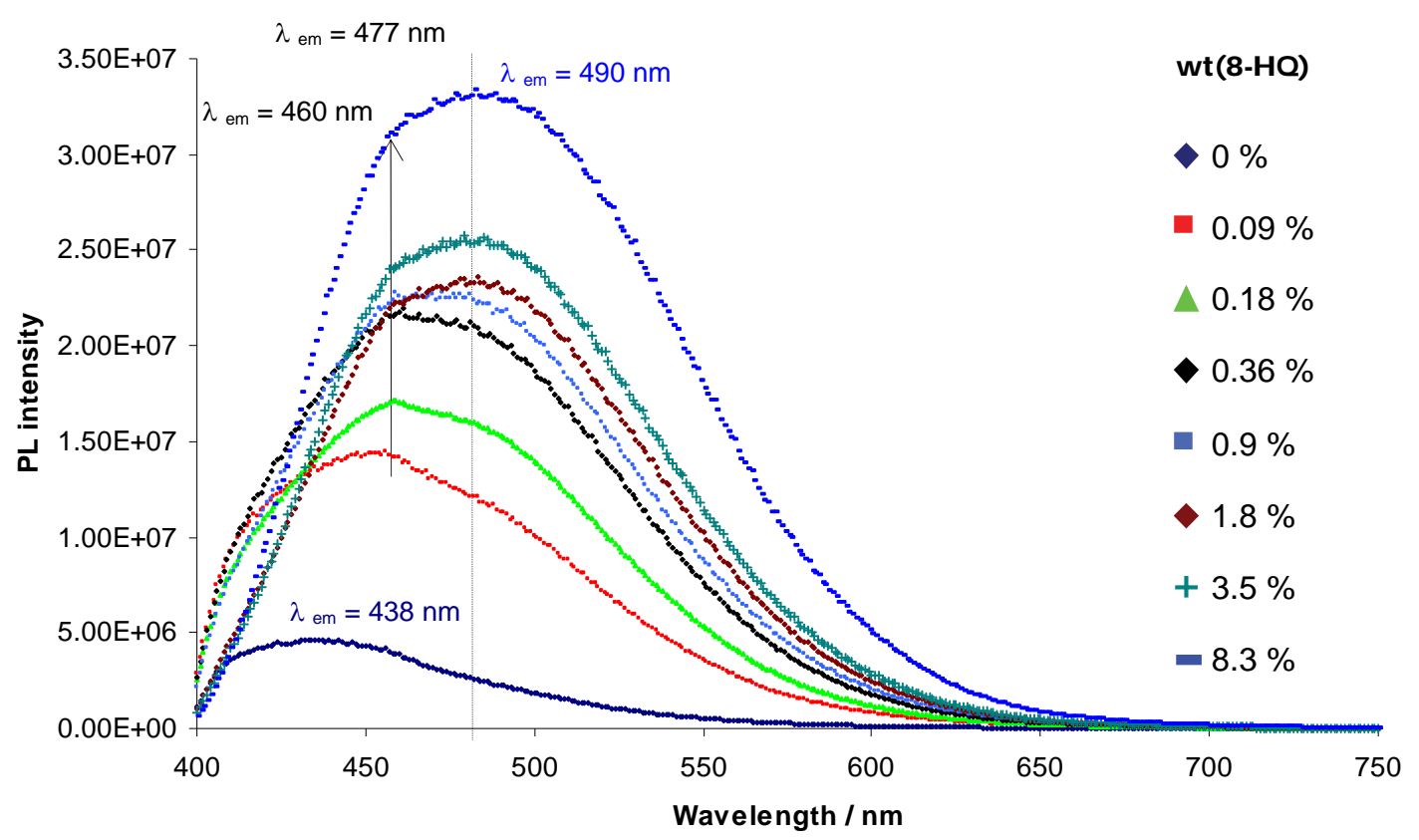

Figure 7.11: PL scans of wool fibre composites produced by the uptake of $\mathrm{ZnCl}_{2}(\mathrm{EtOH})$ onto 8-HQ treated wool $(\mathrm{wt}(8-\mathrm{HQ})=0.1-8 \%)\left(\lambda_{\mathrm{exc}}=350 \mathrm{~nm}\right.$; exciting $/$ emission slit width $\left.=3 \mathrm{~nm}\right)$. Details of sample preparation are liseted in the appendix in table A.21.

The maximum wavelengths obtained by the uptake of $\mathrm{Zn}^{2+}$ onto 8-HQ functionalised wool fibre vary between 460 and $490 \mathrm{~nm}$. This wavelength range for the maximum emission wavelengths is only obtained when the uptake of 8-HQ (first batch process) and zinc (second batch process) was carried out in EtOH solution and absence of water (wool free of water or moisture). The confinement of the emission wavelength until 490 $\mathrm{nm}$ may be related to a limited uptake of 8-HQ onto wool.

The fluorescence of the composite material is characterised by a broad emission peak made-up by a constant emission peak at $460 \mathrm{~nm}$ and an emission peak shifting between 480 and $490 \mathrm{~nm}$ depending on the content of 8-HQ absorbed onto wool. Due to the formation of a broad emission peak made up with at least two emission peaks, the wool fibre composites show a bright white-bluish fluorescence under UV-light (see figure 7.12). Under ambient light, the wool appears also white. However, wool fibre composites with a uptake of 8 -HQ wt $(8-H Q)>1 \%$ show a slightly difference in the appearance. The wool fibre composite obtains an appealing shiny appearance, which however can turns into a greyish colour at higher concentration of 8-HQ (wt(8-HQ) $8 \%)$. 


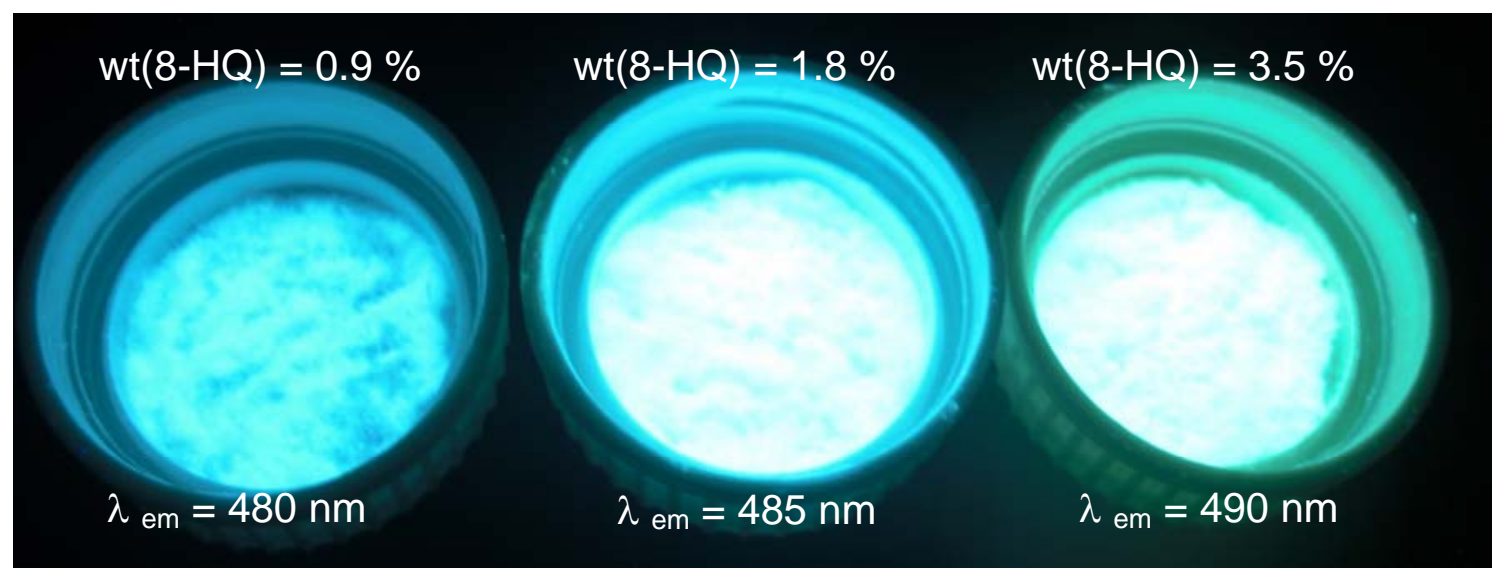

Figure 7.12: Zinc - (8-HQ wool) fibres prepared according dyeing method 1, crushed with liquid nitrogen and observed under UV light $\left(\lambda_{\text {exc }}=366 \mathrm{~nm}\right)$. Maximum emission wavelength and 8-HQ content indicated.

\subsubsection{Dyeing of wool fibre using in-situ method 2}

The in-situ dyeing method 2 involves the uptake of 8-HQ onto zinc doped wool in EtOH solution or gas atmosphere. Similar to the in-situ dyeing method 1, the soaking temperature does not influence the final optical properties of the wool fibre composite, when soaked in solution. However, higher soaking temperature shortens the dyeing bath process and ensures a homogeneous distribution of the precursor materials across the wool. For example, the soaking time of zinc doped wool in 8-HQ EtOH solution was shortened to three days, when soaked between 50 and $70{ }^{\circ} \mathrm{C}$. Besides appropriate soaking times and soaking temperature, the in-situ complex formation of $\mathrm{Zn}(8-\mathrm{Q})_{2}$ and hence optical florescence of the wool fibre composite was highly affected by the 8-HQ concentration (listed in table 7.7) and the choice of the zinc precursor material used such as $\mathrm{ZnCl}_{2}(\mathrm{EtOH}), \mathrm{ZnCl}_{2}\left(\mathrm{H}_{2} \mathrm{O}\right)$ and $\mathrm{Zn}(\mathrm{Ac})_{2}\left(\mathrm{H}_{2} \mathrm{O}\right)$.

\subsubsection{Uptake of zinc onto wool}

For the dyeing method 2, merino wool was functionalised with three precursor materials of zinc; $\mathrm{ZnCl}_{2}(\mathrm{EtOH}), \mathrm{ZnCl}_{2}\left(\mathrm{H}_{2} \mathrm{O}\right)$ and $\mathrm{Zn}(\mathrm{Ac})_{2}\left(\mathrm{H}_{2} \mathrm{O}\right)$. The details of the sample preparation are listed in the appendix in table A.22. In order to increase the uptake of zinc, wool was also pre-treated with $\mathrm{NaOH}, \mathrm{HCl}$ and MSA. This was undertaken to observe differences by the uptake onto wool and also to see difference in the complex formation with 8-HQ. 
The soaking of the wool in $\mathrm{Zn}^{2+}$ solutions should result in the homogenous dispersion of zinc throughout the wool fibre by transcellular and intercellular diffusion. Thereby, the uptake of $\mathrm{Zn}^{2+}$ ions onto wool is concentrated to the hydrophilic compounds of the wool such as the wool matrix inside the cortical cells and protein matrix of $\alpha$-Keratin at the fibre surface.

The uptake of zinc onto wool, however, is general low. EDS mapping did not provide a clear mapping of zinc around the wool. EDS elemental analysis revealed that wool, untreated and modified with $\mathrm{NaOH}$ and $\mathrm{HCl}$, absorbed about $2-5 \mathrm{mg} / \mathrm{g}$ of zinc (wt(Zn) $=0.2-0.5 \%$ metal on weight of wool) in aqueous solution independent on the precursor material. A greater uptake of zinc onto wool is obtained by soaking in EtOH solution. In EtOH solution, the uptake of zinc onto wool was of about $6-8 \mathrm{mg} / \mathrm{g}(0.6-$ $0.8 \%$ metal on weight of wool). The greater uptake can be explained by the absence of a hydration shell. Wool fibre pre-treated with MSA as linker molecule and soaked in $\mathrm{ZnCl}_{2}(\mathrm{EtOH})$ solution reached zinc values onto wool between $9-11 \mathrm{mg} / \mathrm{g}(\mathrm{wt}(\mathrm{Zn})=$ $0.9-1.1 \%$ metal on weight of wool) (see figure 7.13). Here, the use of MSA significantly enhanced the uptake of zinc onto wool. However, the treatment of wool with MSA results in a degradation of the wool fibre. Thereby, fibre damages are caused and several desirable properties of the wool are lost.

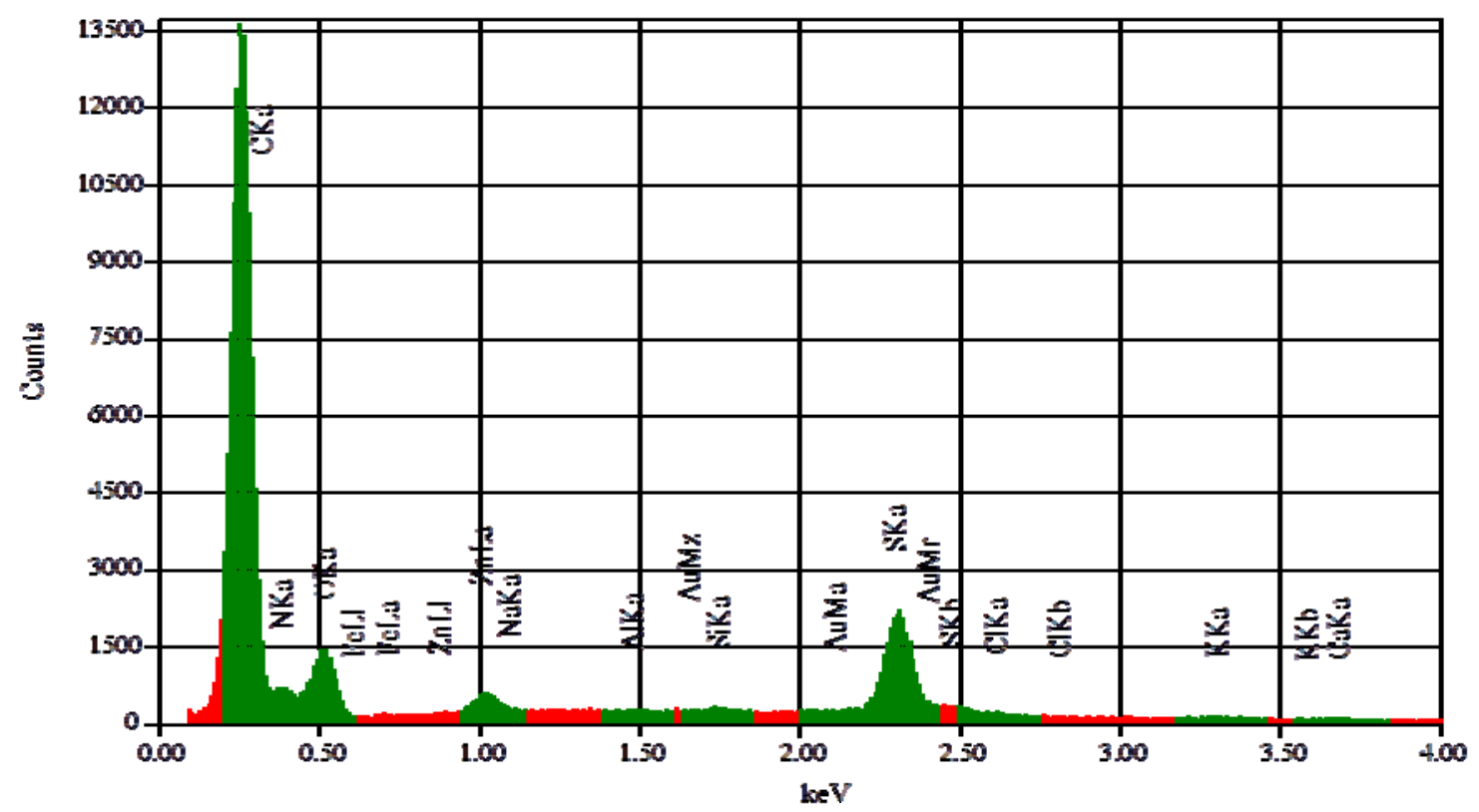

Figure 7.13: EDS elemental analysis of $\mathrm{ZnCl}_{2}$ (EtOH) doped wool pre-treated with MSA. 


\subsubsection{Uptake of 8-HQ (EtOH) onto $\mathrm{ZnCl}_{2}(\mathrm{EtOH})$ doped wool}

Figure 7.14 shows the PL scans $\left(\lambda_{\text {exc }}=350 \mathrm{~nm}\right)$ of $0.1 \mathrm{~g} \mathrm{ZnCl}_{2}(\mathrm{EtOH})$-wool after being soaked in $10 \mathrm{~mL} 8$-HQ $(\mathrm{EtOH})$ solution varying in concentration from $6.25 \cdot 10^{-5}$ to $2.5 \cdot 10^{-3} \mathrm{M}$ at $50^{\circ} \mathrm{C}$ for 3 days. The soaking of $\mathrm{ZnCl}_{2}(\mathrm{EtOH})$ doped wool in $10 \mathrm{~mL}$ $6.25 \cdot 10^{-5} \mathrm{M} 8$-HQ EtOH solution resulted in the formation of a broad emission peak with a straight slope until $460 \mathrm{~nm}$ and an maximum emission wavelength at $490 \mathrm{~nm}$ (see figure 7.14; $\operatorname{wt}(8-\mathrm{HQ})=0.09 \%)$. This emission peak is identical to that observed previously in the PL scan in figure 6.11 shown by wt $(8-\mathrm{HQ})=3.5 \%$. Here, wool was soaked first in $10 \mathrm{~mL} 2.5 \cdot 10^{-3} \mathrm{M} 8$-HQ (EtOH) before being soaked in $10 \mathrm{~mL} 0.01 \mathrm{M}$ $\mathrm{ZnCl}_{2}(\mathrm{EtOH})$ solution according to the in-situ dyeing method 1.

By increasing the concentration of 8 -HQ in the EtOH soaking solution $([8-\mathrm{HQ}]=$ $\left.6.25 \cdot 10^{-5}-1.875 \cdot 10^{-3} \mathrm{M}\right)$, the broad emission peak shifted to the red side of the spectra until a maximum PL intensity at $505 \mathrm{~nm}$ is reached. This wavelength presents a turning point for the optical properties of the wool fibre composite (see figure 7.14; wt $(8-\mathrm{HQ})=$ $2.7 \%$ ). When $\mathrm{ZnCl}_{2}(\mathrm{EtOH})$ doped wool was soaked in higher concentration of 8-HQ $\left(\left([8-\mathrm{HQ}]>1.875 \cdot 10^{-3} \mathrm{M}\right)\right.$, the broad emission peak with a maximum wavelength at $505 \mathrm{~nm}$ loses the straight slope at the base of the curve $(\sim 400 \mathrm{~nm})$ and the slope of the peak starts around $420 \mathrm{~nm}$ (see figure 7.14; wt(8-HQ) $=3.5 \%$ ). By doing this, the maximum emission wavelength at $505 \mathrm{~nm}$ remained unchanged, however, the distinct peak / hump at $460 \mathrm{~nm}$ gradually vanished. With the disappearing of the peak at 460 $\mathrm{nm}$, the fluorescence of the wool fibre composite changed from white to white-greenish.

With ageing (in the soaking solution or dried under ambient air pressure), the maximum emission wavelength at $\lambda_{\mathrm{em}}=505 \mathrm{~nm}$ shifted to higher wavelength accompanied by a reduction of the PL intensity as shown in figure 7.15. The process is accelerated by drying at higher temperature. The emission shift is also noticed by a change of the fluorescence colour from white-greenish to green-yellowish. 


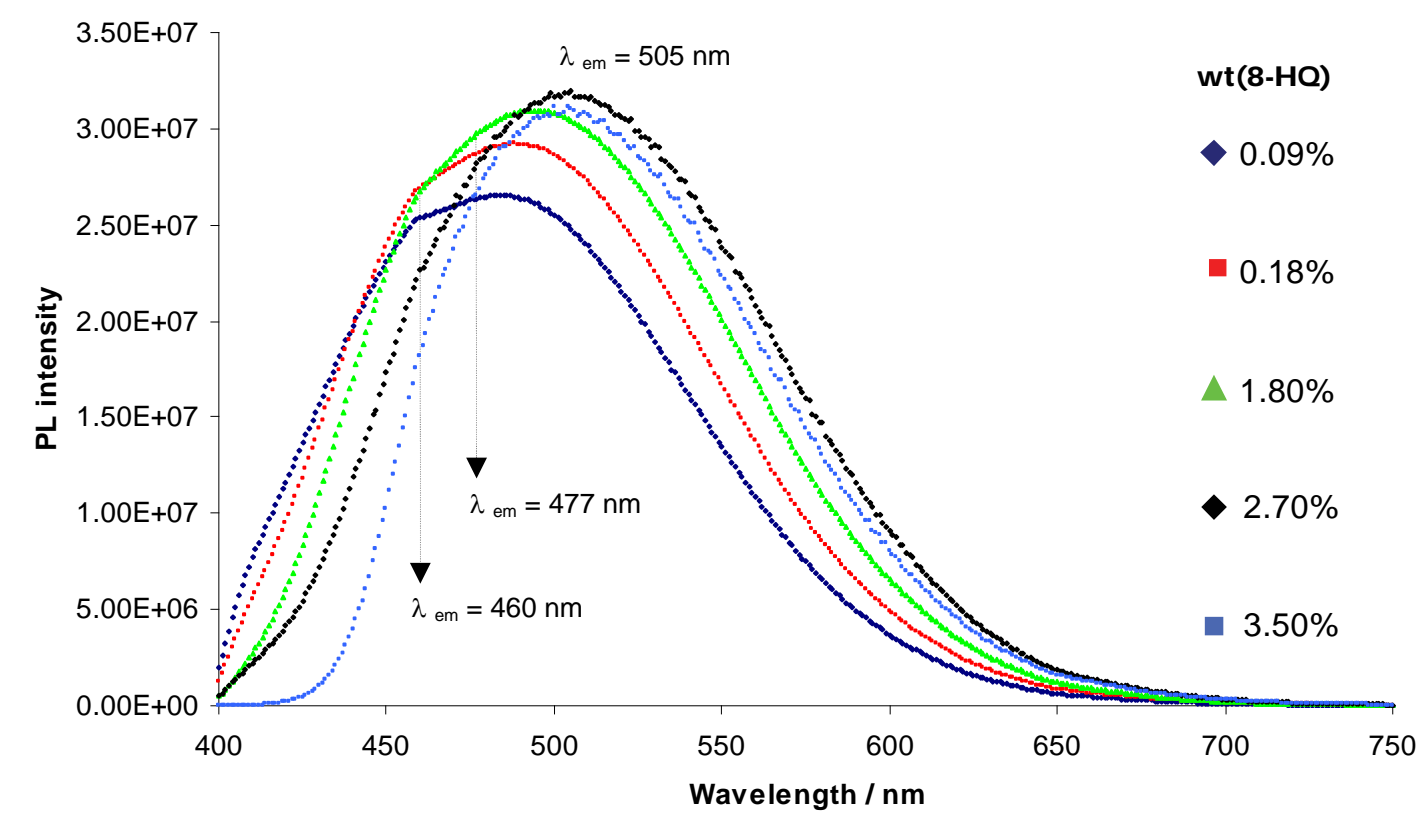

Figure 7.14: PL scan of wool fibre composites produced by soaking $\mathrm{ZnCl}_{2}$ (EtOH) doped wool (zinc content is constant) in $10 \mathrm{~mL}$ 8-HQ (EtOH) solution $\left([8-\mathrm{HQ}]=6.25 \cdot 10^{-5}-2.5 \cdot 10^{-3} \mathrm{M}\right)$ at $50{ }^{\circ} \mathrm{C}$ for 3 days $\left(\lambda_{\text {exc }}=350 \mathrm{~nm}\right.$; exciting $/$ emission slit width $\left.=3 \mathrm{~nm}\right)$. Details of sample preparation are listed in the appendix in table $\mathbf{A} .23$.

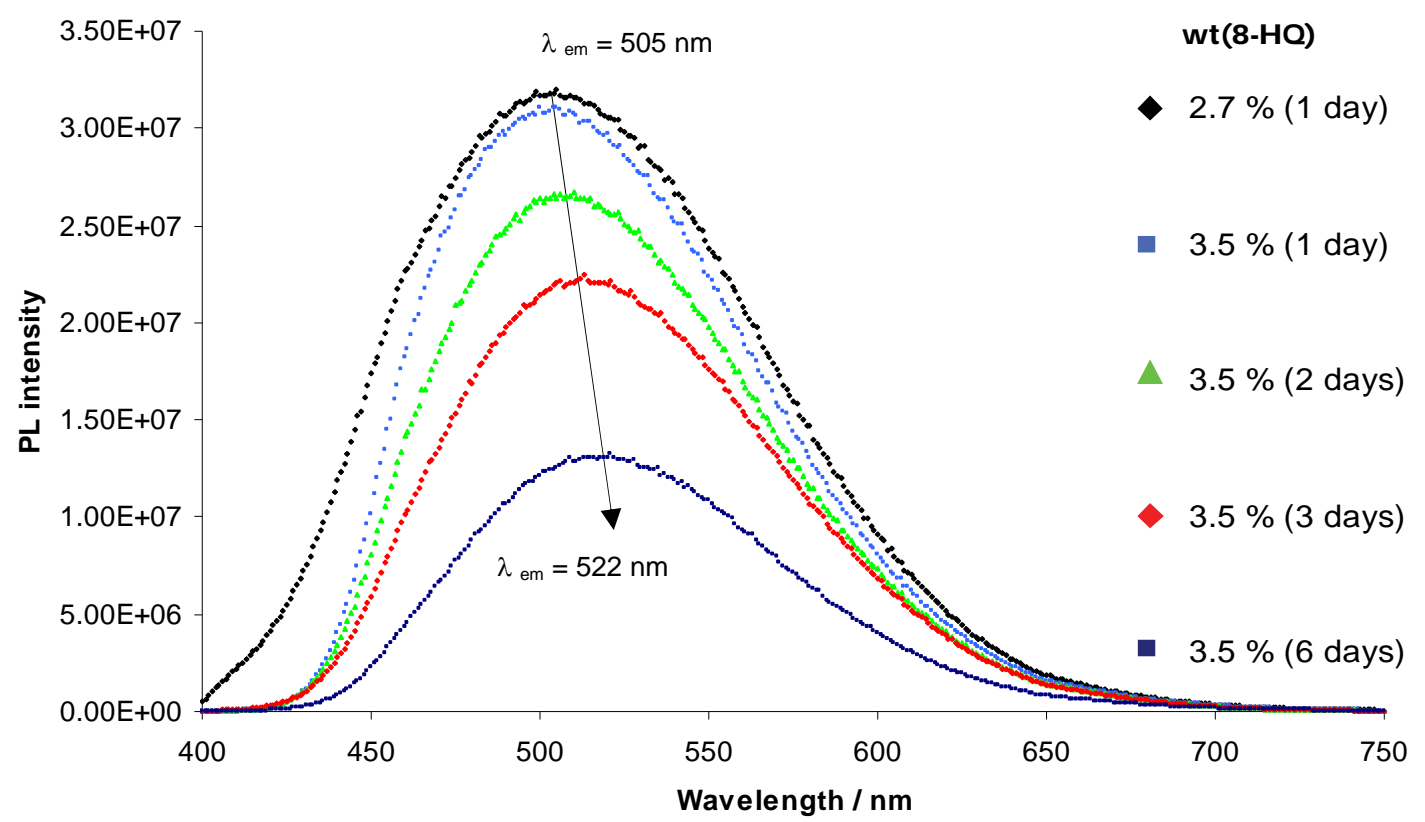

Figure 7.15: PL scan of wool fibre composites with a high uptake of 8-HQ and the effect of ageing on the fluorescence intensity and emission wavelength. The composites were prepared by soaking $0.1 \mathrm{~g}$ Zn doped wool into $10 \mathrm{~mL} \mathrm{8-HQ}(\mathrm{EtOH})$ solution $(0.0025 \mathrm{M})$ at $50^{\circ} \mathrm{C}$ for 3 days $\left(\lambda_{\text {exc }}=350\right.$ $\mathrm{nm}$; exciting / emission slit width $=3 \mathrm{~nm})$.

Wool fibre composites soaked in 8-HQ EtOH solution below $[8-\mathrm{HQ}] \leq 1.875 \cdot 10^{-3} \mathrm{M}$ $(w t(8-H Q) \leq 2.7 \%)$ also decreased slightly their PL intensities with ageing, however the emission peaks with curve shape and maximum emission wavelength remained 
unchanged. Here, it is important to point out that only wool fibre composites underwent a change in their fluorescence over time, when the distinct emission peak / hump at 460 nm was missing.

The maximum wavelength of the wool composite material obtained with this dyeing method can vary in the range of $490-525 \mathrm{~nm}$ depending on the 8-HQ concentration. By applying low concentration of 8 -HQ $\left([8-\mathrm{HQ}]=6.25 \cdot 10^{-5}-1.875 \cdot 10^{-3} \mathrm{M}\right)$, the emission wavelength is located below $505 \mathrm{~nm}$. Here, the fluorescence of the wool fibre composite appears bright white (see figure 7.16; A). With the increment of the 8-HQ concentration in the soaking solution $\left([8-\mathrm{HQ}]=1.875 \cdot 10^{-3} \mathrm{M}\right)$ the bright white fluorescence appears weakened (figure 7.17, B). This is connected with the gradually decrease of the emission peak at $460 \mathrm{~nm}$ with higher 8-HQ concentration (see emission shift in figure 7.14; $\mathrm{wt}(8-\mathrm{HQ})=0.09-2.7 \%)$. The use of higher 8-HQ concentrations $\left(\mathrm{wt}(8-\mathrm{HQ})=3.5 \%\right.$; soaking $\mathrm{ZnCl}_{2}(\mathrm{EtOH})$ doped wool in $10 \mathrm{~mL} 2.5 \cdot 10^{-3} \mathrm{M} 8$-HQ $\mathrm{EtOH})$ resulted in a change of the fluorescence colour from white to green-yellowish (see figure $7.16 ; \mathrm{C}$ ). Here, the emission wavelength shifted to $\lambda_{\mathrm{em}} \sim 515-525 \mathrm{~nm}$ with ageing. This emission shifts is also noticeable by the greenish colour of the wool fibre under ambient light. This wavelength shift is also linked with a reduction of the PL intensity.

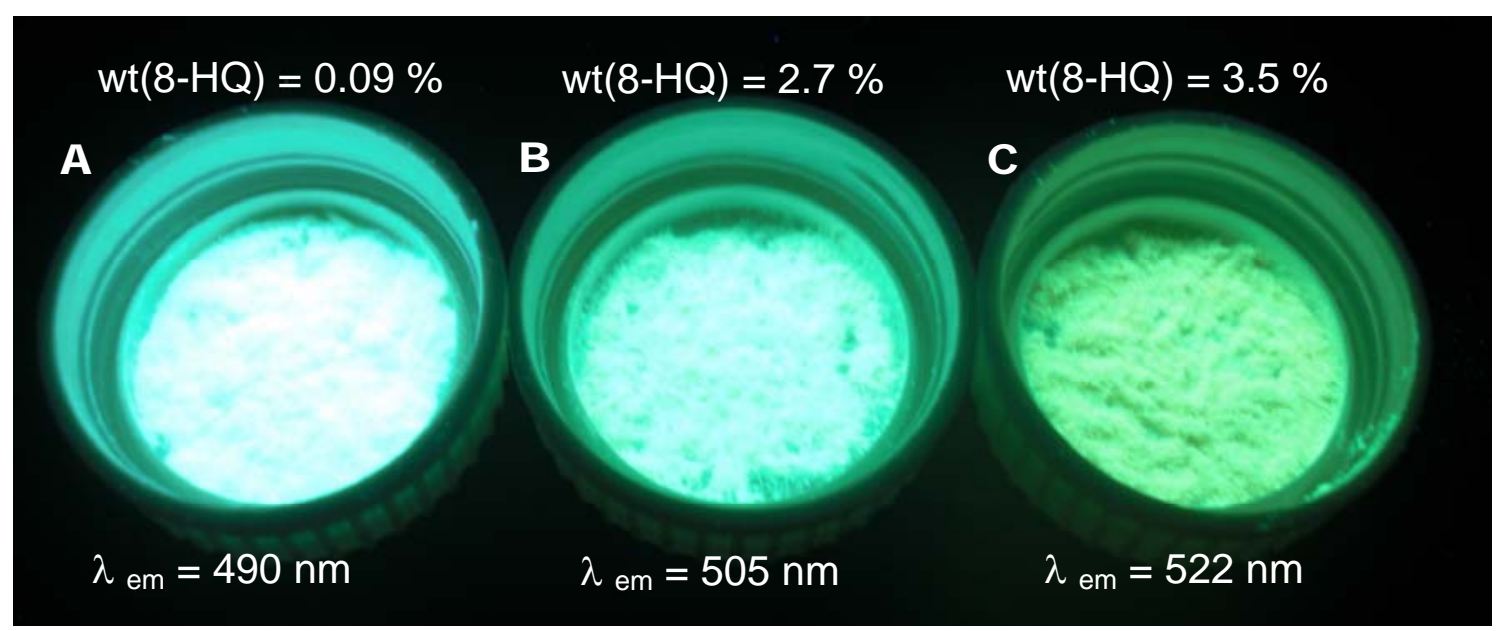

Figure 7.16: 8-HQ - (Zn wool) fibres prepared according dyeing method 2, crushed with liquid nitrogen and observed under UV light $\left(\lambda_{\text {exc }}=366 \mathrm{~nm}\right)$. Maximum emission wavelength and 8-HQ content indicated. 


\subsubsection{Uptake of 8-HQ (EtOH) onto $\mathrm{ZnCl}_{2}\left(\mathrm{H}_{2} \mathrm{O}\right)$ doped wool}

Wool fibres soaked in zinc aqueous solution in the first batch process undergo a change in colour after soaking in 8-HQ EtOH solution. The extent of the change in colour is dependent on the 8-HQ (EtOH) concentration and zinc precursor material used. The colour of the wool fibre composites also darkens gradually with ageing. The colour change of the wool with ageing is also linked with a reduction of the PL intensity of the wool fibre composites.

The PL scans of wool fibre soaked in $\mathrm{ZnCl}_{2}$ aqueous solution are similar to those of $\mathrm{ZnCl}_{2}(\mathrm{EtOH})$ doped wool after soaking in 8-HQ EtOH solution; however undergo a strong wavelength shift with ageing. Figure 7.17 shows $\mathrm{ZnCl}_{2}\left(\mathrm{H}_{2} \mathrm{O}\right)$ doped wool soaked in $10 \mathrm{~mL} 8$-HQ (EtOH) solution varying in concentration in the range of $6.25 \cdot 10^{-5}-1.875 \cdot 10^{-3} \mathrm{M}$ (see table 7.7 ) at $50{ }^{\circ} \mathrm{C}$ for 3 days. The $\mathrm{ZnCl}_{2}\left(\mathrm{H}_{2} \mathrm{O}\right)$ wool fibre composites have been dyed under the same conditions as $\mathrm{ZnCl}_{2}(\mathrm{EtOH})$ doped wool, however the PL scans have been recorded after six days of ageing in ambient air and sunlight exposure.

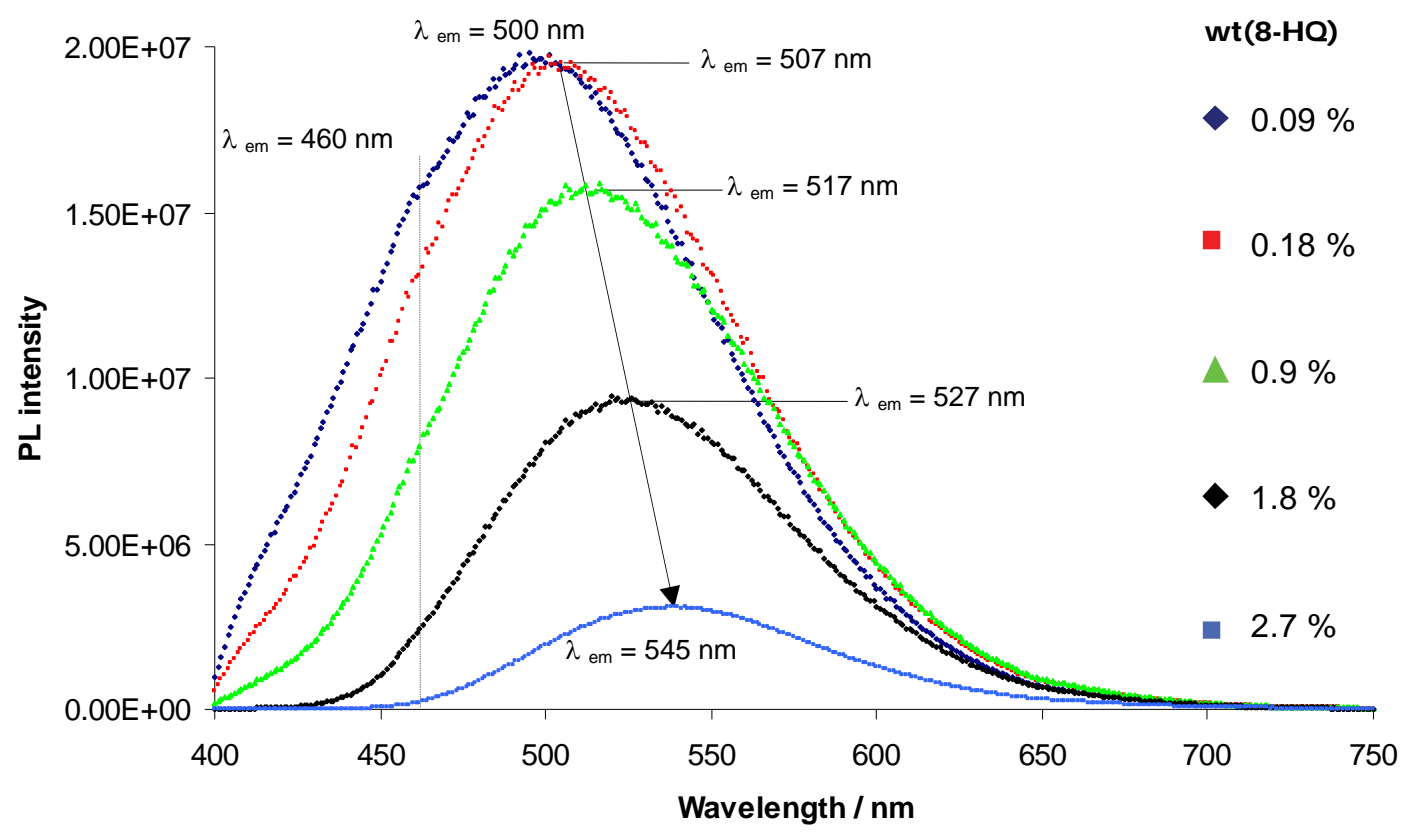

Figure 7.17: PL scans of wool fibre composites produced by soaking $\mathrm{ZnCl}_{2}\left(\mathrm{H}_{2} \mathrm{O}\right)$ doped wool (zinc content is constant) in $10 \mathrm{~mL} \mathrm{8-HQ} \mathrm{(EtOH)} \mathrm{solution}\left([8-\mathrm{HQ}]=6.25 \cdot 10^{-5}-1.875 \cdot 10^{-3} \mathrm{M}\right)$ at $50{ }^{\circ} \mathrm{C}$ for 3 days, The PL scans were obtained after 6 days of ageing $\left(\lambda_{\text {exc }}=350 \mathrm{~nm}\right.$; exciting $/$ emission slit width $=3 \mathrm{~nm}$ ). Details of sample preparation are listed in the appendix in table A.24. 
Here, $\mathrm{ZnCl}_{2}\left(\mathrm{H}_{2} \mathrm{O}\right)$ doped wool obtains a maximum emission wavelength at $500 \mathrm{~nm}$, when soaked in $10 \mathrm{~mL} 6.25 \cdot 10^{-5} \mathrm{M}$ 8-HQ EtOH solution and aged for 6 days in ambient conditions. The PL curve slope starts before $400 \mathrm{~nm}$ and shows a steep increment (see figure 7.17; wt $(8-\mathrm{HQ})=0.09 \%$ ). The emission peak of this wool fibre composite remains constant, however show a slightly reduction in the PL intensity with ageing. The colour of the wool fibre composite under ambient and UV light is white (figure 7.18; A). At higher 8-HQ concentration, the curve slopes gradually flattens and the emission peaks shift to higher wavelength. This is also involved with a colouration of the wool. For example, wool fibre doped with $\mathrm{ZnCl}_{2}\left(\mathrm{H}_{2} \mathrm{O}\right)$ and soaked in $10 \mathrm{~mL}$ $1.25 \cdot 10^{-4} \mathrm{M} 8$-HQ EtOH solution obtains a slight greenish colour under ambient light and UV-light (figure 7.18; B). When the wool composite is excited with $350 \mathrm{~nm}$, a maximum emission wavelength at $\lambda_{\mathrm{em}}=507 \mathrm{~nm}$ is obtained (figure 7.17; $\mathrm{wt}(8-\mathrm{HQ})=$ $0.18 \%)$.

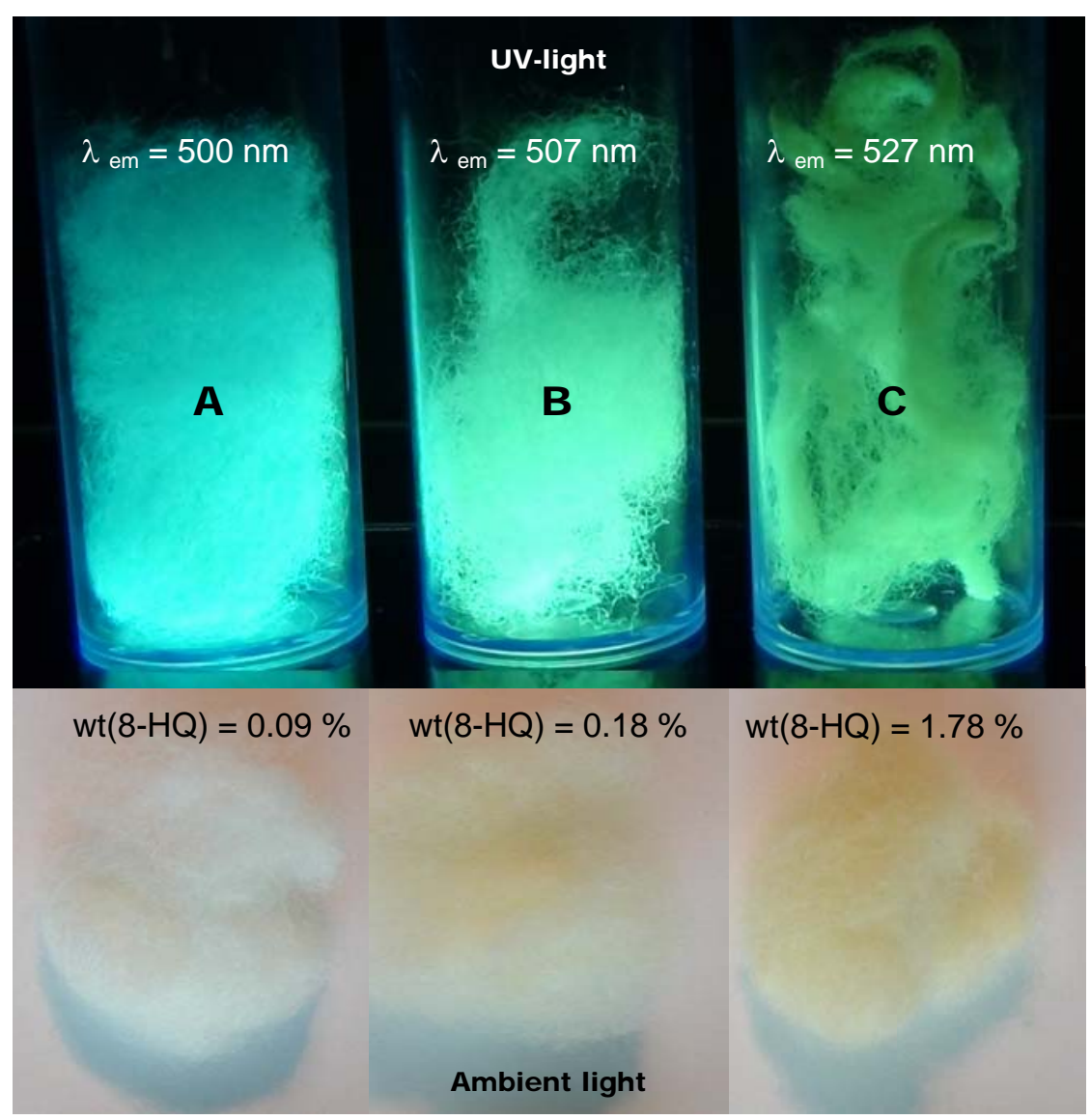

Figure 7.18: Wool doped with $\mathrm{ZnCl}_{2}\left(\mathrm{H}_{2} \mathrm{O}\right)$ and soaked in $10 \mathrm{~mL} \mathrm{6.25} \cdot 10^{-5} \mathrm{M}$ 8-HQ (EtOH) (A), $1.25 \cdot 10^{-4} \mathrm{M}$ 8-HQ (EtOH) (B) and $1.25 \cdot 10^{-3} \mathrm{M} \mathrm{8}-\mathrm{HQ}(\mathrm{EtOH})(\mathrm{C})$ for 3 days at $50{ }^{\circ} \mathrm{C}$ observed under UV-light (above, $\lambda_{\text {exc }}=256 \mathrm{~nm}$ ) and ambient light (below). Pictures were taken after 2 months of ageing. 
The colour change is linked to a stronger shift of the emission peaks to higher wavelengths, when compared with $\mathrm{ZnCl}_{2}(\mathrm{EtOH})$ doped wool samples dyed under the same dyeing bath condition. It is also important to point out that the peak at $460 \mathrm{~nm}$ is less distinctive and the PL intensities are in general lower compared to $\mathrm{ZnCl}_{2}(\mathrm{EtOH})$ wool fibre composites. Wool fibre composites doped with $\mathrm{ZnCl}_{2}\left(\mathrm{H}_{2} \mathrm{O}\right)$ show a blue shift, when the zinc uptake was carried out onto MSA treated wool. For wool fibre composites soaked in $10 \mathrm{~mL} 1.25 \cdot 10^{-3}-1.875 \cdot 10^{-3} \mathrm{M} 8$-HQ EtOH solutions, the peaks are shifted about $15-20 \mathrm{~nm}$ to the left-hand side of the spectrum. Interestingly, the blue shift results in higher PL intensities and the wool fibre composites do not undergo a further colour change with ageing. This, however, is only obtained for wool fibre composites which show a steep increment in the curve slope of the PL scan starting below $400 \mathrm{~nm}$ (see PL scans of $\mathrm{wt}(8-\mathrm{HQ})=0.09$ and $0.18 \%$ in figure 7.17 ). Here, the steep increment of the PL curve is linked with a distinct peak / hump at 460 $\mathrm{nm}$. This peak plays an important role for the optical properties of the wool fibre composites with ageing. Wool fibre composites with a peak / hump at $460 \mathrm{~nm}$ do not undergo a further change in their optical properties. They may only show a slightly reduction in their PL intensities with ageing.

It is important to mention that wool fibre composites with MSA pre-treatment and doped with $\mathrm{ZnCl}_{2}\left(\mathrm{H}_{2} \mathrm{O}\right)$ show no fluorescence properties when soaked in low 8-HQ concentrations $\left([8-\mathrm{HQ}]=6.25 \cdot 10^{-5}-6.25 \cdot 10^{-4} \mathrm{M}\right)$.

\subsubsection{Uptake of 8-HQ (EtOH) onto $\mathrm{Zn}(\mathrm{Ac})_{2}\left(\mathrm{H}_{2} \mathrm{O}\right)$ doped wool}

Wool fibres soaked in $\mathrm{Zn}(\mathrm{Ac})_{2}$ aqueous solution in the first batch process undergo a stronger change in colour, when soaked in 8 -HQ EtOH solution. For example, $\mathrm{Zn}(\mathrm{Ac})_{2}$ doped wool soaked in $10 \mathrm{~mL} 2.5 \cdot 10^{-3} \mathrm{M} 8$-HQ EtOH solution for 3 days at $50{ }^{\circ} \mathrm{C}$ show a cream yellowish colour under ambient light (figure 7.19; A) and intense bright yellow colour under UV-light (figure 7.19; B). When this wool was excited with $350 \mathrm{~nm}$, an emission wavelength around $535 \mathrm{~nm}$ was obtained. 
A

Ambient light
B

\section{UV-light}

Figure 7.19: Wool doped with $\mathrm{Zn}(\mathrm{Ac})_{2}\left(\mathrm{H}_{2} \mathrm{O}\right)$ and soaked in $10 \mathrm{~mL} 2.5 \cdot 10^{-3} \mathrm{M}$ 8-HQ EtOH solution for 3 days at $50{ }^{\circ} \mathrm{C}$. Photographs taken under ambient light $(A)$ and $U V$-light $\left(B ; \lambda_{\text {exc }}=366 \mathrm{~nm}\right)$.

Figure7.20 shows the PL scans of the untreated and MSA pre-treaded wool doped with $\mathrm{Zn}(\mathrm{Ac})_{2}\left(\mathrm{H}_{2} \mathrm{O}\right)$ after soaking in $10 \mathrm{~mL}$ 8-HQ EtOH solution with three different concentrations $\left(\left[8-\mathrm{HQ}=1.25,1.875\right.\right.$ and $\left.2.5 \cdot 10^{-3} \mathrm{M}\right)$ for 3 days at $50{ }^{\circ} \mathrm{C}$. Figure 7.20 also shows the PL scan of $\mathrm{ZnCl}_{2}(\mathrm{EtOH})$ doped wool (black curve) soaked in $10 \mathrm{~mL}$ $1.875 \cdot 10^{-3} \mathrm{M} 8$-HQ EtOH solution $\left(50{ }^{\circ} \mathrm{C}, 3\right.$ days) to compare the different PL scans obtained from $\mathrm{Zn}(\mathrm{Ac})_{2}\left(\mathrm{H}_{2} \mathrm{O}\right)$ doped wool (untreated and MSA treated).

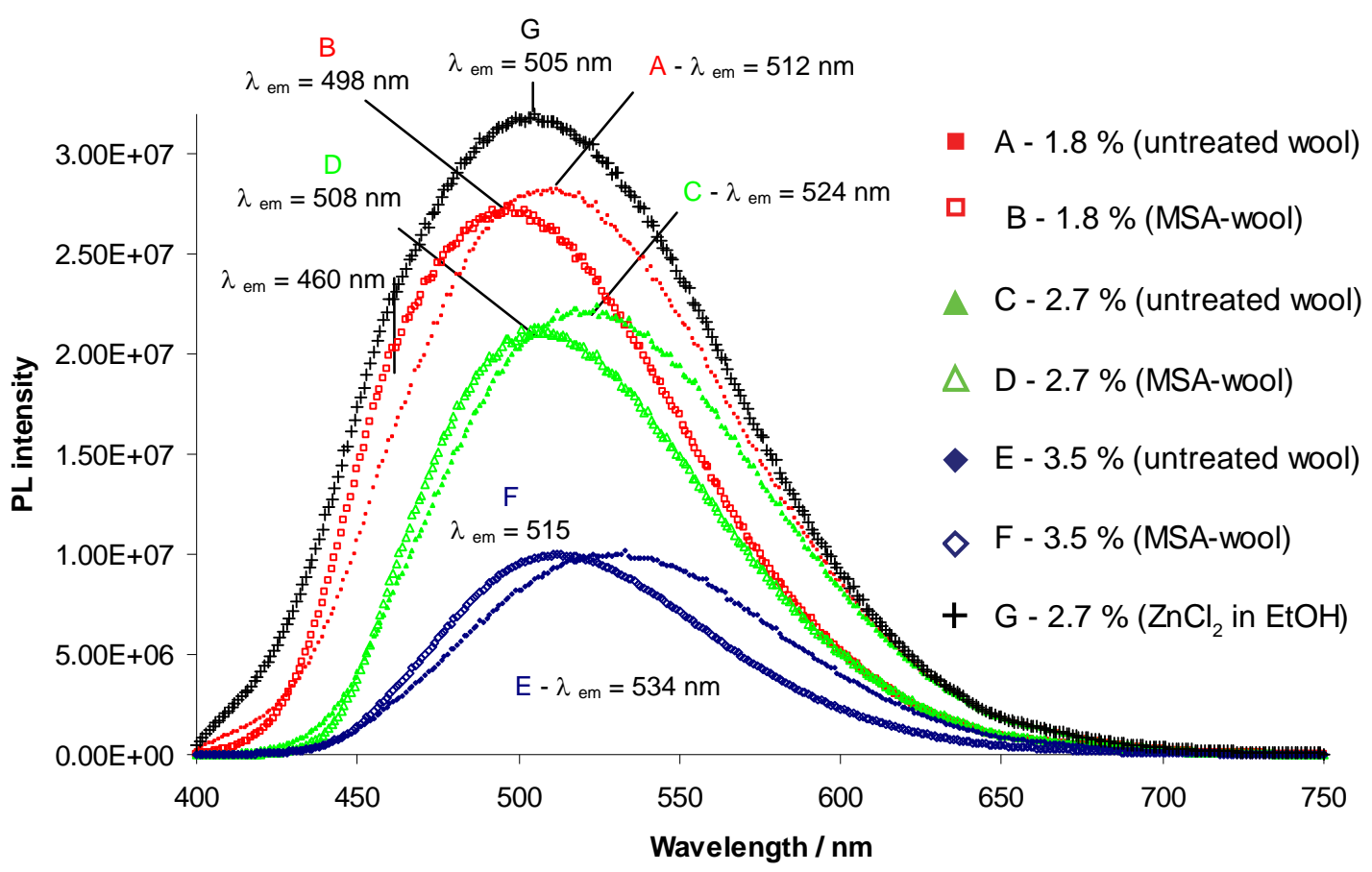

Figure 7.20: PL scan of wool fibre, untreated (solid square, triangle and rhomboid) and pre-treated with MSA (open square, triangle and rhomboid), doped with $\mathrm{Zn}(\mathrm{Ac})_{2}\left(\mathrm{H}_{2} \mathrm{O}\right)$ and soaked in 8-HQ EtOH solution $\left([8-\mathrm{HQ}]=1.25 \cdot 10^{-3}-2.5 \cdot 10^{-3} \mathrm{M}\right)$ and wool fibre doped with $\mathrm{Zn}(\mathrm{Cl})_{2}(\mathrm{EtOH})$ (plus sign) soaked in 8-HQ EtOH solution $\left([8-\mathrm{HQ}]=1.875 \cdot 10^{-3} \mathrm{M}\right)\left(\lambda_{\text {exc }}=350 \mathrm{~nm}\right.$; exciting $/$ emission slit width $=3 \mathrm{~nm})$. Details of sample preparation are listed in the appendix in table A.25. 
Wool doped with $\mathrm{Zn}(\mathrm{Ac})_{2}\left(\mathrm{H}_{2} \mathrm{O}\right)$ and soaked in 8-HQ $(\mathrm{EtOH})$ solution show stronger shifts in their emission peaks to longer wavelengths than $\mathrm{ZnCl}_{2}$ doped wool under constant dyeing bath conditions. For example, $\mathrm{ZnCl}_{2}(\mathrm{EtOH})$ doped wool (figure 7.20; G) show a maximum emission wavelength at $505 \mathrm{~nm}$ after soaking in $10 \mathrm{~mL} 1.875$. $10^{-3} \mathrm{M}$ 8-HQ EtOH solution at $50{ }^{\circ} \mathrm{C}$ for 3 days, whereas $\mathrm{Zn}(\mathrm{Ac})_{2}\left(\mathrm{H}_{2} \mathrm{O}\right)$ doped wool (figure 7.20 ; C) show a maximum emission wavelength at $524 \mathrm{~nm}$ using the same dyeing bath conditions. However, when wool fibre was pre-treated with MSA before the uptake of $\mathrm{Zn}(\mathrm{Ac})_{2}$ and followed by the uptake of 8-HQ, a blue shift of the emission peaks were observed (see figure 7.21; $\rightarrow \mathrm{B}, \mathrm{C} \rightarrow \mathrm{D}$ and $\mathrm{E} \rightarrow \mathrm{F}$ ). These blue shifts of the emission peaks are about $15-20 \mathrm{~nm}$. Due to the blue shift, $\mathrm{Zn}(\mathrm{Ac})_{2}$ doped wool obtain similar emission maximum wavelength as $\mathrm{ZnCl}_{2}(\mathrm{EtOH})$ doped wool only with lower PL intensities.

A further characteristic of $\mathrm{Zn}(\mathrm{Ac})_{2}$ doped wool (independent of untreated or MSA treated wool), is that the colour of the wool fibre composite undergo a further change with ageing. The colour change is visible under ambient and UV light. This effect is also observed by $\mathrm{ZnCl}_{2}(\mathrm{EtOH})$ and $\mathrm{ZnCl}_{2}\left(\mathrm{H}_{2} \mathrm{O}\right)$ doped wool, when soaked in high 8HQ (EtOH) concentrations. The change of the fluorescence with ageing is always indicated by the absence of the distinctive hump / peak at $460 \mathrm{~nm}$ (see figure 7.20; $\mathrm{B}$ and $\mathrm{G})$.

The effect of ageing under ambient conditions and sunlight exposure is shown in the PL scans of figure 7.21. It shows the PL scans of wool fibre composites pre-treaded with MSA and doped with $\mathrm{Zn}(\mathrm{Ac})_{2}\left(\mathrm{H}_{2} \mathrm{O}\right)$ after soaking in $10 \mathrm{~mL} 1.875 \cdot 10^{-3} \mathrm{M}$ 8-HQ EtOH solution for 3 days at $50{ }^{\circ} \mathrm{C}$. 


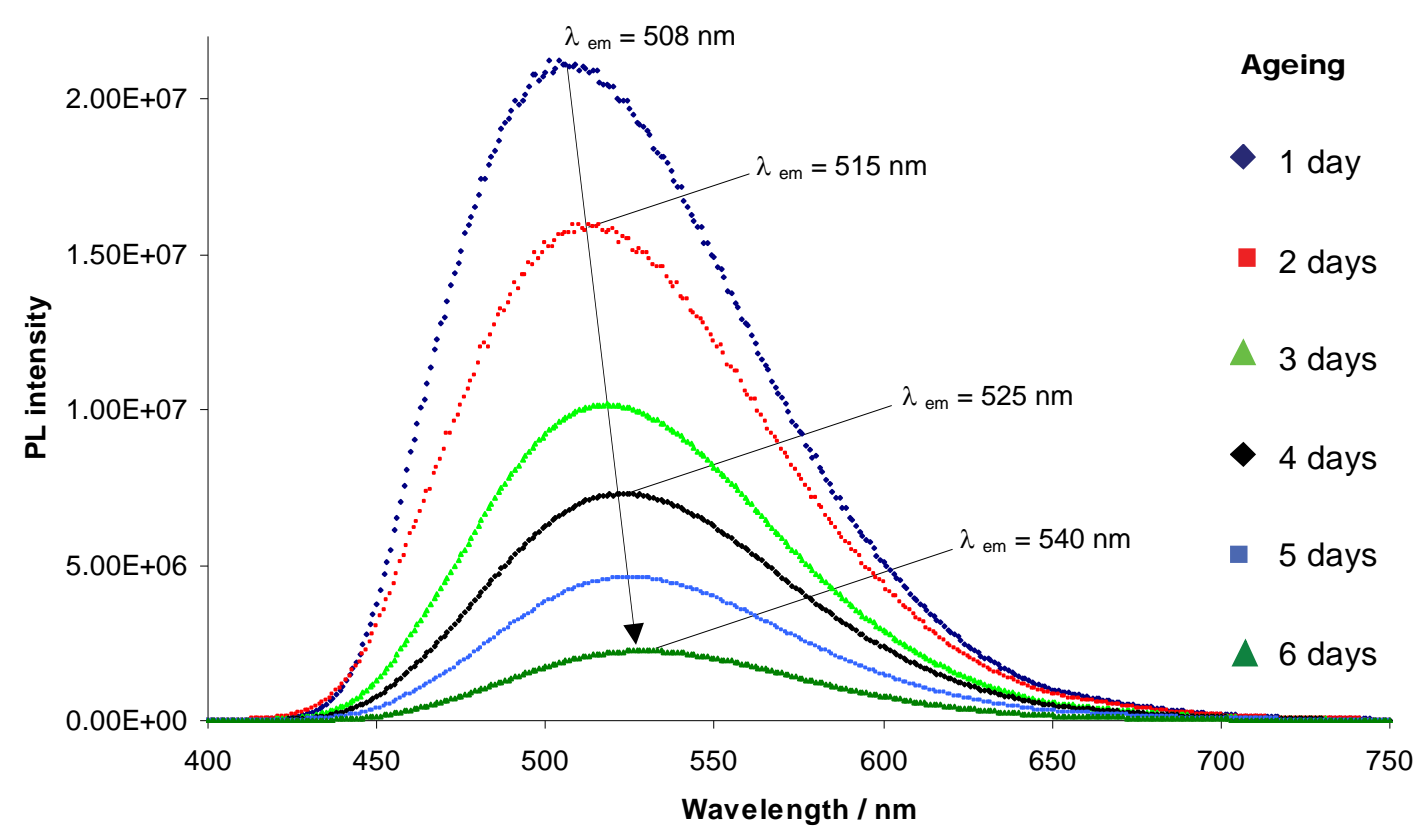

Figure 7.21: PL scan of wool fibre doped with $\mathrm{Zn}(\mathrm{Ac})_{2}\left(\mathrm{H}_{2} \mathrm{O}\right)$ and soaked in $10 \mathrm{~mL} 1.875 \cdot 10^{-3} \mathrm{M} \mathrm{8-}$ HQ EtOH solution $\left(w t(8-H Q)=2.7 \%\right.$ on $0.1 \mathrm{~g}$ wool) at $50{ }^{\circ} \mathrm{C}$ for 3 days. The PL scans were obtained daily after preparation for 6 days $\left(\lambda_{\text {exc }}=350 \mathrm{~nm}\right.$; exciting / emission slit width $\left.=3 \mathrm{~nm}\right)$. The wool fibre was treated with MSA before $\mathrm{Zn}(\mathrm{Ac})_{2}\left(\mathrm{H}_{2} \mathrm{O}\right)$ was absorbed. Details of the sample are listed in the appendix in table A.25; D.

As seen in figure 7.21, the shift of the emission wavelength $(508 \mathrm{~nm})$ to the red end of the spectrum is always linked with a reduction of the PL intensity. With the shift of the maximum emission wavelength, the wool fibre composite shows a stronger yellow colour under ambient light. The maximum shift of the wool fibre composites is located between 540 and $550 \mathrm{~nm}$. In this wavelength range, the wool fibre composite obtains the lowest PL intensity and has a dark yellow colour under ambient light (see figure 7.22).

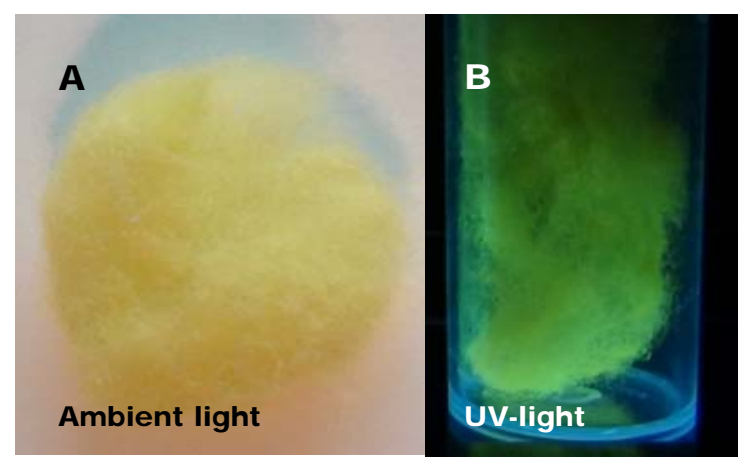

Figure 7.22: Effect of ageing (6 days sun light) on wool pre-treated with MSA, doped with $\mathrm{Zn}(\mathrm{Ac})_{2}$ $\left(\mathrm{H}_{2} \mathrm{O}\right)$ and soaked in $10 \mathrm{~mL} 1.875 \cdot 10^{-3} \mathrm{M}$ 8-HQ EtOH solution for 3 days at $50{ }^{\circ} \mathrm{C}$. Photographs taken under ambient light $(A)$ and $U V$-light $\left(B ; \lambda_{\text {exc }}=366 \mathrm{~nm}\right)$. 


\subsubsection{Uptake of 8-HQ onto zinc doped wool in a gas atmosphere}

Zinc doped wool fibre was also functionalised with the uptake of 8-HQ in gas phase (see method described in 7.2.2.3.4). Depending on the zinc precursor material used for the doping of the wool fibre, different fluorescence colours and emission wavelength are obtained (see figure 7.23).

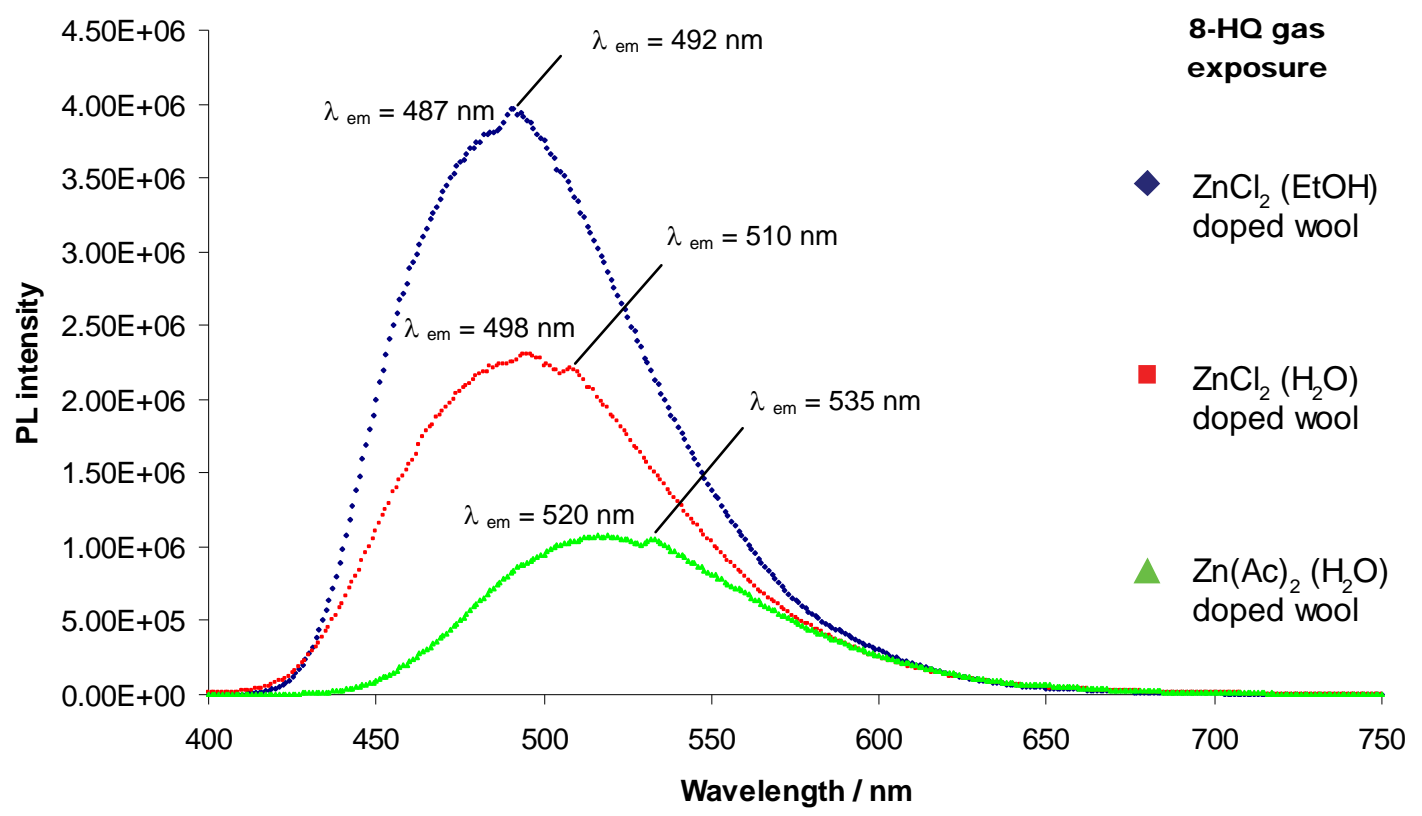

Figure 7.23: $\mathrm{PL}$ scan of wool doped with $\mathrm{ZnCl}_{2}(\mathrm{EtOH})(\mathrm{A}), \mathrm{ZnCl}_{2}\left(\mathrm{H}_{2} \mathrm{O}\right)(\mathrm{B})$ and $\mathrm{Zn}(\mathrm{Ac})_{2}\left(\mathrm{H}_{2} \mathrm{O}\right)(\mathrm{C})$ and exposed to 8-HQ (EtOH) gas for 3 days at $50{ }^{\circ} \mathrm{C}\left(\lambda_{\text {exc }}=260 \mathrm{~nm}\right.$; exciting $/$ emission slit width $=$ $5 \mathrm{~nm}$ ). Details of the $\mathrm{Zn}$-doped wool fibres are listed in the appendix in table A.22.

In general, the fluorescence colour can be controlled by the exposure time of the zinc doped wool to the 8-HQ gas atmosphere. Initially, the wool show a bright white fluorescence, which turns to a yellow fluorescence with prolonged exposure time to 8HQ gas. Wool fibre composite doped with $\mathrm{ZnCl}_{2}\left(\mathrm{H}_{2} \mathrm{O}\right)$ and $\mathrm{Zn}(\mathrm{Ac})_{2}\left(\mathrm{H}_{2} \mathrm{O}\right)$ showed stronger a colour change to green-yellow than $\mathrm{ZnCl}_{2}(\mathrm{EtOH})$ doped wool. The change of the fluorescence colour is also noticeable under ambient light by the yellow colour of the wool fibre composite exhibits. Interestingly, a number of zinc doped wool fibres exposed in 8-HQ gas reveal a second distinctive peak besides the maximum emission peak in their PL scan (indicated in figure 7.23).

In comparison to wool fibre composites dyed in dyeing bath condition according to the dyeing method 1 or 2, zinc doped wool exposed in 8-HQ gas do not show a fluorescence peak below $425 \mathrm{~nm}$. Figure 7.24 illustrates the difference in the PL scans 
of wool fibre composites with a maximum emission wavelength at $490 \mathrm{~nm}$ dyed by the different in-situ methods; soaking 8-HQ - treated wool in $\mathrm{ZnCl}_{2}$ (EtOH) solution (dyeing method 1), soaking zinc doped wool in 8-HQ (EtOH) solution (dyeing method 2) and exposing zinc doped wool in 8-HQ gas atmosphere at 50 and $80{ }^{\circ} \mathrm{C}$ (dyeing method 3), respectively.

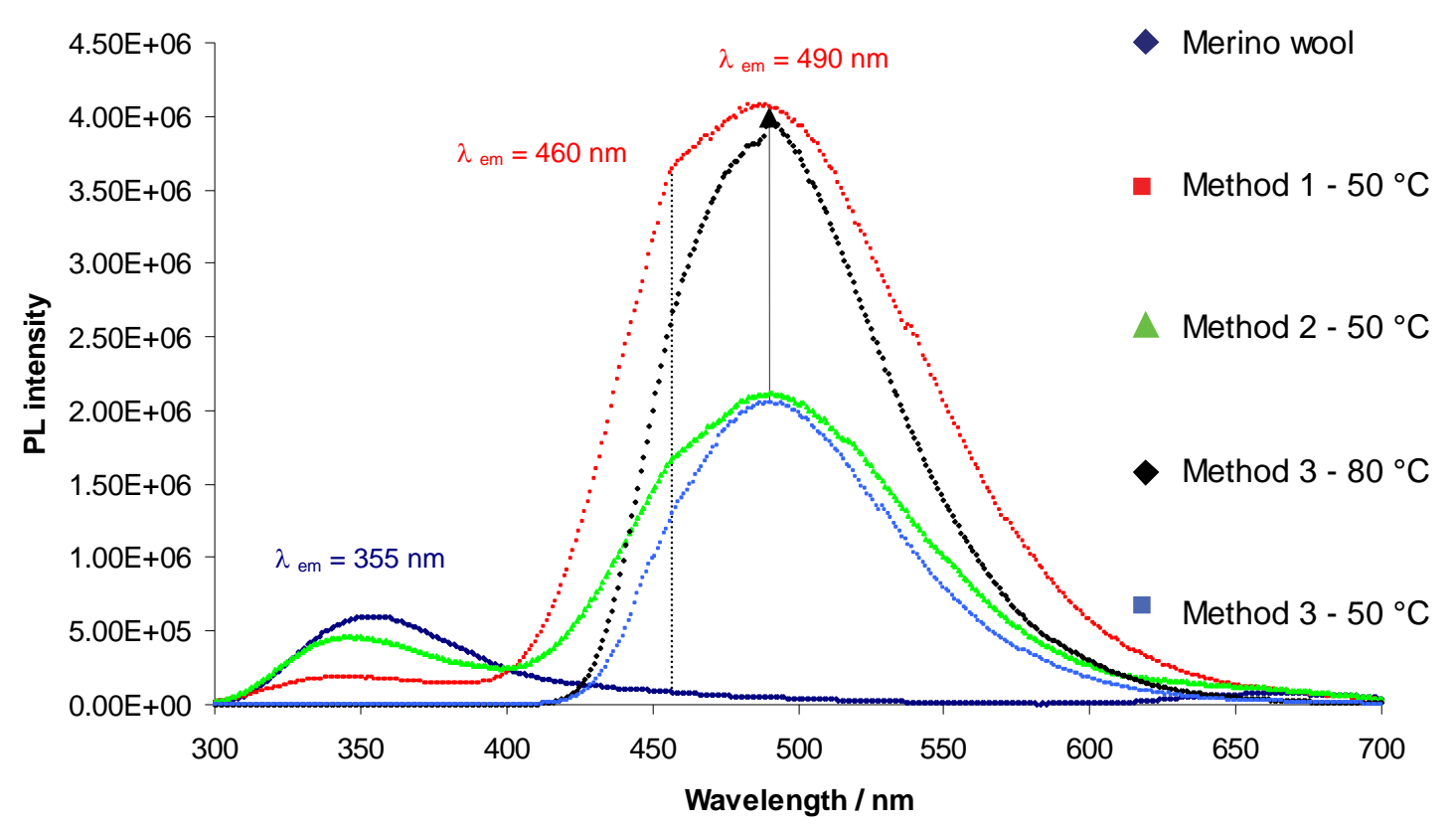

Figure 7.24: PL scans of wool fibre composites dyed using three methods. Method 1: $0.1 \mathrm{~g}$ wool treated with 8-HQ (EtOH) $\left([8-\mathrm{HQ}]=2.5 \cdot 10^{-4} \mathrm{M}\right)$ in a total volume of $10 \mathrm{~mL}$ EtOH and then soaked in $10 \mathrm{~mL} 0.01 \mathrm{M} \mathrm{ZnCl}_{2}(\mathrm{EtOH})$ for 3 days at $50{ }^{\circ} \mathrm{C}$ (sample details are listed in the appendix in table A.21; wt(8-HQ) = $0.36 \%$ ). Method 2: $0.1 \mathrm{~g}$ wool doped with $\mathrm{ZnCl}_{2}(\mathrm{EtOH})$ and then soaked in $10 \mathrm{~mL}$ 8-HQ $(\mathrm{EtOH})$ solution $\left([8-\mathrm{HQ}]=1.25 \cdot 10^{-4} \mathrm{M}\right)$ (sample details are listed in the appendix in table A.23; wt(8-HQ) = $0.18 \%$ ). Method 3: $0.1 \mathrm{~g}$ wool doped with $\mathrm{ZnCl}_{2}(\mathrm{EtOH})$ and exposed in 8-HQ gas for 1 day at 50 (blue line) and $80{ }^{\circ} \mathrm{C}$ (green line) (procedure for 8-HQ gas uptake is described in chapter 7; 7.2.2.3.5); ( $\lambda_{\text {exc }}=260 \mathrm{~nm}$; exciting $/$ emission slit width $\left.=5 \mathrm{~nm}\right)$.

The PL scans in figure 7.24 were measured with an excitation wavelength of $\lambda_{\text {exc }}=260$ $\mathrm{nm}$. It shows that the emission peaks caused by the uptake of 8-HQ in gas phase are narrower than the emission peaks observed in wool fibre doped with $\mathrm{ZnCl}_{2}(\mathrm{EtOH})$ and soaked in 8-HQ $(\mathrm{EtOH})$ solutions $\left([8-\mathrm{HQ}]=1.25 \cdot 10^{-4} \mathrm{M}\right)$, and wool treated with 8-HQ $(\mathrm{wt}(8-\mathrm{HQ})=8.3 \%)$ and soaked in $\mathrm{ZnCl}_{2}(\mathrm{EtOH})$ solution. This difference is caused by the absence of the peak at $460 \mathrm{~nm}$ observed in wool fibre composites dyed in $\mathrm{EtOH}$ solution.

Zinc doped wool obtain higher PL intensities when exposed to 8-HQ gas atmosphere at higher temperature (figure 7.24). In general, prolonged exposure in 8-HQ gas leads to 
the increase and broadening of the emission peak. The extent of shift to longer wavelength, however, is strongly dependent on the 8-HQ concentration in the gas atmosphere. The increment of 8-HQ in the gas atmosphere was easily achieved by adding more 8-HQ treated wool to the sealed glass tube with the $\mathrm{ZnCl}_{2}(\mathrm{EtOH})$ doped wool. By doing so, the fluorescence of the zinc doped wool was tuned from 490 to 520 $\mathrm{nm}$ (see figure 7.25).

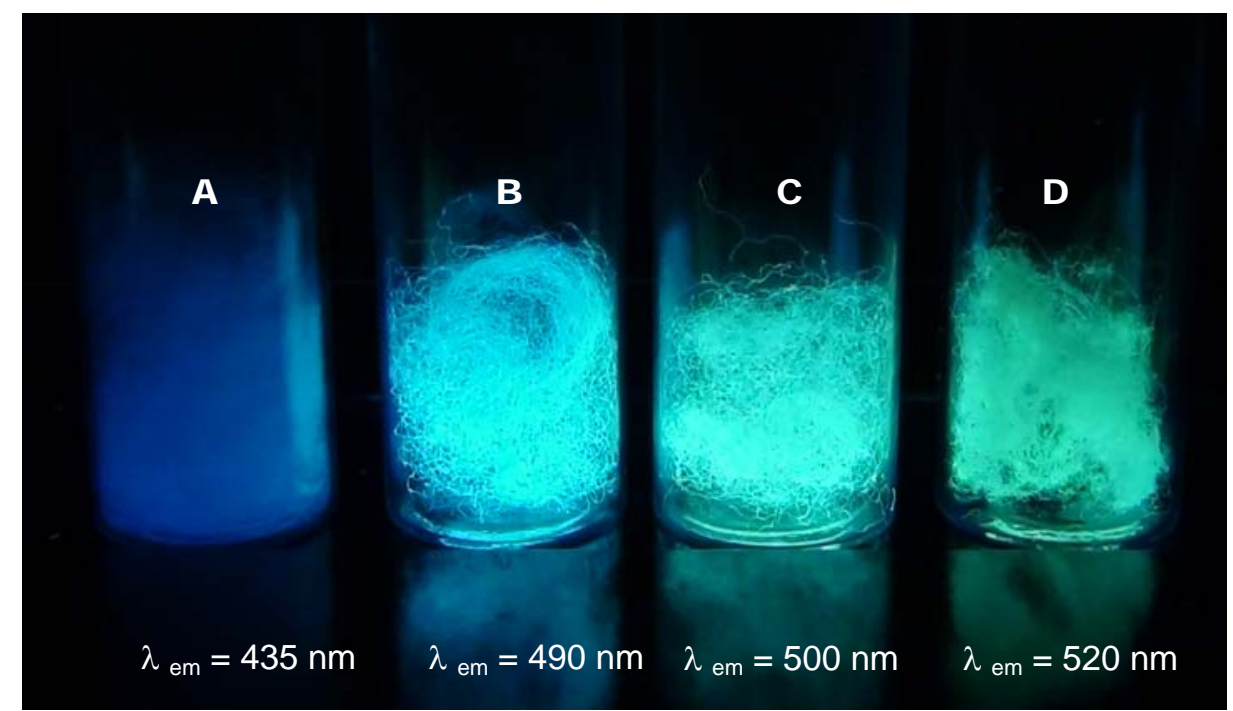

Figure 7.25: Fluorescence of merino wool (A) and wool doped with $\mathrm{ZnCl}_{2}(\mathrm{EtOH})$ and exposed to 8$\mathrm{HQ}$ gas at $80^{\circ} \mathrm{C}$ for 1 day (B), 2 days (C) and 3 days (D) $\left(\lambda_{\text {exc }}=366 \mathrm{~nm}\right)$.

It is important to note, that wool fibre treated with MSA and then soaked in $\mathrm{ZnCl}_{2}$ $(\mathrm{EtOH})$ solution showed the highest PL intensities after being exposed in 8-HQ gas.

\subsubsection{Process parameters of in-situ dyeing method 2}

\subsubsection{Choice of zinc precursor material; $\mathrm{ZnCl}_{2}$ and $\mathrm{Zn}(\mathrm{Ac})_{2}$}

As mentioned before (7.3.3.4), when the zinc uptake was carried out onto MSA treated wool, $\mathrm{Zn}(\mathrm{Ac})_{2}$ doped wool obtain similar emission maximum wavelength as $\mathrm{ZnCl}_{2}$ (EtOH) doped wool. However, both composite materials are still distinguishable by their distinctive colour after being soaked in 8-HQ EtOH solution. Figure 7.26 shows the wool fibre composites of $\mathrm{ZnCl}_{2}(\mathrm{EtOH})$ doped wool (below) and $\mathrm{Zn}(\mathrm{Ac})_{2}\left(\mathrm{H}_{2} \mathrm{O}\right)$ doped wool with MSA pre-treatment (above) after soaking in $10 \mathrm{~mL} 1.875 \cdot 10^{-3} \mathrm{M} 8$ HQ EtOH solution $(w t(8-H Q)=2.7 \%)$. Both composite materials show an emission maximum emission wavelength around $505 \mathrm{~nm}$ (see figure 7.20; D and G). 


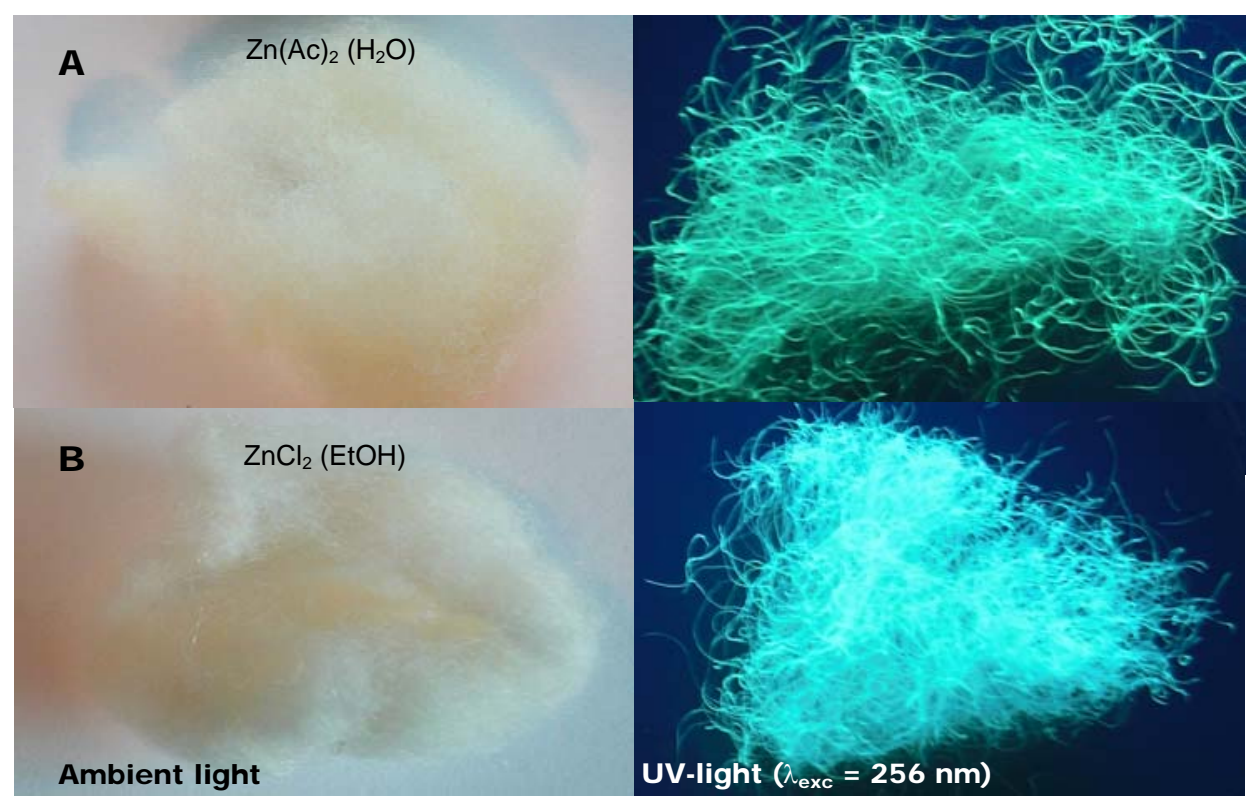

Figure 7.26: Wool pre-treated with MSA and doped with $\mathrm{Zn}(\mathrm{Ac})_{2}\left(\mathrm{H}_{2} \mathrm{O}\right)(\mathrm{A}, \mathrm{B})$ and $\mathrm{ZnCl}_{2}(\mathrm{EtOH})$ doped wool (C, D) soaked in $1.875 \cdot 10^{-3} \mathrm{M}$ 8-HQ EtOH solution at $50{ }^{\circ} \mathrm{C}$ for 3 days. Left: ambient light. Right: UV-light $\left(\lambda_{\text {exc }}=256 \mathrm{~nm}\right)$. Samples shown in A and B are listed in the appendix in table A.25; $D$ and $G$, respectively.

Although both composite materials have the same maximum emission wavelength, they show different fluorescence colour under UV light and ambient light. $\mathrm{Zn}(\mathrm{Ac})_{2}$ doped wool and soaked in 8-HQ EtOH have a characteristic yellowish colour under ambient and a green-yellowish colour under UV-light $\left(\lambda_{\text {exc }}=256 \mathrm{~nm}\right)$, whereas $\mathrm{ZnCl}_{2}$ doped wool and soaked in 8-HQ EtOH is white under ambient light and show a bright white colour under UV-light (see figure 7.26).

When comparing the PL scans of both composite materials (figure 7.20; D and G); it can be observed that the emission peak at $460 \mathrm{~nm}$, present in $\mathrm{ZnCl}_{2}(\mathrm{EtOH})$ doped wool, is missing from $\mathrm{Zn}(\mathrm{Ac})_{2} \mathrm{H}\left({ }_{2} \mathrm{O}\right)$ doped wool. In addition, the PL intensity of $\mathrm{ZnCl}_{2}$ $(\mathrm{EtOH})$ doped wool is higher than of $\mathrm{Zn}(\mathrm{Ac})_{2}\left(\mathrm{H}_{2} \mathrm{O}\right)$ doped wool.

A stronger difference between both composite materials, however, can be seen in their absorbance sans. Figure 7.27 shows the absorbance scans of $\mathrm{ZnCl}_{2}\left(\mathrm{H}_{2} \mathrm{O}\right)$ and $\mathrm{Zn}(\mathrm{Ac})_{2}$ $\left(\mathrm{H}_{2} \mathrm{O}\right)$ doped wool soaked in $10 \mathrm{~mL} 1.25 \cdot 10^{-3}$ and $1.875 \cdot 10^{-3} \mathrm{M} 8-\mathrm{HQ}$ EtOH solution. 


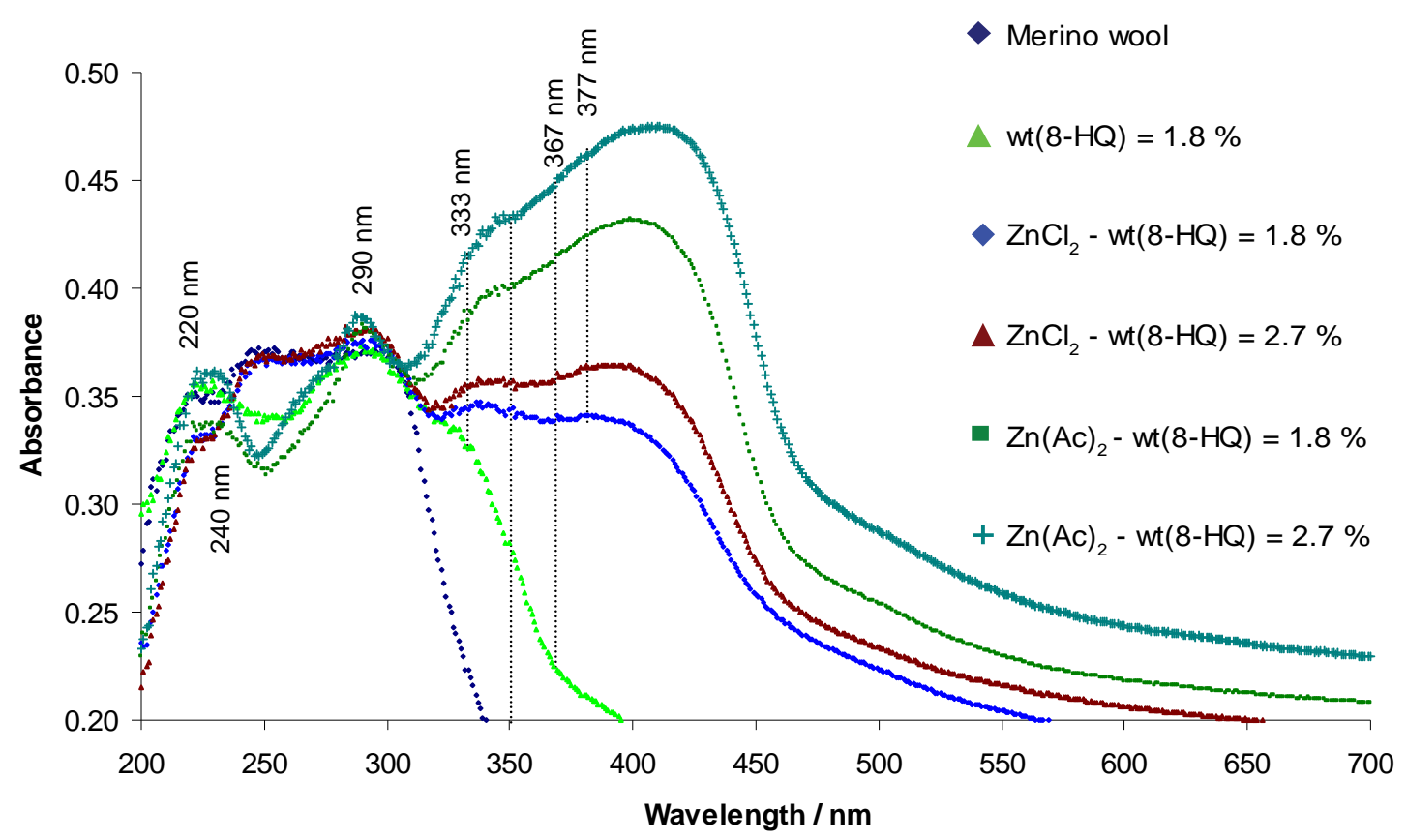

Figure 7.27: Absorbance scan of wool fibre composites functionalised with $\mathrm{ZnCl}_{2}\left(\mathrm{H}_{2} \mathrm{O}\right)$ and $\mathrm{Zn}(\mathrm{Ac})_{2}$ $\left(\mathrm{H}_{2} \mathrm{O}\right)$ and soaked in 8-HQ EtOH solution $\left(\left([8-\mathrm{HQ}]=1.25 \cdot 10^{-4} \mathrm{M}\right.\right.$ and $\left.1.875 \cdot 10^{-4} \mathrm{M}\right)$. Sample details of $\mathrm{wt}(8-\mathrm{HQ})=1.8 \%, \mathrm{ZnCl}_{2}-\mathrm{wt}(8-\mathrm{HQ})=1.8$ and $2.7 \%$ are listed in appendix, table A.19 and $A .24$, respectively. Sample details of $\mathrm{Zn}(\mathrm{Ac})_{2}-\mathrm{wt}(8-\mathrm{HQ})=1.8$ and $2.7 \%$ are listed in table A.25; $A$ and $C$, respectively.

Figure 7.27 shows that a broad absorbance peak between 305 and $475 \mathrm{~nm}$ is formed by the in-situ complex formation of zinc with 8-HQ in EtOH soaking solution. The broad absorbance peak is principally made up of two peaks, which are overlapping. The first peak is located between 305 and $370 \mathrm{~nm}$ and show a maximum absorbance at $350 \mathrm{~nm}$ for $\mathrm{Zn}(\mathrm{Ac})_{2}$ doped wool. The second peak is located between 350 and $475 \mathrm{~nm}$. Here the absorbance peak shifts between 380 and $410 \mathrm{~nm}$ depending on the zinc precursor material and 8-HQ concentration used. The shift to the right hand side of the spectrum leads to the increase and broadening of the absorbance peak. This figure also shows the absorbance scan of merino wool itself and soaked in $10 \mathrm{~mL} 1.25 \cdot 10^{-3} \mathrm{M}$ 8-HQ EtOH solution for 3 days at $50{ }^{\circ} \mathrm{C}$. Merino wool shows a broad absorbance peak between 200 and $350 \mathrm{~nm}$. When treated with $8-\mathrm{HQ}$, the broad absorbance peak of the wool transform into more distinct absorbance peak at 220, 240 and $290 \mathrm{~nm}$. These absorbance peaks are also found in all wool fibre composite materials prepared here. Wool fibre treated with 8-HQ also show a hump at $333 \mathrm{~nm}$.

The absorbance peaks of $\mathrm{Zn}(\mathrm{Ac})_{2}$ doped wool composites are higher and broader than $\mathrm{ZnCl}_{2}$ doped wool composites under constant dyeing bath conditions (figure 7.27). It is 
important to point out that with the increase of the absorbance peak, the colour of the wool turns yellow. Here, the height of the absorbance peaks is a good indicator of the wool fibre colour. Wool fibre composites with a strong absorbance peak between 305 and $475 \mathrm{~nm}\left(\right.$ see $\mathrm{Zn}(\mathrm{Ac})_{2}-\mathrm{wt}(8-\mathrm{HQ})=1.8$ and $2.7 \%$ in figure 7.27$)$ show an intense yellow colour under ambient and UV-light. In contrary, wool fibre composites doped with $\mathrm{ZnCl}_{2}$ show smaller and narrower absorbance peaks in the same wavelength range. The result is a white colour of the wool fibre composite under ambient and UV-light.

It is important to point out that a gap or transition area between 350 and $367 \mathrm{~nm}$ is observed when $\mathrm{ZnCl}_{2}$ doped wool was soaked in a solution of low 8-HQ EtOH concentration. A gap with the same features is observed in the corresponding PL scan. In the PL scan the gap starts at $460 \mathrm{~nm}$ and ends at $467 \mathrm{~nm}$. These emission and absorbance wavelengths are unmistakeable linked to each other and show a constant wavelength differences of $110 \mathrm{~nm}$. Furthermore, this feature was observed in all wool fibre composites studied. The wavelength difference also agrees with the Stoke shift of the second absorbance peak and the maximum emission peak of the wool fibre composites (figure 7.28).

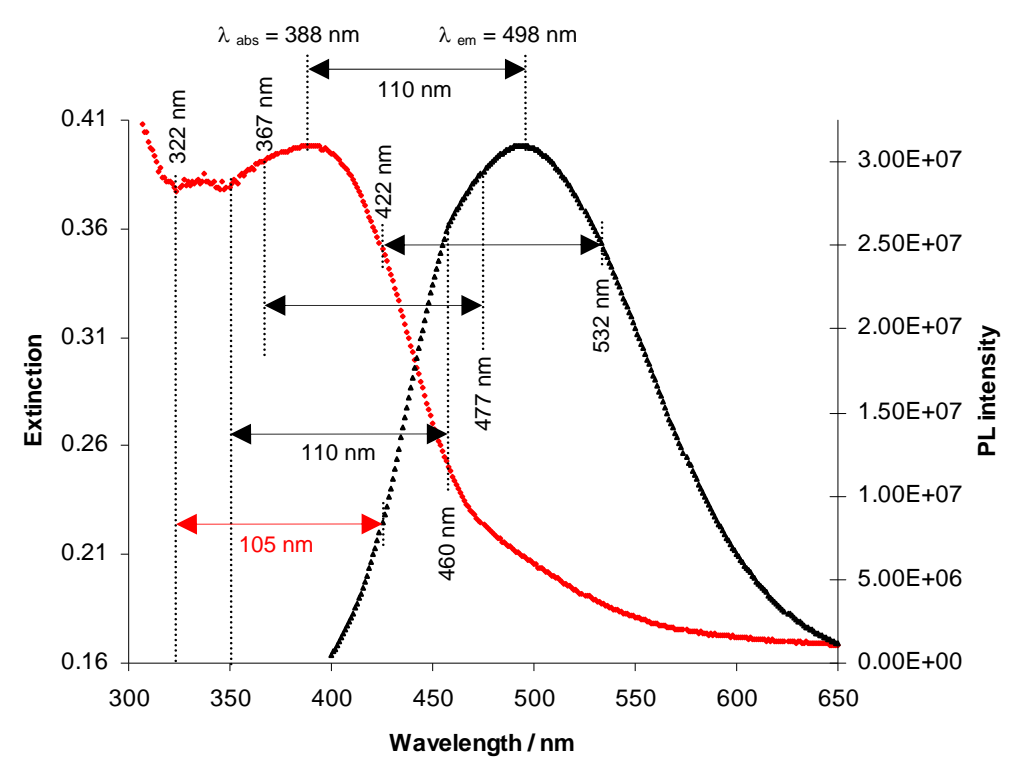

Figure 7.28: Stoke shift of $\mathrm{ZnCl}_{2}$ (EtOH) doped wool soaked in $1.25 \cdot 10^{-3} \mathrm{M}$ 8-HQ (EtOH) (wt(8$\mathrm{HQ})=1.8 \%$ ) at $50{ }^{\circ} \mathrm{C}$ for 3 days. Red: Absorbances scan. Black: PL scan. Details of sample preparation are listed in the appendix in table A.23; wt(8-HQ) = $1.8 \%$.

Interestingly, the hump at $460 \mathrm{~nm}$ in the PL scan is strongly affected by the absorbance peak between 305 and $370 \mathrm{~nm}$. As long an absorbance peak with a maximum absorbance below $350 \mathrm{~nm}$ is shown, the hump at $460 \mathrm{~nm}$ in the PL scans is visible. 
However, with the increase of the 8-HQ concentration, the absorbance peak shifts toward $350 \mathrm{~nm}$. By doing so, the broad emission peak becomes gradually narrower and the hump at $460 \mathrm{~nm}$ disappears.

\subsubsection{Effect of $\mathrm{H}_{2} \mathrm{O}$ added to 8-HQ (EtOH) soaking solution}

The addition of water to the 8-HQ EtOH soaking solution containing zinc doped wool initiates a precipitation reaction of the organometallic chelate complex onto the composite material. Small particles are formed around the wool fibre surface and come easily off by ultrasound treatment or rubbing. Although a large number of crystals lose their adhesion to the wool fibre surface, some particles remain attached to the wool and functionalise the wool fibre with optical fluorescence. The extent of the precipitation reaction is dependent on the amount of water added to the 8-HQ EtOH solution $\left(\mathrm{V}\left(\mathrm{H}_{2} \mathrm{O}\right)=1-5 \mathrm{~mL}\right)$ and soaking temperature $\left(50-80^{\circ} \mathrm{C}\right)$.

Figure 7.29 shows the effect of the addition of $5 \mathrm{~mL}$ water to $10 \mathrm{~mL} 2.5 \cdot 10^{-3} \mathrm{M} 8-\mathrm{HQ}$ EtOH solution containing $0.1 \mathrm{~g} \mathrm{ZnCl}_{2}\left(\mathrm{H}_{2} \mathrm{O}\right) / \mathrm{Zn}(\mathrm{Ac})_{2}\left(\mathrm{H}_{2} \mathrm{O}\right)$ doped wool. Here, 2 × 2.5 $\mathrm{mL} \mathrm{H}_{2} \mathrm{O}$ water was added in a 2 hours time difference after the wool fibre was soaked two days inside the 8-HQ EtOH solution at $50{ }^{\circ} \mathrm{C}$.

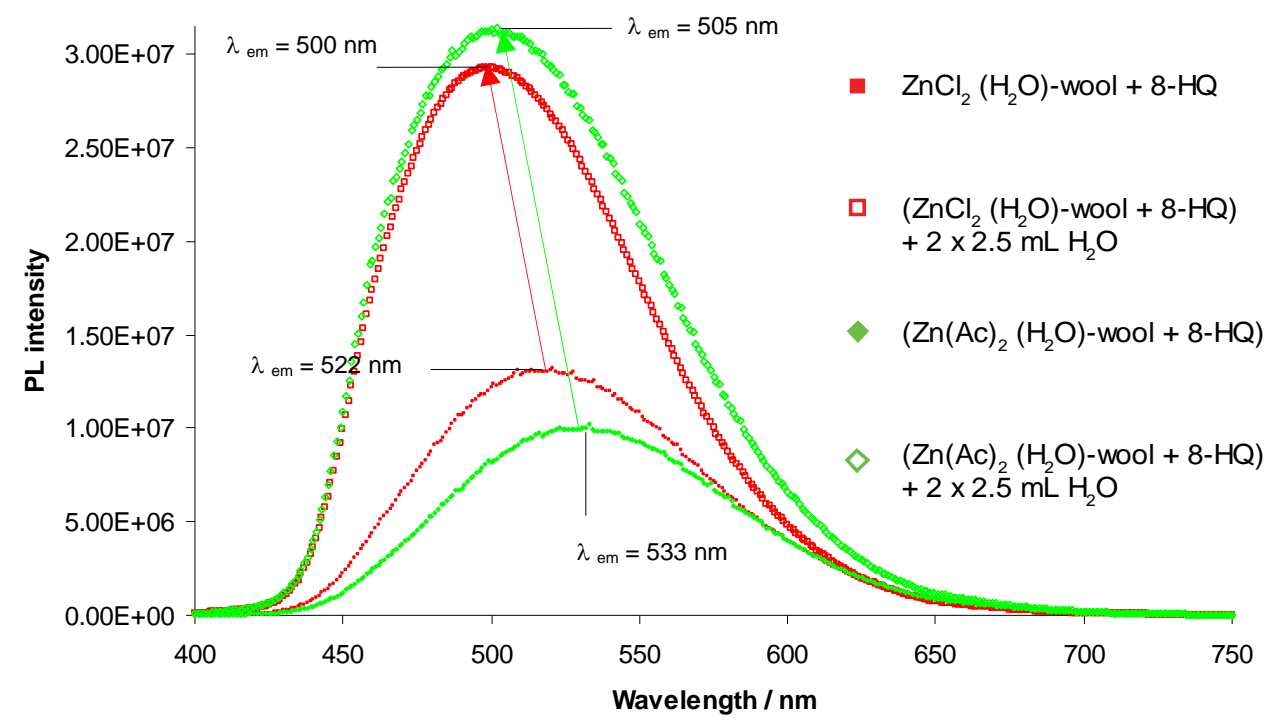

Figure 7.29: Blue shift of the emission wavelength after adding water $\left(2 \times 2.5 \mathrm{~mL} \mathrm{H}_{2} \mathrm{O}\right.$ in a 2 hrs time difference) to $10 \mathrm{~mL} 0.0025 \mathrm{M}$ 8-HQ EtOH solution containing wool doped with $\mathrm{ZnCl}_{2}\left(\mathrm{H}_{2} \mathrm{O}\right)$ (solid red square before, open red square after) and $\mathrm{Zn}(\mathrm{Ac})_{2}\left(\mathrm{H}_{2} \mathrm{O}\right)$ ((solid green rhomboid before, open green rhomboid after $)\left(\lambda_{\text {exc }}=350 \mathrm{~nm}\right.$; exciting $/$ emission slit width $\left.=3 \mathrm{~nm}\right)$. Sample details of $\mathrm{ZnCl}_{2}\left(\mathrm{H}_{2} \mathrm{O}\right)$ - wool are listed in the appendix in table A.24; wt(8-HQ) $=3.5 \%$. Sample details of $\mathrm{Zn}(\mathrm{Ac})_{2}\left(\mathrm{H}_{2} \mathrm{O}\right)$ - wool are listed in table A.25; $\mathrm{E}$. 
The addition of $2 \times 2.5 \mathrm{~mL} \mathrm{H}_{2} \mathrm{O}$ water to the soaking solution with a two hours time difference was undertaken to avoid an instant precipitation reaction of the chelate inside and around the wool. The addition of water resulted in a blue shift of the emission wavelength. It was found that the fluorescence properties of the particles formed by the addition of water show higher PL intensities when the soaking temperature was increased from 50 to $80{ }^{\circ} \mathrm{C}$ before water was added. Thereby, $\mathrm{Zn}(\mathrm{Ac})_{2}$ doped wool obtained a bright yellow greenish fluorescence colour, whereas $\mathrm{ZnCl}_{2}$ doped wool showed a bright white colour after the addition of water.

Independent on the zinc precursor material, the addition of water to 8-HQ soaking solution containing zinc doped wool results in the formation of fluorescent particles around the wool fibre. Here, the fluorescence of the crystals formed around the wool fibre show a maximum wavelength around $505 \mathrm{~nm}$. Interestingly, the particles do not undergo a further colour change with ageing and show in general higher PL intensities than the chelate itself formed by the in-situ reaction inside the wool. The solid precipitate is also easy to re-disperse, when stored in aqueous solution. Figure 7.30 show the fluorescence of particles formed after adding $5 \mathrm{~mL}$ water to $10 \mathrm{~mL} 2.5 \cdot 10^{-3} \mathrm{M}$ 8-HQ EtOH soaking solution containing $\mathrm{ZnCl}_{2}(\mathrm{EtOH})$ doped wool. The fluorescence of the solid precipitate has an emission wavelength around $505 \mathrm{~nm}$ and remains bright after ageing in air and water.

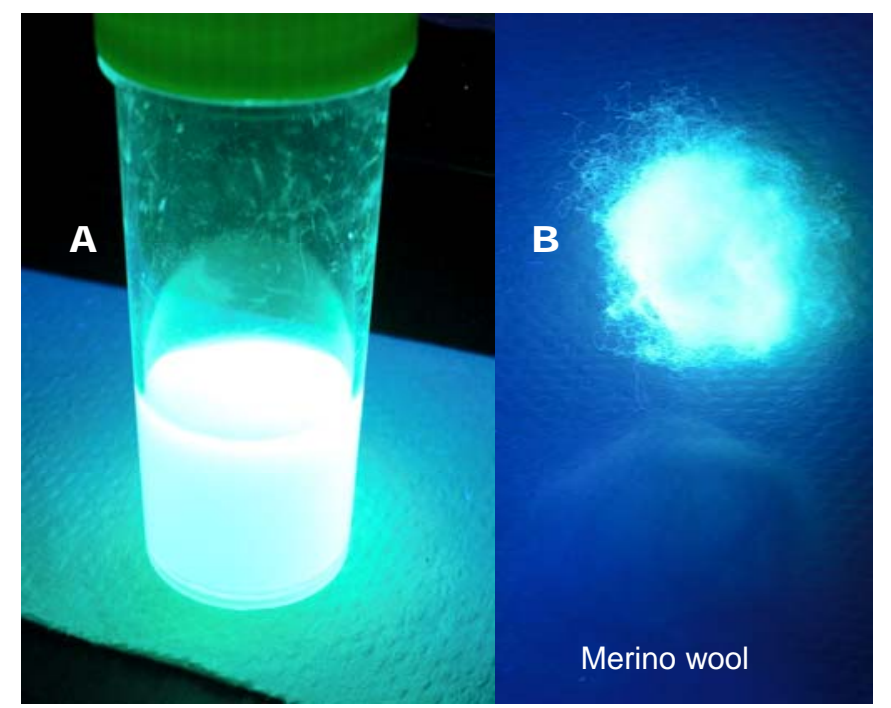

Figure 7.30: Aqueous suspension of $\mathrm{Zn}(8-\mathrm{Q})_{2}$ particles (A) and hydrolysed $\mathrm{Zn}(8-\mathrm{Q})_{2}$ particles around wool (B) under $U V$-light $\left(\lambda_{\text {exc }}=366 \mathrm{~nm}\right)$. Bright fluorescent particles were formed around the wool fibre, when $2 \times 2.5 \mathrm{~mL}$ water were added to $10 \mathrm{~mL} 2.5 \cdot 10^{-3}$ M 8-HQ (EtOH) soaking solution containing $\mathrm{ZnCl}_{2}(\mathrm{EtOH})$ wool fibre at $50{ }^{\circ} \mathrm{C}$. The aqueous suspension of $\mathrm{Zn}(8-\mathrm{Q})_{2}$ was obtained by removing the particles around the wool using ultrasound. 
A typical characteristic of these particles dispersed in solution (figure 7.30) is the absence of a hump at $460 \mathrm{~nm}$ in their PL scan. However, wool fibre composites can reveal a broad emission peak with a hump at $460 \mathrm{~nm}$ after the addition of water to the 8HQ EtOH soaking solution. This is regularly observed for $\mathrm{ZnCl}_{2}$ doped wool fibre composites soaked in lower 8-HQ $(\mathrm{EtOH})$ concentration $\left([8-\mathrm{HQ}]<2.5 \cdot 10^{-3} \mathrm{M}\right)$. Here, however, less fluorescent particles are also formed.

It was found out that pre-treatment procedures of the wool with $\mathrm{NaOH}$ and $\mathrm{HCl}$ do not influence the optical properties of the chelate inside or on the surface of the wool. However, particles formed after the addition of water remained stronger attached to the wool when pre-treated with $\mathrm{NaOH}$. The same examination was obtained, when wool fibre was treated with MSA as linker molecules. Here, fewer particles came off the surface of the wool and the wool fibre composite obtained higher PL intensities with a maximum emission wavelength between 500 and $505 \mathrm{~nm}$.

\subsubsection{Uptake of 8-HQ onto zinc doped wool in water solution}

The use of water as soaking solution for the uptake of 8-HQ onto zinc doped wool also results in the formation of fluorescent particles. Here the particles formed in the chemical surrounding of the wool also come off by ultrasound treatment or rubbing. Nevertheless, a large amount of the solid complex remains chemically attached onto the wool fibre and functionalises it with optical fluorescence. Here, it is important to point out that 8-HQ aggregate in water due to its aromatic molecular structure. However, small quantities of 8-HQ $(\mathrm{EtOH})$ can still be diluted in aqueous solutions without causing a crystallisation reaction as long as the concentration is low ([8-HQ] $\leq 0.003$ $\mathrm{M})$.

For example, $\mathrm{Zn}(\mathrm{Ac})_{2}$ doped wool $\left(\mathrm{wt}\left(\mathrm{Zn}(\mathrm{Ac})_{2}\right) \sim 0.6 \%\right)$ show a bright white greenish fluorescence after being soaked in $10 \mathrm{~mL}$ water with $0.5 \mathrm{~mL} \mathrm{0.025} \mathrm{M} \mathrm{8-HQ} \mathrm{(EtOH)} \mathrm{for}$ two days at $70{ }^{\circ} \mathrm{C}$. The wool was covered by small particles showing an emission wavelength around $500 \mathrm{~nm}$. The PL scan of the wool fibre is identical to the PL scan of $\mathrm{ZnCl}_{2}(\mathrm{EtOH})$ doped wool soaked in $10 \mathrm{~mL} 1.25 \cdot 10^{-3} \mathrm{M} 8$-HQ EtOH solution only with lower PL intensity (see figure 7.15; wt(8-HQ) $=1.8 \%$ ). The addition of $1 \mathrm{~mL}$ $0.025 \mathrm{M} 8$-HQ (EtOH) to the $10 \mathrm{~mL}$ aqueous soaking solution resulted in emission 
wavelength around $505 \mathrm{~nm}$. Thereby, the fluorescence colour turns slightly more greenish. The emission wavelength of the wool composite material corresponds to the maximum emission wavelength of the solid chelate formed by the complex formation of zinc and 8-HQ in the presence of water (see figure 7.7). The maximum emission wavelength obtained in aqueous solution varies between $490-505 \mathrm{~nm}$ depending on the 8-HQ concentration, zinc precursor material and $\mathrm{pH}$ value of the soaking solution. Besides of the restriction of the maximum emission wavelength, the PL scans are not different from wool fibre composites dyed in 8-HQ EtOH solutions. However, bigger differences can be seen in their absorbance spectrum.

Figure 7.31 shows absorbance scans of wool fibre samples covered with the solid chelate. Depending on the dyeing procedure, different absorbance scans are obtained. Here, the curve (A) represents the absorbance scan of wool fibre soaked directly in $\mathrm{Zn}(8-\mathrm{Q})_{2}\left(\mathrm{H}_{2} \mathrm{O}\right)$ suspension for 7 days at $70^{\circ} \mathrm{C}$. The curve (B) represent the absorbance scan of $\mathrm{Zn}(\mathrm{Ac})_{2}$ doped wool soaked in 8-HQ aqueous solution for two days at $70{ }^{\circ} \mathrm{C}$ as described above. The curve $(\mathrm{C})$ represents the absorbance scan of $\mathrm{Zn}(\mathrm{Ac})_{2}$ doped wool after the uptake of 8-HQ in EtOH solution with the addition of $5 \mathrm{~mL} \mathrm{H}_{2} \mathrm{O}$ after being soaked for two days at $50{ }^{\circ} \mathrm{C}$ as described in the previous section (1.3.3.2.5).

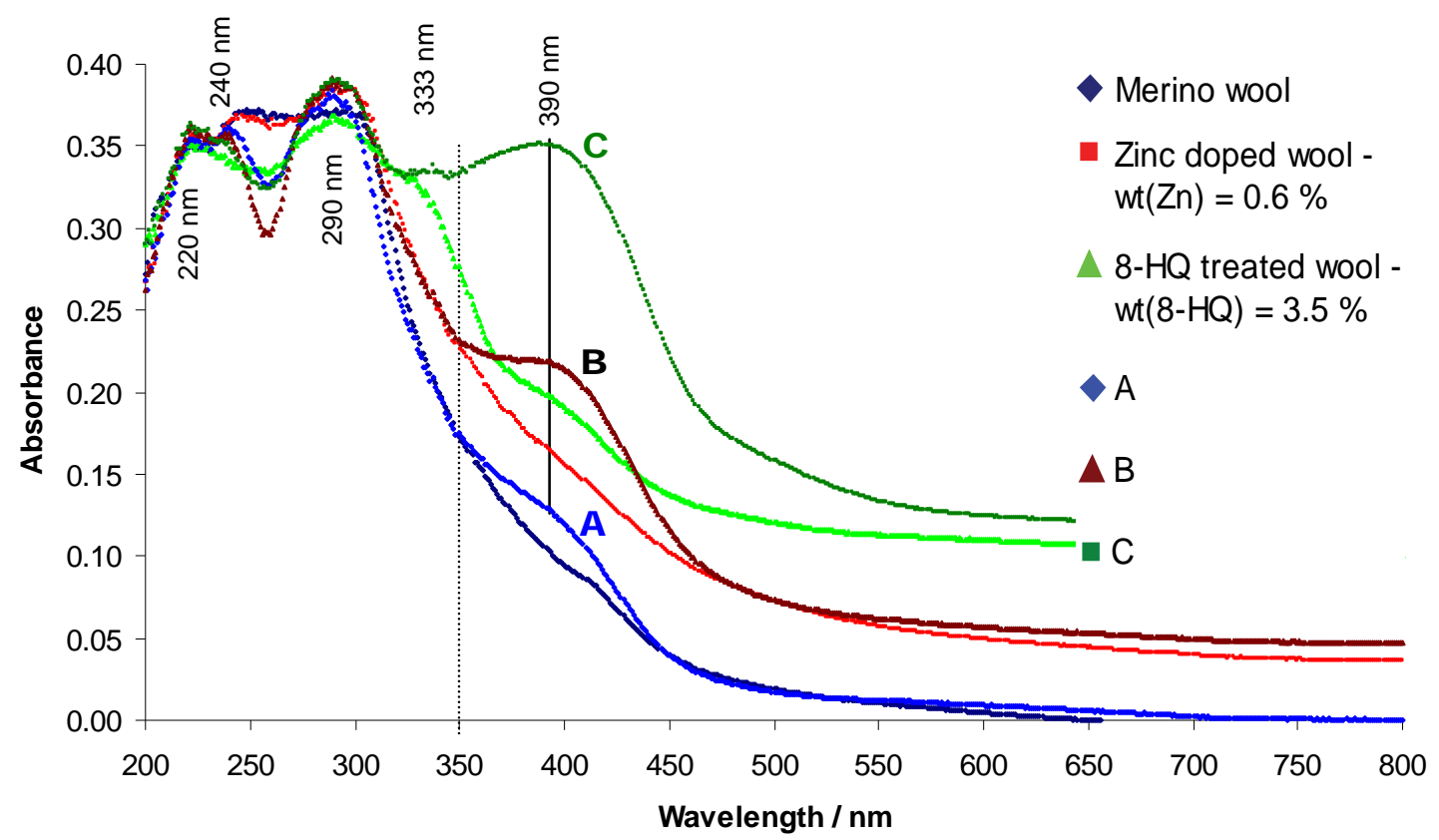

Figure 7.31: Absorbance scans of wool fibre composites dyed with zinc and 8-HQ in the presence of water. A: Wool soaked in aqueous suspension of $\mathrm{Zn}(8-\mathrm{Q})_{2}\left(\mathrm{H}_{2} \mathrm{O}\right)$ ( see table $\mathrm{A.18}$ in appendix). B: Wool soaked in $\mathrm{Zn}^{2+}$ aqueous suspension and followed by the addition of 8-HQ (EtOH). C: Wool doped with zinc and soaked in 8-HQ EtOH solution (see sample $\mathrm{E}$ in the appendix in table A.25) followed by the addition of $\mathrm{H}_{2} \mathrm{O}$. 
The soaking of wool fibre into $\mathrm{Zn}(8-\mathrm{Q})_{2}$ aqueous suspension (figure 7.31 ; curve A) show a similar absorbance scan like wool fibre itself, except a small hump between 360 and $450 \mathrm{~nm}$ appears. The uptake of 8-HQ onto $\mathrm{Zn}(\mathrm{Ac})_{2}$ doped wool in aqueous solution (figure 7.31; curve B) also match with the absorbance scan of $\mathrm{Zn}(\mathrm{Ac})_{2}$ doped wool; however, a distinct peak centred at around $390 \mathrm{~nm}$ is observed. The absorbance curve (C) for $\mathrm{Zn}(\mathrm{Ac})_{2}$ doped wool soaked in 8-HQ EtOH solution with the addition of $\mathrm{H}_{2} \mathrm{O}$ is similar to the absorbance scans of the $\mathrm{ZnCl}_{2}\left(\mathrm{H}_{2} \mathrm{O}\right)$ doped wool soaked in 8-HQ EtOH solution without the addition of $\mathrm{H}_{2} \mathrm{O}$ (see figure $6.28 ; \mathrm{ZnCl}_{2}-\operatorname{wt}(8-\mathrm{HQ})=2.7 \%$ ). However, the absorbance peak between 305 and $475 \mathrm{~nm}$ is narrower and the maximum absorbance peak is centred at around $390 \mathrm{~nm}$.

Here, it is important to point out that wool fibre dyed in EtOH dyeing bath condition reveal always two absorbance peaks between 305 and $475 \mathrm{~nm}$, whereas in aqueous dyeing bath condition only one absorbance peak in the same wavelength range appears. As known, 8-HQ forms with zinc in aqueous solution an insoluble chelate. Figure 7.31 showed that all wool fibre composites covered with the chelate in condensed state have an absorbance peak centred at around $390 \mathrm{~nm}$. Furthermore, all wool fibre composites dyed with 8-HQ and zinc in the presence of water also shows distinct absorbance peaks at 220, 240 and $290 \mathrm{~nm}$. Interestingly, wool fibre composites dyed in aqueous dyeing bath condition do not undergo a further colour change with ageing. However, the PL intensity decreases which may be related to the fact that some of the solid chelate come off the wool fibre.

In contrary, wool fibre composites dyed in EtOH solution with the addition of $\mathrm{H}_{2} \mathrm{O}$ and showing two absorbance peaks between 305 and $475 \mathrm{~nm}$ are more likely affected by a colour change with ageing. This is in particular evident for $\mathrm{Zn}(\mathrm{Ac})_{2}$ doped wool composites dyed in 8-HQ EtOH solution and showing a yellow colour before and after the addition of water. With ageing, the colour of the wool gradually turned to a darker yellow. This colour change was accelerated when the wool fibre composite was soaked again in EtOH solution at $50{ }^{\circ} \mathrm{C}$. However, when soaked in $\mathrm{H}_{2} \mathrm{O}$ at $50{ }^{\circ} \mathrm{C}$, the wool fibre turned to a green colour. Interestingly, the maximum emission wavelength remained unchanged at $505 \mathrm{~nm}$, only the PL intensity decreased. It is also important to note that the PL intensity of the wool fibre composite soaked in EtOH solution was considerable lower than soaked in $\mathrm{H}_{2} \mathrm{O}$. 
The change of colour of the wool fibre composite was also more evident when exposed in sun light. In this case, the colour change to a darker yellow was not shown by a shift of the emission to longer wavelengths, but by a reduction of the PL intensity. It seems that the colour change is connected with the absorbance peak between 305 and $350 \mathrm{~nm}$. This peak is also found in wool fibre treated in 8-HQ EtOH solution, which appears as a distinctive hump at $333 \mathrm{~nm}$ (figure 7.31).

\subsection{Discussion}

The complexation of zinc with 8-HQ in solution and wool shows different PL scans depending on the exciting wavelength used. Zinc and 8-HQ can form the anhydrous chelate $\mathrm{Zn}(8-\mathrm{Q})_{2}$ and the dihydrate chelate $\mathrm{Zn}(8-\mathrm{Q})_{2} \cdot 2 \mathrm{H}_{2} \mathrm{O}$. In none-aqueous solutions, the dihydrate chelate can be further converted into its anhydrous tetramer structure $\left[\mathrm{Zn}(8-\mathrm{Q})_{2}\right]_{4}$. The fluorescence of each chelate structure is determined by the optical band gap between the LUMO and HOMO level. These energy levels, however, are strongly affected by the chemical interaction with the chemical surrounding such as solvent molecules and adjacent chelate structures. Furthermore, the fluorescence of the $\mathrm{Zn}(8-\mathrm{Q})_{2}$ chelate structures are highly affected by crystallisation and precipitation reactions.

\subsubsection{Preparation of $\mathrm{Zn}(8-\mathrm{Q})_{2}$ chelate solution}

The stability of the $\mathrm{Zn}(8-\mathrm{Q})_{2}$ chelate in solution is dependent on the chemical interaction with the solvent molecules. In general, nonpolar solvents stabilise molecular structures with a conjugated $\pi$ - electron system, whereas polar solvents have a destabilization effect.

\subsubsection{Preparation of $\mathrm{Zn}(8-\mathrm{Q})_{2}$ chelate EtOH solution}

The preparation of the $0.001 \mathrm{M} \mathrm{Zn}(8-\mathrm{Q})_{2} \mathrm{EtOH}$ solution using $\mathrm{ZnCl}_{2}(\mathrm{EtOH})$ and 8-HQ $(\mathrm{EtOH})$ as precursor materials, revealed emission peaks at $460 \mathrm{~nm}$ and between 490 and $520 \mathrm{~nm}$, depending on the excitation wavelength (see figure 7.2). For example, when excited with 315 and $330 \mathrm{~nm}$, a maximum emission peak at $460 \mathrm{~nm}$ was obtained. When excited with 350 and $370 \mathrm{~nm}$, emission wavelengths between 490 and $520 \mathrm{~nm}$ were observed. 
In general, the emission wavelength and PL intensity is determined by the molecular structure of the chelate and their chemical interaction with the surrounding such as solvent molecules and adjacent complexes. Here, the emission peaks observed in the PL scans can be attributed to the fluorescence of the dihydrate chelate. It is important to point out, that the complex formation was carried out in Drum EtOH consisting of about $95 \% \mathrm{EtOH}$ and $5 \% \mathrm{H}_{2} \mathrm{O}$.

The fluorescence in a $\mathrm{Zn}(8-\mathrm{Q})_{2}$ chelate is generated by the electronic transition between d-orbitals of the zinc atom (LUMO) and the $\pi$ - MO of 8-HQ (HOMO). Thereby, the geometrical structure of the chelate determines the splitting of the different d-orbitals of the zinc atom and hence the optical band gap between the HOMO and LUMO level of the chelate. The HOMO and LUMO level, however, is also influenced by the chemical interaction of the chelate with the surrounding (solvent molecules) and by intermolecular interaction between adjacent complex structures.

Here, the complex structure of the dihydrate chelate is planar, which promote intermolecular interactions such as dipole-dipole interaction ( $\pi$ - $\pi$ stacking) between the aromatic rings of 8-HQ. Intermolecular interaction usually results in spontaneous arrangement of the chelate in solution due to the attractive and repulsive forces acting on the aromatic ring of 8-HQ molecules. These forces, which can be classified as van der Waals forces, have a stabilization effect on the chelate in solution.

By forming self-assembled associations, however, energy can also be transferred between two adjacent chelates. There are different types of energy transfer mechanisms which can occur between two chelates. For example, intermolecular interaction such as $\pi-\pi$ stacking induced by dipole moments of adjacent aromatic rings of 8-HQ can promote the transfer of energy through a resonance effect. Energy can also be transferred by the formation of a complex state between an excited chelate and a chelate in the ground state (excimer).

The transfer of energy, between a chelate in excited state (donor) and a chelate in ground state (acceptor), usually results in the loss of energy for example via vibration motions. For that reason, weak associations between adjacent chelate structures have the 
potential to change the gap between the HOMO- and LUMO level by lowering the LUMO level or lifting the HOMO level. These changes are shown by a broad emission band at a wavelength longer than that of the chelate without intermolecular forces acting on it [128].

Hence, the emission peak at $460 \mathrm{~nm}$ is most likely attributed to the fluorescence of the monomer dihydrate chelate, whereas the emission shift between 490 and $520 \mathrm{~nm}$ is caused by intermolecular interaction between adjacent dihydrate chelates. The shift of the maximum emission wavelength is determined by the distance between adjacent chelates. For example, short distances between two chelates enhance intermolecular interaction and the formation of weak associations through $\pi$ - $\pi$ stacking or hydrogen bonding. Consequently, shorter distances between two chelates enhance the transfer of energy, which however will further lower the optical band gap of the chelate causing the shift of the maximum emission wavelength to longer wavelengths. Furthermore, shorter distances between adjacent chelates will progressively quench the fluorescence of the monomer chelate.

Hence, the fluorescence properties of the chelate can be tuned by controlling the intermolecular interactions in solution. In theory, intermolecular interaction between molecular structures can be disrupted by dilution, presence of impurities or high temperature. The latter usually breaks weak associations of molecules formed by intermolecular forces. The dilution of the chelate solution with $\mathrm{EtOH}$ will reduce the concentration and increase the distances between adjacent chelates causing a blue shift of the emission wavelength (see figure 7.7). The formation of crystals in solution over time will also reduce the initial concentration of the chelate. For that reason, the crystallisation of the chelate and settlement of these crystals in solution have a strong effect on the PL scans of the chelate solutions (see figure 7.3). For example, when excited with $330 \mathrm{~nm}$, the PL intensity of the emission peak at $460 \mathrm{~nm}$ and the hump at $490 \mathrm{~nm}$ increased after the settlement of the crystals in solution. But, when excited with $370 \mathrm{~nm}$, the PL scans of the chelate solution showed a strong blue shift of the maximum emission wavelength from 520 to $500 \mathrm{~nm}$ after the settlement of the crystals.

As mentioned before, the crystallisation of the chelate reduces the concentration of the chelate in solution and hence increases the distance between individual chelates in 
solution. Consequently, intermolecular interaction and energy transfer processes between adjacent chelates are hindered resulting in a blue shift of the maximum emission wavelength between 490 and $520 \mathrm{~nm}$ (see figure 7.3). Due to the larger distances between adjacent chelates, the PL intensity of the monomer fluorescence also increases shown by an increment of the emission peak at $460 \mathrm{~nm}$ (see figure 7.3).

Interestingly, the dilution of the complex solution with EtOH revealed a further change of the PL scans. The complex solution diluted in EtOH (1:3) revealed two emission peaks around $425 \mathrm{~nm}$ and $555 \mathrm{~nm}$, depending on the exciting wavelength (see figure 7.4). The different peaks observed with different excitation wavelength correspond to different emitting specimen formed by the complex formation of zinc and 8-HQ in EtOH solution. The presence of two distinct emitting species is also indicated by an isoemissive point at exactly $490 \mathrm{~nm}$ which is shown in figure 7.4. The first emission peak around $425-430 \mathrm{~nm}\left(\lambda_{\mathrm{exc}}=260-350 \mathrm{~nm}\right)$ may correspond to the emission peak of the anhydrous $\mathrm{Zn}(8-\mathrm{Q})_{2}$ chelate (see equation (41)). The emission peak at $555 \mathrm{~nm}$ may refer to a weak association of two anhydrous $\mathrm{Zn}(8-\mathrm{Q})_{2}$ chelates. A weak attraction between two anhydrous $\mathrm{Zn}(8-\mathrm{Q})_{2}$ chelates can be created by dipole-dipole interaction, hydrogen bonding or by the formation of excimer states (charge-transfer complexes).

\subsubsection{Preparation of $\mathrm{Zn}(8-\mathrm{Q})_{2}$ chelate aqueous solution}

In aqueous solution, zinc and 8-HQ forms an insoluble precipitate. The precipitation reaction of the chelate is due to the hydrophobic character of the aromatic ring structure of 8-HQ. By converting into a solid state, the chelate formed obtains a higher chemical stability in aqueous solution than complexed with water molecules. In the condensed phase, the lattice energy of the 8-HQ metal complex exceeds the energy which would be obtained by the solvation with water molecules. The conversion in the condensed phase, however, causes the precipitation of the chelate from solution.

Nevertheless, the precipitate shows bright optical fluorescence under UV-light. The fluorescence of the chelate formed in aqueous solution correspond to the dihydrate chelate, $\mathrm{Zn}(8-\mathrm{Q})_{2} \cdot 2 \mathrm{H}_{2} \mathrm{O}$. Normally, the fluorescence of the chelate in the condensed phase would be affected by quenching processes due to the different forces prevailing in 
the solid state. However, these particles show higher PL intensities than $\mathrm{Zn}(8-\mathrm{Q})_{2} \mathrm{EtOH}$ solutions.

The maximum emission wavelength of the chelate compound formed in aqueous solution is restricted between 490 and $505 \mathrm{~nm}\left(\lambda_{\text {exc }}=350 \mathrm{~nm}\right)$. For example, the combination of $\mathrm{ZnCl}_{2}\left(\mathrm{H}_{2} \mathrm{O}\right)$ and 8-HQ $(\mathrm{EtOH})$ in a ratio of 1:2 in aqueous solution $\left(\left[\mathrm{Zn}(8-\mathrm{Q})_{2}\right]=0.001 \mathrm{M}\right)$ result in an emission peak with a maximum emission wavelength around $490 \mathrm{~nm}$, when excited with $350 \mathrm{~nm}$ (see figure 7.7). By adding higher 8-HQ concentration to the zinc aqueous solution, the emission peak gradually increased and broadened. Thereby, the maximum emission wavelength shifted maximal towards $505 \mathrm{~nm}$. On the contrary, the dilution of the chelate aqueous suspension in EtOH resulted in a blue shift of the maximum emission wavelength and a distinct peak at $460 \mathrm{~nm}$ was observed. The blue shift is attributed to the larger distances between the dihydrate chelates obtained after dilution. Larger distances suppress intermolecular interaction and energy transfer processes between adjacent chelates. Furthermore, EtOH molecules stabilize the dihydrate chelate in solution through nonpolar interaction between the ethyl groups of EtOH and the aromatic ring structure of 8-HQ. Therefore, the agglomeration of the dihydrate chelate is suppressed in solution and the fluorescence of the monomer chelate at $460 \mathrm{~nm}$ is observed.

The shift of the maximum emission wavelength from 490 to $505 \mathrm{~nm}$ with the addition of higher 8-HQ concentration is connected with the $\mathrm{pH}$ value of the aqueous suspension. In general, the complex formation of $\mathrm{Zn}(8-\mathrm{Q})_{2}$ and hence optical fluorescence, is influenced by the $\mathrm{pH}$ value.

8-HQ is a bi-functional hydrogen-bonding compound, which act in aqueous and alcohol solution simultaneously as an $\mathrm{H}$-donor at the $\mathrm{OH}$ site (acid group) and an acceptor at the $\mathrm{N}$-atom (basic group). For this reason, it has acid and base properties. The zinc precursor material $\mathrm{ZnCl}_{2}\left(\mathrm{H}_{2} \mathrm{O}\right)$ is a Lewis acid which reduces the $\mathrm{pH}$ value of aqueous solutions. Furthermore, the complex formation of $\mathrm{ZnCl}_{2}\left(\mathrm{H}_{2} \mathrm{O}\right)$ and 8-HQ (EtOH) results in the formation of two $\mathrm{HCl}$ molecules as by-product (see equation (48)), which lowers further the $\mathrm{pH}$ value of the aqueous chelate solution. The formation of $\mathrm{HCl}$ as by-product is illustrated in the following equation. 


$$
\mathrm{ZnCl}_{2} \cdot 2 \mathrm{H}_{2} \mathrm{O}+28 \text { - } \mathrm{HQ}(\mathrm{EtOH}) \quad \rightleftarrows \quad \mathrm{Zn}(8-\mathrm{Q})_{2} \cdot 2 \mathrm{H}_{2} \mathrm{O}+2 \mathrm{HCl}
$$

Acid conditions, however, result in the protonation of the 8-HQ ligand, which prevent further complexation of zinc with 8-HQ (equation (49):

$$
28-\mathrm{HQ}(\mathrm{EtOH})+2 \mathrm{HCl} \rightleftarrows 2[(8-\mathrm{HQ}) \mathrm{H}]^{+}+2 \mathrm{Cl}^{-}
$$

The addition of an alkali agent or higher 8-HQ (EtOH) concentration to the suspension will prevent the $\mathrm{pH}$ decline by acting as an acceptor molecule for protons $\left(\mathrm{H}^{+}\right)$. By doing so, remaining zinc cations can react with 8-HQ and precipitate out as a chelate from aqueous solution. By forming solid particles, energy transfer processes are enhanced causing the shift of the maximum emission wavelength to longer wavelengths. For this reason, the emission shift towards $505 \mathrm{~nm}$ is always shown by an increased precipitation reaction of the chelate in solution.

The precipitation of the chelate may also be affected by ligand exchange reaction in aqueous solution. The exchange of one 8-Q ligand with a water molecule will result in the formation of reactive intermediate structures and free 8-HQ monomers as illustrated in the following equation (50) and (51).

$$
\begin{array}{ll}
{\left[\mathrm{Zn}(8-\mathrm{Q})_{2} \cdot 2 \mathrm{H}_{2} \mathrm{O}\right]+\mathrm{H}_{3} \mathrm{O}^{+}} & \rightleftarrows\left[\mathrm{Zn}(8-\mathrm{Q}) \cdot 3 \mathrm{H}_{2} \mathrm{O}\right]^{+}+8-\mathrm{QH} \\
{\left[\mathrm{Zn}(8-\mathrm{Q}) \cdot 3 \mathrm{H}_{2} \mathrm{O}\right]^{+}+8-\mathrm{QH}} & \rightleftharpoons\left[\mathrm{Zn}(8-\mathrm{Q})(\mathrm{OH}) \cdot 2 \mathrm{H}_{2} \mathrm{O}\right]+[(8-\mathrm{Q}) \mathrm{H}]^{+}
\end{array}
$$

This process is certainly dependent on the temperature and $\mathrm{pH}$ of the surrounding medium. As shown in equation (51), the formation of free 8-HQ molecules can cause the deprotonation of one water ligand of the chelate. This process, also called hydrolysis, may trigger a sol-gel reaction depending on the reaction parameters used. Furthermore, free 8-HQ molecules have a volatile nature in the presence of water. The evaporation of 8-HQ will shift the chemical equilibrium of the balance reaction in (50) to the product side promoting the ligand exchange reaction with water. 
The precipitation reaction in aqueous solution, however, makes it difficult to dye wool fibre with the fluorescent chelate. Nevertheless, wool fibre can be functionalised with optical fluorescence, when wool was soaked first in a zinc aqueous solution and then 8HQ was added. By doing so, it was found that the addition of 8-HQ at higher soaking temperature resulted in the formation of solid particles with brighter fluorescence. Aqueous suspensions of the solid chelate $\mathrm{Zn}(8-\mathrm{Q})_{2}$ show generally a bright white greenish colour under UV light with emission wavelength between 500 and $505 \mathrm{~nm}$. The solid chelate was adsorbed by the wool, however it is not chemical bonded on the wool fibre and can be easily rinsed off by water. Even a large amount of the chelate came off, the wool fibre still showed optical fluorescence after washing. To avoid precipitation and crystallisation reaction in the soaking solution, wool can also be functionalised with zinc and 8-HQ using in-situ approaches.

\subsubsection{Dyeing wool with $\mathrm{Zn}(8-\mathrm{Q})_{2} \cdot 2 \mathrm{H}_{2} \mathrm{O}$ using in-situ approach}

The dihydrate chelate $\mathrm{Zn}(8-\mathrm{Q})_{2} \cdot 2 \mathrm{H}_{2} \mathrm{O}$ can also be formed inside the wool. This was done by the separate uptake of the precursor material zinc and 8-HQ onto wool in dyeing bath conditions. Here, the optical fluorescence was obtained either by the uptake of zinc onto 8-HQ treated wool (in-situ dyeing method 1) or by the uptake of 8-HQ onto zinc doped wool (in-situ dyeing method 2). Because 8-HQ is highly volatile especially at higher temperature, zinc doped wool can also be functionalised with 8-HQ in a gas atmosphere (in-situ dyeing method 3).

Depending on the uptake order of the precursor material, different emission trends of the wool fibre composites were observed in this study. The in-situ dyeing method 1 produced composite material showing emission peaks at 460 and around $490 \mathrm{~nm}$. The in-situ dyeing method 2 produced fluorescent wool with emission peaks at $460 \mathrm{~nm}$ and a maximum emission wavelength between 490 and $505 \mathrm{~nm}$ depending on the 8-HQ soaking concentration in EtOH. Both methods produced wool fibre composites with similar PL scans, however, the in-situ dyeing method 2 required lower 8-HQ concentrations than the in-situ dyeing method 1. Nevertheless, the in-situ dyeing method 1 produced wool fibre composites with maximum emission wavelength below $490 \mathrm{~nm}$, which can not be obtained using in-situ dyeing method 2. The soaking of zinc 
doped wool in low 8-HQ concentration resulted instantaneously in a broad emission peak with a maximum emission wavelength around $490 \mathrm{~nm}$.

Figure 7.32 shows the shift of the maximum emission wavelength between 460 and 505 nm obtained by using both in-situ dyeing methods 1 and 2 .

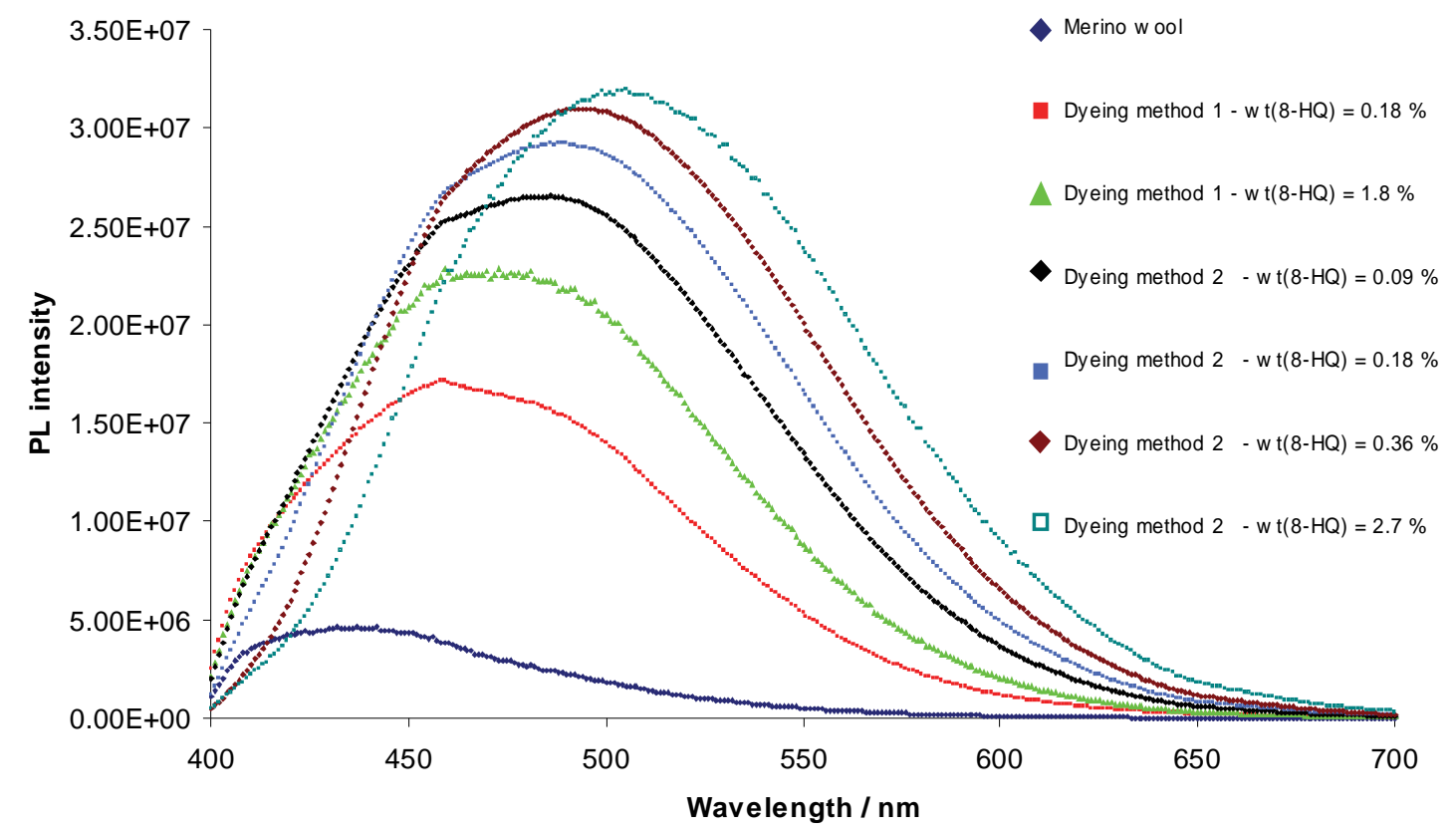

Figure 7.32: Emission shift between 460 and $505 \mathrm{~nm}$ of the maximum emission wavelength of wool fibre composites produced following in-situ approach $1\left(\mathrm{Zn}^{2+}\right.$ uptake onto 8-HQ treated wool) and 2 (8-HQ uptake onto zinc doped wool) using non-aqueous dyeing bath condition and different 8-HQ concentration $\left(\lambda_{\text {exc }}=350 \mathrm{~nm}\right.$; exciting / emission slit width $\left.=3 \mathrm{~nm}\right)$. Sample details are listed in the appendix in table A.21 (dyeing method 1) and table A.23 (dyeing method 2), respectively.

Depending on the in-situ dyeing method and 8-HQ EtOH concentration used, the fluorescence colour of the wool can be tuned from blue-white, bright white, whitegreenish, green and green - yellowish (figure 7.33). This is largely achieved by controlling the uptake of 8-HQ (EtOH) onto wool in the two different in-situ dyeing methods.

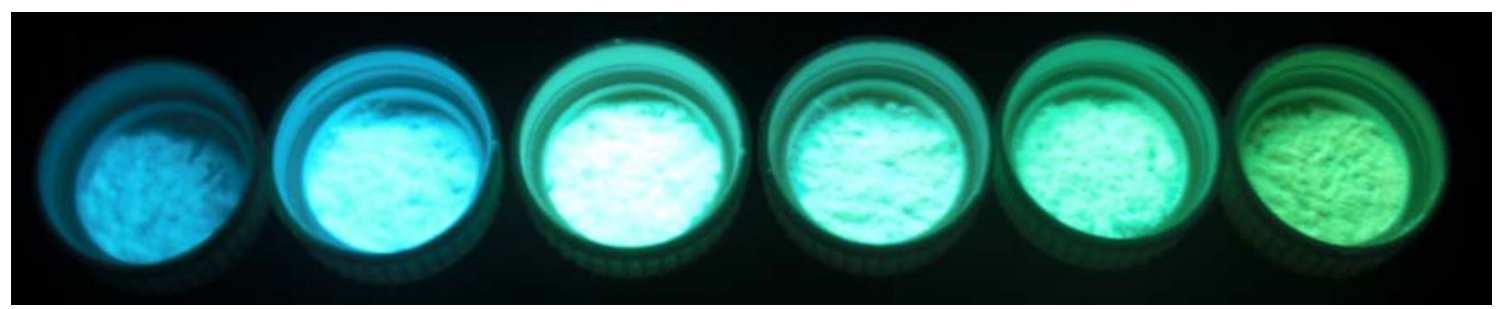

Figure 7.33: Fluorescent colours observed in wool fibre composites functionalised with different concentration of $\mathrm{Zn}(8-\mathrm{Q})_{2}$ using the in-situ dyeing approach 1 and 2 . The concentration varies from small (right) to large (left) $\left(\lambda_{\text {exc }}=366 \mathrm{~nm}\right)$. 
The emission peaks observed in the PL scans of both in-situ dyeing methods (see figure 7.32) corresponds to the dihydrate chelate $\mathrm{Zn}(8-\mathrm{Q})_{2} \cdot 2 \mathrm{H}_{2} \mathrm{O}$ formed by the complexation of zinc with 8-HQ in the chemical environment of the wool fibre. The monomer dihydrate chelate shows fluorescence at $460 \mathrm{~nm}$ and the dimer of the dihydrate chelate shows fluorescence between 490 and $505 \mathrm{~nm}$. The extent of the emission shift between 490 and $505 \mathrm{~nm}$ depends on the intermolecular interaction between adjacent complex structures in wool. For example, an increased formation of the chelate in wool reduces distances between adjacent complex structures, which enhance intermolecular interaction. This results in a stronger stabilization of the chelate in wool and to a higher wavelength shift.

The fluorescence colour of the wool is determined by these emission peaks, which overlap and form a broad emission peak (see figure 7.32). In order to control the fluorescence of the wool fibre composite, it is important to keep track of the penetration and diffusion processes of the precursor materials zinc and 8-HQ throughout the wool fibre. As shown in figure 7.1, wool is made up of hydrophilic and hydrophobic components. It can be assumed that the fluorescence of the dihydrate chelate is influenced by the chemical interaction between the different components of the wool fibre structure. For example, the dihydrate chelate will be stabilized in regions with hydrophobic character. Hydrophobic interaction between the chelate and hydrophobic components of the wool will prevent the agglomeration of the chelate. In the hydrophilic regions, however, the chelate will most likely be stabilized by intermolecular interaction between adjacent chelates. Intermolecular interactions structure such as $\pi-\pi$ stacking and hydrogen bondings, however, enable energy transfer processes which cause the lowering of the optical band gap of the chelate.

Therefore, the fluorescence of the monomer chelate observed at $460 \mathrm{~nm}$ in both dyeing methods can be attributed to the stabilization of the dihydrate chelate within the hydrophobic internal lipid regions of the wool such as the cell membrane complex. On the contrary, the fluorescence of the dimer of the dihydrate chelate between 490 and 505 $\mathrm{nm}$ will occur inside the hydrophilic internal protein structures of the wool such as cortical cells and wool fibre surface. 
The PL intensity and the shift of the maximum emission wavelength is principally dependent on the concentration of 8-HQ absorbed onto wool. However, the uptake of 8HQ is concentrated to different regions of the wool depending on the in-situ dyeing method used. For example, the uptake of 8-HQ in the in-situ dyeing method 1 is largely concentrated to the inner cells of the wool, whereas the uptake of 8-HQ in the in-situ dyeing method 2 is concentrated to the surface of the wool.

In order to understand the formation of the dihydrate chelate in the different regions of the wool, it is important to understand the diffusion processes of the precursor material and their chemical interaction with the wool fibre structure.

\subsubsection{In-situ method 1}

In the in-situ dyeing method 1, the uptake of 8-HQ is concentrated in the inner cells of the wool. The outermost layer of the wool fibre presents initially a diffusion barrier to 8-HQ molecules due to the highly cross-linked protein matrix of $\alpha$-Keratin. For that reason, the uptake of 8-HQ is mostly favoured by intercellular diffusion between gaps of the cuticle cells. In this way, the distribution of 8-HQ is concentrated to the fibre cortex (cell membrane complex and cortical cells) and the endocuticle cells along the inner side of the wool (see figure 7.1). At diffusion equilibrium, 8-HQ will then diffuse to the hydrophilic components of the wool and across the fibre surface by electrostatic interaction. The uptake of zinc $\left(\mathrm{ZnCl}_{2}(\mathrm{EtOH})\right)$ in the second batch process will result in formation of the dihydrate chelate inside the hydrophobic and hydrophilic regions of the wool, where $8-\mathrm{HQ}$ was absorbed by the wool in the first batch process.

The final fluorescence properties of the wool are determined by the complex formation of the dihydrate inside the different region of the wool. For example, a low uptake of 8HQ onto wool will produce wool fibre composites with a maximum emission wavelength at $460 \mathrm{~nm}$. Here, the complex formation of the dihydrate chelate occurs predominantly in the hydrophobic regions of the wool, which stabilize the chelate through hydrophobic interaction. The spatial distances between adjacent chelates are large enough to suppress intermolecular interaction. For this reason, the fluorescence of the monomer chelate dominates. 
A higher uptake of 8-HQ onto wool will expand the distribution of 8-HQ also throughout the hydrophilic regions of the wool. However, the diffusion of 8-HQ within the hydrophilic regions is limited through electrostatic interaction. Furthermore, intermolecular interaction between 8-HQ molecules will most likely occur inside the hydrophilic regions of the wool. Hence, a higher uptake of 8-HQ onto wool will result in an increment and broadening of the emission peak, which is formed by overlap of the monomer chelate's fluorescence at $460 \mathrm{~nm}$ and that of the dimer chelate with a maximum emission wavelength between 480 and $490 \mathrm{~nm}$. The restriction of the maximum emission wavelength at $490 \mathrm{~nm}$ is due to the limited uptake of 8-HQ onto wool.

\subsubsection{In-situ method 2}

The dyeing method 2 also resulted in the in-situ complex formation of the dihydrate complex. However, in this in-situ dyeing method, the formation of chelate is largely directed to the hydrophilic regions of the wool due to the initial uptake of zinc in the first batch process.

Wool is largely made-up of amino acids containing acidic and basic side chains, which enables the chemical binding of metal ions onto wool. For example, zinc can be bound to the protein matrix of $\alpha$-Keratin at the fibre surface through S-linkages. Consequently, the soaking of zinc doped wool in 8-HQ (EtOH) solution (second batch process) will result in the increased formation of the dihydrate chelate within the hydrophilic regions of the wool and at the wool fibre surface due to the complex formation with zinc. Furthermore, few $\mathrm{Zn}^{2+}$ ions absorbed by the wool may diffuse back into solution during the soaking process. However, before zinc will diffuses through the outermost layer of the cuticle cells, it will combine with 8-HQ molecules. Hence, the density of the dihydrate chelate is principally higher at the wool fibre surface. A higher density at the wool fibre surface, however, means that the dihydrate chelates are closely spaced together, which promote intermolecular interaction between adjacent chelates. For this reason, the soaking zinc doped wool in low 8-HQ concentration will instantaneously result in a broad emission peak with a maximum emission wavelength around $490 \mathrm{~nm}$ and a distinct peak / hump at $460 \mathrm{~nm}$. 
The complex formation of the dihydrate chelate can be expanded across the wool fibre using higher 8-HQ soaking concentrations. This consequently increases the formation of the dihydrate chelate and reduces further spatial distances between adjacent chelates. This is demonstrated in the PL scans by an increment and broadening of the emission peak towards $505 \mathrm{~nm}$ (see figure 7.14). It can be assumed that the soaking of zinc doped wool in higher 8-HQ concentrations results in the formation of a $\mathrm{Zn}(8-\mathrm{Q})_{2} \cdot 2 \mathrm{H}_{2} \mathrm{O}$ layer around the wool fibre surface. Thereby, the distances between adjacent dihydrate chelates are gradually reduced with the growth of the $\mathrm{Zn}(8-\mathrm{Q})_{2} \cdot 2 \mathrm{H}_{2} \mathrm{O}$ layer. This promotes attractive intermolecular forces such as electronic $\pi$ - $\pi$ interactions between the aromatic rings of the dihydrate chelate. Because of the attractive intermolecular interaction and less steric hindrance at the fibre surface, the dihydrate chelate can be converted into the anhydrous tetramer structure, which has a crystalline character (see equation (43)).

The conversion into the tetramer complex, however, results in a reduction of the optical band gap. Furthermore, the formation of a homogeneous layer around the wool fibre surface will quench the fluorescence of underlying chelates. For this reason, the conversion process is demonstrated in the PL scans by a decrease of the PL intensity of the wool fibre composite and a red shift of the maximum emission wavelength from 505 towards $535-550 \mathrm{~nm}$, the maximum emission wavelength of the tetramer structure $\left[\mathrm{Zn}(8-\mathrm{Q})_{2}\right]_{4}[137]$ (see figures 7.16, 7.18 and 7.22). The change of the optical band gap is also indicated by the colour change of the wool fibre composite from bright white to green and green-yellowish.

\subsubsection{In-situ method 3}

Since 8-HQ has a highly volatile character, the uptake of 8-HQ onto zinc doped wool was also carried out in a gas atmosphere (dyeing method " 3 "). The uptake of 8-HQ in gas atmosphere is easy to handle by different temperature. Thereby, the uptake is enhanced at higher temperature $\left(80{ }^{\circ} \mathrm{C}\right)$. The fluorescence colour of the wool fibre composite was controlled by the exposure time of zinc doped wool inside the 8-HQ gas atmosphere. Prolonged exposure times resulted in the increment and broadening of the emission peak with emission shift from 490 to $505 \mathrm{~nm}$. This shift also involved a change in fluorescence colour from white to white-greenish. 
Due to the limited penetration of 8-HQ gas through the protein matrix of $\alpha$-Keratin at the wool fibre surface, 8-HQ gas will react predominantly with zinc ions bound to the outermost layer of the wool fibre. By doings so, the dihydrate chelate covers the wool fibre surface and quenches the natural fluorescence of the wool (see figure 7.24). The broadening of the emission peak with prolonged exposure time corresponds to the increased formation of the dihydrate chelate and the emission shift to longer wavelengths indicates enhanced intermolecular interaction between adjacent dihydrate chelates at the wool fibre surface.

Longer exposure times in the 8-HQ gas atmosphere turn the zinc doped wool yellow indicating the formation of the anhydrous tetramer structure $\left[\mathrm{Zn}(8-\mathrm{Q})_{2}\right]_{4}$. The conversion process is also indicated by the reduction of the PL intensity and wavelength shift to $535 \mathrm{~nm}$ (see figure 7.23). It is important to point out that the emission peaks obtained by the uptake of 8-HQ onto zinc-wool in the gas atmosphere are principally narrower than the peaks obtained by the uptake of 8-HQ in EtOH solution (see figure 7.24). Furthermore, a small peak on the right-hand side of the maximum emission wavelength is also visible on the emission peaks (see figure 7.23).

The narrow emission peaks observed is due to the absence of the emission peak at 460 $\mathrm{nm}$. Here, the formation of an homogeneous surface layer around the wool fibre enhance intermolecular interaction between adjacent dihydrate chelate, which consequently quenches the fluorescence of the monomer chelate at $460 \mathrm{~nm}$. The conversion of the dihydrate chelate into the anhydrous tetramer structure $\left[\operatorname{Zn}(8-\mathrm{Q})_{2}\right]_{4}$ is also enhanced at the wool fibre surface due to the lack of steric barrier. Hence, a thin layer of the anhydrous tetramer chelate can be formed on the top of the dihydrate layer shown by a small peak with an emission wavelength that is longer than the maximum emission wavelength. Because of the absence of the emission peak at $460 \mathrm{~nm}$, the emission shift from 490 to $505 \mathrm{~nm}$ is stronger affected by a change of the fluorescence colour from white to white-greenish (see figure 7.26). Nevertheless, due to the large consumption of EtOH solution required for the dyeing method "2", the dyeing method “3” present a more efficient method for dyeing wool fibre with $\mathrm{Zn}(8-\mathrm{Q})_{2} \cdot 2 \mathrm{H}_{2} \mathrm{O}$. 


\subsubsection{Process parameters of in-situ dyeing method 1}

In the in-situ dyeing method 1, the complex formation of the dihydrate chelate is determined by the initial uptake of 8-HQ onto wool in the first batch process.

\subsubsection{Effect of 8-HQ concentration onto fluorescence properties}

As mentioned before, depending on the 8-HQ soaking concentration, the distribution of 8 -HQ is directed to different regions inside the wool. For example, the adsorption of small concentration of 8 -HQ $(\mathrm{wt}(8-\mathrm{HQ})=0.1-0.4 \%)$ is largely directed to the hydrophobic regions of the wool based on hydrophobic interaction. It can be assumed that intermolecular interaction between adjacent dihydrate chelates is widely suppressed due to the attractive hydrophobic interaction with the wool fibre. Therefore, the wool fibre composite will predominately show the fluorescence of the monomer chelate observed at $460 \mathrm{~nm}$ after the uptake of zinc (see figure 7.11).

The soaking of the wool in higher 8-HQ $(\mathrm{EtOH})$ concentration $(\mathrm{wt}(8-\mathrm{HQ})=1-8 \%)$ will expand the adsorption of 8-HQ molecules throughout the hydrophilic regions of the wool and across the wool fibre surface. Here, 8-HQ will associate with the keratinous protein structure by electrostatic interaction. Consequently, the uptake of $\mathrm{ZnCl}_{2}(\mathrm{EtOH})$ in the second batch process will result in an increased formation of the dihydrate chelate across the wool and wool fibre surface. This, however, reduces the spatial distances between the dihydrate chelate, which in turn promote intermolecular interaction between adjacent complex structures. Intermolecular interaction will stabilize the dihydrate chelate within the hydrophilic regions of the wool; however, it will also cause energy transfer processes. Hence, the increment and broadening of the emission peak with a higher uptake of 8-HQ onto wool (see figure 7.10 and 7.11) correspond to a higher content of the dihydrate chelate inside the wool and to an enhanced interaction between closely spaced chelate structures within the hydrophilic regions of the wool.

The fluorescence obtained by intermolecular interaction between two chelates in this insitu dyeing method is restricted between 480 and $490 \mathrm{~nm}$. This restriction is attributed to the limited uptake of 8-HQ onto wool. This is also indicated by the PL scans of wool soaked in different concentration of 8-HQ EtOH solutions (see figure 7.8 and 7.9). 
The PL scans of wool soaked in 8-HQ EtOH solution are very similar to the PL scans of wool fibre composites dyed by the uptake of 8-HQ onto zinc doped wool. The main difference of both composite materials is that the PL intensities of the emission peaks are nearly 10 times lower in 8-HQ treated wool.

Wool fibre naturally contains small traces of zinc embedded as inorganic salt in the fibre structure. The zinc content in merino wool can vary between $0.1-0.2 \mathrm{mg} / \mathrm{g}(0.01$ $-0.02 \%$ metal on weight of wool) depending mainly on its supply in water and food intake [138]. 8-HQ reacts with the zinc salt and form the dihydrate chelate. Depending on the chemical interaction with the wool fibre structure, the fluorescence of the monomer and / or dimer chelate will be observed.

For that reason, wool soaked in low 8-HQ concentrations also shows emission peaks at 460 and $480 \mathrm{~nm}$, which overlap and form a broader peak (see figure 7.8; A). The soaking of the wool in higher concentration of 8 -HQ $\left([8-\mathrm{HQ}]=1.0-2.5 \cdot 10^{-3} \mathrm{M}\right)$, expands the adsorption of 8-HQ molecules throughout the wool fibre (see figure 7.8; B). This also results in a broadening and increment of the emission peak. Here, the progressive transfer of 8-HQ throughout the hydrophilic regions of the wool is reflected by a wavelength shift from 480 to $490 \mathrm{~nm}$. However, the soaking in higher 8-HQ concentration $\left([8-\mathrm{HQ}]>2.5 \cdot 10^{-3} \mathrm{M}\right)$ lead to the reduction of the PL intensity and further wavelength shift from 490 to $500 \mathrm{~nm}$ (see figure 7.7; B; $w \mathrm{wt}(8-\mathrm{HQ})=3.5-5.5$ $\%$ ). A maximum emission shift is reached at $500 \mathrm{~nm}$ and does not change; even after soaking in higher $8-\mathrm{HQ}$ concentration $\left(\left([8-\mathrm{HQ}]>4.0 \cdot 10^{-3} \mathrm{M}\right)\right.$.

Since wool show a saturation effect of 8-HQ, the soaking in higher 8-HQ concentration $(w t(8-H Q) \sim 8 \%)$ will lead to an accumulation of 8-HQ molecules around the wool fibre surface. This is enhanced by the hydrophobic character of the wool fibre surface (fatty acid mono layer). Thereby, the natural fluorescence of the wool fibre and the fluorescence of underlying complexes are quenched by 8-HQ molecules. This is shown by the reduction of the PL intensity of the broad emission peak and by the total quenching of the natural fluorescence of the wool fibre (see figure 7.10; wt $(8-\mathrm{HQ})=8.3$ $\%)$. Furthermore, the colour of the wool turned light greyish, which may refer to the accumulation or degradation of 8-HQ monomers onto wool. 


\subsubsection{Effect of water during the uptake of 8-HQ onto wool}

Wool fibre composites dyed following the in-situ dyeing method 1 show a gradually increment in their emission wavelength and PL intensity with a higher uptake of 8-HQ onto wool in the first batch process. This feature is reproducible, and it was always observed when the experiment was repeated onto untreated wool. However, this was not always observed in wool fibre composites pre-treated with $\mathrm{NaOH}$ and $\mathrm{HCl}$, which PL intensities occasionally fluctuated independently of 8-HQ content. This fluctuation may be caused by the uptake of water absorbed inside the wool.

The uptake of the precursor material is generally dependent on the soaking time and soaking temperature. However, the uptake of 8-HQ onto wool is negatively affected by water during the soaking process. In general, wool can absorb up to $30 \%$ of its weight in water [136]. Water is absorbed inside the cortical cells of the wool by amorphous proteins with high sulphur content. Sulphur attracts water and the cortical cells swells up. The cortical cell can also absorb large amounts of 8-HQ (EtOH), however, a homogeneous diffusion of 8-HQ throughout the wool will be hindered in the presence of water. 8-HQ has a hydrophobic character and in the presence of water, the planar aromatic rings of 8-HQ will stack together by hydrophobic forces to minimise the contact with water. This, however, causes the aggregation of 8-HQ inside and around the wool. The uptake of $\mathrm{ZnCl}_{2}(\mathrm{EtOH})$ followed in the second batch process will then result in the complex formation with 8-HQ in the condensed phase.

In the condensed phase (solid state), however, the chelate is affected by different forces such as hydrophobic, electrostatic and steric forces. These forces have a strong effect on the fluorescence properties of an emitting material, since it can trigger different types of quenching. For that reason, dye aggregation is usually associated with concentration quenching [128]. In general, quenching causes the loss of energy before a radiative relaxation occurs. For example, energy can be converted into vibration motion during the chemical interaction between adjacent chelates and / or with the surrounding (wool). This, however, reduces the fluorescence efficiency of the emitting material and also changes the optical band gap. For this reason, quenching is usually shown by the shift of the maximum emission wavelength to longer wavelengths, which causes the colouration of the wool fibre composite. Here, the aggregation of the chelate inside the wool is typically shown by a patchy green and green-yellow colouration of the wool. 
It is important to note that the aggregation product of 8-HQ has no fluorescence properties under UV-light. Nevertheless, the agglomeration of 8-HQ onto wool was also demonstrated in the PL scans of 8-HQ-wool, when water was used as soaking solution (see figure 7.9). Due to the hydrophobic character of 8-HQ, only small concentrations are dissolvable in water without aggregating. The soaking of the wool in low 8-HQ aqueous solution resulted in similar PL scans to wool soaked in 8-HQ EtOH solution. However, the PL scans of wool soaked in higher concentrations of 8-HQ $\left(\mathrm{H}_{2} \mathrm{O}\right)$ showed stronger fluctuations in the curve shape (see figure 7.9; B, C and D). This irregular curve shape is a clear indication for the aggregation of 8-HQ molecules inside the wool. Furthermore, when the PL scans of wool + 8-HQ $(\mathrm{EtOH})$ and wool $+8-\mathrm{HQ}\left(\mathrm{H}_{2} \mathrm{O}\right)$ are compared, it is evident that the latter has a lower intensity. This is attributed to the quenching effect in condensed phase and to a lower uptake of 8-HQ onto wool in aqueous solution.

To obtain a bright white fluorescence, this dyeing method has to be carried out in EtOH dyeing bath solutions. This involves the uptake of 8-HQ onto wool and the uptake of zinc onto 8-HQ-wool. By doing so, dye aggregation and quenching processes are suppressed.

\subsubsection{Process parameters of in-situ dyeing method 2}

The in-situ dyeing method 2 also produces bright white wool fibre composites with a maximum emission wavelength at around $490 \mathrm{~nm}$. Here, however, the maximum wavelength can be further shifted to longer wavelength depending on the 8-HQ soaking concentration and zinc precursor material used.

\subsubsection{Effect of 8-HQ concentration onto fluorescence properties}

As mentioned before, the complex formation of the dihydrate chelate in this in-situ dyeing method is largely concentrated to the wool fibre surface. The protein matrix of $\alpha$-Keratin at the fibre surface is hydrophilic, which will induce the stabilization of the dihydrate chelate through intermolecular interaction. For this reason, soaking zinc doped wool in low 8-HQ concentration, $10 \mathrm{~mL} 6.25 \cdot 10^{-5} \mathrm{M} 8-\mathrm{HQ}(\mathrm{EtOH})$ (see figure 7.14; $\mathrm{wt}(8-\mathrm{HQ})=0.09 \%)$, resulted instantaneously in a broad emission peak with a 
maximum emission wavelength around $490 \mathrm{~nm}$ and a distinct peak / hump at $460 \mathrm{~nm}$. The soaking of zinc doped wool in higher 8 -HQ concentration, $[8-\mathrm{HQ}]=1.25 \cdot 10^{-4}$ $2.5 \cdot 10^{-4} \mathrm{M}$ (see figure $7.14 ; \operatorname{wt}(8-\mathrm{HQ})=0.18-3.5 \%$ ), resulted in the increment and broadening of the emission peak. This is attributed to the increased formation of the dihydrate chelate across the wool fibre and wool fibre surface. Thereby, the shift of the maximum emission wavelength observed from 490 to $505 \mathrm{~nm}$ corresponds to the shorter distances between the adjacent chelates (figure 7.14). Furthermore, the distinct peak at $460 \mathrm{~nm}$ gradually decreased with the shift of the maximum emission wavelength towards $505 \mathrm{~nm}$. As mentioned before, this emission wavelength corresponds to the fluorescence of the monomer chelate and the absence of this peak indicates that the dihydrate chelates are closely spaced together and interact with each other through van der Waals forces. Intermolecular interactions between chelates, however, quench the fluorescence of the monomer chelate and promote the fluorescence of the dimer chelate. For this reason, the increase of the fluorescence intensity of the dimer between 490 and $505 \mathrm{~nm}$ involves the decline of the fluorescence intensity of the monomer at $460 \mathrm{~nm}$.

However, after reaching the maximum emission wavelength at $505 \mathrm{~nm}$, the fluorescence intensity of the wool fibre composite subsequently decreases again followed by a further wavelength shift. For example, $0.1 \mathrm{~g}$ wool doped with $\mathrm{ZnCl}_{2}\left(\mathrm{H}_{2} \mathrm{O}\right)$ and soaked in $10 \mathrm{~mL} 6.25 \cdot 10^{-5} \mathrm{M} 8$-HQ EtOH solution showed a emission peak between 500 and $505 \mathrm{~nm}$ (see figure 7.17; $\operatorname{wt}(8-\mathrm{HQ})=0.09 \%$ ). The soaking in higher 8-HQ EtOH concentration, however, resulted in the steady decline of the emission peak. The decrease of emission peak was involved with the shift of the maximum emission wavelength to longer wavelengths.

Since a larger amount of zinc ions is bound to the protein matrix of $\alpha$-keratin at the fibre surface, the soaking of zinc doped wool in higher 8-HQ concentration will consequently result in the increased formation of the dihydrate chelate at the wool fibre surface. This, however, reduces further the distances between adjacent chelates which promotes the conversion of the dihydrate chelate into its crystalline anhydrous tetramer structure. The conversion process has a negative effect on the fluorescence efficiency, since the conversion results in the shift of the emission wavelength towards $530-550 \mathrm{~nm}$. This shift is always involved with a reduction of the PL intensity. 
The conversion process occurs with an increased formation of the dihydrate chelate onto wool. Thereby, the absorbance scan of the wool fibre composites provides useful information about the amount of chelate formed by the uptake of 8-HQ onto zinc doped wool. $\mathrm{Zn}(8-\mathrm{Q})_{2}$-wool fibre composites dyed in EtOH solution show two distinctive absorbance peaks above $320 \mathrm{~nm}$. The first absorbance peak around $333 \mathrm{~nm}$ corresponds to 8-HQ and the broad absorbance band above $350 \mathrm{~nm}$ correspond to the dihydrate chelate (see figure 7.27 and 7.28). Here, a high uptake of the chelate onto wool, using in-situ dyeing method 1 and 2, is shown by the increment and broadening of both absorbance bands. Thereby, the borderline between both absorbance peaks is clearly visible at $350 \mathrm{~nm}$.

The constant borderline at $350 \mathrm{~nm}$ corresponds to the optical band gap of the dihydrate chelate, whereas the broad absorbance band above $350 \mathrm{~nm}$ is attributed to electronic transition processes between adjacent chelates. These processes are dependent on the intermolecular interaction between adjacent chelates. Enhanced intermolecular interaction promotes the transfer of energy which is shown by an increment and broadening of the absorbance band. For this reason, the broad absorbance band is often referred to as a charge-transfer band.

In general, there are different types of energy transfer mechanisms which can occur between an emitting material such as electron transfer (energy transfer via the transfer of an electron due to oxidative or reductive processes), energy transfer by the formation of excimer / exiplex states, energy transfer by coulombic interaction (dipole-dipole interaction) and energy transfer by intermolecular orbital overlapping [126-128]. For example, intermolecular interactions such as $\pi$ - $\pi$ stacking induced by dipole moments of the aromatic rings of 8-HQ between adjacent structures, will promote the transfer of energy through resonance effects. Here, the energy transfer based on Coulombic interaction occurs efficiently, when the overlap between emission spectrum and absorption spectrum of the chelate compound is large. The extent of overlap of the absorbance and emission band is determined by the Stoke-shift.

The Stoke shift evaluation of the $\mathrm{Zn}(8-\mathrm{Q})_{2}$ chelate revealed a value of $110 \mathrm{~nm}$ (see figure 7.28). The Stoke shift was obtained by correlating the absorbance peak at $350 \mathrm{~nm}$ with the emission peak at $460 \mathrm{~nm}$ and the maximum absorbance of the charge-transfer 
band with the maximum emission wavelength of the wool fibre composites. For each wool fibre composites, a constant Stoke shift of $110 \mathrm{~nm}$ was obtained. The large Stoke shift implies a small overlap between absorption and emission spectra, which excludes energy transfer via dipole-dipole interaction [128]. It can be assumed that the energy transfer between adjacent aromatic complex structures may mainly occur by the formation of excited complex states (excimer states) between an excited chelate and a chelate in the ground state.

\subsubsection{Effect of water during the uptake of 8-HQ onto zinc-wool}

The conversion process of the dihydrate complex into its crystalline tetramer structure can only occur in non-aqueous solutions. This process is a balance reaction and the addition of water will change the chemical equilibrium. Hence, the hydration of the anhydrous tetramer structure $\left[\mathrm{Zn}(8-\mathrm{Q})_{2}\right]_{4}$ will induce the reverse reaction towards the reactant / educt side and form $\mathrm{Zn}(8-\mathrm{Q})_{2} \cdot 2 \mathrm{H}_{2} \mathrm{O}$ (see equation (43)). This is shown by the formation of fluorescent particles around the wool fibre surface and a strong blue shift of the emission wavelength towards $500-505 \mathrm{~nm}$ (see figure 7.29). The precipitation reaction of the dihydrate chelate around the wool fibre surface is caused due to the hydrophobic character of the chelate. Thereby, particles formed in the presence of water lose their adhesion to wool and come easily off by rubbing. Although a large number of crystals come off the wool fibre surface; some particles remain attached to it and functionalise the wool fibre with optical fluorescence (figure 7.30; B).

The extent of the precipitation reaction depends on the amount of water added to the 8HQ EtOH solution and soaking temperature. Interestingly, the fluorescence of the crystals formed by the hydration of the anhydrous $\left[\mathrm{Zn}(8-\mathrm{Q})_{2}\right]_{4}$ compound is generally brighter (higher PL intensities) than the fluorescence of $\mathrm{Zn}(8-\mathrm{Q})_{2} \cdot 2 \mathrm{H}_{2} \mathrm{O}$ formed by the in-situ reaction inside the wool. The improved PL intensity of the hydrolysed crystals in an aggregated state may be explained by aggregation-induced emission, which is the opposite process of quenching [128]. 


\subsubsection{Effect of drying after dyeing process}

The conversion process of $\mathrm{Zn}(8-\mathrm{Q})_{2} \cdot 2 \mathrm{H}_{2} \mathrm{O}$ into its anhydrous tetramer structure $\left[\mathrm{Zn}(8-\mathrm{Q})_{2}\right]_{4}$ is a gradually process and it is also observed over time in ambient conditions. The formation of the anhydrous tetramer structure is shown by the shift of the emission wavelength from 505 towards $530-550 \mathrm{~nm}$ (see figure 7.15). This shift always involves a reduction of the PL intensity and a colour change to green and green yellowish depending on the mass portion of the dihydrate chelate onto wool.

The conversion process will occur over time, when the distinct peak at $460 \mathrm{~nm}$ in the PL scan of the wool fibre composite is missing. This process can then be enhanced by drying the wool fibre composites at higher temperatures $\left(50{ }^{\circ} \mathrm{C}\right)$. The conversion reaction results in the elimination of eight $\mathrm{H}_{2} \mathrm{O}$ molecules. Drying promote the loss of water molecules through vaporisation, which will enhance the conversion process of the dihydrate chelate into its anhydrous tetramer structure. However, it is important to note that wool fibre composites with emission wavelength below $505 \mathrm{~nm}$ and a distinct peak/ hump at $460 \mathrm{~nm}$ do not undergo a further emissions shift over time. Their fluorescence remains unchanged and bright with ageing.

\subsubsection{Effect of the zinc precursor material onto 8-HQ uptake}

Apart from the 8-HQ concentration, the complex formation of the dihydrate chelate onto wool is also affected by the zinc precursor material used. The dyeing of $\mathrm{Zn}(\mathrm{Ac})_{2}$ doped wool and $\mathrm{ZnCl}_{2}$ doped wool using constant dyeing bath conditions revealed different colours under ambient and UV-light. For example, $0.1 \mathrm{~g}$ wool fibre doped with $\mathrm{Zn}(\mathrm{Ac})_{2}$ show a yellow colour under ambient light and a intense green-yellow fluorescence under UV light after being soaked in $10 \mathrm{~mL} 1.875 \cdot 10^{-3} \mathrm{M} 8$-HQ EtOH solution (see figure 7.26; $\mathrm{A}$ and $\mathrm{B}$ ), whereas $0.1 \mathrm{~g} \mathrm{ZnCl}_{2}$ doped wool reveal a white colour (slightly greenish) and bright white fluorescence (see figure 7.26; C and D).

The differences in their fluorescence colour are shown in their PL scans. $\mathrm{Zn}(\mathrm{Ac})_{2}$ doped wool showed a stronger right-hand shift of the maximum emission wavelength than $\mathrm{ZnCl}_{2}$ doped wool, when soaked under constant dyeing bath conditions. The stronger right-hand shift of the emission wavelength in $\mathrm{Zn}(\mathrm{Ac})_{2}$ doped wool is attributed to an increased formation of the dihydrate chelate onto wool after soaking in 8-HQ EtOH 
solution. Because of the increased formation of the dihydrate chelate, distances between adjacent structures are reduced and intermolecular forces between the aromatic rings of adjacent structures are increased. This, in turn, enhances the conversion of the dihydrate chelate into the anhydrous tetramer structure which is shown by emission wavelengths above $505 \mathrm{~nm}$.

A higher complex formation of the dihydrate chelate onto $\mathrm{Zn}(\mathrm{Ac})_{2}$ doped wool is also demonstrated in the absorbance scans. Wool fibre composites doped with $\mathrm{ZnCl}_{2}$ show a smaller charge transfer band than that functionalised with $\mathrm{Zn}(\mathrm{Ac})_{2}$ when using equal dyeing bath conditions (figure 7.27).

The differences in the PL and absorbance scans are caused by the different acidic characteristics of both zinc precursor materials. $\mathrm{ZnCl}_{2}$ aqueous solution are acidic, whereas $\mathrm{Zn}(\mathrm{Ac})_{2}$ has a milder acidic character in aqueous solution. As shown in the equations (41) and (42), 8-HQ is a mono-protic bidentate chelating ligand and the complex formation with zinc results in the release of two protons $\mathrm{H}^{+}$. Depending on the zinc precursor material used, $\mathrm{ZnCl}_{2}$ or $\mathrm{Zn}(\mathrm{Ac})_{2}$, different by-products are formed, which influence the $\mathrm{pH}$ value of the surrounding differently. For example, when $\mathrm{ZnCl}_{2}$ was used as zinc precursor material, two $\mathrm{HCl}$ molecules are formed as a by-product (see equation (48)). The formation of $\mathrm{HCl}$ will lower the $\mathrm{pH}$ of the surrounding and cause the protonation of 8-HQ. This, however, will inhibit the complex formations of zinc and 8-HQ as mentioned earlier. On the contrary, when $\mathrm{Zn}(\mathrm{Ac})_{2}$ was used as zinc precursor material, the $\mathrm{pH}$ of the surrounding will remain largely unchanged due to the buffer properties of acetic acid (HAc) (see equation (52)).

$$
\mathrm{Zn}(\mathrm{Ac})_{2} \cdot 2 \mathrm{H}_{2} \mathrm{O}+28-\mathrm{HQ}(\mathrm{EtOH}) \quad \rightleftarrows \quad \mathrm{Zn}(8-\mathrm{Q})_{2} \cdot 2 \mathrm{H}_{2} \mathrm{O}+2 \mathrm{HAc}
$$

It is important to point out that wool also act as a buffer due to the wide range of different functional groups of the amino acids. Here, the formation of Hydrogen $\mathrm{H}^{+}$ during the complex formation will be largely absorbed by the functional groups of the wool. Therefore, the difference in the PL scans between $\mathrm{ZnCl}_{2}$ and $\mathrm{Zn}(\mathrm{Ac})_{2}$ doped wool after being soaked in 8-HQ (EtOH) solution, is not only attributed to the $\mathrm{pH}$ difference of both precursor materials. It has to be assumed that the presence of chloride ions inside the wool may interfere the complexation of zinc with 8-HQ. The uptake of $\mathrm{ZnCl}_{2}$ 
onto wool results in the homogenous dispersion of $\mathrm{Zn}^{2+}$ and $\mathrm{Cl}^{-}$ions throughout the wool fibre. $\mathrm{Cl}^{-}$ions may coordinate to $\mathrm{Zn}^{2+}$ ions inside the wool fibre and form a shield, which inhibit the complex formation with 8-HQ. Furthermore the presence of $\mathrm{Cl}^{-}$ions inside the wool may also interrupt intermolecular forces between the organometallic complexes. This interference may also complicate the conversion process of the dihydrate complex into the anhydrous tetramer complex.

Differences in the fluorescence intensity were also observed in the PL scans of $\mathrm{ZnCl}_{2}$ $\left(\mathrm{H}_{2} \mathrm{O}\right)$ doped wool and $\mathrm{ZnCl}_{2}(\mathrm{EtOH})$ doped wool when soaked in 8-HQ (EtOH) solution under the constant dyeing bath conditions. In general, the PL intensities of the $\mathrm{Zn}(8-\mathrm{Q})_{2}$-wool fibre composites doped with zinc ions in aqueous solution in the first batch process are lower than those of wool doped with $\mathrm{ZnCl}_{2}$ in EtOH solution. Wool fibre composites functionalised with $\mathrm{ZnCl}_{2}$ in aqueous solution (figure 7.17) also show a stronger wavelength shift to longer wavelength than wool fibre functionalised with $\mathrm{ZnCl}_{2}$ in EtOH solution (figure 7.14) after being soaked in 8-HQ EtOH solution.

The lower PL intensity and stronger wavelength shift is also recognisable in the colour of the wool fibre composite. For example, $\mathrm{ZnCl}_{2}(\mathrm{EtOH})$ doped wool soaked in $10 \mathrm{~mL}$ $1.25 \cdot 10^{-4} \mathrm{M} 8$-HQ EtOH solution have a white colour under ambient light and a bright white fluorescence under UV light ( $\lambda_{\mathrm{em}}=490 \mathrm{~nm}$, see figure 7.17; A). The white colour of the fluorescence and wool fibre remain unchanged with ageing and sunlight exposure. In contrary, $\mathrm{ZnCl}_{2}\left(\mathrm{H}_{2} \mathrm{O}\right)$ doped wool soaked in $10 \mathrm{~mL} 1.25 \cdot 10^{-4} \mathrm{M} 8$ - $\mathrm{HQ}$ EtOH solution have a white colour with a slightly greenish appearance under ambient light and white greenish fluorescence under UV light $\left(\lambda_{\mathrm{em}}=507 \mathrm{~nm}\right.$; see figure 7.18; B).

These differences in the PL scans of $\mathrm{ZnCl}_{2}(\mathrm{EtOH})$ and $\mathrm{ZnCl}_{2}\left(\mathrm{H}_{2} \mathrm{O}\right)$ doped wool after soaking in 8-HQ EtOH solution is attributed to the different solvation effects of the zinc cations in solution. In aqueous solution, zinc cations are complexed with six water molecules. This hydration shell confines the diffusion of zinc cations throughout the wool and concentrates the uptake of zinc to the hydrophilic regions such as the protein matrix of $\alpha$-keratin at the wool fibre surface. In EtOH solution, however, the uptake of $\mathrm{ZnCl}_{2}$ onto wool is enhanced due to the lack of the hydration shell and zinc cations are 
absorbed by the hydrophilic and hydrophobic regions of the wool. Therefore, the uptake of zinc onto wool is higher in EtOH solution than in aqueous solution (figure 7.13).

The uptake of 8-HQ onto zinc doped wool is also affected by the hydration shell around the zinc cations. As mentioned in the in-situ dyeing method 1, the uptake of 8-HQ onto wool is reduced in the presence of water. Furthermore, the presence of water can lead to the aggregation of $8-\mathrm{HQ}$. This was demonstrated, when $\mathrm{ZnCl}_{2}\left(\mathrm{H}_{2} \mathrm{O}\right)$ and $\mathrm{Zn}(\mathrm{Ac})_{2}$ $\left(\mathrm{H}_{2} \mathrm{O}\right)$ doped wool was soaked in EtOH solution containing a high concentration of 8HQ $\left([8-\mathrm{HQ}]>2.5 \cdot 10^{-3} \mathrm{M}\right)$. The soaking of the zinc doped wool resulted in the crystallisation of 8-HQ around the wool fibre surface. In general, the soaking of the wool in EtOH has a dehydration effect expelling water out of the wool. However, once the water leaves the wool, it will get in contact with 8-HQ at the wool fibre interface causing the aggregation of 8-HQ.

Due to the diffusion process of water outside the wool in EtOH solution, it can be assumed that the dihydrate chelate formed at the wool fibre surface may also undergo dye aggregation. Dye aggregation can trigger different quenching processes resulting in the reduction of the PL intensity. It can also promote the conversion of the dihydrate chelate into its anhydrous tetramer structure shown by wavelength above $505 \mathrm{~nm}$ (see figure 7.21).

Hence, the lower PL intensity and the emission shift to longer wavelength observed in $\mathrm{ZnCl}_{2}\left(\mathrm{H}_{2} \mathrm{O}\right)$ doped wool after being soaked in 8-HQ EtOH solution can be attributed to the lower uptake of 8-HQ onto wool and dye aggregation of the dihydrate chelate at the wool fibre surface. A lower complex formation of zinc with 8-HQ onto $\mathrm{ZnCl}_{2}\left(\mathrm{H}_{2} \mathrm{O}\right)$ doped wool is also observed in the absorbance scans. When comparing wool fibre composites of $\mathrm{ZnCl}_{2}(\mathrm{EtOH})$ and $\mathrm{ZnCl}_{2}\left(\mathrm{H}_{2} \mathrm{O}\right)$ doped wool, the absorbance peak referring to the charge transfer band is smaller and narrower in $\mathrm{ZnCl}_{2}\left(\mathrm{H}_{2} \mathrm{O}\right)$ doped wool than in $\mathrm{ZnCl}_{2}(\mathrm{EtOH})$ doped wool when functionalised under constant dyeing bath conditions in 8-HQ (EtOH) solution. To minimise dye aggregation, it is important to dehydrate zinc doped wool after soaking in aqueous solution. Best results were obtained when zinc doped wool was washed with EtOH and dried at $50{ }^{\circ} \mathrm{C}$. 


\subsubsection{Pre-treatment of wool fibre}

To increase the uptake of zinc onto wool, the wool fibre was subject of different pretreatment methods. This was undertaken, since the uptake of zinc onto wool is generally low. However, pre-treatment methods of the wool fibre with $\mathrm{NaOH}$ and $\mathrm{HCl}$ have not shown any influences on the complex formation of zinc and 8-HQ and hence optical fluorescence. In general, $\mathrm{NaOH}$ and $\mathrm{HCl}$ treatment of the wool remove the fatty acid lipid layer around the wool fibre and expose the underlying protein matrix of $\alpha$-Keratin. By exposing the amino acids at the wool fibre surface, the uptake of zinc ions should be enhanced. However, EDS elemental analysis of zinc doped wool with $\mathrm{NaOH}$ and $\mathrm{HCl}$ pre-treatment have not shown a big differences to untreated wool. Here, it is important to point out that pre-treatment of wool fibre usually have a negative effect on the wool fibre properties. In particular, the pre-treatment of the wool with $\mathrm{NaOH}$ causes a yellowing effect of the wool fibre, when soaked in $\mathrm{NaOH}$ solution at too high temperature. On the other hand, it was found out that the uptake of fluorescent particles formed by zinc and 8-HQ in the presence of water was enhanced, when the wool fibre was pre-treated with $\mathrm{NaOH}$. This was shown by higher PL intensities of the wool fibre composites compared to $\mathrm{HCl}$ and untreated wool.

The highest PL intensities of wool fibre composites dyed according to in-situ dyeing method 2 and 3 were obtained by $\mathrm{ZnCl}_{2}(\mathrm{EtOH})$ doped wool with MSA pre-treatment. The high fluorescence efficiency is correlated to a higher uptake of zinc ions onto wool with MSA as a linker molecule. The greater uptake was also confirmed by the EDS elemental analysis of the corresponding wool fibre composites. A higher uptake of zinc onto wool was also obtained by the treatment of wool with L-cysteine. Although, the PL efficiency of wool fibre composites significantly increases with MSA and L-cysteine as linker molecules, these reagents have a strong acidic character which destroys the fibres of the wool.

Furthermore, acidic linker molecules show a strong effect on the emission wavelength of the wool fibre composites. For example, wool fibre composites doped with $\mathrm{Zn}(\mathrm{Ac})_{2}$ $\left(\mathrm{H}_{2} \mathrm{O}\right)$ show a stronger wavelength shift to the right-hand side of the spectrum than $\mathrm{ZnCl}_{2}$ doped wool. The use of MSA compensate the strong right-hand shift of $\mathrm{Zn}(\mathrm{Ac})_{2}$ doped wool of around $15-20 \mathrm{~nm}$ (see figure 7.20), whereas $\mathrm{ZnCl}_{2}$ doped wool is only 
blue shifted of about $5 \mathrm{~nm}$. This is related to the acidic character of MSA. The complex formation is generally dependent on the $\mathrm{pH}$ of the surrounding medium. Since MSA changes the chemical environment of the wool due to the two carboxyl groups and one thiol group, the complex formation is interfered. Nevertheless, MSA may also have a stabilization effect on the chelate and prevent it from agglomeration.

Here, it is interestingly to point out that wool fibre composites functionalised with MSA show constant emission wavelength with ageing. Usually, wool fibre composites with a high content of the dihydrate chelate are affected by the conversion process of the dihydrate chelate into its anhydrous tetramer structure. As mentioned before, this conversion process has a negative effect on the fluorescence properties, since it results in further emission shift to longer wavelength and reduction of the PL intensity over time. It can be assumed that MSA at the wool fibre surface attract water due to the thiol group. In the presence of water, however, the dihydrate chelate is converted in a solid phase. By converting into a solid phase, the dihydrate usually lose their adhesion to wool and come off. However, the functional groups of MSA will act as linker molecule for the chelate in the condensed phase and bind it to the wool. Furthermore, the conversion process of the dihydrate chelate into its anhydrous tetramer structure is inhibited, when the dihydrate chelate was converted into a solid phase in the presence of water.

The formation of the dihydrate chelate in the solid state is general dependent on the amount of water inside the EtOH solution. As shown in equation (43), the addition of water to the 8-HQ EtOH solution will reverse the conversion process of the dihydrate chelate into its anhydrous tetramer structure. Here, the dihydrate chelate formed after the addition of water will aggregate together and form clumps which finally precipitate as solid particles around the wool fibre. When the wool was pre-treated with MSA, most of the fluorescent particles remain attached to the wool. For this reason, wool fibre composites with MSA show in general higher fluorescence efficiency in the range between 500 and $505 \mathrm{~nm}$ than wool fibre composites without MSA treatment after water was added to the 8-HQ EtOH solution. Furthermore, it was shown that wool fibre composites with MSA can be specifically tuned between 505 and $540 \mathrm{~nm}$ by adjusting the amount of $\mathrm{H}_{2} \mathrm{O}$ added to the 8-HQ soaking solution. 


\subsubsection{Ageing processes and sun light bleaching}

The fluorescence of the $\mathrm{Zn}(8-\mathrm{Q})_{2}$-wool fibre composite is also affected by different ageing processes, which is shown by a colour change of the wool fibre composites over time. For example, $\mathrm{Zn}(\mathrm{Ac})_{2}$ doped wool with a yellow colour turns to a darker yellow with ageing (figure 7.22), whereas $\mathrm{ZnCl}_{2}$ doped wool with a white-greenish colour turns to a darker green colour with ageing. These colour changes are linked to a reduction of the PL intensity and emission shift to longer wavelengths.

The change of the optical properties of the wool fibre composites observed with ageing can be caused by a diversity of processes occurring inside the wool fibre. Such processes could be the conversion process of the dihydrate chelate into the anhydrous tetramer structure, dye aggregation processes and chemical reactions between the chelate and the wool. For example, the chelate may be affected by oxidation / reduction process inside the wool occurring over time. However, the colour change with ageing was more obvious when the wool fibre composite was exposed in sun light.

It was found that the extent of the colour change through photo-bleaching is strongly dependent on the uptake of 8-HQ onto wool. When wool was soaked in 8-HQ (EtOH) solution, the absorbance spectrum reveals a small peak / hump at around $333 \mathrm{~nm}$ (see figure $6.31 ; \operatorname{wt}(8-\mathrm{HQ})=1.8 \%$ ). This absorbance peak refers to 8-HQ onto wool.

Zinc doped wool soaked in 8-HQ EtOH solution also reveals an absorbance peak in the same wavelength range next to the broad charge transfer band. Here, the absorbance peak of 8-HQ onto wool increases and broadens, when zinc doped wool was soaked in EtOH solution with higher concentration of 8-HQ. The type of zinc precursor material also determined the extent of the absorbance peaks. The highest absorbance peak of 8HQ onto wool was obtained with $\mathrm{Zn}(\mathrm{Ac})_{2}$ doped wool followed by $\mathrm{ZnCl}_{2}$ (EtOH) doped wool and finally $\mathrm{ZnCl}_{2}\left(\mathrm{H}_{2} \mathrm{O}\right)$ doped wool (see figure 7.27). Here, the uptake of 8-HQ may be enhanced due to intermolecular interaction between free 8-HQ molecules and the dihydrate chelate formed by the complexation of zinc with 8-HQ. Hence, wool fibre composites with a high content of the dihydrate chelate will have a higher uptake of free 8-HQ than wool fibre composites with a low content of the dihydrate chelate. The uptake of 8-HQ is generally shown by the absorbance peak between 320 and $350 \mathrm{~nm}$. 
Free 8-HQ molecules, however, are sensitive to sun exposure and form degradation products. The degradation products have quenching properties and reduce the fluorescence intensity of the composite material. For this reason, wool fibre composites with a low uptake of 8-HQ onto wool are more resistant to sun light exposure. For example, wool fibre composites dyed according to the in-situ dyeing method "1" generally remain their fluorescence brightness over time and under sunlight exposure, because of the limited uptake of 8-HQ onto wool in the first batch process.

Furthermore, wool fibre composites dyed in aqueous solution also show stable fluorescence colours over time. Here, wool fibre composites do not show an absorbance peak between 320 and $350 \mathrm{~nm}$, when $\mathrm{Zn}$ doped wool was dyed with 8-HQ in aqueous solution (figure 7.31; A and B). However, the uptake of 8-HQ onto zinc doped wool in aqueous solution results always in the formation of fluorescent particles around the wool fibre surface. Due to hydrophobic character, the dihydrate chelate forms clumps to minimise the contact with water. This results in the precipitation of the chelate at the wool fibre surface. Although, these particles can easily come off by rubbing or after ultrasonic bath treatment, large amounts of particles remain on the wool and functionalise it with optical fluorescence. Wool fibre can also be functionalised directly with these fluorescent particles. Here, the absorbance scan only shows the absorbance peak of the charge transfer band above $350 \mathrm{~nm}$ (figure 7.31, A). It is important to point out that the formation of the anhydrous tetramer structure $\left[\mathrm{Zn}(8-\mathrm{Q})_{2}\right]_{4}$ is prevented in solution and with ageing, when water was used as solvent for the uptake of 8-HQ onto zinc doped wool.

The uptake of 8-HQ onto wool is affected in the presence of water. In the presence of water, 8-HQ has a highly volatile character and also forms clumps to minimise the contact with water. A lower uptake of 8-HQ onto wool was also shown in the absorbance scans, when zinc doped wool was hydrated before soaking in an 8-HQ in EtOH solution. The hydration of the wool resulted in a smaller and narrower absorbance peak between 320 and $350 \mathrm{~nm}$. The hydration of the wool, however, can cause dye aggregation of the dihydrate chelate inside the wool shown by lower PL intensity and emission shifts to longer wavelengths. 
In summary, photo-bleaching and dye-aggregation will result in the quenching of the fluorescence intensity of the final composite material. Therefore, the use of water in the in-situ dyeing methods should be avoided. Also, thorough washing is necessary to remove all loosely bound 8-HQ molecules from the wool fibre material. Furthermore, the conversion process of the dihydrate chelate into its anhydrous tetramer structure will change the fluorescence properties over time. This conversion process usually takes place when zinc doped wool was soaked in higher 8-HQ concentration. Therefore, the uptake of 8-HQ onto zinc doped wool should be low.

\subsubsection{Anti-microbial activity of wool dyed with $\mathrm{Zn}(8-\mathrm{Q})_{2}$}

8-HQ as well as their metal complexes exhibit antiseptic, disinfectant, and pesticide properties [139]. Preliminary assessment of the anti-microbial properties of the wool fibre composite with $\mathrm{Zn}(8-\mathrm{Q})_{2}$ was conducted. Two bacteria, Staphylococcus aureus and Escherichia coli were used in this assessment and promising results were obtained. However, a more detailed assessment that considers different contents of the chelate onto wool and the effect of sun light bleaching on the anti-microbial action should be conducted in future studies.

\subsection{Conclusions}

Wool fibre can be successful dyed with the fluorescent chelate $\mathrm{Zn}(8-\mathrm{Q})_{2} \cdot 2 \mathrm{H}_{2} \mathrm{O}$ using the in-situ dyeing method 1 and 2. Both methods differ in the uptake order of the precursor materials zinc and 8-HQ onto wool conducted in two separated batch processes. The fluorescence of the dihydrate is determined by the fluorescence of the monomer chelate at $460 \mathrm{~nm}$ and the fluorescence of the dimer between 480 and $505 \mathrm{~nm}$. The emission wavelength of the dimer depends on the spatial distance between two dihydrate chelate interacting with each other.

Both methods result in the production of wool fibre composites with bright white fluorescence. Here, the fluorescence of the monomer and dimer chelate overlaps which lead to a bright white fluorescence. Depending on the in-situ dyeing method used, the fluorescence of the monomer and dimer chelate can be tuned. Thereby, the emission of the wool fibre composite was principally controlled by the uptake of 8-HQ onto wool in 
both in-situ dyeing methods. The uptake of 8-HQ is directed to different regions of the wool depending on the in-situ dyeing method used. Thereby, the complex formation of the dihydrate chelate occurs in the different regions of the wool.

In the in-situ dyeing method 1, the uptake of 8-HQ is concentrated to the inner cells of the wool with a hydrophobic character. However, soaking wool in higher 8-HQ (EtOH) concentration also resulted in the gradually diffusion of 8-HQ from the hydrophobic regions into the hydrophilic regions of the wool through electrostatic interaction. In the hydrophobic regions of the wool, 8-HQ is stabilized by hydrophobic interaction, however; in the hydrophilic regions of the wool, 8-HQ will be spaced closely together and stabilized by intermolecular interaction. The uptake of 8-HQ onto wool is limited, which also limit the complex formation of the dihydrate chelate. Hence, the uptake of zinc in the second batch process will result in the complex formation of the dihydrate chelate in the different regions of the wool, where 8-HQ was initially absorbed. The fluorescence of the monomer chelate will dominate in the regions of the wool with a hydrophobic character, whereas the fluorescence of the dimer chelate occurs in the hydrophilic regions of the wool.

The uptake of 8-HQ in the in-situ dyeing method 2 is largely concentrated to the surface of the wool fibre. The initial uptake of zinc onto wool in the first batch process is mostly directed to the protein matrix of $\alpha$-keratin at the wool fibre surface. Hence, the soaking in 8-HQ (EtOH) solution results in the immediate complex formation of the zinc cations with 8 -HQ at the wool fibre surface. The fluorescence of the dihydrate chelate is strongly dependent on the spatial distances between adjacent chelates. Hence, an increased formation of the chelate at the wool fibre surface reduces the spatial distances between adjacent dihydrate chelate structures and promoting the fluorescence of the dimer chelate.

By getting closer together, the fluorescence of the monomer chelate $(460 \mathrm{~nm})$ will be gradually quenched and at a certain uptake of the dihydrate chelate onto the wool fibre surface the distances between adjacent chelates are short enough to form a homogeneous layer around the wool fibre surface. The formation of a homogeneous layer, however, promotes the conversion of the dihydrate chelate into its anhydrous tetramer structure $\left[\mathrm{Zn}(8-\mathrm{Q})_{2}\right]_{4}$, when non-aqueous dyeing bath condition is applied. The 
conversion process, however, has a negative effect on the fluorescence properties of the wool fibre composite and is shown by a colour change of the wool to green-yellow. The conversion process of the dihydrate chelate into its anhydrous tetramer structure is a chemical equilibrium reaction. Therefore, the hydration of the wool fibre composite results in the reconversion of the anhydrous tetramer compound into the dihydrate chelate. This, however, occurs in the chemical surrounding of the wool. Furthermore, the dihydrate chelate forms clumps to minimise the contact with water. The solid chelate precipitates around the wool fibre and shows strong fluorescence with emission wavelength between 500 and $505 \mathrm{~nm}$. The solid chelate formed in the chemical surrounding of the wool has stronger fluorescence brightness than those formed in solution.

The fluorescence colour of the wool fibre composite, however, is further affected by different process parameters. For example, the complex formation is strongly affected by the $\mathrm{pH}$ value of the surroundings. The complexation of zinc with two 8-HQ molecules causes the dissociation of two protons. Depending on the counter ions of the zinc precursor material, different acidic by-products are formed which affect the $\mathrm{pH}$ of the surrounding medium. Acidic conditions, however, lead to the protonation of the 8HQ ligand which suppress the complex formation of $\mathrm{Zn}(8-\mathrm{Q})_{2} \cdot 2 \mathrm{H}_{2} \mathrm{O}$. Thereby, zinc cations complexed with water and surrounded by chloride ions are also stronger affected by a $\mathrm{pH}$ change during the complex formation. The modification of the wool fibre with acidic linker molecules will also suppress the complexation of zinc with 8-HQ.

Furthermore, water absorbed by the wool can confine the uptake of 8-HQ in the different regions of the wool due to the hydrophobic character. Water can also cause the aggregation of 8-HQ and the dihydrate chelate inside the wool. This, however, will trigger different quenching processes which result in the emission shift to longer wavelengths and reduce the PL intensity of the wool fibre composite. The uptake of 8HQ onto zinc doped wool is also affected by dye aggregation, when wool was doped with zinc in aqueous solution. Furthermore, chloride ions form $\mathrm{ZnCl}_{2}$, diffusing along with the zinc cation throughout the wool, can interfere with the complex formation of $\mathrm{Zn}(8-\mathrm{Q})_{2} \cdot 2 \mathrm{H}_{2} \mathrm{O}$. Also, the uptake of free 8-HQ onto the wool fibre composite has a negative effect on the fluorescence properties of the wool fibre composites. Free 8-HQ molecules are not stable in sun light and degrade. The degradation products act as 
quencher molecules causing a change in colour with ageing. The colour change is involved with an emission wavelength to the red side of the spectrum and reduction of the PL intensity. Photo-degradation, however, can be predominantly avoided by a low complex formation of the dihydrate chelate onto wool.

8-HQ is highly volatile, especially at higher temperature, zinc doped wool can also be functionalised with 8-HQ in a gas atmosphere. This method results in the complex formation of $\mathrm{Zn}(8-\mathrm{Q})_{2} \cdot 2 \mathrm{H}_{2} \mathrm{O}$ at the wool fibre surface and it can be easily controlled by the exposure time of the wool fibre composite inside the 8-HQ gas atmosphere. Furthermore, this dyeing method has the potential to be used in a larger scale. Here, it is important to note that the in-situ dyeing methods conducted in solution were easily upscaled to $10 \mathrm{~g}$ wool.

Future research should focus on finishing treatment of the fluorescent fibres using polymers such as methacrylamide, acrylic acid, ethyl acrylate and so on. These compounds will form a hydrophobic coat on the fibres which will improve their resistance against the damaging effect of water and sunlight and therefore the fluorescene activity of the chelate can be extended considerably. 


\section{Chapter 8}

\section{Concluding remarks}

The goal of this project was to bind quantum dots to wool and paper fibres to produce new quantum dot - fibre materials that exhibit tuneable optical fluorescence, for potential use in textiles, and packaging and labelling papers. This project was divided in two sections, synthesis and characterisation of quantum dots and development of new fluorescent composite materials using fluorophores and natural fibrous substrate.

The nanotechnology presented in first part of the thesis dealt with the synthesis and characterisation of metal oxide quantum dots. The goal was to produce fluorescent quantum dots made of non-toxic or low toxicity materials, so they are compatible with human skin and suitable for the use in textiles and other everyday products. Copper (I) oxide $\left(\mathrm{Cu}_{2} \mathrm{O}\right)$ and zinc oxide $(\mathrm{ZnO})$ were used as metal oxide semiconductors due to their non-toxicity in their bulk form, simple production using wet chemical synthesis methods and scalability. However, the synthesis of metal oxide quantum dots with intense, stable, spectroscopically pure fluorescent colours is difficult to achieve.

Initially, copper (I) oxide quantum dots were synthesised by reducing aqueous copper nitrate $\left(\mathrm{Cu}\left(\mathrm{NO}_{3}\right)_{2}\right)$ solution with sodium borohydride $\left(\mathrm{NaBH}_{4}\right)$ in the presence of trisodium citrate $\left(\mathrm{Na}_{3} \mathrm{C}_{6} \mathrm{H}_{5} \mathrm{O}_{7}\right)$ and poly(methacrylic acid sodium salt) (PMAA-Na) at 99 $100{ }^{\circ} \mathrm{C}$. The sample preparation resulted in the formation of small spherical nanocrystals (5 - $10 \mathrm{~nm}$ ) with a super lattice structure. These small nanocrystals have strong affinity to form cube-shaped particles of $70-250 \mathrm{~nm}$, depending on synthesis conditions. The size-distribution of the cubes can be controlled by changing simple process parameters such as stirring time, reaction temperature, concentration of stabilizer agents, concentration of reducing agent $\mathrm{NaBH}_{4}$ and the use of dopants.

The arrangement of the small nanocrystals into nanocubes has a strong effect on the light absorption and scattering properties of the $\mathrm{Cu}_{2} \mathrm{O}$ suspension. Suspensions containing mainly large polycrystalline nanocubes revealed a dichroic effect. Here, the suspension was yellow-green in reflected light, whereas in transmitted light the 
suspension was red-purple and transparent. This phenomenon is caused by Surface Plasmon Resonance (SPR). SPR shows characteristic absorbance peaks in the visible range, which can be correlated with the physical dimension of the nanocubes. Thereby, the size of the nanocubes and intermolecular interaction between the nanocubes determined the wavelength (range) of the adsorbed and scattered light and the ratio of light absorption and light scattering.

The SPR effect was only observed when the quantum dots were arranged into nanocubes following a self-assembled stabilization mechanism. However, the association of the quantum dots inside the cubes is based on weak electrostatic interactions and hydrogen bondings. These bondings can be easily broken by diluting the suspension with water or adding ionic surfactants. However, with the collapse of the self-assembled structures, the light absorption and scattering properties are lost. For this reason, further investigation is needed to reinforce the cube-shaped structure of $\mathrm{Cu}_{2} \mathrm{O}$ particles and gain a higher chemical stability. By doing so, $\mathrm{Cu}_{2} \mathrm{O}$ nanocubes can be incorporated in polymers to create composite materials with a dichroic behaviour. This material can be used, for example, in the preparation of anti-counterfeiting bank notes or it can be incorporated in plastics products to create discrete barcodes to mark valuables or commercial goods.

The $\mathrm{Cu}_{2} \mathrm{O}$ quantum dot suspension did not show optical fluorescence and hence $\mathrm{Cu}_{2} \mathrm{O}$ was not suitable to functionalise wool and paper fibre. For this reason, a second metal oxide semiconductor, $\mathrm{ZnO}$, was studied. $\mathrm{ZnO}$ quantum dots were prepared by a sol-gel method adding an alkali reducing agent, $\mathrm{NaBH}_{4}$, to a zinc chloride aqueous solution complexed with polyvinylpyrrolidine (PVP) at a reaction temperature of $70{ }^{\circ} \mathrm{C}$. This sol-gel method produced $\mathrm{ZnO}$ quantum dots with an average size of $10-12 \mathrm{~nm}$. These quantum dots are spherical and ellipsoidal in shape and form larger polycrystalline particles in the shape of round rice grains varying between $100 \mathrm{~nm}$ and $1 \mu \mathrm{m}$. By sticking together, the nanocrystals reduce their surface-to-volume ratio and gain higher stability in aqueous suspension. ZnO suspensions prepared according to this sol-gel method are white under ambient light and yellow-green under UV light.

The fluorescence analysis of the $\mathrm{ZnO}$ suspension showed the characteristic band-edge emission at $380 \mathrm{~nm}$, which refer to the radiative recombination of the electron-hole pair 
(exciton) in spherical $\mathrm{ZnO}$ quantum dots. The band-edge emission, however, was strongly affected by ageing. Ageing resulted in the progressive reduction of the bandedge emission and the rise of a broad emission peak around $560 \mathrm{~nm}$. This broad peak refers to the defect emission of $\mathrm{ZnO}$ quantum dots caused by surface defects. These surface defects incorporate surface related energy states within the band gap and quench the band-edge emission by trapping the excited electron or hole with the surface related defect states during the radiative recombination of the exciton.

The defect emission strongly depends on the surface coverage of the $\mathrm{ZnO}$ nanocrystals. $\mathrm{ZnO}$ quantum dots were coated by PVP chains, which passivate the surface area of the quantum dots and confine the particle growth of the quantum dots through steric hindrance. The stability of the surface coverage, however, strongly depends on the type of bonds between functional groups of the polymer and surface atoms. The charge of metal oxides is known to be frequently delocalised over the whole particle surface resulting in the formation of weak chemical bonds such as hydrogen bond. Weak chemical bonding, however, support the loss of polymer chains giving rise to surface defects and hence to the defect emission. For this reason, an increment of the fluorescence intensity of the defect emission was observed in the $\mathrm{ZnO}$ suspension for the first two to three days after preparation. However, with the loss of capping agents, attractive van der Waals forces between the quantum dots increased and pulled the nanocrystal closer together. Thereby, the quantum dots can adhere strongly together and this process is irreversible. This, in turn, enables non-radiative recombination processes that reduce the defect emission (quenching) and the intensity of the fluorescence decreases after three days of ageing.

It was observed that the agglomeration of the quantum dots into larger polycrystalline particles has a positive effect on the fluorescence intensity of the defect emission. By being enclosed inside a polymer host matrix, further condensation processes between particles are inhibited. Here, the particles are held together by van der Waals forces such as weak electrostatic interaction and hydrogen bondings between the polymer chains of adjacent nanocrystals. However, the self-assembled structures are not stable and split in halves, which makes it difficult to control the size and shape of the particles. Also, the stability of the polycrystalline particles is strongly affected by dilution, which breaks the self-assembled structure apart and quantum dots remain mono-dispersed in solution. 
When PMAA-Na was used as a polymeric dispersant, $\mathrm{ZnO}$ quantum dots also agglomerated and formed larger polycrystalline particles. These particles, however, were significantly smaller and the fluorescence intensity of the defect emission was lower. A reduction in the size of the polycrystalline particles and the fluorescence intensity of the defect emission is caused by a stronger surface coverage around the quantum dots. PMAA-Na contains negative charged carboxylic groups in their polymer chains which passivate the quantum dots surface area by electrostatic interaction. This charged surface layer also interferes with processes that lead to network forming reactions between quantum dots.

Interestingly, $\mathrm{ZnO}$ prepared in the presence of $\mathrm{CeCl}_{3}$ showed only one sharp and intense band-edge emission at around $380 \mathrm{~nm}$, when excited with $\lambda_{\text {exc }}=260 \mathrm{~nm}$. The defect emission was not observed with the ageing of the suspension; however, a shift of the band-edge emission to longer wavelength was observed ( 385 nm). This shift is caused by Ostwald ripening process leading to the growth of the $\mathrm{ZnO}$ quantum dots in suspension. These results obtained with $\mathrm{Ce}^{3+}$ are interesting, worth of further research but beyond the scope of this thesis.

The second part of this thesis project dealt with development of new fluorescent composite materials which combine the properties of $\mathrm{ZnO}$ quantum dots with those of wool and paper fibres. ZnO quantum dots were chosen for the preparation of quantum dot - fibre composites because of their fluorescence in the UV and visible range of the light and uncomplicated synthesis via a sol-gel method. For the development of $\mathrm{ZnO}$ quantum dot - fibre composites, strategies such as in-situ and building block approach were explored. Here, it was important to identify the best processing strategy to obtain fluorescence and a strong chemical bonding between the inorganic and organic phase. This is crucial to ensure long life-time stability of the composite material.

In the in-situ approach, $\mathrm{ZnO}$ quantum dots were formed in the presence of wool using the sol-gel method developed in this thesis (Chapter 4). The in-situ method relied on cross-link reactions between the quantum dots and wool fibre during nucleation and particle growth. In the building block approach, $\mathrm{ZnO}$ quantum dots were attached to wool and paper fibre either directly or through the use of linker molecules. Different chemical techniques were used to functionalise the substrate material or the quantum 
dot surface with linker molecules and organic ligands to increase the compatibility between both components. However, none of these approaches resulted in the production of fibre composites showing fluorescence in the visible range. The building block and in-situ approach produced ZnO quantum dot - wool composites with a sharp emission peak in the UV range. This emission peak referred to the band-edge emission of the $\mathrm{ZnO}$ quantum dots ( $380 \mathrm{~nm})$. The attachment of $\mathrm{ZnO}$ quantum dots to bleached Kraft paper quenched completely the fluorescence of the bleached paper fibre, without changing the white appearance of the fibres under ambient light. Here, the paper fibres were coated by $\mathrm{ZnO}$ quantum dots which absorb UV-light and release the excitation energy through non-radiative recombination processes.

Although the attachment of quantum dot to fibrous material did not result in visible fluorescence, $\mathrm{ZnO}$ quantum dots show UV filtering properties which shield the fibres from dangerous UV radiation and protect them from photo-degradation. Also, preliminary studies revealed anti-microbial action of this new composite material and hence it possesses great potential in the fabrication of anti-microbial textiles.

To tune the fluorescence of the $\mathrm{ZnO}$ quantum dot - fibre composite into the visible range, the use of 8-hydroxyquinoline (8-HQ) as a fluorescent ligand for the $\mathrm{ZnO}$ quantum dot surface was explored. Depending on the concentration of 8-HQ and other parameters such as choice of solvent, soaking temperature and time, the fluorescence of $\mathrm{ZnO}$ quantum dot - paper and wool composites were tuned from white, light green to yellow-green. The modification of the ZnO quantum dot surface with 8-HQ, however, promotes the agglomeration of the quantum dots at the interface of the organic interface. This is due to the hydrophobic character of 8-HQ which leads to the formation of larger particles. Depending on the size of the particles, they lose their chemical attachment to the fibre and easily come off by friction. In paper fibre composites, the fluorescent particles filled the spaces and crevices between the fibres, which resulted in a uniform coating of the composite material. However, the fluorescence intensity of wool fibre composites gradually decreased with ageing due to leaching. To avoid this, an alternative procedure to functionalise wool with optical fluorescence using zinc and 8HQ was developed. 
In this dyeing method, the fluorescent chelate $\mathrm{Zn}(8-\mathrm{Q})_{2} \cdot 2 \mathrm{H}_{2} \mathrm{O}$ was formed inside the wool by the uptake of zinc and 8-HQ in two separated batch processes. Depending on the uptake order, concentration of the precursor materials and dyeing bath conditions, wool fibre was functionalised with a strong white fluorescence. The white fluorescence was obtained by the overlap of at least two emission peaks at $460 \mathrm{~nm}$ (blue light) and between 490 and $505 \mathrm{~nm}$ (green light). The wavelength of the green emission light is strongly dependent on the uptake of 8-HQ onto wool. For example, by applying higher concentration of 8-HQ, a further shift to longer wavelengths was observed. This, however, changed the colour of the wool under ambient and UV-light and the wool showed a white-greenish to green-yellowish fluorescence depending on the 8-HQ concentration used in the soaking solution.

The fluorescence activity of the wool fibre composites soaked in high concentration of 8-HQ (EtOH) was negatively affected by sun light exposure. Besides the in-situ complex formation of the chelate, soaking wool fibre in high 8-HQ concentration also result in the uptake of free 8-HQ molecules onto wool. Free 8-HQ, however, is sensitive to sun-light exposure and form degradation products which quench the fluorescence of the chelate. The quenching effect also involves a colour change, and a red shift of the emission wavelength to the red side of the spectrum and reduction of the PL intensity is observed. This feature can be extremely useful as an indicator of sun light exposure for products that are sensitive to light.

As shown in this thesis the use of quantum dots to functionalise wool and paper fibre with optical fluorescence has a great potential for the development of new composite material. The synthesis of metal oxide quantum dots using elements of low toxicity is feasible; however, obtaining bright fluorescent colours is difficult and has not been achieved yet. The latter is an aspect that should be further explored. For instance, metal oxide quantum co-precipitated with ZnS (see chapter 5) show strong fluorescent colours in the visible range depending on the composition of the metal oxide. This fluorescence remains stable showing bright intense colour, which is a feature of great interest. 


\section{References}

(1) C. Burda, X. Chen, R. Narayanan, M.E. El-Sayed. Chemistry and Properties of nanocrystals of different shapes. Chem. Rev., (2005); 105: 1025 - 1102.

(2) James H. Johnston, Kerstin A. Burridge and Fern M. Kelly. The Formation and Binding of Gold Nanoparticles onto Wool Fibres. Advanced Materials and Nanotechnology, (2009); 1151: 189-192.

(3) Fern $\mathrm{M}$ Kelly and James $\mathrm{H}$ Johnston. Colored and Functional Silver Nanoparticle Wool Fiber Composites. ACS Appl. Mater. Interfaces, (2011); 3 (4): 1083-1092.

(4) James H Johnston and Kerstin A Lucas. Nanogold Synthesis in Wool Fibres: Novel Colourants. Gold Bulletin, (2011); 44(2): 85-89.

(5) J.L. West, N.J. Halas. Application of nanotechnology to biotechnology. Elsevier Verlag, (2000); 11 (2): 215 - 217.

(6) M. Bruchez, M. Moronne, P. Gin, S. Weiss and A.P. Alivisatos. Semiconductor Nanocrystals as Fluorescent Biological Labels. Science. (1998); 281: 2013-2015.

(7) U. Resch-Genger, M. Grabolle, Quantum dots versus organic dyes as fluorescent labels. Nature Methods Review. (2008); 5: 763-775.

(8) S.G. Murray. Dyes and fluorescent whitening agents for paper in Paper Chemistry. Springer Science+Business Media B.V. (1995); 161 - 193.

(9) T.T. Baker. Fluorescent dyes and the application of fluorescence to textiles. Journal of the society of dyers and colourist. (1953); 69 (4): 109 - 112.

(10) R.M. Christie. Fluorescent dyes. Review of Progress in Colouration. (1993); 23.

(11) Y. Lin, R. Weissleder, C.H. Tung. Synthesis and properties of sulfhydrylreactive near infrared cyanine fluorochromes for fluorescence imaging. Molecular Imaging. (2003); 2(2): 87 - 92

(12) G.Y. Mitronova, V. N. Belov, M. L. Bossi, C. A. Wurm, L. Meyer, R. Medda, G. Moneron, S. Bretschneider, C. Eggeling, S. Jakobs, S. W. Hell. New Fluorinated Rhodamines for Optical Microscopy and Nanoscopy. Chemistry - A European Journal. (2010); 16: 4477-4488.

(13) R.W. Sabnis, G.J. Kazemi, D.W. Rangnekar. Synthesis and application of 4 (coumarin-3-yl)-thiophenes. Phosphorous, Sulfur, and Silicon. (1992); 71: 1-6

(14) M.F. Esteves. Optical brighteners effect on white and coloured textiles. World Textile Conference - 4th AUTEX Conference. (2004).

(15) G. Calzaferri. Particle in a one-dimensional box as a model for the description of conjugated systems. CHIMIA. (1987); 41: 248 - 250.

(16) A. G. M. Shaloub, Visible Spectra of Conjugated Dyes: Integrating Quantum Chemical Concepts with Experimental Data. J. Chem. Ed. (1997); 74: 1317.

(17) C. J. Ballhausen, J. Chem. Ed. (1979); 56: 194-197, 215-218,357-361.

(18) H.P. Latscha, H.A. Klein, Chemie basiswissen, Teil I, Springer-Verlag Berlin Heidelberg (1994); sechste Auflage.

(19) P.E. Burrows, S.R. Forrest and M.E. Thompson. Prospects and applications for organic light-emitting diodes. Current Opinion in Solid State Mater. Sci. (1997); 2: $236-243$.

(20) AI Ekimov, AL Efros, AA. Onushchenko. Quantum size effect in semiconductor microcrystals. Solid State Commun (1985); 56: 921 - 924. 
(21) L. Brus. Electronic wave-functions in semiconductor clusters: experiments and theory. J. Phys. Chem. (1986); 90: 25555 - 2560.

(22) R. Rossetti, L. Brus. Electron-hole recombination emission as a probe of surface chemistry in aqueous cadmium sulfide colloids. J. Phys. Chem. (1982); 86:4470 $-4472$.

(23) A.P. Alivisatos. Semiconductor clusters, nanocrystals, and quantum dots. Science. (1996); 271: 933 - 937.

(24) F. Chandezon, C. Reynaud. Fluorescent Semiconducting Nanocrystals Show Their Colours. Marterials Science Division Report, CEA Grenoble. (2005).

(25) V.M. Ustinov, A.E. Zhukov, A.Yu. Egorov, N.A. Maleev. Quantum Dot Lasers. Oxford University Press, Oxford (2003).

(26) E. Arici, N.S. Sariciftci. Hybrid Solar Cells. Encyclopaedia of Nanoscience and Nanotechnology. (2004); 10: 1-16.

(27) J. Drbohlavova, V. Adam, R. Kizek and J. Hubalek. Quantum dots Characterisation, preparation and usage in biological systems. Int. J. Mol. Sci. (2009); 10: 656 - 657.

(28) A.A. Balandin and K. L. Wang; Handbook of Semiconductor Nanostructures and Nanodevices. American Scientific Publishers. (2006); 2: 295 - 334.

(29) P.Y. Yu, M. Cardona. Fundamentals of Semiconductors: Physics and Materials Properties. 3. Auflage. Springer, Berlin, 2004.

(30) J. Cox. A quantum paintbox. Chemistry in Britain. (2003); 21 - 25.

(31) B. Enright and D. Fitzmaurice. Spectroscopic determination of electron and hole effective masses in a nanocrystalline semiconductor film. J. Phys. Chem. (1996); 100 (3): 1027 - 1035.

(32) M. Grundmann. The physics of semiconductors. Springer Verlag (2010)

(33) P.W. Atkins, Physikalische Chemie. Wiley-VCH: Weinheim, New York, Basel, Cambridge, Tokyo. (1996).

(34) He Hu, Weihua Zhang; Synthesis and properties of transition metals and rareearth metals doped ZnS nanoparticles. Optical materials. (2006); 28: 536-550.

(35) M. Shimizu and T. Hiyama. Organic fluorophores exhibiting highly efficient photoluminescence in the solid state. Chem. Asian J. (2010); 5: 1516 - 1531.

(36) Aaron C. Small and James H. Johnston: Novel Hybrid Materials of Cellulose and Doped ZnS Nanoparticles. Current Applied Physics, (2008); 8: 512-515.

(37) Treasury, NZ Economic and Financial Overview, (2012).

(38) J. Jolivet. Metal Oxide Chemistry and Synthesis. From Solution to Solid State. Chicester. Wiley \& Sons (2000).

(39) M. Henry, J.-P. Jolivet, J. Livage. Aqueous Chemistry of Metal Cations Hydrolysis, Condensation and Complexation. Structure and Bonding (1922); 77: 153-206.

(40) J. Livage, M. Henrz, C. Sanchez. Sol-Gel Chemistry of Transition Metal Oxides. In Prog. Solid St. Chem. (1988), 18, 259-341.

(41) Q. Shang, H. Yu, X. Kong, H. Wang, X. Wang, Y. Sun, Y. Zhang, Q. Zeng. Green and red up-conversion emissions of $\mathrm{Er}^{3+}-\mathrm{Yb}^{3+}$ Co-doped $\mathrm{TiO}_{2}$ nanocrystals prepared by the sol-gel method. Journal of Luminescence (2008); 128:1211-1216.

(42) B. Hojjati, R. Sui, P.A. Charpentier. Synthesis of $\mathrm{TiO}_{2} / \mathrm{PAA}$ nanocomposite by RAFT polymerization. Polymer (2007); 48: 5850-5858.

(43) C.J. Brinker, G.W. Scherer. Sol-gel science: the physics and chemistry of sol-gel processing. Academic Press. (1990). 
(44) D. Bimberg, M. Grundman, N.N. Ledenstov. Quantum Dot Heterostructures. Wiley, Chichester (1999).

(45) A. Mohammad, A Morshed, Md. Hasan Ikbal and S.M. Kamrul Hasan. Impact of Nanotechnology in the Arena of Textile Apparel Finishing. Textile Today, (2010); July-August.

(46) O. Masala, R. Seshadri; Synthesis routes for large volumes of nanoparticles, Annu. Rev. Mater. Res. (2004); 34: 41 - 81

(47) E. Arici, D. Meissner, F. Schäffler, N.S. Sariciftci. Core/shell nanomaterials in photovoltaics. International Journal of Photoenergy. (2003); 5: 199 - 208.

(48) M. Green. Solution routes to III-V semiconductor quantum dots. Current Opinion in Solid State and Material Science. (2002); 6: 355-363

(49) T. Sugimoto. Preparation of monodispersed colloidal particles. Advances in Colloid and Interface Science. (1987); 28: 65 - 108.

(50) I. Capek. Preparation of Metal Nanoparticles in Water-in-oil (w/o) Microemulsions. Adv. Colloid Interface Science, 110, 49-74 (2004).

(51) A. Henglein and M. Giersig; Formation of Colloidal Silver Nanoparticles: Capping Action of Citrate. J. Phys. Chem. B (1999), 103, 9533-9539.

(52) G. Kickelbick. Hybrid materials. Synthesis, Characterisation, and Application. Wiley-VCH Verlag, Weinheim, (2007).

(53) H. Forward, The Chemical and Physical Structure of Merino Wool, CSIRO.

(54) H. Lindley, Chemistry of Natural Fibres, Plenum Press, London (1977).

(55) G.A. Smook; Handbook for Pulp \& Paper Technolgists, Angus Wilde Publications (1992).

(56) H. Höcker, Wool: Science and Technology, W.S. Simpson and G.H. Crawshaw, Woolhead Publishing Limited, Cambridge (2002).

(57) J. Bradbury, The Structure and Chemistry of Keratin Fibres, in Andvances In Protein Chemistry, (1973); 111-210.

(58) G. Rogers, Annals of the New York Academy of Science, (1973); 83, 378-399.

(59) A. Haly, Textile Research Journal, (1957); 27: 82-84.

(60) J. Leeder, Wool Science Review, (1986); 63: 3-35.

(61) J. Church, G. Corino, and A. Woodhead, Biopolymers, (1996); 42(1): 7-17.

(62) A. Negri, H. Cornell, and D. Rivett, Textile Research Journal, (1993): 63(2): 109-115.

(63) N. King and J. Bradbury, Australian Journal of Biological Sciences, (1968); 21: 375-384.

(64) C. Carr, I. Leever, and A. Hughes, Textile Research Journal, (1986); 56: 457461.

(65) A. Negri, H. Cornell, and D. Rivett, Textile Research Journal, (1992); 62: 381387.

(66) D.H. Simmonds. Aust.J. Biol. Sci. (1955); 8: 537.

(67) G. Mazingue, P. Ponchel, and J. Lubrez, Proceedings from the Applied Polymer Conference, (1971).

(68) K. Markison and J. Leed, Textile Research Journal, (1973); 44: 856.

(69) L. Jones, D. Rivett, and D. Tucker, Wool and Related Mammalian Fibres, in Handbook of Fibre Chemistry, M. Lewin and E. Pearce, Marcel Dekker INC, New York, (1998).

(70) R. Crawford, Lignin Biodegration and Transformation, J. W. Sons Eds., New York, (1981).

(71) N.R. Jana, Z.L. Wang, T.K. Sau, T. Pal. Seed-mediated growth method to prepare cubic copper nanoparticles. Current Science: (2000); 79: 1367 - 1370. 
(72) S. Ram, C. Mitra. Formation of stable $\mathrm{Cu}_{2} \mathrm{O}$ nanocrystals in a new orthorhombic crystal structure. Material Science and Engineering (2001); A304-306: 805-809.

(73) S. Xu, X. Song, C. Fan, G. Chen, W. Zhao, T. You, S. Sun. Kinetically controlled synthesis of $\mathrm{Cu}_{2} \mathrm{O}$ microcrystals with various morphologies by adjusting pH value. Journal of Crystal Growth (2007); 305: 3-7.

(74) J. Zhu, Y. Wang, X. Wang, X. Yang, L. Lu. A conv. Method for preparing shape-controlled nanocrystalline $\mathrm{Cu}_{2} \mathrm{O}$ in a polyol or water/polyol system. Powder technology. (2008); 181: 249-254

(75) S. Anandan, S. Yang. Emergent methods to synthesize ad characterize semiconductor $\mathrm{CuO}$ nanoparticles with various morphologies -an overview. Journal of Experimental Nanoscience (2007); 2: 23-56.

(76) D. Bera, L. Qian, S. Sabui, S. Santra, P.H. Holloway. Photoluminescence of $\mathrm{ZnO}$ quantum dots produced by a sol-gel process. Optical Materials (2008); 30: 1233-1239.

(77) Kirill L. Levine, Jude O. Iroh and Peter B. Kosel. Synthesis and Properties of the Nanocomposite of Zinc Oxide and Poly(amic acid). Appl. Surf. Sci. (2004); 230, 24-33.

(78) M. Sato, A. Kawata, S. Morito, Y. Sato, I. Yamaguchi. Preparation and properties of polymer/zinc oxide nanocomposites using functionalized zinc oxide quantum dots. European Polymer Journal (2008); 44: 3430-3438.

(79) W. Yude, Z. Shuo, W. Xinghui, L. Qingju. Synthesis and optical properties of nano-ZnO particles/mesostructured $\mathrm{SnO}_{2}$ composite. Materials Chemistry and Physics (2006); 98: 121-124.

(80) H. Hu, W. Zhang. Synthesis and properties of transition metals and rare-earth metals doped ZnS nanoparticles. Optical Materials (2006); 28: 536-550.

(81) R. Kho, C.L. Torrez-Martínez, R.K. Mehra. A simple colloidal synthesis of gram-quality production of water-soluble ZnS nanocrystal powders. Journal of Colloidal and Interface Science (2000); 227: 561-566.

(82) W.Q. Peng, S.C. Qu, G.W. Cong, Z.G. Wang. Concentration effect of $\mathrm{Mn}^{2+}$ on the photoluminescence of ZnS:Mn nanocrystals. Journal of Crystal Growth (2005); 279: 454-460.

(83) T. Charinpanitkul, A. Chanagul, J. Dutta, U. Rungsardthong, W. Tanthapanichakoon. Effect of cosurfactant on ZnS nanoparticle synthesis in microemulsion. Science and Technology of Advance Materials (2005): 6: 266271.

(84) T. Trinade. Nanocrystalline Semiconductors: Synthesis, Properties and Perspective (review). Chem. Mater. (2001); 13: 3843-3858.

(85) M. Yin, C. Wu, Y. Lou, C. Burda, J.T. Koberstein, Y. Zhu, S. O’Brien. Copper oxide nanocrystals. Journal of the American Chemical Society (2005); 127: 9506-9511.

(86) F. Du, J. Liu, Z. Guo. Shape controlled synthesis of $\mathrm{Cu}_{2} \mathrm{O}$ and its catalytic application to synthesize amorphous carbon nanofibres. Materials Research Bulletin (2009); 44: 25-29.

(87) J.Y. Xiang, J.P. Tu, Y.F. Yuan, X.H. Huang, Y. Zhou, L. Zhang. Improved electrochemical performances of core-shell $\mathrm{Cu}_{2} \mathrm{O} / \mathrm{Cu}$ composite prepared by a simple one-step method. Electrochemistry Communications (2008); 11: 262265.

(88) Y.C. Zhang, J.Y. Tang, G.L. Wang, M. Zhang, X.Y. Hu. Facile synthesis of submicron $\mathrm{Cu}_{2} \mathrm{O}$ and $\mathrm{CuO}$ crystallites from a solid metallorganic molecular precursor. Journal of Crystal Growth (2006); 294: 278-282. 
(89) J.C. Park, J. Kim, H. Kwon, H. Song. Gram-scale synthesis of $\mathrm{Cu}_{2} \mathrm{O}$ nanocubes and subsequent oxidation to $\mathrm{CuO}$ hollow nanostructures for lithium-ion battery anode materials. Advance Materials (2008); 20: 1-5.

(90) Y. Lu, C. Chen, M.H. Lin. Effect of hydrogen plasma treatment on the electrical properties of sputtered N-doped cuprous oxide films. This Solid Films (2005); 480-481: 482-485.

(91) H. Li, Y. Ni, Y. Cai, L. Zhang, J. Zhou, J. Hong, X. Wei. Ultrasound-assisted preparation, characterisation and properties of porous $\mathrm{Cu}_{2} \mathrm{O}$ microtubes. Journal of Materials Chemistry (2009); 19: 594-597.

(92) D. Sun, N. Miyatake and Hung-Jue Sue; Transparent PMAA/ZnO nanocomposite films based on colloidal $\mathrm{ZnO}$ quantum dots. IOP Publishing, Nanotechnology. (2007); 18: 215606.

(93) R. Feign, D.H. Napper. Depletion stabilization and depletion flocculation. Journal of Colloidal and Interface Science. (1980); 75(2): 525 - 541.

(94) D.H. Napper, Polymeric Stabilization of Colloidal Dispersions. Academic Press, London. (1983).

(95) T. Sato, R. Ruch. Stabilization of colloidal dispersion by polymer adsorption. Marcel Dekker Inc., New York, (1980).

(96) E.J. Verwey, J.Th.G. Overbeek. Theory of the Stability of Lyophobic Colloids. Elsevier, Amsterdam. (1948).

(97) B.V. Derjaguin, L.Landau. DLVO forces. Acta Physiochim. URSS. (1941); 14: 633-662.

(98) A. Liang, Q. Liu, G. Weng, Z. Jiang. The surface-plasmon-resonance effect of nanogold/silver and its analytical applications. Elsevier Science B.V., Amsterdam. (2012); 37: 32 - 47.

(99) Younan Xia, Naomi J. Halas. Shape-Controlled Synthesis and Surface Plasmonic Properties of Metallic Nanostructures. Material Research Society Bulletin. (2005); 30(5): 338-348.

(100) D C. Daniel and D. Astruc. Gold nanoparticles: assembly, supramolecular chemistry, quantum-size-related properties, and applications toward biology, catalysis, and nanotechnology. Chemical Reviews. (2004); 104: 293-346

(101) P. Mulvaney, Langmuir. Surface Plasmon Spectroscopy of Nanosized Metal. Particles. (1996); 12: 788 - 800.

(102) O.A. Yeshchenko, I.M. Dmitruk, A.M. Dmytruk, A.A. Alexeenko. Influence of annealing conditions on size and optical properties of copper nanoparticles embedded in silica matrix. Materials Science and Engineering B (2007); 137: 247-254.

(103) D. Harnath, S. Sahai and P. Joshi. Tuning of emission in zinc oxide quantum dots. Appl. Physics letters. (2008); 92: 233113.

(104) K. Borgohain, S. Mahamuni. Luminescence behaviour of chemically grown ZnO quantum dots. Semiconductor Science and Technology (1998); 13: 11541157.

(105) S.D. Kshirsagar, V.V. Nikesh, S. Mahamuni. Exciton structure in sodium doped zinc oxide quantum dots. Applied physics letters (2006); 89: 053120.

(106) D.S. Bohle, C.J. Spina. The relationship of oxygen binding and peroxide sites and the fluorescent properties of zinc oxide semiconductor nanocrystals. Journal of the American Chemical Society (2007); 129: 12380-12381.

(107) Y. Fu, X. Du, S.A. Kulinich, J. Qiu, W. Qin, R. Li, J. Sun, J. Liu. Stable aqueous dispersion of $\mathrm{ZnO}$ quantum dots with strong blue emission via simple solution route. Journal of the American Chemical Society (2007); 129: 16029-16033. 
(108) D.W. Bahnemann, C. Kormann, M.R. Hoffmann. Preparation and characterization of quantum size zinc oxide: A detailed spectroscopy studie. J. Phys. Chem. (1987); 91: 3789-398.

(109) K.G. Kanade, B.B. Kale, J. Baeg, S.M. Lee, C.W. Lee, S, Moon, H. Chang. Self-assembled aligned $\mathrm{Cu}$ doped $\mathrm{ZnO}$ nanoparticles for photocatalytic hydrogen production under visible light radiation. Materials Chemistry and Physics (2007); 102: 98-104.

(110) C.L. Yang, J.N. Wang, W.K. Ge, L. Guo, S.H. Yang, D.Z. Shen. Enhanced ultraviolet emission and optical properties in polyvinyl pyrrolidone surface modified ZnO quantum dots. Journal of applied physics (2001); 90: 4489-4494.

(111) G. Kalyuzhny, R.W. Murray.Ligand effects on optical properties of CdSe nanocrystals. J. Phys. Chem. B. (2005); 109(15): 7012 - 7021.

(112) R.H. Wang, J.H. Xin, X.M. Tao. UV-blocking property of dumbbell-shaped ZnO crystallites on cotton fabrics. Inorganic Chemistry (2005); 44: 3926-3930.

(113) H. Koga, T. Kitaoka, H. Wariishi. In situ synthesis of silver nanoparticles on zinc oxide whiskers incorporates in a paper matrix for antibacterial applications. Journal of Materials Chemistry (2009); 19: 2135-2140.

(114) R. Wang, J.H. Xin, X.M. Tao, W.A. Daoud. ZnO nanorods grown on cotton fabrics at low temperature. Chemical Physics Letters (2004); 398: 250-255.

(115) H. Koga, T. Kitaoka, H. Wariishi. In situ synthesis of Cu nanocrystals on ZnO whiskers embedded in a microstructured paper composite for autothermal hydrogen production. Chemical Communications (2008); 43: 5616-5618.

(116) J. Leeder and J. Rippon, Journal of the Society of Dyers and Colourists, (1985); 101: 11-16.

(117) A. Negri, H. Cornell, and D. Rivett, Australian Journal of Agricultural Research, (1991); 42: 1285-1292.

(118) L. Jones and D. Rivett, Micron (1997); 28: 469-485.

(119) G. Giro, M. Cocchi, P. Di Marco. Role played by cell configuration and layer preparation in LEDs based on hydroxyquinoline metal complexes and a triphenyl-diamine derivative (TPD). Synthetic Metals (1999); 102,(1-3): 10181019.

(120) Y. Hamada, T. Sano, M. Fujita, T. Fujii, Y. Nishio, and K. Shibata. Organic electroluminescent devices with 8-hydroxyquinoline derivative-metal complexes as an emitter. Japanese Journal of Applied Physics, Part 2, (1993); 32(4): L514L515.

(121) M. Ghedini, M. La Deda, I. Aiello, and A. Grisolia. Synthesis and photophysical characterisation of soluble photoluminescent metal complexes with substituted 8-hydroxyquinolines. Synthetic Metals (2003); 138 (1-2): 189-192.

(122) H. Pan, F. Liang, C. Mao, J. Zhu, H. Chen. Highly luminescent Zinc (II) - Bis (8-hydroxyquinoline) complex nanorods: sonochemical synthesis, characterisation and protein sensing. Journal of Physical Chemistry, B (2007); 111: 5767-5772.

(123) F. Papadimitrakopoulos, X. Zhang and K.A. Higginson. Chemical and morphological stability of $\mathrm{Alq}_{3}$ : Effects in light-emitting devices. IEEE Journal of selected topics in quantum electronics (1998); 4: 49-57.

(124) V. Krishnakumar, R. Nagalakshmi, P. Janaki. Growth and spectroscopic characterisation of a new organic nonlinear optical crystal-8-hydroxyquinoline. Spectrochimica Acta, Part A (2005); 61: 1097-1103. 
(125) L. S. Sapochak, F. E. Benincasa, R. S. Schofield et al. Electroluminescent zinc(II) bis(8-hydroxyquinoline): structural effects on electronic states and device performance. Journal of the American Chemical Society (2002); 124 (21): 6119-6125.

(126) T. Förster. Energiewanderung und Fluoreszenz. Die Naturwissenschaften. (1946); 6: $166-175$.

(127) E. Margapoti, V. Shukla, A. Valore. Excimer emission in single layer electroluminescent devices based on [ir(4, 5-diphenyl-2-methylthiazolo) ${ }_{2}$ (5methyl-1, 10-phenanthroline) $]^{+}\left[\mathrm{PF}_{6}\right]^{-}$. Journal of Physical Chemistry C (2009); 113 (28): 12517-12522.

(128) M. Shimizu. T. Hiyama. Organic Fluorophores Exhibiting Highly Photoluminescence in the Solid State. Chem. Asain J. (2010); 5: 1516-1531.

(129) P.L. Choyke, R. Alford, H.M. Simpson, J. Duberman. Toxicity of organic fluorophores used in molecular imaging: literature review. Molecular Imaging. (2009); 8 (6): 341 - 354.

(130) J.R. Kanofsky and P.D. Sima. Structural and environmental requirements for quenching of singlet oxygen by cyanine dyes. Photochemistry and Photobiology. (2000); 71: 361-368.

(131) Y. Kai, M. Moraita, N. Yasuka, and N. Kasai, The crystal and molecular structure of anhydrous zinc 8-quinolinolate complex, $\left[\mathrm{Zn}\left(\mathrm{C}_{9} \mathrm{H}_{6} \mathrm{NO}\right)_{2}\right]_{4}$. Bulletin of the Chemical Society of Japan (1985); 58(6): 1631-1635.

(132) L. S. Sapochak, A. Falkowitz, K. F. Ferris, S. Steinberg, and P. E. Burrows. Supramolecular structures of zinc (II) (8-quinolinolato) chelates. Journal of Physical Chemistry B, (2004); 108 (25): 8558-8566.

(133) B. S. Xu, Y. Y. Hao, H. Wang, H. F. Zhou, X. G. Liu, and M. W. Chen. The effects of crystal structure on optical absorption/photoluminescence of bis(8hydroxyquinoline)zinc. Solid State Communications, (2005); 136 (6): 318-322.

(134) R. Kienle, G. Royer, and H. McCleary, American Association of Textile Chemists and Colorists, (1945); 42-53.

(135) M. Martí, R. Ramirez, C. Barba, L. Coderch, J. Parra. Influence of Internal Lipid on Dyeing of Wool fibres. Textile Research Journal, (2010); 80(4): 365-373.

(136) P.R. Brady. Penetration Pathways of Dyes into Natural Protein Fibres. Proceedings of $8^{\text {th }}$ Int. Wool Text. Res. Conf., Christchurch, NZ, February (1990); 4: 217-226.

(137) V. K. Shukla, J. Maitra. Investigations of Different Phases Responsible for Changes in Optical Properties of Organic Semiconducting Device Material Thin Films. Journal of Materials. (2013); Article ID 690237, 5 pages.

(138) B. Patkowska-Sokola, Z. Dobrzanski, K. Osman, R. Bodkowski, K. Zygadlik.The content of chosen chemical elements in wool of sheep of different origins and breeds. Archiv Tierzucht 52 (2009); 4: 410-418.

(139) V. Prachayastittiku, S. Prachayasittikul, S. Ruchirawat, V.Prachayasittikul. 8Hydroxyquinolines: a review of their metal chelating properties and medicinal applications. Drug Des Devel Ther. (2013); 7: 1157 - 1178. 


\section{Appendix}

\section{List of samples}

Table A.1: $\mathrm{Cu}_{2} \mathrm{O}$ quantum dots samples prepared according to a wet chemical reduction method (see chapter 2; 2.1.3.1). Variable process parameter: trisodium citrate concentrations. Constant process parameters: $\mathrm{T}=99^{\circ} \mathrm{C}$ and stirring time $=30 \mathrm{~min}$.

\begin{tabular}{|c|c|c|c|c|c|}
\hline$\left[\mathrm{Na}_{3} \mathrm{C}_{6} \mathrm{H}_{5} \mathrm{O}_{7}\right] / \mathrm{M}$ & $0.3 \cdot 10^{-3}$ & $0.33 \cdot 10^{-3}$ & $0.36 \cdot 10^{-3}$ & $0.39 \cdot 10^{-3}$ & $0.48 \cdot 10^{-3}$ \\
\hline $\mathrm{V}\left(\mathrm{H}_{2} \mathrm{O}\right) / \mathrm{mL}$ & 46 & 45.95 & 45.9 & 45.85 & 45.7 \\
\hline $\mathrm{V}\left(0.03 \mathrm{M} \mathrm{Na}_{3} \mathrm{C}_{6} \mathrm{H}_{5} \mathrm{O}_{7} \cdot 2 \mathrm{H}_{2} \mathrm{O}(\right.$ aq. $\left.)\right) / \mathrm{mL}$ & 0.5 & 0.55 & 0.6 & 0.65 & 0.8 \\
\hline $\mathrm{V}\left(0.01 \mathrm{M} \mathrm{Cu}\left(\mathrm{NO}_{3}\right)_{2}(\mathrm{aq}).\right) / \mathrm{mL}$ & 2.5 & 2.5 & 2.5 & 2.5 & 2.5 \\
\hline \multirow{2}{*}{$\begin{array}{l}\mathrm{V}(5 \mathrm{ppm} \mathrm{PMAA-Na(aq.))} / \mathrm{mL} \\
\mathrm{V}\left(2 \mathrm{M} \mathrm{NaBH}{ }_{4} \text { in triethyl glycole dimethyl- }\right. \\
\text { ether })) / \mathrm{mL}\end{array}$} & 0.5 & 0.5 & 0.5 & 0.5 & 0.5 \\
\hline & 0.5 & 0.5 & 0.5 & 0.5 & 0.5 \\
\hline
\end{tabular}

Table A.2: $\mathrm{Cu}_{2} \mathrm{O}$ quantum dots samples prepared according to a wet chemical reduction method (see chapter 2; 2.1.3.1). Variable process parameters: copper salt precursor, polymeric dispersant and reaction temperature. Constant process parameter: stirring time $=\mathbf{3 0} \mathrm{min}$.

\begin{tabular}{|c|c|c|c|}
\hline Sample name & A & B & C \\
\hline $\mathrm{V}\left(\mathrm{H}_{2} \mathrm{O}\right) / \mathrm{mL}$ & 46.25 & 46.25 & 46.25 \\
\hline $\mathrm{V}\left(0.03 \mathrm{M} \mathrm{Na}_{3} \mathrm{C}_{6} \mathrm{H}_{5} \mathrm{O}_{7} \cdot 2 \mathrm{H}_{2} \mathrm{O}\right.$ (aq. $\left.)\right) / \mathrm{mL}$ & 0.5 & 0.5 & 0.5 \\
\hline $\mathrm{V}\left(0.01 \mathrm{M} \mathrm{Cu}\left(\mathrm{NO}_{3}\right)_{2}\right.$ (aq. $\left.)\right) / \mathrm{mL}$ & 2.5 & 0 & 2.5 \\
\hline $\mathrm{V}\left(0.01 \mathrm{M} \mathrm{CuCl}_{2}(\right.$ aq. $\left.)\right) / \mathrm{mL}$ & 0 & 2.5 & 0 \\
\hline $\mathrm{V}(5 \mathrm{ppm}$ PMAA-Na(aq.)) /mL & 0.5 & 0.5 & 0 \\
\hline $\mathrm{V}(5 \mathrm{ppm} \operatorname{PVP}(\mathrm{aq}).) / \mathrm{mL}$ & 0 & 0 & 0.5 \\
\hline $\begin{array}{l}\mathrm{V}\left(2 \mathrm{M} \mathrm{NaBH}_{4} \text { in triethyl glycole }\right. \\
\text { dimethylether })) / \mathrm{mL}\end{array}$ & 0.25 & 0.25 & 0.25 \\
\hline Reaction temperature $/{ }^{\circ} \mathrm{C}$ & 96 & 99 & 99 \\
\hline
\end{tabular}


Table A.3: $\mathrm{Cu}_{2} \mathrm{O}$ quantum dots samples prepared according to a wet chemical reduction method (see chapter 2; 2.1.3.1). Variable process parameters: copper salt precursor, polymeric dispersant and reaction temperature. Constant process parameter: stirring time $=\mathbf{3 0} \mathbf{m i n}$.

\begin{tabular}{|c|c|c|}
\hline Sample name & A & B \\
\hline $\mathrm{V}\left(\mathrm{H}_{2} \mathrm{O}\right) / \mathrm{mL}$ & 45.7 & 44.7 \\
\hline $\mathrm{V}\left(0.01 \mathrm{M} \mathrm{Cu}\left(\mathrm{NO}_{3}\right)_{2}\right.$ (aq. $\left.)\right) / \mathrm{mL}$ & 3 & 3 \\
\hline V(5 ppm PMAA-Na(aq.)) / mL & 0.5 & 0.5 \\
\hline $\mathrm{V}(0.01 \mathrm{M} \mathrm{ZnCl}$ (aq.)) $/ \mathrm{mL}$ & 0 & 1 \\
\hline $\mathrm{V}\left(0.01 \mathrm{M} \mathrm{NiCl}_{2}\right.$ (aq.) $) / \mathrm{mL}$ & 0.3 & 0.3 \\
\hline $\mathrm{V}\left(1 \mathrm{M} \mathrm{NaBH}_{4}\right.$ (aq.) $) / \mathrm{mL}$ & 0.5 & 0.5 \\
\hline Reaction temperature $/{ }^{\circ} \mathrm{C}$ & 99 & $100 *$ \\
\hline Stirring time / min & 30 & 30 \\
\hline
\end{tabular}

* Reaction medium was refluxed at $100^{\circ} \mathrm{C}$ using overheated silicone oil bath $\left(\mathrm{T} \sim 140^{\circ} \mathrm{C}\right)$

Table A.4: $\mathrm{Cu}_{2} \mathrm{O}$ quantum dots samples prepared according to a wet chemical reduction method (see chapter 2; 2.1.3.1). Variable process parameter: sodium borohydride. Constant process parameters: $\mathrm{T}=99^{\circ} \mathrm{C}$ and stirring time $=30 \mathrm{~min}$.

\begin{tabular}{|c|c|c|c|c|c|c|c|}
\hline Sample name & A & B & C & $\mathrm{D}$ & E & $E^{*}$ & $\begin{array}{c}\mathrm{E}+ \\
\mathrm{NaOH}\end{array}$ \\
\hline $\mathrm{V}\left(\mathrm{H}_{2} \mathrm{O}\right) / \mathrm{mL}$ & 46.3 & 46.25 & 46.2 & 46.1 & 46 & 46 & 46 \\
\hline $\mathrm{V}\left(0.03 \mathrm{M} \mathrm{Na}_{3} \mathrm{C}_{6} \mathrm{H}_{5} \mathrm{O}_{7} \cdot 2 \mathrm{H}_{2} \mathrm{O}(\right.$ aq. $\left.)\right) / \mathrm{mL}$ & 0.5 & 0.5 & 0.5 & 0.5 & 0.5 & 0.5 & 0.5 \\
\hline $\mathrm{V}\left(0.01 \mathrm{M} \mathrm{Cu}\left(\mathrm{NO}_{3}\right)_{2}\right.$ (aq.) $) / \mathrm{mL}$ & 2.5 & 2.5 & 2.5 & 2.5 & 2.5 & 0 & 2.5 \\
\hline $\mathrm{V}\left(0.01 \mathrm{M} \mathrm{CuCl}_{2}\right.$ (aq.)) $/ \mathrm{mL}$ & 0 & 0 & 0 & 0 & 0 & 2.5 & 0 \\
\hline V(5 ppm PMAA-Na(aq.))/mL & 0.5 & 0.5 & 0.5 & 0.5 & 0.5 & 0.5 & 0.5 \\
\hline $\mathrm{V}\left(1 \mathrm{M} \mathrm{NaBH}_{4}(\mathrm{aq}).\right) / \mathrm{mL}$ & 0.2 & 0.25 & 0.3 & 0.4 & 0.5 & 0.5 & 0.5 \\
\hline $\mathrm{V}(0.01 \mathrm{M} \mathrm{NaOH}(\mathrm{aq}).) / \mathrm{mL}$ & 0 & 0 & 0 & 0 & 0 & 0 & 0.25 \\
\hline
\end{tabular}

Table A.5: $\mathrm{Cu}_{2} \mathrm{O}$ quantum dots samples doped with impurities (see chapter 2; 2.1.3.1). Variable process parameters: dopants, stirring time and addition of trisodium citrate. Constant process parameter: $\mathrm{T}=99^{\circ} \mathrm{C}$.

\begin{tabular}{|c|c|c|c|c|}
\hline Sample name & $\mathrm{Zn} / \mathrm{Mn}$ & $\mathrm{Zn} / \mathrm{Co}$ & $\mathrm{Zn}$ & Zn/Citrate \\
\hline $\mathrm{V}\left(\mathrm{H}_{2} \mathrm{O}\right) / \mathrm{mL}$ & 44.7 & 44.7 & 45 & 42.5 \\
\hline $\mathrm{V}\left(0.03 \mathrm{M} \mathrm{Na}_{3} \mathrm{C}_{6} \mathrm{H}_{5} \mathrm{O}_{7} \cdot 2 \mathrm{H}_{2} \mathrm{O}(\mathrm{aq}).\right) / \mathrm{mL}$ & 0 & 0 & 0 & 0.5 \\
\hline $\mathrm{V}\left(0.01 \mathrm{M} \mathrm{Cu}\left(\mathrm{NO}_{3}\right)_{2}\right.$ (aq.) $) / \mathrm{mL}$ & 3 & 3 & 3 & 3 \\
\hline V(5 ppm PMAA-Na(aq.)) / mL & 0.5 & 0.5 & 0.5 & 0.5 \\
\hline $\mathrm{V}\left(0.01 \mathrm{M} \mathrm{ZnCl}_{2}\right.$ (aq.)) $/ \mathrm{mL}$ & 1 & 1 & 1 & 3 \\
\hline $\mathrm{V}\left(0.01 \mathrm{M} \mathrm{NiCl}_{2}\right.$ (aq.) $) / \mathrm{mL}$ & 0 & 0 & 0 & 0 \\
\hline $\mathrm{V}\left(0.01 \mathrm{M} \mathrm{MnCl}_{2}\right.$ (aq.) $) / \mathrm{mL}$ & 0.3 & 0 & 0 & 0 \\
\hline $\mathrm{V}\left(0.01 \mathrm{M} \mathrm{CoCl}_{2}\right.$ (aq.) $) / \mathrm{mL}$ & 0 & 0.3 & 0 & 0 \\
\hline $\mathrm{V}\left(1 \mathrm{M} \mathrm{NaBH}{ }_{4}(\mathrm{aq}).\right) / \mathrm{mL}$ & 0.5 & 0.5 & 0.5 & 0.5 \\
\hline Stirring time / min & $10 / 30$ & $10 / 30$ & 30 & 30 \\
\hline
\end{tabular}


Table A.6: ZnO quantum dots samples prepared according to a sol-gel method (see chapter 2; 2.1.3.2). Variable process parameter: borohydride precursor. Constant process parameters: $\mathbf{T}=\mathbf{7 0}$ ${ }^{\circ} \mathrm{C}$ and stirring time $=\mathbf{3 0} \mathrm{min}$.

\begin{tabular}{|c|c|c|}
\hline Sample name & $A$ & B \\
\hline $\mathrm{V}\left(\mathrm{H}_{2} \mathrm{O}\right) / \mathrm{mL}$ & 71.5 & 71.5 \\
\hline $\mathrm{V}(1 \mathrm{M} \mathrm{ZnCl}$ (aq.) $) / \mathrm{mL}$ & 1 & 1 \\
\hline V(5 ppm PVP(aq.)) /mL & 2 & 2 \\
\hline $\begin{array}{l}\mathrm{V}\left(2 \mathrm{M} \mathrm{NaBH}_{4} \text { in triethyl glycole }\right. \\
\text { dimethylether })) / \mathrm{mL}\end{array}$ & 0.5 & 0 \\
\hline V(2 M LiBH ${ }_{4}$ in THF)) /mL & 0 & 0.5 \\
\hline
\end{tabular}

Table A.7: ZnO quantum dots samples prepared according to a sol-gel method (see chapter 2; 2.1.3.2). Variable process parameter: polymeric dispersant. Constant process parameters: $\mathrm{T}=70^{\circ} \mathrm{C}$ and stirring time $=\mathbf{3 0} \mathrm{min}$.

\begin{tabular}{|c|c|c|c|}
\hline Sample name & A & B & C \\
\hline $\mathrm{V}\left(\mathrm{H}_{2} \mathrm{O}\right) / \mathrm{mL}$ & 71.5 & 71.5 & 71.5 \\
\hline $\mathrm{V}\left(1 \mathrm{M} \mathrm{ZnCl}{ }_{2}(\mathrm{aq}).\right) / \mathrm{mL}$ & 1 & 1 & 1 \\
\hline V(5 ppm PVP(aq.)) /mL & 2 & 0 & 1 \\
\hline V(5 ppm PMAA-Na(aq.)) /mL & 0 & 2 & 1 \\
\hline $\begin{array}{l}\mathrm{V}\left(2 \mathrm{M} \mathrm{NaBH}_{4} \text { in triethyl glycole }\right. \\
\text { dimethylether })) / \mathrm{mL}\end{array}$ & 0.5 & 0.5 & 0.5 \\
\hline
\end{tabular}

Table A.8: ZnO quantum dots samples prepared according to a sol-gel method (see chapter 2; 2.1.3.2). Variable process parameter: dopants. Constant process parameters: $\mathrm{T}=70^{\circ} \mathrm{C}$ and stirring time $=30 \mathrm{~min}$.

\begin{tabular}{lcc}
\hline Sample name & ZnO & ZnO/Ce \\
\hline $\mathrm{V}\left(\mathrm{H}_{2} \mathrm{O}\right) / \mathrm{mL}$ & 71.5 & 70.5 \\
$\mathrm{~V}(1 \mathrm{M} \mathrm{ZnCl}$ (aq.) $) / \mathrm{mL}$ & 1 & 1 \\
$\mathrm{~V}(5 \mathrm{ppm} \mathrm{PVP(aq.)}) / \mathrm{mL}$ & 2 & 2 \\
$\mathrm{~V}\left(0.01 \mathrm{M} \mathrm{CeCl}_{2}(\mathrm{aq}).\right) / \mathrm{mL}$ & 0 & 1 \\
$\mathrm{~V}\left(2 \mathrm{M} \mathrm{NaBH}_{4}\right.$ in triethyl glycole & 0.5 & 0.5 \\
dimethylether $)) / \mathrm{mL}$ & & \\
\hline
\end{tabular}

Table A.9: ZnO quantum dots samples prepared according to a sol-gel method (see chapter 2; 2.1.3.2). Variable process parameter: PVP concentration. Constant process parameters: $\mathrm{T}=70{ }^{\circ} \mathrm{C}$ and stirring time $=30 \mathrm{~min}$.

\begin{tabular}{lccccc}
\hline Sample name & A & B & C & D & $E$ \\
\hline $\mathrm{V}\left(\mathrm{H}_{2} \mathrm{O}\right) / \mathrm{mL}$ & 72 & 71.5 & 71 & 70.5 & 68.5 \\
$\mathrm{~V}(1 \mathrm{M} \mathrm{ZnCl}($ aq. $)) / \mathrm{mL}$ & 1 & 1 & 1 & 1 & 1 \\
$\mathrm{~V}(5 \mathrm{ppm} \mathrm{PVP}($ aq. $)) / \mathrm{mL}$ & 1.5 & 2 & 2.5 & 3 & 5 \\
$\mathrm{~V}\left(2 \mathrm{M} \mathrm{NaBH}_{4}\right.$ in triethyl glycole & 0.5 & 0.5 & 0.5 & 0.5 & 0.5 \\
dimethylether $)) / \mathrm{mL}$ & & & &
\end{tabular}


Table A.10: ZnO quantum dots samples prepared according to a sol-gel method (see chapter 2; 2.1.3.2). Variable process parameter: $\mathrm{NaBH}_{4}$ concentration. Constant process parameters: $\mathrm{T}=$ $70^{\circ} \mathrm{C}$ and stirring time $=30 \mathrm{~min}$.

\begin{tabular}{|c|c|c|c|c|}
\hline Sample name & A & B & $\mathrm{C}$ & D \\
\hline $\mathrm{V}\left(\mathrm{H}_{2} \mathrm{O}\right) / \mathrm{mL}$ & 71.75 & 71.5 & 71.25 & 71 \\
\hline $\mathrm{V}(1 \mathrm{M} \mathrm{ZnCl}$ (aq.) $) / \mathrm{mL}$ & 1 & 1 & 1 & 1 \\
\hline V(5 ppm PVP(aq.)) /mL & 2 & 2 & 2 & 2 \\
\hline $\begin{array}{l}\mathrm{V}\left(2 \mathrm{M} \mathrm{NaBH}_{4} \text { in triethyl glycole }\right. \\
\text { dimethylether) }) / \mathrm{mL}\end{array}$ & 0.25 & 0.5 & 0.75 & 1 \\
\hline
\end{tabular}

Table A.11: ZnS quantum dots samples prepared according to a wet chemical precipitation method (see chapter 2; 2.1.3.3). Variable process parameter: dopants.

\begin{tabular}{|c|c|c|c|c|c|}
\hline & Sample name & A & $\mathrm{B}$ & $\mathrm{C}$ & D \\
\hline \multirow[t]{9}{*}{$1^{\text {st }}$ step } & $\mathrm{V}\left(\mathrm{H}_{2} \mathrm{O}\right) / \mathrm{mL}$ & 37.8 & 46.3 & 46.3 & 45 \\
\hline & $\mathrm{V}\left(0.01 \mathrm{M} \mathrm{Cu}\left(\mathrm{NO}_{3}\right)_{2}\right.$ (aq. $\left.)\right) / \mathrm{mL}$ & 0 & 3 & 0 & 0 \\
\hline & $\mathrm{V}\left(0.01 \mathrm{M} \mathrm{MnCl}_{2}\right.$ (aq.) $) / \mathrm{mL}$ & 0 & 0 & 3 & 3 \\
\hline & $\mathrm{V}(5 \mathrm{ppm}$ PMAA-Na(aq.)) /mL & 2 & 0.5 & 0.5 & 0.5 \\
\hline & $\mathrm{V}\left(0.1 \mathrm{M} \mathrm{ZnCl}_{2}\right.$ (aq.) $) / \mathrm{mL}$ & 10 & 0 & 0 & 1 \\
\hline & $\mathrm{V}\left(0.01 \mathrm{M} \mathrm{NiCl}_{2}\right.$ (aq.) $) / \mathrm{mL}$ & 0 & 0 & 0 & 0.3 \\
\hline & $\mathrm{V}\left(2 \mathrm{M} \mathrm{NaBH}_{4}\right.$ in triethyl glycole dimethylether $\left.)\right) / \mathrm{mL}$ & 0.2 & 0.2 & 0.2 & 0.2 \\
\hline & Reaction temperature $/{ }^{\circ} \mathrm{C}$ & 70 & 99 & 99 & 99 \\
\hline & Stirring time / min & 5 & 5 & 5 & 5 \\
\hline \multirow[t]{3}{*}{$2^{\text {nd }}$ step } & $\mathrm{V}(1 \mathrm{M} \mathrm{ZnCl}$ (aq.) $) / \mathrm{mL}$ & 5 & 5 & 5 & 5 \\
\hline & $\mathrm{V}\left(1 \mathrm{M} \mathrm{Na}_{2} \mathrm{~S}\right.$ (aq. $\left.)\right) / \mathrm{mL}$ & 5 & 5 & 5 & 5 \\
\hline & Reaction temperature ${ }^{*(1)} /{ }^{\circ} \mathrm{C}$ & 70 & 99 & 99 & 99 \\
\hline
\end{tabular}


Table A.12: $\mathrm{ZnO}$ quantum dot - wool fibre composites developed according to the in-situ dyeing method (see chapter 2; 2.2.1). Variable process parameter: capping agents. Constant process parameters: $\mathrm{m}($ wool $)=0.2 \mathrm{~g}, \mathrm{~V}_{\text {tot }}=50 \mathrm{~mL}$, soaking temperature $=70^{\circ} \mathrm{C}$ for $24 \mathrm{hrs}$ and then room temperature for 7 days.

\begin{tabular}{|c|c|c|c|}
\hline Sample name & $\begin{array}{l}\mathrm{ZnO} \text { - wool } \\
\text { (L-cysteine) }\end{array}$ & $\begin{array}{l}\mathrm{ZnO}-\text { wool } \\
\text { (PMAA-Na) }\end{array}$ & ZnO - wool (PVP) \\
\hline $\mathrm{V}\left(\mathrm{H}_{2} \mathrm{O}\right) / \mathrm{mL}$ & 48 & 48 & 48 \\
\hline $\mathrm{m}$ (merino wool) / g & 0.2 & 0.2 & 0.2 \\
\hline $\mathrm{V}(1 \mathrm{M} \mathrm{ZnCl}$ (aq.)) /mL & 1 & 1 & 1 \\
\hline V(5 ppm PVP(aq.)) /mL & 0 & 0 & 2 \\
\hline $\mathrm{V}(5 \mathrm{ppm}$ PMAA-Na(aq.)) /mL & 0 & 2 & 0 \\
\hline V(0.005 M L-cysteine (aq.) / mL & 0.5 & 0 & 0 \\
\hline $\begin{array}{l}\mathrm{V}\left(2 \mathrm{M} \mathrm{NaBH}_{4} \text { in triethyl glycole }\right. \\
\text { dimethylether) }) / \mathrm{mL}\end{array}$ & 0.5 & 0.5 & 0.5 \\
\hline Soaking temperature $/{ }^{\circ} \mathrm{C}$ & $\begin{array}{l}70^{\star(1)} \\
70^{\star(2)} \\
\mathrm{RT}^{\star(3)}\end{array}$ & $\begin{array}{l}70^{\star(1)} \\
70^{\star(2)} \\
\mathrm{R}^{\star}{ }^{\star(3)}\end{array}$ & $\begin{array}{l}70^{\star(1)} \\
70^{\star(2)} \\
\mathrm{RT}^{\star(3)}\end{array}$ \\
\hline
\end{tabular}

${ }^{*(1)}$ : wool was soaked in $0.02 \mathrm{M} \mathrm{ZnCl}_{2}$ aqua solution at $70{ }^{\circ} \mathrm{C}$ for $3 \mathrm{hrs}$

*(2): wool was soaked in the reaction medium at $70{ }^{\circ} \mathrm{C}$ for 24 hrs after the addition of $0.5 \mathrm{~mL} 2 \mathrm{M} \mathrm{NaBH}_{4}$

${ }^{*}(3)$ : wool was soaked in the reaction medum at room temperature (RT) for 7 days after soaking for $24 \mathrm{hrs}$ at $70{ }^{\circ} \mathrm{C}$

Table A.13: ZnO quantum dot - wool fibre composites developed according to the building block dyeing method (chapter 2; 2.2.2.3). Variable process parameter: merino wool (untreated and pretreated with $\mathrm{NaOH}, \mathrm{HCl}$, MSA and 8-HQ). Constant process parameters: $\mathrm{m}($ wool $)=0.1 \mathrm{~g}, \mathrm{~V}_{\text {tot }}=20$ $\mathrm{mL}$ and soaking temperature $=70^{\circ} \mathrm{C}$ for 3 days.

\begin{tabular}{lc}
\hline Sample name & ZnO-wool \\
\hline $\mathrm{V}(\text { ZnO suspension })^{\star(1)} / \mathrm{mL}$ & 20 \\
$\mathrm{~m}(\text { merino wool })^{\star(2)} / \mathrm{g}$ & 0.1 \\
Soaking temperature $/{ }^{\circ} \mathrm{C}$ & 70 \\
Soaking time $/$ days & 3 \\
\hline
\end{tabular}

${ }^{(1)}$ : ZnO quantum dot suspension was obtained using procedure described in chapter 2; 2.1.3.2.

${ }^{*}{ }^{(2)}$ : merino wool untreated and pretreated with $\mathrm{NaOH}, \mathrm{HCl}$. Pre-treatment procedure described in chapter 2; 2.2.2.1

Table A.14: 8-HQ - wool fibre composites (see preparation in chapter 2; 2.2.2.1.4). Variable process parameter: 8-HQ uptake. Constant process parameters: $\mathrm{m}($ wool $)=0.1 \mathrm{~g}, \mathrm{~V}_{\text {tot }}=10 \mathrm{~mL}$ and soaking temperature $=50^{\circ} \mathrm{C}$ for 1 day.

\begin{tabular}{lcc}
\hline Sample name & wt $(8-\mathrm{HQ})=0.15 \% \mathrm{wt}(8-\mathrm{HQ})=0.35 \%$ \\
\hline $\mathrm{V}(\mathrm{EtOH}) / \mathrm{mL}$ & 9.9 & 9.75 \\
$\mathrm{~m}($ merino wool $) / \mathrm{g}$ & 0.1 & 0.1 \\
$\mathrm{~V}(0.001 \mathrm{M}$ 8- $\mathrm{HQ}(\mathrm{EtOH})) / \mathrm{mL}$ & 0.1 & 0.25 \\
Soaking temperature $/{ }^{\circ} \mathrm{C}$ & 50 & 50 \\
Soaking time $/$ hrs & 24 & 24 \\
\hline
\end{tabular}


Table A15: Surface modification of ZnO quantum dots with MSA-wool and 8-HQ.

\begin{tabular}{|c|c|c|}
\hline Sample name & A & B \\
\hline $\mathrm{V}(\mathrm{ZnO} \text { suspension })^{\star^{(1)}} / \mathrm{mL}$ & 75 & 75 \\
\hline $\mathrm{m}$ (untreated wool) / g & 0.5 & 0 \\
\hline $\mathrm{V}\left(0.5 \mathrm{M} \mathrm{MSA}\left(\mathrm{H}_{2} \mathrm{O}\right)\right) / \mathrm{mL}$ & 0.5 & 0 \\
\hline $\mathrm{V}(0.025 \mathrm{M}$ 8-HQ (EtOH)) / mL & 0 & 0.5 \\
\hline Soaking temperature $/{ }^{\circ} \mathrm{C}$ & $\mathrm{RT}^{\star(3)}$ & 70 \\
\hline Soaking time / days & 7 & 0 \\
\hline
\end{tabular}

Table A.16: ZnO quantum dot - wool fibre composites functionalised with 8-HQ (procedure described in chapter 2; 2.2.2.3). Variable process parameter: 8-HQ concentration. Constant process parameters: $m(w o o l)=0.1 \mathrm{~g}, V_{\text {tot }}=20 \mathrm{~mL}$ and soaking temperature $=50^{\circ} \mathrm{C}$ for 3 days.

\begin{tabular}{lc}
\hline Sample name & $\begin{array}{c}(\mathrm{ZnO}-\text { wool })+ \\
8-\mathrm{HQ}\end{array}$ \\
\hline $\mathrm{V}(\mathrm{EtOH}) / \mathrm{mL}$ & 19 \\
$\mathrm{~m}(\mathrm{ZnO}-\mathrm{wool}){ }^{*(1)} / \mathrm{g}$ & 0.1 \\
$\mathrm{~V}(0.025 \mathrm{M}$ 8- $\mathrm{HQ}(\mathrm{EtOH})) / \mathrm{mL}$ & 1 \\
Soaking temperature $/{ }^{\circ} \mathrm{C}$ & 50 \\
Soaking time / days & 3
\end{tabular}

${ }^{*}{ }^{(1)} \mathrm{ZnO}$ - wool was prepared according to the building block approach described in chapter 2.2.2.3. The details of the $\mathrm{ZnO}$-wool sample preparation are shown in table A.13.

Table A.17: ZnO quantum dot - paper fibre composites developed according to the building block dyeing methods (see chapter 2 ; 2.2.2.4). Variable process parameter: 8-HQ concentration and soaking temperature. Constant process parameters: $\mathrm{m}$ (paper) $=0.1 \mathrm{~g}$ and $\mathrm{V}_{\text {tot }}=80 \mathrm{~mL}$.

\begin{tabular}{|c|c|c|c|c|}
\hline Sample name & $\begin{array}{c}\text { ZnO- } \\
\text { paper - A }\end{array}$ & B & C & $\mathrm{D}$ \\
\hline $\mathrm{m}$ (bleached Kraft paper) ${ }^{*^{(1)}} / \mathrm{g}$ & 0.1 & 0.1 & 0.1 & 0.1 \\
\hline $\mathrm{V}(\mathrm{ZnO} \text { suspension })^{\star(2)} / \mathrm{mL}$ & 75 & 75 & 75 & 75 \\
\hline V(0.025 M 8-HQ (EtOH)) / mL & 0 & 0.1 & 1 & 1 \\
\hline Soaking temperature $/{ }^{\circ} \mathrm{C}$ & 70 & 70 & 70 & 100 \\
\hline Soaking time & 7 days $^{\star(3)}$ & $30 \mathrm{~min}$ & $30 \mathrm{~min}$ & $30 \mathrm{~min}$ \\
\hline
\end{tabular}

${ }^{*}{ }^{(1)}: 0.1 \mathrm{~g}$ bleached paper was converted into paper pulp using $5 \mathrm{~mL}$ water and Turrax high speed homogenizer before dispersing in the $\mathrm{ZnO}$ quantum dot suspension

${ }^{*}{ }^{(2)}$ : ZnO quantum dot suspension was obtained using procedure described in chapter 2; 2.1.3.2.

${ }^{*}{ }^{(3)}$ : Paper pulp in $\mathrm{ZnO}$ quantum dot suspension was stirred for $30 \mathrm{~min}$ at $70{ }^{\circ} \mathrm{C}$, cooled down and then gentle agitated for 7 days at room temperature. 
Table A.18: Preparation of 0.001 M Zn(8-Q) $)_{2} \cdot 2 \mathrm{H}_{2} \mathrm{O}$ in EtOH (A) and $\mathrm{H}_{2} \mathrm{O}$ (B) solution. Constant process parameter: $\mathrm{V}_{\text {tot }}=10 \mathrm{~mL}$.

\begin{tabular}{|c|c|c|}
\hline Sample name & A & B \\
\hline $\mathrm{V}(\mathrm{EtOH}) / \mathrm{mL}$ & 8 & 0 \\
\hline $\mathrm{V}\left(\mathrm{H}_{2} \mathrm{O}\right) / \mathrm{mL}$ & 0 & 8 \\
\hline $\mathrm{V}\left(0.01 \mathrm{M} \mathrm{ZnCl} \mathrm{EtOH}_{2}\right) / \mathrm{mL}$ & 1 & 0 \\
\hline $\mathrm{V}\left(0.01 \mathrm{M} \mathrm{ZnCl}_{2}\left(\mathrm{H}_{2} \mathrm{O}\right)\right) / \mathrm{mL}$ & 0 & 1 \\
\hline $\mathrm{V}(0.025 \mathrm{M} 8-\mathrm{HQ}(\mathrm{EtOH}))^{\star(1)} / \mathrm{mL}$ & 1 & 1 \\
\hline
\end{tabular}

${ }^{*(1)}$ : For the preparation of $0.001 \mathrm{M} \mathrm{Zn}(8-\mathrm{Q})_{2} \cdot 2 \mathrm{H}_{2} \mathrm{O}$ solutions, a slightly higher concentration of 8-HQ was used to ensure that all zinc cation are complexed with 8-HQ

Table A.19: List of 8-HQ - wool fibre composites with different uptake of 8-HQ (EtOH) onto wool (see procedure in chapter 2; 2.3.4). Variable process parameter: 8-HQ uptake. Constant process parameters: $\mathrm{m}($ wool $)=0.1 \mathrm{~g}, \mathrm{~V}_{\text {tot }}=10 \mathrm{~mL}$, solvent $=\mathrm{EtOH}$ and soaking temperature $=50{ }^{\circ} \mathrm{C}$ for 3 days.

\begin{tabular}{|c|c|c|c|c|c|c|c|}
\hline \multirow{2}{*}{$\frac{\text { Sample name }}{\mathrm{V}(\mathrm{EtOH}) / \mathrm{mL}}$} & \multicolumn{7}{|c|}{$\begin{array}{l}\text { wt(8-HQ) wt(8-HQ) wt(8-HQ) wt(8-HQ) wt(8-HQ) wt(8-HQ) wt(8-HQ) } \\
=0.15 \%=0.35 \%=0.7 \%=1.0 \%=1.5 \%=1.8 \%=2 \%\end{array}$} \\
\hline & 9.9 & 9.75 & 9.5 & 9.25 & 9.0 & 8.75 & 8.5 \\
\hline $\mathrm{m}$ (merino wool) / g & 0.1 & 0.1 & 0.1 & 0.1 & 0.1 & 0.1 & 0.1 \\
\hline V(0.001 M 8-HQ (EtOH)) / mL & 0.1 & 0.25 & 0.5 & 0.75 & 1.0 & 1.25 & 1.5 \\
\hline Soaking temperature $/{ }^{\circ} \mathrm{C}$ & 50 & 50 & 50 & 50 & 50 & 50 & 50 \\
\hline Soaking time / days & 3 & 3 & 3 & 3 & 3 & 3 & 3 \\
\hline Sample name & $\begin{array}{l}\text { wt(8-HQ } \\
=3 \%\end{array}$ & $\begin{array}{l}v t(8-H Q \\
=3.5 \%\end{array}$ & $\begin{array}{c}\text { wt(8-HQ) } \\
=5.5 \%\end{array}$ & & & & \\
\hline $\mathrm{V}(\mathrm{EtOH}) / \mathrm{mL}$ & 8.0 & 7.5 & 6.0 & & & & \\
\hline $\mathrm{m}$ (merino wool) / g & 0.1 & 0.1 & 0.1 & & & & \\
\hline V(0.001 M 8-HQ (EtOH)) / mL & 2.0 & 2.5 & 4.0 & & & & \\
\hline Soaking temperature $/{ }^{\circ} \mathrm{C}$ & 50 & 50 & 50 & & & & \\
\hline Soaking time / days & 3 & 3 & 3 & & & & \\
\hline
\end{tabular}

Table A.20: List of 8-HQ - wool fibre composites with different uptake of 8-HQ $\left(\mathrm{H}_{2} \mathrm{O}\right)$ onto wool (see procedure in chapter 2; 2.3.4). Variable process parameter: 8-HQ uptake. Constant process parameters: $\mathrm{m}($ wool $)=0.1 \mathrm{~g}, \mathrm{~V}_{\text {tot }}=10 \mathrm{~mL}$, solvent $=\mathrm{H}_{2} \mathrm{O}$ and soaking temperature $=50{ }^{\circ} \mathrm{C}$ for 7 days.

\begin{tabular}{|c|c|c|c|c|c|c|c|}
\hline Sample name & $\begin{array}{c}\text { wt(8-HQ) } \\
=0.1 \%\end{array}$ & $\begin{array}{c}\text { wt(8-HQ) } \\
=0.2 \%\end{array}$ & $\begin{array}{l}\text { wt(8-HQ) } \\
=0.3 \%\end{array}$ & $\begin{array}{c}\text { wt(8-HQ) } \\
=0.4 \%\end{array}$ & $\begin{array}{c}\text { wt(8-HQ) } \\
=0.6 \%\end{array}$ & $\begin{array}{c}\text { wt(8-HQ) } \\
=0.8 \%\end{array}$ & $\begin{array}{c}\text { wt(8-HQ) } \\
=1 \%\end{array}$ \\
\hline $\mathrm{V}(\mathrm{EtOH}) / \mathrm{mL}$ & 9.9 & 9.75 & 9.5 & 9.25 & 9.0 & 8.75 & 8.5 \\
\hline $\mathrm{m}$ (merino wool) / g & 0.1 & 0.1 & 0.1 & 0.1 & 0.1 & 0.1 & 0.1 \\
\hline $\mathrm{V}\left(0.003 \mathrm{M} 8-\mathrm{HQ}\left(\mathrm{H}_{2} \mathrm{O}\right)\right) / \mathrm{mL}$ & 0.25 & 0.5 & 0.75 & 1.0 & 1.5 & 2.0 & 2.5 \\
\hline Soaking temperature $/{ }^{\circ} \mathrm{C}$ & 50 & 50 & 50 & 50 & 50 & 50 & 50 \\
\hline Soaking time / days & 7 & 7 & 7 & 7 & 7 & 7 & 7 \\
\hline
\end{tabular}


Table A.21: List of 8-HQ - wool fibre composites soaked in $10 \mathrm{~mL} 0.01 \mathrm{M} \mathrm{ZnCl}_{2}$ (EtOH) solutions at $50{ }^{\circ} \mathrm{C}$ for 3 days (see procedure in chapter 2 ; 2.3.6). Variable process parameter: 8-HQ uptake onto wool. Constant process parameters: $\mathrm{m}(\mathrm{wool})=0.1 \mathrm{~g}, \mathrm{~V}_{\text {tot }}=10 \mathrm{~mL}$ and soaking temperature $=$ $50{ }^{\circ} \mathrm{C}$ for 3 days.

\begin{tabular}{|c|c|c|c|c|c|c|}
\hline \multirow{3}{*}{$\overline{1^{\text {st }} \text { batch process }}$} & \multirow{2}{*}{ Sample name } & \multicolumn{5}{|c|}{$w t(8-H Q)=w t(8-H Q)=w t(8-H Q)=w t(8-H Q)=$} \\
\hline & & & $0.09 \%$ & $0.18 \%$ & $0.27 \%$ & $0.36 \%$ \\
\hline & $\mathrm{V}(\mathrm{EtOH}) / \mathrm{mL}$ & & 9.75 & 9.5 & 9.25 & 9 \\
\hline & $\mathrm{m}$ (merino wool) / g & & 0.1 & 0.1 & 0.1 & 0.1 \\
\hline & $\mathrm{V}(0.0025 \mathrm{M}$ 8-HQ (EtOH)) / mL & & 0.25 & 0.5 & 0.75 & 1 \\
\hline & Soaking temperature $/{ }^{\circ} \mathrm{C}$ & & 50 & 50 & 50 & 50 \\
\hline & Soaking time / days & & 3 & 3 & 3 & 3 \\
\hline \multirow[t]{4}{*}{$2^{\text {nd }}$ batch process } & $\mathrm{V}(0.01 \mathrm{M} \mathrm{ZnCl} 2(\mathrm{EtOH})) / \mathrm{mL}$ & & 10 & 10 & 10 & 10 \\
\hline & Soaking temperature $/{ }^{\circ} \mathrm{C}$ & & 50 & 50 & 50 & 50 \\
\hline & Soaking time / days & & 3 & 3 & 3 & 3 \\
\hline & Sample name & $\begin{array}{l}\text { wt(8-HQ) } \\
=0.9 \%\end{array}$ & $\begin{array}{c}\text { wt(8-HQ) } \\
=1.8 \%\end{array}$ & $\begin{array}{l}\text { wt(8-HQ) } \\
=2.7 \%\end{array}$ & $\begin{array}{c}\text { wt(8-HQ) } \\
=3.5 \%\end{array}$ & $\begin{array}{c}\text { wt(8-HQ) } \\
=8.3 \%\end{array}$ \\
\hline \multirow{5}{*}{$1^{\text {st }}$ batch process } & $\mathrm{V}(\mathrm{EtOH}) / \mathrm{mL}$ & 9.75 & 9.5 & 9.25 & 9 & 7.5 \\
\hline & $\mathrm{m}$ (merino wool) / g & 0.1 & 0.1 & 0.1 & 0.1 & 0.1 \\
\hline & $\mathrm{V}(0.025 \mathrm{M}$ 8-HQ (EtOH)) / mL & 0.25 & 0.5 & 0.75 & 1 & 2.5 \\
\hline & Soaking temperature $/{ }^{\circ} \mathrm{C}$ & 50 & 50 & 50 & 50 & 50 \\
\hline & Soaking time / days & 3 & 3 & 3 & 3 & 3 \\
\hline \multirow{3}{*}{$2^{\text {nd }}$ batch process } & $\mathrm{V}\left(0.01 \mathrm{M} \mathrm{ZnCl}{ }_{2}(\mathrm{EtOH})\right) / \mathrm{mL}$ & 10 & 10 & 10 & 10 & 10 \\
\hline & Soaking temperature $/{ }^{\circ} \mathrm{C}$ & 50 & 50 & 50 & 50 & 50 \\
\hline & Soaking time / days & 3 & 3 & 3 & 3 & 3 \\
\hline
\end{tabular}

Table A.22: List of $\mathrm{Zn}$ - wool fibre composites with different zinc precursor materials (see procedure in chapter 2; 2.3.3). Variable process parameters: wool (untreated, NaOH-, HCl-, MSAand L-cysteine pre-treated) and zinc precursor $\left(\mathrm{ZnCl}_{2}(\mathrm{EtOH}), \mathrm{ZnCl}_{2}\left(\mathrm{H}_{2} \mathrm{O}\right)\right.$ and $\left.\mathrm{ZnAc}_{2}\left(\mathrm{H}_{2} \mathrm{O}\right)\right)$. Constant process parameters: $m($ wool $)=0.1 \mathrm{~g}, \mathrm{~V}_{\text {tot }}=10 \mathrm{~mL}, \mathrm{c}\left(\mathrm{Zn}^{2+}\right)=0.01 \mathrm{M}$ and soaking temperature $=50^{\circ} \mathrm{C}$ for 2 days.

\begin{tabular}{lccc}
\hline Sample name & $\begin{array}{c}\mathrm{ZnCl}_{2} \\
(\mathrm{EtOH})\end{array}$ & $\begin{array}{c}\mathrm{ZnCl}_{2} \\
\left(\mathrm{H}_{2} \mathrm{O}\right)\end{array}$ & $\begin{array}{c}\mathrm{Zn}(\mathrm{Ac})_{2} \\
\left(\mathrm{H}_{2} \mathrm{O}\right)\end{array}$ \\
\hline $\mathrm{m}(\text { merino wool })^{*(1)} / \mathrm{g}$ & 0.1 & 0.1 & 0.1 \\
$\mathrm{~V}(0.01 \mathrm{M} \mathrm{ZnCl} 2(\mathrm{EtOH})) / \mathrm{mL}$ & 10 & 0 & 0 \\
$\mathrm{~V}\left(0.01 \mathrm{M} \mathrm{ZnCl}\left(\mathrm{H}_{2} \mathrm{O}\right)\right) / \mathrm{mL}$ & 0 & 10 & 0 \\
$\mathrm{~V}\left(0.01 \mathrm{M} \mathrm{Zn}(\mathrm{Ac})_{2}\left(\mathrm{H}_{2} \mathrm{O}\right)\right) / \mathrm{mL}$ & 0 & 0 & 10 \\
Soaking temperature $/{ }^{\circ} \mathrm{C}$ & 50 & 50 & 50 \\
Soaking time / days & 2 & 2 & 2 \\
*(1): The uptake of zinc onto wool was also carried out onto NaOH-, HCl-, MSA-, and L-cysteine pre- \\
treated wool to maximise the uptake of zinc onto wool.
\end{tabular}


Table A.23: List of $\mathrm{ZnCl}_{2}(\mathrm{EtOH})$ - wool fibre composites soaked in $10 \mathrm{~mL}$ 8-HQ (EtOH) solutions at $50{ }^{\circ} \mathrm{C}$ for 3 days (see procedure in chapter 2; 2.3.5). Variable process parameter: c(8-HQ $(\mathrm{EtOH})$. Constant process parameters: $\mathrm{m}($ wool $)=0.1 \mathrm{~g}, \mathrm{~V}_{\text {tot }}=10 \mathrm{~mL}$ and soaking temperature $=50$ ${ }^{\circ} \mathrm{C}$ for 3 days.

\begin{tabular}{|c|c|c|c|c|c|}
\hline \multirow{2}{*}{$\begin{array}{l}\text { Sample name } \\
\mathrm{V}(\mathrm{EtOH}) / \mathrm{mL}\end{array}$} & \multicolumn{5}{|c|}{$\begin{array}{l}\text { wt(8-HQ) wt(8-HQ) wt(8-HQ) wt(8-HQ) wt(8-HQ) } \\
=0.09 \%=0.18 \%=1.8 \%=2.7 \%=3.5 \%\end{array}$} \\
\hline & 9.75 & 9.5 & 9.5 & 9.25 & 9.0 \\
\hline $\mathrm{m}\left(\mathrm{ZnCl}_{2}(\mathrm{EtOH})-\right.$ wool $^{*^{(1)}} / \mathrm{g}$ & 0.1 & 0.1 & 0.1 & 0.1 & 0.1 \\
\hline $\mathrm{V}(0.0025 \mathrm{M}$ 8-HQ (EtOH)) / mL & 0.25 & 0.5 & 0 & 0 & 0 \\
\hline V(0.025 M 8-HQ (EtOH)) / mL & 0 & 0 & 0.5 & 0.75 & 1.0 \\
\hline Soaking temperature $/{ }^{\circ} \mathrm{C}$ & 50 & 50 & 50 & 50 & 50 \\
\hline Soaking time / days & 3 & 3 & 3 & 3 & 3 \\
\hline
\end{tabular}

${ }^{*}(1)$ : The uptake of zinc onto wool was also carried out onto NaOH-, HCl-, MSA-, and L-cysteine pretreated wool to maximise the uptake of zinc onto wool.

Table A.24: List of $\mathrm{ZnCl}_{2}\left(\mathrm{H}_{2} \mathrm{O}\right)$ - wool fibre composites soaked in $10 \mathrm{~mL}$ 8-HQ (EtOH) solutions at $50{ }^{\circ} \mathrm{C}$ for 3 days (see procedure in chapter 2; 2.3.5). Variable process parameter: c(8-HQ (EtOH). Constant process parameters: $\mathrm{m}($ wool $)=0.1 \mathrm{~g}, \mathrm{~V}_{\text {tot }}=10 \mathrm{~mL}$ and soaking temperature $=50{ }^{\circ} \mathrm{C}$ for 3 days.

\begin{tabular}{|c|c|c|c|c|c|c|}
\hline \multirow{2}{*}{$\frac{\text { Sample name }}{\mathrm{V}(\mathrm{EtOH}) / \mathrm{mL}}$} & \multicolumn{6}{|c|}{$\begin{array}{l}\text { wt(8-HQ) wt(8-HQ) wt(8-HQ) wt(8-HQ) wt(8-HQ) wt(8-HQ) } \\
=0.09 \%=0.18 \%=0.9 \%=1.8 \%=2.7 \%=3.5 \%\end{array}$} \\
\hline & 9.75 & 9.5 & 9.75 & 9.5 & 9.25 & 9.0 \\
\hline $\mathrm{m}\left(\mathrm{ZnCl}_{2}\left(\mathrm{H}_{2} \mathrm{O}\right)-\text { wool }\right)^{\star(1)} / \mathrm{g}$ & 0.1 & 0.1 & 0.1 & 0.1 & 0.1 & 0.1 \\
\hline $\mathrm{V}(0.0025 \mathrm{M}$ 8-HQ (EtOH)) / mL & 0.25 & 0.5 & 0 & 0 & 0 & 0 \\
\hline $\mathrm{V}(0.025 \mathrm{M}$ 8-HQ (EtOH)) / mL & 0 & 0 & 0.25 & 0.5 & 0.75 & 1.0 \\
\hline Soaking temperature $/{ }^{\circ} \mathrm{C}$ & 50 & 50 & 50 & 50 & 50 & 50 \\
\hline Soaking time / days & 3 & 3 & 3 & 3 & 3 & 3 \\
\hline
\end{tabular}

Table A.25: List of $\mathrm{Zn}(\mathrm{Ac})_{2}\left(\mathrm{H}_{2} \mathrm{O}\right)$ - wool fibre composites soaked in $10 \mathrm{~mL}$ 8-HQ (EtOH) solutions at $50{ }^{\circ} \mathrm{C}$ for 3 days (see procedure in chapter 2; 2.3.5). Variable process parameter: c(8-HQ $\left(\right.$ EtOH). Constant process parameters: $\mathrm{m}(\mathrm{wool})=0.1 \mathrm{~g}, \mathrm{~V}_{\text {tot }}=10 \mathrm{~mL}$ and soaking temperature $=$ $50^{\circ} \mathrm{C}$ for 3 days.

\begin{tabular}{|c|c|c|c|c|c|c|c|}
\hline Sample name & $A$ & B & $\mathrm{C}$ & $\mathrm{D}$ & $E$ & $\mathrm{~F}$ & G \\
\hline $\mathrm{V}(\mathrm{EtOH}) / \mathrm{mL}$ & 9.5 & 9.5 & 9.25 & 9.25 & 9.0 & 9.0 & 9.25 \\
\hline$m\left(\mathrm{Zn}(\mathrm{Ac})_{2}\left(\mathrm{H}_{2} \mathrm{O}\right)-\right.$ wool $) / \mathrm{g}$ & 0.1 & 0 & 0.1 & 0 & 0.1 & 0 & 0 \\
\hline$m\left(\mathrm{Zn}(\mathrm{Ac})_{2}\left(\mathrm{H}_{2} \mathrm{O}\right)-\mathrm{MSA}-\text { wool }\right)^{\star(1)} / \mathrm{g}$ & 0 & 0.1 & 0 & 0.1 & 0 & 0.1 & 0 \\
\hline $\mathrm{m}\left(\mathrm{ZnCl}_{2}(\mathrm{EtOH})\right)-$ wool / g & 0 & 0 & 0 & 0 & 0 & 0 & 0.1 \\
\hline V(0.025 M 8-HQ (EtOH)) / mL & 0.5 & 0.5 & 0.75 & 0.75 & 1.0 & 1.0 & 0.75 \\
\hline Soaking temperature $/{ }^{\circ} \mathrm{C}$ & 50 & 50 & 50 & 50 & 50 & 50 & 50 \\
\hline Soaking time / days & 3 & 3 & 3 & 3 & 3 & 3 & 3 \\
\hline
\end{tabular}

${ }^{*(1)}$ : The uptake of zinc onto wool was also carried out onto MSA pre-treated wool to maximise the uptake of zinc onto wool. 